DESIGN OF A HIGH-PRESSURE RESEARCH FLOW LOOP FOR THE

EXPERIMENTAL INVESTIGATION OF LIQUID LOADING IN GAS WELLS

\author{
A Thesis \\ by \\ JUAN JOSE FERNANDEZ ALVAREZ
}

\author{
Submitted to the Office of Graduate Studies of \\ Texas A\&M University \\ in partial fulfillment of the requirements for the degree of \\ MASTER OF SCIENCE
}

December 2008

Major Subject: Petroleum Engineering 


\title{
DESIGN OF A HIGH-PRESSURE RESEARCH FLOW LOOP FOR THE EXPERIMENTAL INVESTIGATION OF LIQUID LOADING IN GAS WELLS
}

\author{
A Thesis \\ by \\ JUAN JOSE FERNANDEZ ALVAREZ
}

\author{
Submitted to the Office of Graduate Studies of \\ Texas A\&M University \\ in partial fulfillment of the requirements for the degree of \\ MASTER OF SCIENCE
}

\begin{abstract}
Approved by:
Co-Chairs of Committee, Gioia Falcone Catalin Teodoriu

Committee Member, Gerald Morrison

Head of Department, Stephen A. Holditch
\end{abstract}

December 2008

Major Subject: Petroleum Engineering 


\begin{abstract}
Design of a High-Pressure Research Flow Loop for the Experimental Investigation of Liquid Loading in Gas Wells. (December 2008)

Juan Jose Fernandez Alvarez, B.S., La Universidad del Zulia

Co-Chairs of Advisory Committee: Dr. Gioia Falcone

Dr. Catalin Teodoriu
\end{abstract}

Liquid loading in producing gas wells is the inability of the produced gas to remove produced liquids from the wellbore. A review of existing flow loops worldwide revealed that specialized areas of research such as liquid loading in gas wells are still lacking dedicated test facilities. This project presents the design of a new dedicated facility to be located at the TowerLab at the Richardson building with adequate operating conditions to reproduce the flow regimes encountered prior to and after the onset of liquid loading in gas wells. The facility consists of a compressed air system, pipelines for air and water, a pressure vessel containing glass beads, an injection manifold, and flow control and monitoring devices.

Our results show that three compressors working in parallel is the most technical and economic configuration for the TowerLab based on the overall costs provided by the supplier, the footprint but most importantly the flexibility. The design of the pressure vessel required a cylindrical body with top and bottom welded-flat head covers with multiple openings to minimize its weight. The pipelines connecting major equipment and injection manifold located at the pressure vessel were selected based on the superficial velocities for air and water. These values also showed the need for independent injection using two manifolds instead of commingling flow through a tee joint. The use of digital pressure gauges with an accuracy of 0.05 to $25 \%$ and coriolis or vortex meters to measure air flowrate is also suggested. For the water line, installation of turbine meters results in the most economic approach. 


\section{DEDICATION}

To my parents, sister and my entire family, for their unlimited support and encouragement throughout this work 


\section{ACKNOWLEDGEMENTS}

I would like to express my sincere gratitude to my advisors Dr. Gioia Falcone and Dr. Catalin Teodoriu for giving me the opportunity to be a part of their team and this project, for their encouragement to learn and to provide meaningful results. Their knowledge and suggestions were fundamental throughout this work.

Thanks also go to the gas well unloading group: He Zhang, Gopi Chava, Meher Surendra, Han-Young Park and Fouad Solomon for being such a great team to work with. Thank you to my very good friends, Jerome Rajnauth and Arnaldo Espinel with whom I shared not only knowledge but most importantly created a good friendship. I would also like to thank the department faculty and staff for making my time at Texas A\&M University a great experience.

Thanks to Dr. Gerald Morrison for serving on my committee. Finally, thanks to the Crisman Institute at the Department of Petroleum Engineering at Texas A\&M University for funding this study. 


\section{TABLE OF CONTENTS}

Page

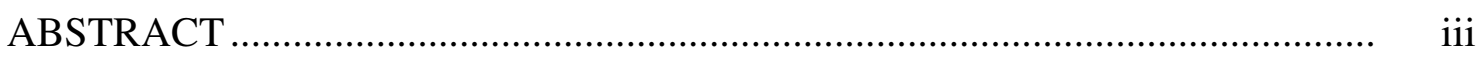

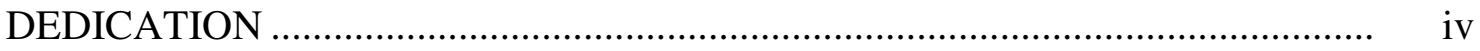

ACKNOWLEDGEMENTS ...............................................................

TABLE OF CONTENTS ...................................................................... vi

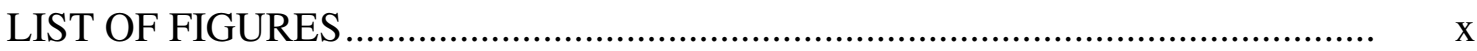

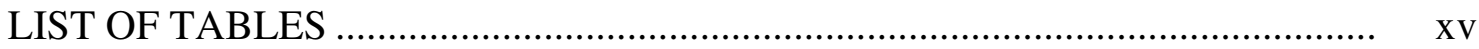

\section{CHAPTER}

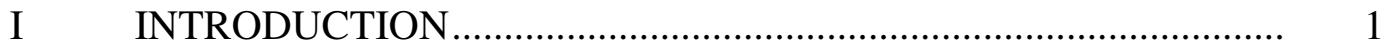

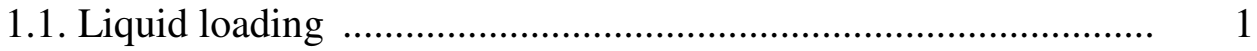

1.2. Problem description............................................................. 5

1.3. Research objectives ....................................................... 6

1.4. Structure of the thesis ............................................................ 7

II $\quad$ LITERATURE REVIEW ........................................................ 8

2.1. Flow loops around the world - Technical specifications and equipment aspects

2.1.1. The Foundation for Scientific and Industrial Research at the Norwegian Institute of Technology - SINTEF........... 8

2.1.2. Institute for Energy and Technology - IFE ...................... 12

2.1.3. Norsk Hydro multiphase flow loop............................... 17

2.1.4. Southwestern Research Institute - SwRI.......................... 22

2.1.5. NEL wet gas flow loop ............................................. 24

2.1.6. Colorado Engineering Experimental Station Inc-CEESI.. 27

2.1.6.1. Wet gas metering facility................................. 27

2.1.6.2. Multiphase flow loop .......................................... 29

2.1.7. K-Lab ..................................................................... 30

2.1.8. Boussens flow loop ..................................................... 32

2.1.9. IFP multiphase flow loop ........................................... 35

2.1.10. The LOTUS rig ..................................................... 36

2.1.11. TAMU TowerLab ........................................................ 39

2.2. Summary and comparison of hardware and major equipment

of existing multiphase research flow loops ............................... 40 
2.3. Typical operating conditions in gas fields experiencing liquid loading

2.4. The reality of liquid loading reproduced in a laboratory environment...

2.5. A dedicated research flow loop for liquid loading investigation ..

III DESIGN OF A NEW DEDICATED FACILITY FOR THE INVESTIGATION OF LIQUID LOADING IN GAS WELLS

3.1. Design factors for mimicking liquid loading at TAMU TowerLab 52

3.2. Defined range in operating conditions for TAMU TowerLab .... 53

3.3. Design options for the boosting system ................................. 54

3.3.1. Multiphase pump ..................................................... 54

3.3.2. Compressor and water pump ..................................... 56

3.3.3. Compressor - water pump - tank ............................. 58

3.4. Technical analysis of the compressor - water pump- tank ......... 59

3.5. Facility design ................................................................... 66

3.5.1. Pressure vessel ….................................................. 66

3.5.1.1. Design pressure and temperature ..................... 67

3.5.1.2. Selection of construction materials .................... 67

3.5.1.3. Non-corrosive service ..................................... 68

3.5.1.4. Corrosion allowance ....................................... 69

3.5.1.5. Joint efficiencies .............................................. 69

3.5.1.6. ASME Code, Section VIII, Division 1 vs. 2 ....... 70

3.5.1.7. Pressure vessel configuration ............................ 71

3.5.1.8. Shell thickness ................................................... 74

3.5.1.9. Bottom flat head cover ...................................... 77

3.5.1.10. Nozzles ....................................................... 79

3.5.1.10.1. Nozzles attached to the shell ........... 81

3.5.1.10.2. Flange-pipe attachment in nozzles ... 83

3.5.1.11. Flange attached to shell body ......................... 85

3.5.1.12. Gasket facing details and bolt calculations ....... $\quad 86$

3.5.1.12.1. Gasket selection ............................. 86

3.5.1.12.2. Bolt sizing and material selection .... 88

3.5.1.13. Top cover head ........................................... $\quad 90$

3.5.1.14. Nozzle attached to flat head ........................... 92

3.5.1.15. Static seals for nozzle-tubing assembly ............ 92

3.5.1.16. Weight of top flat head and alternatives ............ 94

3.5.1.16.1. Openings with bolted covers ........... 94

3.5.1.16.2. Additional options for weight reduction ......................................... 96

3.5.1.17. Vessel support ............................................ 97 
3.5.1.17.1. Support-leg columns

3.5.1.17.2. Base plates and bolts ..................... 106

3.5.1.18. Lifting lugs ............................................... 107

3.5.2. Design of the compressed air system ........................... 108

3.5.2.1. Technical considerations and compressed air needs for the TowerLab .................................... 109

3.5.2.1.1. Air quality ........................................ 110

3.5.2.1.2. Establishing compressor capacity ....... 113

3.5.2.1.3. Load pattern demand ......................... 114

3.5.2.1.4. Required pressure ........................... 115

3.5.2.1.5. Location ........................................ 116

3.5.2.1.6. Packaged compressors ...................... 120

3.5.2.1.7. Inlet and discharge piping ................. 120

3.5.2.1.8. Distribution system ........................... 120

3.5.2.1.9. Compressor selection ....................... 122

3.5.2.1.9.1. Reciprocating …............. 126

3.5.2.1.9.2. Lubricated screw ............ 128

3.5.2.1.9.3. Lubricated-free screw....... 130

3.5.2.1.9.4. Centrifugal ..................... 132

3.5.2.1.10. Advantages and disadvantages ........ 134

3.5.2.1.11. Compressors commercially available 135

3.5.3. Injection manifold and piping design ........................... 145

3.5.3.1. Injection manifold ....................................... 145

3.5.3.1.1. Internal pipe diameter ........................ 147

3.5.3.2. Pressure drop in manifold ............................... 154

3.5.3.3. Piping system ............................................... 161

3.5.3.3.1. Liquid line ..................................... 162

3.5.3.3.2. Air line ........................................ 162

3.5.3.3.3. Pipe joints ...................................... 163

3.5.3.3.4. Pipe support and hangers .................. 165

IV MONITORING AND INSTRUMENTATION ........................... 167

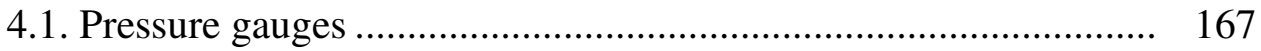

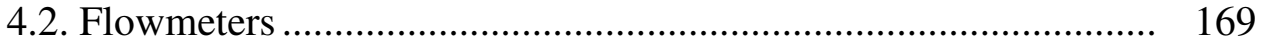

V CONCLUSIONS AND RECOMMENDATIONS ........................ 172

5.1. Conclusions ................................................................ 172

5.2. Recommendations ............................................................. 173

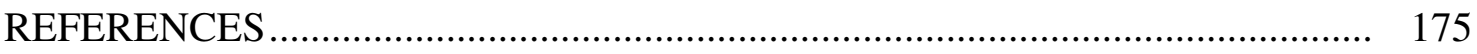




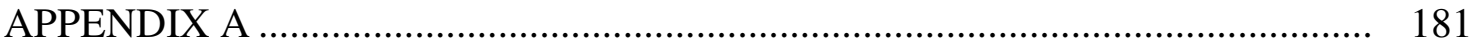

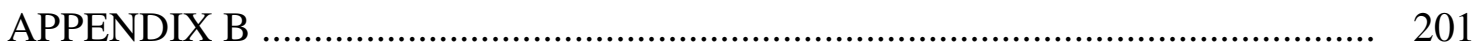

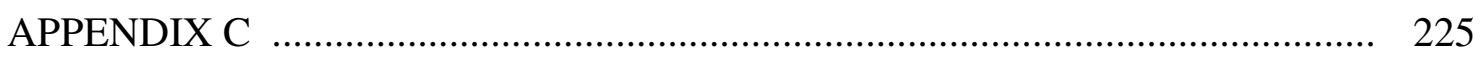

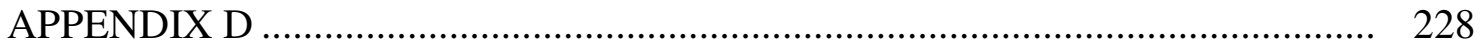

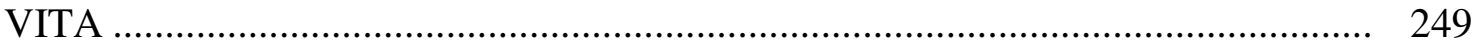




\section{LIST OF FIGURES}

FIGURE Page

1.1 a) $1^{\text {st }}$ stage, b) $2^{\text {nd }}$ stage, c) $3^{\text {rd }}$ stage and d) $4^{\text {th }}$ and final stage.................. 2

2.1 SINTEF multiphase flow loop and plant layout.................................... 9

2.2 Schematic drawing of IFE's multiphase flow loop ............................. 12

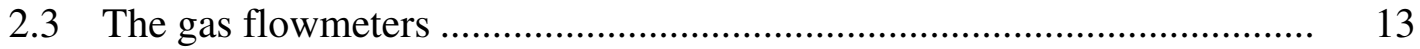

2.4 a) The gas/compressor pump (multiphase pump) and b) behind left: large dosage pump; front left: water pump; green pump: small dosage pumps, front right: oil pump ........................................................ 15

2.5 (a) The optical acrylic and (b) inlet mixing section .............................. 16

2.6 (a) Slug catcher at the outlet of the test section and (b) gas/liquid (top) and oil/water separators ..................................................................... 16

2.7 The tiltable section of the flow loop in the horizontal position................. 18

2.8 Main parts of the multiphase flow loop ............................................. 19

2.9 The 3 in flow loop and the choke test section .................................... 20

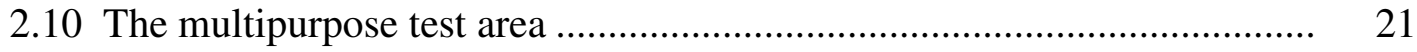

2.11 Range of possible test conditions .................................................... 23

2.12 Schematic diagram of NEL wet gas loop ............................................ 25

2.13 Simplified block diagram of major components .................................. 27

2.14 Schematic of K-Lab test loop......................................................... 30

2.15 Flow circulation diagram ......................................................... 32

2.16 Supporting and hoisting framework for the 6 in test pipe ........................ 34

2.17 IFP multiphase flow loop layout ..................................................... 35

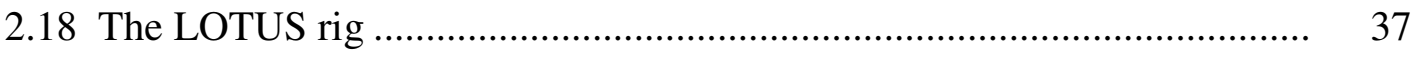

2.19 Schematic layout of the proposed modification of the LOTUS rig .......... 38

2.20 TowerLab stretches through 10 floors of the Richardson building, beginning in the basement under the first floor shown here 
FIGURE Page

2.21 Vertical height for different flow loops.............................................. 41

2.22 Maximum gas flowrate for different flow loops ................................ 41

2.23 Vertical length vs. pressure .............................................................. 42

2.24 Vertical length vs. maximum gas rate ................................................... 42

2.25 Depth distribution of United States gas wells ...................................... 44

2.26 Production tubing sizes distribution in reported cases within the US ....... 45

2.27 Three commonly used well configurations in South Texas .................... 46

2.28 Vertical flow regimes during liquid loading ...................................... 47

2.29 Downscaling of a gas well from at the TowerLab ................................ 49

2.30 Pressure vessel to recreate effects of the near-wellbore region................ 50

2.31 Distribution mechanism ............................................................... 50

3.1 Location of required operating conditions ....................................... 54

3.2 Case No.1 will include flow from the water and air supply through a multiphase pump to the tubing ........................................................ 55

3.3 Bornemann multiphase pump would provide additional boosting pressure prior to entering the injection manifold ................................... 56

3.4 In the compressor - water pump system, fluids will flow directly from the inlet valves to the tubing with no additional pressure ............... 57

3.5 In the tank configuration, air and water will flow directly through two different flow lines when leaving the air and water container ................. 58

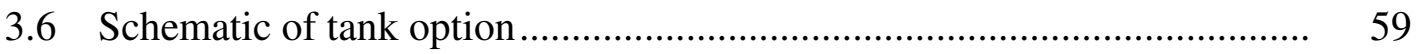

3.7 Final volume of air and water inside the tank after compression vs. initial air pressure on the supply side ................................................... 64

3.8 Pressure drawdown with time .......................................................... 65

3.9 Principal loads on the pressure vessel ............................................... 66

3.10 (a) Basement at Richardson building and (b) location of pressure vessel . 72

3.11 TowerLab main dimensions from $2^{\text {nd }}$ to $10^{\text {th }}$ floor................................. 72

3.12 Proposed location of pressure vessel in the TowerLab .......................... 73 
FIGURE Page

3.13 Pressure vessel expected appearance .............................................. 74

3.14 Option of torispherical head at bottom .......................................... 78

3.15 Acceptable types of welded nozzles to shells and heads ....................... 79

3.16 Areas available for reinforcement in openings .................................... 80

3.17 Radial flow to be expected from the eight nozzles .............................. 81

3.18 Full and raised-face flanges......................................................... 83

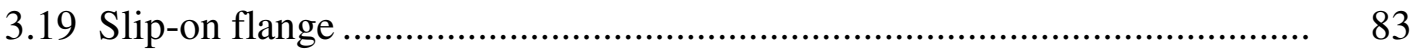

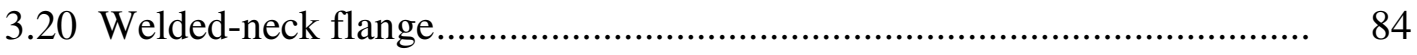

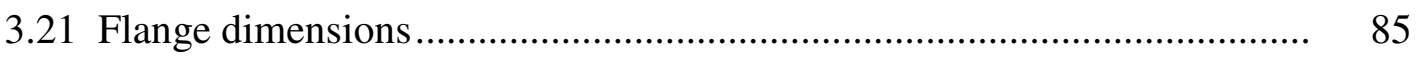

3.22 Temperature-pressure guidelines for common gasket materials .............. 87

3.23 Flange configuration ................................................................... 90

3.24 Dimensional data and forces for a blind flange................................... 91

3.25 Sealing between nozzle and tubing on top cover ................................. 93

3.26 Extrusion gap limits for two different hardness materials ....................... 94

3.27 (a) Original design compared to (b) alternative option .......................... 95

3.28 Overall weight of pressure vessel according to different design options... 97

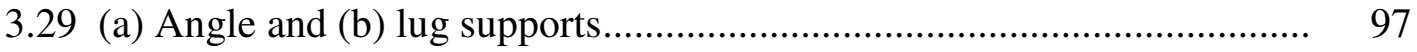

3.30 Weld configuration of support legs ................................................ 99

3.31 Top and lateral view of vessel ..................................................... 100

3.32 Equivalent static force for dynamic flow ........................................... 100

3.33 Distribution of lateral forces on columns ......................................... 103

3.34 Leg configuration for horizontal force distribution............................. 103

3.35 Angle parameters ............................................................................. 106

3.36 Lug design for lifting purposes .................................................... 108

3.37 Compressed air system and its components ...................................... 109

3.38 Examples of using multiple compressors to meet different demands ....... 114

3.39 Pressure profile for a system ........................................................ 116

3.40 Possible location for compressor.................................................. 117 
FIGURE Page

3.41 Logistics for compressor delivery .................................................. 118

3.42 Opening located outside the Richardson building...................................... 119

3.43 Proposed location of compressed air package .......................................... 119

3.44 Basic compressor types .................................................................. 122

3.45 Screening of compressor types.............................................................. 124

3.46 Compressor operating ranges ................................................................ 125

3.47 Proposed solutions........................................................................... 125

3.48 Basic principle of operation of reciprocating compressors ......................... 126

3.49 (a) Single and (b) double acting reciprocating compressors ..................... 127

3.50 Single-stage oil-injected screw compressor ............................................... 129

3.51 Two-stage lubricated-free screw compressor........................................... 131

3.52 Centrifugal compressor ……............................................................... 132

3.53 Screening of compressor manufacturers ………...................................... 135

3.54 Kaeser Compressors ........................................................................... 136

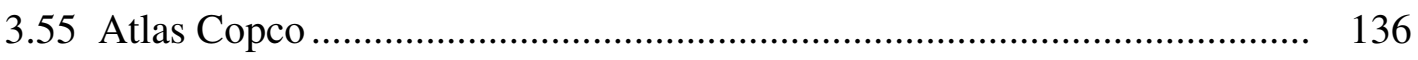

3.56 Cameron Compressor ………………………..................................... 137

3.57 Sullair Compressors .......................................................................... 137

3.58 Ingersoll Rand ............................................................................... 138

3.59 CompAir Compressors …………................................................ 138

3.60 Discharge pressure and air flowrate range according to Solomon et al. ${ }^{16}$ The green square groups values of pressure at the discharge of the compressor whereas the red square groups the air flowrate...................... 139

3.61 Initial and running costs for the first year ............................................... 140

3.62 Running costs for five years of operation ................................................ 140

3.63 Capacity and power for each option........................................................ 141

3.64 Compressor costs over a ten-year life period ............................................ 141

3.65 Ranking of options based on different criteria .......................................... 143

3.66 Kepner-Tregoe decision analysis results .................................................... 144 
FIGURE Page

3.67 Analytic Hierarchy Process results ............................................... 144

3.68 Initial configuration of injection manifold ....................................... 145

3.69 Problems with initial design ...................................................... 145

3.70 Proposed solutions for manifold design .............................................. 146

3.71 Recommended manifold designs .................................................. 147

3.72 Red area represents the region covered by the pressure and flowrate range and the blue points inside define the internal diameter range ........ 148

3.73 Independent injection manifolds for two configurations ........................ 149

3.74 Moody friction factor …......................................................... 156

3.75 Pressure drop as a function of pipe length (water) ............................ 156

3.76 Compressed air system pressure drop due to friction ............................ 159

3.77 Schematic of piping system......................................................... 161

3.78 Water supply system ................................................................ 162

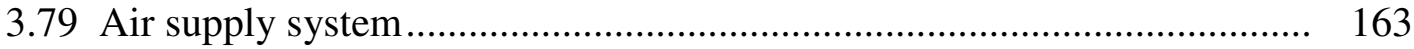

3.80 Typical welded joints. (a) Butt weld; (b) socket weld ........................... 164

3.81 Flanged joint ................................................................................. 164

3.82 Typical structure attachments. (a) Insert; (b) beam clamp; (c) bracket..... 165

3.83 Typical pipe hangers. (a) Adjustable band hanger; (b) adjustable clevis hanger; (c) long tangent U-bolt and (d) saddles ................................. 166

4.1 Range in price as a function of accuracy.......................................... 168

4.2 Installation of model DPG7000 on pressure vessel ............................. 169 


\section{LIST OF TABLES}

TABLE Page

2.1 Operating parameters at SINTEF .................................................... 10

2.2 Instrument summary list................................................................. 11

2.3 General specifications of the flow loop............................................ 13

2.4 Overview of process and scientific instruments.................................. 14

2.5 Overview of pumps ......................................................................... 15

2.6 Multiphase flow loop main characteristics......................................... 18

2.7 Main control instrumentation ....................................................... 22

2.8 Characteristics of SwRI multiphase flow looop.................................... 23

2.9 Wet gas test facility specifications .................................................. 26

2.10 Gas velocities and GVF capacity for 2 in. schedule 80 pipe................... 29

2.11 Gas velocities and GVF capacity for 4 in. schedule 80 pipe................... 30

$2.12 \mathrm{~K}-\mathrm{Lab}$ operating specifications ...................................................... 31

2.13 Major equipment for liquid injection and gas compression .................... 40

3.1 Design factors .......................................................................... 52

3.2 Technical specifications ............................................................ 56

3.3 Results for different values of pressure ............................................... 64

3.4 Material selection guide ............................................................. 68

3.5 Mechanical properties ......................................................................... 69

3.6 Application of joint efficiencies ................................................. 70

3.7 Shell thicknesses ......................................................................... 76

3.8 MAWP, MAP, HTP ............................................................................ 77

3.9 Thickness and MAWP, MAP, HTP for bottom flat head cover .............. 77

3.10 Thickness and MAWP, MAP, HTP for torispherical head option ............ 78

3.11 Pipe properties and dimensions ....................................................... 82

3.12 Nozzle neck thickness calculations and pressure limitations ................... 82 
TABLE Page

3.13 Configuration of the nozzle ........................................................... 82

3.14 Flange properties .................................................................. 84

3.15 Gasket characteristics ................................................................ 86

3.16 Bolt cross-sectional area required and sizing information ...................... 89

3.17 Nozzle neck thickness calculations and pressure limitations ................... 92

3.18 Configuration of the nozzle ........................................................... 92

3.19 Bolt details for upper openings ........................................................ 95

3.20 Bolt details for bottom opening .................................................. 95

3.21 Configurations for weight reduction ............................................... 96

3.22 Advantages and disadvantages between angle and lug support............... 98

3.23 Support leg recommendations ......................................................... 98

3.24 Column parameters .................................................................. 104

3.25 Stresses to determine weld sizes ................................................... 105

3.26 Angle main dimensions ............................................................... 107

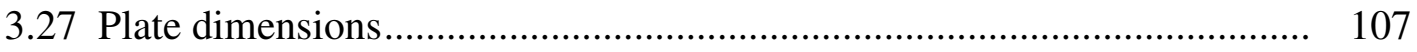

$3.28 \mathrm{Lug}$ data for pressure vessel ........................................................ 108

3.29 Types of air quality .................................................................. 110

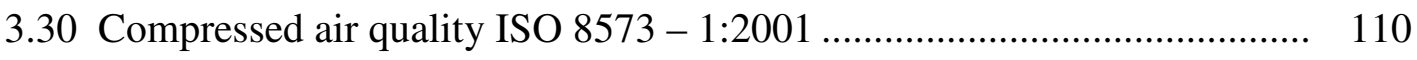

3.31 Advantages and disadvantages of non-lubricated compressors ............... 111

3.32 Industry classification for air quality for common applications................ 112

3.33 Compressor operating limits ....................................................... 123

3.34 Operating conditions for compressor selection ................................ 123

3.35 Compressor characteristics ............................................................ 126

3.36 Advantages and disadvantages of each type of compressor ................... 134

3.37 Energy costs ........................................................................ 142

3.38 Pipe internal diameter range for air and water ................................... 148

3.39 Thread allowance for pipe wall thickness calculation for ANSI B31.3 .... 151

3.40 Basic allowance stress for grade B seamless pipe, psi ......................... 151 
TABLE Page

3.41 Data for different pipe sizes ........................................................ 151

3.42 Design factor, F ................................................................... 152

3.43 Temperature derating factor, T................................................... 152

3.44 Results in wall thickness using ANSI B31.3 and ANSI B31.8 ............... 153

3.45 Pipe internal roughness .............................................................. 155

3.46 Major pressure drops in manifold ............................................... 157

3.47 Pressure drop due to fittings ........................................................... 157

3.48 Pressure drop in manifold for air flow ................................................. 160

3.49 Pressure drop due to fittings ......................................................... 160

3.50 Recommended rod size for individual pipes ........................................ 165

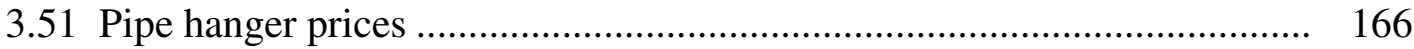

4.1 Location and quantity of pressure gauges for TAMU TowerLab ........... 167

4.2 Comparison of different pressure gauges ........................................... 168

4.3 Comparison between different flowmeter elements............................. 167 


\section{CHAPTER I INTRODUCTION}

Natural gas is one of the principle sources of energy for many of our day-to-day needs and activities. It is a vital component of the world's supply of energy being one of the cleanest, safest, and most useful of all energy sources. Due to the strong domestic and international demand, it has become commercially attractive since the 1950s. ${ }^{1}$ However, the deliverability from existing gas reservoirs continues to decline and the scarcity of promising prospects has increased the need to maximize gas recovery from every gas well.

As natural gas is produced from a reservoir, the simultaneous flow of gas, liquid hydrocarbons and water is a common situation for both land and offshore production systems. $^{2}$ In vertical wells, water and intermediate hydrocarbons can condense in liquids in the wellbore depending on the current composition of the gas during production. As long as the gas flow rate is sufficiently high to maintain annular mist flow, liquids can then be lifted and carried out from the well.

Liquid loading limits current productivity of $90 \%$ of the natural gas wells in the USA. $^{3}$ Removal of water and hydrocarbon liquids from gas wells is increasingly recognized as an important topic for mature gas reservoirs where the continuous decline in reservoir pressure not only triggers the alteration of the flow regime, switching the annular mist flow to churn flow, but also dramatically decreases the liquid lifting capacity of the flowing gas.

\subsection{Liquid loading}

We consider a well to be liquid loaded when the fraction by volume of liquids present in the gas flow path is higher than would be present in a mist flow situation. ${ }^{4}$ As natural gas is produced from depletion-drive reservoirs, the energy available to remove the produced-accumulated liquids, condensates and formation water to the surface

This thesis follows the style of SPE Journal. 
declines. This energy eventually becomes low enough that flow rates decrease below a certain critical velocity, and fluids produced with the gas flow stream begin to fall back no longer being carried to the surface. In cases where the flow stream is not sufficient to continuously transport the well liquids upward in the wellbore, a wellbore liquid loading condition begins.

It is conceived that generally this process occurs in such a way that some of the liquid in the flow stream coalesces along the conduit wall and moves downward as a liquid film. ${ }^{5}$ Initially, liquids start to accumulate in the casing below the end of the tubing where the cross-sectional area is relatively larger, and the velocities are correspondingly smaller than in the tubing. The continuous accumulation of liquids results in an increase in hydrostatic back-pressure on the reservoir, flowing bottom hole pressure (FBHP) and multiphase flow instability and flow regime changes.

In most cases, if this situation is not monitored or controlled, the wellbore will accumulate sufficient fluids to balance and overcome the available reservoir energy completely, dramatically inhibiting or stopping gas production. ${ }^{6}$ Furthermore, the economic impact attributed to abandoned gas wells include and is not limited to lost reserves during competitive situations, reduced ultimate recovery estimates and most importantly reduced cash flow and profitability. The process of liquid loading as described by Neves et al. ${ }^{7}$ can be summarized in four stages:

1) After the well has been completed and production begins, a gas well has enough energy, due to high initial reservoir pressure and gas flow rate, to carry the liquids all the way to the surface (Fig. 1.1(a)). At this stage the gas velocity is greater than or equal to the critical velocity required to continuously remove the liquids in the gas stream.

2) As production continues, natural reservoir pressure declines producing a decrease in gas flow rate which directly induces a decrease in gas velocity until reaching and falling down the critical gas velocity value. Consequently, liquid droplets suspended in the gaseous phase will begin to move downward and start accumulating at the 
wellbore restricting the effective flow area for the gas impeding its continuous production (Fig. 1.1(b)).

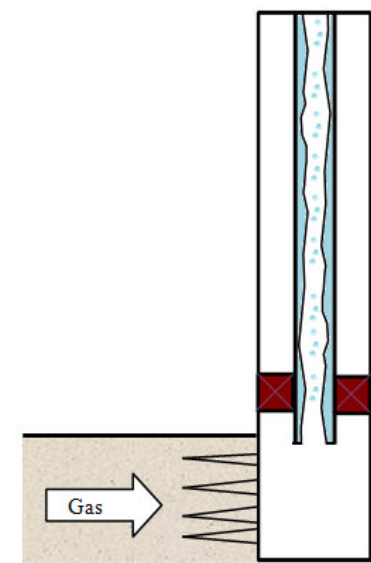

(a)

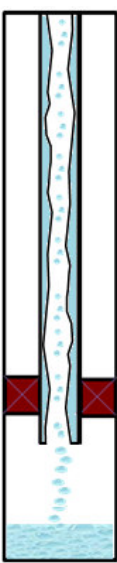

(b)

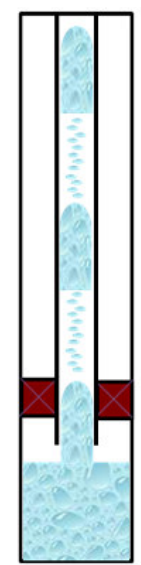

(c)

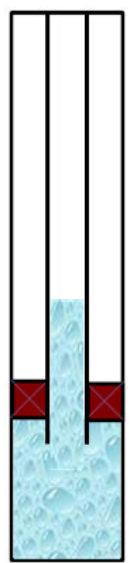

(d)

Fig 1.1- (a) $1^{\text {st }}$ stage, (b) $2^{\text {nd }}$ stage, (c) $3^{\text {rd }}$ stage and (d) $4^{\text {th }}$ and final stage

3) Followed by the accumulation of the liquids, is an initial increase in gas flow rate because of the reduction of the effective area for the gas phase to flow which results in a larger pressure drop across the accumulated liquids downhole. The pressure drop increases until the downstream pressure reaches the pressure necessary to transport the liquids up the tubing (Fig. 1.1 (c)).

4) A well cycles back and forth between the last two stages; however the time differential between produced liquid slugs at the surface become greater as a consequence of the time required by the reservoir to reach a pressure high enough to blow the liquid slugs up the string. Eventually, the backpressure at the sandface originated by the liquids that have accumulated at the bottom overcome the available reservoir energy causing the well to load up and die (Fig. 1.1 (d)).

A well that produces at subcritical velocities may load up and cease flowing under its own potential due to an increase in the liquid accumulation in the wellbore. As demonstrated by Sutton et al. ${ }^{8}$ and Dousi et al. ${ }^{9}$ liquid loading is not always identifiable because as loading occurs, the well may still produce for a significant period of time at subcritical velocities since reaching and moving below this fixed value does not 
necessarily mean a sudden end of production. However, once liquid loading has been recognized, the best approach is to attack the problem immediately.

Liquid loading is not only attributed to low energy reservoirs alone, but exists in prolific reservoirs with high gas liquid ratios (GLR). It can be critical in tight gas formations and may even be induced early in the integrated gas well production system by poor well planning design and completion methods.

Today, the aspect of liquid loading is emphasized depending on the discipline. For the production engineer there is an interest in developing a methodical approach for the selection of a suitable deliquification method as well as the timing of the intervention at a given point in the well's depletion.

Bondurat et al. ${ }^{10}$ discuss the recent developments in the research area of well deliquification techniques. Lea and Nickens ${ }^{4}$ and Stephenson et al. ${ }^{11}$ describe the wellestablished techniques to alleviate the effects of liquid loading. Foaming the liquid water can enable the gas to lift water from the well. Using smaller tubing or creating a lower wellhead pressure sometimes can keep mist flow. The well can be unloaded by gas lifting or pumping the liquids out of the well. Heating the wellbore can prevent liquid condensation. Downhole injection of water into an underlying disposal zone could be another option. Yamamoto et al. ${ }^{1}$ recognizes that each of these technologies has its niche, but deciding the most optimum strategy depends on a collection of factors including composition of reservoir fluid, operating pressures and temperatures, and of course economics.

Liquid loading is not always obvious and recognizing its presence is not necessarily an easy task. Symptoms indicating liquid loading as discussed by Lea and Nickens ${ }^{4}$ include: presence of recorded pressure spikes through gas measuring devices, erratic production and increase in decline rate, tubing pressure decreases, pressure surveys showing a sharp distinct change in pressure gradient, annular heading and the sudden cease in liquid production.

Loading up of liquid in the wellbore has been recognized as one of the most severe problems in gas production. Accurate prediction is of critical importance to 
timely take the most optimum approach to deal with this situation. Although several investigators ${ }^{6,9,12,13,14,15,16}$ have suggested methods to predict liquid loading, results from these models often show discrepancies and in some cases they are not easy to use because of the difficulties they involve.

\subsection{Problem description}

Liquid loading is a problem that has been present in the oil and gas business for a long time. Previous work by Guo et al. ${ }^{12}$, Turner et al. ${ }^{13}$, Coleman et al. ${ }^{14}$, and Nosseir et $a l{ }^{15}$ consisted of experimental efforts that provided empirical correlations to evaluate the possible initiation of loading in gas wells.

Standard models used to predict and analyze liquid loading in gas wells are only applicable to steady-state flow; however, liquid loading corresponds to unsteady-state flow conditions, both in the well and in the near-wellbore region of the reservoir.

A more reliable approach would be to use a transient multiphase flow wellbore model that accounts for the dynamics in the near-wellbore region via transient boundary conditions. A dynamic, integrated (reservoir/wellbore) system modeling approach would predict conditions for transition from an acceptable production flow regime (annular or mist flow) to an unacceptable regime (churn flow) that triggers liquid loading in the wellbore. ${ }^{17}$ Achieving these objectives, requires rigorous empirical studies of transient multiphase flow under laboratory conditions that attempt to mimic well behavior under liquid loading.

However, reproducing an integrated reservoir/wellbore system in a laboratory environment implies downscaling of the system's geometry and flow parameters. Given the wide range of possible operating conditions in the field, no flow loop can be representative of all possible situations. Even when experiments in a given flow loop are believed to be sufficiently exhaustive for a specific study area, the conditions encountered in real life applications can be different from those recreated in the research facility.

A review of existing flow loops worldwide has revealed that some specialized areas of research such as liquid loading in gas wells are still lacking dedicated test 
facilities. ${ }^{18}$ Having an experimental facility with adequate vertical height and diameter of the test section to reproduce the flow regimes encountered prior to and after onset of liquid loading in gas wells is necessary to conduct experimental studies to mimic the dynamic boundary conditions that exist between the reservoir and wellbore during liquid loading. This will require a compression and boosting system capable of circulating fluids at flow rates, pressures and high gas volume fractions (GVF) similar to real gasfield production situations, as well as, technology and instrumentation for controlling and monitoring key two-phase flow parameters such as pressure, temperature, and flow rates among others.

Additionally, the facility should allow the creation of the flow resistance represented by the reservoir, for which the common practice has been to use a series of valves to simulate permeability variations. Nevertheless, flow from a real reservoir to the wellbore goes through a porous medium and in the case of multiphase flow, relative permeability must be taken into consideration.

To recreate the effect of the near-wellbore region, an experimental option would be to attach a cylindrical pressure vessel containing tightly packed spherical glass beads to the base of a vertical multiphase flow loop. Flow of fluids into the porous medium may be provided by entry points symmetrically aligned at the pressure vessel using an injection manifold with adequate geometry to ensure proper dispersion of the fluids.

\subsection{Research objectives}

The main objective of this study is to design a high-pressure research flow loop for the experimental investigation of liquid loading problems in gas wells. Further objectives involve:

1. Technical and economic evaluation of different compression and pumping systems for the optimum selection of the equipment configuration capable of delivering the operating conditions required to run the experiments.

2. Design of all the pipe work necessary to circulate the fluids from major equipment to delivery points at the pressure vessel, including single or multiple injection manifolds. 
3. Design of the pressure vessel that will include the porous medium used to mimic the near-wellbore region of the reservoir which will be connected to the bottom of the vertical test section.

4. Selection and recommendation of the appropriate instrumentation to monitor key performance indicators for the compression and pumping system.

5. Elaboration of layout drawings of the entire system including the parts to be manufactured.

\subsection{Structure of the thesis}

In CHAPTER II state-of-the-art multiphase flow research facilities are reviewed emphasizing the available ranges of operating conditions, hardware used to circulate fluids and instrumentation to monitor and gather data. This review further identifies a niche for the design of a dedicated facility operating in a region not yet covered by current existing flow loops worldwide. Typical operating conditions in gas field as a function of pressure, flow rates and geometry are described leading to recognition of a need for downscaling to address the reality of liquid loading reproduced in a laboratory environment.

CHAPTER III describes the design of a new dedicated facility for the investigation of liquid loading in gas wells. The range of working pressures and flow rates for the flow loop is defined and the evaluation of alternative configurations for the compression and boosting system are carried out. Selection of major equipment and design of mechanical elements are explained in detail.

CHAPTER IV summarizes the selection and recommendation of key instrumentation devices such as pressure gauges and flowmeters including a comparison table between different manufacturers and equipment performance. Final recommendations on flow control devices are also mentioned.

In CHAPTER V, conclusions are drawn for the work described in this thesis and recommendations made for future work. 


\section{CHAPTER II}

\section{LITERATURE REVIEW}

Downscaling of petroleum systems to a laboratory environment requires establishing a fixed envelope of operating conditions that allows experimental work to be performed to study phenomena related to a specific area of research. In the case of multiphase research flow loops, the process is accompanied by the installation of major equipment and hardware that may include but is not limited to compressed air systems, water pumps, multiphase pumps and static vessels used as separators.

Commercial and non-commercial research flow loops operate around the world addressing multiple problems within the oil and gas industry. Different configurations can be found; however, the equipment and hardware used to provide the testing fluid into the flow loop tend not to vary significantly. This investigation presents a survey of facilities with focus on oil and gas multiphase flow to identify technical specifications of the equipment commonly used in a flow loop, and the main characteristics of each installation.

\subsection{Flow loops around the world - Technical specifications and equipment aspects}

\subsubsection{The Foundation for Scientific and Industrial Research at the Norwegian Institute of Technology - SINTEF.}

SINTEF's multiphase flow laboratory located in Trondheim, Norway, was established in 1982. Currently, it includes a small high pressure flow loop for hydrate analyses, a medium scale flow loop, the flow wheel laboratory and a large scale flow loop.

The main activity at SINTEF is to provide facilities for the experimental research of multiphase flow and flow-assurance related issues. Results from different studies have contributed to the development of multiphase flow simulators being the latter an ongoing activity. Furthermore, this facility is also suited for testing both process equipment and instrumentation prior their employment in the field. 
The large scale multiphase flow loop is an industrial scale facility with explosion control (see Fig. 2.1). The main features are a 1,640 ft long horizontal test section that ends in a $197 \mathrm{ft}$ high riser tower. The pipe diameter is 8 in. with additional 4 in. and 12 in. diameter lines. A $164 \mathrm{ft}$ long bent section is inserted in the horizontal line starting at $460 \mathrm{ft}$ from the mixing point with the purpose of simulating terrain effects. The pipe slopes $5^{\circ}$ from the main axis ( $82 \mathrm{ft} 5^{\circ}$ outward, $82 \mathrm{ft} 5^{\circ}$ inward) and it may be rotated to generate a low point, a horizontal line, or a high point. In addition, the last $230 \mathrm{ft}$ upstream of the riser inlet, starting at $1,083 \mathrm{ft}$ from the mixing point, may be dipped $1^{\circ}$ or $2^{\mathbf{o}}$ down to introduce terrain slugging in the riser. ${ }^{19}$ All parts are made of carbon steel.
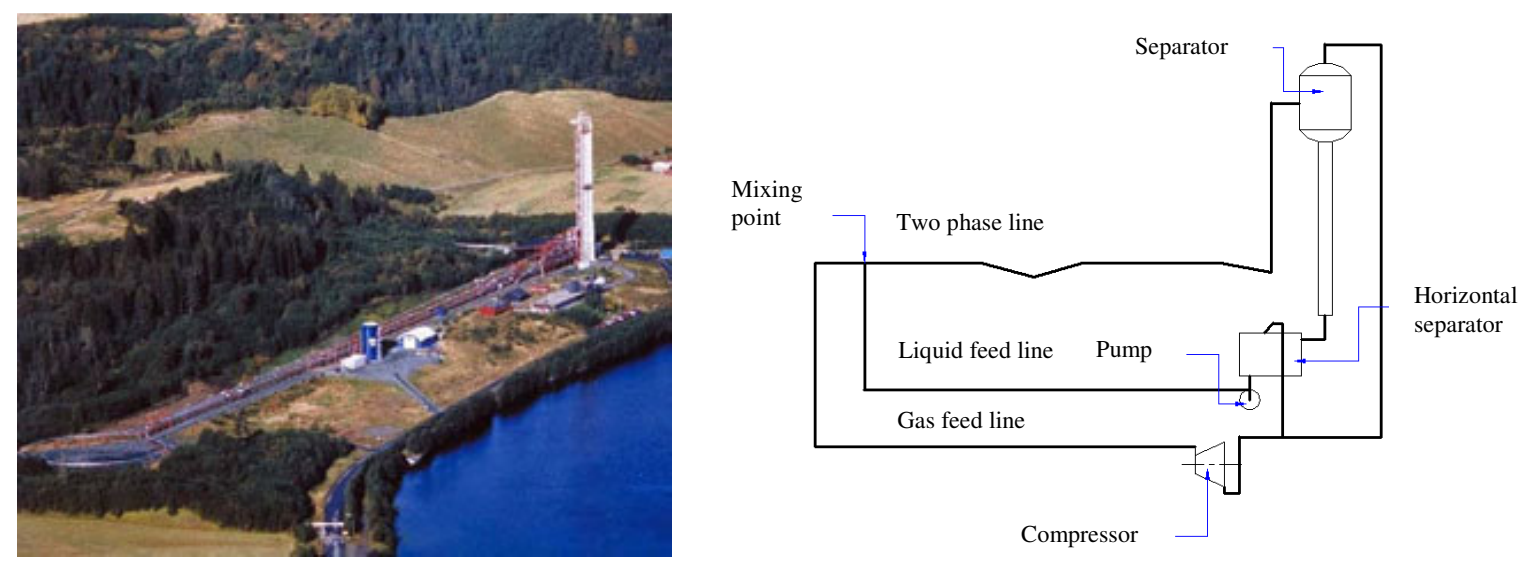

Fig. 2.1- SINTEF multiphase flow loop and plant layout ${ }^{19,20}$

The two-phase flow from the riser enters the top separator where the initial gasliquid separation takes place. The liquid then falls through a drop leg to the ground level where the horizontal separator is placed; consequently the residual gas is released at this point and the liquid is pumped back to the mixing point.

There are three centrifugal pumps working in parallel handling a total liquid rate of 1,980 gpm with total power input of $443 \mathrm{hp}$. The gas flows through a vertical scrubber at the compressor suction where it is recompressed prior entering the mixing point. The compressor is a four-cylinder, double acting, reciprocating unit working at a maximum operational pressure of 1,300 psi for a maximum flow rate of $930 \mathrm{scfm}$. The normal 
operational temperature in the loop is $86^{\circ} \mathrm{F}$. A summary of the operating parameters is presented in Table 2.1.

Table 2.1- Operating parameters at SINTEF ${ }^{19}$

\begin{tabular}{|c|c|c|}
\hline Horizontal line, $\mathrm{ft}$ & \multicolumn{2}{|c|}{$1,640 \mathrm{ft}$} \\
\hline Riser, $\mathrm{ft}$ & \multicolumn{2}{|c|}{$\begin{array}{c}170 \mathrm{ft} \text { (177 ft when downward inclination is } \\
\text { included) }\end{array}$} \\
\hline Pipe diameter, in. & \multicolumn{2}{|c|}{8} \\
\hline Liquid superficial velocity, ft/s & \multicolumn{2}{|c|}{0.5 to 15} \\
\hline Gas superficial velocity, $\mathrm{ft} / \mathrm{s}$ & \multicolumn{2}{|c|}{0.8 to 40} \\
\hline Pressure, psi & \multicolumn{2}{|c|}{290 to 1,305} \\
\hline \multirow{3}{*}{ Liquid } & \multicolumn{2}{|c|}{ Naphtha/Diesel/Lube oil } \\
\hline & Density, $\mathrm{lb} / \mathrm{ft}^{3}$ & Viscosity, $\mathrm{cP}$ \\
\hline & 41 to 54 & 0.2 to $20 \mathrm{cP}$ \\
\hline \multirow{3}{*}{ Gas } & \multicolumn{2}{|c|}{ Nitrogen } \\
\hline & Density, $\mathrm{lb} / \mathrm{ft}^{3}$ & Viscosity, $\mathrm{cP}$ \\
\hline & 1.25 to 7 & 0.01 to $0.02 \mathrm{cP}$ \\
\hline
\end{tabular}

The system was designed to simulate two-phase flow in a nearly horizontal line, with some terrain effects on the sea floor, finishing in a platform riser. The 8 in. pipe diameter represents a medium-size two-phase flow line considered as the most optimum choice from a cost-benefit evaluation which also sets practical limits for the length and height dimensions.

Currently, naphtha, diesel and lube oil are used in the facility. They are easy to handle, they can be stored in atmospheric tanks and the viscosity range is large enough to cover most practical applications. Nitrogen was selected to be used as the experimental gas. Nitrogen has an appropriate molecular weight, a low solubility in hydrocarbon liquids and is inert. A maximum system pressure of 1,450 psi produces a gas-liquid density ratio corresponding to 2,540 psi for natural gas.

Flow velocities cover all flow types of commercial interest; however it is not possible to obtain the maximum liquid and gas velocities simultaneously since the total pressure drop in the loop is limited to approximately 218 psi.

Experiments are typically run for a fixed geometry and a given fluid combination for which the temperature is regulated to $86 \pm 33^{\circ} \mathrm{F}$ to minimize fluid property 
fluctuations. Hence, the gas and liquid flow rates become the essential input variables during each experiment. The volumetric flow rates are measured with turbine and vortex flowmeters for the liquid and gas respectively, each feed line having two metering runs in parallel to cover the actual metering range. The flow temperature is measured at four locations along the two-phase line and also at the flowmeters. Table 2.2 presents a summary of the instruments used for measurements.

Table 2.2- Instrument summary list ${ }^{19}$

\begin{tabular}{|l|l|l|}
\hline Instrument & Manufacturer & Location \\
\hline Liquid flowmeter & Fisher Controls Ltd. & $\begin{array}{l}3 \text { in. Turbine } \\
6 \text { in. Turbine, liquid feed line }\end{array}$ \\
\hline Gas flowmeter & Neptune Measurement Ltd. & $\begin{array}{l}3 \text { in. Vortex } \\
6 \text { in. Vortex, gas feed line }\end{array}$ \\
\hline Pressure & Rosemount Inc. & Reference line \\
\hline Pressure differential (DP) & Rosemount Inc. & $\begin{array}{l}\text { Flow meters } \\
\text { Two-phase line (6X) } \\
\text { Separator }\end{array}$ \\
\hline DP-Quick & RDP Electronics Ltd. & Two-phase line (2X) \\
\hline Temperature & Gould & $\begin{array}{l}\text { Flow meters } \\
\text { Two-phase line (4X) }\end{array}$ \\
\hline Gamma densitometers & Gamma Instruments A/S & Two-phase line (8X) \\
\hline
\end{tabular}

There are six stations all together for pressure measurements along the two-phase test section; four on the horizontal line and two on the riser. The pressure is also measured at the flowmeters and in the top of the separator. The stations on the horizontal runs have two differential pressure transmitters; one having a maximum range of $30 \mathrm{psi}$, the other 290 psi. In the riser, only high-range transmitters are used.

The gamma densitometers are the most vital instruments used to determine the flow pattern, liquid holdup, slug front velocity and slug-length frequency. A total of eight densitometers along the line, five on the horizontal section and three on the riser are installed in the loop. In addition, special instrumentation is installed as needed and special sections exist for optical measurements and visual observation. 


\subsubsection{Institute for Energy Technology - IFE}

The flow loop is a closed multiphase oil-water-gas flow loop with an $82 \mathrm{ft}$ long test section with an internal diameter of a 3.94 in. The two liquid phases are ordinary tap water and oil. A schematic outline of the test facility is illustrated in Fig. 2.2.

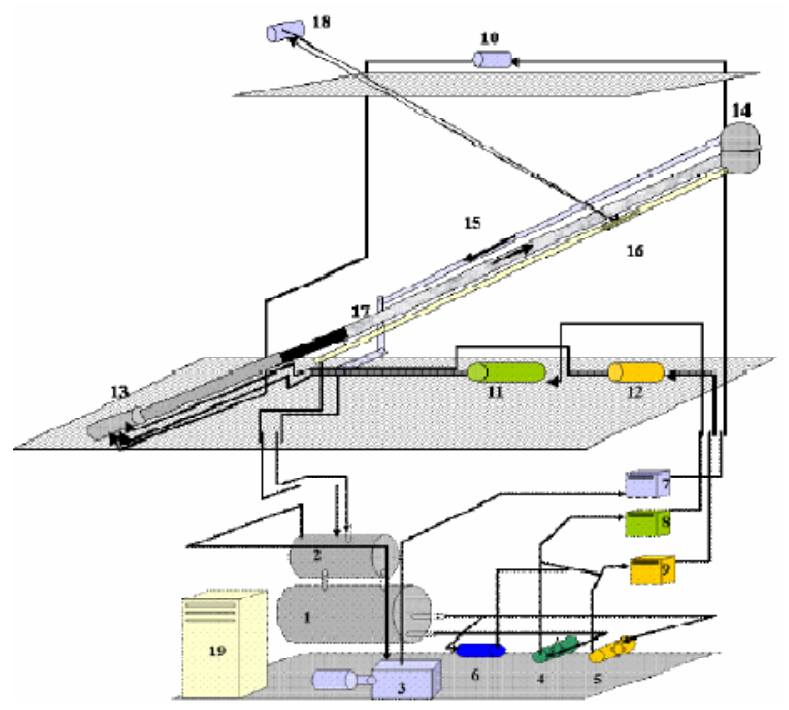

1. Oil-water separator 10. Gas turbine meter

2. Gas-liquid sep. $\quad$ 11. Water E.M. meter

3. Gas compressor 12. Oil coriolis meter

4. Water pump 13. Inlet mix, section

5. Oil pump 14. Slug catcher

6. Helical pump 15. Return pipe, gas

7. Heat exchanger, gas 16. Return pipe, liq.

8. Heat ex., water 17. Test section

9. Heat exchanger, oil 18. Winch

19. Main switch board

Fig.2.2- Schematic drawing of IFE's multiphase flow loop ${ }^{21}$

Since the test rig is not explosion-proof, the oil phase must have a high flame point. The test rig normally utilizes a high molecular weight gas, $\mathrm{SF}_{6}$ (sulphur hexafluoride), which has a density of $3.12 \mathrm{lb} / \mathrm{ft}^{3}$ at $145 \mathrm{psi}$, to simulate high pressure flow conditions found in oil and gas pipelines. However, by using the dense gas, gas densities that correspond to natural gas at a pressure as high as 942 psi can be achieved. Nitrogen has also been used as the gas phase. The pipes are in stainless steel 316L and some sections are made in transparent acrylic.

The gas is cooled after compression and an automatic control system maintains a constant gas temperature at the inlet of the test section. The oil and water are circulated through the loop by centrifugal pumps or, for very low flow rates, by dosage pumps. A screw pump is also available to be used for high viscosity liquids or for liquids where 
exposure to high shear is not desired. The flow rates are adjusted to the desired values by combined use of throttling valves and variable speed motors of the pumps. The main specifications for the loop are given in Table 2.3.

Table 2.3- General specifications of the flow loop ${ }^{21}$

\begin{tabular}{|l|l|}
\hline Maximum pressure, $\mathrm{psi}$ & 145 \\
\hline Test section diameter, in. & 3.94 \\
\hline Test section length, $\mathrm{ft}$ & $\begin{array}{l}82 \mathrm{ft} \text { inclination } 0 \text { to } \\
49\end{array}$ \\
\hline Superficial gas velocity, $\mathrm{ft} / \mathrm{s}$ & 0.98 to 39.37 \\
\hline Superficial oil velocity, $\mathrm{ft} / \mathrm{s}$ & 0 to 7 \\
\hline Superficial water velocity, $\mathrm{ft} / \mathrm{s}$ & 0 to 7 \\
\hline Inclination of test section: & 0 to $90^{\circ}$ \\
\hline
\end{tabular}

The flow loop is permanently equipped with instrumentation necessary for basic multiphase flow experiments. The basic instrumentation consists of flowmeters (see Fig. 2.3), temperature sensors, and pressure gauges that are used to monitor flow conditions during an experiment.

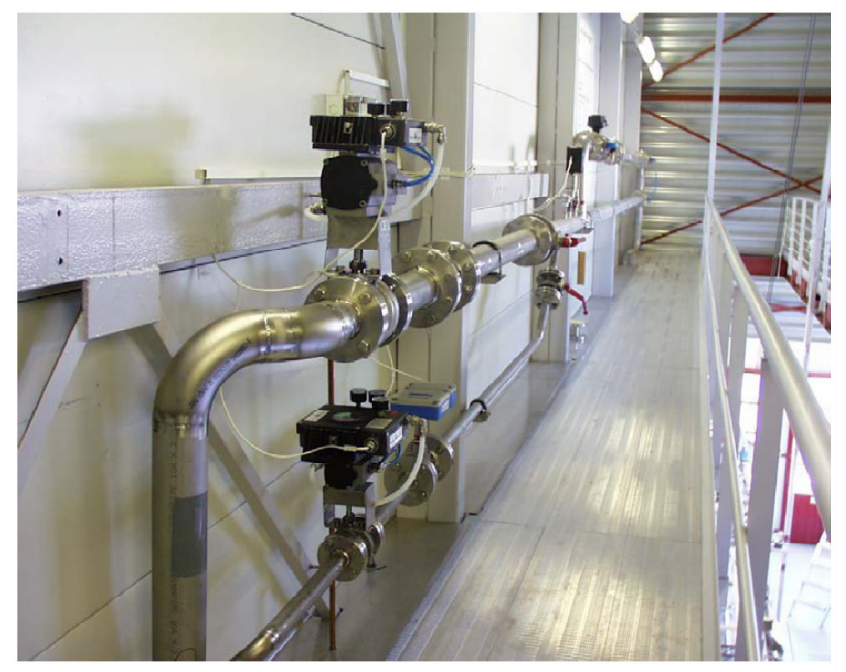

Fig. 2.3-The gas flowmeters ${ }^{21}$

In addition, the test section is equipped with equipment to measure pressure drops, phase fractions and water wetting of the pipe wall. Two specially made spool pieces are also available for accommodation of hot-film probes (wall shear stress 
measurements) and an iso-kinetic probe (droplet flux measurements), respectively. A brief overview of the various instruments available is found in Table 2.4.

Table 2.4- Overview of process and scientific instruments

\begin{tabular}{|l|l|}
\hline Measurement & Measurement device \\
\hline Oil flow rate, ft & $\begin{array}{l}\text { Danfoss Coriolis meter/ Type MASSFLO 1000 } \\
\text { Accuracy + 0.3\% of range }\end{array}$ \\
\hline Water flow rate & $\begin{array}{l}\text { Fisher \& Porter Electromagnetic Flow meter Type 10 Dx } \\
\text { 3311A }\end{array}$ \\
\hline Gas flow rate & $\begin{array}{l}\left.\text { Instromet DN80 Turbine meter/ Type Q-775-F (25 to } 400 \mathrm{~m}^{3} / \mathrm{h}\right) \\
\text { Fisher \& Porter Vortex meter/ Type 10V-1 }\end{array}$ \\
\hline $\begin{array}{l}\text { Pressure gradient (SMART } \\
\text { transducers), psi }\end{array}$ & $\begin{array}{l}\text { Fuji Electric Differential Pressure } \\
\text { Type FHC W11 W1 AKCAY }\end{array}$ \\
\hline $\begin{array}{l}\text { Pressure gradient (transducers } \\
\text { with high frequency response) }\end{array}$ & Druck LPX \\
\hline $\begin{array}{l}\text { Hold-up } \\
\text { Single energy (Am-source) broad beam gamma densitometer. } \\
\text { Built at IFE } \\
\text { Dual energy (Ba-source) broad beam gamma densitometer. } \\
\text { Built at IFE }\end{array}$ \\
\hline Wall wetting & Tonductance ring probe. Built at IFE \\
\hline Local droplet fraction & Hot-film system based on tsi-IFA 100 electronics and software \\
\hline Wall shear stress & S-VHS Video recorder with 25 frames/s \\
\hline Visualization &
\end{tabular}

A multiphase pump is used to circulate/compress the gas (see Fig. 2.4 (a)). This pump can cope with nearly $100 \%$ gas flow. The small amount of liquid drops that follows the gas, particularly at high gas flow rates, is removed in a scrubber downstream of the pump. For the liquid phases several choices are available. The "default" is a pair of relatively high capacity centrifugal pumps, one for each liquid; several dosage pumps (see Fig. 2.4(b)) used for low liquid flow rates, and, as previously mentioned, a low shear screw pump. 


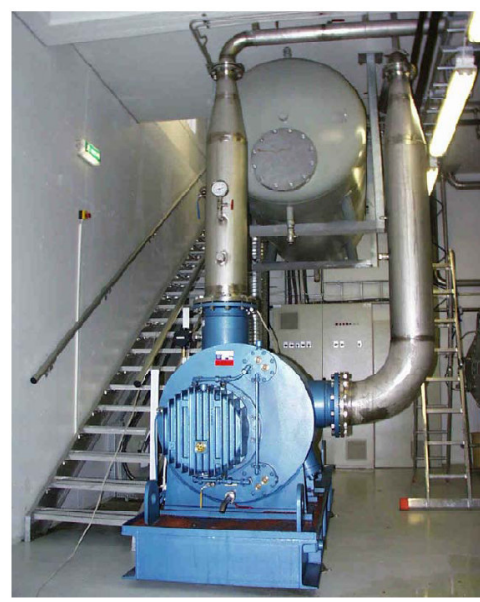

(a)

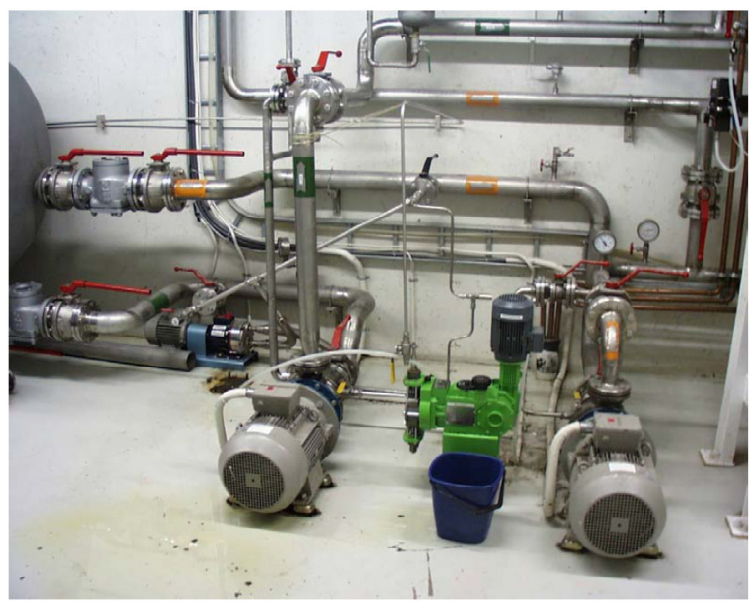

(b)

Fig. 2.4- a) The gas /compressor pump (multiphase pump) and b) behind left: large dosage pump; front left: water pump; green pump: small dosage pumps; front right: oil pump ${ }^{21}$

Frequency converters enable the speed to be smoothly adjusted for most of these pumps. These pumps all have lifting head higher than 98 to $131 \mathrm{ft}$, which is well above the pressure drop in the loop, even at very high flow rates and steep inclinations. Table 2.5 gives an overview of the different pumps and the size of their respective motors.

Table 2.5- Overview of pumps ${ }^{21}$

\begin{tabular}{|c|c|c|c|c|}
\hline Manufacturer & Type & Model & Capacity, gpm & $\begin{array}{l}\text { Motor } \\
\text { hp }\end{array}$ \\
\hline $\begin{array}{l}\text { Bornemann } \\
\text { multiphase pump }\end{array}$ & $\begin{array}{l}\text { Two-spindle screw } \\
\text { pump }\end{array}$ & MW7T.3ZK-85 & 1760 & 87 \\
\hline $\begin{array}{l}\text { (2X) Siemens \& } \\
\text { Hirsch liquid pumps }\end{array}$ & Centrifugal pump & ZLKC50200/1800 & 440 & 17 \\
\hline OBL dosage pumps & $\begin{array}{l}\text { Positive displacement } \\
\text { pump }\end{array}$ & Type XRN6.38A85 & 0.15 & 0.13 \\
\hline $\begin{array}{l}\text { PULSAR dosage } \\
\text { pump }\end{array}$ & Membrane & $\begin{array}{c}\text { PULSAFEEDER } \\
25 \mathrm{HJ}\end{array}$ & 0.4 & 0.74 \\
\hline $\begin{array}{l}\text { Wanner Engineering } \\
\text { Inc. Dosage pump }\end{array}$ & $\begin{array}{l}\text { Membrane Plunger } \\
\text { pump }\end{array}$ & $\begin{array}{c}\text { Hydra-Cell } \\
\text { G10XDSGSNEMB }\end{array}$ & 1.3 & 2 \\
\hline $\begin{array}{l}\text { Bornemann liquid } \\
\text { pump }\end{array}$ & Eccentric helical rotor & E2H 2650 & 220 & 15 \\
\hline
\end{tabular}


The piping is mainly in stainless steel 316L including spool pieces made in transparent acrylic in the test section (Fig. 2.5(a)). The test section has a pipe diameter of 3.94 in. The piping between the pumps and the mixing section (Fig. 2.5 (b)) has diameters between 1.57 and 3.94 in.

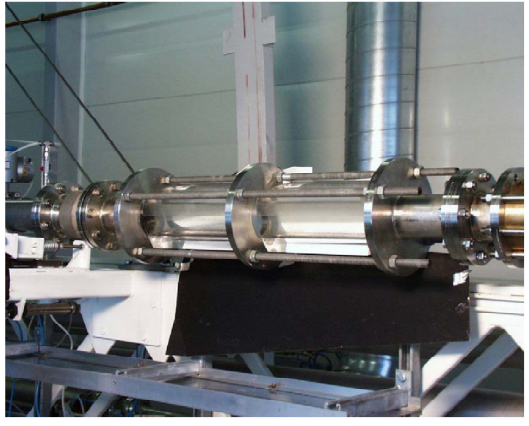

(a)

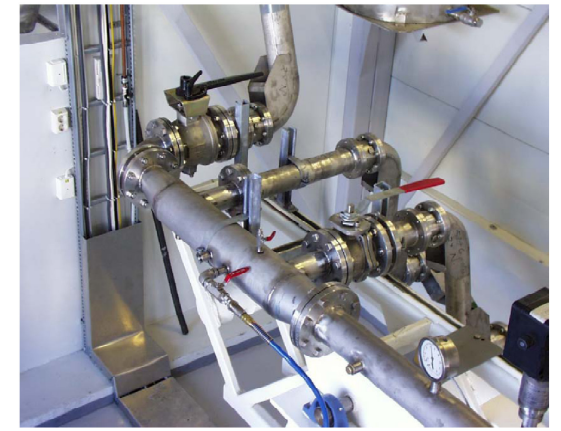

(b)

Fig. 2.5- a) The optical acrylic and b) inlet mixing section ${ }^{21}$

The return pipes from the test section outlet have diameters in the range of 4.72 and 7.87 in. Separation of the phases is achieved by means of a slug catcher (see Fig. 2.6 (a)) and 2 separators (Fig. 2.6 (b)).

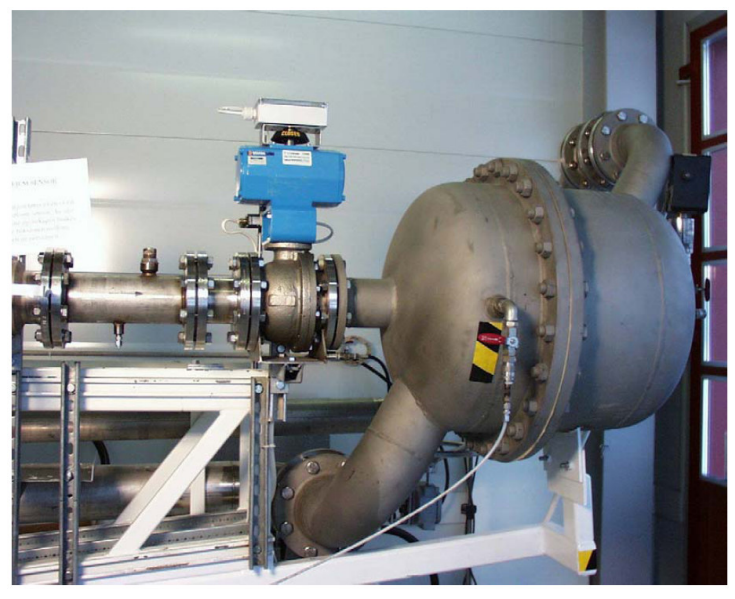

(a)

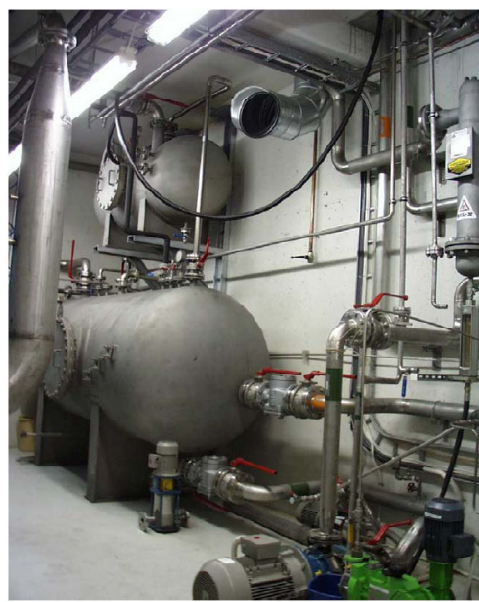

(b)

Fig. 2.6- a) Slug catcher at the outlet of the test section and b) gas/liquid (top) and oil/water separators ${ }^{21}$ 
The slug catcher is located at the outlet end of the downstream test section and is in charge of the initial separation of the gas and the liquids. Following the first stage of separation, gas and liquids are recombined in the gas-liquid separator from which two lines depart, one to the multiphase pump and another line to the oil-water separator. The sizes of the slug catcher, gas-liquid and liquid-liquid separator are 28, 140 and $7 \mathrm{ft}^{3}$ respectively.

\subsubsection{Norsk Hydro multiphase flow loop}

Norsk Hydro ASA decided in 1984 to construct their first experimental test rig for the investigation of the processes of transport behavior of live and recombined crude oil systems. Conceptually the idea was to establish an environment for the in-house modeling of multiphase flow and gas/oil/water separation where the effect of water and the peculiarity of hydrocarbon systems compared to current models for oil systems could be studied.

A range of test rigs have been built ranging from transparent oil-water flow loop to a high pressure multiphase flow loop. Sizes vary facilitating the experimental study of oil and water processes, making emphasis on transport phenomena. Norsk Hydro has a 7,250 psi titanium laboratory dispersion characterization rig for determining fluid separation characteristics.

Norsk Hydro ASA's multiphase flow loop was built in 1991 and ready for operation in 1994 to investigate multiphase phenomena such as the characterization of fluid separations, emulsions, foaming, transport behavior, phase dispersions, droplet break-up and coalescence, multiphase gas/oil/water flow, wax precipitation, hydrate precipitation, corrosion, effects on inhibitors, and the qualification and testing of equipment and instrumentation.

The multiphase flow loop is suited to realistic process conditions handling pressures and temperatures that make it possible to create test conditions representative for those found in oil and gas fields. Gas, oil and water are available, which allows recombination of hydrocarbon fluids and saling water, which represents realistic fluid systems. This is in great contrast to small-scale lab facilities that are constrained by 
using only air and water at low pressure and fluid flow rates. The technical specifications of the rig are given in Table 2.6.

Table 2.6- Multiphase flow loop main characteristics ${ }^{22}$

\begin{tabular}{|l|l|}
\hline Number of phases & Three (gas, oil, water) \\
\hline Water capacity, gpm & 0 to 176 (Superficial velocity $=0$ to $8 \mathrm{ft} / \mathrm{s})$ \\
\hline Oil capacity, bbl/d & 0.3 to 176 (Superficial velocity $=0$ to $8 \mathrm{ft} / \mathrm{s}$ ) \\
\hline Liquid capacity gpm & 0 to 264 (Superficial velocity $=0$ to $11 \mathrm{ft} / \mathrm{s})$ \\
\hline Gas capacity, scfm & 0.2 to 121 (Superficial velocity $=0$ to $38 \mathrm{ft} / \mathrm{s}$ ) \\
\hline Maximum pressure, psi & 1,595 \\
\hline Maximum pressure drop, psi & 580 \\
\hline Temperature range, ${ }^{\circ} \mathrm{F}$ & 23 to 284 \\
\hline Inner tube diameter, in. & 3 \\
\hline Flow loop length, $\mathrm{ft}$ & 656 \\
\hline Tilt & $-6^{\circ}$ to $+10^{\circ}$ \\
\hline Material & Duplex steel \\
\hline Water phase & Water with optional content of salt, $\mathrm{MeOH}$ or Glycol \\
\hline Oil phase & Crude Oil \\
\hline Gas phase & Hydrocarbon gas \\
\hline
\end{tabular}

The loop contains a three-phase separator, circulating pumps and a heat exchanger to control the temperature. The loop itself has three optional test loops connected: the flow loop, the choke test section, and the multipurpose test area. Fig. 2.7 shows the outer end of the $394 \mathrm{ft}$ long flow loop.

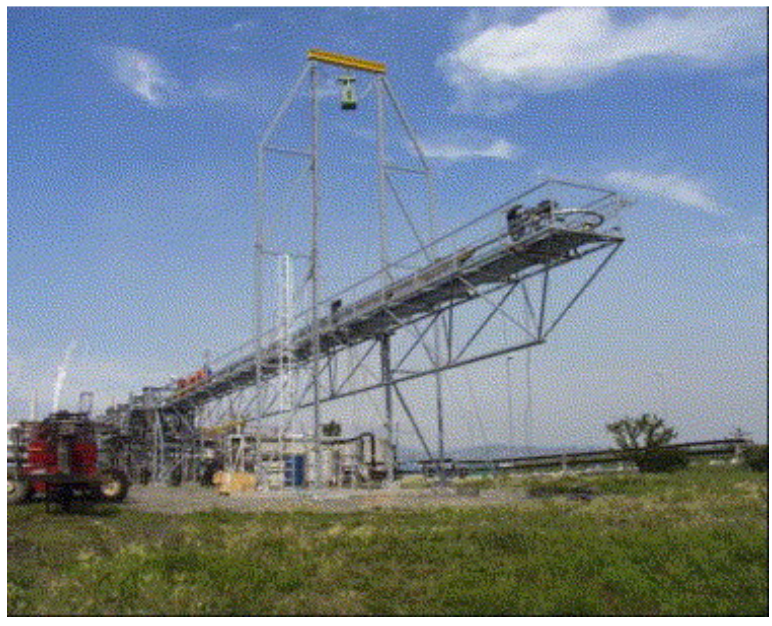

Fig. 2.7- The tiltable section of the flow loop in the horizontal position ${ }^{22}$ 
The three phase separator acts as a central reservoir from which the three phases flow independently to individual pumps/circulator, fitted with variable speed controllers to a mixing point. The multiphase flow loop has two different mixing points, the Y-junction and the T-junction and also allows adding chemicals into the system to perform tests with the addition of different types of inhibitors (corrosion, hydrate, flow improvers, etc.).

As depicted by Fig. 2.8, the mixed flow from the test section enters the three phase separator. After separation takes place, the gas phase leaves the separator at the top and flows through the gas circulator and cooler to then be directed whether to the multipurpose test area or the three-phase mixing point upstream from the test section.

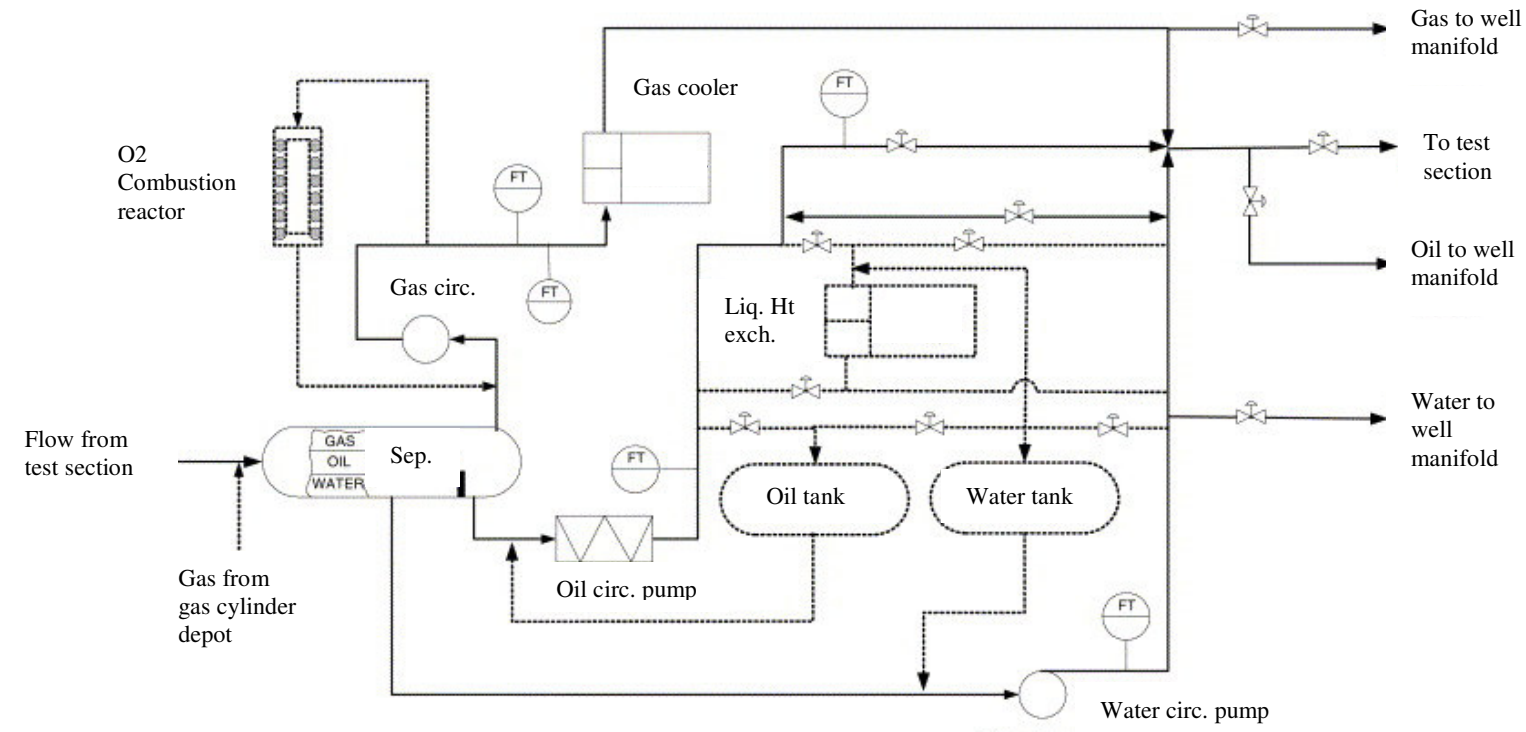

Fig. 2.8- Main parts of the multiphase flow loop ${ }^{22}$

Oil flows from the separator to a circulating pump to then flow through or bypass the liquid heat exchanger. After this stage, the oil phase is mixed with the water at a T-junction or in the three-phase Y-junction. Water flows from the separator to the water circulating pump and can optionally flow through the liquid heat exchanger to then be directed to the well manifold in the multipurpose test area or to the test sections via the T-junction or the Y-junction. 
The flow loop and the test section are illustrated in Fig. 2.9. The mixture flows from the separator to the $197 \mathrm{ft}$ long horizontal part of the test section and if required, the flow can then be directed to the choke test section following a multiphase pump before arriving at the multipurpose test area or the choke test section where different chokes can be tested. The flow can return to the separator or enter the inclined section which continues straight ahead for $130 \mathrm{ft}$ before a U-turn brings the flow back to the separator.

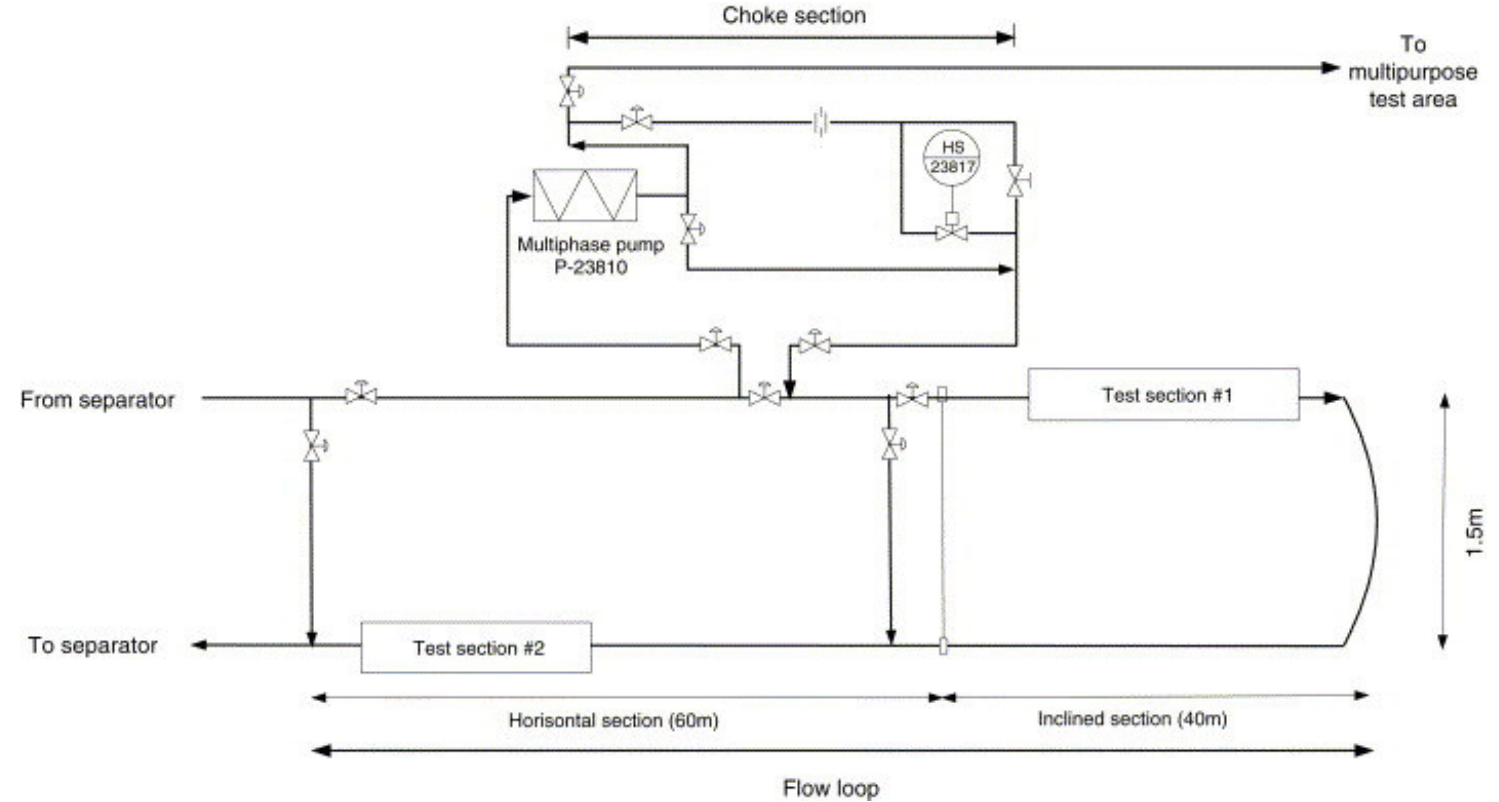

Fig. 2.9- The 3 in. flow loop and the choke test section ${ }^{22}$

Fig. 2.9 also shows two test section areas placed conveniently to avoid nothing but pipe effects. The first test section is placed more than $880 \mathrm{~L} / \mathrm{D}$ from the last bend, and the second test section is placed $700 \mathrm{~L} / \mathrm{D}$ from the bend in the inclined section, and $190 \mathrm{~L} / \mathrm{D}$ from the inclined section bypass.

The multipurpose test area as seen in Fig. 2.10 shows the flow coming from the separator in two optional ways, either through the well manifold or via the multiphase pump in the choke section to finally be directed to the separator. The test area is a $164 \mathrm{ft}$ x $65 \mathrm{ft}$ area situated on the ground, making it easy to connect equipment. 


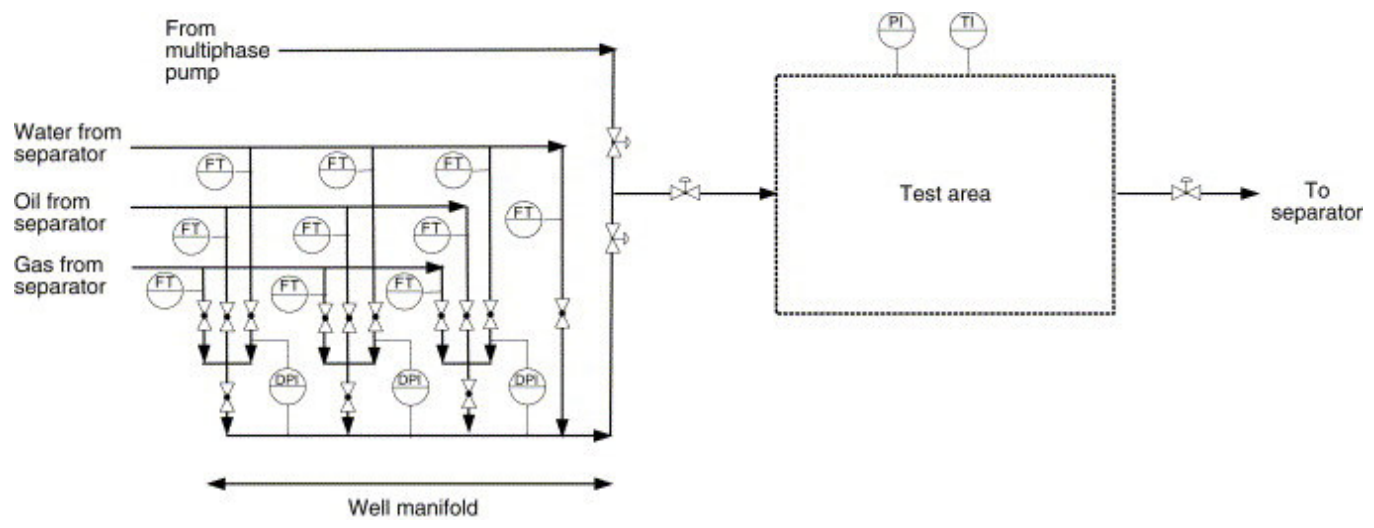

Fig. 2.10- The multipurpose test $\operatorname{area}^{22}$

The test facility was built of duplex steel, W.Nr 1.4462, X2CrNiMoN, 22-5 UNS S31803. All process wetted parts are duplex steel, Incolloy 825 or Hastelloy C-276. Monel K-500 was used as the material in the glands and graffiti spiral glands with interior and exterior rings in Monell K-500 are used to connect process piping.

The separator in the flow loop has a volume of $78 \mathrm{ft}^{3}$ and has a built-in splash plate on the inlet which reduces the incoming momentum caused by the arrival of the fluids. The operational conditions are determined by controlling the level of the liquids, therefore the addition of buffer tanks was considered to maintain the desired water and oil levels in the vessel.

A Bertin peripheral compressor, Type CP 310.1.008 was installed in the facility. The sealing system is a John Crane back to back mechanical seal with an API pressurized support plan. A Bornemann type W6.5ZX1-30/324008 twin-screw pump was selected to increase the pressure of the oil phase based on its low shear that avoids production of droplets. The pump is fitted with a single Burgmann seal and the leaks are collected in a dedicated container to avoid contamination of the area.

The water pump is an APV canned glandless leakage proof centrifugal pump of type HMD type hp 2E SM RES-125 fitted with a magnetic drive coupling. The booster pump is of the type Bornemann, type MPC208X1-57, fitted with a single unpressurized Burgmann mechanical seal which leaks are collected in a dedicated container. This pump raises the pressure of the multiphase stream by approximately 580 psi. 
The test facility incorporates pressure transmitters, temperature sensors, flow transducers, level measurements and gas detectors due to the complexity of the process and the safety issues that need to be addressed.

Full control of the specific flow rates of each phase and control of the fluids leaving the separator is of vital importance during the experimental work, therefore five coriolis mass flow meters (no calibration is necessary) are installed to measure the liquids leaving the separator as well as indicating the fluid densities.

A turbine meter is installed to measure gas at high flow rates while a V-cone differential pressure flow meter is installed to measure to measure low rates. The main instrumentation is depicted in Table 2.7.

Table 2.7- Main control instrumentation ${ }^{22}$

\begin{tabular}{|l|l|l|}
\hline Instrument & \multicolumn{1}{|c|}{ Model } & \multicolumn{1}{c|}{ Description } \\
\hline Oil flow meter & Rheonik Micro Motion & Coriolis mass flow meter \\
\hline Water flow meter & Rheonik Micro Motion & Coriolis mass flow meter \\
\hline Liquid flow rate & Endress-Hauser & Coriolis mass flow meter \\
\hline Gas flow rate & Instromet SM-RI & Turbine gas flow meter \\
\hline Gas flow rate & Fisher Rosemount & V-cone, differential pressure flow meter \\
\hline Pressure transmitter & Rosemount 2088G4A2 & \\
\hline $\begin{array}{l}\text { Temperature } \\
\text { transmitter }\end{array}$ & $\begin{array}{l}\text { Pt-100, INOR TRA } \\
\text { R6X }\end{array}$ & \\
\hline $\begin{array}{l}\text { Separator level } \\
\text { indicator }\end{array}$ & Sentech Profile Gauge & 40 capacitance probes \\
\hline
\end{tabular}

\subsubsection{Southwestern Research Institute - SwRI}

The facility is used for research and testing in areas such as flow assurance, wet gas metering, multiphase flow metering, and subsea pipeline design and operation. Several possible configurations combined with a wide range of flow rates, pressures, and temperatures make this a versatile facility with unique capabilities in multiphase flow research and testing.

The flexibility of the multiphase flow loop allows conducting experimental investigations using different testing fluids to simulate specific situations in the oil and gas industry. Natural gas, nitrogen, crude oil, condensates, refined liquid, fresh water, saltwater and oil and water combined are readily available at the facility. 
Flow lines can be pressurized from 100 psi up to 3,600 psi with a temperature range of $40^{\circ} \mathrm{F}$ to $120^{\circ} \mathrm{F}$. When two phase flow studies are carried out, a GVF from $0 \%$ to $100 \%$, as well as, water cut within the same range can be achieved. The maximum differential pressure across the test section is set to be 100 psi. Table 2.8 presents a summary of the principal features of the facility.

Table 2.8- Characteristics of SwRI multiphase flow loop

\begin{tabular}{|l|l|}
\hline Reference meters & Gas: Coriolis meters $(0.5,1,1.5$ and 2 in.) \\
& Liquid: Coriolis meters $(0.5,1,1.5,2$ and 3 in.) \\
\hline Instrumentation & $\begin{array}{l}\text { Temperature, pressure, differential pressure } \\
\text { Gas volume fraction, (GVF) } \\
\text { Water cut } \\
\text { Deposition and holdup monitoring by gamma ray densitometer } \\
\text { High-pressure optical system to view inside pressurized lines }\end{array}$ \\
\hline Data acquisition & $\begin{array}{l}\text { Digital interface is used for most loop instruments to give improved } \\
\text { accuracy and reliability in sensor measurements. } \\
\text { Analog, HART, Modbus, and Foundation Fieldbus interfaces are available } \\
\text { for connection of customer test articles. }\end{array}$ \\
\hline Flow rate range, scfm & 0 to 120 (gas and liquid combined) \\
\hline
\end{tabular}

The combined flow rates of gas and liquid are constrained to be within the total volumetric limit of $120 \mathrm{scfm}$. The range of possible test conditions is inside the boundaries shown in red in Fig. 2.11.

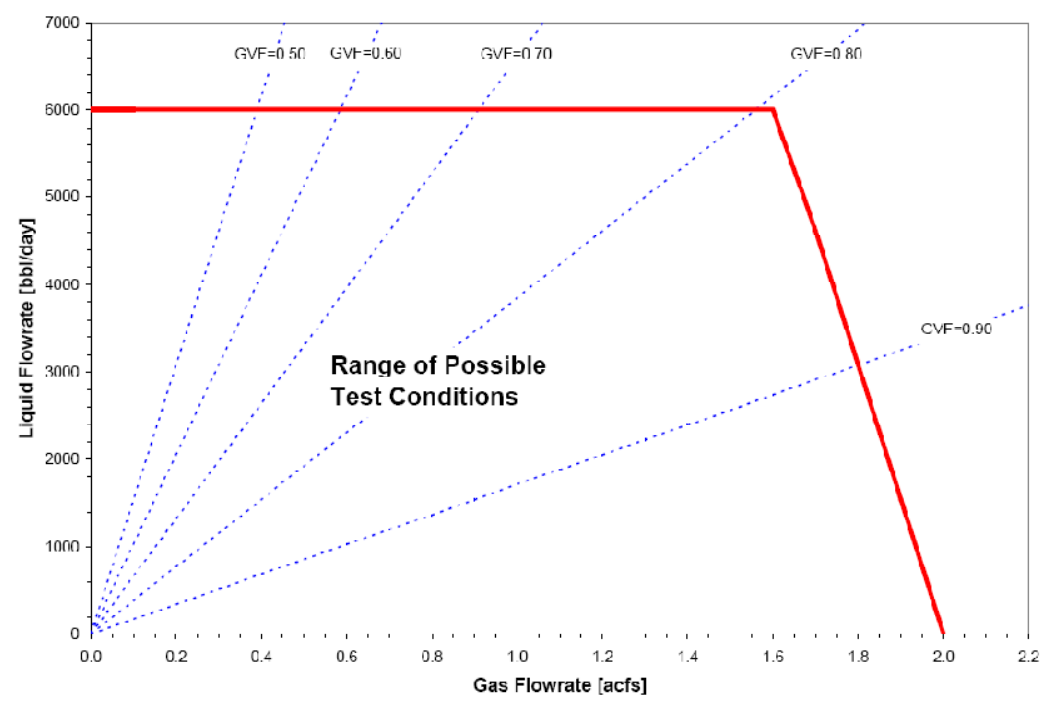

Fig. 2.11- Range of possible test conditions ${ }^{23}$ 
Standard gas and liquid are metered independently and combined upstream of the test section. Typical projects include equipment and instrument development and testing. The test section differential pressures can be set up to $200 \mathrm{psi}$ at flow rates up to $60 \mathrm{scfm}$ (gas and liquid combined); typical projects have included hydrate formation and dissociation studies requiring large pressure differentials.

Part of the tubing in the flow loop is in a water bath specialized test section consisting of $1,200 \mathrm{ft}$ of $1 \mathrm{in}$. coiled tubing for testing projects that include flow assurance and inhibitor evaluation and gel strength estimation. The loop also counts with a specialized test section in which solids can be recirculated without being crushed or sheared in a pump. Typical projects have included studies of precipitation, deposition, agglomeration, and rheology of solid-liquid-gas transport streams.

\subsubsection{NEL wet gas flow loop}

The NEL high-pressure wet-gas test facility, commissioned in 1999, is a highGVF two-phase gas-liquid flow facility designed to simulate gas flows with small quantities of liquids present in the stream, such as found in gas/condensate and very high GOR fields. The facility is a closed-loop recirculating system built around a high pressure gas-liquid separator, which stores the working fluids (Nitrogen and a kerosene substitute as the test fluids). ${ }^{24}$

Each phase is pumped and metered separately prior to mixing. Two test lines are available with horizontal and vertical configurations to suit the equipment under test. The facility is enclosed in a dedicated testing building which provides a comfortable and safe working environment.

The nitrogen and kerosene are stored at a pressure up to a maximum of $914 \mathrm{psi}$ in a gas-liquid separator with a volume of approximately $396 \mathrm{ft}^{3}$ that is linked to a nominally 6 in. diameter flow loop. The nitrogen is drawn from the separator outlet by a $268 \mathrm{hp}$ Howden encapsulated centrifugal gas blower up to a maximum (dry-gas) flow rate of $824 \mathrm{scfm}$ and is then cooled using a chilled-water heat exchanger positioned directly downstream of the blower. Temperature control is maintained within a $33{ }^{\circ} \mathrm{F}$ band around the set point. The cooled gas then passes through the reference flowmeter 
and into the test section. Fig. 2.12 is a schematic of the facility followed by a description of its main components.

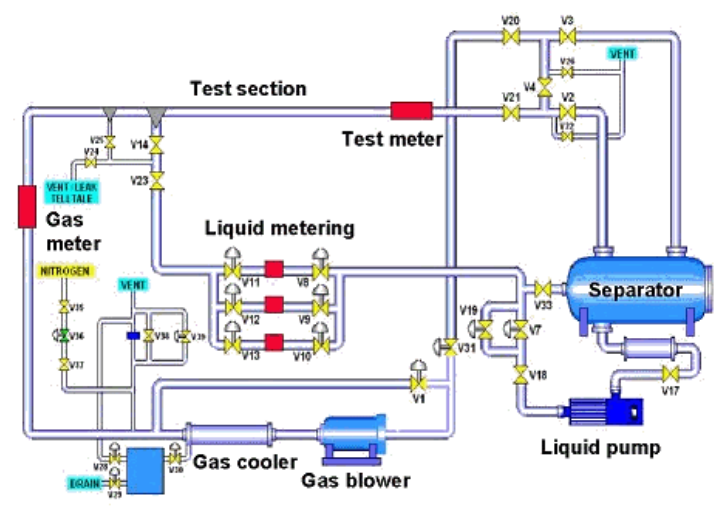

Fig. 2.12- Schematic diagram of NEL wet gas loop ${ }^{24,25}$

(1) Wet gas separator

(2) Separator outlet

(3) Separator intlet

(4) Ball valve

(5) Ball valve

(6) Junction

(7) Blower by-pass line

(8) Blower

(9) Gas cooling system
(10) Pressurizing/depressurizing system

(11) Flow conditioner

(12) Dry gas reference meter

(13) Pump

(14) Flow meters

(15) Liquid injectors

(16-17) Test piece

(18) Automatic recirculation valve

(19) Separator pipe

The liquid is drawn from the bottom of the separator using a $174 \mathrm{hp} \mathrm{11-stage}$ Ingersoll-Dresser centrifugal pump capable of delivering a maximum flow rate of $352 \mathrm{gpm}$. Prior entering the pump inlet the liquid passes through another chilled-water heat exchanger, which allows the fluid temperature to be controlled to a level better than $32^{\circ} \mathrm{F}$ of the setpoint, and generally to a value within $33^{\circ} \mathrm{F}$ of the gas temperature.

The liquid flow rate is controlled using a pair of control valves trimmed to allow as wide a range of flow rates to be obtained as possible in relation to the ranges of the 
three reference $0.5,1$ and 3 in. turbine meters used on the liquid injection line capable of metering from 0.66 to $7 \mathrm{gpm}, 7$ to $66 \mathrm{gpm}$, and 22 to $660 \mathrm{gpm}$ respectively.

After passing through the reference metering section the liquid is injected into the gas stream approximately 60D upstream of the test meter section of the flow loop. The two-phase flow pattern within the test line can be viewed using a modified deep-sea video camera which is useful for observing the transition between stratified-wavy and annular-mist flow regimes. The stable two-phase mixture passes through the meter under test and is then returned to the gas- liquid separator.

The maximum achievable liquid volume fraction from the facility is dependent on both the gas velocity required and the pressure drop within the test section, but is generally in the range 0.1 to $5 \%$. All reference measurements and instrumentation are fully traceable. The uncertainties in the gas and liquid reference mass flow rates are $0.4 \%$ and $0.15 \%$. Table 2.9 presents a summary of the wet gas test facility specifications.

Table 2.9- Wet gas test facility specifications ${ }^{26}$

\begin{tabular}{|l|l|}
\hline \multicolumn{2}{|c|}{ Fluids/Flow rate } \\
\hline Gas (Nitrogen), scfm & Up to 824 \\
\hline Liquid (Kerosene or water) & $0 \%$ to $10 \%$ by volume \\
\hline Uncertainty & Gas flow $<0.5 \%$ \\
\hline \multicolumn{2}{|c|}{ Operating conditions } \\
\hline \multicolumn{2}{|c|}{ Reference Instrumentation } \\
\hline Line pressure, psi & 0 to 910 \\
\hline Line temperature, ${ }^{\circ} \mathrm{F}$ & 60 to 77 (controlled to $32^{\circ} \mathrm{F}$ ) \\
\hline Line sizes, in. & 2 to 8 \\
\hline Horizontal line length, ft & 49 \\
\hline Vertical line height, ft & 6 in. ultrasonic and turbine meters \\
\hline \multicolumn{2}{|c|}{ Instrumentation } \\
\hline Gas & $0.5,1$ and 3 in. turbine meters \\
\hline Liquid & Offline instrumentation \\
\hline \multicolumn{2}{|c|}{ Density measurement } \\
\hline Test instrumentation & Viscosity measurement \\
\hline Temperature, pressure, $\Delta \mathrm{p}$ & \\
\hline High speed $\Delta \mathrm{p} /$ pressure & \\
\hline Subsea visualization camera & \\
\hline Gamma densitometer &
\end{tabular}




\subsubsection{Colorado Engineering Experimental Station Inc - CEESI}

\subsubsection{Wet gas metering facility}

In 1997 the Colorado Engineering Experiment Station Inc. (CEESI) began construction of a multiphase test facility laboratory in Nunn, Colorado. Initially the facility was designed for two phase flow studies consisting on natural gas and hydrocarbon liquids. Fig. 2.13 is a simplified block diagram that shows the location of the major components of the wet-gas research loop.

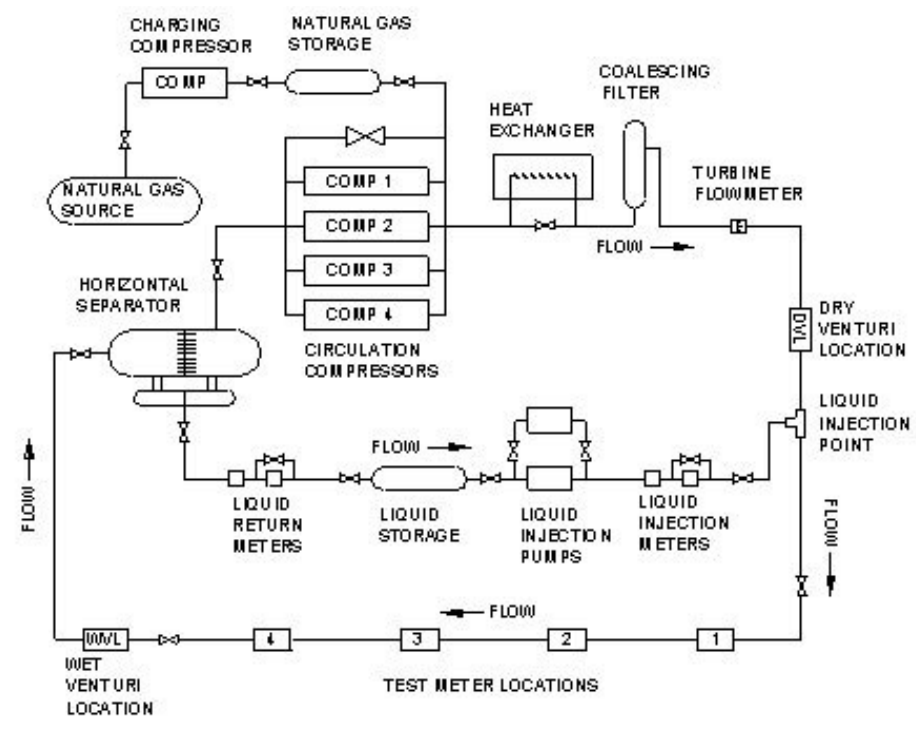

Fig.2.13- Simplified block diagram of major components ${ }^{27}$

The system was designed to use lean natural gas as the gaseous fluid with a methane mole fraction near 0.94. Initially, the liquids considered were water (pure) and gas condensate to simulate condensate gas wells. The average gas velocity is set to be $40 \mathrm{ft} / \mathrm{s}$ based on a 4 in. schedule 80 pipe; however, higher velocities are easily achievable for many operating conditions. The maximum liquid flow rate corresponds to $20 \mathrm{gpm}$ which sets a maximum limit on the liquid load for high pressure, high velocity operation that is less than 0.5 .

The test pressure within the multiphase research loop can be between 100 and 1440 psi up to 1500 psi. The minimum compressor suction pressure is $80 \%$ of the discharge pressure which limits the maximum pressure drop. The temperature within the 
system is designed with the objective of maintaining a stable operating temperature (dependent on the ambient environment) for the duration of the test.

A charging compressor is used to pressurize the research loop to the desired operating pressure for the test being conducted. The normal operating pressure range is between 100 to $1,440 \mathrm{psi}$. Once the loop is pressurized, any combination of the four positive displacement compressors can be used to circulate the natural gas around the research loop at the desired velocity. ${ }^{27}$

Three identical headers are installed adjacent to the compressors to reduce flow pulsations that may affect the results of an experiment and to make up for losses that will occur invariably during a test. The size of each is $12 \mathrm{in}$. by $45 \mathrm{ft}$ long, the pressure rating is 1,440 psi. Gas velocities of 7 to $90 \mathrm{ft} / \mathrm{s}$ can be obtained in a 4 in. pipe. The obtainable velocities in other size pipes vary inversely with the pipe area.

Both a turbine meter and a subsonic venturi measure the mass flow rate of the natural gas. The difference in mass flow rate between these two meters is monitored; and if the difference exceeds a specified amount, the data is scrutinized for detrimental effects such as pulsation. If the difference is within tolerance, than all other meters installed in the research loop can be compared to the natural gas mass flow rate as measured by the turbine meter.

The hydrocarbon liquid, which resides in the liquid storage vessel, can be injected into the gas stream by positive displacement pumps (triplex pumps). Coriolis meters measure the liquid mass flow rate and the density of the injected liquid. The gas stream carries the liquid through the meter test locations to the horizontal separator where it is then returned to the liquid storage vessel. Coriolis meters again measure the mass flow rate and the density of the returned liquid. When the injected liquid mass flow rate is equal to the return liquid mass flow rate and all pressures and temperatures within the loop are constant with time; the system is at a steady state condition where data can be acquired. At this condition, the liquid "hold-up" within the piping sections has occurred. Once the steady state conditions exist within the research loop, the effect of liquid entrained in the gas stream upon the flow meter can be determined. 
For the separation of the two phase, a conventional vertical and horizontal double barrel separator are located upstream of the compressor suction header and dry gas flow measurement section respectively. The vertical separator is 40 in. by $8.5 \mathrm{ft}$ tall while the horizontal is $14 \mathrm{in}$. by $11 \mathrm{ft}$ long. Both separators can withstand the maximum pressure of the system rated at 1440 psi.

\subsubsection{Multiphase flow loop}

The multiphase research loop is designed as a single pass liquid system. The gas is circulated around the loop, but the liquid is passed through the multiphase meter only once. The liquids (water and oil) are individually measured and injected into the gas stream upstream of the multiphase meter. The gas stream conveys with the liquids through the test meter and then into a large separator. The gas from the separator is returned to the compressors and recirculated around the loop with very little or no liquid carry-over at the proposed gas velocities.

Tables 2.10 and 2.11 show the gas velocities and GVF capabilities for two pipe sizes. The tables contain "X" at those conditions that can be obtained while the "-" are those conditions that can be obtained when additional capacity is added.

Table 2.10- Gas velocities and GVF capacity for 2 in. schedule 80 pipe $^{28}$

\begin{tabular}{|c|c|c|c|c|c|c|c|}
\hline Gas Velocity, ft/s & GVF & GVF & GVF & GVF & GVF & GVF & GVF \\
& 0.0 & 0.99 & 0.98 & 0.96 & 0.94 & 0.92 & 0.90 \\
\hline 4.9 & $\mathrm{X}$ & $\mathrm{X}$ & $\mathrm{X}$ & $\mathrm{X}$ & $\mathrm{X}$ & $\mathrm{X}$ & $\mathrm{X}$ \\
\hline 9.8 & $\mathrm{X}$ & $\mathrm{X}$ & $\mathrm{X}$ & $\mathrm{X}$ & $\mathrm{X}$ & $\mathrm{X}$ & $\mathrm{X}$ \\
\hline 15 & $\mathrm{X}$ & $\mathrm{X}$ & $\mathrm{X}$ & $\mathrm{X}$ & $\mathrm{X}$ & $\mathrm{X}$ & $\mathrm{X}$ \\
\hline 20 & $\mathrm{X}$ & $\mathrm{X}$ & $\mathrm{X}$ & $\mathrm{X}$ & $\mathrm{X}$ & $\mathrm{X}$ & $\mathrm{X}$ \\
\hline 25 & $\mathrm{X}$ & $\mathrm{X}$ & $\mathrm{X}$ & $\mathrm{X}$ & $\mathrm{X}$ & $\mathrm{X}$ & - \\
\hline 30 & $\mathrm{X}$ & $\mathrm{X}$ & $\mathrm{X}$ & $\mathrm{X}$ & $\mathrm{X}$ & - & - \\
\hline 35 & $\mathrm{X}$ & $\mathrm{X}$ & $\mathrm{X}$ & $\mathrm{X}$ & $\mathrm{X}$ & - & - \\
\hline 40 & $\mathrm{X}$ & $\mathrm{X}$ & $\mathrm{X}$ & $\mathrm{X}$ & $\mathrm{X}$ & - & - \\
\hline 45 & $\mathrm{X}$ & $\mathrm{X}$ & $\mathrm{X}$ & $\mathrm{X}$ & - & - & - \\
\hline 50 & $\mathrm{X}$ & $\mathrm{X}$ & $\mathrm{X}$ & $\mathrm{X}$ & - & - & \\
\hline 55 & $\mathrm{X}$ & $\mathrm{X}$ & $\mathrm{X}$ & $\mathrm{X}$ & - & - & \\
\hline 60 & $\mathrm{X}$ & $\mathrm{X}$ & $\mathrm{X}$ & $\mathrm{X}$ & - & - & \\
\hline 65 & $\mathrm{X}$ & $\mathrm{X}$ & $\mathrm{X}$ & $\mathrm{X}$ & - & & \\
\hline 70 & $\mathrm{X}$ & $\mathrm{X}$ & $\mathrm{X}$ & - & - & & \\
\hline
\end{tabular}


Table 2.11- Gas velocities and GVF capacity for 4 in. schedule 80 pipe $^{28}$

\begin{tabular}{|c|c|c|c|c|c|c|c|}
\hline Gas Velocity, ft/s & GVF & GVF & GVF & GVF & GVF & GVF & GVF \\
& 0.0 & 0.99 & 0.98 & 0.96 & 0.94 & 0.92 & 0.90 \\
\hline 4.9 & $\mathrm{X}$ & $\mathrm{X}$ & $\mathrm{X}$ & $\mathrm{X}$ & $\mathrm{X}$ & $\mathrm{X}$ & $\mathrm{X}$ \\
\hline 9.8 & $\mathrm{X}$ & $\mathrm{X}$ & $\mathrm{X}$ & $\mathrm{X}$ & $\mathrm{X}$ & - & - \\
\hline 15 & $\mathrm{X}$ & $\mathrm{X}$ & $\mathrm{X}$ & $\mathrm{X}$ & - & - & \\
\hline 20 & $\mathrm{X}$ & $\mathrm{X}$ & $\mathrm{X}$ & - & - & & \\
\hline 25 & $\mathrm{X}$ & $\mathrm{X}$ & $\mathrm{X}$ & - & & & \\
\hline 30 & $\mathrm{X}$ & $\mathrm{X}$ & $\mathrm{X}$ & - & & & \\
\hline 35 & $\mathrm{X}$ & $\mathrm{X}$ & - & - & & & \\
\hline 40 & $\mathrm{X}$ & $\mathrm{X}$ & - & & & & \\
\hline 45 & $\mathrm{X}$ & $\mathrm{X}$ & - & & & & \\
\hline 50 & $\mathrm{X}$ & $\mathrm{X}$ & - & & & & \\
\hline
\end{tabular}

\subsubsection{K-Lab}

Located near Haugesund in the Stavanger area, the Karsto metering and technology Laborary, or K-Lab, is adjacent to Norway's first natural-gas-processing plant. It receives natural gas from across the Norwegian Trench from the Statfjord complex and after processing it sends it on to Emden, West Germany.

K-Lab enables calibration of any type of high-pressure, gas-metering device through the operation of a closed loop with working pressure and temperature ranges from 290 to 2263 psia, and $68-140^{\circ} \mathrm{F}$ above ambient, respectively. A schematic layout of the loop is shown in Fig. 2.14

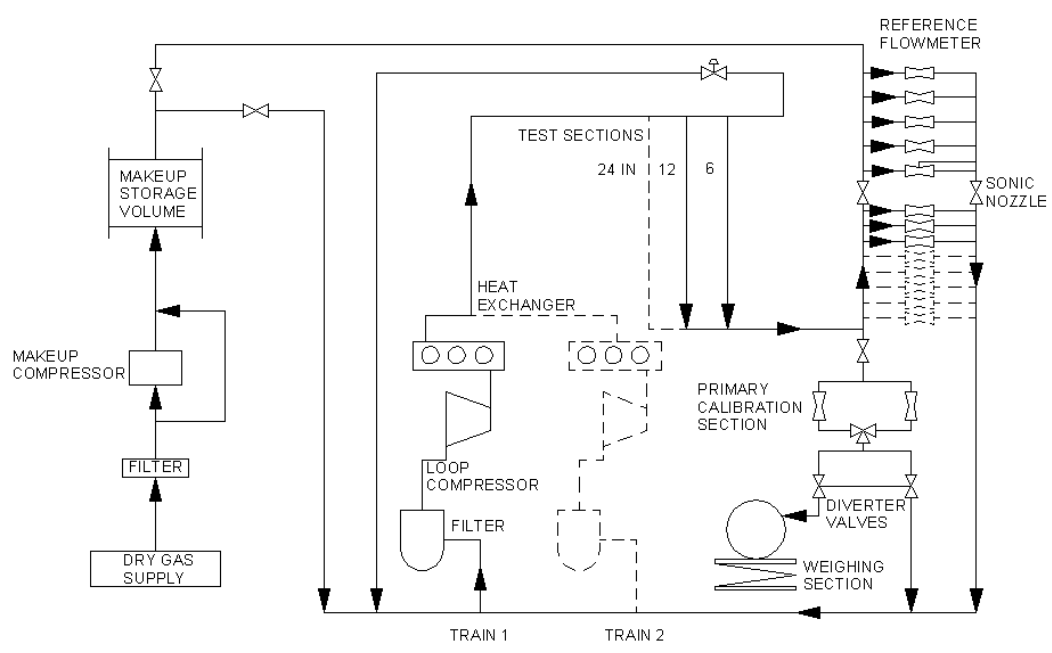

Fig. 2.14- Schematic of K-Lab test loop 
The K-Lab facility includes a main control room, two centrifugal compressors with a 4,023 hp driver, and buildings for flow metering and primary calibration system. The test area can accommodate straight pipe lengths up to $328 \mathrm{ft}$ where fully modular 6 , 12 and 24 in. pipe diameter test sections are located for equipment testing purposes.

The two centrifugal compressors and associated cooling train are installed to provide a maximum volume flow rate of 318,812.96 scfm, and corresponding maximum mass flow rate of $287 \mathrm{lb} / \mathrm{s}$. Dry mechanical seals in the gas compressors are installed to provide an oil-free environment for the gas phase, that if present, would probably alter the calibration of the sonic nozzles in an indeterminate manner, and gradually alter the composition of the gas in the loop as the test proceeds.

As previously indicated, devices in the test section are compared against a bank of sonic nozzles which are used as reference flow meters. These sonic nozzles are individually calibrated within the loop using a gravimetric primary calibration system. The main operating specifications of the facility are summarized in Table 2.12.

Table 2.12- K-Lab operating specifications ${ }^{29}$

\begin{tabular}{|l|l|}
\hline Design pressure, $\mathrm{psi}$ & 2611 \\
\hline Maximum test section pressure, $\mathrm{psi}$ & 2263 \\
\hline Maximum mass flow rate per compressor, $\mathrm{scfm}$ & $159,406.48$ \\
\hline Gas temperature, ${ }^{\circ} \mathrm{F}$ & 68 to 140 \\
\hline Modular test sections, in. & 4 to 24 \\
\hline Reference flowmeter & Sonic nozzles \\
\hline Accuracy, $\%$ & \pm 0.25 \\
\hline
\end{tabular}

The K-Lab loop has a diverter system based on two 3 in. ball valves having a common actuator used to divert the flow from the test loop into the weigh tank and back again during a primary calibration of a sonic nozzle. The maximum design diversion time is set at $0.030 \mathrm{sec}$.

The mass of gas is obtained by weighing the emptied tank and the tank full of gas respectively. To carry out this each time, the weigh tank must be disconnected from the pipework so that it is freely floating on the balance. 
To measure density, Solatron 7810 or 7811 density meters in pockets in the inlet header to the primary calibration section are used. This measure is then corrected using measured pressure, and temperature to obtain the value of density just upstream of the sonic nozzle being calibrated. The main purpose of K-Lab is to carry out accurate calibration of gas-flow meters with an uncertainty in mass flow measurement of less than $0.25 \%$.

A computerized system is used to acquire the data from the scientific instruments around the loop during the latest run. In addition, this system produces test reports, calibration reports, keeps track of instrument calibrations, and has an archiving system to store safely all the results.

\subsubsection{Boussens flow loop}

The two phase flow test loops was designed and built with the objective of gathering data to obtain more realistic and precise models in two-phase fluid flow in horizontal, inclined and vertical pipes. Fig. 2.15 shows the facility layout.

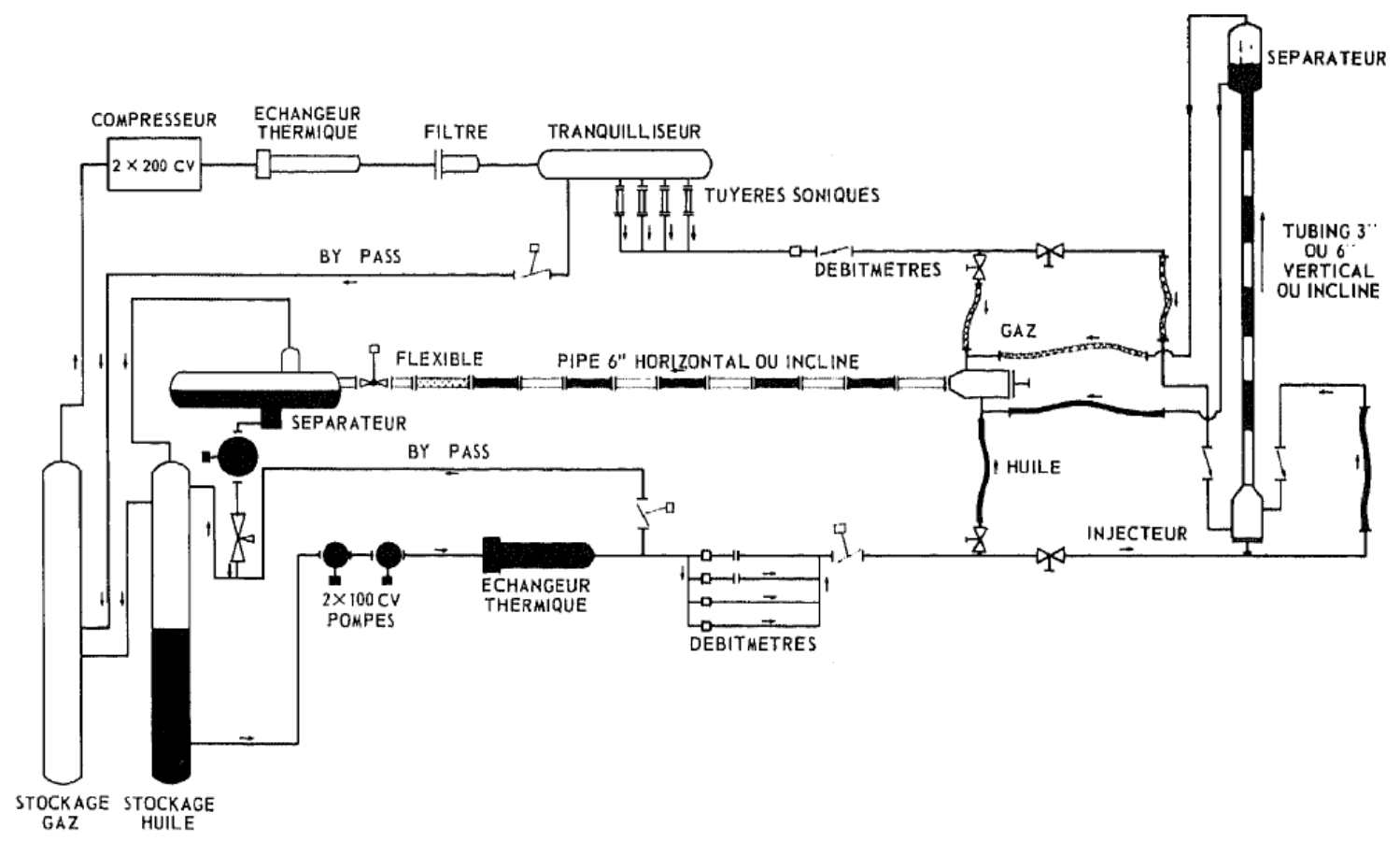

Fig. 2.15- Flow circulation diagram ${ }^{30}$ 
The loop works with two liquid hydrocarbons under pressure used so as to cover the widest possible range of Reynold numbers. A light condensate (specific weight 43 $\mathrm{lb} / \mathrm{ft}^{3}$, viscosity $\left.0.2 \mathrm{cP}\right)$ and a heavy gasoil $\left(25^{\circ} \mathrm{F}\right.$, specific weight $56 \mathrm{lb} / \mathrm{ft}^{3}$, viscosity $20 \mathrm{cP}$ ). The natural gas used consists of about $90 \%$ of methane.

The facility covers a wide domain of parameters such as: slopes, flow speeds, gas oil ratio (GOR), specific weights, viscosities and interfacial tension of the fluids. The installation was completed in 1981 by a second loop with a diameter of 3 and 6 in. intended for testing multiphase flow in vertical and highly inclined pipes to model fluid behavior in oil wells.

The configuration of the system and the operating conditions in the loop have allowed recognizing the characteristics of the principal flow regimes encountered in multiphase flow such as: stratified, annular, slug and bubble flow which equally contribute to set up the specifications of the loop for fluid testing and the specifications for the instrumentation required. The principal characteristics of the loop are:

- $\quad$ Nominal diameter 6 in. (inside diameter 5.748 in.)

- Length of the loop: $394 \mathrm{ft}$ slope adjustable of +7 until 5\% (or +4 until $-3^{\circ}$ )

- $\quad$ Maximum working pressure: 725 psi

These features are optimum from a research standpoint since the operating conditions and hardware are similar with most of the production tubing diameters used in fluid production and transportation, considering safety, precision in measurements and cost issues. The length of the loop, is the one needed to allow for the formation and measurement of the multiphase flow regimes encountered in common horizontal pipes. The chosen slopes remain variable with the construction of a metallic framework inclinable and easily maneuverable. At last, the working pressure allows the location of portholes for observation of flow regimes facilitating the interpretation of the signals and the elaboration of multiphase flow regime maps.

The loop is constituted by steel pipes with a thickness 0.433 in. assembled by flanges and supported by a metallic structure constituted of 4 articulated sections 
(see Fig. 2.16). A flexible pipe of $98 \mathrm{ft}$ rolled up in a half spiral shape allows regulating the slope without dismantling the loop.

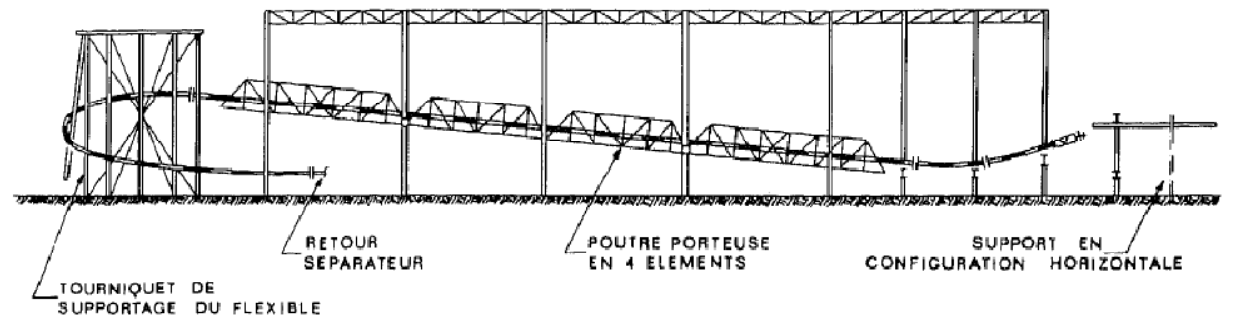

Fig. 2.16- Supporting and hoisting framework for the 6 in. test pipe $\mathrm{e}^{30}$

The vertical section of the loop accommodates tubing with nominal diameter of 3 and 6 in. The length of the section is $82 \mathrm{ft}$ with a slope adjustable of 0 to $90^{\circ}$ without dismantling the system. These features result from a deep dimensional analysis that showed the need to operate the loops in similar conditions to those found in real wells where tubings have common diameters in the order of $3 \mathrm{in}$. and have at least $82 \mathrm{ft}$ in length so that flow regimes can be established during the experimentation.

The 2 testing tubings are installed simultaneously on a beam in a metallic structure of $98 \mathrm{ft}$ long and $10 \mathrm{ft}$ wide, equipped with access while the experiments are run. Two hydraulic jacks of 90 tons allow inclining the structure from the horizontal to the vertical axis in $15 \mathrm{~min}$. A balanced weight of 17 tons allows balancing the structure to maintain the chosen position. Fluid injection and return flow from the vertical section are possible by means of a flexible connection in the $6 \mathrm{in}$. horizontal section. The flow speed in the 3 in. tubing can attain $66 \mathrm{ft} / \mathrm{s}$ for the gas and $33 \mathrm{ft} / \mathrm{s}$ for the oil simultaneously.

The separator, compressor, and fluid flow regulation and supply are set up based on the following required operating conditions in the loop:

- Pressure: between 73 and 725 psi while experiments are being conducted

- Oil flow rate (light condensate or heavy gasoil) from 0 to 30,191 bpd

- Natural gas flow rate from 0 to $235 \mathrm{scfm}$ at testing conditions. 
Two tanks are used to store and supply the gas and the oil to the flow loop. The oil is injected using two-100 hp Centrifugal pumps. A heat exchanger is located downstream of the centrifugal pumps before the liquid arrives at the horizontal-inclined or vertical section. As for the gas phase, two $200 \mathrm{hp}$ centrifugal compressors are installed in place from which air departs and goes through a heat exchanger and filters before arriving at the tranquilizer. Two separators are located in the facility, one at the top of the vertical section and one downstream of the horizontal section. The separators count with great capacity and thermal inertia to stabilize the operating pressure and temperature assuring the thermodynamic balance of the oil and gas phases.

Measurements of oil and gas flow rates that are very influential parameters for experimentation purposes are performed simultaneously by a large quantity of flow meters. A mini-computer manages the acquisition of the measures of about 40 sensors located in the process to gathered data related to pressure, temperature, flow rates, level of fluids in the separator, etc.

\subsubsection{IFP multiphase flow loop}

The facility was designed as a closed loop equipped with a multiphase pump driven by a variable speed motor. Fig 2.17 shows the layout at IFP.

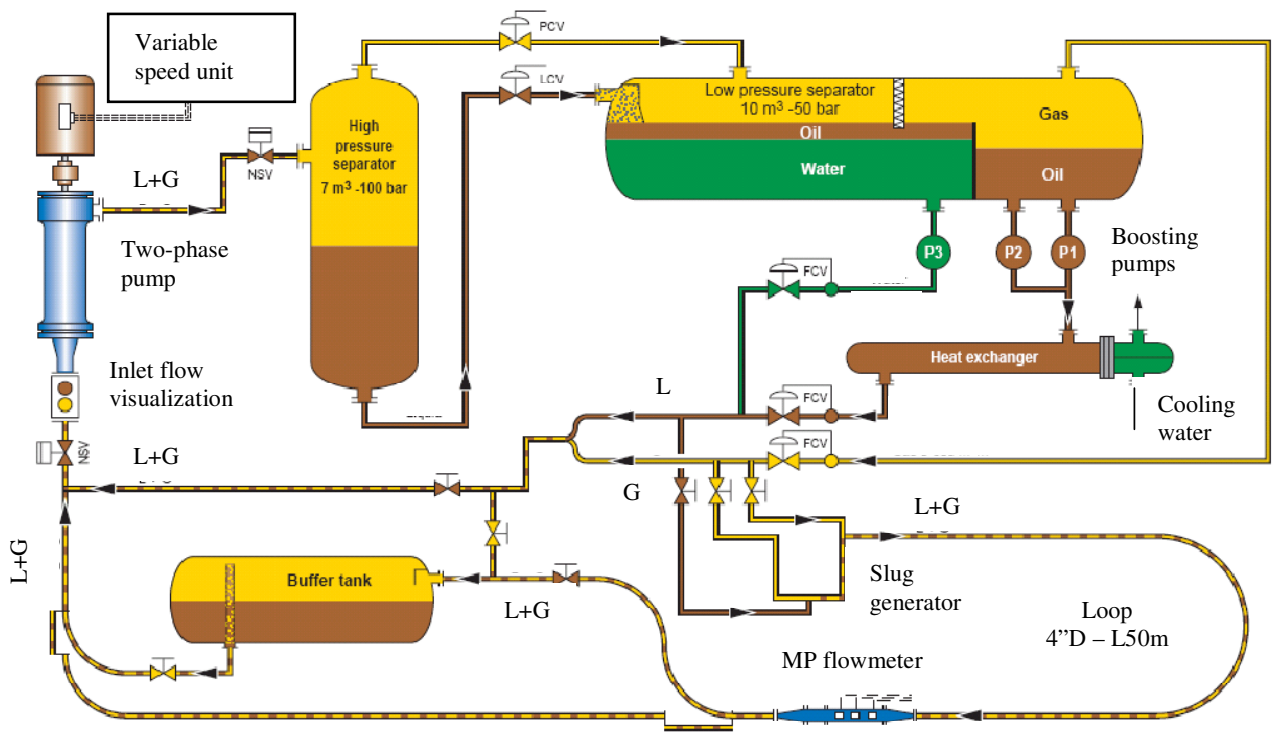

Fig. 2.17- IFP multiphase flow loop layout ${ }^{31}$ 
Two large capacity separators are set up in series downstream of the pump to ensure multiphase flow separation. The horizontal separator is a tri-phase separator therefore a liquid phase of two components is achievable. Testing fluids include domestic fuel oil, water, and nitrogen or methane.

Three boosting pumps are placed downstream of the horizontal separator to increase liquid flow rates if necessary and the temperature, in the case of the oil phase, can be controlled using a water heat exchanger. The test section is $164 \mathrm{ft}$ in length with a pipe internal diameter of 4 in. The loop also comprises a system of valves and forks used to drive the multiphase mixture towards different configurations of the loop. Additional equipment includes a pool for underwater testing, a buffer tank and a slug generator.

The facility can be operated within a range of static pressures from 44 psi up to 725 psi which allows reproducing various multiphase flow regimes. The flow rates of the liquid and gas phase are independent of the static pressure chosen for testing since the flows are circulated in a closed loop. Total liquid flow rates from 23 to $584 \mathrm{gpm}$ and gas flow rates up $208 \mathrm{scfm}$ at line conditions can be measured.

\subsubsection{The LOTUS rig}

The LOTUS (LOng TUbe System) rig facility was installed at Imperial College in 1992 and spans four floors of the Pilot Plant Laboratory in the Chemical Engineering Department. The multiphase flow loop counts with a long vertical copper tube with an internal diameter of $1.25 \mathrm{in}$. and length of approximately $34.45 \mathrm{ft}$. The entire tube length is made up of sections that are connected together by flanges to accommodate various measurement devices. To provide the alignment and straightness of the vertical section of the loop, a rigid carbon steel beam was conveniently installed at the top of the facility.

Originally, the system was fed with air and water from the bottom to the top. The air is provided from the college main supply at a pressure of 90 psi passing through orifice plates used to meter the air flow rate by means of differential pressure transducers. Water is pumped, either by a 2 or an $11 \mathrm{hp}$ pump from a tank to the system following a flow metering step via flow rotameters. The two phase mixture is passed 
from the tube sections back to the water tank which also serves as separator, where air is then discharged to the atmosphere and the water is recirculated throughout de system. Fig. 2.18 depicts the configuration of the LOTUS rig.

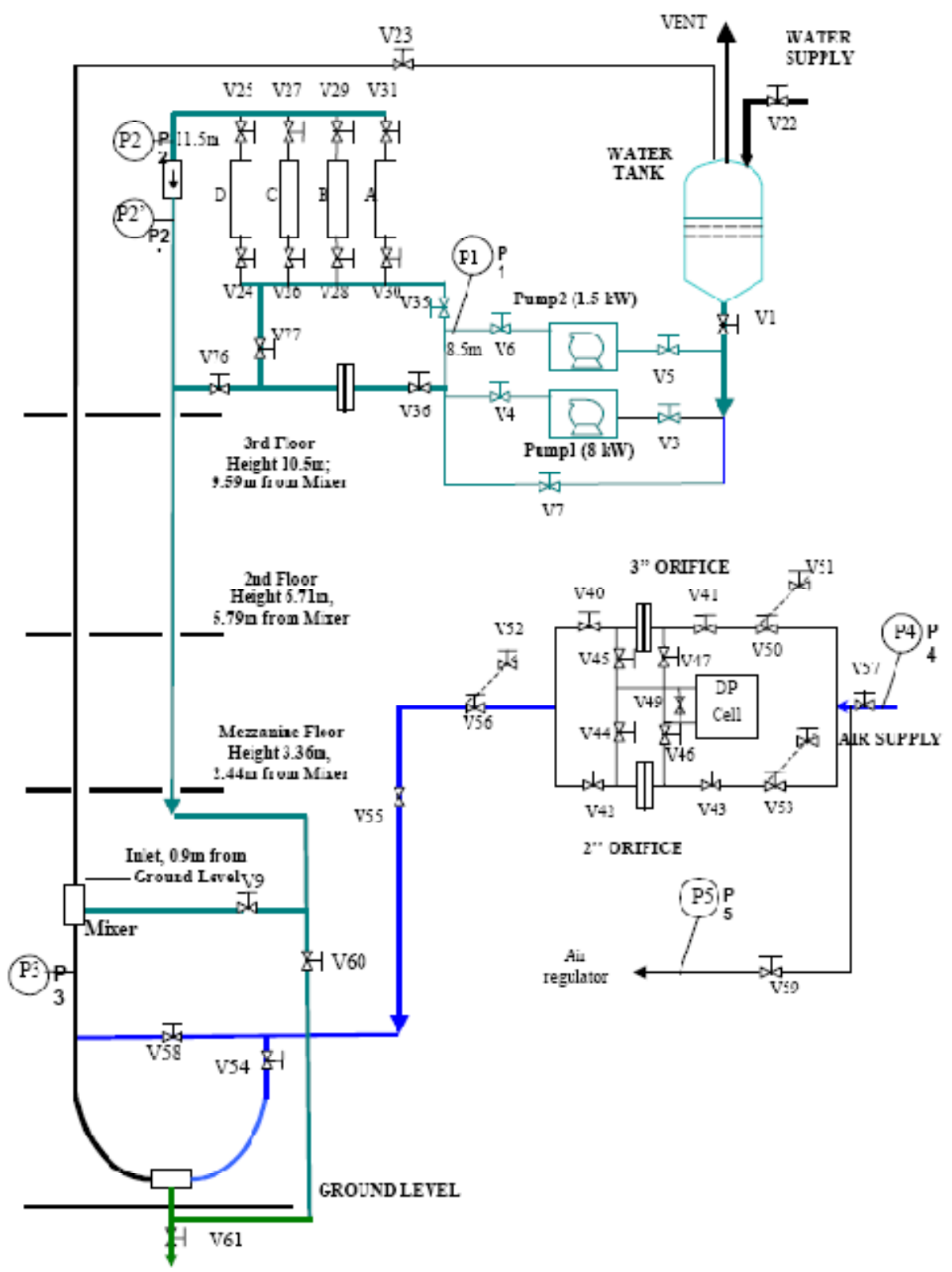

Fig. 2.18- The LOTUS rig $^{32}$

Even though this facility does not work with medium-high pressures (400 to $700 \mathrm{psi}$ ), a major retrofit proposed by Constantini et al. ${ }^{33}$ involved providing 
water injection into the system using compressed air through a pressure vessel (rated at $90 \mathrm{psi}$ ) to simulate the reservoir. In addition, to account for permeability variations in the reservoir, the idea was to use a cylindrical porous medium contained in a pressurized vessel to reproduce the near-wellbore region. Fig. 2.19 illustrates idea behind the proposed modification.
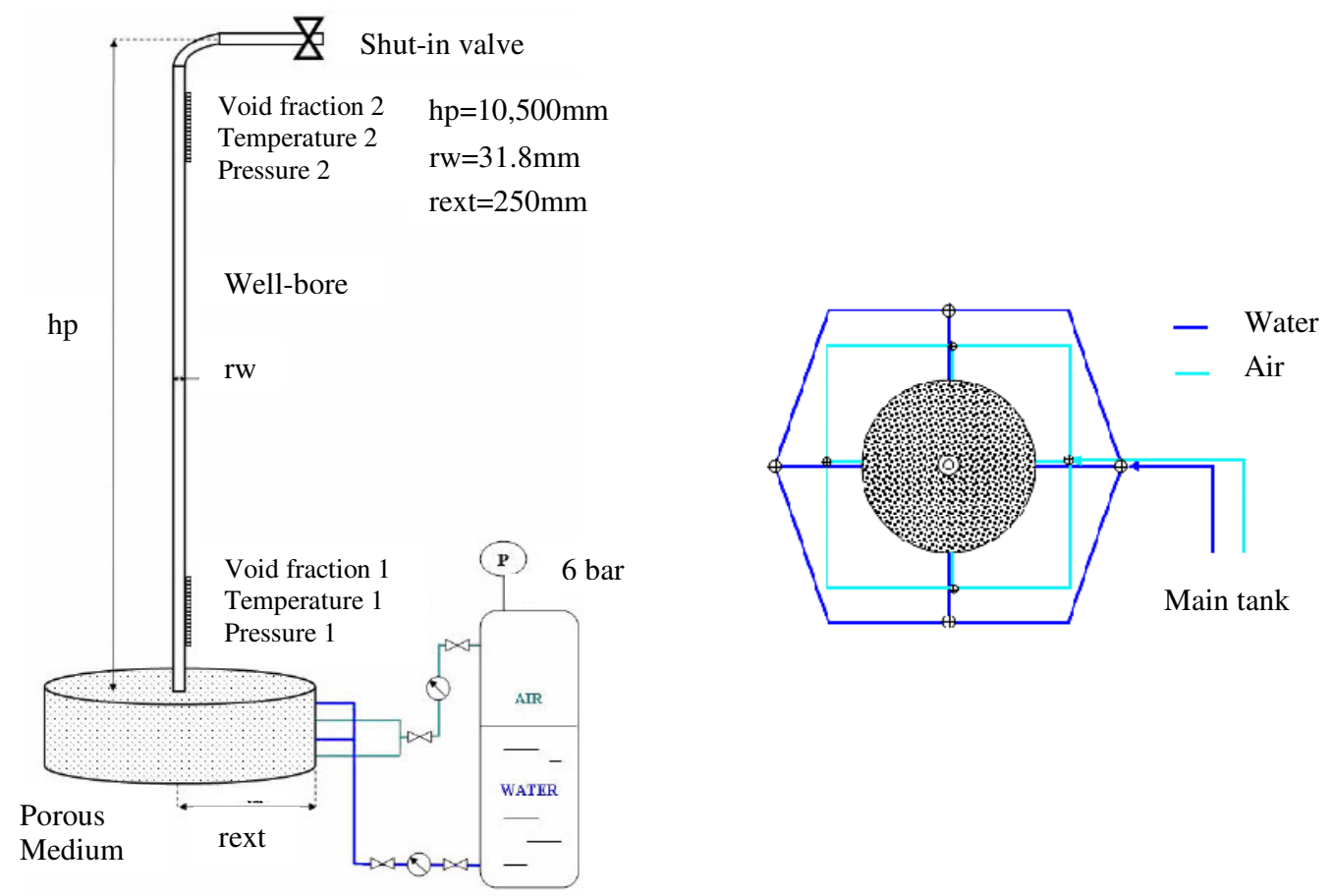

Fig. 2.19- Schematic layout of the proposed modification of the LOTUS rig

An option for the artificial porous medium was to use glass beads accurately located in a Plexiglass cylinder of $5 \mathrm{ft}$ in length and 20 in. internal diameter. The cylinder would also have four symmetrical entrance points for the inflow of water and gas from the main tank, positioned alternately along the height of the vessel to ensure proper distribution and dispersion of the flowing phases. An internal cylinder core, comprised of a slotted screen, would provide a no-return barrier for the micro glass beads and represent a perforation interval effect to the flow.

Air and water flows could be monitored by turbine meters and stabilized to the required rates by opening the regulation valves. After the flow stabilizes, the surface 
valve could be closed to simulate the well shut-in. Consequently the well inflow would be expected to decay as a function of the pressure drop that occurs through the porous medium as the top pressure rises.

Despite the interesting approach behind Costantini's et al. ${ }^{33}$ proposal, no modifications to the LOTUS rig have been carried out up to this date.

\subsubsection{TAMU TowerLab}

The TowerLab at the Joe C. Richardson building in the petroleum engineering department starts from the basement and stretches $140 \mathrm{ft}$ almost covering the entire height of the building. It has an available surface area of $56 \mathrm{ft}^{2} / \mathrm{floor}$ for piping and instrumentation (see Fig. 2.20). These unique features of the TowerLab facility present an attractive opportunity to conduct innovative research in areas related to integrated oil and gas production systems.

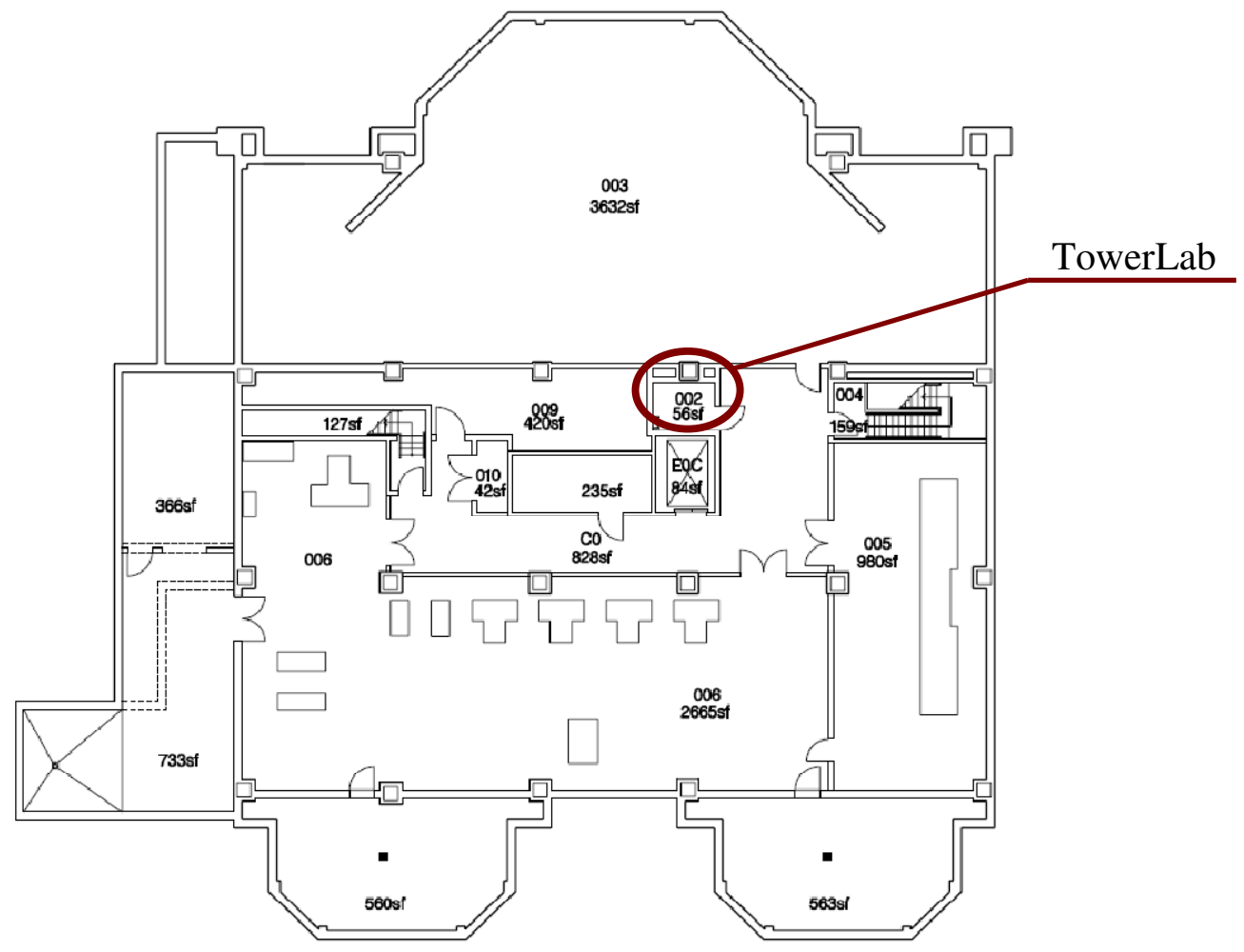

Fig. 2.20- TowerLab stretches through 10 floors of the Richardson Building, beginning in the basement under the first floor shown here 
Currently the maximum working pressure at the TowerLab is 120 psi; however, this investigation proposes an upgrade in terms of higher operating pressures and flow rates for the testing fluids to be used.

\subsection{Summary and comparison of hardware and major equipment of exiting multiphase research flow loops}

Table 2.13 illustrates the maximum pressure as well as the equipment used to handle the liquid and gas phase for the flow loops described in this investigation.

Table 2.13- Major equipment for liquid injection and gas compression

\begin{tabular}{|l|c|c|c|}
\hline Flow loop & $\begin{array}{c}\text { Maximum } \\
\text { Pressure, } \\
\text { psi }\end{array}$ & Liquid phase & Gaseous phase \\
\hline $\begin{array}{l}\text { SouthWestern } \\
\text { Reseach Institute } \\
\text { (SWRI) }\end{array}$ & 3600 & Centrifugal pumps & Centrifugal compressor \\
\hline $\begin{array}{l}\text { NORSK } \\
\text { HYDRO }\end{array}$ & 1595 & $\begin{array}{c}\text { Centrifugal pumps and twin } \\
\text { screw pump (for oil) }\end{array}$ & Centrifugal compressor \\
\hline CEESI & 1500 & Positive displacement pump & $\begin{array}{c}\text { 4 stage Reciprocating } \\
\text { compressor + 4 single } \\
\text { stage reciprocating } \\
\text { compressors }\end{array}$ \\
\hline SINTEF LSL & 1305 & Centrifugal pump & Reciprocating compressor \\
\hline NEL & 910 & Centrifugal pump & Centrifugal gas blower \\
\hline Boussens & 725 & Centrifugal pumps & Centrifugal compressor \\
\hline IFP & 725 & \multicolumn{2}{|c|}{ Two-phase pump pump } \\
\hline IFE & 145 & $\begin{array}{c}\text { Centrifugal and dosage pumps } \\
\text { and screw pump }\end{array}$ & $\begin{array}{c}\text { Multiphase pump (twin } \\
\text { screw) }\end{array}$ \\
\hline SINTEF MSL & 117 & Centrifugal pumps & $\begin{array}{c}\text { Multiphase pump (twin } \\
\text { screw) }\end{array}$ \\
\hline LOTUS & 90 & Centrifugal pumps & Compressed tank \\
\hline
\end{tabular}

Fig. 2.21 shows the vertical height for the multiphase research flow loops mentioned in Table 2.12. As indicated by Falcone et al. ${ }^{18}$, the maximum length of a flow loop affects the development of different flow regimes, particularly when transient flow is investigated. It is evident by looking at Fig. 2.21 that the vertical height of the test section available at the TAMU TowerLab is competitive when compared to other facilities. 


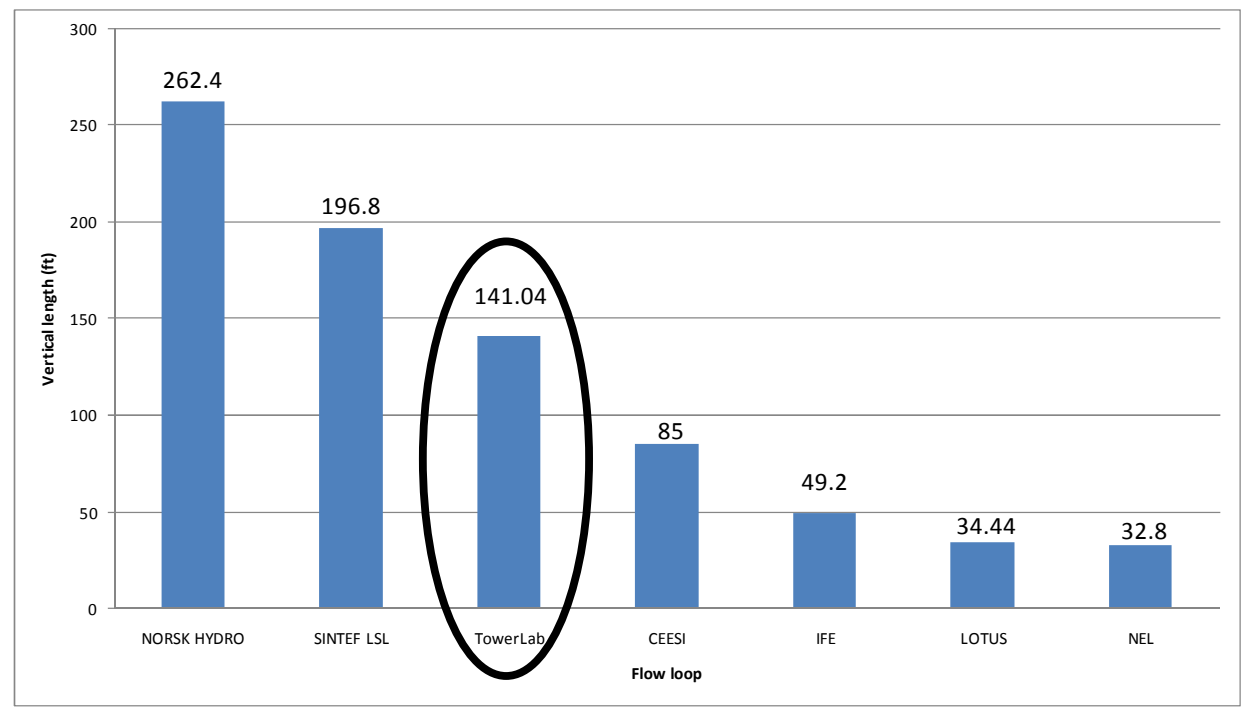

Fig. 2.21- Vertical height for different flow loops

The range of flow regimes that can be reproduced in a flow loop is related to the flow rates that can be circulated in the system. The maximum reported flow rates of gas for the flow loops identified for this study are given in Fig. 2.22. It is clear that achieving a rate, as the one indicated in Fig 2.22 for the TAMU TowerLab, would give this facility adequate conditions to conduct experiments related to gas fluid flow.

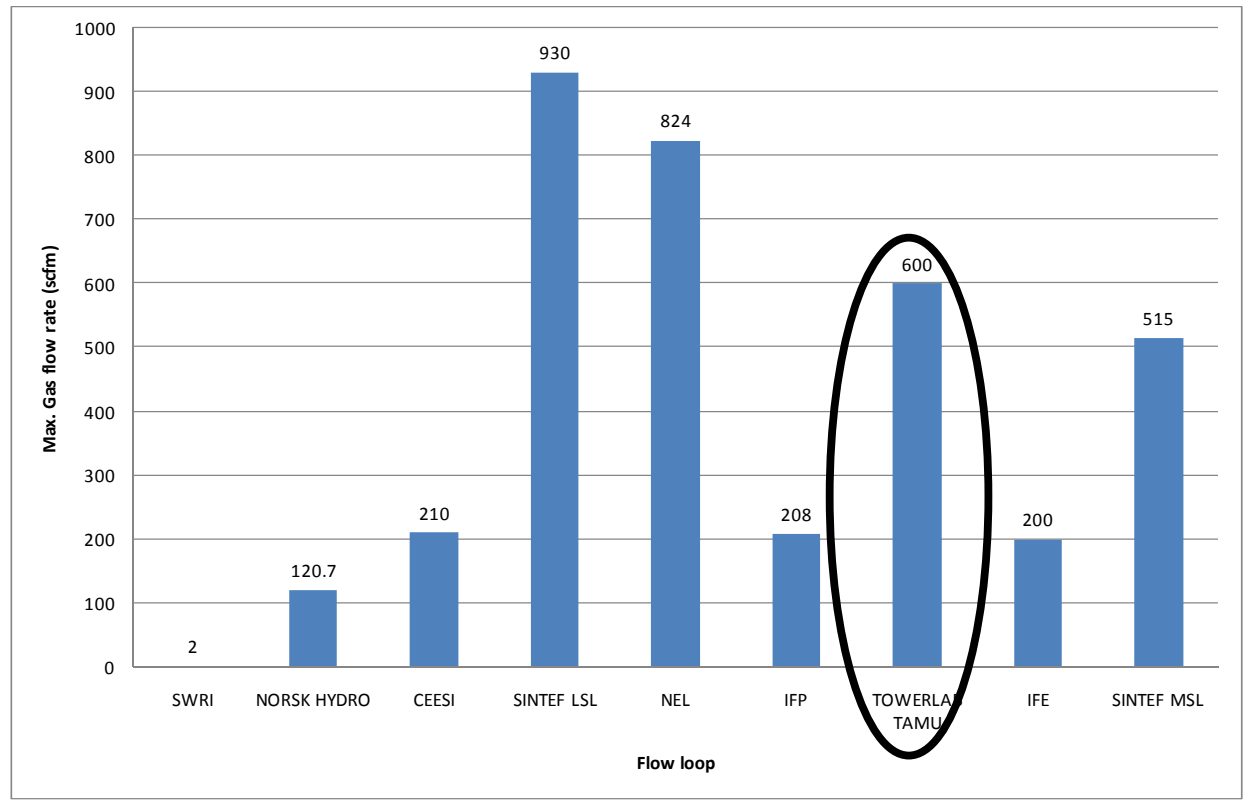

Fig. 2.22- Maximum gas flow rate for different flow loops 
In Fig. 2.23 the vertical length as a function of maximum operating pressures is presented. The highlighted portion of the graph shows that the TAMU TowerLab working within the proposed range in pressures would operate in a region not yet covered by any flow loop.

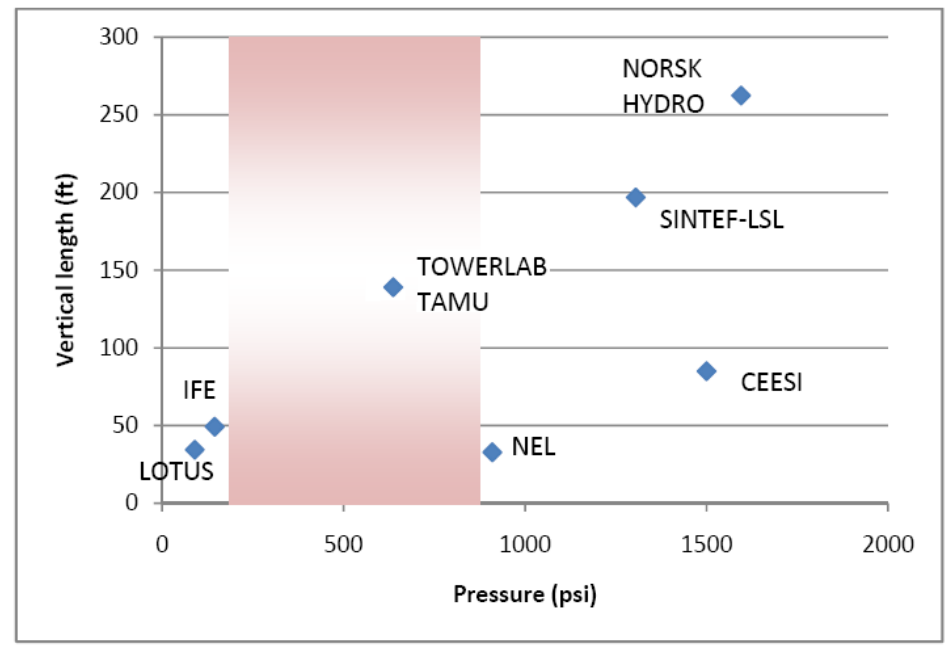

Fig. 2.23- Vertical length vs. pressure

In addition, comparing the vertical height as a function of gas flow rate in Fig. 2.24, it is noticeable that the TAMU TowerLab would also cover an area where currently available research flow loops do not operate.

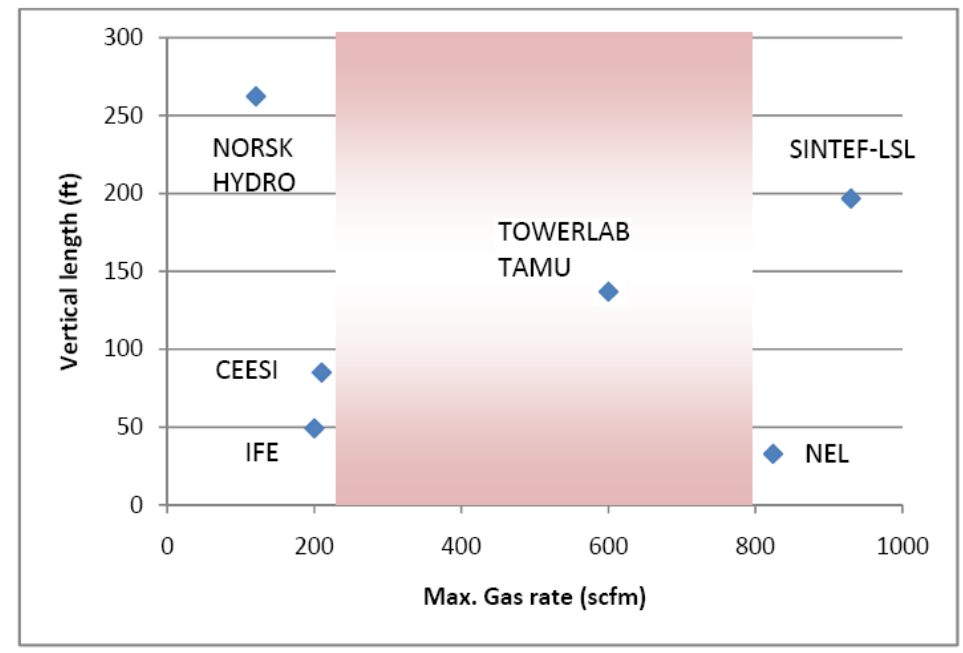

Fig. 2.24- Vertical length vs. maximum gas rate 
Results of the extensive review presented in this study indicate a niche in operating conditions in which the TAMU TowerLab could be operating; however, the final values for an experimental facility have to be selected based on the type of research to be conducted.

Since the TAMU TowerLab is intended to be used for the experimental investigation of liquid loading, values of pressure and flow rates including adequate geometry and hardware should allow mimicking the conditions that precede and follow liquid loading in a gas well. Consequently, the reality of reproducing liquid loading in a laboratory environment needs to be addressed focusing on the conditions both in operation and geometry found in real gas field which represent the basis for the downscaling process.

\subsection{Typical operating conditions in gas fields experiencing liquid loading}

When producing liquids from a gas well, the density, size and shape of the liquid droplets, mainly determine the minimum gas velocity required to lift these droplets up

the tubing string. ${ }^{34}$ However, other issues such as the hardware installed in the well and the values of pressure that may have been initially imposed at the surface may also influence the early beginning of accumulation of liquids in the wellbore.

Typical operating conditions in a gas well vary from field to field even within the same reservoir. The string design, completions, and all the hardware that may be necessary to locate downhole is designed not only to withstand the maximum loads that may be encountered throughout the life of the well at the lowest cost, but also to guarantee the maximum production of hydrocarbons. It is then the challenge for the well completion engineer to find an optimum diameter at which liquid loading can be prevented while maximizing production.

Even though the most optimum casing design and completions may have been proposed and executed in a gas well, the flow is often or will eventually be hindered due to water loading. Engineers consider the phenomena of liquid loading not only in their designs prior to drilling and completing a gas well, but also when it comes to evaluate the different strategies to manage the field. 
To determine and continuously monitor typical operating conditions, as well as, their interaction with the hardware installed in the well is a common practice in gas field management. In most cases, monitoring and estimating optimum well intervention times, lead to an increase in the corresponding ultimate cumulative gas production, hence the need to take remedial actions that prevent and delay the occurrence of liquid loading is most likely to be mandatory.

North American gas wells are, in general, relatively deep; 60\% are deeper than 5,000 ft. The depth distribution is illustrated in Fig. 2.25. Most wells are completed with relatively small casing with common sizes of 4-1/2 in. and 5-1/2 in.; however, in some cases wells are cased with $3-1 / 2$ in. or 2-7/8 casing to reduce completion costs as well as to avoid encountering liquid loading at early stages of production.

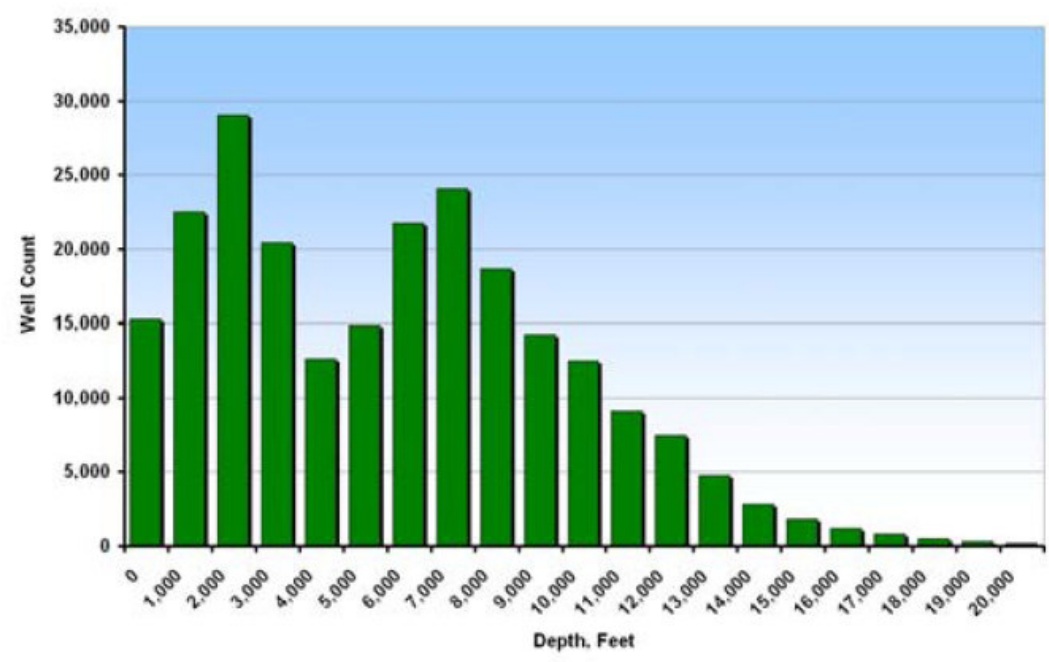

Fig. 2.25- Depth distribution of United States gas wells ${ }^{10}$

Case histories in the literature ${ }^{4}$ and in reported cases found in the SPE library, show operating conditions and hardware used in gas fields affected by liquid loading. Gas flow rates and volumes of water produced are particular for every field, but a common tendency is observed in the tubing size used in gas well completions. 
Fig. 2.26 shows a distribution of typical production tubing sizes found in 16 reported cases.

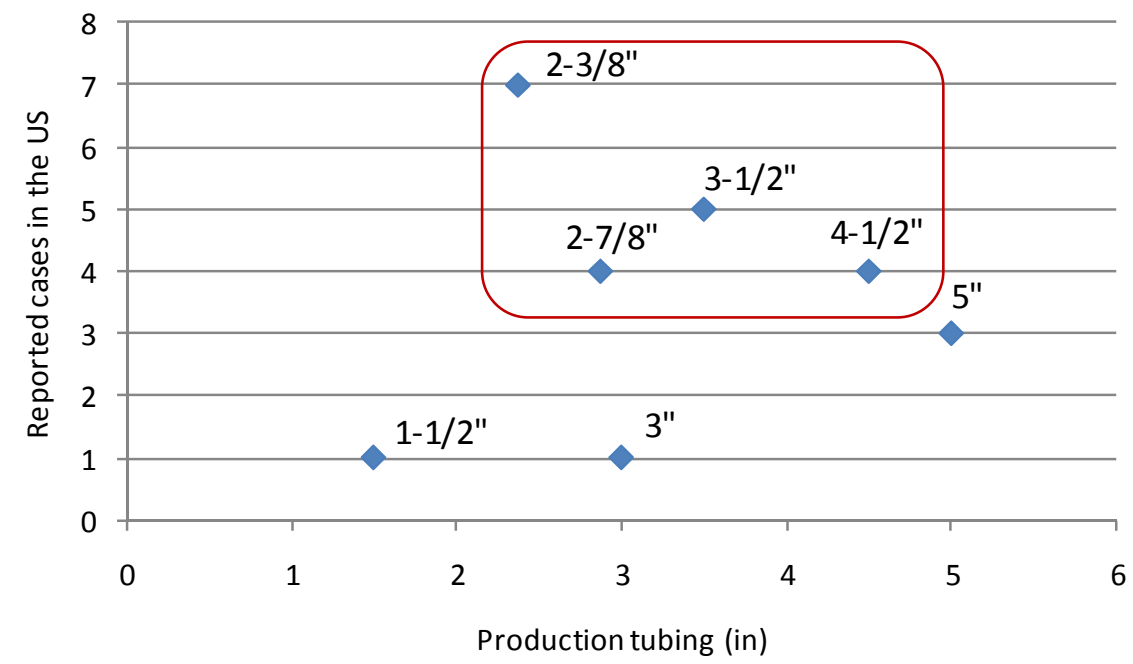

Fig. 2.26- Production tubing sizes distribution in reported cases within the US

In places like the Intermediate Shelf gas play in southwest Texas, production tubings are either 1.90 in. or 2-3/8 in., and in some wells the 2-3/8 in. have been replaced by $1.61 \mathrm{in}$. sizes to increase the gas velocity. The same strategy proved to be successful in gas wells located in the Permian Basin, where 2-1/2 in. tubings were replaced by 2 in. sizes. $^{35}$

Reducing the tubing diameter is often accompanied by some other dewatering technique, as in the case of the South Burns Chapel field in northern West Virginia, ${ }^{36}$ where gas wells required plunger lift in production tubings of 2-3/8 in.

Gas wells located in the western sedimentary basin, as well as Oklahoma in the Hugoton and Panema fields, are completed with 2-3/8 in. production tubing along with automated unloading programs. ${ }^{37,38}$

Challenges are also faced in areas such as the Wilcox formation in south Texas where Y-type completions ${ }^{39}$ (see Fig. 2.27) using production tubings of 3-1/2 in. have been used to allow successful gas production. A similar approach but with conventional completions with $3-1 / 2$ or $2-7 / 8$ in. tubing, is used to produce gas fields in East Texas. ${ }^{40}$ 

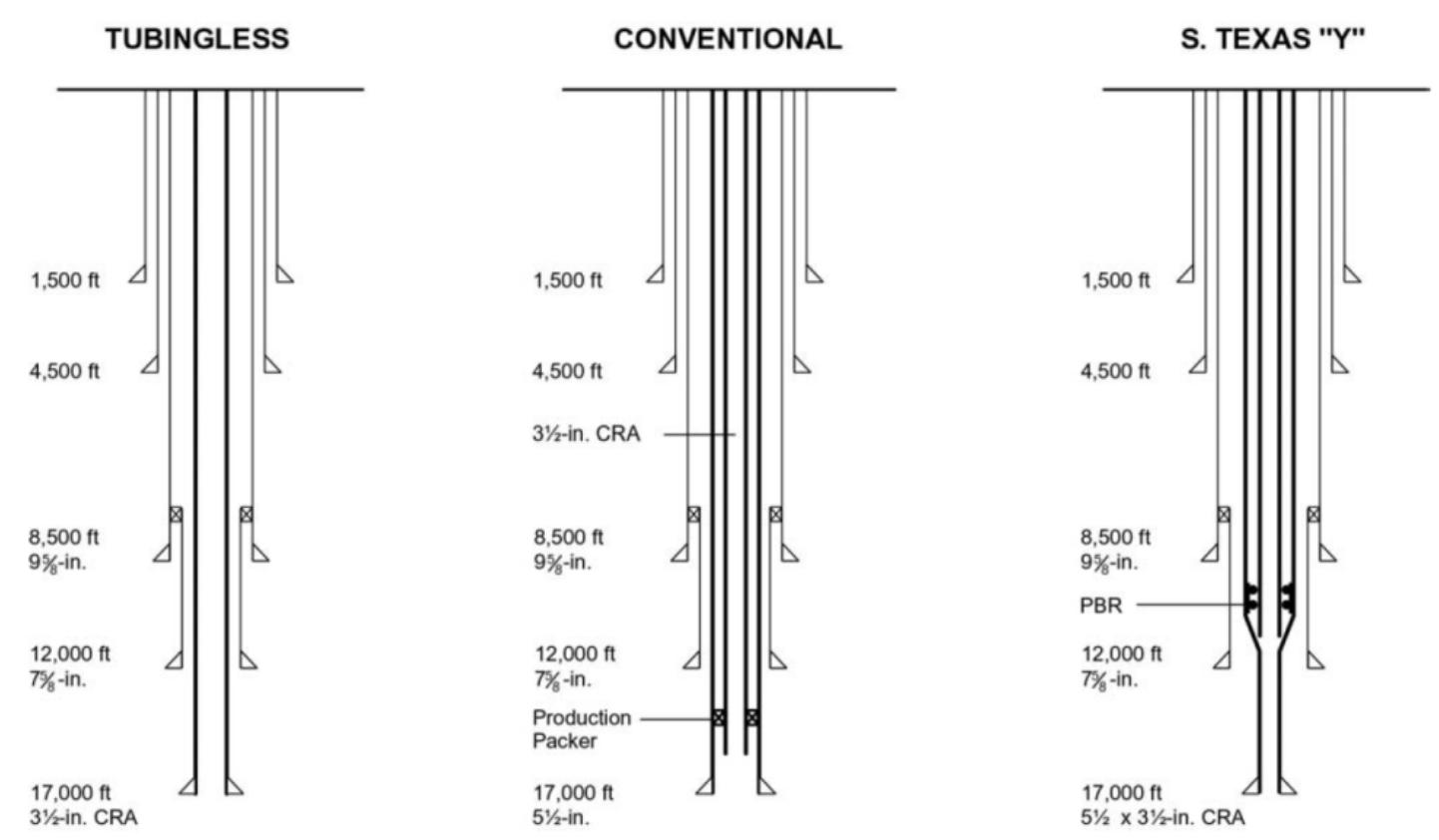

Fig. 2.27- Three commonly used well configurations in South Texas ${ }^{39}$

The reality of hardware used in gas well completions is similar when exiting the US boundaries. In South America, specifically Argentina, tubing sizes are in the range of $3-1 / 2$ to $5-1 / 2$ in. for new developments, and $2-3 / 8$ to $2-7 / 8$ in. for mature fields. In Indonesia, completions are found in the range of 2-3/8 to 3-1/2 in., and in areas like the North Sea and Australia, tubings can also be found to be 4 and 5-1/2 to 9-5/8 in. respectively.

As a summary, the optimum tubing diameter will be dictated mainly by production targets and its size is subject to replacements as field depletion occurs with time. However, from a research standpoint, as Fig. 2.26 shows, production tubing used in gas well completions within the US and other countries varies, in most cases, from $2-3 / 8$ to $4-1 / 2$ in. Therefore, if a size is to be selected for a facility that aims to study gaswell-liquid loading issues in a more realistic manner, using standard tubing included in this range is recommended. 


\subsection{The reality of liquid loading reproduced in a laboratory environment}

A flow loop is an experimental facility where a limited amount of fluid is continuously circulated in a closed loop for investigating different phenomena characterizing fluid flow in pipes. In a multiphase flow loop, each phase must have its own pump or compressor so that the flow rate can be individually controlled. Obviously this implies that there must be a mixing point through a device (usually just a specially shaped $\mathrm{T}$ or $\mathrm{Y}$-junction) and a separator to ensure that the phases are properly separated before they are once again fed into the pumps and compressor respectively. Flow rates are measured on the single phase branches of the loop just downstream of the pumps and compressor.

The mixing device, or mixing point, usually defines the starting point of the loop after which the measurement section is usually placed at different deviations from vertical through horizontal. The test section may be anything that needs to be tested, and the pipe is designed to be as long and straight as possible, therefore stable and fully developed flow is achieved.

Lea, Nickens and Wells ${ }^{4}$ describe the several flow regimes that a gas well undergoes throughout its life cycle. As illustrated in Fig. 2.28, annular mist flow is achieved at beginning of production; however, as gas rate decreases, slug-annular transition (churn flow), slug flow and bubble flow are experienced in the production tubing. The oil and gas industry still lacks an understanding of flow pattern transitions, especially transitions to churn flow that triggers liquid loading.

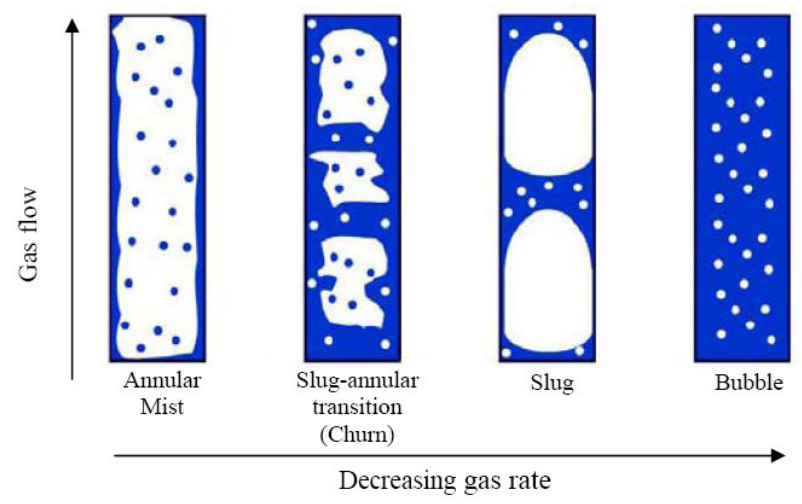

Fig.2.28- Vertical flow regimes during liquid loading (after Lea and Nickens, 2003) 
Toma et $a .^{2}$ attempted to improve the comprehensive understanding of instability effects of flow pattern transitions in oil and gas production systems from laboratory experiments and field data. The predicted flow pattern transitions were obtained using a $10 \mathrm{ft}$ long, $0.75 \mathrm{in}$. diameter tube, and 3 to $5 \mathrm{KHz}$ pressure transducers to record voltage data as a function of fluctuation in bottomhole pressure.

Although this approach is a step in the right direction, the pressure ranges of (0.72 to $0.78 \mathrm{psi}$ ) used to quantify the flow pattern transitions is not statistically significant to draw firm conclusions on the boundary criteria for flow pattern transitions that depend on pressures and densities of gas/liquid flow. Furthermore, the tubing size dimensions may have limited the full development of a flow pattern, thereby limiting range of applicability of the models developed from empirical data.

Experiments on flow pattern transitions have been carried out in relatively short tubes with the flow pattern boundaries being established by changing input flows. Since experiments are actually difficult to perform on actual wells, conditions for flow transitions are assumed similar to those occurring in short tubes with relatively constant phase flow rates. However, considerable uncertainties about flow regime behavior remain in these situations, particularly with respect to churn flow. Models developed with the appropriate physics and satisfied closure relationships could provide more understanding of liquid loading in gas wells.

\subsection{A dedicated research flow loop for liquid loading investigation}

From a research standpoint it is evident that there is a significant difference between the modeling of hydrocarbon processes in a laboratory environment compared to the existing operating conditions encountered in real oil and gas fields. However, this breach can be narrowed by designing a state-of-the-art facility operating under more realistic conditions of pressure, flow rates and temperatures as found in real gas wells. Nevertheless, one has to realize that the exact representation of an existing field is difficult to be entirely accomplished.

Flexibility is an important characteristic the multiphase flow loop should incorporate at an early stage of the design; however, as a result of the high capital 
investment needed to build a facility to study multiple problems within the area of multiphase flow, making a flow loop $100 \%$ flexible is neither technical nor economically feasible. The approach by most laboratories is to design and build their facilities establishing a range in operation (pressure and temperature) and fluids to be handled by their flow loops.

Current models applied within the area of multiphase flow have been obtained and validated via research flow loops. However, new investigations are continuously being carried to out to improve existing correlations or to propose new methods. This creates a need for more problem-oriented research flow loops.

A dedicated facility for the experimental investigation of liquid loading is proposed in this study. The range in operating conditions has been identified based on a review of multiphase flow loops around the world. These values are validated in Chapter III guaranteeing that conditions that precede and follow liquid loading can be achieved.

Fig. 2.29 shows the proposed downscaling of a gas well completion for the TAMU TowerLab.
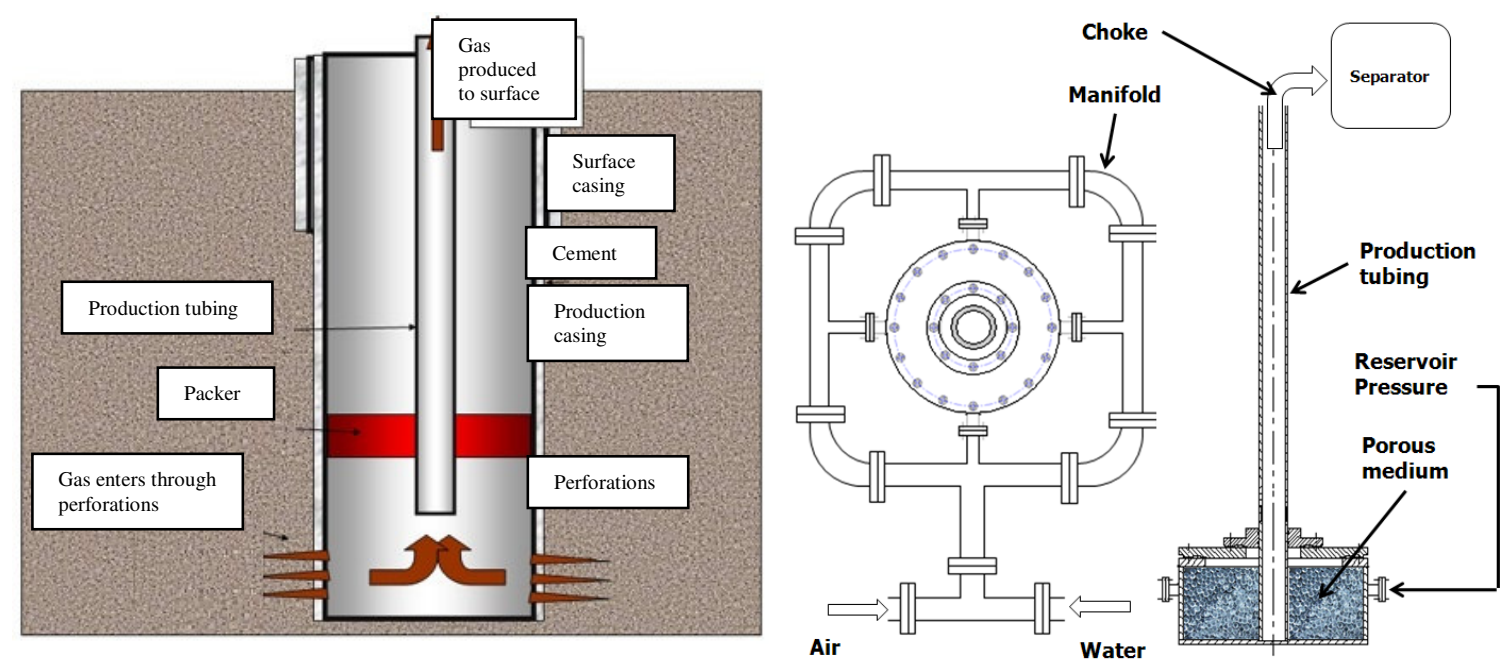

Fig. 2.29- Downscaling of a gas well at the TowerLab

To recreate the effect of the near-wellbore region, an experimental option is to attach a cylindrical pressure vessel containing tightly packed spherical glass beads to the 
base of the vertical multiphase flow loop. Glass beads are fairly uniform in grain size, low cost, readily available, and evidence of their use to conduct fluid flow experiments has been well documented. ${ }^{41,42}$ Average porosity is in the order of $38 \%$, with a range in permeability from 1.2 to 29 darcy. The permeability of glass beads is dependent on the size and packing of the beads. Fig. 2.30 is a front view section of the pressure vessel containing glass beads tightly packed.

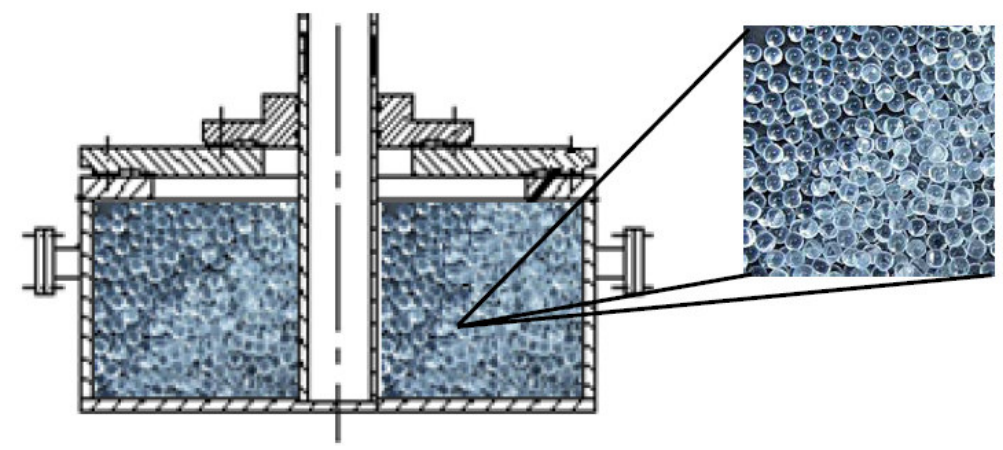

Fig. 2.30- Pressure vessel to recreate effects of the near-wellbore region

Flow of fluids into the porous medium may be provided by entry points symmetrically aligned at the pressure vessel by means of a distribution mechanism that ensures proper dispersion of the fluids. Fig. 2.31 illustrates the use of a single injection manifold where the testing fluids would mix prior their arrival at the pressure vessel.

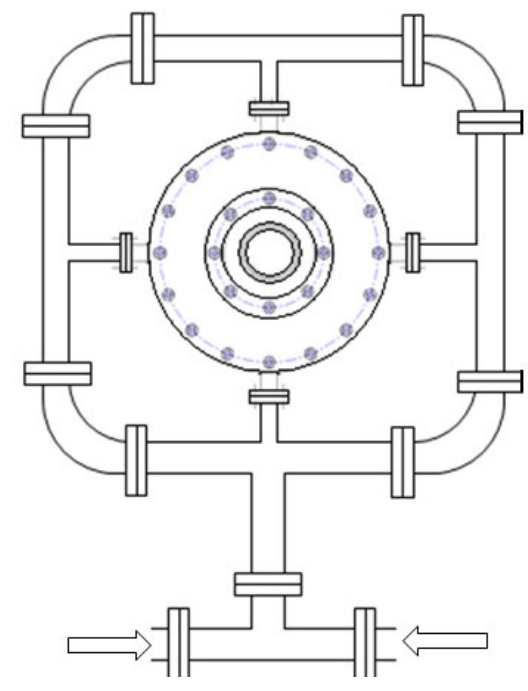

Fig. 2.31- Distribution mechanism 
The phases initially considered as testing fluids are air and water. This will demand using a compression and boosting system capable of delivering the fluids at the required operating conditions. Control and monitoring will be performed using flow or mass meters. During transient flow periods, measurements of void fraction, temperatures and pressures will have to be measured at different locations across the flow loop.

The assembly is designed as to attempt mimicking the dynamics of the near wellbore region to provide boundary conditions for the development of predictive transient multiphase flow models. 


\section{CHAPTER III}

\section{DESIGN OF A NEW DEDICATED FACILITY FOR THE INVESTIGATION OF LIQUID LOADING IN GAS WELLS}

Chapter II summarizes the proposed configuration of the research flow loop for TAMU TowerLab. The comparison of operating parameters between research facilities around the world identified a niche for the values in pressure and flow rates to be handled by the facility; however, this required further verification to design the mechanical elements in the loop and assure the proper selection of the equipment in charge of supplying the testing fluids.

\subsection{Design factors for mimicking liquid loading at TAMU TowerLab}

Solomon et $a .^{17}$ conducted a sensitivity analysis using a commercial wellbore/reservoir simulator along with Response Surface Methodology (RMS) to minimize cost and investigate different design parameter settings to recreate liquid loading under laboratory conditions. Table 3.1 presents the design variables investigated which included tubing diameter sizes, glass beads packing permeability, cylindrical pressure vessel dimensions, and operating pressures.

\section{Table 3.1- Design factors $^{17}$}

\begin{tabular}{|l|c|}
\hline Factor & Numerical range \\
\hline Permeability, darcy & 0.01 to 6,000 \\
\hline Tubing diameter, in. & 2.72 to 4.5 \\
\hline Compressor discharge pressure, psia & 470 to 650 \\
\hline Pressure vessel radius, $\mathrm{ft}$ & 1 to 6 \\
\hline Pressure vessel height, $\mathrm{ft}$ & 0.7 to 3 \\
\hline Water Gas ratio, stb/MMscf (constant) & 3,300 \\
\hline Surface choke pressure, psia & 20 to 400 \\
\hline
\end{tabular}

A range in glass bead packing permeability of 0.01 to 6,000 darcy accommodates different sizes of glass beads and their packing orientation. While conducting the study, it was realized that low-permeability ranges ( 0.01 to 0.05 darcy) resulted in low air-flow 
rates (300 to $600 \mathrm{scf} / \mathrm{D}$ ), whereas the higher range (500 to 6,000 darcy) yielded high flow rates (5 to 7MMscf/D) where frictional and turbulence effects are of concern. ${ }^{16}$

The typical diameter of glass beads used to perform fluid flow experiments ranges from $250 \mu \mathrm{m}$ to $3 \mathrm{~mm}$, with permeability and porosity values of 29 to 1,200 darcy units and 38 to $46 \%$ respectively.

The premise behind the tubing size diameters investigated was that they had to be typical for gas-producing wells. Nonetheless, this required the analysis on the effect of the deliverability of the system, considering other variables.

A compressor discharge pressure range of 470 to 650 psi was considered to evaluate the impact on the flow potential of the system including a constant Water Gas Ratio fixed at 3,300 STB/MMscf.

For the design of the pressure vessel, it was important to make optimal use of the available area at the basement $\left(56 \mathrm{ft}^{2}\right)$. The dimensions of the vessel had to be carefully analyzed to guarantee safe operations, and adequate space for the installation of flow metering and pressure monitoring devices. As for the surface chokes, a range of 20 to 400 psi was investigated in combination with other system design factors.

A multiple response optimization covered in appendix $\mathrm{C}$ identified options to minimize cost and maximize system performance. The recommended design factor combination was to use 3 in. tubing with a 500 psi compressor and cylindrical pressure vessel dimensions of radius $4.85 \mathrm{ft}$ and a height of $2 \mathrm{ft}$. These parameters were used to perform system calculations using the wellbore/reservoir simulator to validate their applicability to simulate liquid loading in TAMU TowerLab. Appendix C includes the results from generating IPR (Inflow Performance Relatinship) and VLP (Vertical Lift Performance) curves which confirmed the feasibility of using the parameters considered in the optimization process.

\subsection{Defined range in operating conditions for TAMU TowerLab}

A compressed air system providing a discharge pressure between 470 to 650 psi with gas rates in the order of 400 to $650 \mathrm{scf} / \mathrm{min}$ along with water injected at a rate of $100 \mathrm{gpm}$ was considered by Solomon et al. ${ }^{17}$ This study considered the conditions of the 
fluids measured at the nozzle ports located on the external entry points of the pressurized vessel. Fig. 3.1 shows the location.
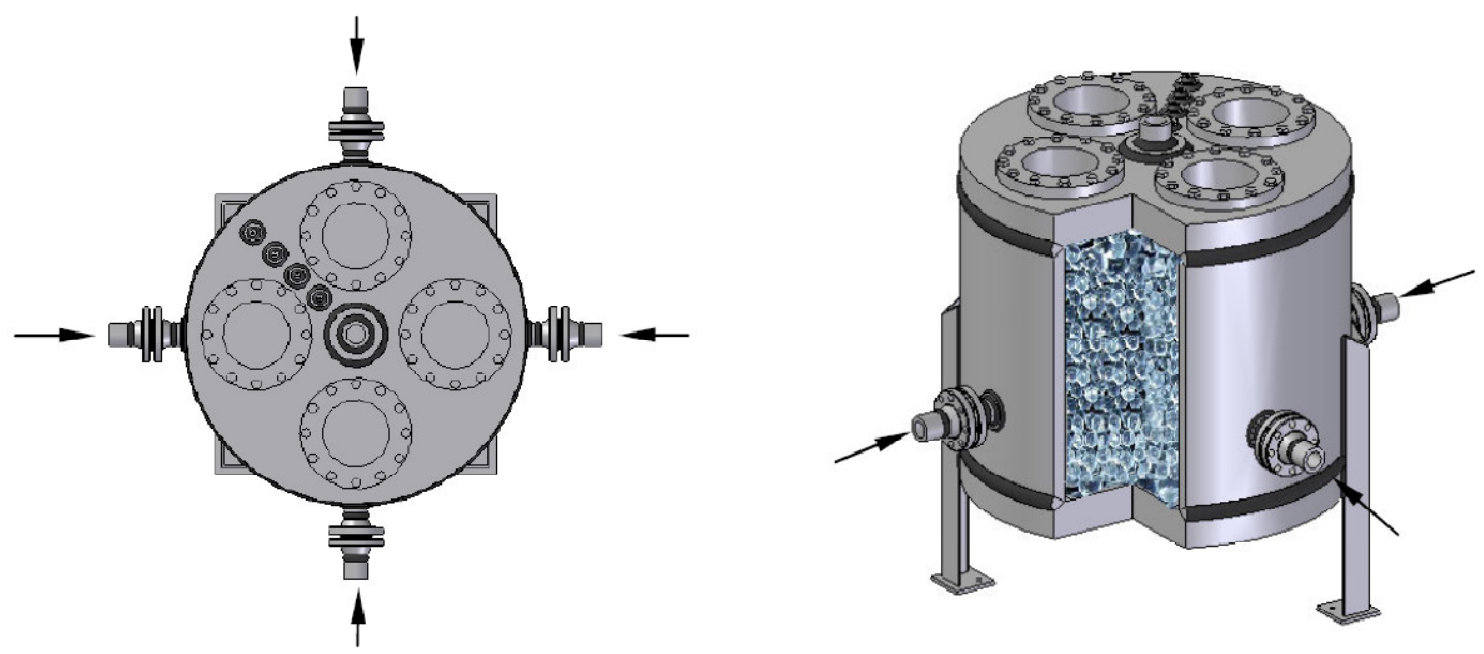

Fig. 3.1- Location of required operating conditions

\subsection{Design options for the boosting system}

Three possible arrangements for compression and boosting systems for conducting liquid loading experiments are identified. The configurations include a compressor and water pump; one would also include a multiphase pump and another a compressed tank.

\subsubsection{Multiphase pump}

The idea behind the first option is to provide air and water flow rates required by the system as a commingled mixture prior to entering the injection manifold. Selection of a compressor and water pump capable of delivering the required operational parameters (flow rates and delivery pressures) will consider the constraints fixed by inlet conditions for a multiphase pump.

Fig. 3.2 is a schematic of the flow path starting from the equipment to the delivery point. A single or a two-stage compression unit (1) for flexibility purposes will be considered. Water (2) and air will flow through the pipelines where flow meters (3) 
and valves $(4,5)$ will provide metering for data gathering and flow control. Commingling of the fluids will occur at a T-junction (6) prior to the inlet at the suction port of the multiphase pump (7). The mixture will then be boosted and will flow through a symmetrically aligned manifold (8) to arrive with the required operational conditions at the bottom section of the vertical tubing (9).

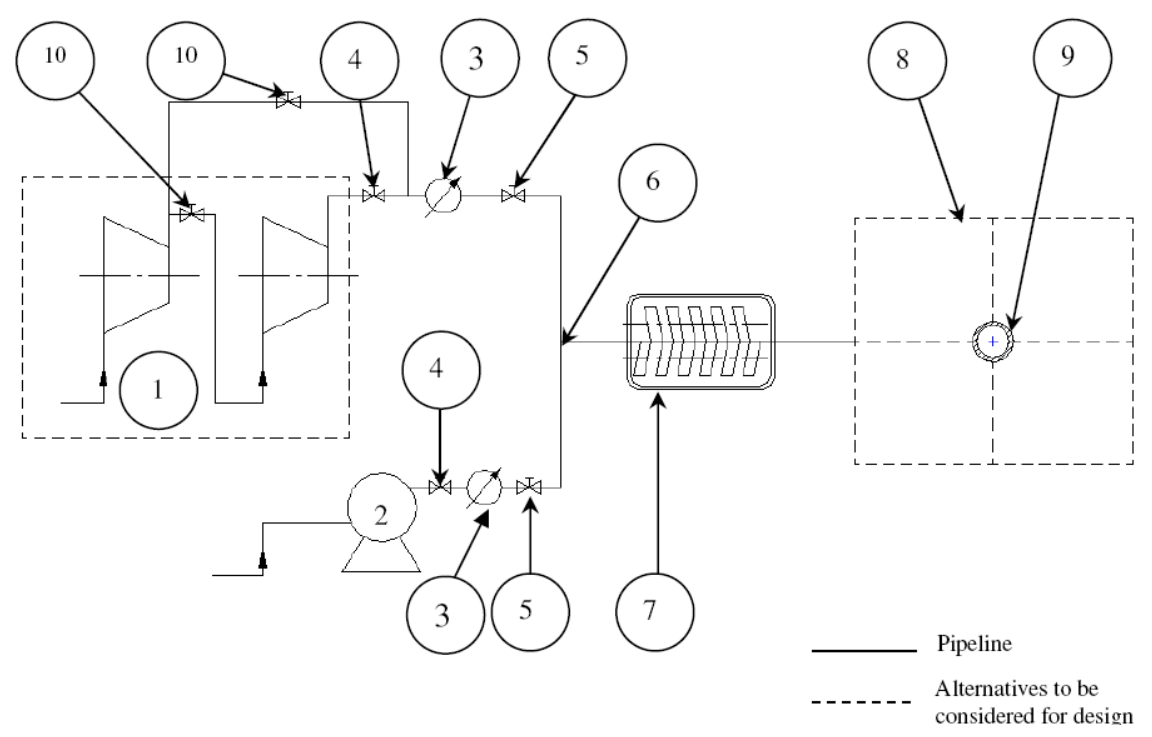

\section{Fig. 3.2- Case No. 1 will include flow from the water and air supply through a multiphase pump to the tubing}

Legend:

1 - Single/multiple stage compression

2 - Water phase pump

3 - Rotameter (Turbine meters)

4 - Regulation valve

5 - Non-return valve
$6-\mathrm{T}$ joint

7 - Multiphase pump

8 - Manifold

9 - Bottom section of tubing

10 - Shut off valves (by-pass)

Operational constraints for the multiphase pump such as maximum gas volume fraction $(\mathrm{GVF})$ and available head will be taken into consideration for proper equipment design and ultimate flow assurance. The main attraction of the multiphase pump option is that the system would not require too high compression-pumping specifications as the multiphase pump would take care of the final boosting. Several manufacturers supply 
multiphase pumps. Among the list, Bornemann Pumps is considered one of the leaders in multiphase pumping solutions and already has provided equipment for other projects within our department. Fig. 3.3 shows a multiphase pump and Table 3.2 provides the technical specifications of the equipment.

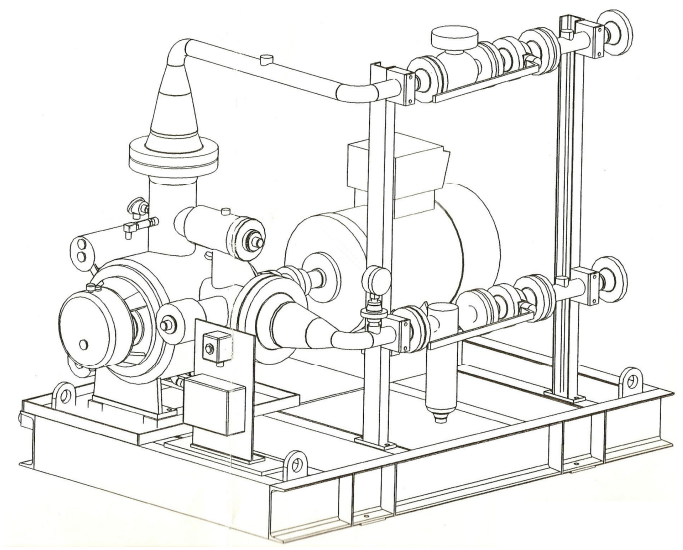

Fig. 3.3- Bornemann multiphase pump would provide additional boosting pressure prior to entering the injection manifold ${ }^{43}$

Table 3.2- Technical specifications ${ }^{43}$

\begin{tabular}{|l|l|}
\hline Model & MW 6.5zk-37 \\
\hline Velocity, rpm & 1000 to 2000 \\
\hline Flow rate, scf/min & 1,060 to 2,472 \\
\hline Discharge Pressure, psi & 116 to 130 \\
\hline Max. Temperature, ${ }^{\circ} \mathrm{F}$ & 176 \\
\hline Power, hp @ 176 psi differential pressure: & 14 to 44 \\
\hline GVF, \%: & 0 to 98 \\
\hline
\end{tabular}

The use of this arrangement will ultimately provide further opportunities for evaluating and monitoring the performance and efficiency of the equipment, especially the multiphase pump. This is particularly relevant considering that multiphase pumping is still regarded by many as a technology still under development.

\subsubsection{Compressor and water pump}

For the compressor and water pump option, both equipment $(1,2)$ are delivering, separately or commingled $(3,4,5,6)$, the required flow rates and pressures at the 
Furthermore, this option involves less equipment, therefore minimizing maintenance complexity and costs. Nevertheless, that larger equipment may be necessary to provide the required operational parameters, presents not only economical issues, but also constraints related to physical space available; however, it is thought by flow loop engineers that it is much less expensive to increase capacity of a single or multiple stage compressor and pump than to add a multiphase unit to the loop.

\subsubsection{Compressor - water pump - tank}

In Fig. 3.5 a compressor (1) provides air to a tank (2) filled with a certain amount of water. The system is then pressurized (by means of a water pump) until reaching the required operating value. Both phases leave the vessel at the same pressure and they can be either mixed $(3,4)$ or distributed separately $(5,6)$ prior to entering the tubing $(7)$. Fig. 3.5 illustrates the system configuration.

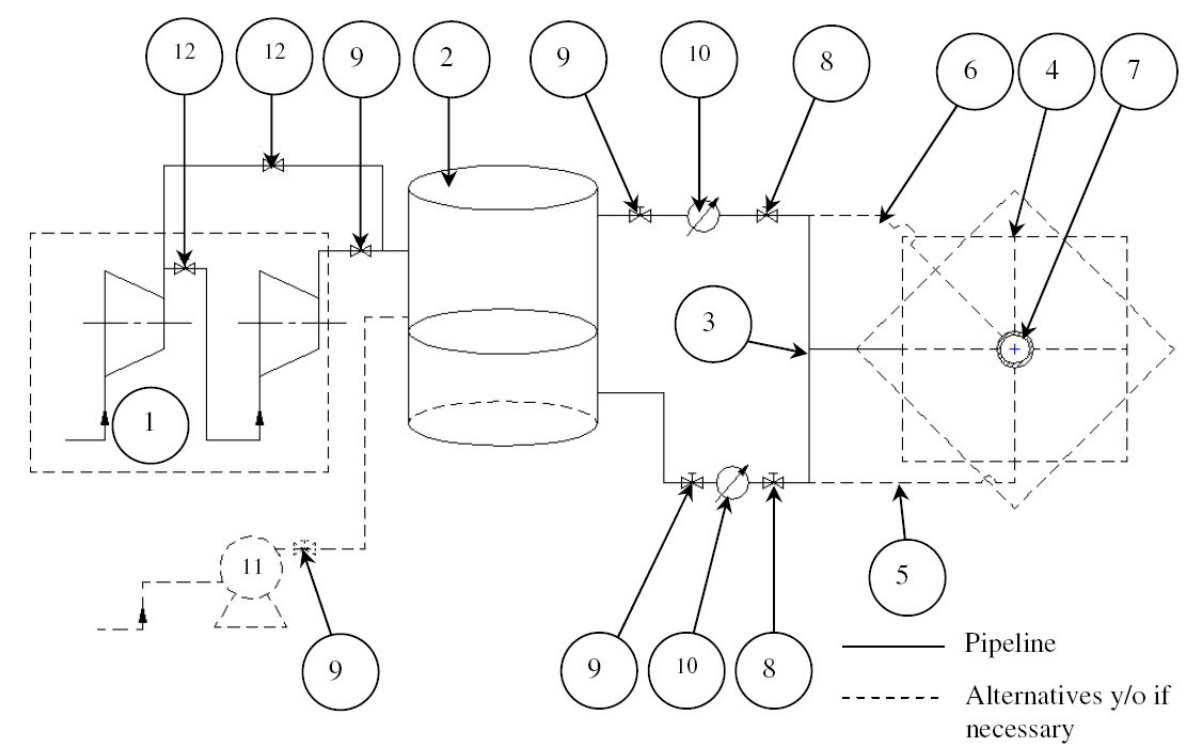

Fig. 3.5- In the tank configuration, air and water will flow through two different flow lines when leaving the air and water container

Legend:

1 - Single/multiple stage compression

2 - Tank

$3-$ T-joint

4 - Two phase manifold
5 - Water Manifold

6 - Air Manifold

7 - Bottom section of tubing

8 - Non-return valve
9 - Regulation valve

10 -Turbine meters

11 - Water phase pump

12 - Shut off valves 
From an economic standpoint, the tank option is the least expensive since a water pump downstream from the process will not be necessary as the pressure of the liquid will be provided by the air phase at the tank. In addition, a multiphase pump will not be needed. Despite these advantages, further evaluation is required to assess the impact of equipment sizing as compared to the physical space available. Moreover, this design will have to guarantee safety and proper fluid flow control due to the inherent problem of a transient condition while conducting the experiments.

\subsection{Technical analysis of the compressor - water pump - tank}

As previously mentioned, this arrangement considers a tank supplying air and water at the same pressure. The tank needs to have inlet entries both for air and water and for safety reasons, a pressure relief valve as well as drain port should be incorporated in the design (see Fig. 3.6). The initial conditions are specified by an initial pressure inside the tank as a consequence of pressurized air provided by a low capacity compressor located on site. After maximum pressure of air is achieved inside the tank, water will start to be pumped inside the tank to increase the air pressure up to the desired operating value.

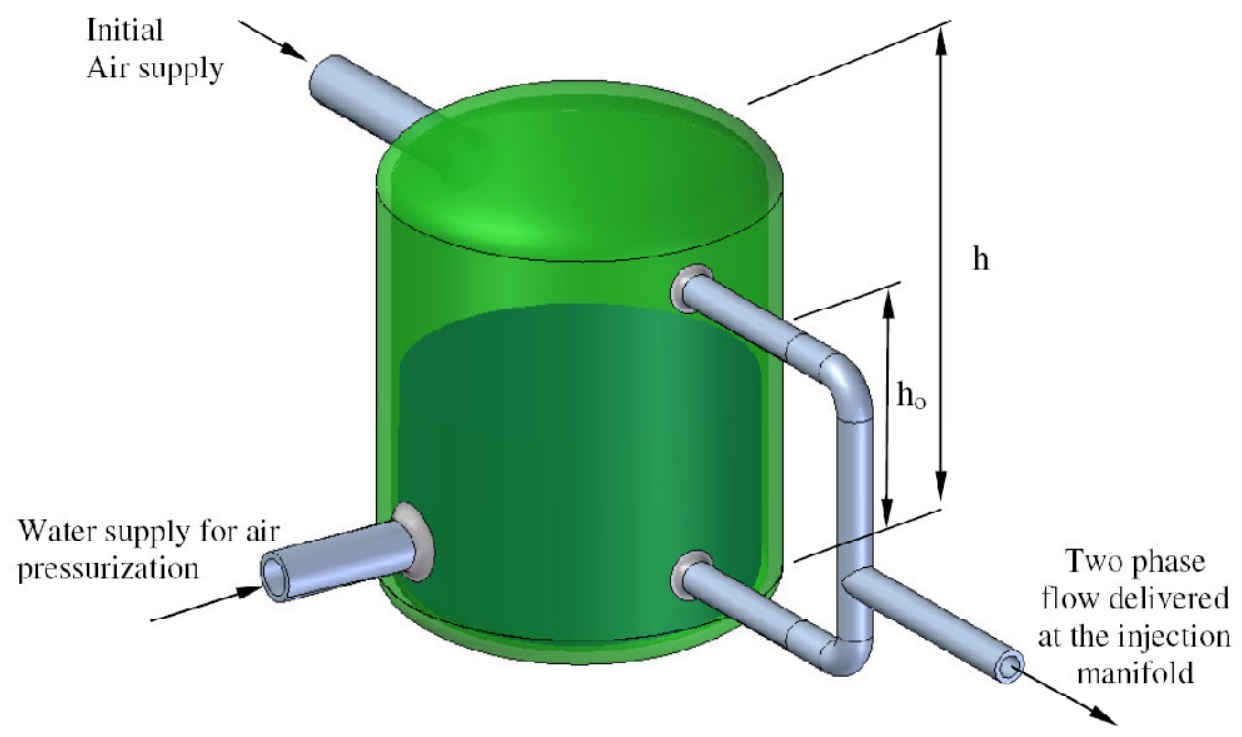

Fig. 3.6- Schematic of tank option 
The configuration of the tank has to be defined, as well as, the deliverability of the water pump; however sensitivities on these parameters could be performed to verify the time required for the air phase to reach approximately 650 psi. Furthermore, the final volume of the air when the operating pressure is achieved has to be adequate enough so the experiments can be conducted during a reasonable time at the required flow rates.

For the initial conditions and a final time " $\mathrm{t}$ ":

$\mathrm{P}_{\mathrm{i}} \mathrm{V}_{\mathrm{i}}=\mathrm{P}_{\mathrm{t}} \mathrm{V}_{\mathrm{t}}$

$\mathrm{P}_{\mathrm{i}} \mathrm{V}_{\mathrm{i}}=\mathrm{P}_{\mathrm{t}}\left(\mathrm{V}_{\mathrm{i}}-\mathrm{q} \Delta \mathrm{t}\right)$

$\mathrm{V}_{\mathrm{air}}=\mathrm{V}_{\mathrm{i}}-\mathrm{qt}$

$\mathrm{V}_{\mathrm{air}}=\mathrm{A}_{\text {Tank }}\left(\mathrm{h}-\mathrm{h}_{\mathrm{o}}\right)-\mathrm{qt}$

Where:

$\mathrm{P}_{\mathrm{i}}=\quad$ initial pressure of air, psia

$\mathrm{V}_{\mathrm{i}}=$ initial volume of air, $\mathrm{ft}^{3}$

$\mathrm{P}_{\mathrm{t}}=$ final air pressure, psia

$\mathrm{V}_{\mathrm{t}}=$ final volume of air, $\mathrm{ft}^{3}$

$\mathrm{q}=\quad$ liquid rate, $\mathrm{ft}^{3} / \mathrm{min}$

$\mathrm{t}=$ Time, $\min$

$\mathrm{h}=\quad$ vessel height, $\mathrm{ft}$

$\mathrm{h}_{\mathrm{o}}=\quad$ initial level of liquid inside the vessel, $\mathrm{ft}$

According to the Ideal gas Law:

$\mathrm{PV}=\mathrm{nRT}$

Where:

$\mathrm{n}=\quad$ number of moles in the gas, lb.mol

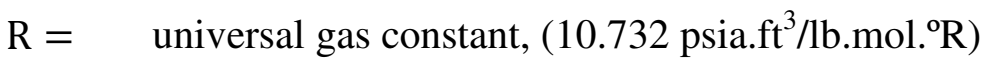

$\mathrm{T}=$ Temperature, ${ }^{\circ} \mathrm{R}$ 
For the pressurization process, the mass of air is constant, which means that $n$ (mass of air/molecular weight of air) is also constant. In addition, since both volume and pressure change at the same time, it could also be assumed that the temperature throughout the process remains constant. This situation leads to the following result:

$\mathrm{P}_{\mathrm{i}} \mathrm{V}_{\mathrm{i}}=\mathrm{nRT}=\mathrm{constant}$

If we want to know how pressure and volume change with time, let us find the derivative of both variables with respect to time.

$\frac{d(P V)}{d t}=\frac{d(n R T)}{d t}$
$\frac{P d V}{d t}+\frac{V d P}{d t}=0$
$\frac{P d V}{d t}=-\frac{V d P}{d t}$

Finally we get:

$\mathrm{dV}=-\frac{\mathrm{VdP}}{\mathrm{P}}$

But we also know that the change of volume of air with time can be defined as:

$\mathrm{V}_{\mathrm{air}}=\mathrm{A}_{\text {Tank }}\left(\mathrm{h}-\mathrm{h}_{\mathrm{o}}\right)-\mathrm{qt} \quad$ from equation No.3.4

$\mathrm{V}_{\mathrm{air}}=\mathrm{V}_{\mathrm{i}}-\mathrm{qt}$

Differentiating with respect to time

$\mathrm{dV}_{\text {air }}=-\mathrm{qdt}$

Substituting equation 3.8 in 3.7 we get:

$-\mathrm{qdt}=-\frac{\mathrm{VdP}}{\mathrm{P}}$ 
From the ideal gas equation:

$\mathrm{V}=\frac{\mathrm{nRT}}{\mathrm{P}}$

Substituting equation 3.10 in 3.9 then:

$-\mathrm{qdt}=-\frac{\mathrm{nRTdP}}{\mathrm{P}^{2}}$

Rearranging equation 3.11 and integrating we get the following result:

$\mathrm{dt}=\frac{\mathrm{nRTdP}}{\mathrm{qP}^{2}}$

$\int_{t_{0}}^{t_{1}} d t=\frac{n R T}{q} \int_{P_{0}}^{P_{1}} \frac{d P}{P^{2}}$

$\mathrm{t}=-\frac{\mathrm{nRT}}{\mathrm{q}}\left(\frac{1}{\mathrm{P}_{1}}-\frac{1}{\mathrm{P}_{0}}\right)$

Assuming the following conditions:

- Tank inside diameter: 22.73 in. for a standard 24 in. OD casing

- Height: 140ft - Entire height at TowerLab

- Water flow rate: $20 \mathrm{gpm}\left(2.674 \mathrm{ft}^{3} / \mathrm{min}\right)$

- No liquid inside the tank

- Initial pressure: $20 \mathrm{psi}$

- Air density @ $20 \mathrm{psi}=0.177 \mathrm{lb} / \mathrm{ft}^{3}$

- Required pressure: 650 psi

- Pump will supply: 650 psi plus hydrostatic head of final column of water

$A_{\text {tank }}=\frac{\pi}{4}(22.73 \mathrm{ft})^{2}=2.818 \mathrm{ft}^{2}$

$\mathrm{V}_{\text {air inside tank }}=2.818 \mathrm{ft}^{2} \times 140 \mathrm{ft}=394.506 \mathrm{ft}^{3}$

Mass of air $=0.177 \frac{\mathrm{lb}}{\mathrm{ft}^{3}} \times 394.506 \mathrm{ft}^{3}=69.827 \mathrm{lb}$ 


$$
\begin{aligned}
& \mathrm{n}=\frac{69.827 \mathrm{lb}}{29 \frac{\mathrm{lb}}{\mathrm{lb} \cdot \mathrm{mol}}}=2.408 \mathrm{lb} . \mathrm{mol} \\
& \mathrm{R}=10.732 \frac{\mathrm{psia} \cdot \mathrm{ft}^{3}}{\mathrm{lb} \cdot \mathrm{mol}^{\circ} \mathrm{R}} \\
& \mathrm{T}=70^{\circ} \mathrm{F}=530^{\circ} \mathrm{R}
\end{aligned}
$$

Substituting the results in equation No. 3.12

$$
\begin{aligned}
& \mathrm{t}=-\frac{2.408 \mathrm{lb} . \mathrm{mol} \times 10.7323 \frac{\mathrm{psia} \cdot \mathrm{ft}^{3}}{\mathrm{lb} \cdot \mathrm{mol}^{\mathrm{o}} \mathrm{R}} \times 530^{\mathrm{o}} \mathrm{R}}{2.674 \mathrm{ft}^{3} / \mathrm{min}}\left(\frac{1}{35 \mathrm{psia}}-\frac{1}{665 \mathrm{psia}}\right) \\
& \mathrm{t}=140 \mathrm{~min}
\end{aligned}
$$

However, it is also important to get the final volume of water required to reach 650 psi.

$$
\begin{aligned}
& \mathrm{V}_{\text {water }}=\mathrm{qt} \\
& \mathrm{V}_{\text {water }}=2.674 \mathrm{ft}^{3} / \mathrm{min} \times 140 \mathrm{~min}=374 \mathrm{ft}^{3}
\end{aligned}
$$

From these results we see that the volume of water almost occupies the entire volume available inside the tank leaving little volume of air to conduct experiments. Based on the initial assumptions, the column of water is $133 \mathrm{ft}$; therefore the pump should provide 708 psi considering the hydrostatic head inside the tank. If higher volumes of air are to be accomplished, as equation 3.4 and 3.12 show, the only solution is to increase the initial pressure in the tank from 20 psi to a value that would allow achieving the 708 psi with enough air remaining in the vessel at the end of the pressurization process.

First, let us establish a range of initial pressures in the vessel from 20 psi to 200 psi. The next step is to determine the density of air at for each point, followed by the moles and mass of air and finally the time with the corresponding volumes of water and air inside the tank at the end of the process. Table 3.3 and Fig. 3.7 show the results.

$$
\mathrm{P} \rightarrow \rho_{\text {air }} \rightarrow \mathrm{n} \rightarrow \mathrm{m}_{\text {air }} \rightarrow \mathrm{t}, \mathrm{V}_{\text {air,final }}, \mathrm{V}_{\text {water,final }}
$$


Table 3.3- Results for different values of pressures

\begin{tabular}{|c|c|c|c|c|c|}
\hline $\begin{array}{c}\text { Initial } \\
\text { pressure, psi }\end{array}$ & Air density, $\mathbf{l b} / \mathbf{f t}^{\mathbf{3}}$ & Time, $\mathbf{m i n}$ & $\begin{array}{c}\mathbf{V}_{\text {ini,air, }} \\
\mathbf{f t}^{\mathbf{3}}\end{array}$ & $\begin{array}{c}\mathbf{V}_{\text {water, }}, \\
\mathbf{f t}^{\mathbf{3}^{3}}\end{array}$ & $\begin{array}{c}\mathbf{V}_{\text {end,air }}, \\
\mathbf{f t}^{\mathbf{3}}\end{array}$ \\
\hline 20 & 0.177 & 140 & 395 & 374 & 21 \\
\hline 50 & 0.330 & 133 & 395 & 355 & 39 \\
\hline 70 & 0.432 & 128 & 395 & 343 & 51 \\
\hline 100 & 0.585 & 122 & 395 & 325 & 70 \\
\hline 150 & 0.840 & 110 & 395 & 295 & 100 \\
\hline 200 & 1.095 & 99 & 395 & 264 & 130 \\
\hline
\end{tabular}

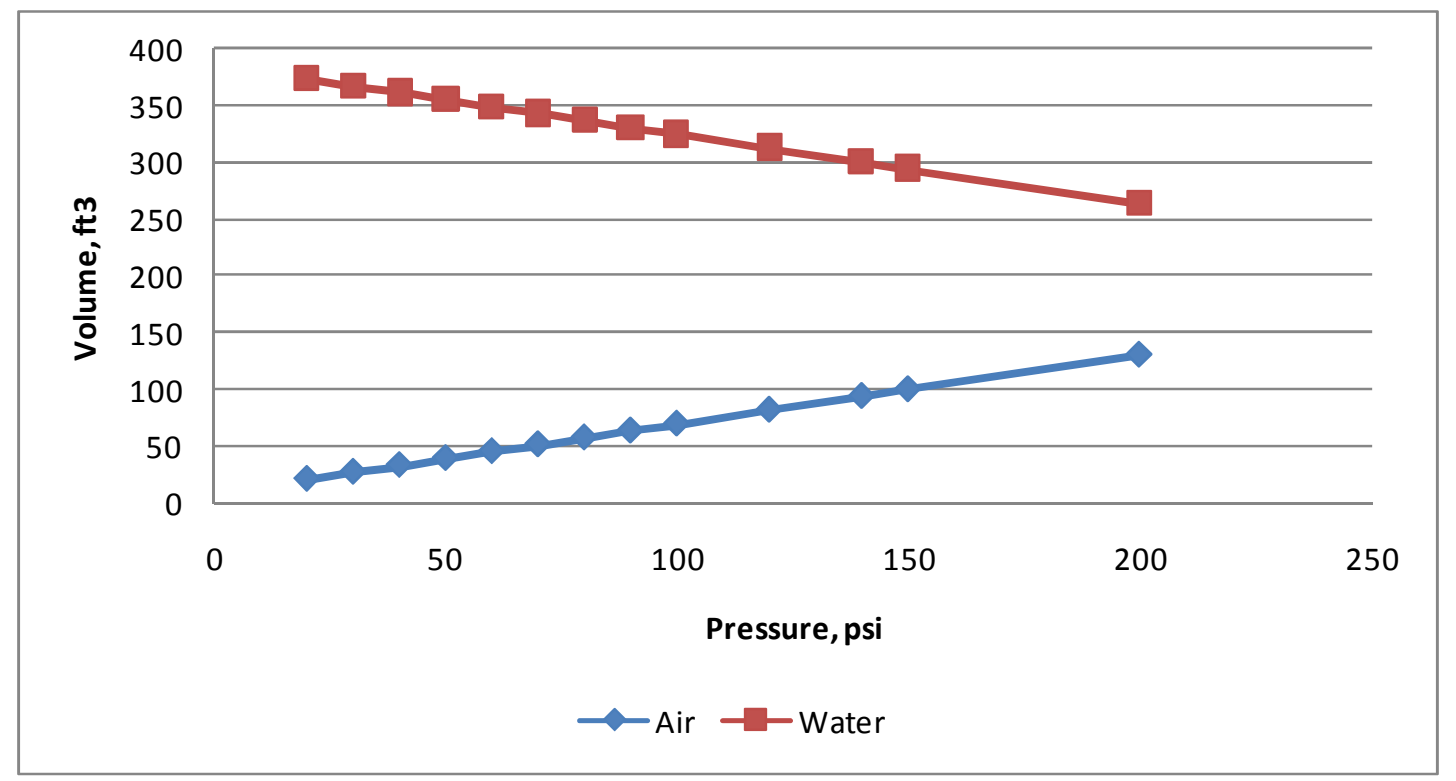

Fig. 3.7- Final volume of air and water inside the tank after compression vs. initial air pressure on the supply side

After observing this behavior, if a higher final volume of air is needed prior the initiation of experiments, the initial pressure of the air supply will have to be increased to a value that guarantees maintaining the operating flow rate throughout the loop during the time it may be desired.

If this approach results in a technical and economic option, one final calculation has to be performed to determine the time it takes for the air stream to decrease its pressure once it is open to flow at a fixed flow rate of approximately $600 \mathrm{ft}^{3} / \mathrm{min}$. 
As the process begins the mass of air in the tank will start decreasing, then:

$\mathrm{m}_{\mathrm{f}, \operatorname{tank}}=\mathrm{m}_{\mathrm{o}}-\dot{\mathrm{m}}_{\mathrm{air}} \mathrm{t}$

$\mathrm{m}_{\mathrm{o}}=\rho_{\text {air@650psi }} \times \mathrm{V}_{\mathrm{f}, \text { air }}$

The volume of air inside the tank is fixed at $130 \mathrm{ft}^{3}$ but as air is released, the density changes and the mass of air decreases with time. Rearranging equation 3.13 to get the time we get the following expression:

$\mathrm{t}=\frac{\mathrm{m}_{\mathrm{f}, \operatorname{tank}}-\mathrm{m}_{\mathrm{o}}}{\dot{\mathrm{m}}_{\mathrm{air}}}$

Results presented in Fig. 3.8 show that the pressure drops dramatically once the air is released into the flow loop at the operating flow rate. This condition makes design option No.3 unlikely to be used as the mechanism to supply compressed air to the flow loop. Even though this option is the least expensive of all three designs, from a technical standpoint, it does not satisfy the required parameters for the system.

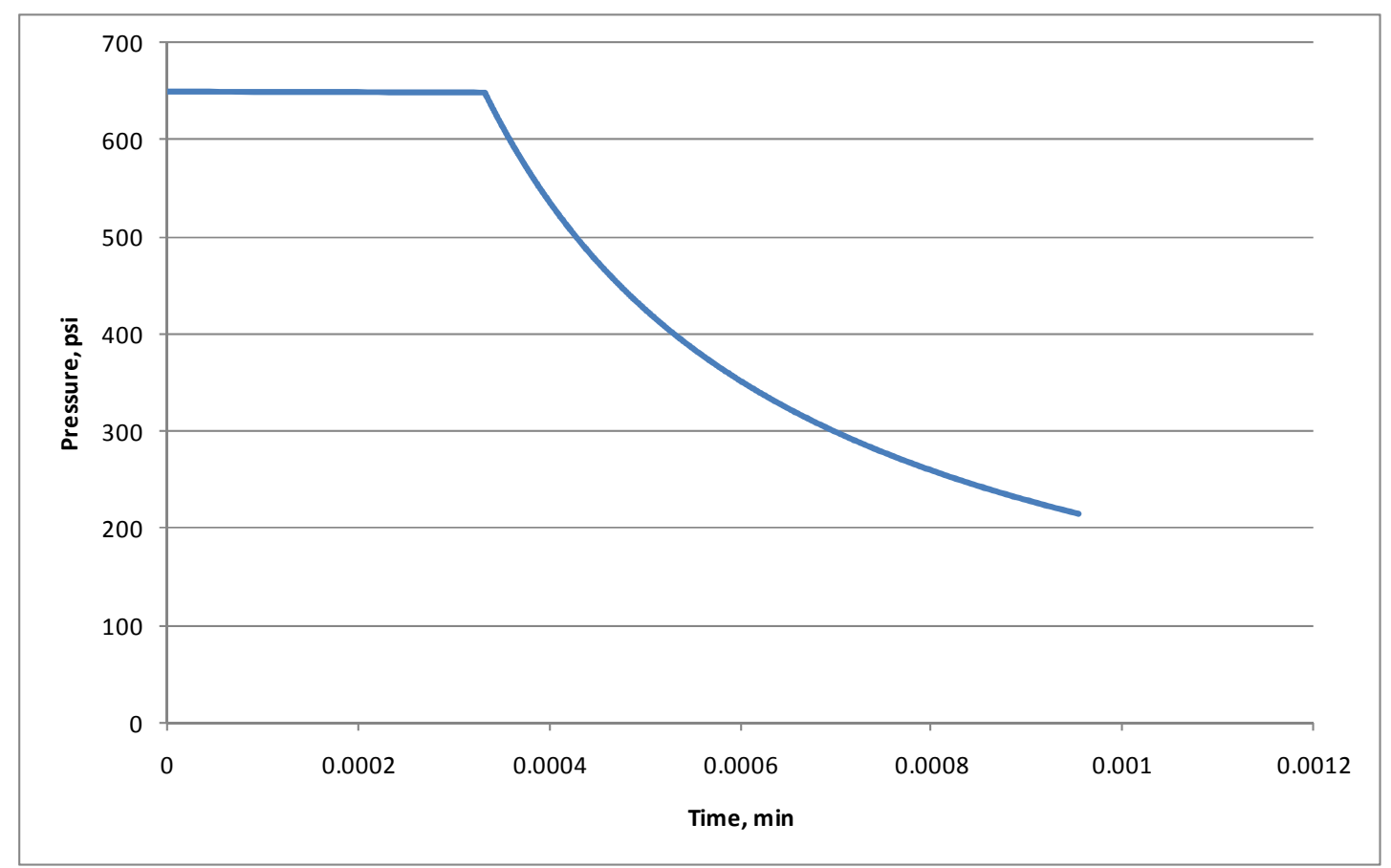

Fig. 3.8- Pressure drawdown with time 


\subsection{Facility design}

The approach to designing the facility that will accommodate all the major equipment and components for the operation of the multiphase flow loop at TowerLab began by establishing the operating conditions indicated at the beginning of this chapter.

Since one of the main challenges of this project is to properly size the compressed air system, the recommended methodology is to start the design process at the pressure vessel going upstream trough the injection manifold, pipe lines, instrumentation equipment until reaching the compressor.

\subsubsection{Pressure vessel}

The first step in the design of the pressure vessel that will serve as a container of the artificial porous medium is to define the different loads that the component will be subject to during its operation. The principal loads, as depicted in Fig. 3.9, can be summarized as:
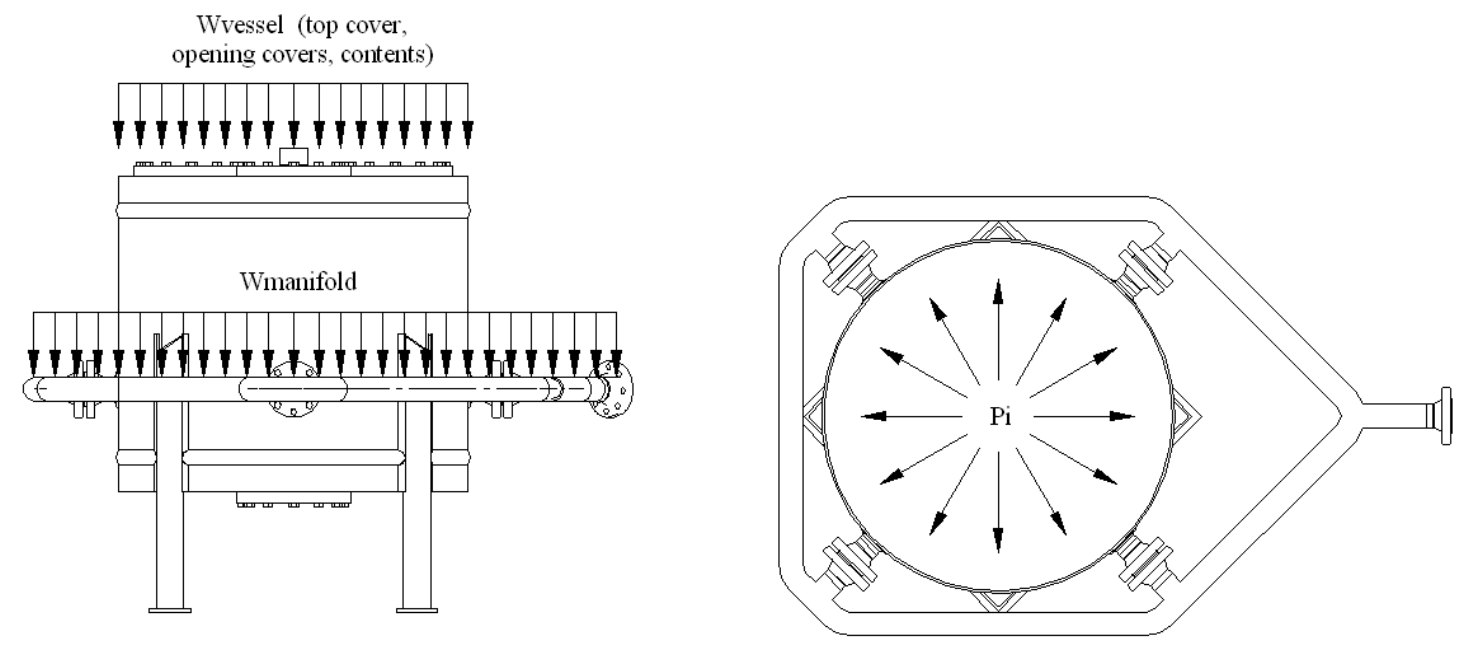

Fig. 3.9- Principal loads on the pressure vessel

- The internal pressure at the corresponding temperature of the fluids.

- The weight of the vessel and tubing attached at the different nozzle ports.

- The weight of the contents inside the vessel. 


\subsubsection{Design pressure and temperature}

The design pressure and temperature are the parameters used to determine thicknesses of the pressure vessel walls. A $10 \%$ overpressure is usually considered, and in the case of temperature, $50^{\circ} \mathrm{F}$ can be added to the maximum temperature of the

operating fluid. ${ }^{44}$ A pressure of 580 psi given at the discharge of the compressed air system is being considered for the design as a result of the evaluation of different compressed air system suppliers explained in detail later in this chapter.

Working fluids: $\quad$ Air and water

Design pressure: $\quad 595$ psia $+10 \% \quad=\quad 655$ psia

Design temperature: $70^{\circ} \mathrm{F}+50^{\circ} \mathrm{F} \quad=\quad 120^{\circ} \mathrm{F}$

\subsubsection{Selection of construction materials}

The selection of construction materials for code pressure vessels has to be made from code approved material specifications. There are many factors to be considered in selecting the most suitable materials. They include:

- Corrosion resistance

- Strength requirements for design temperature and pressure

- Cost

- Ready market availability

- Fabricability

- Quality of future maintenance

Generally, process equipment is designed for a certain minimum service life under specific operating conditions. Based on a corrosion rate in mils ( 0.001 in.) per year, a total corrosion allowance is established which is added to the calculated required thickness.

The selected material must be suitable for services of different levels of severity from the standpoint of pressure, temperature, corrosive environments, etc. However, since the choice of material for a vessel depends primarily on the service environment, it would seem practical to classify construction materials according to service: 
noncorrosive, with corrosion rates negligible or very low and definitely established (for carbon steel, a maximum of $1 / 4$ in. total; otherwise an alternative material with a better corrosion resistance is used); or corrosive, requiring special materials other than carbon steels or low-alloy steels.

Having established the importance of selecting adequate materials for the design and construction of a pressurized vessel, it is then convenient to characterize the environment under which the experimental investigations will be conducted in the TowerLab facility.

The working fluids, handled in the flow loop at the corresponding design operating conditions make it possible to classify the experimental environment in the TowerLab as a non-corrosive one. Nevertheless, corrosion allowance must be considered in design calculations before selecting the final nominal thicknesses for the different elements involved.

\subsubsection{Non-corrosive service}

In addition to corrosion resistance, the fundamental material selection criteria are design temperature and design pressure. In the range of intermediate temperatures (from $+33^{\circ} \mathrm{F}$ to $+800^{\circ} \mathrm{F}$ ), low-carbon steels are sufficient. Up to about $800^{\circ} \mathrm{F}$ they behave essentially in an elastic manner. Table 3.4 presents typical materials for intermediate temperatures, along with recommendations on where to be used in the vessel design. Table 3.5 illustrates mechanical properties for the most commonly used.

Table 3.4- Material selection guide ${ }^{44}$

\begin{tabular}{|c|c|c|c|c|c|}
\hline $\begin{array}{c}\text { Design } \\
\text { Temperature } \\
{ }^{\mathbf{0}} \mathbf{F}\end{array}$ & Material & Plate & Pipe & Forgings & Bolting/Nuts \\
\hline 33 to 60 & Carbon & $\begin{array}{c}\text { SA-516-AII } \\
\text { SA-515-AII } \\
\text { SA to } 775\end{array}$ & $\begin{array}{c}\text { SA-53-B } \\
\text { SA-106-B }\end{array}$ & $\begin{array}{c}\text { SA-105 } \\
\text { SA-181-60,70 }\end{array}$ & $\begin{array}{c}\text { SA-193-B7 with } \\
\text { SA-194-2H }\end{array}$ \\
\hline
\end{tabular}


Table 3.5- Mechanical properties ${ }^{45}$

\begin{tabular}{|l|l|c|c|c|}
\hline \multirow{2}{*}{ Element } & Material & \multicolumn{3}{|c|}{ Mechanical properties } \\
\cline { 2 - 5 } & Designation & Min. TS, ksi & Min. YS, ksi & S@ 200'F, ksi \\
\hline \multirow{5}{*}{ Plate } & SA-515-60 & 60 & 32 & 17.1 \\
\cline { 2 - 5 } & SA-515-70 & 70 & 38 & 20 \\
\cline { 2 - 5 } & SA-516-55 & 55 & 30 & 15.7 \\
\cline { 2 - 5 } & SA-516-60 & 60 & 32 & 17.1 \\
\cline { 2 - 5 } & SA-516-70 & 70 & 38 & 20 \\
\hline \multirow{3}{*}{ Pipe } & SA-53-B & 60 & 35 & 17.1 \\
\cline { 2 - 5 } & SA-106-B & 60 & 35 & 17.1 \\
\cline { 2 - 5 } & SA-106-C & 70 & 40 & 20 \\
\hline \multirow{3}{*}{ Forgings } & SA-105 & 70 & 36 & 20 \\
\cline { 2 - 5 } & SA-234-WPB & 60 & 35 & 25.1 \\
\hline Bolt & SA-193-B7 & 125 & 105 & \\
\hline
\end{tabular}

TS $=$ Tensile Strength, YS = Yield Strength, $\mathrm{S}=$ Maximum allowable Stress

\subsubsection{Corrosion allowance}

In pressure vessel design, even when a corrosive reaction is not likely to take place because of the inherent non-corrosive properties of the working fluids and the operating conditions controlling the application process, experience has demonstrated that a fully non-corrosive environment is unlikely to be encountered in any process. As a consequence, designers usually consider adding an additional value to the required thickness to account for corrosion effect. Megyesy ${ }^{44}$ and Bednar ${ }^{46}$ recommend adding $0.125 \mathrm{in}$. and $0.250 \mathrm{in}$. for non-corrosive and corrosive applications respectively to the calculated required thickness of the component under investigation.

\subsubsection{Joint efficiencies}

The joint efficiency is strictly related to welded joints, expressed as a numerical quantity used in the design of a joint as a multiplier of the appropriate allowable stress value taken from the applicable material in table 3.5. The joint efficiency decreases the resistance of the material to withstand a specific value of pressure. Using a value different of unity will increase the thickness required in the element. 
Table 3.6 shows the application of joint efficiencies depending on the welding process and the extend of radiography required. For the calculations to be performed on the vessel design, case No.2 with full extend radiography is considered and different arrangements of top and bottom covers will be analyzed to select the most adequate configuration. Nevertheless, the joint efficiency for those cases will be considered to be unity because they will be seamless heads.

Table 3.6- Application of joint efficiencies ${ }^{47}$

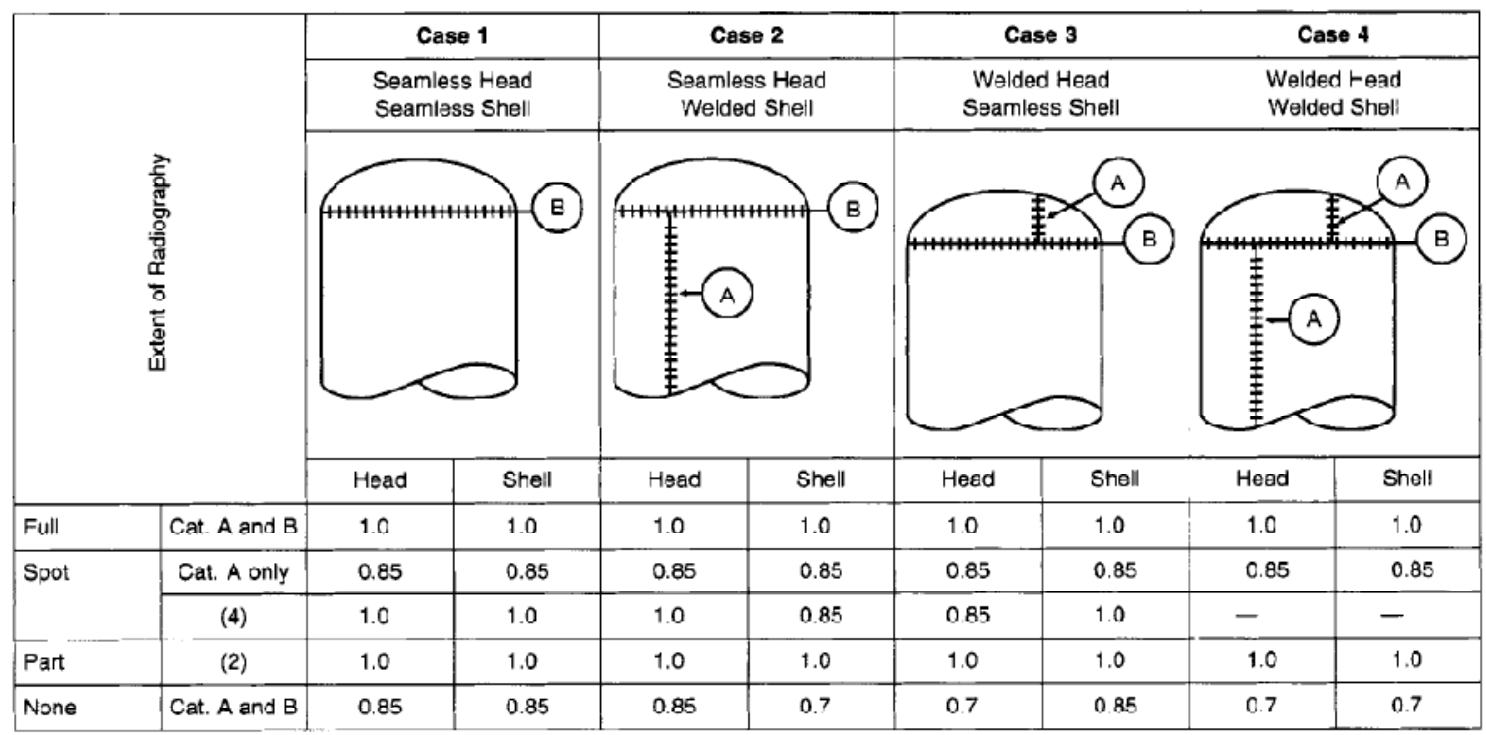

\subsubsection{ASME Code, Section VIII, Division 1 vs. 2}

ASME Code, Section VIII, Division 1 does not explicitly consider the effects of combined stress. Neither does it give detailed methods on how stresses are combined. ${ }^{47}$ ASME Code, Section VIII, Division 2, on the other hand, provides specific guidelines for stresses, how they are combined, and allowable stresses for categories of combined stresses. Division 2 is designed by analysis whereas Division 1 is design by rules.

Division 2 stress analyses consider all stresses in a triaxial state combined in accordance with the maximum shear stress theory. Division 1 and the procedures covered by this methodology consider a biaxial state of stress combined in accordance with the maximum stress theory. Consequently, Division 2 is more intended to be used 
in complex scenarios (e.g. a nuclear reactor) whereas Division 1 for simpler pressure vessel configurations as in the case for the pressure vessel needed for the TowerLab.

The rules as per ASME VIII Division covered in this study are the following:

- UG-27 Thickness of shells under internal pressure

- UG-32 Formed heads, pressure on concave side

- UG-34 Unstayed flat heads and covers

- UG-36 Openings in Pressure Vessels

- UG-37 Reinforcement required for openings in shells and formed heads

- UG-39 Reinforcement required for openings in flat heads

- UG-40 Limits of reinforcement

- UG-41 Strength of reinforcement

- UG-44 Flange and pipe fittings

- UG-45 Nozzle neck thickness

- UG-46 Inspection openings

- UW-9 Design of welded joints

- UW-12 Joint efficiencies

- UW-16 Minimum requirements for attachment welds at openings

- Mandatory appendix 1. Supplementary design formulas

- Mandatory appendix 2. Rules for bolted flange connections with ring type gaskets

\subsubsection{Pressure vessel configuration}

The pressure vessel will be located at the basement of the Richardson building. The first task is to identify the dimensions of the space available for the proper installation and operation of the equipment. Fig 3.10(a) is a top view of the Richardson building and Fig. 3.10(b) highlights the location for the vessel with the main dimensions. 


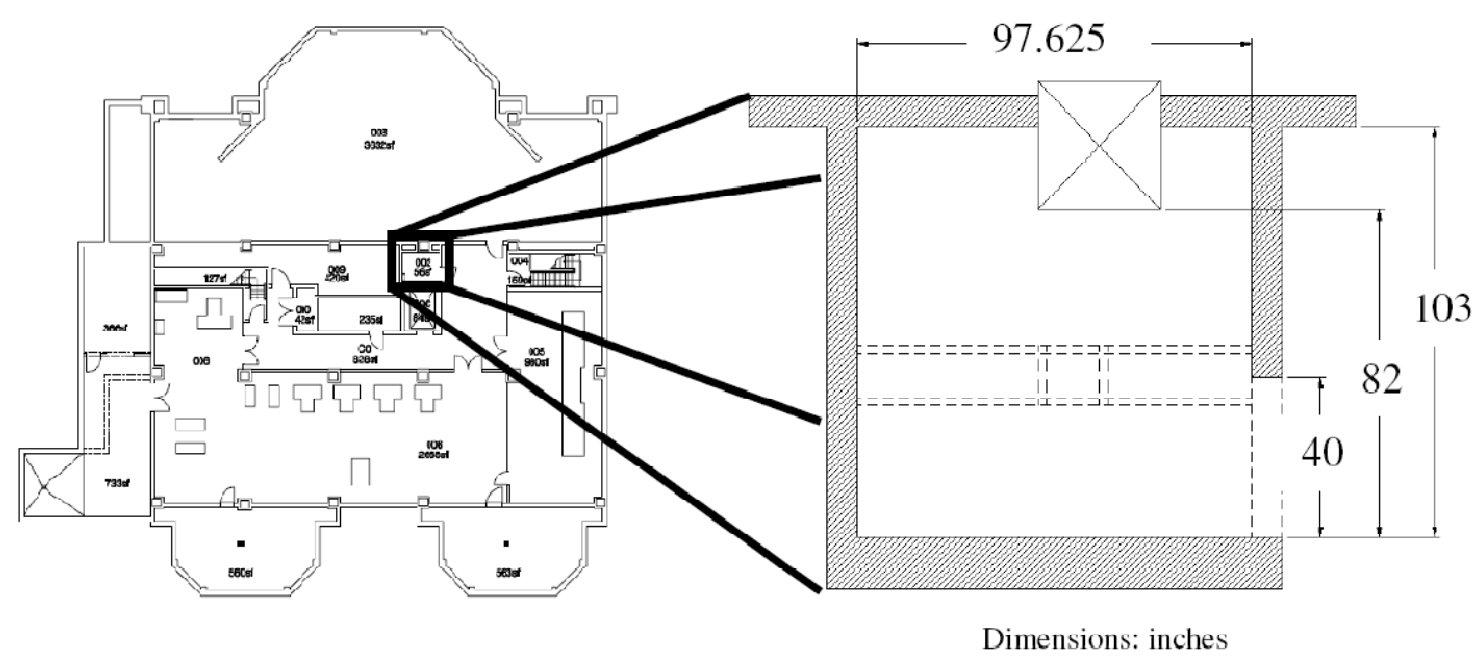

(a)

(b)

Fig. 3.10- (a) Basement at Richardson building and (b) location of pressure vessel

However, it is important to mention that the area in the basement does not extend throughout the height of the building that is why measurements of every floor are mandatory to guarantee a free path for the tubing. Fig. 3.11 is a top view of the TowerLab from $2^{\text {nd }}$ to $10^{\text {th }}$ floor.

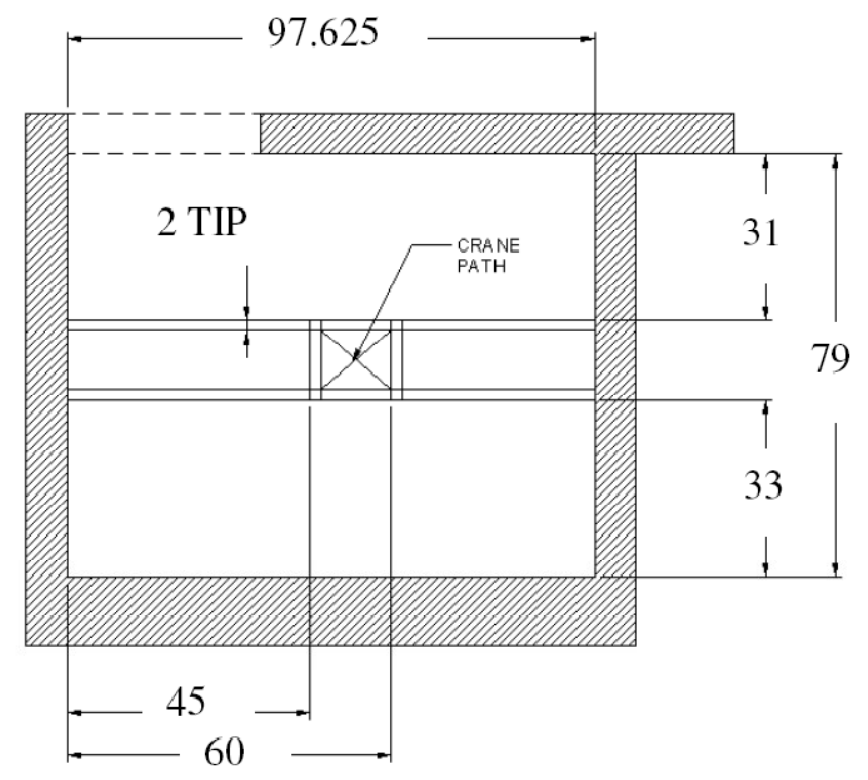

Fig. 3.11- TowerLab main dimensions from $2^{\text {nd }}$ to $10^{\text {th }}$ floor 
Three openings could be used to attach the tubing; however, the central opening is used by the crane installed at the roof of the building. This leaves only two options from which the one closest to the wall has to be eliminated because of its impact limiting the radius of the vessel. As Fig. 3.11 shows, these openings have I beam structures conveniently located at both sides (on every floor) useful for holding and maintaining the verticality of the pipe sections.

Considering that the tubing is located as indicated in Fig. 3.12, and that the geometrical center is to be shared by both the pressure vessel and the tubing, it is now possible to estimate the maximum internal diameter of the container knowing its location with respect to the space available, both at every floor and at the basement.

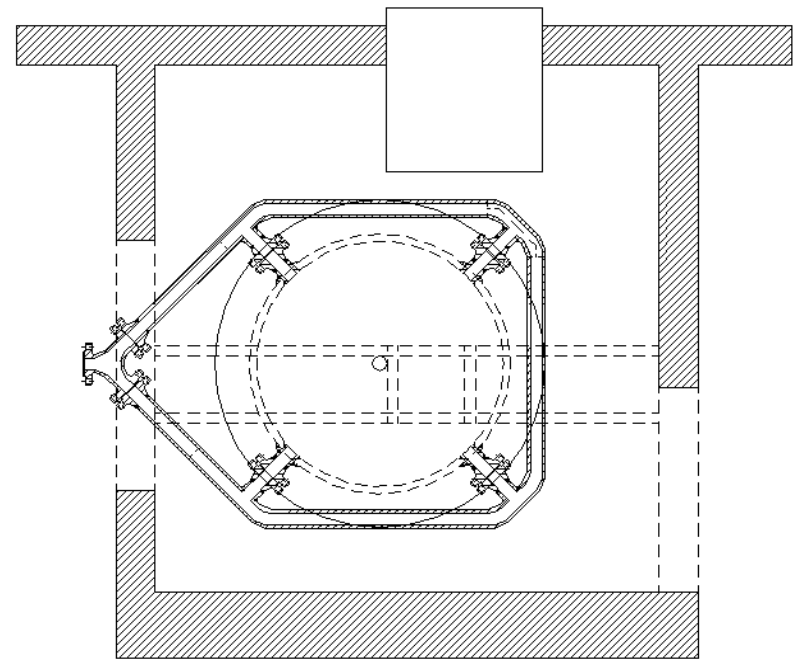

Fig. 3.12- Proposed location of pressure vessel in the TowerLab

Geometrical parameters initially defined by the analysis conducted by Solomon et $a l .{ }^{17}$ indicated an internal radius of $5 \mathrm{ft}$ (60 in.) and uniform height of $3 \mathrm{ft}$ (36 in.). However, it is obvious that the value for internal diameter had to be modified because of lack of space. Nevertheless, the objective is to provide the largest possible diameter based on the physical constraints imposed by the system. 
Fig 3.13 shows that a $2 \mathrm{ft}$ ( 24 in.) internal diameter vessel with the proposed design for the manifold consisting of 3 in. nominal pipe and flanges along with angle columns for weight support would result in the best option. This modification was reviewed by Solomon et al. ${ }^{17}$, and results indicated these dimensions to be adequate.

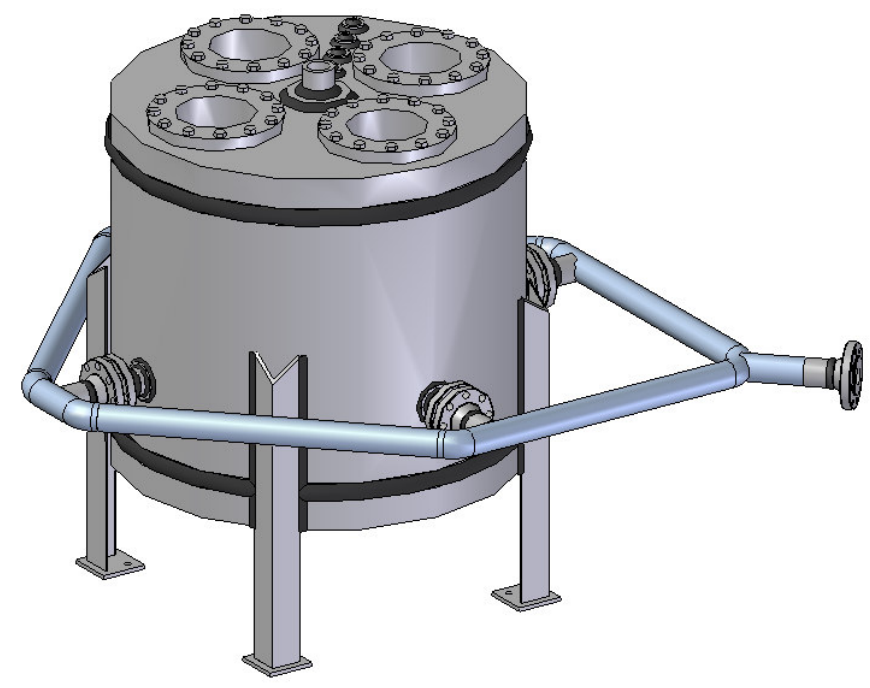

Fig. 3.13- Pressure vessel expected appearance

\subsubsection{Shell thickness}

UG-27 dictates calculation of the circumferential, longitudinal and compressive stresses to select the minimum thickness required. The governing equations for the three stresses are the following:

- Circumferential stress, $\mathrm{t}_{\mathrm{c}}$ :

$t_{c}=\frac{P R_{i}}{S E_{c}-0.6 P}+\frac{\frac{H g}{144} R_{i}}{S E_{c}-0.6\left(\frac{H g}{144}\right)}$

- Longitudinal stress, $\mathrm{t}_{\mathrm{l}}$ :

$t_{l}=\frac{P R_{i}}{2 S E_{l}+0.4 P}-\frac{W_{v}+W_{c}}{\pi D_{i} S E_{l}} \pm \frac{M_{b}}{\pi R_{i}^{2} S E_{l}}$ 
- Compressive stress, $\mathrm{t}_{\mathrm{C}}$ :

$\mathrm{t}_{\mathrm{C}}=\frac{\mathrm{W}_{\mathrm{v}}}{\pi \mathrm{D}_{\mathrm{i}} \mathrm{SE}_{\mathrm{l}}} \pm \frac{\mathrm{M}_{\mathrm{b}}}{\pi \mathrm{R}_{\mathrm{i}}^{2} \mathrm{SE}_{\mathrm{l}}}$

Where:

$\mathrm{P}=\quad$ design pressure, $\mathrm{psi}$

$\mathrm{R}_{\mathrm{i}}=$ internal radius, corroded condition, in.

$E_{c}=$ circumferential joint efficiency

$\mathrm{H}=\quad$ vessel internal height, $\mathrm{ft}$

$\mathrm{g}=\quad$ density of contents, $1 \mathrm{bm} / \mathrm{ft}^{3}$

$\mathrm{E}_{1}=\quad$ longitudinal joint efficiency

$\mathrm{W}_{\mathrm{v}}=\quad$ weight of vessel, $1 \mathrm{bm}$

$\mathrm{W}_{\mathrm{c}}=\quad$ weight of contents, $\mathrm{lbm}$

$\mathrm{D}_{\mathrm{i}}=$ internal diameter, corroded condition, in.

$\mathrm{M}_{\mathrm{b}}=$ bending moment, lbf. in.

Equipment attached to the vessel can also cause unsymmetrical distribution of the loading due to weight resulting in bending stresses. This unsymmetrical arrangement of small equipment such as pipes and openings may be neglected, but since the shell is to be connected to a manifold surrounding the external diameter of this component (see Fig. 3.13), we can then determine the thickness required in the shell based on the following equation.

$\mathrm{t}_{\mathrm{r}}=\frac{12 \mathrm{~W}_{\mathrm{e}}}{\mathrm{R}_{\mathrm{m}} \pi S E}$ 
Final results are presented in table 3.7.

Table 3.7- Shell thicknesses

\begin{tabular}{|l|c|c|c|c|}
\hline Stress & $\begin{array}{c}\text { Thickness } \\
\text { required, in. }\end{array}$ & $\begin{array}{c}\text { Governing } \\
\text { thickness, in. }\end{array}$ & $\begin{array}{c}\text { Corrosion } \\
\text { allowance, in. }\end{array}$ & $\begin{array}{c}\text { Nominal } \\
\text { thickness }\end{array}$ \\
\hline Circumferential & 0.806 & & & \\
\cline { 1 - 2 } Longitudinal & 0.383 & 0.806 & 0.125 & 1.000 \\
\cline { 1 - 2 } Compressive & 0.005 & & & \\
\hline Eccentric load & 0.448 & & & \\
\hline
\end{tabular}

The following step is to calculate the maximum allowable working pressure, the maximum allowable pressure and the hydrostatic test pressure.

- Maximum allowable working pressure (MAWP), $\mathrm{P}_{\mathrm{w}}$ :

$P_{w}=\frac{S_{D T} E t_{s c}}{R_{c}+0.6 t_{s c}}$

- Maximum allowable pressure (MAP), $\mathrm{P}_{\mathrm{M}}$ :

$P_{M}=\frac{S_{a} E t_{s n}}{R_{n}+0.6 t_{s n}}$

- Hydrostatic test pressure (HTP), $\mathrm{P}_{\mathrm{S}}$ :

$\mathrm{P}_{\mathrm{s}}=1.5 \mathrm{P}_{\mathrm{M}}$ or $1.3 \mathrm{P}_{\mathrm{w}}\left[\frac{\mathrm{S}_{\mathrm{a}}}{\mathrm{S}_{\mathrm{DT}}}\right]$

Where:

$\mathrm{S}_{\mathrm{DT}}=$ maximum allowable stress at design temperature, psi

$\mathrm{t}_{\mathrm{sc}}=$ thickness of shell corroded, in.

$\mathrm{R}_{\mathrm{c}}=$ internal radius corroded, in.

$\mathrm{S}_{\mathrm{a}}=$ maximum allowable stress at ambient temperature, psi

$\mathrm{t}_{\mathrm{sn}}=$ thickness of shell new, in.

$\mathrm{R}_{\mathrm{n}}=$ internal radius new, in.

$\mathrm{E}=\quad$ joint efficiency 
Table 3.8 shows the results:

Table 3.8- MAWP, MAP, HTP

\begin{tabular}{|l|c|}
\hline Condition & Pressure, $\mathbf{p s i}$ \\
\hline Maximum allowable working pressure & 710 \\
\hline Maximum allowable pressure & 813 \\
\hline Hydrostatic test pressure & 1,220 \\
\hline
\end{tabular}

\subsubsection{Bottom flat head cover}

The governing equation to determine the minimum thickness for a flat head according to UG-34 is:

$\mathrm{t}_{\mathrm{r}}=\mathrm{d} \sqrt{\frac{\mathrm{CP}}{\mathrm{SE}}}$

Where:

$\mathrm{t}_{\mathrm{r}}=$ Thickness required, in.

$\mathrm{C}=$ Factor, see appendix A, Fig. A.2

$\mathrm{d}=$ Internal diameter corroded, as per Fig. A.2

Table 3.9 presents the thickness and the values for MAWP, MAP, HTP.

Table 3.9-Thickness and MAWP, MAP, HTP for bottom flat head cover

\begin{tabular}{|c|c|c|}
\hline Thickness required, in. & Corrosion allowance, in. & Nominal thickness, in. \\
\hline 4.524 & 0.125 & 4.750 \\
\hline \multicolumn{3}{|c|}{ Pressures } \\
\hline MAWP, psi & MAP, psi & HTP, psi \\
\hline 684 & 730 & 1,093 \\
\hline
\end{tabular}

It is evident, that the thickness obtained for the bottom flat cover will make this element considerably heavy ( 1.2 ton for $0.284 \mathrm{lb} / \mathrm{in}^{3}$ steel) adding a lot of weight on the total configuration of the vessel. One alternative is to design a torispherical head for which the governing equation for $\mathrm{L} / \mathrm{r}=16-2 / 3$, as per $\mathrm{UG}-32$, is the following:

$\mathrm{t}_{\mathrm{r}}=\frac{0.885 \mathrm{PL}}{\mathrm{SE}-0.1 \mathrm{P}}$ 
Where:

$\mathrm{L}=$ Inside spherical or crown radius, in.

$r=$ Knuckle radius, in.

Fig. 3.14 illustrates the shape of the bottom cover of the pressure vessel if a torispherical head is to be selected.

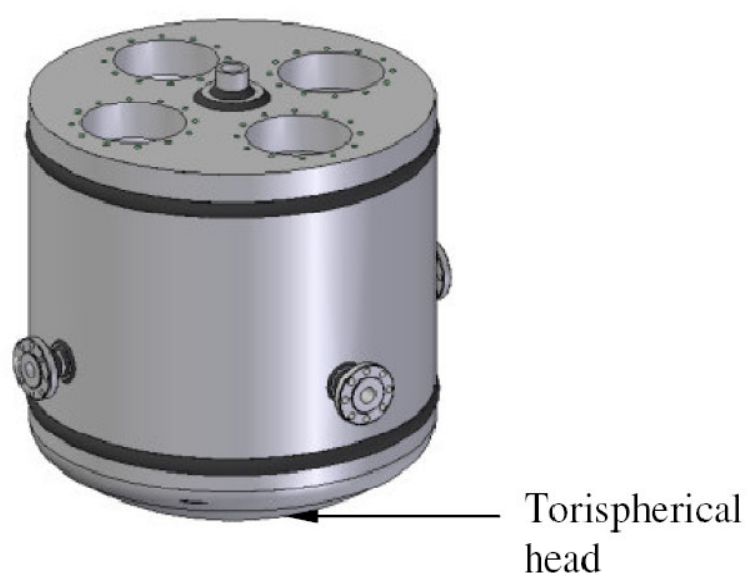

Fig. 3.14- Option of torispherical head at bottom

Table 3.10 shows the thickness and the values for MAWP, MAP and HTP. It is evident the impact this alternative has on the overall weight of the configuration. However, as air and water are injected to recreate liquid loading, the denser fluid could start depositing at the bottom head as a consequence of its geometry. This would create difficulties for lifting the liquid phase while running the experiments.

Table 3.10-Thickness and MAWP, MAP, HTP for torispherical head option

\begin{tabular}{|c|c|c|c|c|}
\hline $\mathbf{L}$ & $\mathbf{r}$ & $\begin{array}{c}\text { Thickness } \\
\text { required, in. }\end{array}$ & $\begin{array}{c}\text { Corrosion allowance, } \\
\text { in. }\end{array}$ & $\begin{array}{c}\text { Nominal } \\
\text { thickness, in. }\end{array}$ \\
\hline 38 & 2.28 & 1.104 & 0.125 & 1.250 \\
\hline \multicolumn{5}{|c|}{ Pressures } \\
\hline \multicolumn{2}{|c|}{ MAP, psi } \\
\hline \multicolumn{2}{|c|}{740} & HTP, psi \\
\hline
\end{tabular}


The total reduction in weight is estimated to be:

Weight of flat head $=$

Weight of ASME flange and dish cover $=$

Reduction $=$
$2,650 \mathrm{lb} \quad(1.2$ ton $)$

$1,002 \mathrm{lb} \quad(0.45 \mathrm{ton})$

$62 \%$

This option is attractive from a logistics standpoint; however the experimental constraint previously described rules it out.

\subsubsection{Nozzles}

Four entry points have been considered for the attachment of nozzles to communicate fluids in and out of the pressure vessel. Four nozzles are located on the shell body and one on the top flat head cover. The rules for reinforcement of openings are taken from the ASME Code, ${ }^{45}$ UG-36 trough UG-44. The basic requirement is that around the opening the vessel must be reinforced with an equal amount of metal which has been cut out of the opening. The reinforcement in any case may be an integral part of the vessel and nozzle or may be an additional reinforcement pad. Fig 3.15 depicts all the possible configurations for attaching nozzles to pressure vessels.

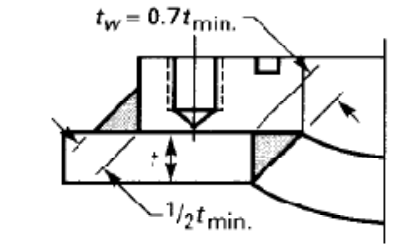

(p)
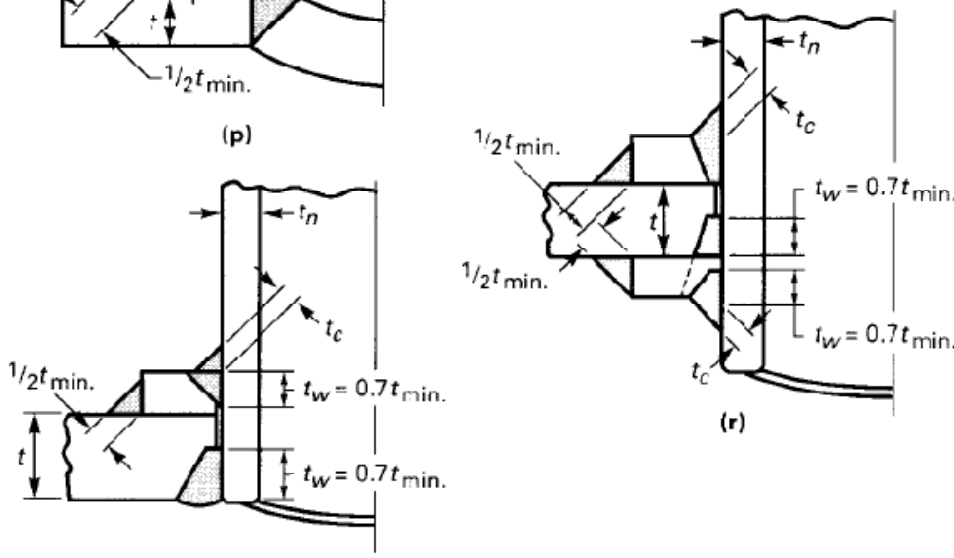

(r)

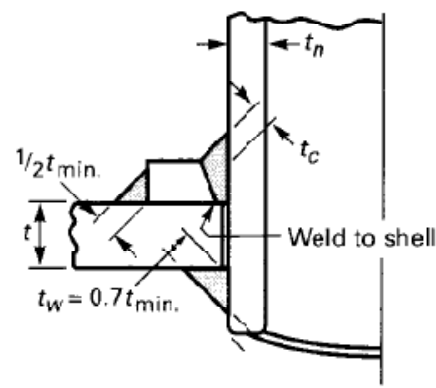

(s)

(q)

Fig. 3.15- Acceptable types of welded nozzles to shells and heads ${ }^{43}$ 
These rules; however, need further refinements as follows (see Fig 3.16):

- It is not necessary to replace the actually removed amount of metal, but only the amount which is required to resist the internal pressure $\left(\mathrm{A}_{\mathrm{r}}\right)$. This required thickness of the vessel at the opening is usually less than at other points of the shell or head.

- The plate used and nozzle neck usually are thicker than would be required according to calculation. The excess in the vessel wall $\left(\mathrm{A}_{1}\right)$ and nozzle wall $\left(A_{2}\right)$ serve as reinforcements. Likewise the inside extension of the opening $\left(\mathrm{A}_{3}\right)$ and the area of the weld metal $\left(\mathrm{A}_{41+42+43}\right)$ can also be taken into consideration as reinforcement.

- The reinforcement must be within a limit

- The area of reinforcement must be proportionally increased if its stress value is lower than that of the vessel wall

- The area requirement for reinforcement must be satisfied for all planes through the center of opening and normal to the vessel surface

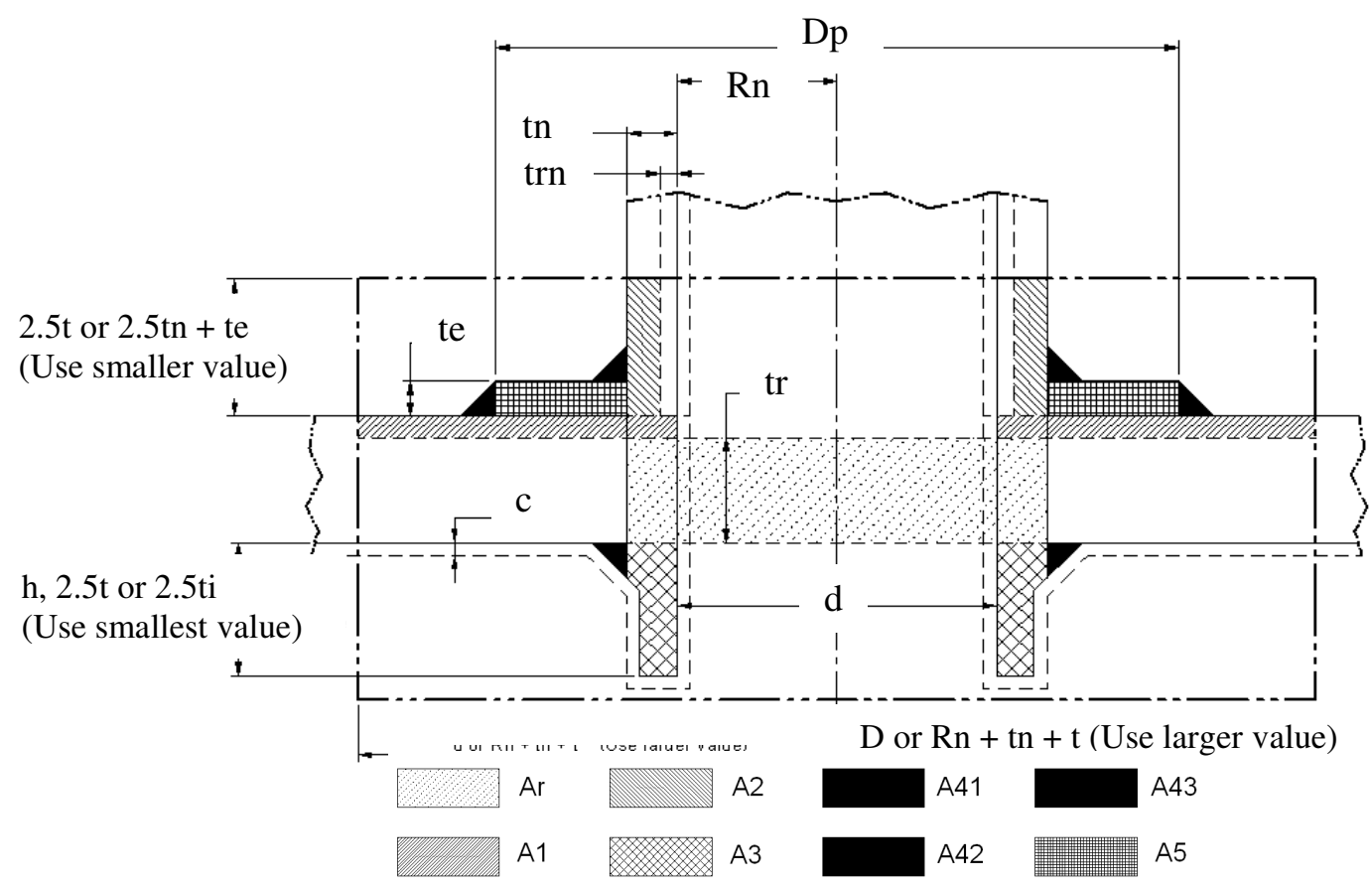

Fig. 3.16- Areas available for reinforcement in openings 
The required cross-sectional area of the reinforcement then must be the required area for the shell or head to resist the internal pressure, $\left(A_{r}\right)$. If the sum of the areas available for reinforcement $\left(A_{1}+A_{2}+A_{3}+A_{41+42+43}\right)$ is equal or greater than the area to be replaced $\left(A_{r}\right)$, the opening is adequately reinforced; otherwise the difference must be supplied by reinforcing pad $\left(\mathrm{A}_{5}\right)$

Some manufacturers follow a simple practice using reinforcing pads with a crosssectional are which is equal to the metal area actually removed for the opening. ${ }^{46}$ This practice results in oversized reinforcement, but with the elimination of calculations.

\subsection{Nozzles attached to the shell}

As a result from the analysis of Solomon et $a l .{ }^{17}$ superficial velocities were calculated at the entry points for both water and air to determine the internal diameter required by the injection manifold. This chapter includes the results for the manifold design which showed that two manifolds are recommended for the final assembly.

Eight entry points are considered to allow for a path for the fluids inside the vessel. They are uniformly spaced with respect to the geometric center of the vessel (see Fig. 3.17) to facilitate an evenly distribution of the injected phases so that a more realistic condition of radial flow in porous medium can be achieved. The governing equation to calculate the required thickness for both pipes is the same as for the shell design (equation 3.16) considering only circumferential stresses as this is the most severe condition.

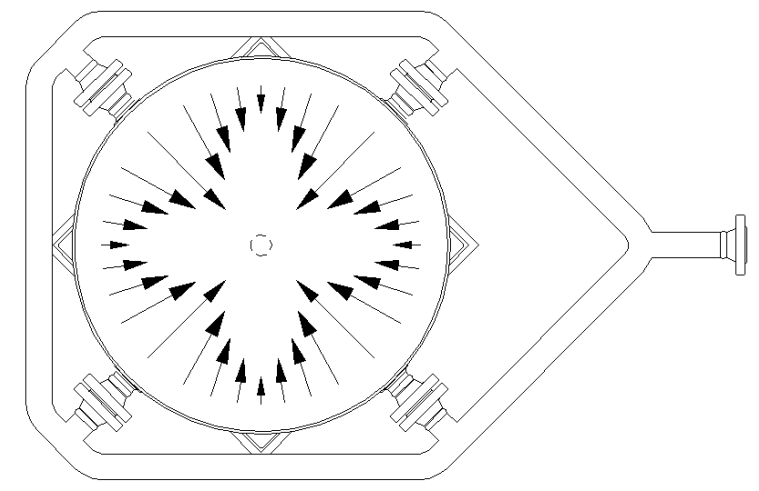

Fig. 3.17- Radial flow to be expected from the eight nozzles 
The pipe size selected for the manifold circulating water is 3 in. schedule 80 with an internal diameter of 2.9 in. For the manifold transporting air, the pipe corresponds to 1.5 schedule 80 with 1.5 in. internal diameter. Thickness calculations as per ASME code $^{45}$ showed that non-standard pipe will have to be indicated for both cases. Tables 3.11 and 3.12 show the pipe properties, as well as, the results for the thickness and pressure calculations.

Table 3.11- Pipe properties and dimensions

\begin{tabular}{|c|c|c|c|c|}
\hline Fluid & $\begin{array}{c}\text { External } \\
\text { diameter, in. }\end{array}$ & $\begin{array}{c}\text { Internal } \\
\text { diameter, in. }\end{array}$ & Material & $\begin{array}{c}\text { Maximum } \\
\text { allowable stress, psi }\end{array}$ \\
\hline Air & 3 & 1.5 & SA-106-C & 20,000 \\
\hline Water & 4 & 2.9 & SA-106-C & 20,000 \\
\hline
\end{tabular}

Table 3.12- Nozzle neck thickness calculations and pressure limitations

\begin{tabular}{|c|c|c|c|c|c|c|}
\hline Fluid & $\begin{array}{c}\text { Nozzle neck } \\
\text { thickness } \\
\text { required, in }\end{array}$ & $\begin{array}{c}\text { Corrosion } \\
\text { allowance, } \\
\text { in. }\end{array}$ & $\begin{array}{c}\text { Nominal } \\
\text { thickness, } \\
\text { in. }\end{array}$ & $\begin{array}{c}\text { MAWP, } \\
\text { psi }\end{array}$ & MAP, psi & HTP, psi \\
\cline { 1 - 2 } Air & 0.154 & 0.125 & 0.600 & 7,947 & 9,932 & 14,897 \\
\hline Water & 0.177 & 0.177 &
\end{tabular}

Calculations to verify the need of reinforcement at each nozzle is also presented. Table 3.13 shows that for the air line it will not be necessary whereas for the case of the water manifold it has to be designed.

Table 3.13- Configuration of the nozzle

\begin{tabular}{|c|c|c|c|c|}
\hline Fluid & $\begin{array}{c}\text { Area } \\
\text { required, } \text { in }^{2}\end{array}$ & $\begin{array}{c}\text { Area available, } \\
\text { in }^{2}\end{array}$ & $\begin{array}{c}\text { Pad dimensions, } \\
\text { in. }\end{array}$ & $\begin{array}{c}\text { Area } \\
\text { provided, } \text { in }^{2}\end{array}$ \\
\hline Air & 1.411 & 1.680 & N/A & 1.680 \\
\hline Water & 2.540 & 1.624 & $5.5 \times 4 \times 0.5$ & 2.762 \\
\hline
\end{tabular}




\subsection{Flange-pipe attachment in nozzles}

The two main types of plain-faced flanges are full-face and raised-face flanges (see Fig. 3.18), furthermore, there are several configurations for the flange pipe attachment available in the market. Following is a description of two of the most commonly used.
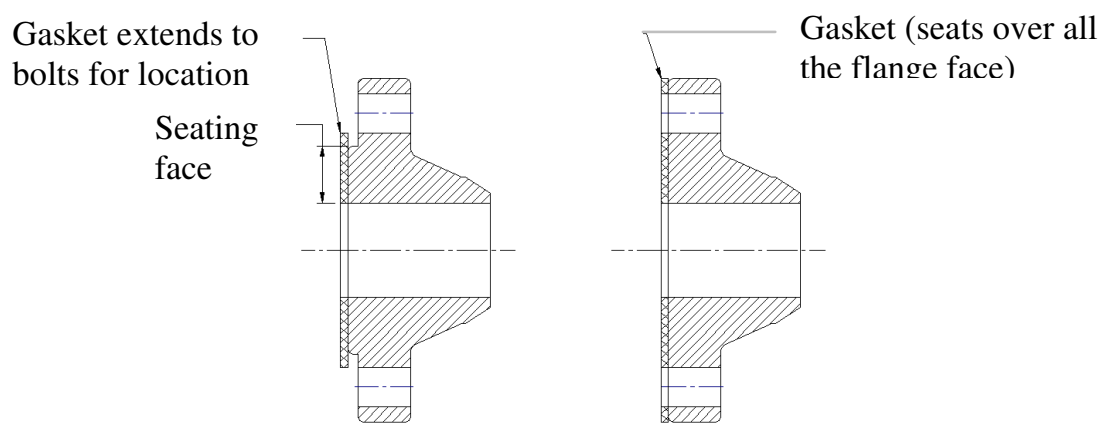

Fig. 3.18- Full and raised-face flanges

- Slip-on flange: The slip-on flange fits over the end of the pipe (see Fig. 3.19). The flange is attached to the pipe by two circumferential fillet welds where the inner weld is not to be omitted because the strength of the assembly would be impaired and the gap would be subject to crevice corrosion under certain process conditions. When subject to bending, the welds are subjected to high stresses. The welds are difficult to inspect by radiographic methods and the flange faces can distort during the welding process, causing sealing difficulties. If the clearance between the flange and the pipe is high, then the integrity of the assembly relies heavily on the weld.

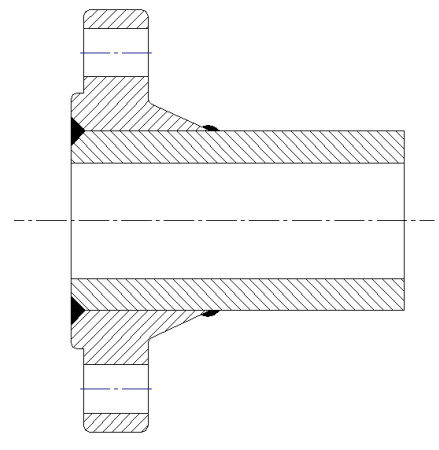

Fig. 3.19- Slip-on flange 
- Welded-neck flange: The welded-neck flange incorporates a taper that leads from the flange to the pipe diameter as illustrated in Fig 3.20. It can be seen that the transition from the thick flange to the thin pipe wall is eased.

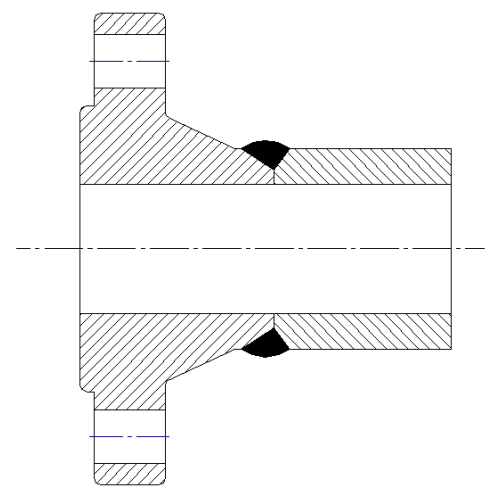

Fig. 3.20- Welded-neck flange

One circumferential butt weld is used to connect the flange to the pipe. The butt weld is a high integrity weld that can be inspected readily by radiographic methods if required. The weld is located away from regions of maximum stress if the flange is subjected to bending. The weld neck flange is usually a forging which should have good, consistent material properties. Therefore there are several factors that combine to make the weld neck flange a high-integrity flange. The weld neck flange is widely specified and is in common use. It is more suitable than the slip-on flange for high-pressure, hightemperature, and cyclic loading conditions.

Following these recommendations, a weld neck flange is considered for the design of the pipe-flange attachment. Table 3.14 provides information for the selected flange. Dimensional information is available in ASME B16.5. ${ }^{48}$

Table 3.14- Flange properties ${ }^{48}$

\begin{tabular}{|c|c|c|}
\hline Material & Class & Max. working pressure, psi @ 200 \\
\hline SA-105 & 400 & 905 \\
\hline
\end{tabular}




\subsubsection{Flange attached to shell body}

The maximum commercially available flange according to pipe nominal sizes is 24 in. This implies that a customized design following the governing rules in appendix 2 from the ASME Code ${ }^{45}$ is required to define the final dimensions of the flange. These rules apply specifically to the design of bolted flange connections with gaskets that are entirely within the circle enclosed by the bolt holes and with no contact outside this circle. The hub thickness of the weld neck flange design shall also comply with the minimum thickness requirements in subsection A of the ASME division.

Appendix A presents the steps for the flange design including bolts and gasket selection based on the stresses produced by the forces and bending moments due to the internal pressure in the vessel. Fig 3.21 illustrates the principal dimensions of the design.

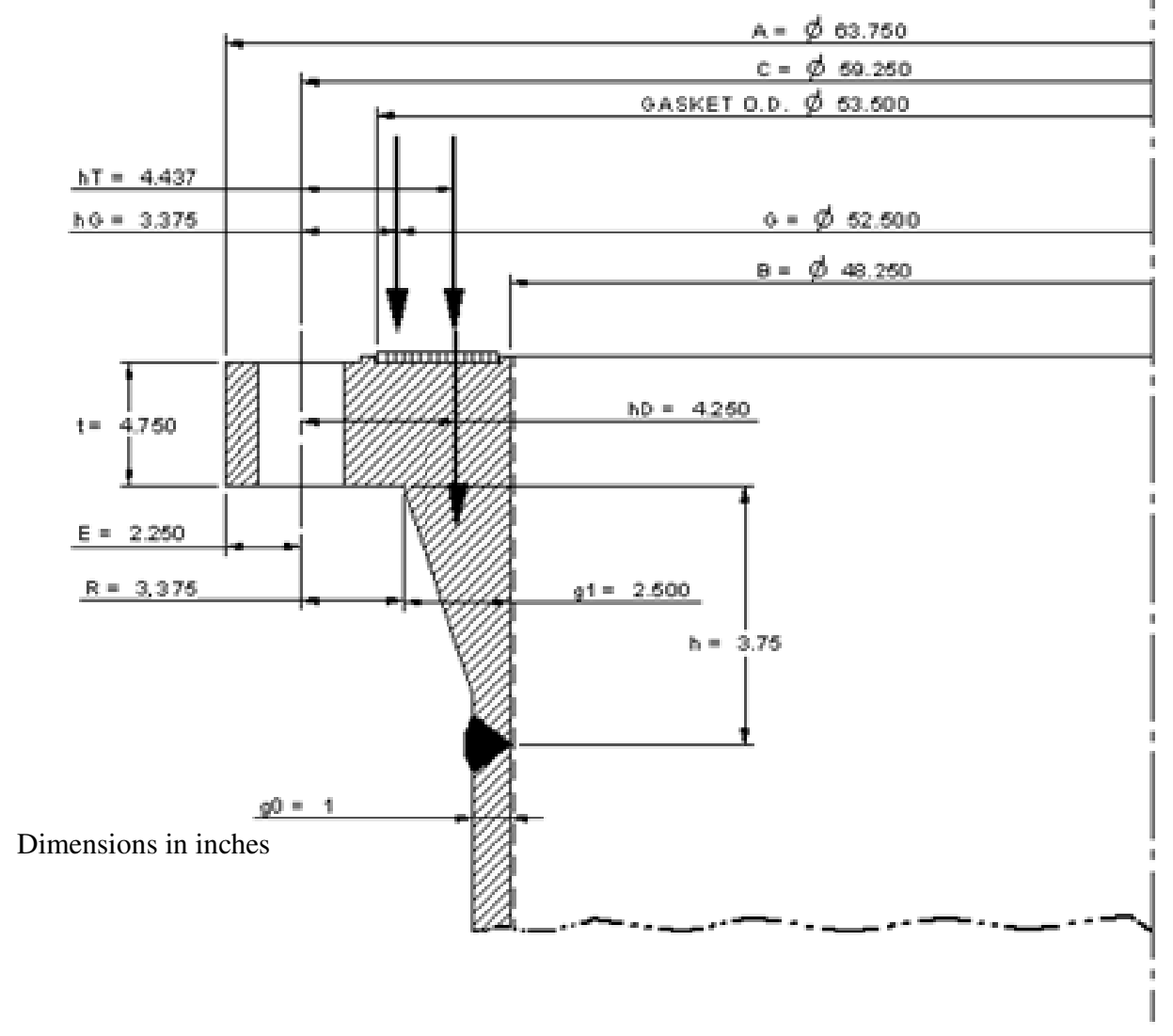

Fig. 3.21- Flange dimensions 


\subsubsection{Gasket-facing details and bolt calculations}

\subsection{Gasket selection}

ASME Code for Pressure Vessels, Section VIII, Division 1, appendix. 2, is the most commonly used design guide for gasketed joints. An important part of this code focuses on two factors: an $m$ factor, called the gasket material factor, which is associated with the hydrostatic end force, and a $y$ factor, which is the minimum seating stress associated with a particular gasket material. The $m$ or maintenance factor is essentially a safety factor to increase the clamping load to such an amount that the hydrostatic end force does not unseat the gasket to the point of leakage. The $y$ or yield factor is used to determine the clamp force required to be applied to a gasket to ensure that is seats properly to provide a seal.

Table 3.15 presents the principal features of different types of non metallic gaskets based on the operating conditions in our process.

Table 3.15-Gasket characteristics ${ }^{49}$

\begin{tabular}{|l|c|c|l|l|}
\hline Material & $\begin{array}{c}\text { Operating } \\
\text { Limitations } \\
{ }^{\mathbf{0}} \mathbf{F} / \mathbf{p s i}\end{array}$ & $\begin{array}{c}\text { Compressibility, } \\
\mathbf{\%}\end{array}$ & \multicolumn{1}{|c|}{ Advantages } & Disadvantages \\
\hline $\begin{array}{l}\text { Compressed } \\
\text { asbestos }\end{array}$ & $1,022 /$ & 8 to 10 & $\begin{array}{l}\text { Tough and durable, } \\
\text { dimensionally stable, } \\
\text { relatively incompressible, } \\
\text { good steam and hot water } \\
\text { resistance }\end{array}$ & $\begin{array}{l}\text { Less suitable for } \\
\text { lighter bolt loading }\end{array}$ \\
\hline $\begin{array}{l}\text { Asbestos- } \\
\text { free }\end{array}$ & $842 / 2,900$ & 7 to 17 & $\begin{array}{l}\text { Exceptional low chloride } \\
\text { content }\end{array}$ & $\begin{array}{l}\text { More expensive than } \\
\text { compressed asbestos }\end{array}$ \\
\hline Graphite & $932 / 4,351$ & 35 to 40 & $\begin{array}{l}\text { Wide chemical resistance, } \\
\text { good physical properties } \\
\text { non-toxic superior } \\
\text { performance on steam for } \\
\text { applications up to 572 }\end{array}$ & $\begin{array}{l}\text { Relatively high cost, } \\
\text { fragile }\end{array}$ \\
\hline
\end{tabular}

Additional issues concerning the selection of the gasket are related to fugitive emissions, compatibility with the process fluid, temperature and the internal pressure. This last parameter is of critical importance because the gasket not only has to resist the 
operating pressure in the process but also the maximum pressure which in most cases is the test pressure.

Fig. 3.22 is a distribution of gasket capabilities based on internal pressure and temperature.

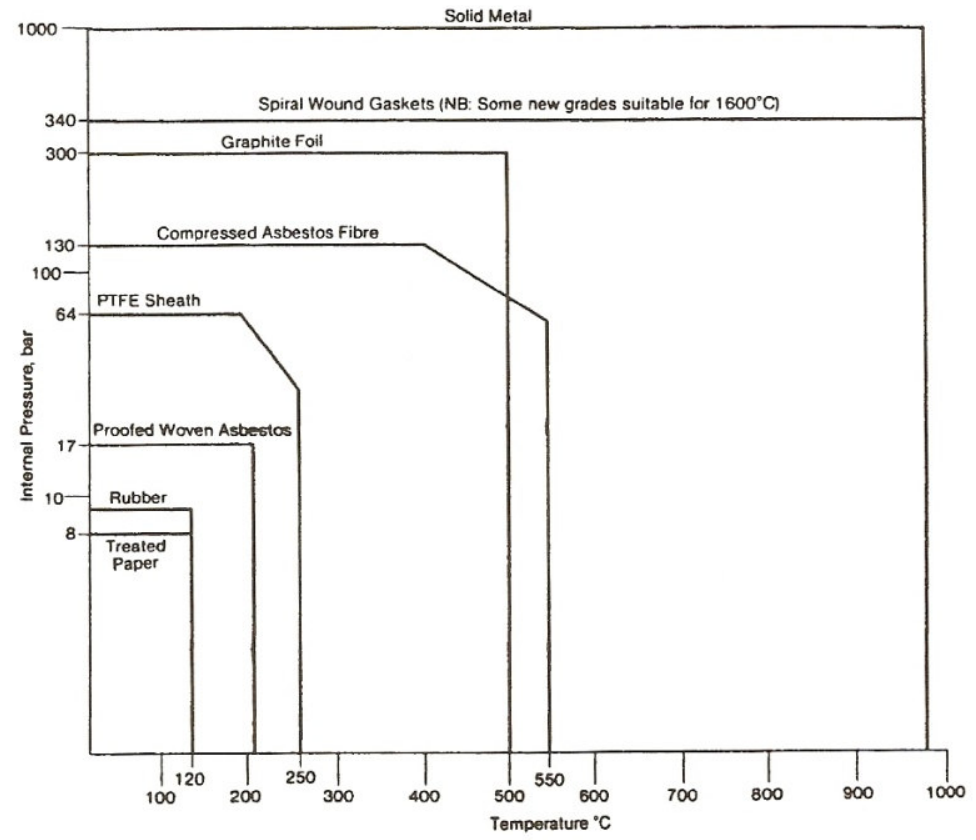

Fig. 3.22- Temperature-pressure guidelines for common gasket materials ${ }^{47}$

Selection of a compressed asbestos fiber gasket seems as a good option because of its overall resistance to pressure and temperature. However, asbestos is considered a harmful material and its use has been minimized. Nevertheless, for the purpose of this investigation values of $m$ and $y$ for compressed asbestos will be used. Once a manufacturer is identified, $m$ and $y$ should be updated to verify the adequacy of the initial assumption.

For most jointing materials, stress relaxation resistance decreases with increasing gasket thickness; therefore, it is sensible to choose a gasket as thin as possible. Thompson ${ }^{47}$ provides an equation to serve as a guide to determine the minimum thickness, $\delta$, for flat non-metallic gaskets: 
$\delta=\frac{8 \mathrm{R}_{\mathrm{a}} 100}{\mathrm{C}_{\mathrm{omp}}}$

Where:

$\delta=\quad$ minimum thickness, in.

$\mathrm{R}_{\mathrm{a}}=\quad$ flange surface finish, in. (see Table A.1 in appendix A)

$\mathrm{C}_{\mathrm{omp}}=$ compressibility, $\%$, see Table 3.15

$\delta=0,0125$ in

This means that using a gasket of 1/16 in. nominal thickness should guarantee a safety operation.

\subsection{Bolt sizing and material selection}

The bolts in a flange assembly provide the compressive force on the gasket to prevent leakage. The compressive force is transmitted through each flange to squeeze the gasket, thus a flange joint relies on bolts for its integrity.

The required bolt load for the operating conditions $\mathrm{W}_{\mathrm{m} 1}$ shall be sufficient to resist the hydrostatic end force $\mathrm{H}$ exerted by the maximum allowable working pressure and/or design pressure, on the area bounded by the diameter of gasket reaction, and in addition, to maintain on the gasket or joint-contact surface a compression load $\mathrm{H}_{\mathrm{p}}$, which experience has shown to be sufficient to assure a tight joint.

$\mathrm{W}_{\mathrm{m} 1}=\mathrm{H}+\mathrm{H}_{\mathrm{p}}$

$\mathrm{W}_{\mathrm{m} 1}=\frac{\pi \mathrm{G}^{2}}{4} \mathrm{P}+2 \mathrm{~b} \pi \mathrm{GmP}$

Where:

$\mathrm{b}=$ effective gasket or joint-contact-surface seating width, in.

$\mathrm{G}=$ diameter at location of gasket load reaction, in.

$\mathrm{m}=$ gasket maintenance factor 
Furthermore, the minimum initial load considered to be adequate for proper seating of the gasket is a function of the gasket material, and the effective gasket or contact area to be seated.

$\mathrm{W}_{\mathrm{m} 2}=\mathrm{b} \pi \mathrm{Gy}$

Where:

$\mathrm{b}=$ effective gasket or joint-contact-surface seating width, in.

$\mathrm{G}=$ diameter at location of gasket load reaction, in.

$\mathrm{y}=$ gasket yield factor, psi

The total required cross-sectional area of bolts $\mathrm{A}_{\mathrm{m}}$ required for both the operating conditions and gasket seating is the greater of the values of $A_{m 1}$ and $A_{m 2}$ defined as follows:

$A_{\mathrm{m} 1}=\frac{W_{\mathrm{m} 1}}{\mathrm{~S}_{\mathrm{b}}}$
$\mathrm{A}_{\mathrm{m} 2}=\frac{\mathrm{W}_{\mathrm{m} 2}}{\mathrm{~S}_{\mathrm{a}}}$

Where:

$\mathrm{A}_{\mathrm{m} 1}=$ total cross-sectional area of bolts at root of thread at operating conditions, in ${ }^{2}$

$\mathrm{A}_{\mathrm{m} 2}=$ total cross-sectional area of bolts at root of thread for gasket seating, $\mathrm{in}^{2}$

$\mathrm{S}_{\mathrm{b}}=\quad$ allowable bolt stress at design temperature, psi

$\mathrm{S}_{\mathrm{a}}=\quad$ allowable bolt stress at atmospheric conditions, psi

According to table 3.4, the recommended material to be used for the bolts is SA-193-B7; however, an alternative using SA-540-B22 was also considered with the purpose of reducing the number and size of bolts. The results are presented in Table 3.16

Table 3.16- Bolt cross-sectional area required and sizing information

\begin{tabular}{|l|c|c|c|c|c|}
\hline Material & $\mathbf{S}_{\mathbf{a}}=\mathbf{S}_{\mathbf{b}}, \mathbf{k s i}$ & $\mathbf{A}_{\mathbf{m} 1}, \mathbf{i n}^{\mathbf{2}}$ & $\mathbf{A}_{\mathbf{m} 2}$, in $^{\mathbf{2}}$ & Bolt size & No. of bolts \\
\hline SA-193-B7 & 25 & 65.270 & 5.277 & 2 & 30 \\
\hline SA-540-B22 & 31 & 52.642 & 4.256 & 2 & 24 \\
\hline
\end{tabular}


Evaluating results shown in Table 3.16 the final recommendation would be to use 30 bolts manufactured in SA-193-B7. The configuration of the flange and its main components are illustrated in Fig. 3.23.
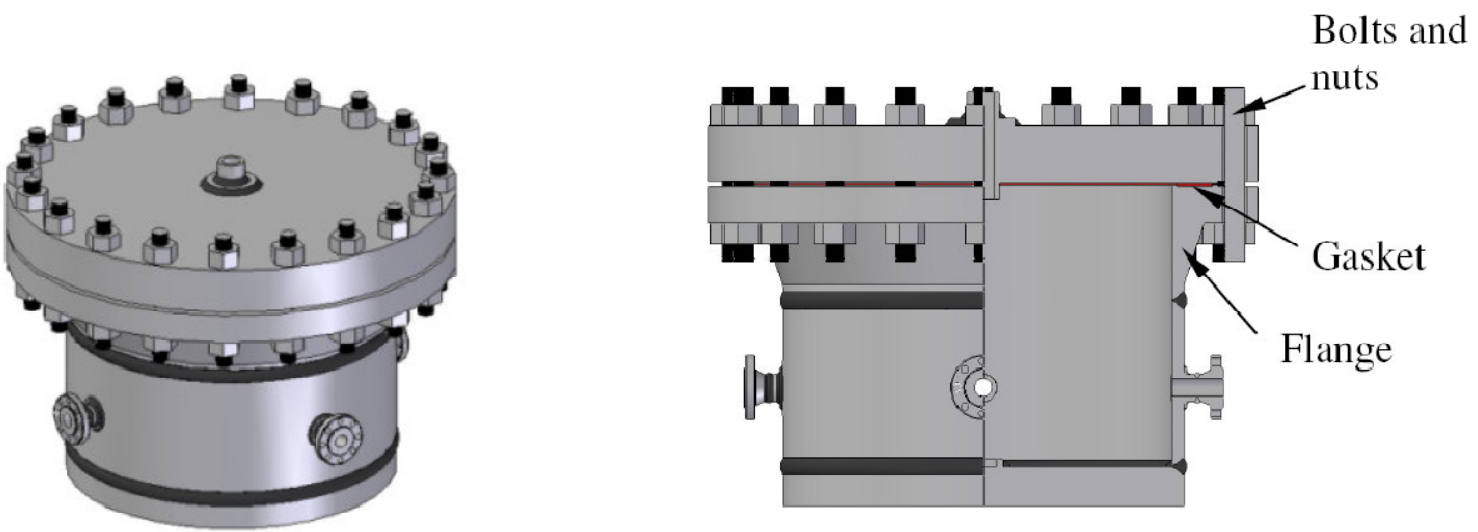

Fig. 3.23- Flange configuration

\subsubsection{Top cover head}

As in some of the elements of the pressure vessel, experimental conditions have imposed fixed conditions for the overall configuration of the recipient. For the top cover head, a flat head-type shape as seen in Fig. 3.23 is suggested because of the need of keeping a uniform thickness of the artificial porous medium and also because of pressure measurement devices intended to be installed in this location.

As per UG-34, the governing equations to calculate the thickness required for the operating and seating conditions are:

$$
\begin{aligned}
& \mathrm{t}_{\mathrm{ro}}=\mathrm{G} \sqrt{\frac{\mathrm{CP}}{\mathrm{SE}}+\frac{1.9 \mathrm{Wh}_{\mathrm{G}}}{\mathrm{SEG}^{3}}} \\
& \mathrm{t}_{\mathrm{rg}}=\mathrm{G} \sqrt{\frac{1.9 \mathrm{Wh}_{\mathrm{G}}}{\mathrm{SEG}^{3}}}
\end{aligned}
$$


Where:

$\mathrm{t}_{\mathrm{ro}}=$ thickness required for the operating condition, in.

$\mathrm{t}_{\mathrm{rg}}=$ thickness required for gasket seating, in.

$W=W_{m 1}$ and $\left(A_{m}+A_{b}\right) S_{a} / 2$ for the operating and gasket seating conditions respectively, lbf

$A_{b}=$ cross-sectional area at root diameter of bolt, in ${ }^{2}$

$\mathrm{h}_{\mathrm{G}}=$ radial distance from gasket load reaction to the bolt circle, in. (see Fig. 3.24)

$\mathrm{G}=\quad$ diameter of gasket load reaction, in. (see Fig. 3.24)

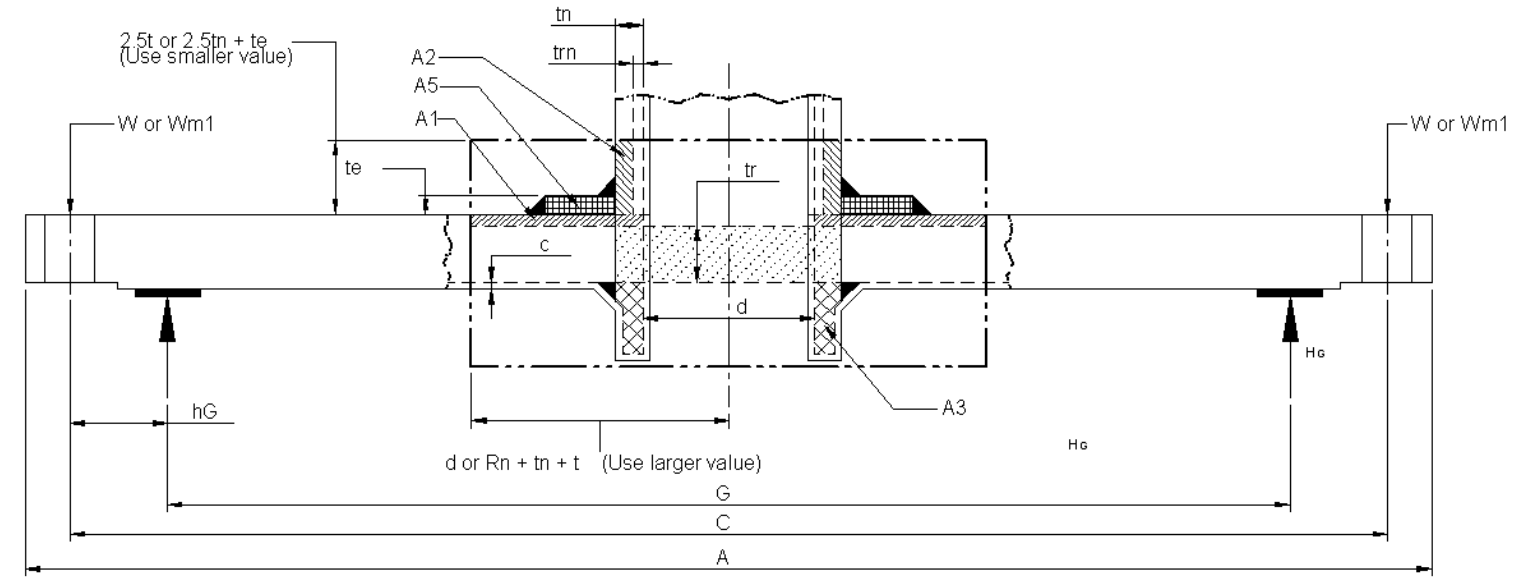

Fig. 3.24-Dimensional data and forces for a blind flange

Designating SA-516-70 as the material for fabrication (same material for the shell body) the required thicknesses in both situations are:

$\mathrm{t}_{\text {ro }}=6.083 \mathrm{in}$.

$\mathrm{t}_{\mathrm{rg}}=2.271 \mathrm{in}$.

Since the top head will have an opening for the connection of the vertical tubing, a nozzle was designed to guarantee the integrity of the system. The nominal thickness was finally determined to be 6.5 in. The corresponding values of MAWP, MAP and HTP are: 
$\mathrm{MAWP}=742 \mathrm{psi}$

$\mathrm{MAP}=782 \mathrm{psi}$

$\mathrm{HTP}=1,172 \mathrm{psi}$

\subsubsection{Nozzle attached to flat head}

For the opening calculations, the same approach was used as in 3.4.1.9 using SA-106-C to estimate the required thickness of the nozzle and to verify the need for a reinforcement element. The results are presented in Tables 3.17 and 3.18 where it can be seen that a pad was needed for the opening. Detail calculations are presented in appendix A.

Table 3.17- Nozzle neck thickness calculations and pressure limitations

\begin{tabular}{|c|c|c|c|c|c|}
\hline $\begin{array}{c}\text { Nozzle neck } \\
\text { thickness } \\
\text { required, in. }\end{array}$ & $\begin{array}{c}\text { Corrosion } \\
\text { allowance, in. }\end{array}$ & $\begin{array}{c}\text { Nominal } \\
\text { thickness, in. }\end{array}$ & MAWP, psi & MAP, psi & HTP, psi \\
\hline 0.177 & 0.125 & 0.625 & 6,266 & 8,525 & 12,787 \\
\hline
\end{tabular}

Table 3.18- Configuration of the nozzle

\begin{tabular}{|c|c|c|c|c|}
\hline $\begin{array}{c}\text { Area } \\
\text { required, } \text { in }^{2}\end{array}$ & $\begin{array}{c}\text { Weld size, } \\
\text { in. }\end{array}$ & $\begin{array}{c}\text { Area } \\
\text { available, in }\end{array}$ & $\begin{array}{c}\text { Pad } \\
\text { dimensions, in. }\end{array}$ & $\begin{array}{c}\text { Area } \\
\text { provided, in }\end{array}$ \\
\hline 9.518 & 0.750 & 7.936 & $8 \times 4.125 \times 0.75$ & 11.498 \\
\hline
\end{tabular}

\subsubsection{Static seals for nozzle-tubing assembly}

Elastomeric o-rings are probably the most common form of general purpose static seal, the groove design is relatively simple, but with compliance with some-well developed design rules is necessary to achieve a reliable seal.

From calculations performed in 3.4.1.14, it is evident that the overall area provided with the pad over exceeds the area required to secure the opening; however, it is necessary to account for the material that will be removed from the internal nozzle wall to create grooves for the installation of o-rings to seal the fluids inside the vessel as shown in Fig. 3.25. 


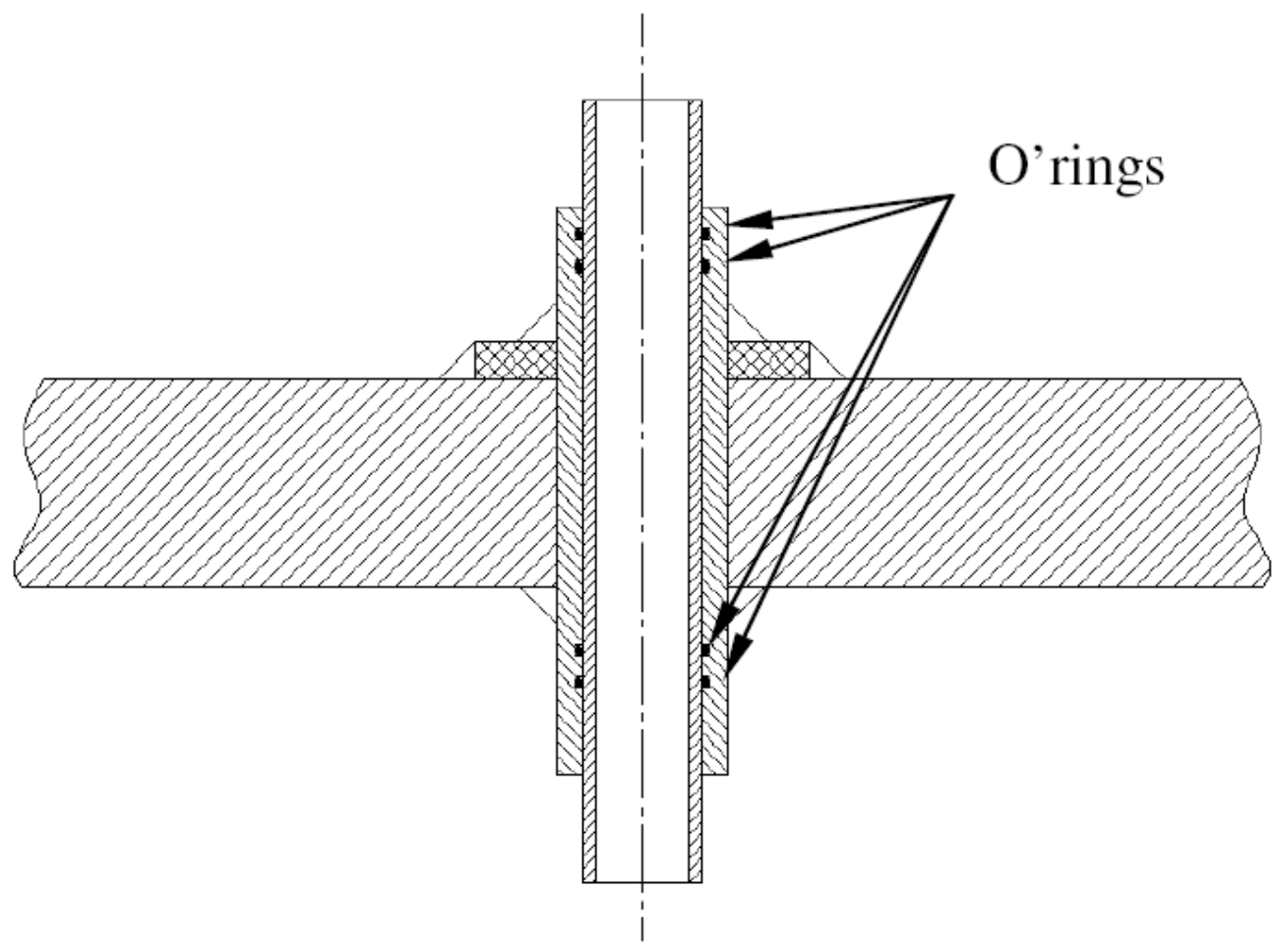

Fig. 3.25- Sealing between nozzle and tubing on top cover

Fig. 3.26 shows four static o-ring seals size 336 section 0.210 in. based on the external diameter of a 2-7/8 in. tubing. The area removed for a standard groove for a 336 o-ring is $0.097 \mathrm{in}^{2}$, therefore the area provided is:

$\mathrm{A}=(11.889-4 \times 0.097) \mathrm{in}^{2}=11.498 \mathrm{in}^{2}>9.518 \mathrm{in}^{2}$

The material selection of an o-ring is based on the same criteria used for gaskets (see 3.4.1.12.1). Among the several parameters, pressure, temperature and chemical compatibility are the most important issues. According to the fluids and existing operating conditions in the process, the most technical and economic solution is fluorocarbon (viton ${ }^{\circledR}$ ) with 72 or 90 shore A hardness. Viton has a maximum operating temperature resistance of $400^{\circ} \mathrm{F}$ and according to Fig. 3.26 a backup ring to prevent extrusion will not be necessary based on the design pressure in the system and the extrusion gap between tubing and nozzle. 


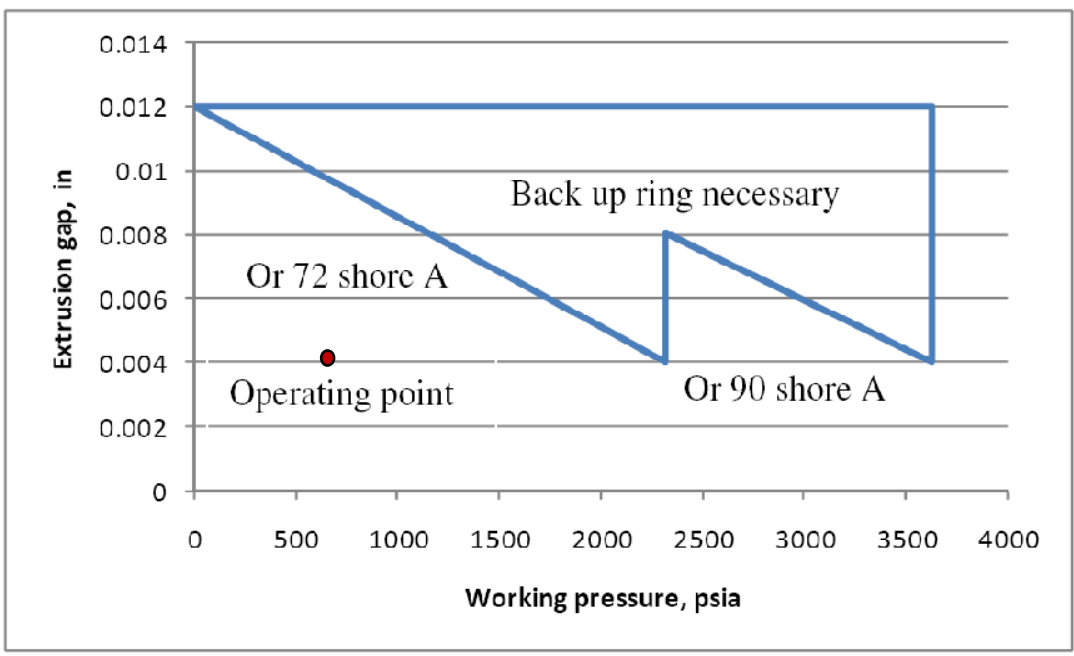

Fig. 3.26- Extrusion gap limits for two different hardness materials ${ }^{50}$

\subsubsection{Weight of top flat head and alternatives}

Reducing the weight of the vessel from an economic and logistics standpoint is of critical importance to facilitate its mobility and installation in the TowerLab. Currently, a 1-ton crane can be used for lifting equipment; however plans of getting larger capacity equipment are to be evaluated.

An alternative to lower the overall weight of the vessel is to create equally space openings on the top cover closed by bolted plates and to design of a torispherical head for the bottom cover. Even though this results in the lightest configuration, it does not guarantee a constant thickness of the porous medium.

\subsection{Openings with bolted covers}

For this option, calculations to determine the size and amount of bolts as per appendix 2 in ASME Code, as well as, the selection of the static seals is recommended. The diameter of the openings was estimated at 13.375 in.

Since these covers are removable to provide access to the interior of the vessel, using o-rings result in the most technical and economic solution to seal the fluids instead of flat gaskets. The material is Fluorocarbon size 382 shore 72 or 90 shore A.

Table 3.19 presents the results for the required thickness of the covers and the size and number of bolts. 
Table 3.19- Bolt details for upper openings

\begin{tabular}{|c|c|c|c|c|c|c|}
\hline $\begin{array}{c}\text { Thickness } \\
\text { required of } \\
\text { cover, in }\end{array}$ & $\begin{array}{c}\text { Hole } \\
\text { diameter, in. }\end{array}$ & $\mathbf{W}_{\mathbf{m} \mathbf{1}}, \mathbf{l b f}$ & $\begin{array}{c}\text { Bolt } \\
\text { material }\end{array}$ & $\begin{array}{c}\mathbf{A}_{\mathbf{m}}, \\
\mathbf{i n}^{\mathbf{2}}\end{array}$ & $\begin{array}{c}\text { Bolt size, } \\
\text { in. }\end{array}$ & No. of bolts \\
\hline 1.5 & 13.375 & $91,911.125$ & SA-197-B7 & 3.676 & 0.875 & 10 \\
\hline
\end{tabular}

As shown in 3.4.1.9., the required thickness resulted in 4.75 in. If alternatively, a flat head welded to the shell body is designed instead of using a flange bolted head, the original thickness could be decreased by 1.75 in. (6.5 to 4.75 in.). In addition, the external diameter could also be reduced by making it equal to the external diameter of the cylindrical shell. Fig 3.27, presents the idea of this alternative as compared to the original design.

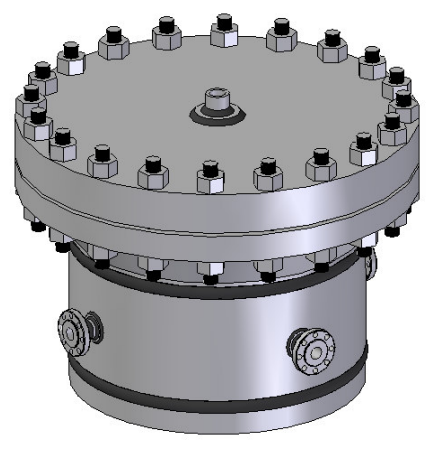

(a)

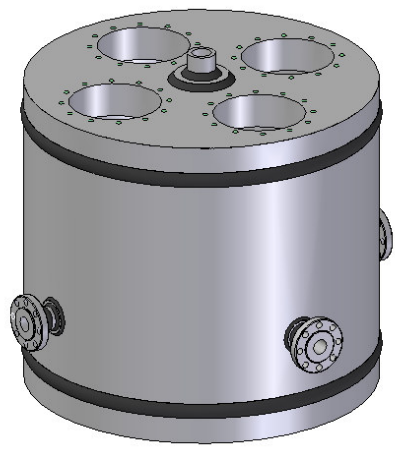

(b)

Fig. 3.27 (a) Original design compared to (b) alternative option

Additionally, the bottom flat head will have an opening designed not only to reduce the overall weight of this element, but also to provide access to remove the glass beads inside the vessel. Table 3.20 presents the results for the required thickness of the cover and the size and number of bolts.

Table 3.20- Bolt details for bottom opening

\begin{tabular}{|c|c|c|c|c|c|c|}
\hline $\begin{array}{c}\text { Thickness } \\
\text { required of } \\
\text { cover, in. }\end{array}$ & $\begin{array}{c}\text { Hole } \\
\text { diameter, in. }\end{array}$ & $\begin{array}{c}\mathbf{W}_{\mathbf{m} 1}+\text { Glass } \\
\text { beads and } \\
\text { liquid weight, } \\
\text { lbf }\end{array}$ & $\begin{array}{c}\text { Bolt } \\
\text { material }\end{array}$ & $\begin{array}{c}\mathbf{A}_{\mathbf{m}}, \\
\mathbf{i n}^{2}\end{array}$ & $\begin{array}{c}\text { Bolt size, } \\
\text { in. }\end{array}$ & No. of bolts \\
\hline 1.5 & 13.375 & $92,550.606$ & SA-197-B7 & 3.702 & 0.875 & 10 \\
\hline
\end{tabular}




\subsection{Additional options for weight reduction}

Besides the case previously presented other options were evaluated to guarantee that the chosen design resulted in the most technical and economic option for the facility. Table 3.21 shows the weight comparison of the different alternatives considering the possible weight reduction as a function of the base case (see appendix A).

Table 3.21- Configurations for weight reduction

\begin{tabular}{|c|c|c|}
\hline Option & Descripion & Figure \\
\hline 1 & $\begin{array}{l}\text { Original design } \\
\text { - Bolted flange } \\
\text { - Flat top cover } \\
\text { - Flat bottom cover }\end{array}$ & \\
\hline 2 & $\begin{array}{l}\text { Original desing with torispherical head on bottom } \\
\qquad 11 \% \text { Weight reduction }\end{array}$ & \\
\hline 3 & $\begin{array}{l}\text { Original design with four openings on top cover and } \\
\text { one opening on bottom cover } \\
\qquad \mathbf{8 \%} \text { Weight reduction }\end{array}$ & \\
\hline 4 & $\begin{array}{l}\text { Same as option No.2 but with torispherical head on } \\
\text { bottom } \\
\qquad \mathbf{1 8 \%} \text { Weight reduction }\end{array}$ & \\
\hline 5 & $\begin{array}{l}\text { Flat head cover welded to shell body (elimination of } \\
\text { bolted flange) with flat head on bottom. } \\
\qquad \mathbf{4 6 \%} \text { Weight reduction }\end{array}$ & \\
\hline 6 & $\begin{array}{l}\text { Same as option No.2 but with torispherical head on } \\
\text { bottom } \\
\qquad \mathbf{5 6 \%} \text { Weight reduction }\end{array}$ & \\
\hline
\end{tabular}


Fig. 3.28 presents the weight comparison of the six options. It is also indicated that option 5 results in the best choice since the weight is greatly reduced and the thickness of the porous medium is kept constant throughout the vessel radius.

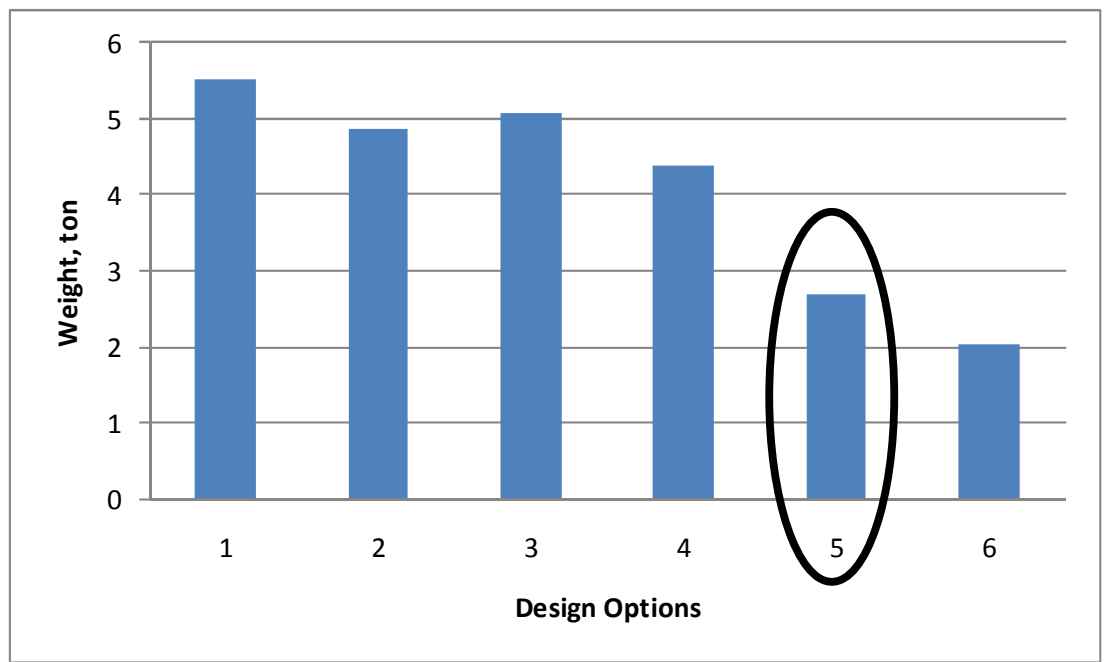

Fig. 3.28- Overall weight of pressure vessel according to different design options

\subsubsection{Vessel support}

Fig. 3.29 shows two common types of support designs for vertical pressure vessels. Selecting the right candidate not only depends on the overall resistance of the structure as compared to existing loads, but also on logistics (installation in place) and elements within the vicinity of the vessel that could be jeopardized by an inadequate support design.
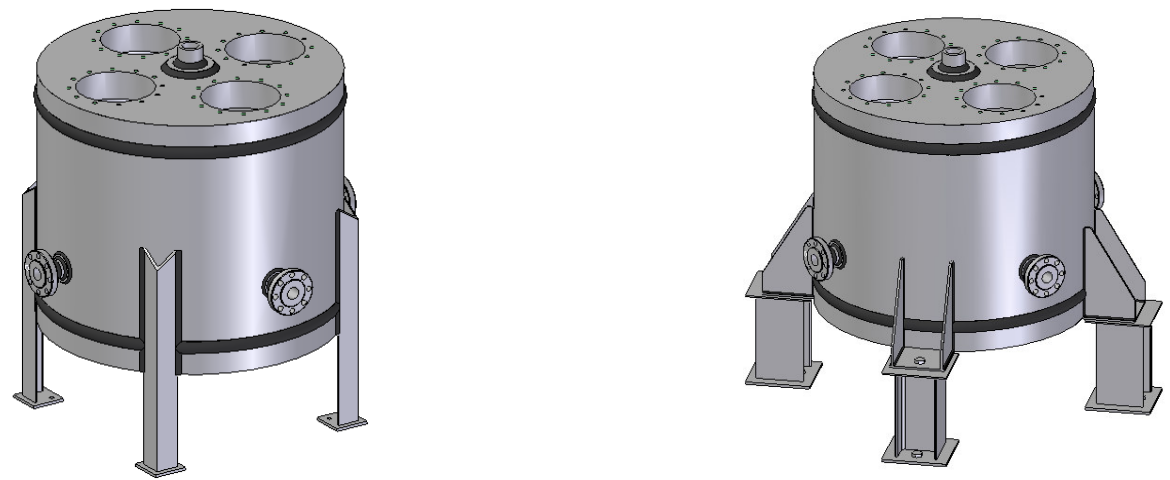

Fig. 3.29- (a) Angle and (b) lug supports 
Table 3.22 presents advantages and disadvantages of using angle or lug supports for the vessel. The final decision was to use angle beams due to the simplicity of the design and the small radial space required as compared to using lugs.

Table 3.22- Advantages and disadvantages between angle and lug support

\begin{tabular}{|c|c|c|}
\hline Configuration & Advantages & Disadvantages \\
\hline Angle column & $\begin{array}{l}\text { - Good overall axial compression } \\
\text { resistance. }\end{array}$ & $\begin{array}{l}\text { - May complicate logistics of } \\
\text { installation and mobility because } \\
\text { legs are already attached to the } \\
\text { vessel. }\end{array}$ \\
\hline Lugs & $\begin{array}{l}\text { - High axial compression } \\
\text { resistance, suitable for larger } \\
\text { loads. } \\
\text { - May facilitate installation and } \\
\text { mobility of vessel prior its final } \\
\text { installation. }\end{array}$ & $\begin{array}{l}\text { - May complicate fabrication } \\
\text { process (not as simple as the angle } \\
\text { beam column) and costs could be } \\
\text { higher. } \\
\text { - } \\
\text { Requires larger radial space. }\end{array}$ \\
\hline
\end{tabular}

Small and medium-sized vertical vessels, located on the ground and limited to the dimensions given in Table 3.23 are usually supported on uniformly spaced columns called support legs. To allow good access under the vessel, even for larger-diameter vessels the number of the support legs is held to four, braced against the wind, unless a larger number of legs cannot be avoided.

Table 3.23-Support leg recommendations ${ }^{46}$

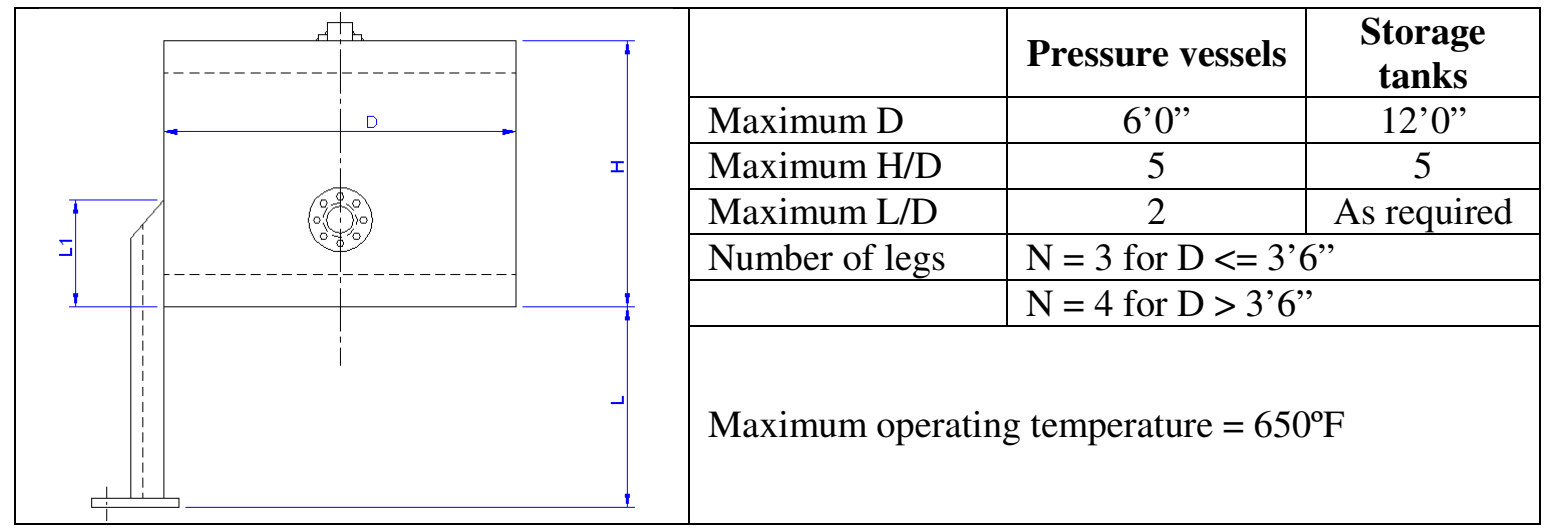


The structural shapes used for support legs are equal leg angles. The two different ways to weld the angle supports to the vessel are shown in Fig. 3.30. The position in (a) offers greater moment of inertia in resisting external loads on the vessel. For the support design considered in this investigation angle beams will be welded to the vessel according to option (b).

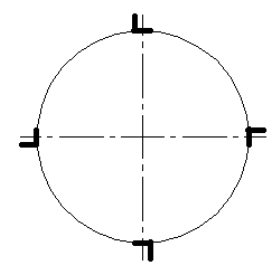

(a)

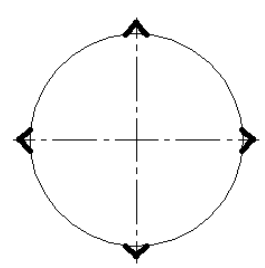

(b)

\section{Fig. 3.30- Weld configuration of support legs}

The immediate task is to determine by a stress analysis the required dimensions of the following parts:

- Support-leg columns

- Base plate

- Leg-to-shell weld size
- Leg-to-base plate weld size

- Stresses in the vessel shell at supports

- Size of anchor bolts

\subsection{Support-leg columns}

The loads imposed on the support legs are vertical and horizontal, due to the vessel weight and the force exerted by the fluids as they approach they injection manifold surrounding the pressure vessel.

Generally there are no additional moments from piping or other equipment to be considered. The tops of the support legs are assumed to be welded to a rigid vessel wall that is actually flexible. The anchor bolts are initially pretightened, and as long as some compression between the base plates and the foundation exists due to weight and the initial bolt load, the vessel will have a tendency to overturn about the axis A-A (Fig. 3.31) as a neutral axis and the reactions in the columns due to the overturning moment $\mathrm{Mb}$ will be proportional to the distance from the axis A-A. 

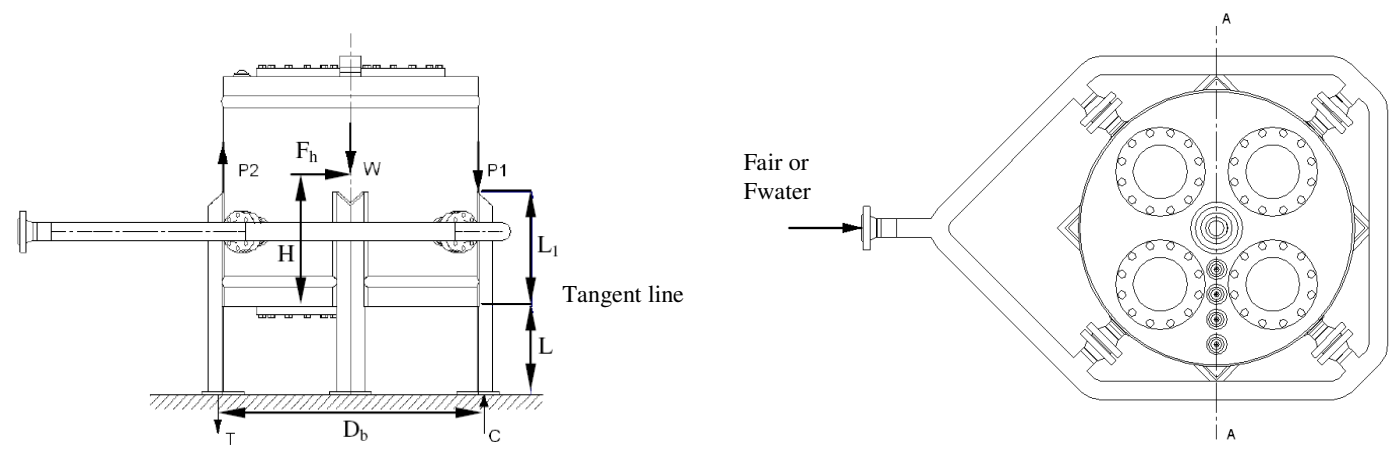

Fig. 3.31 Top and lateral view of vessel

The vertical reactions $\mathrm{C}$ in compression and $\mathrm{T}$ in tension at the support base consist of (a) vessel weight, assumed to be equal to W/N for every leg, and (b) reaction to the overturning moment, $\mathrm{Mb}$.

(a) Vessel weight and contents

The vessel weight corresponds to 5,934 lbs (without bolted covers -4 on top and 1 on bottom). However, we assumed 14,000 lbs to include additional elements attached to the vessel. In addition, the weight of contents was calculated as the total volume inside the vessel available for both the glass beads and water. The weight of the vertical tubing inside the vessel will be absorbed by support devices to be located on every floor in the building. These devices will also work absorbing the axial force exerted on the tubing during the experiments. The final weight " $\mathrm{W}_{\mathrm{o}}$ " is:

$\mathrm{W}_{\mathrm{o}}=\mathrm{W}_{\text {vessel }}+\mathrm{W}_{\mathrm{c}}=14,000+8,236 \mathrm{lbs}=22,240 \mathrm{lbs}$

(b) Reaction to the overturning moment, $\mathrm{M}_{\mathrm{b}}$ :

Fig. 3.32 illustrates the equivalent static force produced by dynamic flow.

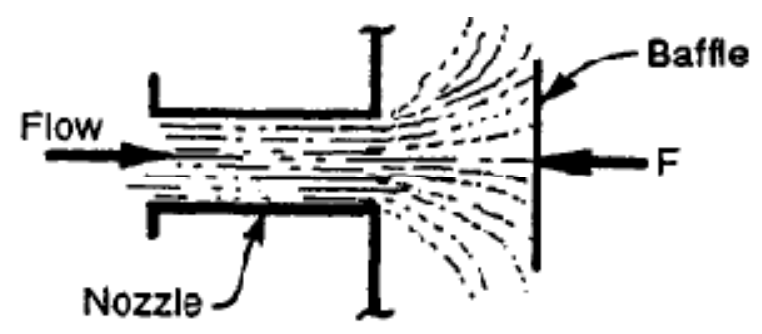

Fig. 3.32- Equivalent static force for dynamic flow ${ }^{47}$ 
For any fluid in a conduit, the static force when in contact with a wall can be estimated by:

$\mathrm{F}_{\text {fluid }}=\frac{\mathrm{A} \rho_{\text {fluid }} \mathrm{V}^{2}}{\mathrm{~g}}$

Where:

$\mathrm{F}_{\text {fluid }}=\quad$ Static force, $\mathrm{lbf}$

$\mathrm{A}=\quad$ Area, $\mathrm{ft}^{2}$

$\rho_{\text {fluid }}=$ Density of air and/or water, $1 \mathrm{bm} / \mathrm{ft}^{3}$

$\mathrm{V}=\quad$ Velocity, $\mathrm{ft} / \mathrm{s}$

$\mathrm{g}=\quad$ Acceleration of gravitational, $\mathrm{ft} / \mathrm{s}$

As seen in Fig. 3.32 fluids are driven to the injection manifold by means of a Yconnection, nevertheless the case as depicted in Fig. 3.33 was considered for this study.

The approach consisted in calculating the force when water and air are running independently through the pipeline, and then use the maximum value as the horizontal force for the support design. The results for both air and water are the following:

$\mathrm{F}_{\text {air }}=0.128 \mathrm{lbf}$

$\mathrm{F}_{\text {water }}=0.01 \mathrm{lbf}$

Then, the maximum horizontal force, $\mathrm{F}_{\mathrm{h}}$, is:

$\mathrm{F}_{\mathrm{h}}=0.128 \mathrm{lbf}$

The overturning moment at base and tangent line are calculated as:

$\mathrm{M}_{\mathrm{b}}=(\mathrm{L}+\mathrm{H}) \mathrm{F}_{\mathrm{h}}$

$\mathrm{M}_{\mathrm{t}}=\mathrm{F}_{\mathrm{h}} \mathrm{H}$

The maximum total axial load at the leeward side (compression) for the operating condition is:

$\mathrm{C}_{\mathrm{o}}=\frac{\mathrm{W}_{\mathrm{o}}}{\mathrm{N}}+\frac{4 \mathrm{M}_{\mathrm{b}}}{\mathrm{ND}_{\mathrm{b}}}$ 
Where:

$\mathrm{W}_{\mathrm{o}}=\quad$ total weight of vessel and contents, lbm

$\mathrm{N}=\quad$ number of legs

$\mathrm{M}_{\mathrm{b}}=\quad$ overturning moment at base, lbf.ft

$\mathrm{D}_{\mathrm{b}}=\quad$ distance between legs, in. (see Fig 3.32)

The maximum axial load on the side subject to tension for the operating conditions is:

$\mathrm{T}_{\mathrm{o}}=-\frac{\mathrm{W}_{\mathrm{o}}}{\mathrm{N}}+\frac{4 \mathrm{M}_{\mathrm{b}}}{\mathrm{ND}_{\mathrm{b}}}$

(c) Eccentric loads

The eccentric loads $\mathrm{P}_{1}$ and $\mathrm{P}_{2}$ at column top for the operating conditions are:

$P_{1}=\frac{W_{o}}{N}+\frac{4 M_{a}}{N D_{b}}$
$P_{2}=-\frac{W_{o}}{N}+\frac{4 M_{a}}{N D_{b}}$

Where:

$\mathrm{M}_{\mathrm{a}}=$ moment at tangent line, lbf.ft

(d) Lateral forces on legs

For computing the lateral load per column from the force $F_{h}$, the deflections at the base of all columns under load $F_{h}$ are approximately equal to:

$\mathrm{F}=\frac{\mathrm{F}_{\mathrm{h}} \mathrm{I}}{\sum \mathrm{I}}$

Where $I$ is the moment of inertia of the column cross section about the axis perpendicular to the direction of the horizontal force and $\sum \mathrm{I}$ is the summation of the moments of inertia of all column cross sections about the axes perpendicular to the direction of $F_{h}$. The results are illustrated in Fig. 3.33. 


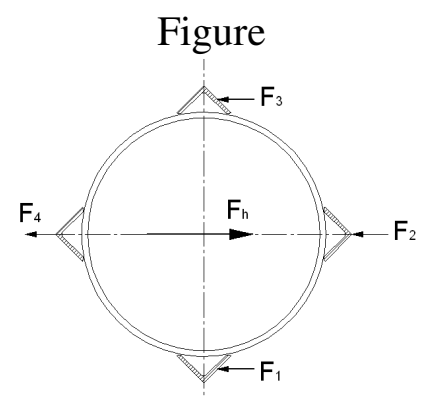

Inertia

$$
\begin{aligned}
& \mathrm{I}_{1}=\mathrm{I}_{3}=6.944 \mathrm{in}^{4} \\
& \mathrm{I}_{2}=\mathrm{I}_{4}=1.776 \mathrm{in}^{4} \\
& \mathrm{~F}_{1}=\mathrm{F}_{3}=0.051 \mathrm{lbf} \\
& \mathrm{F}_{2}=\mathrm{F}_{4}=0.013 \mathrm{lbf}
\end{aligned}
$$

Fig. 3.33- Distribution of lateral forces on columns

(e) Combined column stress in compression

In this step we need to calculate the axial force $f_{a}$, and bending stress $f_{b}$ for every leg.

$f_{a}=\frac{C_{n}}{A}$

Where the vertical load distribution and bending moments for every leg, $C_{n}$ and $\mathrm{M}_{\mathrm{n}}$ are:

$\mathrm{C}_{\mathrm{n}}=\frac{\mathrm{W}_{\mathrm{o}}}{\mathrm{N}} \pm \frac{4 \mathrm{M}_{\mathrm{n}}}{\mathrm{ND}_{\mathrm{b}}}$

$M_{n}=P_{n} e \pm F_{n} L$

Fig. 3.34 shows the situation describing the horizontal force acting on a single leg.

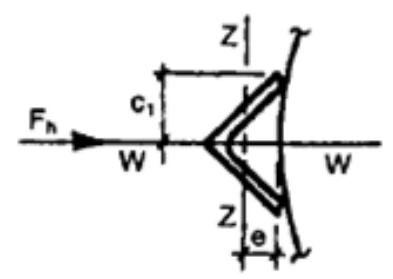

Fig. 3.34- Leg configuration for horizontal force distribution ${ }^{47}$

The corresponding equation is given by:

$\mathrm{f}_{\mathrm{b}}=\frac{\mathrm{M}_{\mathrm{n}} \mathrm{C}_{1}}{\mathrm{I}}$ 
Since the allowable unit stress $F_{a}$ in a column for axial loads only is not equal to the allowable unit stress $F_{b}$ in bending in absence of an axial load, we need to know the value at which the total combined stress in a column be limited to. A widely used procedure is the straight line interaction method used in AISC handbook. This method of determining the limit stress, without determining the principal stresses, is based on the assumption that if a certain percentage of the strength of a member has been used for axial compression load, the remaining percentage may be used for bending:

$\frac{f_{a}}{F_{a}} \leq 0.15 \Rightarrow \frac{f_{a}}{F_{a}}+\frac{f_{b}}{F_{b}} \leq 1$

$\frac{f_{a}}{F_{a}}>0.15 \Rightarrow \frac{f_{a}}{F_{a}}+\frac{C_{m} f_{b}}{\left[1-\frac{f_{a}}{F^{\prime}}\right] F_{b}} \leq 1$

For good design the expression on the right should be close to one. The value of $\mathrm{F}_{\mathrm{b}}$ can be taken as $0.6 \mathrm{~F}_{\mathrm{y}}$, the yield strength; the reduction factor $\mathrm{C}_{\mathrm{m}}$ conservatively equals one. The allowable stress $\mathrm{F}_{\mathrm{a}}$ as well as $\mathrm{F}_{\mathrm{e}}{ }_{\mathrm{e}}$ can be obtained directly from the AISC Manual, they depend on the slenderness ratio $\mathrm{kl} / \mathrm{r}$ and $\mathrm{kl} / \mathrm{r}_{\mathrm{b}}$ and the yield strength $\mathrm{F}_{\mathrm{y}}$. Considering ASTM A36 (yield stress of $36 \mathrm{ksi}$ ) as the chosen material for the structural shape, we obtain the results shown in Table 3.24:

Table 3.24- Column parameters

\begin{tabular}{|c|c|c|c|c|}
\hline Parameter & Leg 1 & Leg 2 & Leg 3 & Leg 4 \\
\hline Vertical load, C, lbf & \multicolumn{4}{|c|}{5,692} \\
\hline Bending moment, M, lbf.in. & \multicolumn{4}{|c|}{6,972} \\
\hline Axial stress, $\mathrm{f}_{\mathrm{a}}, \mathrm{psi}$ & \multicolumn{4}{|c|}{1,990} \\
\hline Bending stress, $\mathrm{f}_{\mathrm{b}}, \mathrm{psi}$ & 2,890 & 11,100 & 2,840 & 11,101 \\
\hline Total compressive stress, psi & 4,830 & 13,090 & 4,830 & 13,092 \\
\hline $\mathrm{f}_{\mathrm{a}} / \mathrm{F}_{\mathrm{a}}$ & \multicolumn{4}{|c|}{0.095} \\
\hline $\mathrm{f}_{\mathrm{b}} / \mathrm{F}_{\mathrm{b}}$ & 0.13 & 0.51 & 0.13 & 0.51 \\
\hline $\mathrm{f}_{\mathrm{a}} / \mathrm{F}_{\mathrm{a}}+\mathrm{f}_{\mathrm{b}} / \mathrm{F}_{\mathrm{b}}(<1)$ & 0.23 & 0.60 & 0.23 & 0.60 \\
\hline
\end{tabular}


(f) Shear load in welds attaching legs

To properly size the fillet welds for attaching the leg supports to the shell body, we must determine the shear load and select a material and weld size suitable for the load conditions.

$f_{s}=\frac{\mathrm{P}_{1}}{2 \mathrm{~h}}=\frac{\mathrm{lb}}{\text { in. of weld }}$

The bending stress is given by:

$f_{b}=\frac{\left[\frac{\mathrm{C}_{\mathrm{o}} \mathrm{D}_{\mathrm{b}}}{2}+\frac{\mathrm{F}_{\mathrm{h}} \mathrm{L}}{4}\right]}{\mathrm{Z}_{\mathrm{w}}}=\frac{\mathrm{lb}}{\text { in. of weld }}$

Where $Z_{w}$ is the linear section modulus of the weld (see Table A.8 in appendix A).

The total force in the weld is finally calculated by:

$f_{t}=\sqrt{f_{s}^{2}+f_{b}^{2}}$

The weld size is calculated based on the electrode type as:

$w=\frac{f_{t}}{E_{s}}$

The final results based on electrode E70 combined with A36 structural steel are presented in Table 3.25

Table 3.25- Stresses to determine weld sizes

\begin{tabular}{|l|l|}
\hline Shear load, lb/in.weld & 116 \\
\hline Bending stress, lb/in.weld & 356 \\
\hline Modulus, in ${ }^{3}$ & 192 \\
\hline Total stress, lb/in.weld & 374 \\
\hline E60, lbf/in weld size & 2,390 \\
\hline Weld size required, in. & 0.157 \\
\hline Final weld size, in. & $3 / 16$ \\
\hline
\end{tabular}




\subsection{Base plates and bolts}

Base plates are primarily used to distribute large, concentrated vertical loads into the concrete foundation so that the concrete is not overstressed and to accommodate the anchor bolts. They are shop welded to the support legs.

The loads used in connection with angle support legs are too low and practical dimensions for base plates are usually selected. Under an axial column load alone the bearing pressure between the base plate and the concrete base is assumed to be uniformly distributed. The total downward load on the foundation is the sum of the initial tensile load in the anchor bolts and the column loads. The size of the anchor bolts is usually not too large, and the effect of the tensile load is neglected. ${ }^{48}$

For the design of the base plate, we could neglect the effects of the overturning moments only considering the axial loads. The minimum thickness of the plate is calculated by:

$t=\sqrt{\frac{3 f_{c L^{2}}}{F_{b}}}$

Where:

$\mathrm{f}_{\mathrm{c}}=\quad$ Bearing pressure, $\mathrm{psi}$

$\mathrm{L}=\mathrm{m} \quad$ See Figure 3.35, in.

$\mathrm{F}_{\mathrm{b}}=\quad$ Allowable bending stress, psi

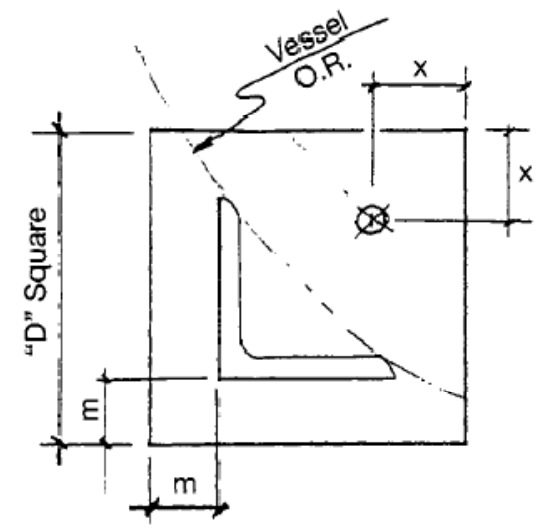

Fig. 3.35- Angle parameters ${ }^{47}$ 
$f_{c}=\frac{W o}{D^{2}}$

Dimensions for typical angle beams are presented in Table 3.26. Table 3.27 shows the results.

Table 3.26- Angle main dimensions ${ }^{47}$

\begin{tabular}{|l|c|c|c|c|}
\hline Leg size, in & D, in. & $\mathbf{x , \text { in. }}$ & $\mathbf{m}$, in. & Min. thickness, in. \\
\hline $2 \times 2$ & 4 & 1.5 & 1 & $1 / 2$ \\
\hline $2.5 \times 2.5$ & 5 & 1.5 & 1.25 & $1 / 2$ \\
\hline $3 \times 3$ & 6 & 1.75 & 1.5 & $1 / 2$ \\
\hline $4 \times 4$ & 8 & 2 & 2 & $5 / 8$ \\
\hline $5 \times 5$ & 9 & 2.75 & 2 & $5 / 8$ \\
\hline $6 \times 6$ & 10 & 3.5 & 2 & $3 / 4$ \\
\hline
\end{tabular}

Table 3.27- Plate dimensions

\begin{tabular}{|l|l|}
\hline Angle section & $4 \times 4 \times 3 / 8$ \\
\hline Bearing pressure, $\mathrm{f}_{\mathrm{c}}, \mathrm{psi}$ & 347 \\
\hline $\mathrm{L}=\mathrm{m}$, in. & 2 \\
\hline Allowable bending stress, $\mathrm{F}_{\mathrm{b}}$ & 21,600 \\
\hline Thickness, $\mathrm{t}$, in. & 0.438 \\
\hline Final thickness & $5 / 8$ \\
\hline
\end{tabular}

As for the bolts to be used on the plates, if:

$W_{o}>\frac{4 M_{b}}{D_{b}}$

then no uplift occurs and anchor bolts should be made a minimum of $3 / 4$ in. diameter. ${ }^{47}$ Additionally the governing forces on the plate will be mainly axial due to the weight of the vessel, therefore the weld sizes between the angle and plates could be the same as the one used between the angle and shell body.

\subsubsection{Lifting lugs}

Lifting lugs are mechanical elements attached to pressure vessels that allow their transportation and installation at the desired location. Lifting lugs can be installed in 
different areas of the vessel; however the position and final number of lugs depend on the configuration, whether vertical or horizontal and the maximum weight of the arrangement. Lifting lugs are commercially available and they have already been designed for different loads. Fig. 3.36 shows a typical lug and Table 3.28 provides information for the lug chosen for the vessel. Table A.9 in appendix A shows additional data for other sizes.
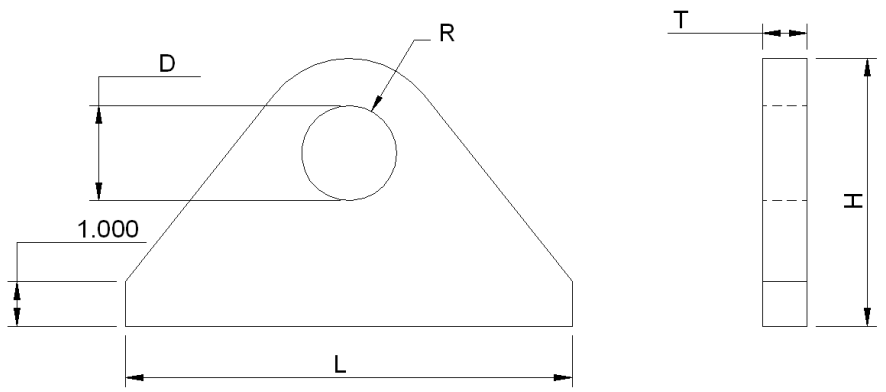

Fig. 3.36- Lug design for lifting purposes

Table 3.28- Lug data for pressure vessel

\begin{tabular}{|l|c|}
\hline $\begin{array}{l}\text { Vessel } \\
\text { weight }\end{array}$ & 30,000 \\
\hline No. of lugs & 2 \\
\hline $\mathbf{D}$, in. & $1-3 / 8$ \\
\hline $\mathbf{T}$, in. & 1 \\
\hline $\mathbf{R}$, in. & $6-1 / 8$ \\
\hline $\mathbf{H}$, in. & 10 \\
\hline $\mathbf{L}$, in. & $\begin{array}{c}\text { Full penetration } \\
\text { with } 1 / 2 \text { fillet }\end{array}$ \\
Weld (min.) & \\
\hline
\end{tabular}

\subsubsection{Design of the compressed air system}

The TowerLab involves the installation of static, as well as dynamic equipment for achieving the required operating conditions for the system to be able to mimic liquid loading under similar conditions as those found in real gas wells; however one of the critical elements of the facility is the compressed air system. 
A modern industrial compressed air system is composed of several major subsystems and many sub-components. Major sub-systems include the compressor, prime mover, controls, treatment equipment and accessories, and the distribution system. The compressor is the mechanical device that takes in ambient air and increases its pressure. The prime mover powers the compressor. Controls serve to regulate the amount of compressed air being produced. The treatment equipment removes contaminants from the compressed air, and accessories keep the system operating properly. The distribution system is used to transport the air where it is needed. Fig. 3.37 shows a representative industrial compressed air system and its components.

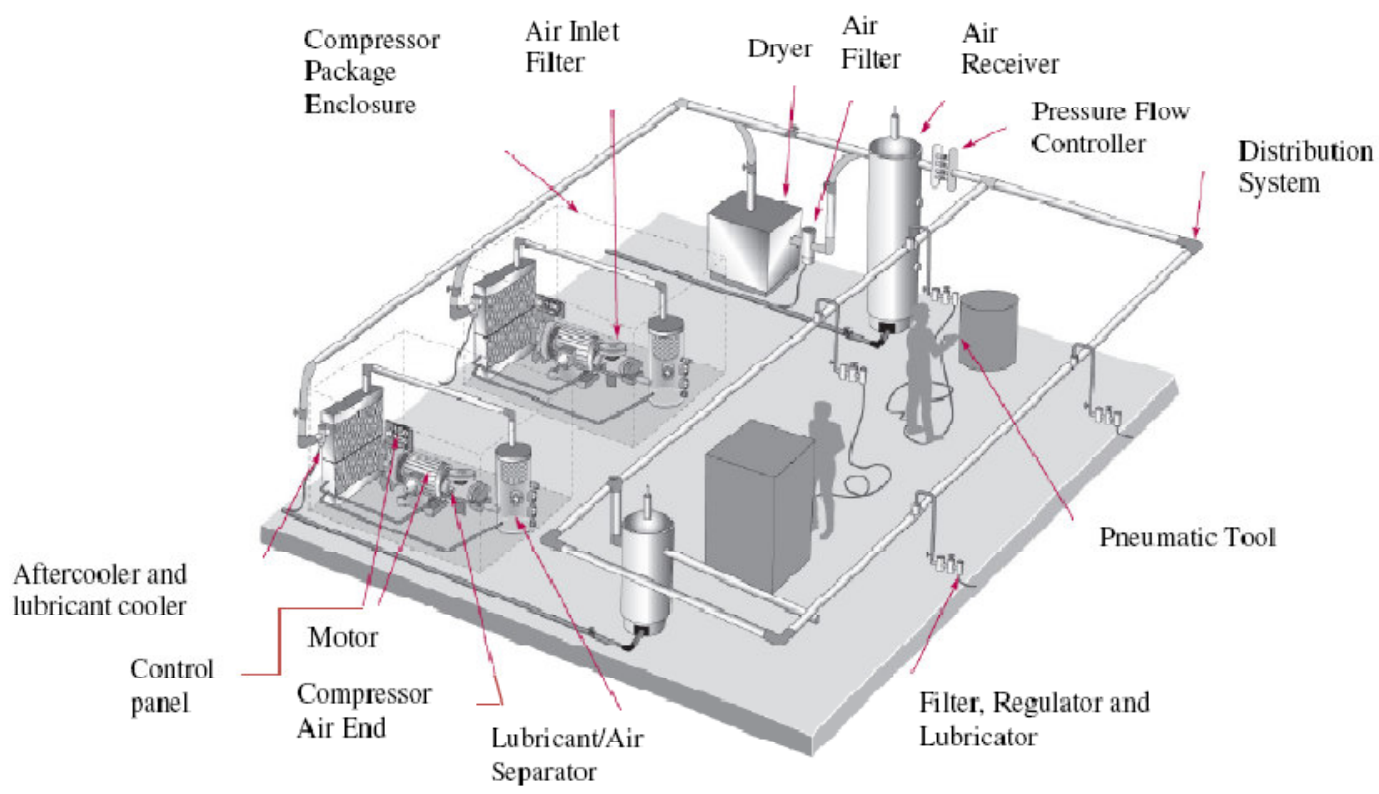

Fig. 3.37- Compressed air system and its components ${ }^{51}$

The object of installing a compressed air system is to provide air at the point of application in sufficient quantity and quality, and with adequate pressure for efficient operation of the system.

\subsubsection{Technical considerations and compressed air needs for the TowerLab}

There are important considerations in the design of the compressed air system to be installed at the TowerLab. A typical compressed air system is usually defined by the 
air quality, quantity, load pattern and level of pressure required by the application being studied. Analyzing these needs carefully will ensure that a compressed air system is configured properly.

\subsection{Air quality}

The quality of the compressed air produced by a system can range from plant air to high quality breathing air. Different end-users require different levels of air quality (see Table 3.29). Dryness and contaminant level are the two key factors used to distinguish low from high quality air. Higher quality air usually requires additional equipment, which not only increases initial capital investment, but also makes the overall system more expensive to operate in terms of energy consumption and maintenance costs.

Table 3.29- Types of air quality ${ }^{52}$

\begin{tabular}{|l|l|}
\hline \multicolumn{1}{|c|}{ Air Quality } & \multicolumn{1}{c|}{ Applications } \\
\hline Breathing air & $\begin{array}{l}\text { Hospital air systems, refill diving tanks, respirators for cleaning and/or grit blasting } \\
\text { and spray painting }\end{array}$ \\
\hline Process air & Food and pharmaceutical process air, electronics \\
\hline Instrument air & Laboratories, paint spraying, powder coating, climate control \\
\hline Plant air & Air tools, general plant air \\
\hline
\end{tabular}

Maintaining air quality is so important that the International Standards Organization (ISO) developed six compressed air quality classes, as defined by ISO8573-1 illustrated in Table 3.30.

Table 3.30- Compressed air quality ISO 8573-1:2001 ${ }^{52}$

\begin{tabular}{|c|c|c|c|c|c|}
\hline $\begin{array}{l}\text { Quality } \\
\text { Class }\end{array}$ & 0.1 to 0.5 micron & $\begin{array}{c}\text { Solids } \\
\text { nber of Particles }\end{array}$ & 1 to 5 micron & $\begin{array}{c}\text { Water } \\
\text { Pressure Dew } \\
\text { Point } \\
{ }^{\circ} \mathrm{C}\end{array}$ & $\begin{array}{c}\text { Oil \& Oil } \\
\text { Vapor } \\
\text { mg/m }\end{array}$ \\
\hline 0 & \multicolumn{5}{|c|}{ As specified by the end user or manufacturer, and more stringent than Class 1} \\
\hline 1 & 100 & 1 & 0 & -70 & 0.01 \\
\hline 2 & 100,000 & 1,000 & 10 & -40 & 0.1 \\
\hline 3 & N/A & 10,000 & 500 & -20 & 1 \\
\hline 4 & N/A & N/A & 1,000 & 3 & 5 \\
\hline 5 & N/A & N/A & 20,000 & 7 & N/A \\
\hline 6 & N/A & N/A & N/A & 10 & N/A \\
\hline
\end{tabular}


When selecting a compressor, consideration is to be given to the level of air quality required. In the case of lubricant-free air, this can be achieved with either lubricant-free compressors, or with lubricant-injected compressors that have additional separation and filtration equipment. Lubricant-free compressors usually cost more to install and have higher maintenance costs. Lubricant-injected compressors while cheaper to purchase have the additional capital, energy and maintenance costs of separation and filtration equipment. Table 3.31 lists some of the characteristics of the two compressor types in terms of the advantages and disadvantages.

\section{Table 3.31- Advantages and disadvantages of non-lubricated and lubricated} compressors

\begin{tabular}{|c|c|c|}
\hline Compressor & Non-Lubricated & Lubricated \\
\hline Advantages & $\begin{array}{l}\text { - More efficient and thus lower running } \\
\text { cost. } \\
\text { - May require fewer filters and oil } \\
\text { changes. } \\
\text { - Longer operational life. } \\
\text { - Often preferred when manufacturing } \\
\text { sensitive products such as food and } \\
\text { pharmaceuticals }\end{array}$ & $\begin{array}{l}\text { - Considerably lower capital cost. } \\
\text { - Simple plant. } \\
\text { - Oil provides an important } \\
\text { cooling effect. } \\
\text { - Lower speeds/temperatures than } \\
\text { oil-free compressors }\end{array}$ \\
\hline Disadvantages & $\begin{array}{l}\text { - Capital cost is normally greater } \\
\text { - Routine servicing costs are usually } \\
\text { higher } \\
\text { - Multi-stage compression necessary to } \\
\text { reach higher pressures. } \\
\text { - Compressors are more complex }\end{array}$ & $\begin{array}{l}\text { - } \text { More frequent rebuilds } \\
\text { - } \text { More filter maintenance and oil } \\
\text { changes required. } \\
\text { - Greater treatment capital and } \\
\text { running costs (due to pressure } \\
\text { drop across filters) }\end{array}$ \\
\hline
\end{tabular}

In addition to understanding the air quality needs and compressor types, efficient methods to reduce contaminants need to be investigated. Prior to the compression cycle an air compressor inhales water vapor, dirt, and atmospheric pollution. During the process the volume of air reduces, causing the level of contamination to increase. Additionally, further contaminants such as oil vapor or wear particles can be introduced by certain types of compressors during the compression process.

This concentration of contaminants means that the compressed air can rarely be used without some form of treatment. A wide range of filtration and drying equipment is 
available to improve air quality. Choosing an appropriate area to install the compressor is of critical importance so that exposure to contaminants present in the environment surrounding the equipment is minimized.

The quality of air required for the TowerLab flow loop could be classified, according to ISO standards, as Class 3 or 4 . Table 3.32 describes common applications for different air quality classes.

Table 3.32- Industry classification for air quality for common applications ${ }^{\mathbf{5 2}}$

\begin{tabular}{|c|c|c|}
\hline Class & Description & Applications \\
\hline $\begin{array}{l}\text { IN1 } \\
\text { Instrument Grade Air: } \\
\text { ISO Class 2.1.1 }\end{array}$ & $\begin{array}{lllr}\text { Efficient } & \text { removal of solid } \\
\text { particulates } & \text { and oil. ISO Class } 1 . \\
\text { Pressure } & \text { dewpoint will be } \\
\text { maintained } & & & \end{array}$ & $\begin{array}{l}\text { Instrumentation, process, oil and } \\
\text { gas, chemical, electronics }\end{array}$ \\
\hline $\begin{array}{l}\text { IN1 Odor Free } \\
\text { Instrument Grade Air: } \\
\text { ISO Class } 2.1 .1 \text { odor } \\
\text { free }\end{array}$ & $\begin{array}{l}\text { Efficient removal of solid } \\
\text { particulates, oil and oil vapor. ISO } \\
\text { Class 1. Pressure dewpoint will be } \\
\text { maintained }\end{array}$ & $\begin{array}{l}\text { Pharmaceutical, food and } \\
\text { beverage, clean rooms }\end{array}$ \\
\hline $\begin{array}{l}\text { IN2 } \\
\text { Instrument Grade Air } \\
\text { ISO Class 2.2.1 }\end{array}$ & $\begin{array}{lllr}\text { Efficient } & \text { removal of } & \text { solid } \\
\text { particulates } & \text { and oil. ISO Class } 2 . \\
\text { Pressure } & \text { dewpoint will be } \\
\text { maintained } & & & \end{array}$ & $\begin{array}{l}\text { Instrumentation, process, oil and } \\
\text { gas, chemical, electronics. }\end{array}$ \\
\hline $\begin{array}{l}\text { IN2 Odor Free } \\
\text { Instrument Grade Air: } \\
\text { ISO Class 2.2.1 } \\
\text { Odor free }\end{array}$ & $\begin{array}{l}\text { Efficient removal of solid } \\
\text { particulates, oil and oil vapor. ISO } \\
\text { Class 2. Pressure dewpoint will be } \\
\text { maintained }\end{array}$ & $\begin{array}{l}\text { Pharmaceutical, food } \\
\text { beverage, clean rooms }\end{array}$ \\
\hline $\begin{array}{l}\text { IG4 } \\
\text { Industrial Grade Air: } \\
\text { ISO 2.4.1 }\end{array}$ & $\begin{array}{l}\text { Efficient removal of solid } \\
\text { particulates and oil. ISO Class } 4 . \\
\text { Pressure dewpoint or a } 30 \% \text { (or less) } \\
\text { Relative Humidity (RH) will be } \\
\text { maintained. }\end{array}$ & $\begin{array}{l}\text { General manufacturing, metal } \\
\text { stamping, air tool use, forging, } \\
\text { assembly, painting and finishing }\end{array}$ \\
\hline $\begin{array}{l}\text { IG4 Odor Free } \\
\text { Industrial Grade Air: } \\
\text { ISO 2.4.1 Odor free }\end{array}$ & $\begin{array}{l}\text { Efficient removal of solid } \\
\text { particulates and oil vapor. ISO Class } \\
\text { 4. Pressure dewpoint or a } 30 \% \text { (or } \\
\text { less) Relative Humidity (RH) will be } \\
\text { maintained. }\end{array}$ & $\begin{array}{l}\text { Food and beverage, raw material } \\
\text { mixing }\end{array}$ \\
\hline $\begin{array}{l}\text { IG6 } \\
\text { Industrial Grade Air: } \\
\text { ISO 2.6.1 }\end{array}$ & $\begin{array}{l}\text { Efficient removal of solid } \\
\text { particulates and oil. ISO Class } 6 . \\
\text { Pressure dewpoint or a } 50 \% \text { (or less) } \\
\text { Relative Humidity (RH) will be } \\
\text { maintained. }\end{array}$ & $\begin{array}{l}\text { Sand blasting, } \\
\text { construction }\end{array}$ \\
\hline
\end{tabular}


An oil-free flow of air guarantees a more controlled environment for the two phase flow experiments. Oil-free compressors are more common in larger systems and lubricated compressors for smaller systems. An oil-free compressor avoids adding oil from the compression process into the supply air and may seem the obvious choice. However, lubricated compressors; usually installed in smaller systems; with the right filters can produce air of equally high quality. That is why we believe that the best technical and economic solution is to use a lubricated machine with filters and drain ports strategically installed between the compressor and injection manifold, so that the initial and running costs could be minimized.

\subsection{Establishing compressor capacity}

The capacity of a compressor can be expressed in volumetric or mass flow terms. The volumetric flow required for the compressed air system to be properly sized is a function of the nature of the experiments that are carried out in a facility. Defining the true demand in a compressed air system can be difficult but it is a critical first step. Air demand often fluctuates significantly; however, the system should be sized based on the average flow demand.

Determination of the average air consumption is facilitated by the use of the concept of "load factor" defined as the ratio of actual air consumption to the maximum continuous full-load, each measured in cubic feet of free air per minute. However, since this investigation deals with a specific requirement of air flow rate at the injection manifold, using the load factor concept may not be necessary.

To comply with the process needs, adequate equipment sizing is to be carefully analyzed. Results may indicate the use of one large compressor operating at full load or multiple smaller compressors with sequencing controls to allow for an efficient operation at times for higher flow rates at the supply end.

Compressor capacity and discharge pressure go together when selecting a compressor for a specific application. Consequently, both parameters will influence the initial screening for the compressor selection. 


\subsection{Load pattern demand}

The demand for compressed air can vary widely depending on what the compressed air is used for. Demand patterns can be relatively constant, stepped or widely fluctuating. As compressors are more efficient when operating at or near full load, it is more efficient to use a combination of compressors and controls (including variable speed technology) to meet a varying demand than to use one large compressor running at part load for most of the time.

From a production engineering standpoint, it is well known that when a well is in operation, the surface pressure is fixed due to surface equipment conditions mainly dictated by the separator; however, the reservoir pressure can vary as well as the flow rate of the fluids being produced.

Consequently, a flow loop designed to conduct experiments related to liquid loading in a laboratory environment, must then be equipped with a system flexible enough to allow the researcher to carry on sensitivity analyses on variables of interest while leaving others fixed. As a result, the compressed air system must account for possible fluctuations in the air demand at the application points. Fig. 3.38 shows some examples of equipment arrangement recommended for different types of demands.

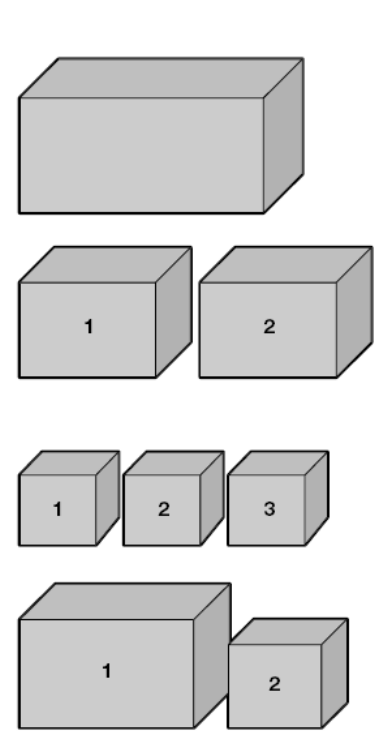

Best met with Example load profiles

One single machine

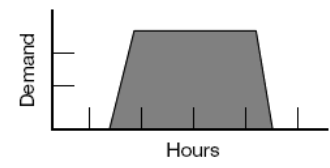

Two equal machines

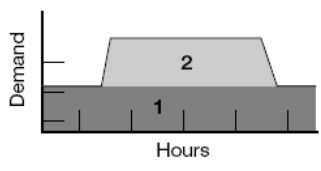

Three equal machines

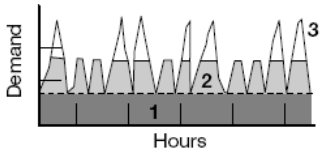

One base load machine and one topping up machine

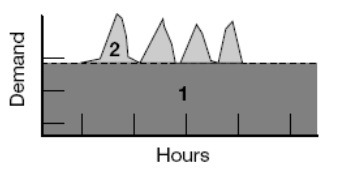

Fig. 3-38- Examples of using multiple compressors to meet different demands ${ }^{53}$ 
The TAMU TowerLab requires a maximum value of pressure and flow rate. Based on these values, an initial screening on possible compressor types and configurations is to be performed. However, the final decision on whether to use a single or multiple units will depend on the technical and economic evaluation of options from commercially available equipment supplied by different compressor manufacturers.

\subsection{Required pressure}

When designing and operating a system it is important to correctly evaluate the amount of pressure required. Air must be delivered to the point of use at the desired pressure and in the right condition. Too low a pressure will impair air from lifting the liquid droplets within the main gas phase and too high a pressure may avoid visualizing flow regime patterns which are of critical importance critical while running the experiments. Furthermore, the design of static equipment such as tubing, pipelines and the pressure vessel containing the artificial porous medium could be overdesigned, hence increasing initial and operating costs.

Different air pressures could be required when operating the flow loop. However, the system should be sized for the maximum operating pressured for the process. As a result, if lower pressures are to be indicated, supplying pressure regulators with the equipment to keep the supply pressure to the desired value will result in savings. Alternatively, the system may be able to be divided in two, with a high pressure network and a low pressure section by using a small compressor operating at full load and a booster, to provide both, low and high pressure values, if this should be necessary.

A way to analyze a compressed air system is to draw a pressure profile. A pressure profile shows the pressure drops through a system. These pressure measurements give feedback for control adjustments, determine pressure drops across components, and help determine system operating pressures. The tools required for measurement are matched, calibrated pressure gauges or differential pressure gauges. Fig. 3.39 is a layout of a system illustrating the expected pressure profile. 


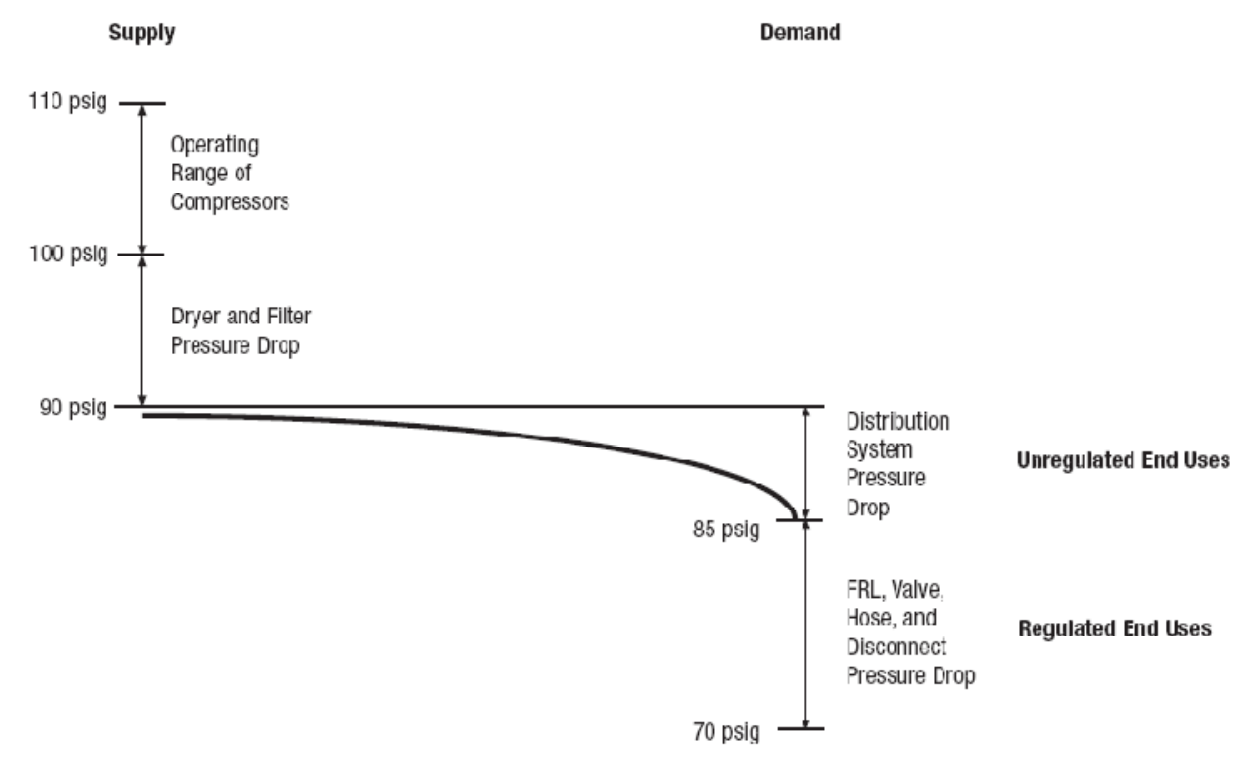

Fig. 3.39- Pressure profile for a system ${ }^{53}$

When designing an air compressed system, minimizing the demand plays a key role in the overall efficiency of the system. It is very important to correctly size the equipment, as oversized air compressors are extremely inefficient. This is because most systems use more energy per unit volume of air produced when operating at part load. While air compressor efficiency generally increases with size, due to lower part-load efficiency, it is usually more efficient to run a smaller compressor at full load rather than a large one at low load.

\subsection{Location}

The air compressor location should be as close as possible to the point where the compressed air is to be used, in a dry, clean, cool, and well ventilated area keeping it away from dirt, vapor, and volatile fumes that may clog the intake filter and valves. If a dry, clean space is unavailable, a remote air intake is recommended.

The location of the compressor is an important parameter prior the selection and installation of the compressed air equipment. Having an outdoor or indoor environment eventually impacts the air inlet conditions at the beginning and continuous operation of the machine. Furthermore, an outdoor location may involve an special design for a 
successful operation. In the case of an indoor installation, provision for proper ventilation and an adequate air supply must be guaranteed along with enough space around the system for maintenance purposes.

Selecting the final location is related to the specific application for which the compressed system is designed for, and in most situations, like in the case of this investigation, can have a big impact on selecting the type of compressor.

The initial approach for the TowerLab was to define whether an indoor or outdoor installation was recommended. Since the flowloop starts with air and water being supplied to the injection manifold s located at the basement of the Richardson Building, the decision was to exhaust all possible resources to locate both the compressed air system and water supply indoors. Following this decision, selecting the specific site to locate the compressor represented one of the biggest challenges of the project, because even though area is available for a standard unit, the logistics involving transporting and installing the equipment at the Basement does not represent an easy task.

Initially, it was believed the unit could be installed in the core room right next to the TowerLab so reduction in pipe work from the compressor to the injection manifold could be accomplished; however, looking at the dimensions of one of the machines offered (Fig. 3.40) it was obvious that we had to evaluate other options.

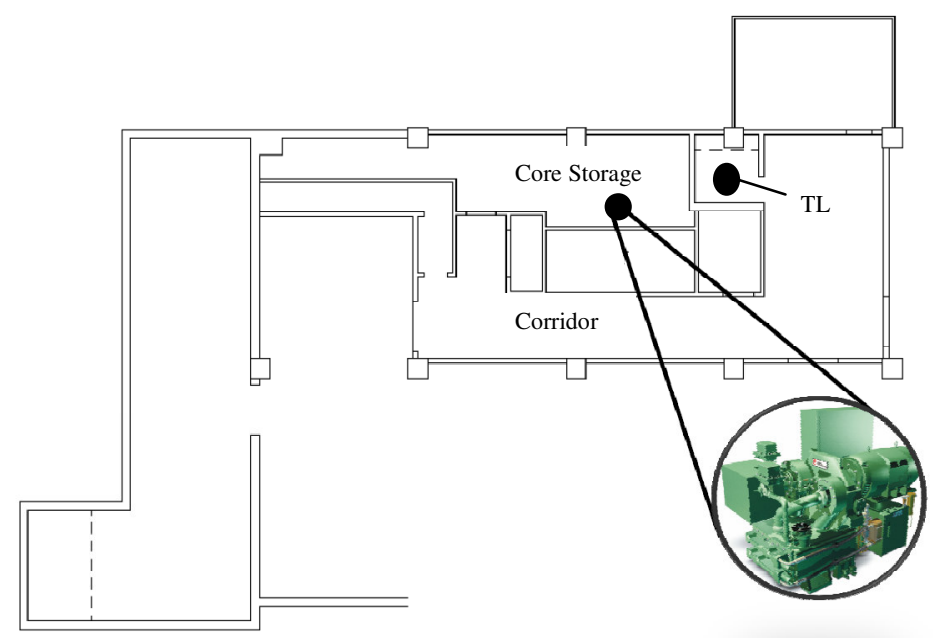

Fig. 3.40- Possible location for compressor 
Picturing the compressor being delivered by the chosen manufacturer, and thinking about the logistics to mobilize and install it at the basement (see Fig. 3.41), helped in selecting the most adequate area to locate the machine.

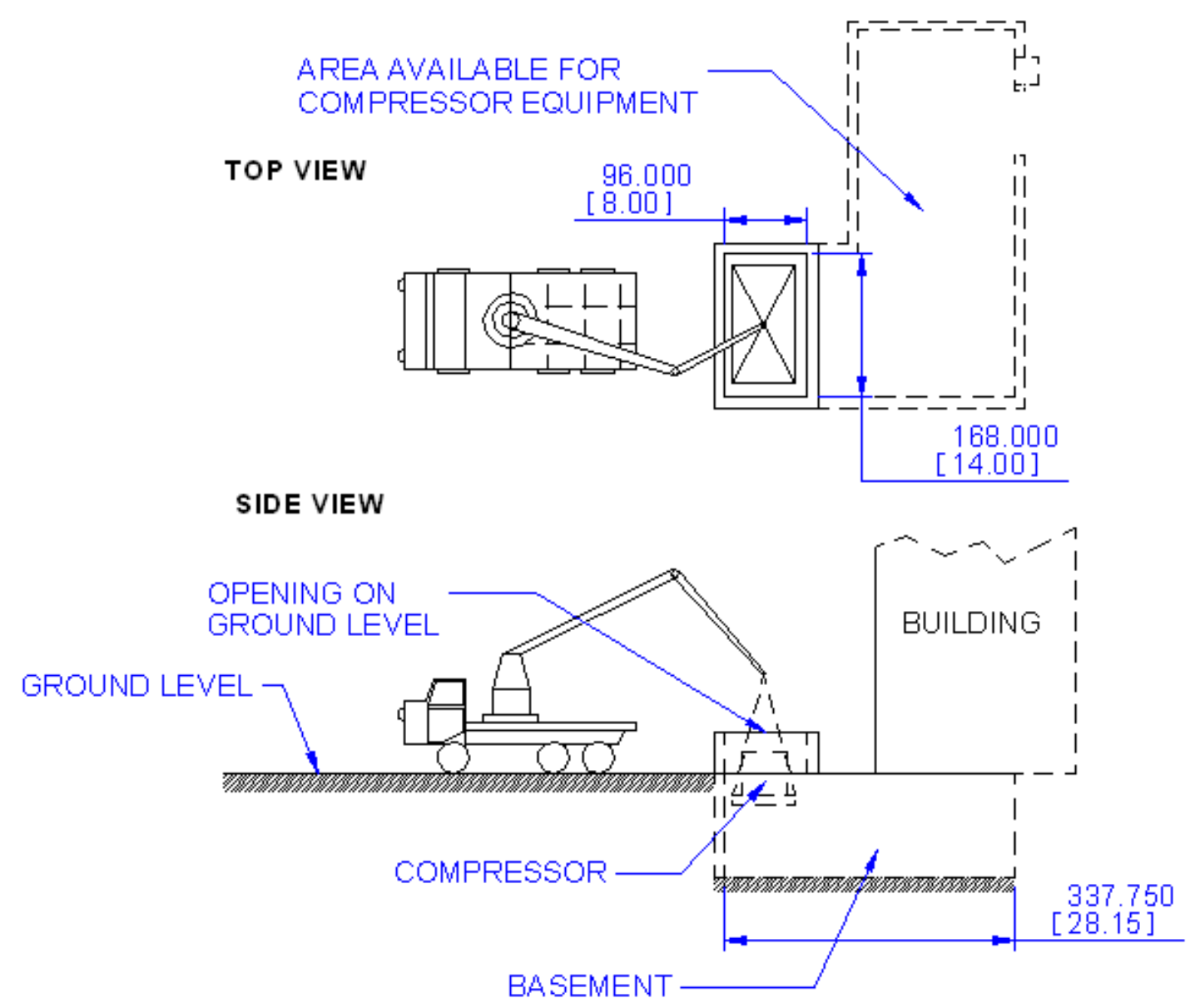

Fig. 3.41- Logistics for compressor delivery

The final decision was to install the compressed air system in the storage room located in the basement. This room has an area of $733 \mathrm{in}^{2}$ and also allows for the manufacturer to bring the equipment down the basement through an opening (see Figs. 3.42 and 3.43) located outside the building. The opening has the dimensions as depicted in Fig. 3.41; however, it is technically feasible to lower the unit using a crane. This 
situation will more than likely require the foundation (if necessary) to be placed aligned with the axis of movement of the compressor while being lowered by the crane.
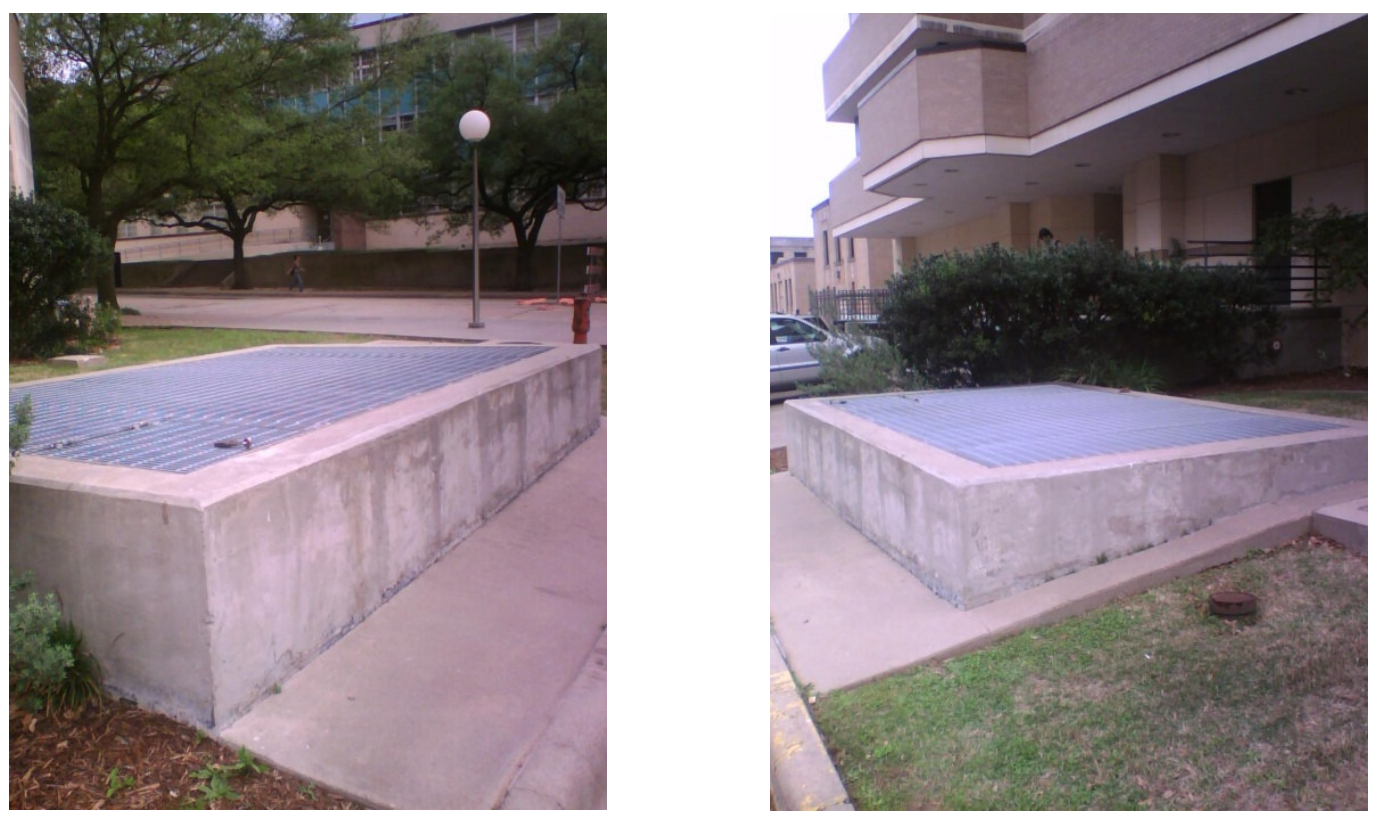

Fig. 3.42- Opening located outside the Richardson building

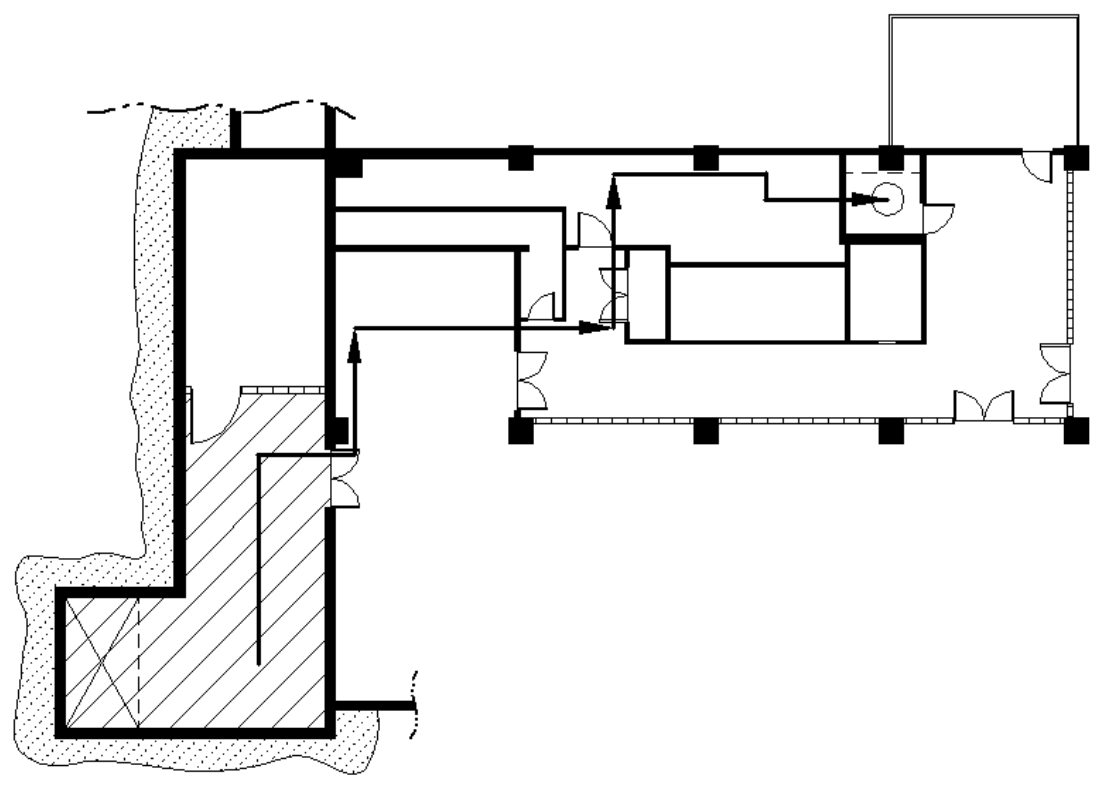

Fig. 3.43- Proposed location of compressed air package 


\subsection{Packaged compressors}

There is a general recognition that a factory engineered air compressor as a package, tested before it leaves the compressor manufacturer, offers many advantages such as having a single-point system responsibility with the manufacturer, a single purchase order, a single shipment, and a minimum of installation time after delivery. That is why the main approach was to contact packaged compressor manufacturers to get options for the compressed air system for the facility.

\subsection{Inlet and discharge piping}

A clean, cool, dry air supply is essential to the satisfactory operation of a compressor. Wherever possible the compressor inlet air should be taken from the outside air. The open end of the inlet pipe must be well hooded to prevent rain from entering the filter. The filter should take air from at least $6 \mathrm{ft}$ or more above the ground, or roof, and should be located several feet away from any wall to minimize pulsating effect on the structure.

Inlet air pressure has a direct and substantial effect on compressor capacity and horsepower. Based on a barometric pressure of 14.7 psia, an increase in inlet pressure of only 0.14 psia will increase compressor capacity by $1 \%$. It is recommended that the piping be fabricated with a sufficient number of flanged joints so that it can be dismantled easily for cleaning.

The discharge piping is considered to be the piping between the compressor and after-cooler, if one is used. If an after-cooler is not used, the discharge pipe should run directly to the receiver or the process. The discharge pipe should be as short and direct as possible with long radius elbows where bends are necessary, and should have a few fittings as possible.

\subsection{Distribution system}

Any drop in pressure between the compressor and the point of use is an irrecoverable loss. The distribution system is, therefore, one of the most important elements of the compressed air system. 
Pipe sizes should be large enough that the pressure drop between the receiver (if needed) and the point of use will not exceed $10 \%$ of the initial pressure. Fittings offering least resistance to flow, such as long radius elbows, should be selected.

All piping should be sloped so that it drains towards a drop leg or moisture trap so that condensation may be removed. The slope of the lines should always be away from the compressor and a slope of about one fourth of an inch per foot may be used with drains provided at all low points.

All overhead piping must be well supported to relieve the compressor from pipe strains. The discharge piping of a loaded compressor may be vibrating from a combination of mechanically transmitted forces and pulsations. If mechanical vibrations are unacceptable, these usually can be corrected by installing flexible connections at the compressor discharge, thus isolating the compressor movement from the piping.

Three types of piping strains must be considered:

- Strains due to dead weight of the piping itself

- Strains due to expansion or contraction of the piping as it undergoes temperature change

- Strains due to internal pressure within the piping

Adequate piping support is necessary to prevent excessive dead loads on the flanged joints located in different sections of the pipe work. Pipe supports should normally be of the flexible type and they should be located as close as possible to the flange connections.

As a summary, a satisfactory piping arrangement can normally be obtained by giving proper attention to an adequate support for all parts of the piping system, allowing for expansion in a manner that will avoid piping strains on the compressor (individual conditions will determine whether expansion joints, expansion loops, or bends will be required) and installing a sufficient number of anchors so that the direction and magnitude of expansion are controlled. 


\subsection{Compressor selection}

Compressed air can be supplied by different types of equipment. The choice depends on the amount, pressure and quality of air a system requires. Many modern industrial air compressors are sold "packaged" with the compressor, drive motor, and many of the accessories mounted on a frame for ease of installation. Fig.3.44 shows the two basic compressor types: positive-displacement and dynamic, which, in most cases, are available in both lubricated and non-lubricated forms.

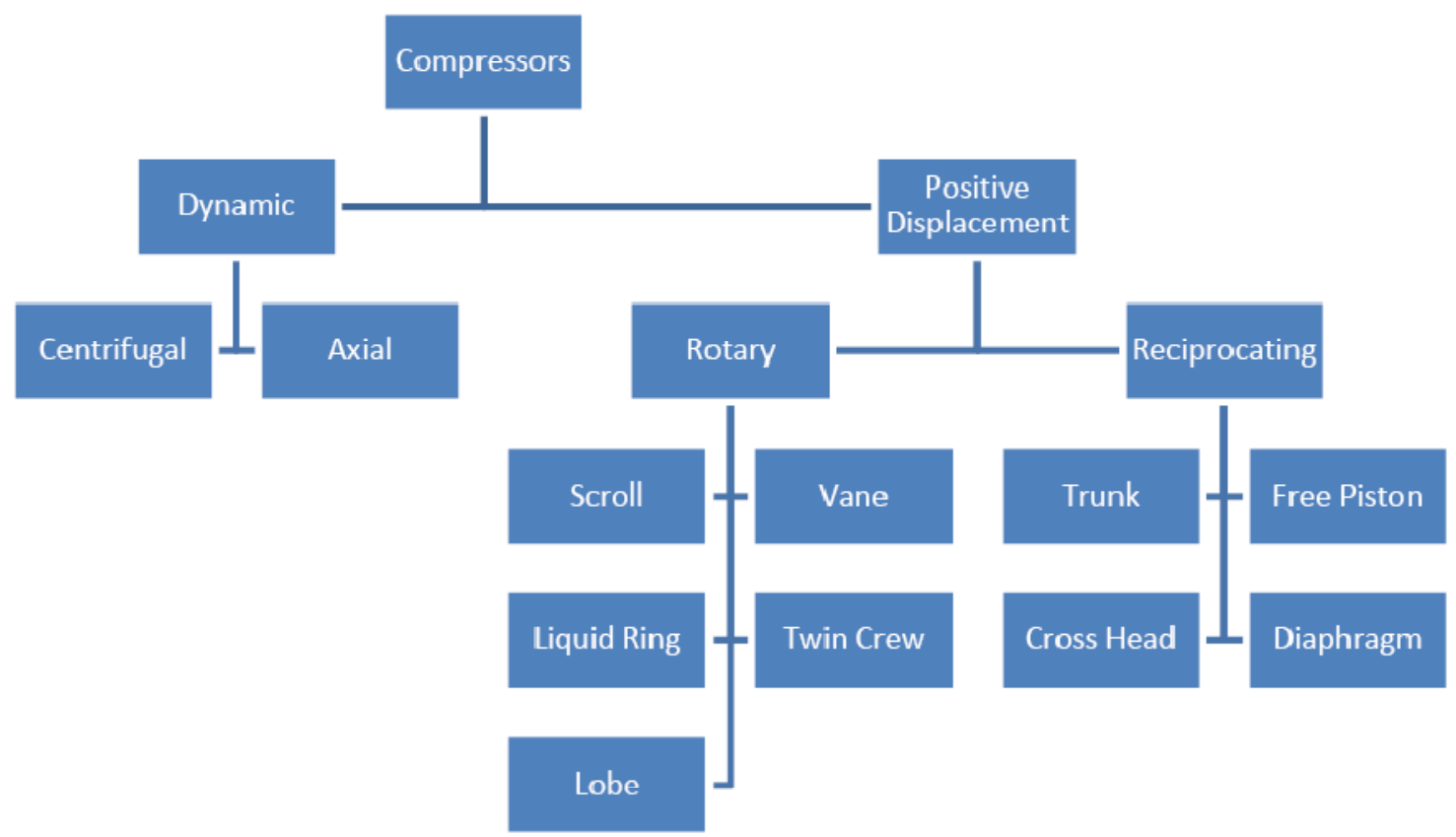

Fig. 3.44- Basic compressor types

In the positive-displacement type, a given quantity of air or gas is trapped in a compression chamber and the volume which it occupies is mechanically reduced, causing a corresponding rise in pressure prior to discharge. At constant speed, the air flow remains essentially constant with variations in discharge pressure. Dynamic compressors impart velocity energy to continuously flowing air or gas by means of impellers rotating at very high speeds. The velocity energy is changed into pressure energy both by the impellers and the discharge volutes or diffusers. In the centrifugal- 
type dynamic compressors, the shape of the impeller blades determines the relationship between air flow and the pressure (or head) generated. Table 3.33 provides information related to volume and pressure capabilities for the different types of technologies.

Table 3.33- Compressor operating limits ${ }^{54}$

\begin{tabular}{|l|c|c|c|c|}
\hline Type & $\begin{array}{c}\text { Min. suction } \\
\text { volume } \\
\left(\mathbf{f t}^{\mathbf{3}} / \mathbf{m i n}\right)\end{array}$ & $\begin{array}{c}\text { Max. suction } \\
\text { volume } \\
\left(\mathbf{f t}^{\mathbf{3}} / \mathbf{m i n}\right)\end{array}$ & $\begin{array}{c}\text { Max. } \\
\text { Pressure } \\
\text { ratio }\end{array}$ & $\begin{array}{c}\text { Max. } \\
\text { discharge } \\
\text { pressure } \\
(\mathbf{p s i})\end{array}$ \\
\hline Axial compressors & 29,430 & 588,578 & $5(1)$ & 73 \\
\hline $\begin{array}{l}\text { Axial centrifugal } \\
\text { compressors }\end{array}$ & 29,430 & 588,578 & $10(1)$ & 145 \\
\hline Centrifugal compressors & $294(2)$ & 176,573 & $100(1)$ & 8702 \\
\hline $\begin{array}{l}\text { Reciprocating lubricated } \\
\text { compressor }\end{array}$ & 18 & 8,829 & 1,000 & 43,511 \\
\hline $\begin{array}{l}\text { Reciprocating non- } \\
\text { lubricated compressor }\end{array}$ & 18 & 8,829 & 100 & 1,450 \\
\hline Diaphragm compressor & 0.58 & 294 & 300 & 36,260 \\
\hline $\begin{array}{l}\text { Oil-free screw } \\
\text { compressor }\end{array}$ & 294 & $11,772(3)$ & $5(4)$ & 580 \\
\hline Oil-injected screw & 29 & 5,886 & 20 & $653(6)$ \\
\hline $\begin{array}{l}\text { Positive displacement } \\
\text { blowers }\end{array}$ & 29 & 38,258 & 2 & $435(5)$ \\
\hline $\begin{array}{l}\text { Sliding vane } \\
\text { compressors }\end{array}$ & 59 & 2,943 & 10 & 145 \\
\hline
\end{tabular}

(1) Dependent upon $\mathrm{M}_{\mathrm{w}}$ of gas

(2) Discharge volume minimum $59 \mathrm{ft}^{3} / \mathrm{min}$

(4) Up to 10 possible with liquid injection

(3) Up to $785 \mathrm{ft}^{3} / \mathrm{min}$ possible, but relatively inefficient

(5) Depending upon blower type

(6) $1,088 \mathrm{psi}$ in development

As mentioned in 3.1, Solomon et al. ${ }^{17}$ estimated the values of pressure and air flow rate necessary to conduct the experiments at TAMU TowerLab. The range in operating conditions is illustrated in Table 3.34 .

Table 3.34- Operating conditions for compressor selection

\begin{tabular}{|c|c|}
\hline Pressure, $\mathbf{p s i}$ & Air flow rate, $\mathbf{f t}^{\mathbf{3}} / \mathbf{m i n}$ \\
\hline 470 to 650 & 400 to 600 \\
\hline
\end{tabular}

Once the capacity and pressure requirements are known, an initial screening can be performed to identify possible compressor types for the TowerLab. 
Fig 3.45 shows the results:
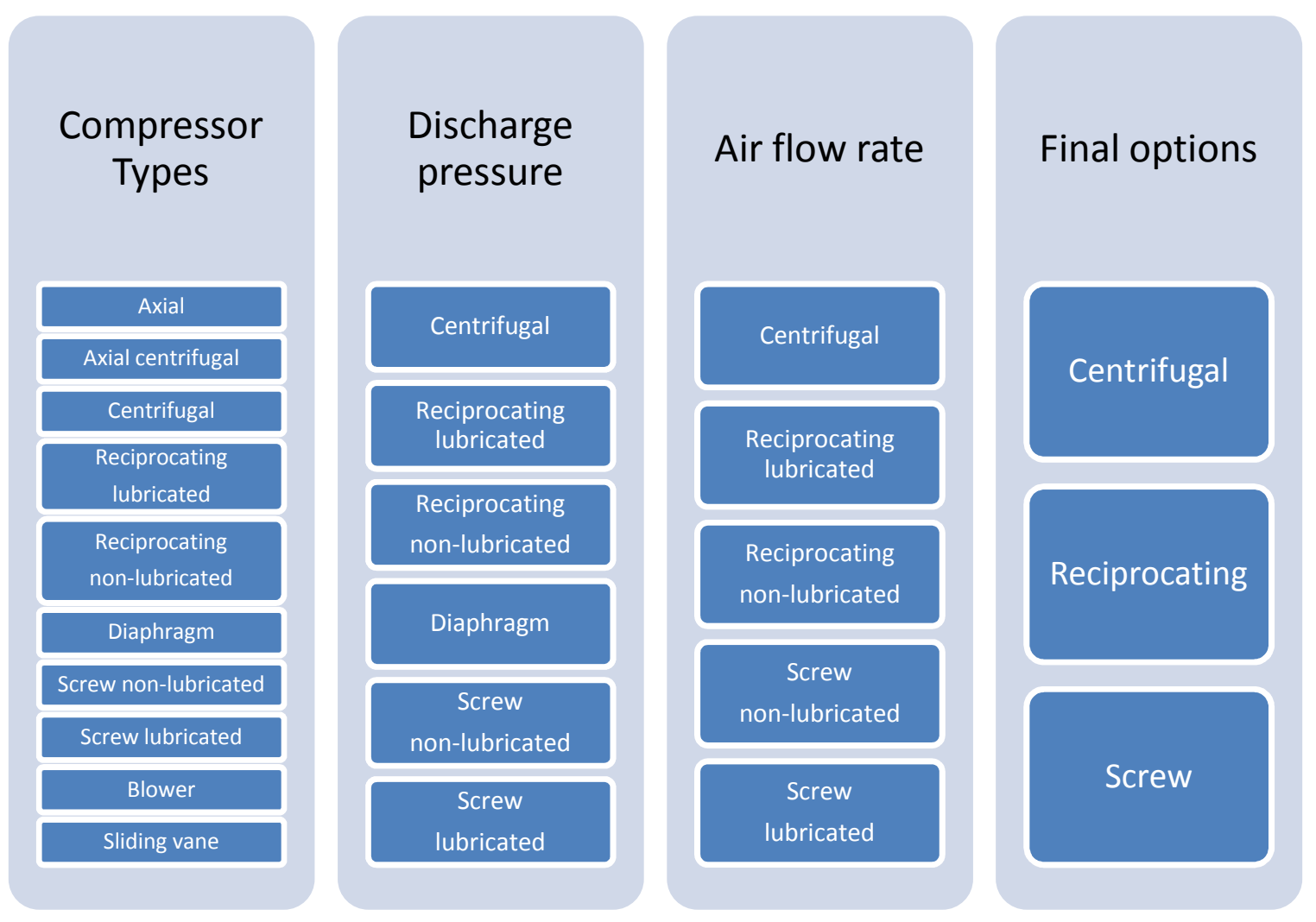

Fig. 3.45-Screening of compressor types

According to the literature, it is possible to use a centrifugal (surge problems might occur with varying flows), reciprocating or screw compressor. However, other references $^{55}$ suggest that the screw compressor by itself is not capable of delivering the discharge pressure needed in the system (see Fig 3.46), therefore these machines are usually accompanied by boosters downstream to supply the additional extra pressure. 


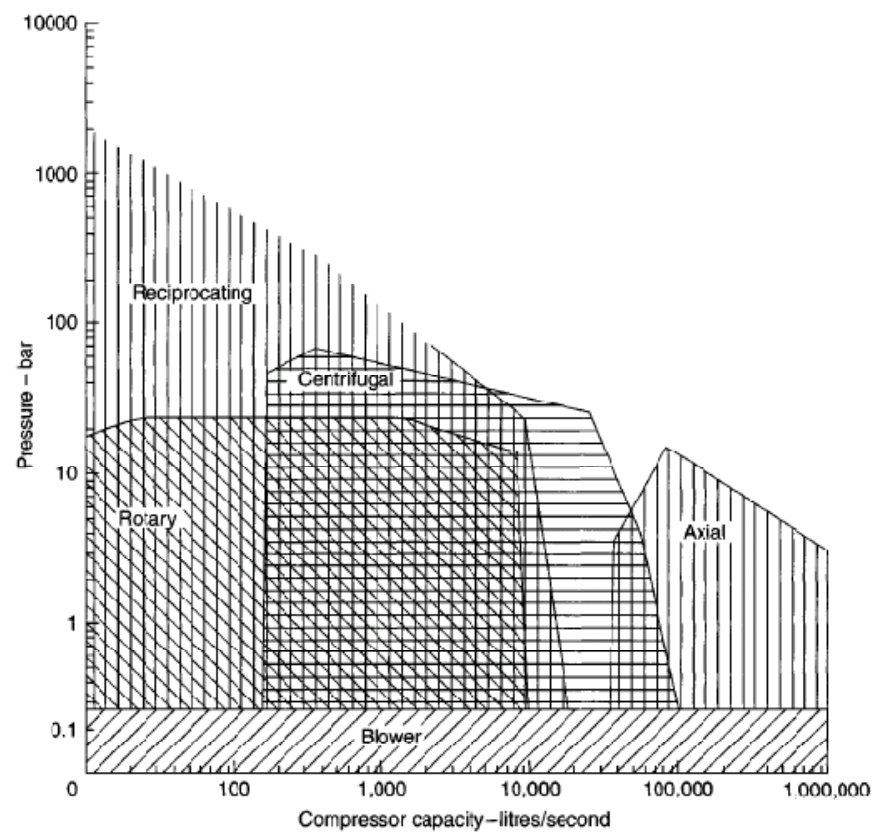

Fig. 3.46- Compressor operating ranges ${ }^{55}$

This situation suggests that an additional option could be to evaluate the combination between compressor technologies if this results to be commercially, as well as, technically and economically feasible. The proposed solutions are summarized in Fig. 3.47, and their main characteristics of each technology are described in Table 3.35.
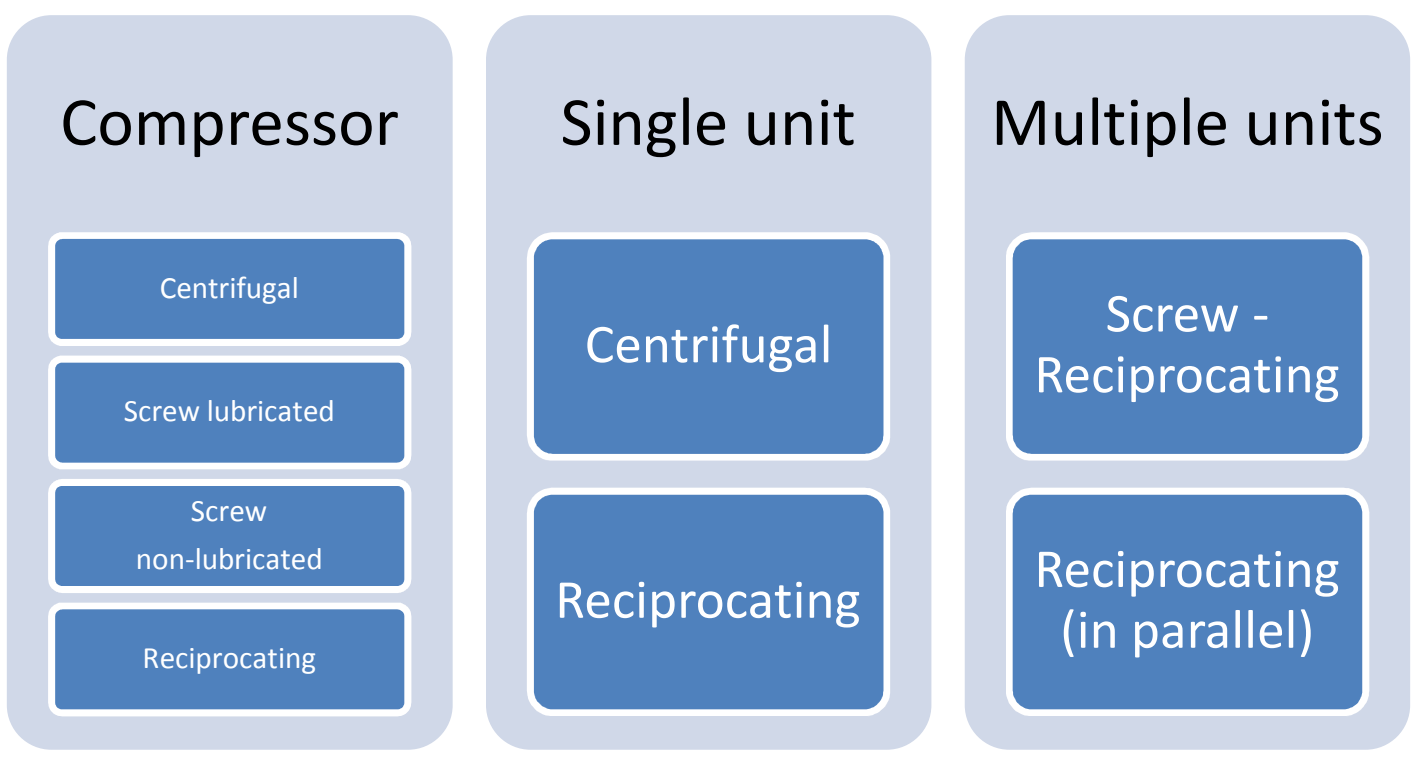

Fig. 3.47- Proposed solutions 
Table 3.35- Compressor characteristics ${ }^{56}$

\begin{tabular}{|c|c|c|c|c|c|}
\hline \multirow{3}{*}{ Characteristics } & \multirow{3}{*}{ Reciprocating } & \multicolumn{3}{|c|}{ Rotary screw } & \multirow{3}{*}{ Centrifugal } \\
\hline & & \multicolumn{2}{|c|}{ Lubricated } & \multirow{2}{*}{$\begin{array}{c}\text { Oil-free } \\
\text { Two } \\
\text { stage }\end{array}$} & \\
\hline & & $\begin{array}{c}\text { Two } \\
\text { stage }\end{array}$ & $\begin{array}{c}\text { Two } \\
\text { stage }\end{array}$ & & \\
\hline Initial cost & Fair & Excellent & Good & Fair & Fair \\
\hline Installation cost & Fair & Excellent & Excellent & Good & Good \\
\hline Maintenance & Fair & Good & Good & Good & Good \\
\hline Operating cost & Excellent & Good & Excellent & Fair & Good \\
\hline Air quality & Fair & Excellent & Excellent & Excellent & Excellent \\
\hline $\begin{array}{l}\text { Cooling system } \\
\text { flexibility }\end{array}$ & Fair & Excellent & Excellent & Excellent & Fair \\
\hline $\begin{array}{l}\text { Integral heat system } \\
\text { flexibility }\end{array}$ & Fair & Excellent & Excellent & Excellent & Fair \\
\hline $\begin{array}{l}\text { Sensitivity to inlet } \\
\text { contaminants }\end{array}$ & Good & Excellent & Excellent & Good & Fair \\
\hline $\begin{array}{l}\text { Capacity turndown } \\
\text { capability }\end{array}$ & Good & Excellent & Excellent & Fair & Fair \\
\hline $\begin{array}{l}\text { Auxiliary air inlet } \\
\text { receiver need }\end{array}$ & Yes & No & No & Yes & Yes \\
\hline $\begin{array}{l}\text { Operating cost, } \\
\mathrm{hp} / 100 \mathrm{ft}^{3} / \mathrm{min}\end{array}$ & 20 to 21 & 24 to 25 & 21 to 23 & 27 to 30 & 21 to 27 \\
\hline
\end{tabular}

\subsection{Reciprocating}

Also commonly referred to as a positive displacement compressor, a reciprocating compressor takes in a volume of air, and via a piston connected to a crankshaft forces that initial volume of air into a smaller volume. Taken on its simplest terms the governing physics behind this operation is depicted by Fig.3.48.
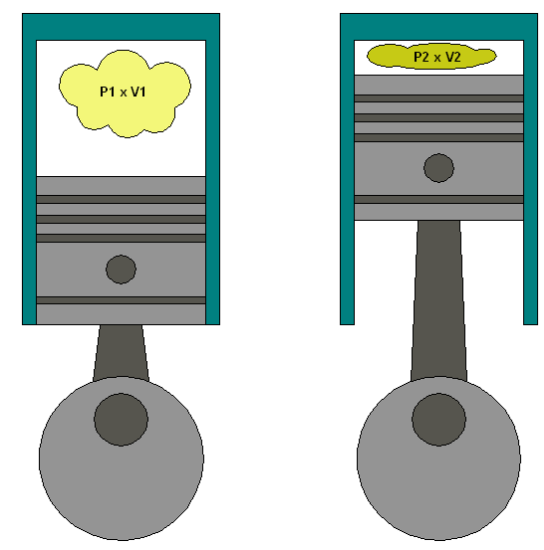

Fig. 3.48- Basic principle of operation of reciprocating compressors 
The flow capacity of the compressor is then dictated by the size of the cylinder volume, and the pressure ratio is controlled by the piston stroke. Reciprocating air compressors cover a broad range of output capacity. They are typically found in applications from $1 \mathrm{hp}$ to more than $600 \mathrm{hp}$. The practical limit for a single stage of reciprocating compressors is typically considered to be 5 to 6 ratios. To achieve higher discharge pressures the process is simply repeated in a second compressor piped in series with the first to achieve two-stages of compression.

One advantage of a reciprocating compressor arises from its push-pull action. Compression can be configured to take place on one or both sides of the piston. If only one side of the piston is performing the compression, the process is referred to as single acting. If both sides of the piston are used, the process is referred to as double acting (see Fig. 3.49). To ensure that the highest efficiency of compression is achieved an effective seal between the sliding piston and stationary cylinder is required.

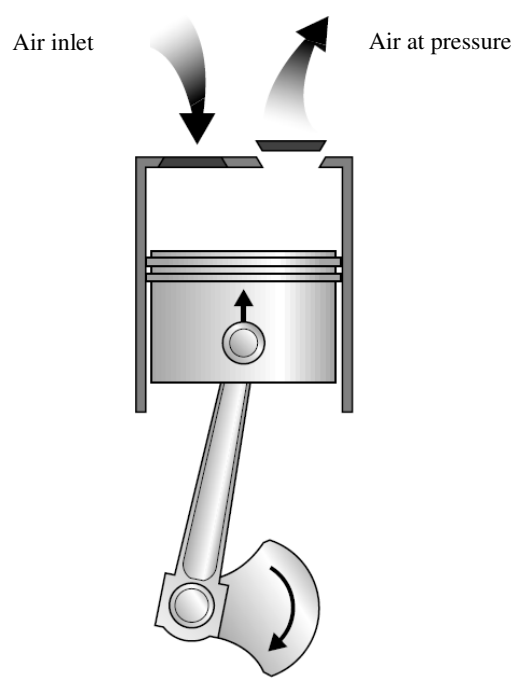

(a)

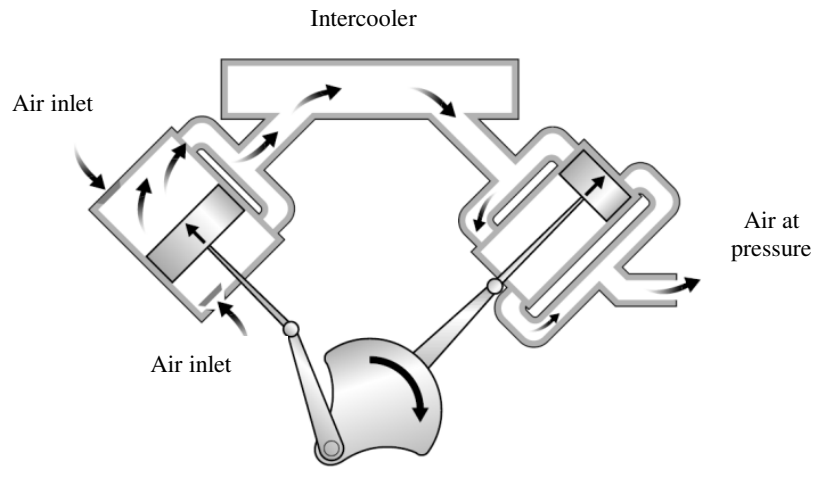

(b)

Fig 3.49- (a) Single and (b) double acting reciprocating compressors ${ }^{53}$

Although there are commercially available oil-free reciprocating compressors, it is more typical to see lubricated (also referred to as oil-flooded) machines. Introducing lubricating oil into the cylinder reduces the wear between the piston and cylinder wall 
but has the undesirable effect of mixing and carrying over into the compressed air stream. As a result, if the process using the air is not tolerant of the presence of lubricant in the air a downstream separator is required to remove the oil from the air stream.

Typically, oil separators are sized and selected based upon the end use of the air. That end use dictates the oil removal rate and efficiency. The primary benefit of reciprocating compressors is their simplicity and initial low cost. The drawback is the frequency of piston and cylinder maintenance and the work involved to replace these parts. From this perspective, compressor availability must be considered.

In the case of oil lubricated machines, the significant drawback of the additional cost for oil separation systems and maintenance of those systems to produce air quality acceptable for the required process must be calculated into cost of ownership. Due to the relatively harsh nature of their inherent operating characteristics reciprocating compressors often require more substantial foundations than centrifugal and rotary screw compressors, and may not be suitable where noise emissions are an issue. Nevertheless, they are the most energy efficient, both at full and part loads. ${ }^{53}$

\subsection{Lubricated screw}

The lubricant-injected rotary screw compressor powered by an electric motor has become a dominant type of industrial compressor for a wide variety of applications. It consists of two intermeshing rotors in a stator housing having an inlet port at one end and a discharge port at the other. The male rotor has lobes formed helically along its length while the female rotor has corresponding helical grooves or flutes. The number of helical lobes and grooves may vary in otherwise similar designs.

Air flowing in through the inlet port fills the spaces between the lobes on each rotor. Rotation then causes the air to be trapped between the lobes and the stator as the inter-lobe spaces pass beyond the inlet port. As rotation continues, a lobe on one rotor rolls into a groove on the other rotor and the point of intermeshing moves progressively along the axial length of the rotors, reducing the space occupied by the air, resulting in increased pressure. Compression continues until the inter-lobe spaces are exposed to the 
discharge port when the compressed air is discharged. Fig. 3.50 shows the compression process in a lubricated screw compressor.
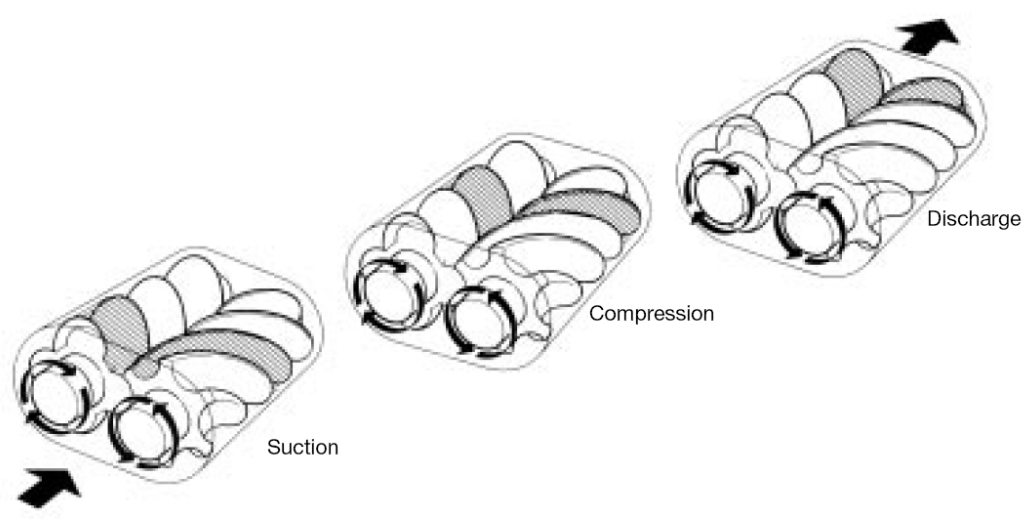

Fig. 3.50- Single-stage oil-injected screw compressor ${ }^{53}$

Lubricant is injected into the compression chamber during compression and serves three basic functions:

a) It lubricates the intermeshing rotors and associated bearings

b) It takes away most of the heat caused by compression

c) It acts as a seal in the clearances between the meshing rotors and between rotors and stator.

The lubricant may be a hydrocarbon product, but most compressors now use cleaner and longer life synthetic lubricants, including diesters, polyglycols, polyalphaolefins, polyol esters, and silicon-based lubricants suitable for a wider range of temperatures. A mixture of compressed air and injected lubricant leaves the air end and is passed to a sump/separator where the lubricant is removed from the compressed air. Directional and velocity changes are used to separate most of the liquid. The remaining aerosols in the compressed air then are separated by means of a coalescing filter, resulting in only a few parts per million (ppm) of lubricant carry-over (usually in the range of 2 to $5 \mathrm{ppm}$ ).

A minimum pressure device, often combined with a discharge check valve, prevents excessive velocities through the separator element until a normal system pressure is achieved at start-up. Most lubricant-injected rotary screw compressor 
packages use the air pressure in the lubricant sump/separator, after the discharge of the air end, to circulate the lubricant through a filter and cooler prior to reinjection to the compression chamber. Some designs may use a lubricant pump.

Generally, suitable lubricant temperature and viscosity are required for proper lubrication, sealing, and to avoid condensation in the lubricant sump. It also is necessary to avoid excessive temperatures, which could result in a breakdown of the lubricant and reduced life. In addition to lubricant cooling, an after-cooler is used to cool the discharged air and a moisture separator removes the condensate. In water-cooled designs, water-cooled heat exchangers with water control valves also are available on most rotary screw compressor packages.

Single-stage, lubricant-injected, rotary screw compressor packages are available from 3 to $900 \mathrm{hp}$, or 8 to $5,000 \mathrm{ft}^{3} / \mathrm{min}$, with discharge pressures from 50 to $250 \mathrm{psig}$. Two-stage versions can reduce specific power and some can achieve discharge pressures up to 500 psig.

These compressors are usually the lowest cost to install, for large volumes of compressed air. To ensure maximum efficiency of screw compressors, it is important to correctly size the compressor and apply internal and external control systems for part load conditions. Variable output and variable speed drives are usually available from most suppliers.

\subsection{Lubricated-free screw}

Non-lubricated rotary compressors are usually more efficient than oil-injected machines, mainly because they are two-stage units. They generally have a higher capital cost, but longer lifetime than lubricated compressors. Oil-free rotary machines are often used when high quality air is required. These machines are highly applicable where space is restricted or the foundations are not substantial. The female rotors in oil-free compressors are driven separately by gears (unlike lubricated screw compressors where the male rotor directly drives the female rotor) as seen in Fig. 3.51. 


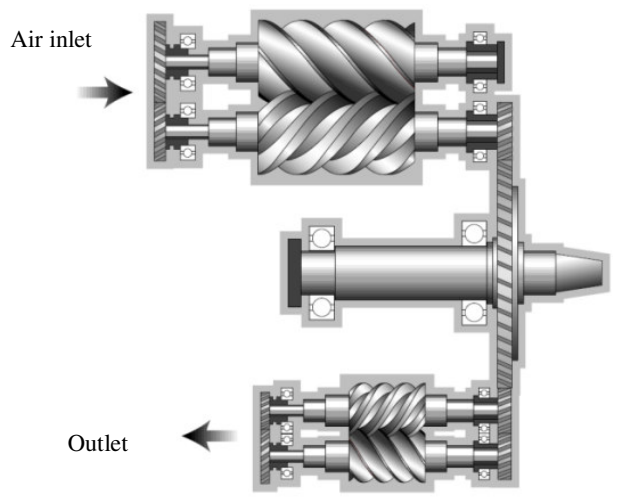

Fig. 3.51- Two-stage lubricated-free screw compressor ${ }^{53}$

The principle of compression in lubricant-free rotary screw compressors is similar to that of the lubricant-injected rotary screw compressors but, without lubricant being introduced into the compression chamber. Two distinct types are available: the dry-type and the water-injected type.

In the dry-type, the intermeshing rotors are not allowed to touch and their relative positions are maintained by means of lubricated timing gears external to the compression chamber. Since there is no injected fluid to remove the heat of compression, most designs use two stages of compression with an intercooler between the stages and an after-cooler after the second stage. The lack of a sealing fluid also requires higher rotation speeds than for the lubricant-injected type.

Dry-type, lubricant-free rotary screw compressors have a range from 25 to 4,000 hp or 90 to $20,000 \mathrm{ft}^{3} / \mathrm{min}$. Single-stage units operate up to $50 \mathrm{psig}$, while two-stage can achieve up to $150 \mathrm{psig}$. In the water-injected type, similar timing gear construction is used, but water is injected into the compression chamber to act as a seal in internal clearances and to remove the heat of compression. This allows pressures in the 100 to 150 psig range to be accomplished with only one stage. The injected water, together with condensed moisture from the atmosphere, is removed from the discharged compressed air by a conventional moisture separation device. Similar to the lubricant-injected type, lubricant-free rotary screw compressors generally are packaged with all necessary accessories. 
Lubricant-free rotary screw compressors utilize lubricant for bearings and gears, which are isolated from the compression chamber. The lubricant also may be used for stator jacket cooling in air-cooled units. Typically, a lubricant pump is directly driven from a shaft in the gearbox, assuring lubricant flow immediately at start-up and during run-down in the event of power failure. A lubricant filter, typically with 10 micron rating, protects bearings, gears, and the lubricant pump from damage.

The cooling system for the dry-type, lubricant-free rotary screw compressor normally consists of an air cooler after each stage and a lubricant cooler. These may be water-cooled or air-cooled, radiator-type.

\subsection{Centrifugal}

A centrifugal air compressor (Fig. 3.52) has a continuously flowing air stream which has velocity energy, or kinetic energy, imparted to it by an impeller, or impellers, which rotate at speeds that, can exceed 50,000 revolutions per minute (rpm).

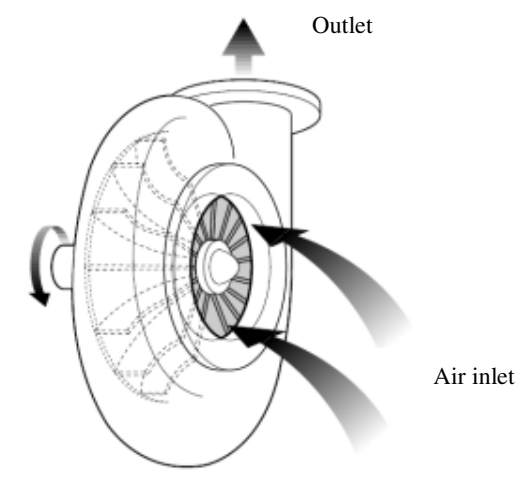

\section{Fig. 3.52- Centrifugal compressor ${ }^{53}$}

Approximately one half of the pressure energy is developed in the impeller with the other half achieved by converting the velocity energy to pressure energy as the air speed is reduced in a diffuser and volute. The most common centrifugal air compressor is one with two to four stages for pressures in the 100 to 150 psig range. A water-cooled intercooler and separator between each stage returns the air temperature to approximately ambient temperature and removes condensed moisture before entering the 
next stage. An after-cooler cools the air from the final stage and a moisture separator removes the moisture prior to air delivery to distribution.

The inherent characteristic of centrifugal air compressors is that as system pressure decreases, the compressor's flow capacity increases. The steepness of the pressure head/capacity curve is dependent upon the impeller design. The more the impeller blades lean backwards from the true radial position, the steeper the curve.

Most standard centrifugal air compressor packages are designed for an ambient temperature of $95^{\circ} \mathrm{F}$ and near sea level barometer pressure. The dynamic nature of the centrifugal compressor results in the pressure head generated by each impeller increasing as the air density increases. The compressor mass flow and actual cubic feet per minute (acfm) capacity at a given discharge pressure increases as the ambient temperature decreases. Typically, a capacity control system is provided with the compressor to maintain the desired capacity and to operate within the motor horsepower limits. The control system regulates the air flow by means of an inlet throttle valve or inlet guide vanes.

Centrifugal air compressors range from around 300 to more than $100,000 \mathrm{ft}^{3} / \mathrm{min}$ but the more common air compressors are from 1,200 to 5,000 $\mathrm{ft}^{3} / \mathrm{min}$ with discharge pressures up to $125 \mathrm{psig}$. Special designs may address higher values of pressure when high flow rate conditions are needed. These may have several impellers in line on a single shaft or with separate impellers integrally geared.

Centrifugal air compressors provide lubricant-free air delivery as there is no lubricant in the compression chambers. Lubrication for speed increasing gears and the special high-speed shaft bearings is kept away from the compression chambers by means of shaft seals, which may also have air purge and vent connections.

Centrifugal air compressors are high-speed rotating machines and as such, shaft vibration monitoring is mandated to record operational trends and protect the equipment. Automatic control of the compressors is typical and has been greatly improved by the use of microprocessors, which monitor the pressure/capacity/temperature characteristics as well as main-drive motor current draw. 


\subsection{Advantages and disadvantages}

The advantages and disadvantages of the three types of compressor under consideration are described in Table 3.36.

Table 3.36- Advantages and disadvantages of each type of compressor

\begin{tabular}{|c|c|c|}
\hline Compressor & Advantages & Disadvantages \\
\hline $\begin{array}{l}\text { Reciprocating } \\
\text { (Single acting air- } \\
\text { cooled) } \\
\text { Operating Efficiency: } \\
22 \text { to } 24 \mathrm{~kW} / 100 \mathrm{cfm}^{*}\end{array}$ & $\begin{array}{l}\text { - Small size and weight } \\
\text { - Generally can be located close to point- } \\
\text { of-use avoiding lengthy piping runs and } \\
\text { pressure drops } \\
\text { - Do not require separate cooling systems } \\
\text { - Simple maintenance procedures. }\end{array}$ & $\begin{array}{l}\text { - Lubricant carryover as piston rings wear, } \\
\text { which should be avoided } \\
\text { - Relatively high noise } \\
\text { - Relatively high cost of compression } \\
\text { - Generally are designed to run not more } \\
\text { than } 50 \text { percent of the time. } \\
\text { - Generally compress and store the air in a } \\
\text { receiver at a pressure higher than required } \\
\text { at the point-of use. }\end{array}$ \\
\hline $\begin{array}{l}\text { Reciprocating } \\
\text { Double acting water } \\
\text { cooled } \\
\text { Operating Efficiency: } \\
15 \text { to } 16 \mathrm{~kW} / 100 \mathrm{cfm} *\end{array}$ & $\begin{array}{l}\text { - Efficient compression, particularly with } \\
\text { multi-stage compressors } \\
\text { - Three-step (0-50-100 percent) or five- } \\
\text { step (0-25-50- } 75-100 \text { percent) capacity } \\
\text { controls, allowing efficient part-load } \\
\text { operation } \\
\text { - Relatively routine maintenance } \\
\text { procedures. }\end{array}$ & $\begin{array}{l}\text { - Relatively high first cost compared with } \\
\text { equivalent rotary air compressors } \\
\text { - Relatively high space requirements } \\
\text { - Lubricant carryover on lubricant cooled } \\
\text { units } \\
\text { - Relatively high vibrations require high } \\
\text { foundation costs } \\
\text { - Seldom sold as complete independent } \\
\text { packages } \\
\text { - Require flywheel mass to overcome torque } \\
\text { and current pulsations in motor driver } \\
\text { - Repair procedures require some training } \\
\text { and skills. }\end{array}$ \\
\hline $\begin{array}{l}\text { Screw } \\
\text { Lubricant-Injected } \\
\text { Operating Efficiency: } \\
18 \text { to } 19 \mathrm{~kW} / 100 \mathrm{cfm} \text {, } \\
\text { single-stage* } \\
16 \text { to } 17 \mathrm{~kW} / 100 \mathrm{cfm} \text {, } \\
\text { two-stage* }\end{array}$ & $\begin{array}{l}\text { - Compact size and complete package } \\
\text { - Economic first cost } \\
\text { - Vibration-free operation does not } \\
\text { require special foundation } \\
\text { - Part-load capacity control systems can } \\
\text { match system demand } \\
\text { - Lower end temperatures } \\
\text { - Quiet and simple operation. } \\
\text { - Routine maintenance includes lubricant } \\
\text { and filter changes. }\end{array}$ & $\begin{array}{l}\text { - Less efficient full- and part-load operation } \\
\text { compared with water-cooled reciprocating } \\
\text { air compressors } \\
\text { - Lubricant carryover into delivered air } \\
\text { requires proper maintenance of } \\
\text { air/lubricant separator and the lubricant } \\
\text { itself. }\end{array}$ \\
\hline $\begin{array}{l}\text { Screw } \\
\text { Lubricant-Free } \\
\text { Operating Efficiency: } \\
18 \text { to } 22 \mathrm{~kW} / 100 \mathrm{cfm} *\end{array}$ & $\begin{array}{l}\text { - } \text { Completely packaged } \\
\text { - Quiet and simple operation } \\
\text { - Lower end temperatures } \\
\text { - Designed to deliver lubricant-free air } \\
\text { - Reduced air treatment } \\
\text { - Do not require any special foundations. }\end{array}$ & $\begin{array}{l}\text { - Significant premium over lubricant- } \\
\text { injected type } \\
\text { - Less efficient than lubricant-injected type } \\
\text { - Higher initial cost } \\
\text { - Limited to load/unload capacity control } \\
\text { and VSD } \\
\text { - Higher maintenance costs than lubricant- } \\
\text { injected type over the life of the machine. }\end{array}$ \\
\hline $\begin{array}{l}\text { Centrifugal } \\
\text { Operating Efficiency: } \\
16 \text { to } 20 \mathrm{~kW} / 100 \mathrm{cfm}^{*}\end{array}$ & $\begin{array}{l}\text { - Completely packaged for plant or } \\
\text { instrument air up through } 500 \mathrm{hp} \\
\text { - Relative first cost improves as size } \\
\text { increases } \\
\text { - Designed to deliver lubricant-free air } \\
\text { - Do not require any special foundations. } \\
\text { - Quiet } \\
\text { - Energy efficient }\end{array}$ & $\begin{array}{l}\text { - Sensitive to dirt and air } \\
\text { - Relatively high cost } \\
\text { - Limited capacity control modulation, } \\
\text { requiring unloading for reduced capacities } \\
\text { - High rotational speeds require special } \\
\text { bearings, sophisticated monitoring of } \\
\text { vibrations and clearances } \\
\text { - Specialized maintenance considerations. }\end{array}$ \\
\hline
\end{tabular}




\subsection{Compressors commercially available}

The initial approach for this project was to select the right technology (compressor type) based on the operating conditions (Table 3.34). Once the technologies were defined, we contacted several manufacturers to get proposals for the facility. Fig. 3.53 shows a list of the companies, and also illustrates the screening process that led us to the final suppliers considered for this project.
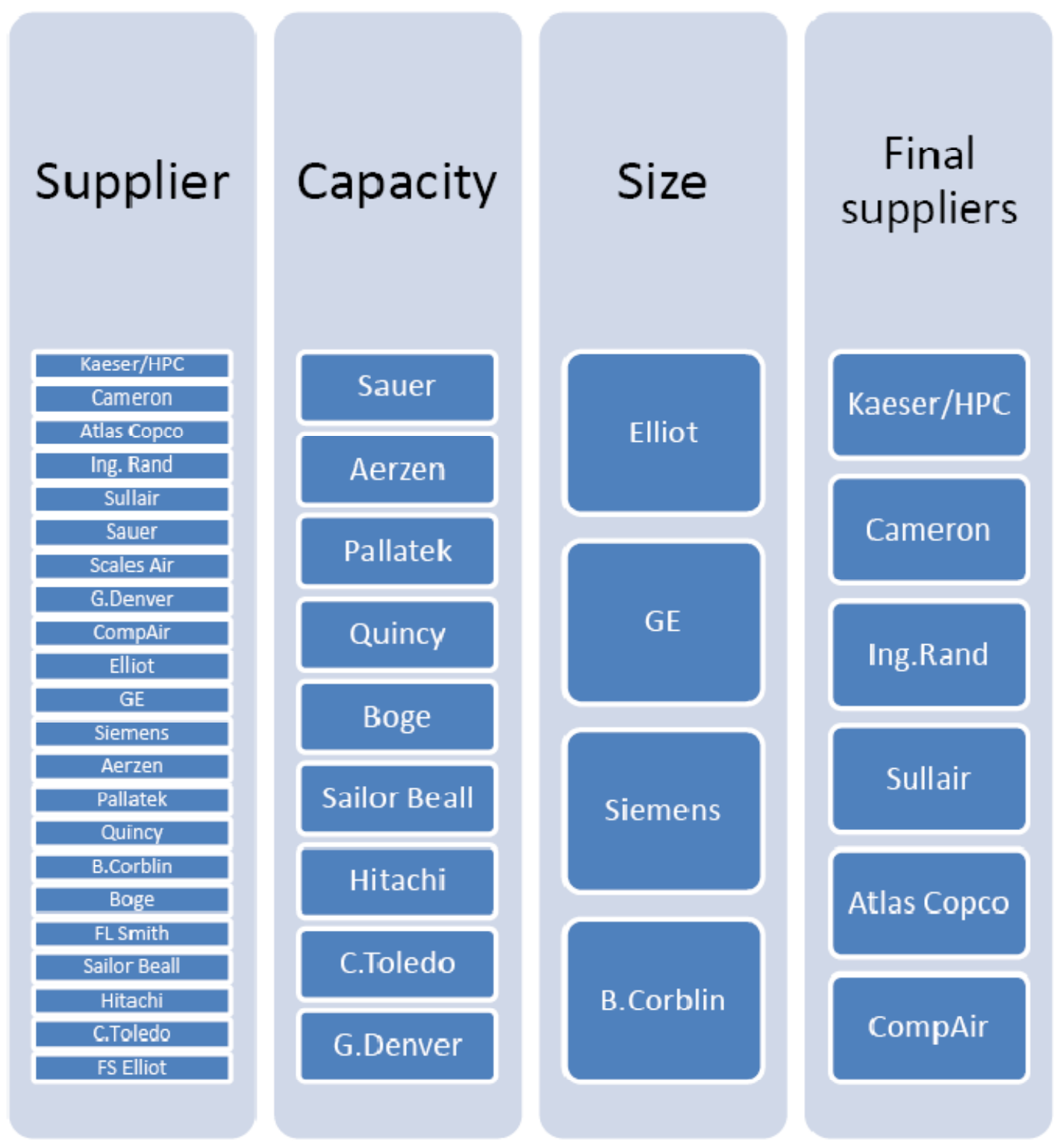

Fig. 3.53- Screening of compressor manufacturers 
Some of the proposals involve using single or multiple units, all delivering different values of pressure and flow rates within the operating conditions range. Figs. 3.54 up to 3.59 are diagrams of each alternative:

(a) Kaeser compressor: Rotary screw compressor feeds booster downstream.

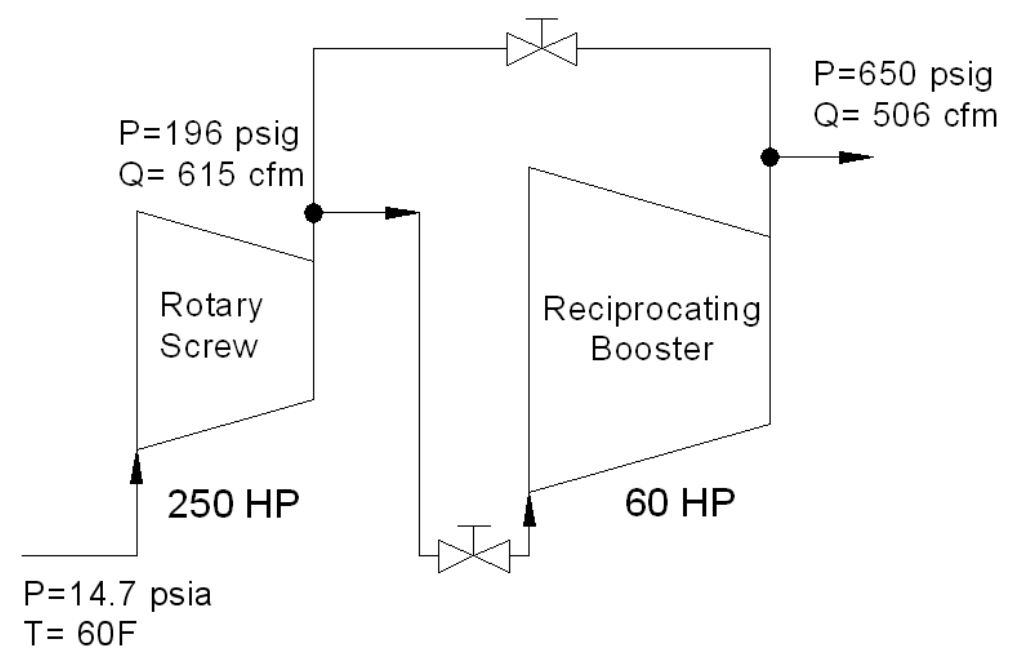

Fig. 3.54- Kaeser Compressors

(b) Atlas Copco: Rotary crew compressor feeds booster downstream.

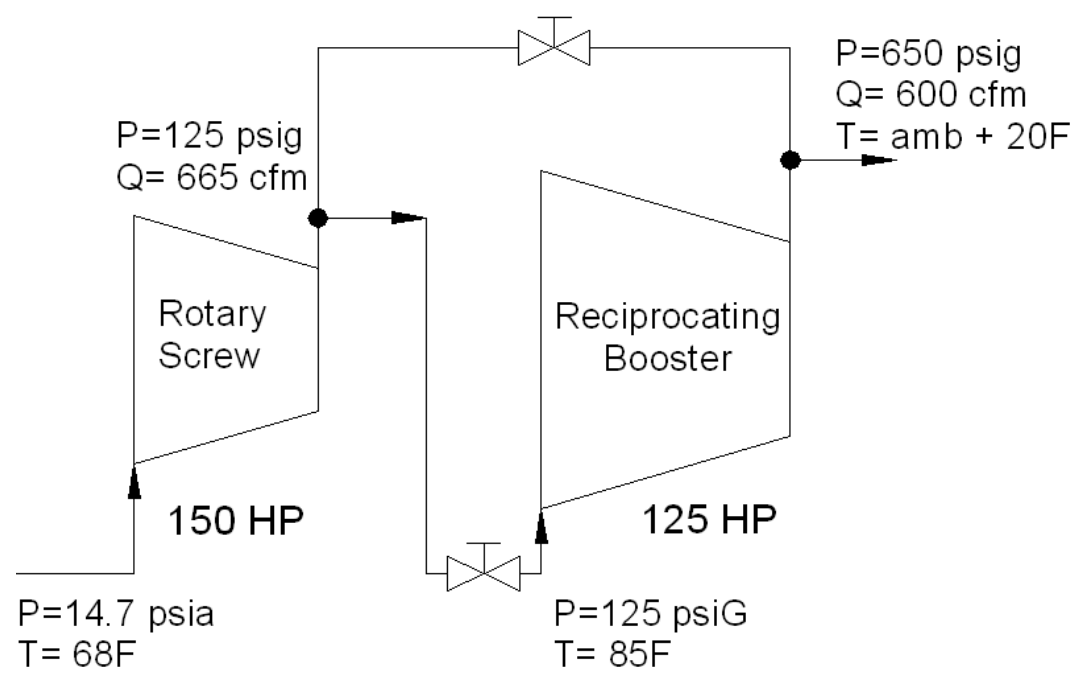

Fig. 3.55- Atlas Copco 
(c) Cameron: Centrifugal compressor delivers required conditions as a single unit.

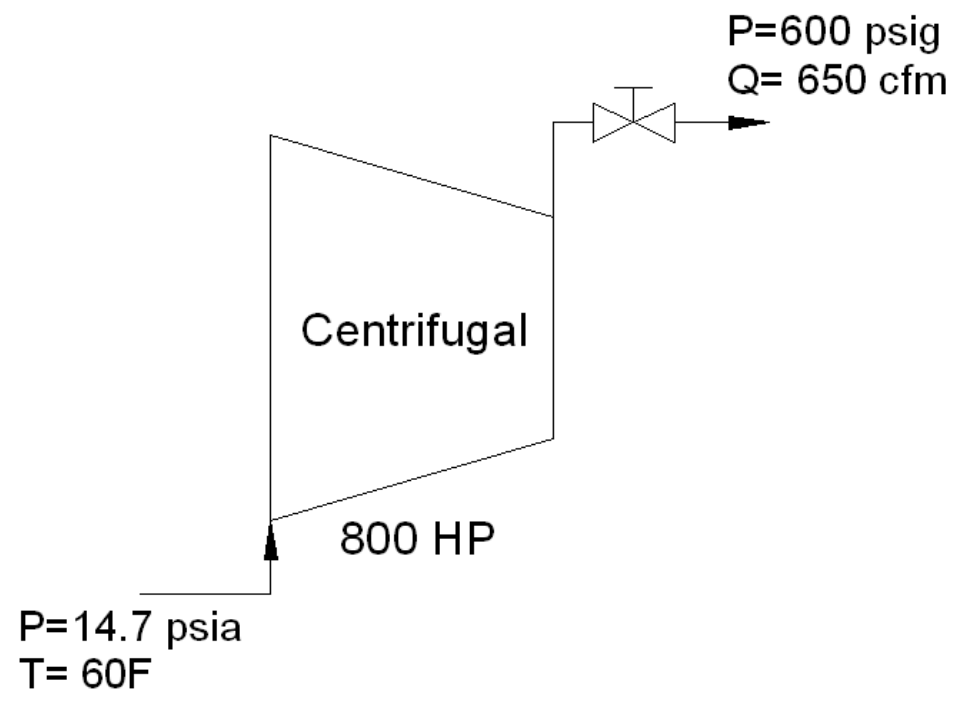

Fig. 3.56- Cameron Compressors

(d) Sullair compressors: high pressure feeder is followed by a booster downstream.

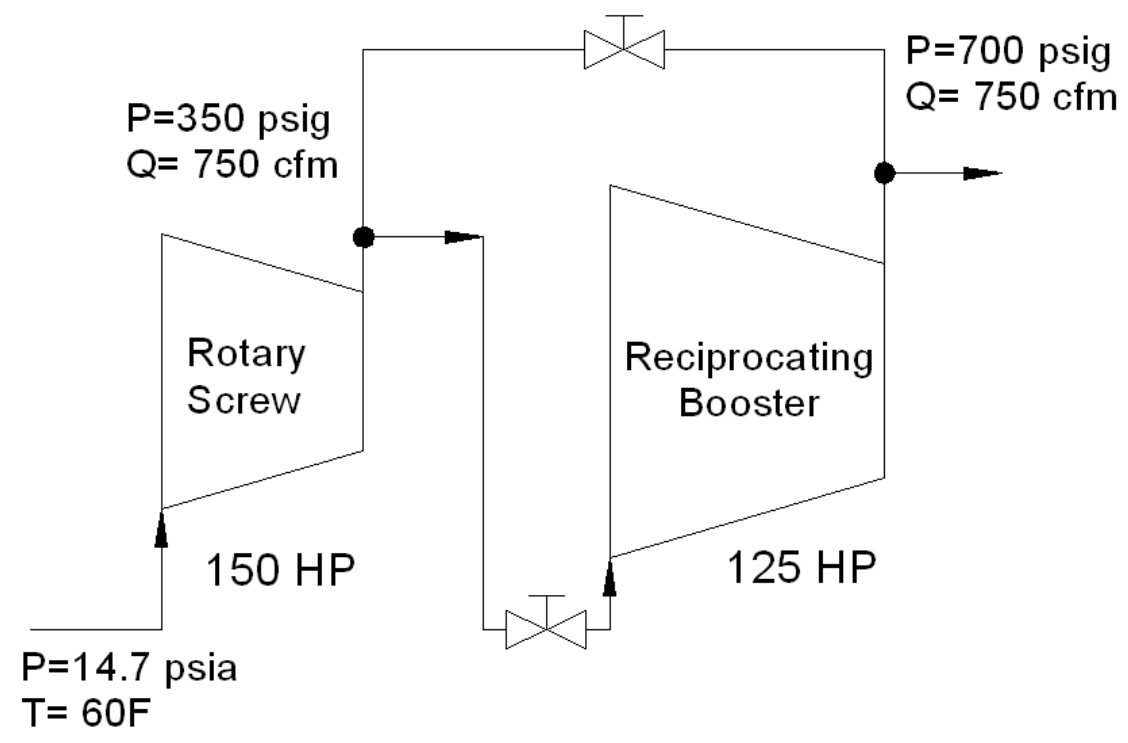

Fig. 3.57- Sullair Compressors 
(e) Ingersol Rand: Rotary screw feeds booster downstream.

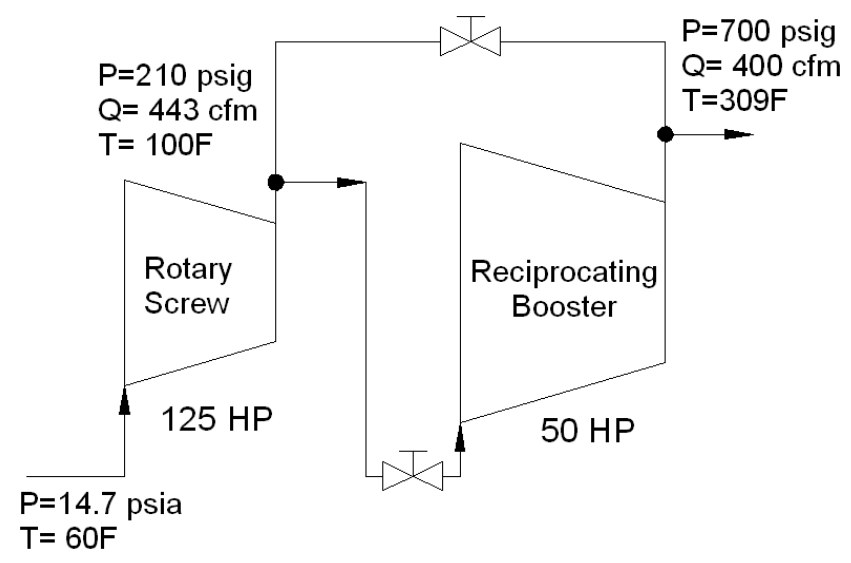

Fig. 3.58- Ingersoll Rand

(f) CompAir: Three reciprocating units installed in parallel

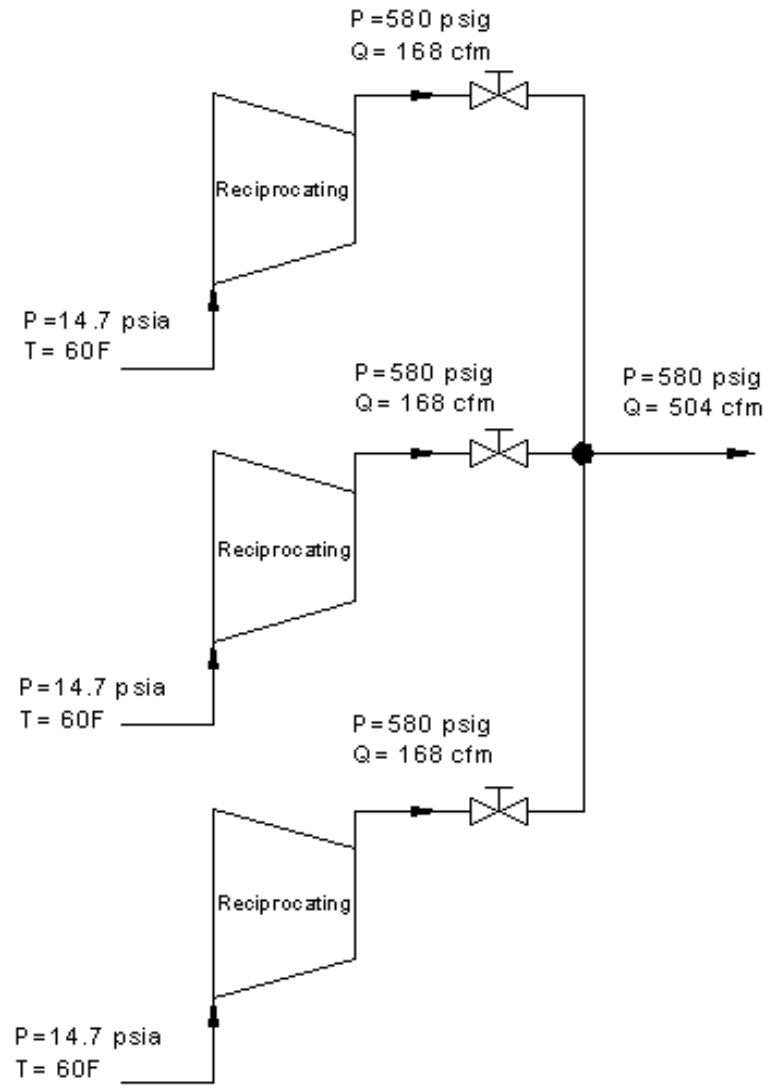

Fig. 3.59- CompAir Compressors 
Following are a series of charts describing the following parameters for the proposed options:

- $\quad$ Operating conditions - Fig. 3.60

- Initial cost (equipment only) and running costs (1st year) - Fig. 3.61

- Initial cost (equipment only) and running costs (5 years) - Fig. 3.62

- Capacity $\left(\mathrm{ft}^{3} / \mathrm{min}\right)$ and power required (hp) - Fig. 3.63

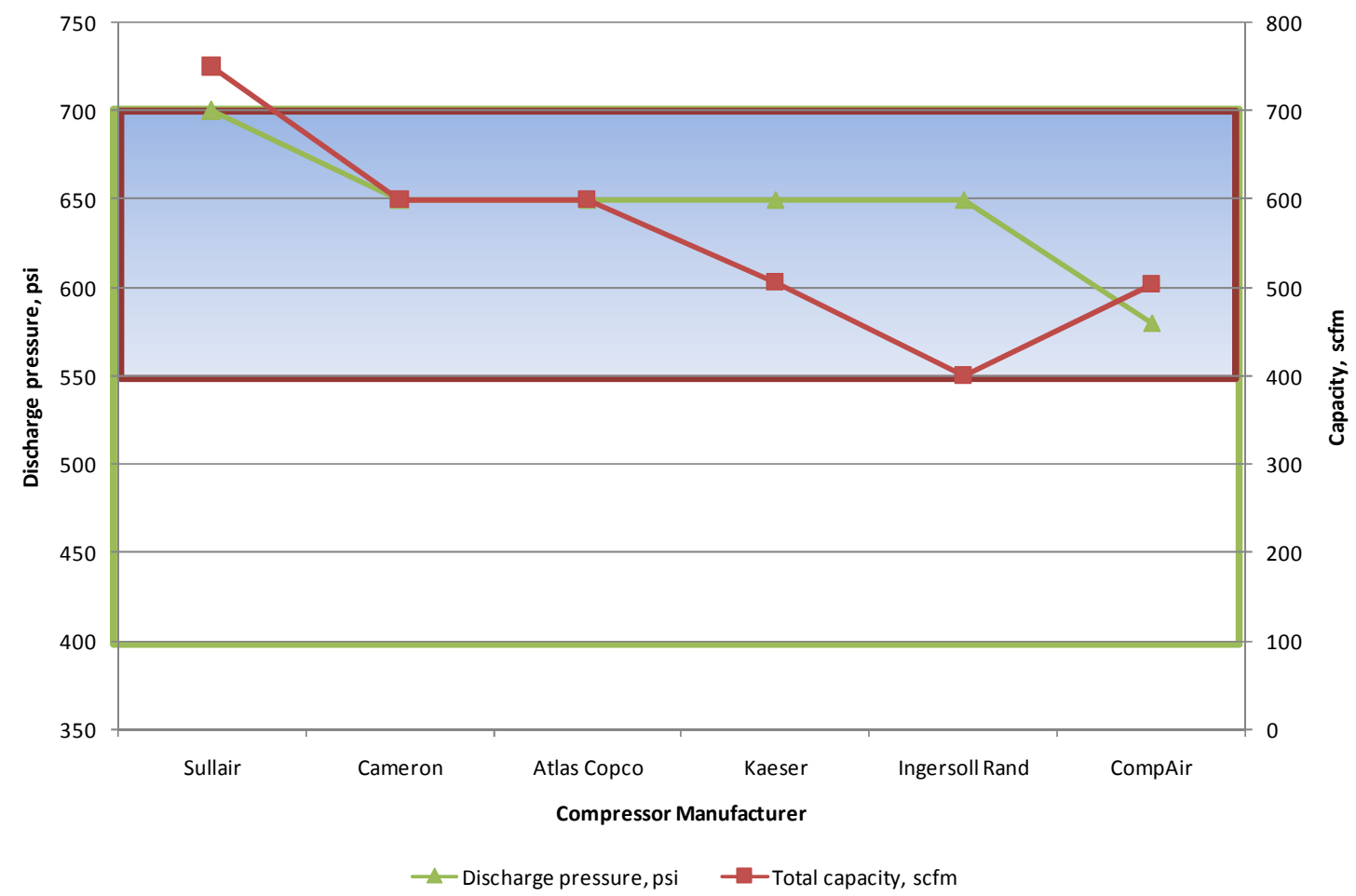

Fig. 3.60- Discharge pressure and air flowrate range according to Solomon et al. ${ }^{17}$ The green square groups values of pressure at the discharge of the compressor whereas the red square groups the air flowrate 


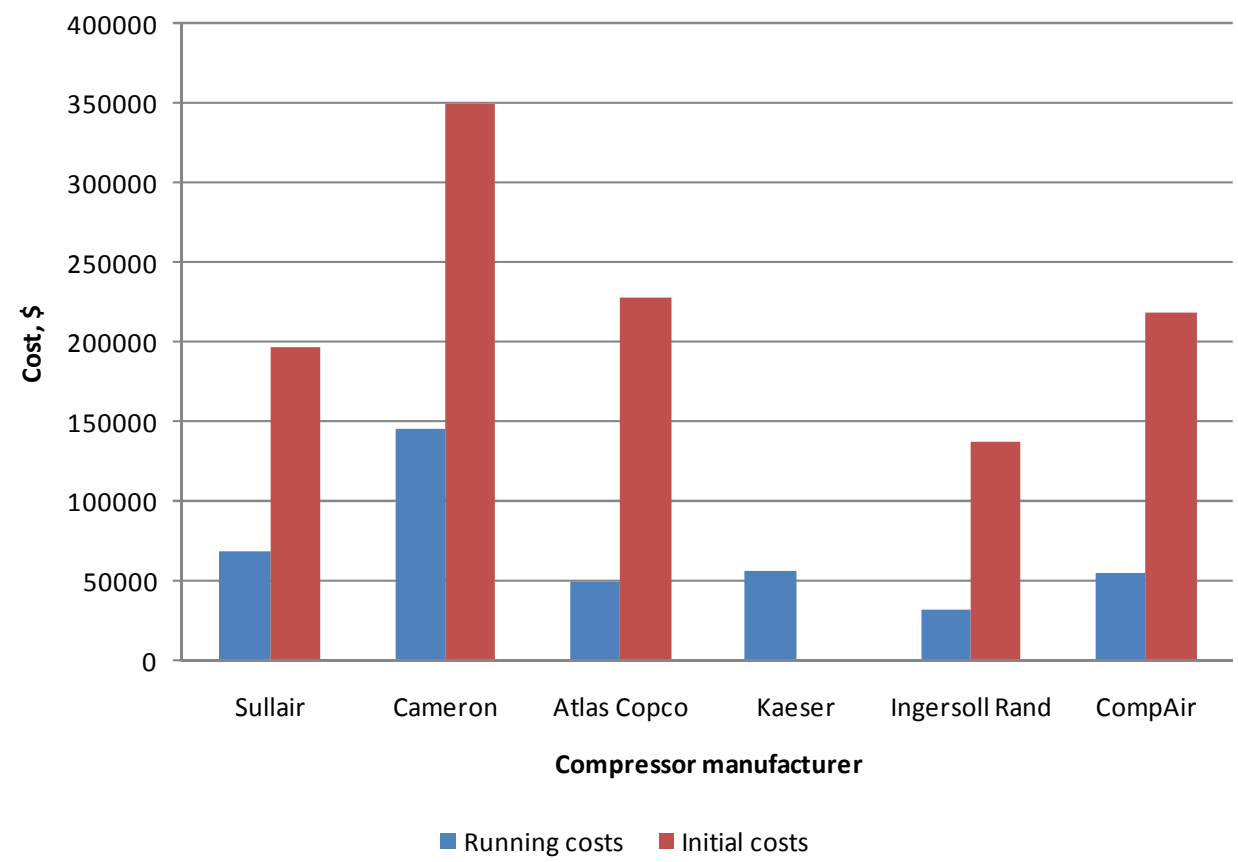

Fig. 3.61- Initial and running costs for the first year

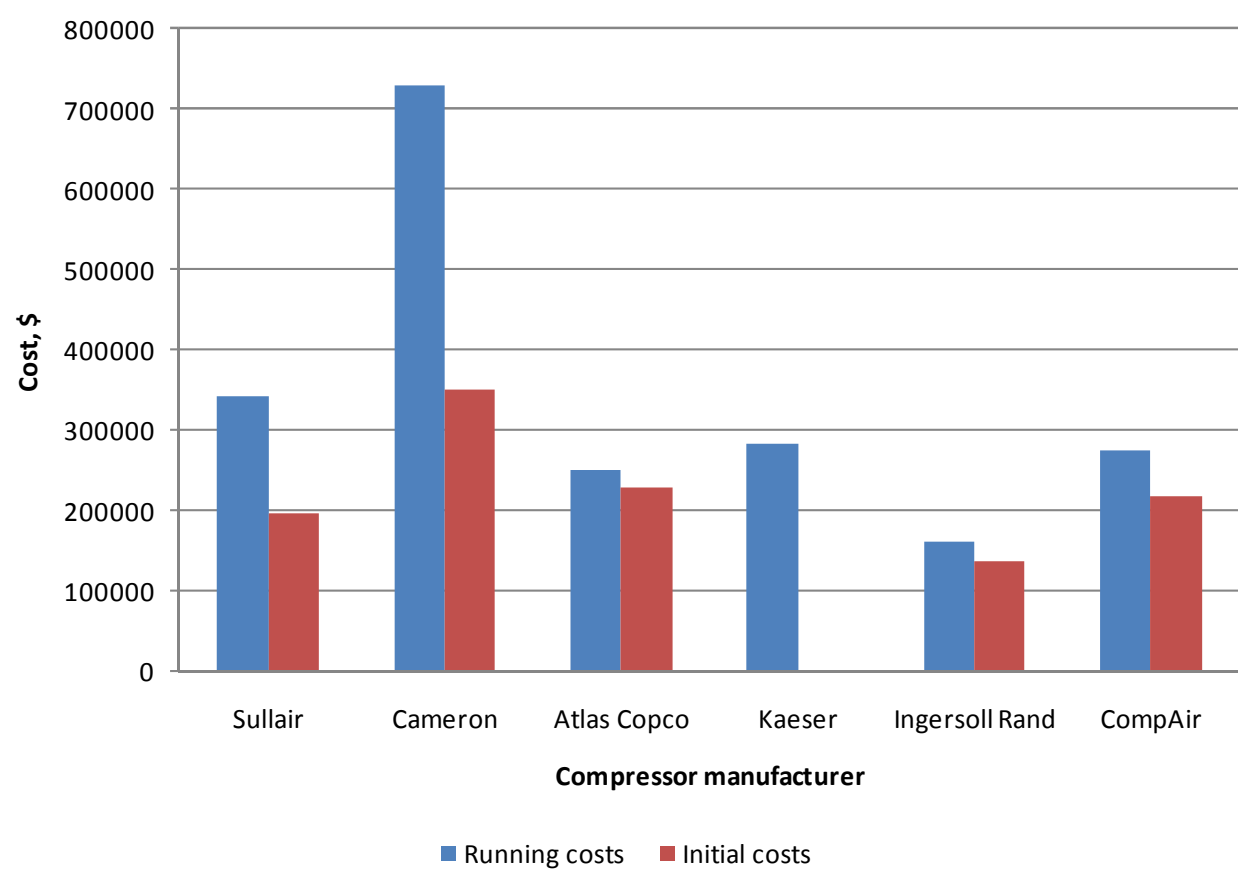

Fig. 3.62- Running costs for five years of operation 


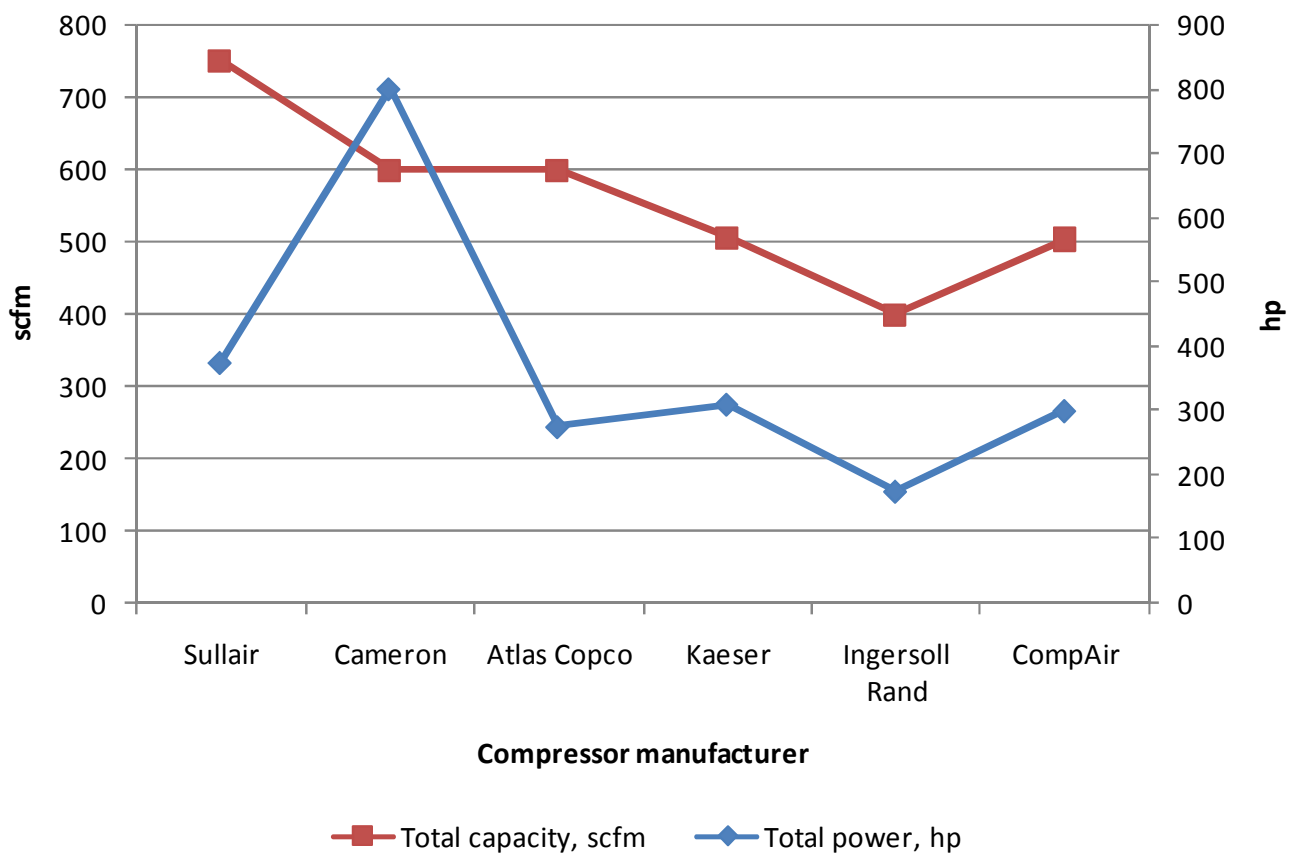

Fig. 3.63- Capacity and power for each option

Fig. 3.55 and 3.57 clearly show that costs are by far the largest expense of owning and operating a compressed air system. The initial cost is a key factor to assess the equipment supplier; however, the most important parameter to consider is the energy costs which as depicted in Fig. 3.64 can account for more than $70 \%$ of the overall compressor cost.

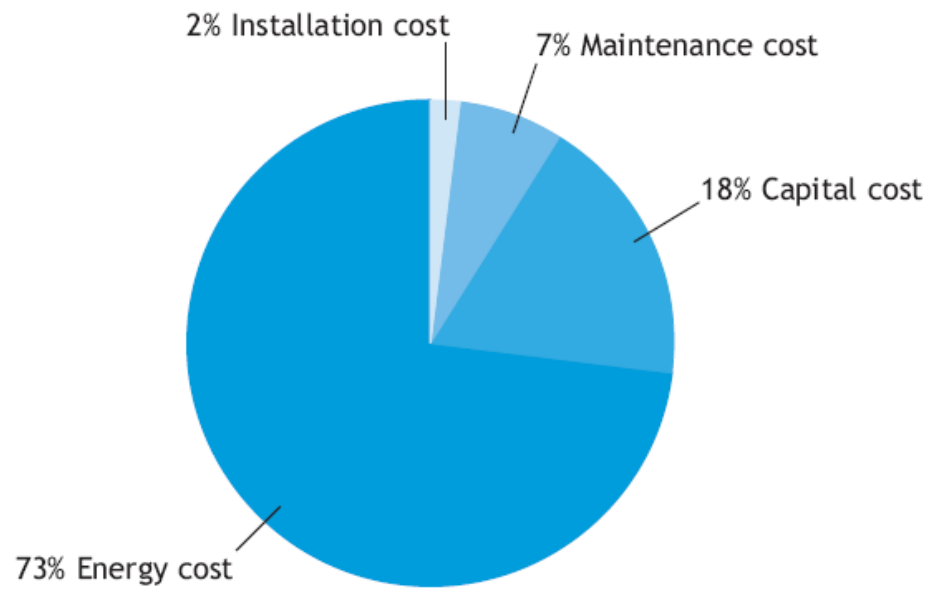

Fig. 3.64- Compressor costs over a ten-year life period ${ }^{57}$ 
Added to this are the installation cost and annual maintenance costs, which can be 10 percent or more of the initial cost of the system.

The running costs for a specific compressed air system is a function of the following parameters:

- Compressor motor nameplate rating (bhp)

- Motor nameplate efficiency (or an estimate of efficiency)

- Annual hours of operation (hours/year)

- Cost of electricity in dollars per kilowatt-hour (\$/kW-hr).

$$
\text { Energy cost }=\frac{(\mathrm{bhp}) \times \text { factor } \times(\mathrm{t}) \times(\text { Electricity cost }) \times(\% \text { of full load })}{\text { Motor Efficiency }}
$$

Considering 8 hours a day of operation during 20 days a month for one year, we obtain the results shown in Table 3.37 and Fig 3.61. The cost of electricity is provided by the Physical Plant at Texas A \& M University at a rate of $0.122 \$ / \mathrm{kWh}$.

Table 3.37- Energy costs

\begin{tabular}{|c|c|c|c|c|c|c|c|}
\hline Manufacturer & Equipment & hp & Efficiency & Days & hours & $\begin{array}{c}\text { Time } \\
\text { (hr) }\end{array}$ & $\underset{\$}{\text { Annual Costs, }}$ \\
\hline \multirow[t]{2}{*}{ Sullair } & Feeder & 300 & 0.962 & 20 & 8 & 1920 & \multirow{2}{*}{$68,421.119$} \\
\hline & Booster & 75 & 0.941 & 20 & 8 & 1920 & \\
\hline Cameron & Centrifugal & 800 & 0.958 & 20 & 8 & 1920 & $145,923.206$ \\
\hline \multirow[t]{2}{*}{ Atlas } & Feeder & 150 & 0.958 & 20 & 8 & 1920 & \multirow{2}{*}{$50,161.102$} \\
\hline & Booster & 125 & 0.958 & 20 & 8 & 1920 & \\
\hline \multirow{2}{*}{ Kaesser } & Feeder & 250 & 0.955 & 20 & 8 & 1920 & \multirow{2}{*}{$56,722.872$} \\
\hline & Booster & 60 & 0.955 & 20 & 8 & 1920 & \\
\hline \multirow{2}{*}{$\begin{array}{l}\text { Ingersoll } \\
\text { Rand }\end{array}$} & Feeder & 125 & 0.945 & 20 & 8 & 1920 & \multirow{2}{*}{$32,359.822$} \\
\hline & Booster & 50 & 0.945 & 20 & 8 & 1920 & \\
\hline CompAir & 3-units & 300 & 0.95 & 20 & 8 & 1920 & $55,182.012$ \\
\hline
\end{tabular}

Let us rank the alternatives based on the following criteria:

- Initial costs - Lowest to highest

- Running costs - Lowest to highest

- High pressure - Arrangement with highest to lowest pressure 
- High flow rate - Arrangement with highest to lowest flow rate

- Total power - Lowest to highest power required

- Efficiency based on hp/100 scf/min delivered - Lowest to highest

The results are depicted in Fig. 3.65
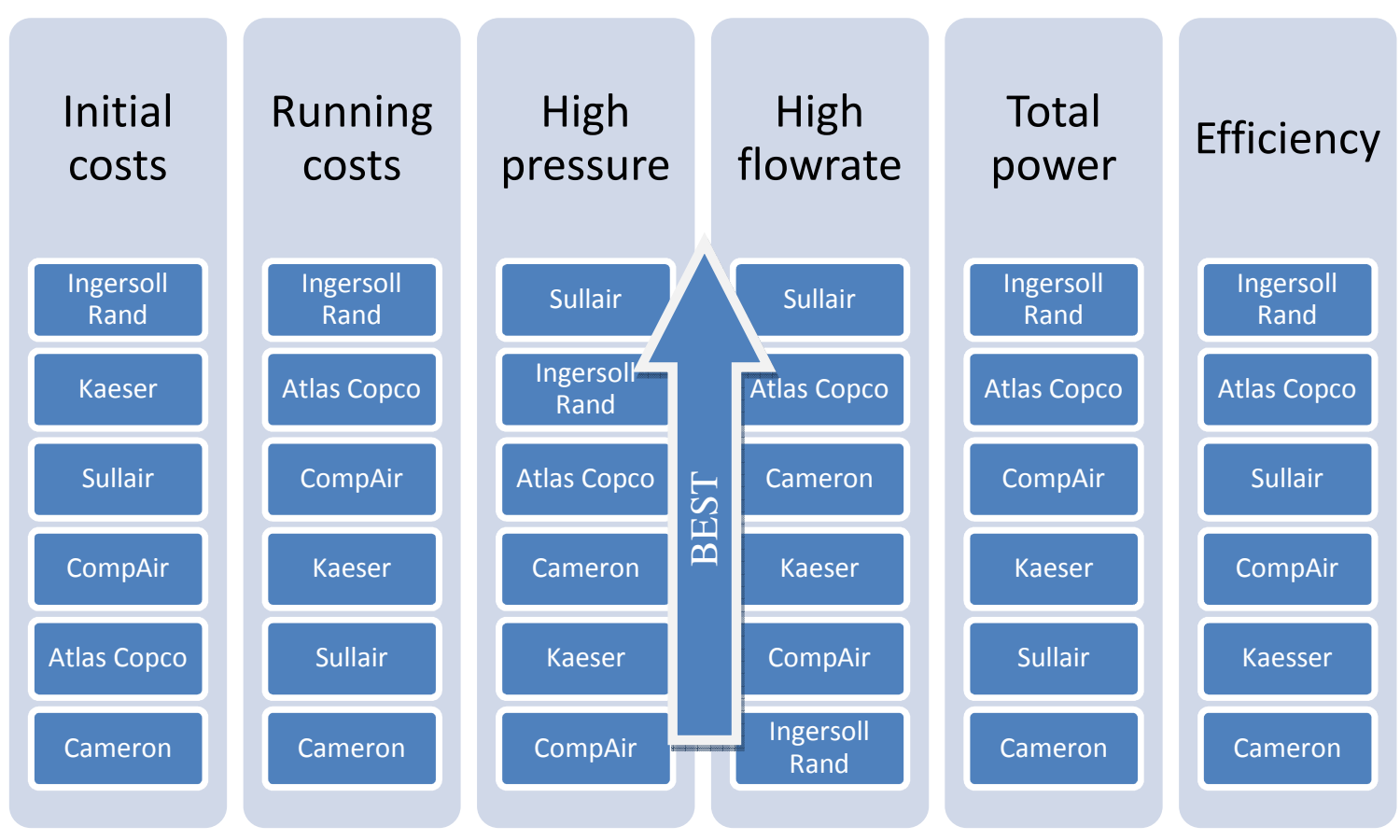

Fig. 3.65- Ranking of options based on different criteria

It is evident that depending on each criteria different options are possible; however, using a scoring method would narrow our search for the most technical and economic option. Appendix B presents the complete comparison between the several proposals received by different equipment manufacturers. The information gathered served as the basis for the application of two weighted scoring methods, the Kepner Tregoe decision analysis and the analytic hierarchy process. Appendix B also includes the methodology followed for the final selection of the compressed air system provider. 
Figs. 3.66 and 3.67 show the results for both techniques, where CompAir results in the most attractive option for the TAMU TowerLab with the highest amount of points and overall percentage given by the two scoring methods used in this study.

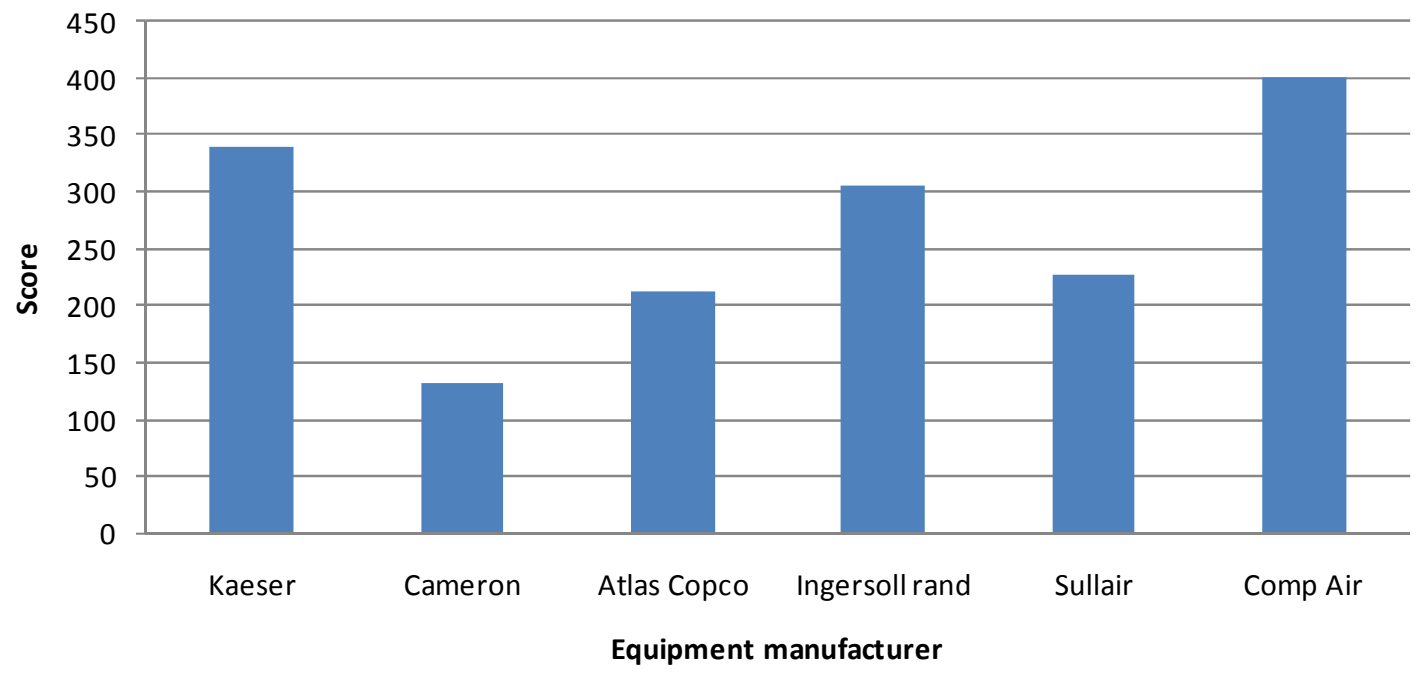

Fig. 3.66- Kepner-Tregoe decision analysis results

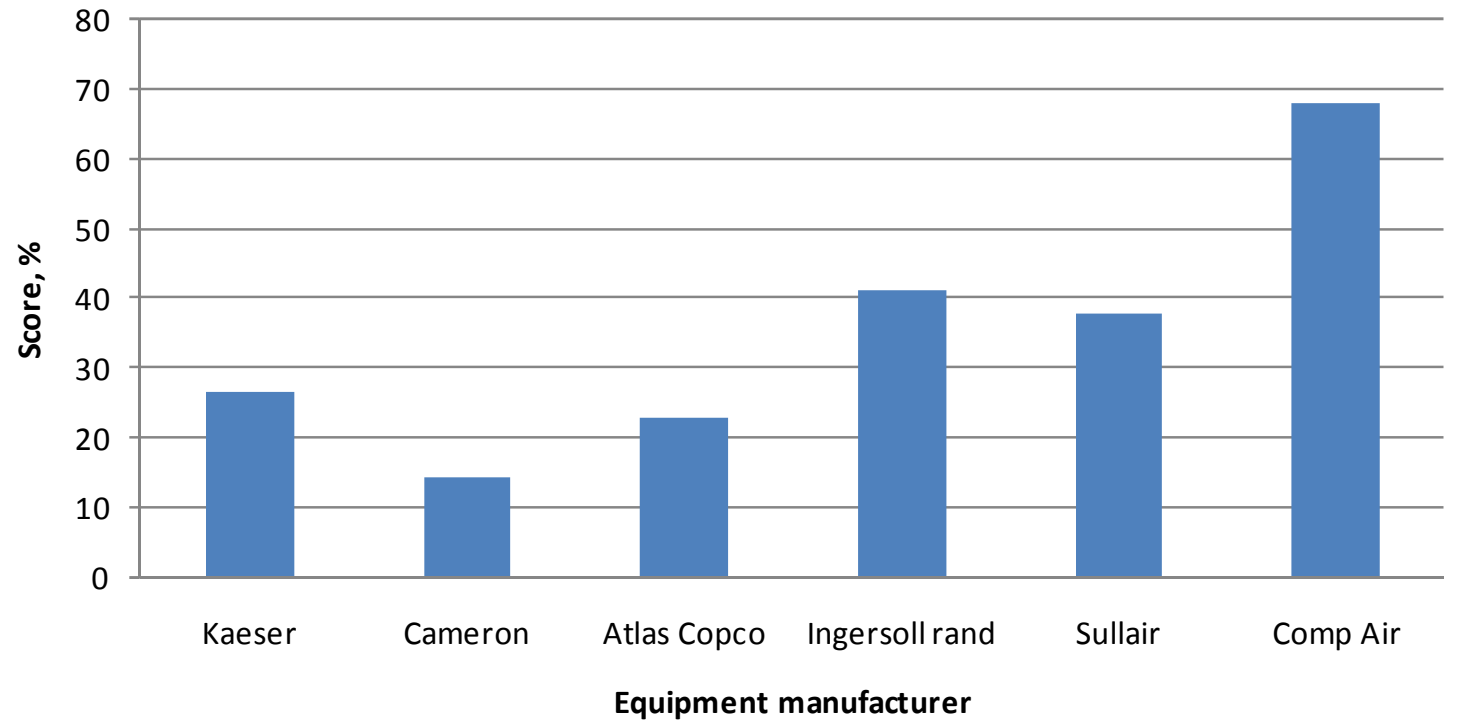

Fig. 3.67- Analytic Hierarchy Process results 


\subsubsection{Injection manifold and piping design}

\subsubsection{Injection manifold}

The manifold is the element in charge of distributing the fluids provided by the compressed air and water systems to the artificial porous medium inside the pressure vessel. It is connected to the container through four equally-spaced entry points to propitiate a radial-distributed pressure and flow. Figure 3.68 shows the initial idea for the design.

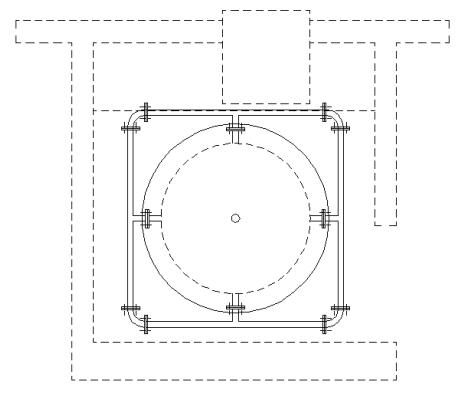

Fig. 3.68- Initial configuration of injection manifold

After approximated dimensions were used for the final sketch, it was realized that a better designed was needed because of space constraints imposed by the TowerLab. Figure 3.69 illustrates the problems encountered with the space available. Additionally, the first entry point is shown aligned with the T-joint carrying air and water. This creates a point of higher pressure and flow rate as compared to the other ports.

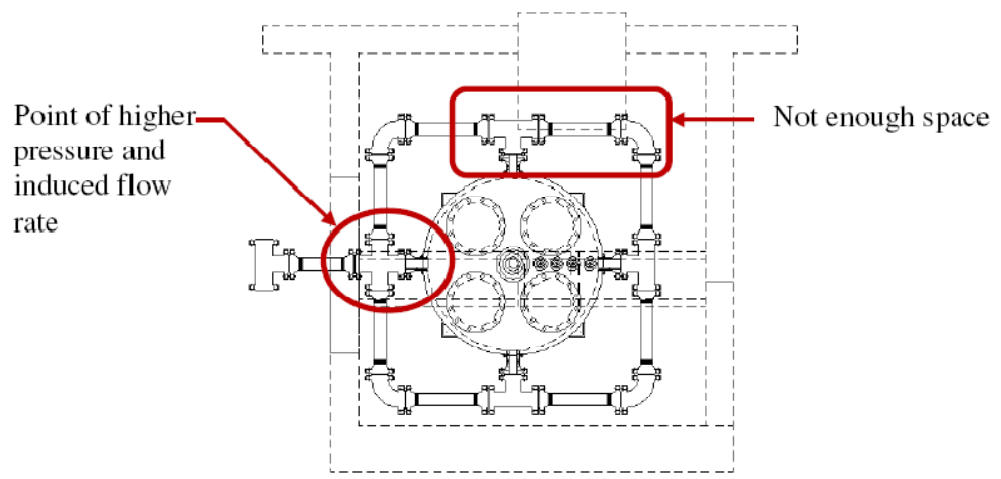

Fig. 3.69- Problems with initial design 
Figure 3.70 illustrates alternatives to the original design to address the problem encountered with physical space limitations.
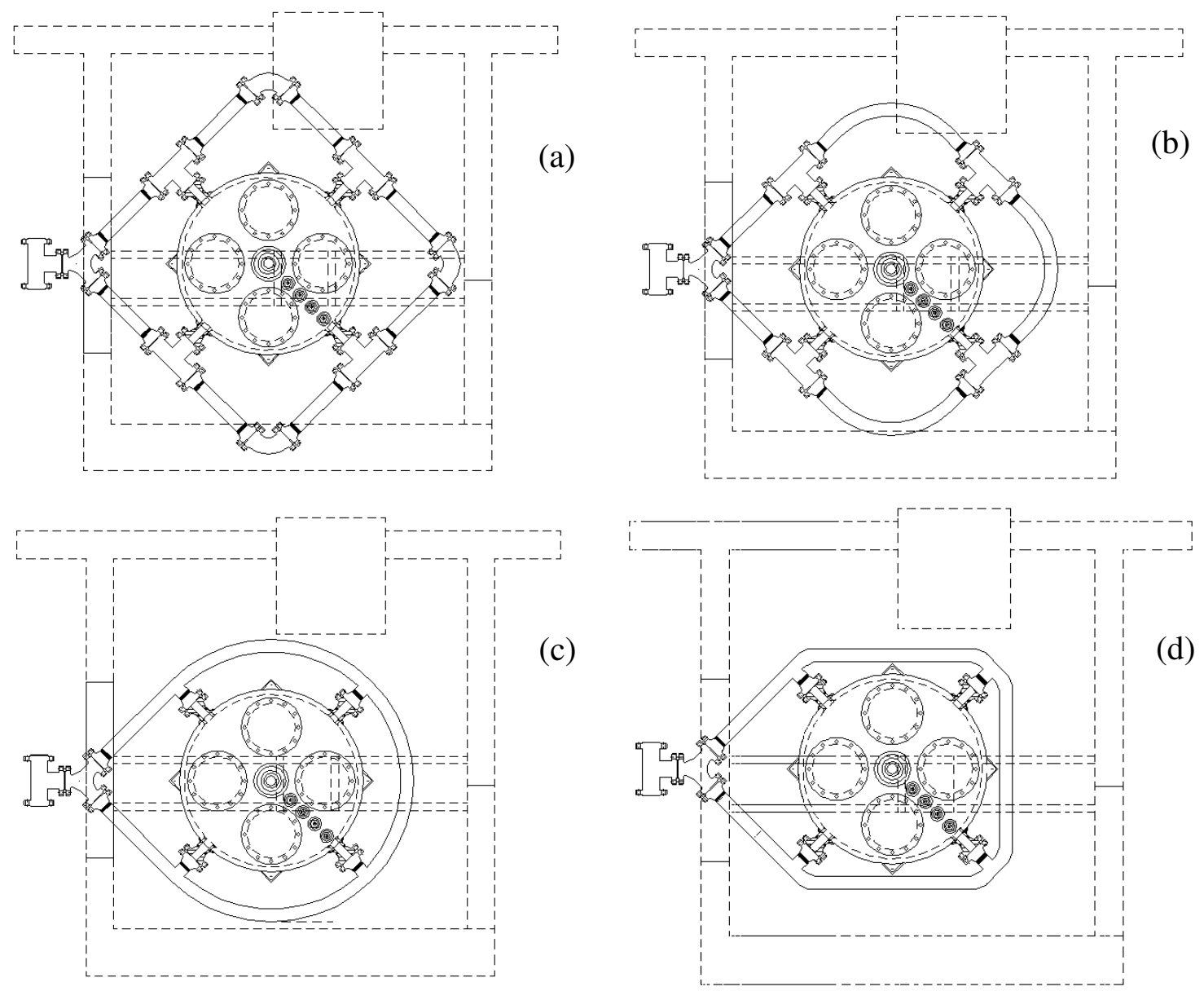

Fig. 3.70- Proposed solutions for manifold design

Options (a) and (b) provide a more uniform distribution of the flow; however, the problem with space is still evident. Alternatively, options (c) and (d) can be located in place without any physical constraints and they both allow for a better flow path than the previous options. The feasibility of fabrication is yet to be evaluated with pipe manufacturers. Figure 3.71 shows the two configurations. 

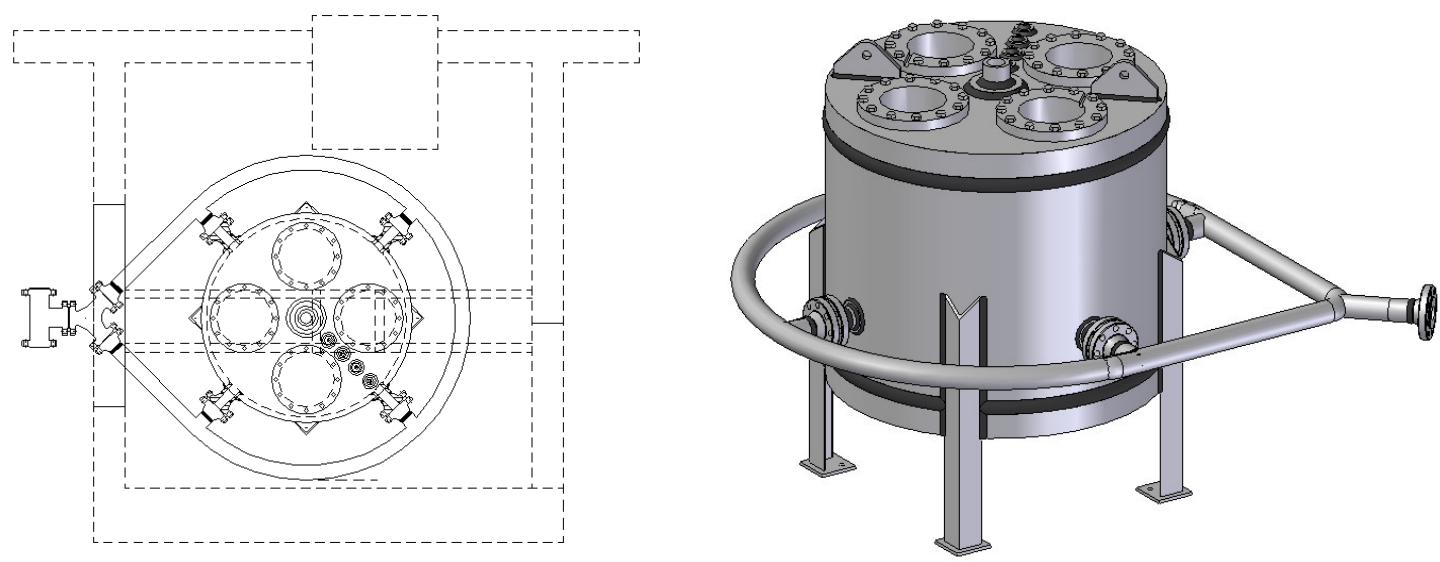

(c)
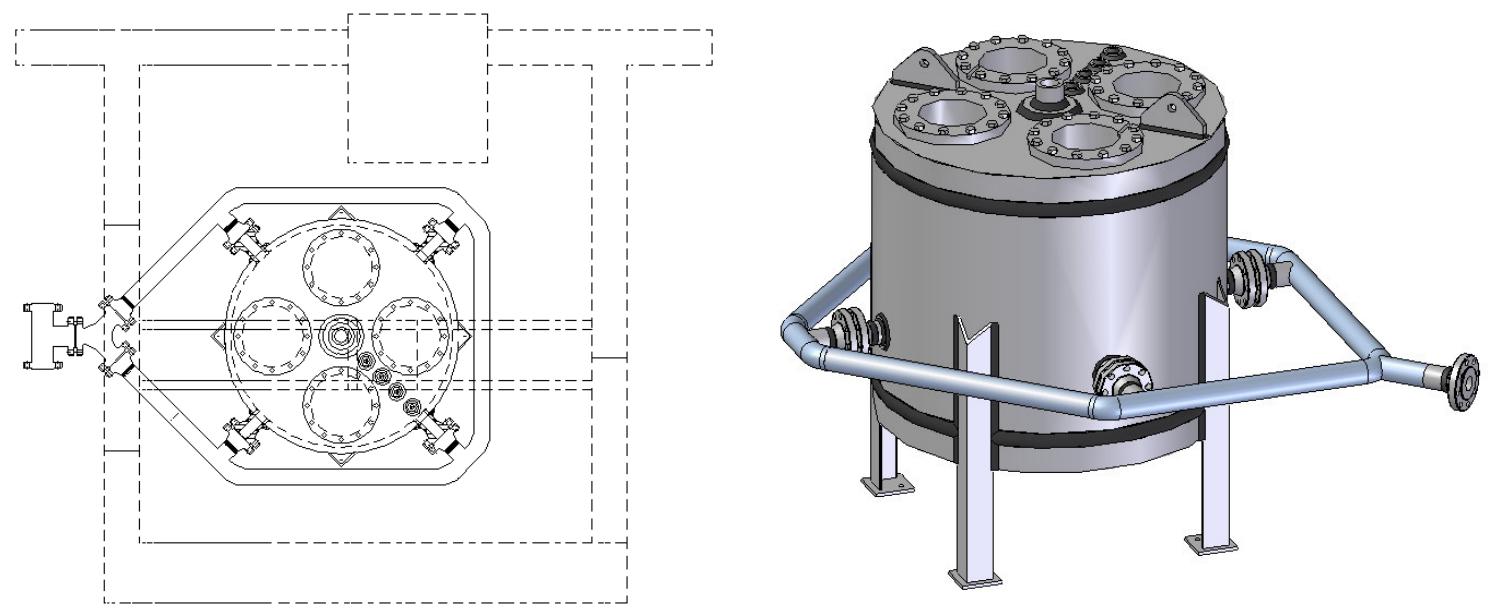

(d)

Fig. 3.71- Recommended manifold designs

\subsection{Internal pipe diameter}

The design of the injection manifold requires selection of an adequate internal diameter for the distribution of both air and water at the entry points located at the pressure vessel. When choosing a line size it is necessary to consider both pressure drop and velocity of flow. Normally, pressure drop is not a governing criterion in production facility piping systems, since most of the pressure drop occurs across a control valve and there is relatively little pressure drop in the line compared to that available in the process. 
The initial design considers a single manifold to transport water and air into the vessel; however, evaluation of the internal diameter based on the superficial velocities required for both phases might dictate the need of independent injection manifolds. The water and air superficial velocities were obtained based on the results by Solomon et $a l .{ }^{17}$ where parameters, such as, permeability, tubing size, pressure (at injection points), vessel radius, choke and water gas ratio were varied to identify recommended ranges in operation for different scenarios.

Table 3.38 presents the results for the range in internal diameter required for the water and air pipelines. Fig 3.72 shows the range that can be covered based on the operating pressure and flow rate delivered by the compressed air system (see appendix C for more details).

Table 3.38- Pipe internal diameter range for air and water

\begin{tabular}{|l|c|c|}
\hline \multirow{2}{*}{ Fluid } & \multicolumn{2}{|c|}{ Pipe diameter range, in. } \\
\cline { 2 - 3 } & Min. & Max. \\
\hline Air & 0.532 & 1.660 \\
\hline Water & 2.844 & 3.030 \\
\hline
\end{tabular}

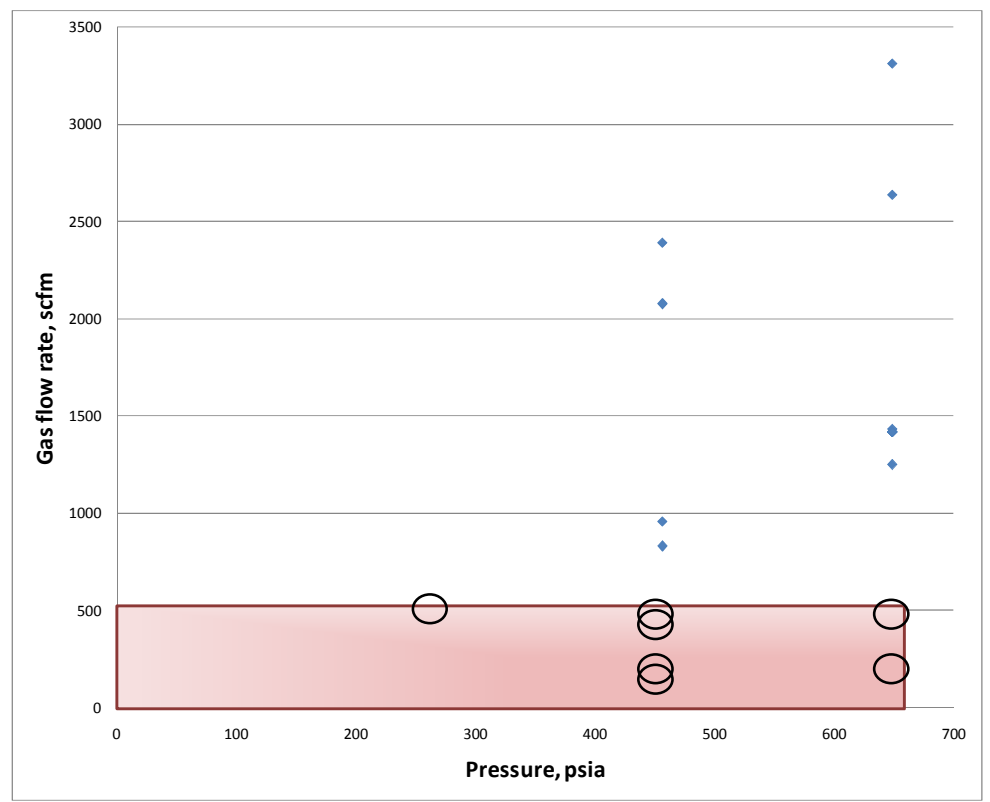

Fig. 3.72- Red area represents the region covered by the pressure and flowrate range and the blue points inside define the internal diameter range 
The difference between sizes for the air and water lines suggests that independent injection, as shown in Fig. 3.73, might be necessary so that the superficial velocities required in the system can be achieved. However, if a single manifold is desired, more investigation on how air and water would behave in a shared pipe should be carried out accounting for the actual phase distribution in the pipe.
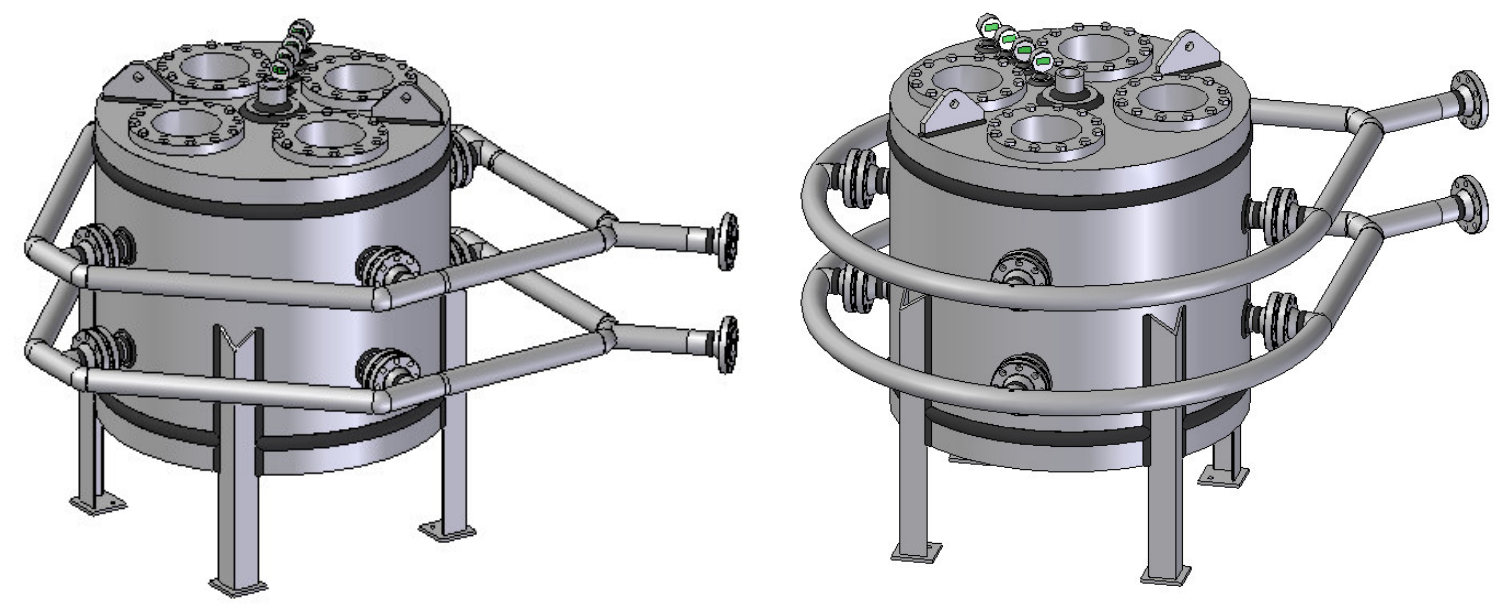

Fig. 3.73- Independent injection manifolds for two configurations

The points inside the region presented in Fig. 3.72 correspond to a range in internal diameter that goes from 0.742 to 1.5 in. for the air injection manifold. Based on this result, the installation of a $1.5 \mathrm{in}$. is recommended. For the case of the water line, we should select an internal diameter within the range provided in table 3.37.

Once the internal diameters have been selected, calculations to determine the wall thickness based on the existing operating conditions of the system are needed. There are different standards used in calculating the required wall thickness of a pipe. The following is a list of the standards used in the United States:

a) ANSI B 31.1- Power Piping: This standard deals with steam and is required by the US Coast Guard in all rigs.

b) ANSI B 31.3 - Chemical Plant and Petroleum Refinery piping: This standard is required by the US Minerals Management Service for offshore platforms in 
federal waters. It is also used extensively for offshore facilities in state waters and for offshore facilities in other parts of the world.

c) ANSI B 31.4 - Liquid Petroleum Transportation Piping Systems: This standard is normally used in onshore oil production facilities.

d) ANSI B 31.8 - Gas Transmission and Distribution Piping Systems: This standard is normally used for gas lines in onshore production facilities and when transporting or distributing gas. In general, the US Department of Transportation has adopted this standard for gas pipelines.

ANSI B31.1 and ANSI B31.3 use the same equation to calculate the required wall thickness. ANSI B31.4 is actually a subset of ANSI B31.8 when it comes to calculating wall thickness. Therefore, from a wall thickness standpoint, only ANSI B31.3 and ANSI B31.8 are in common use. In general, but not always, ANSI B31.3 is the more severe in calculating required wall thickness.

- $\quad$ ANSI B31.3 - Chemical Plant and Petroleum Refinery piping

The wall thickness specified by ANSI B 31.3 can be calculated by:

$t=\left[t_{c}+t_{t h}+\frac{P d_{o}}{2(S E+P Y)}\right]\left[\frac{100}{100-\text { Tol }}\right]$

Where:

$\mathrm{t}=\quad$ required wall thickness, in.

$\mathrm{t}_{\mathrm{c}}=$ corrosion allowance, in. (normally 0.05 in.)

$t_{t h}=$ thread or groove depth, in. (Table 3.39)

$\mathrm{P}=\quad$ internal pipe pressure, $\mathrm{psi}$

$\mathrm{d}_{\mathrm{o}}=$ pipe outside diameter, in.

$\mathrm{S}=\quad$ allowable stress for pipe material, psi (Table 3.40)

$\mathrm{E}=\quad$ longitudinal weld joint factor (1.00 for seamless, 0.85 for ERW)

$\mathrm{Y}=$ factor $\left(0.4\right.$ for ferrous materials below $\left.900^{\circ} \mathrm{F}\right)$

Tol $=$ manufacturers' allowed tolerance (12.5\% for API 5L up to 20 in. diameter) 
Table 3.39- Thread allowance for pipe wall thickness calculations for ANSI B31.3

\begin{tabular}{|c|c|}
\hline Nominal pipe size (in.) & $\mathbf{t}_{\text {th }}$ \\
\hline $1 / 4-3 / 8$ & 0.05 \\
\hline $1 / 2-3 / 4$ & 0.06 \\
\hline $1-2$ & 0.08 \\
\hline $2-1 / 2-20$ & 0.11 \\
\hline
\end{tabular}

Table 3.40- Basic allowable stress for grade B seamless pipe, psi

\begin{tabular}{|c|c|}
\hline Temperature, $^{\mathbf{F}}$ & ASTM A106 (SA106), psi \\
\hline-20 to 400 & 20,000 \\
\hline 500 & 18,900 \\
\hline 600 & 17,300 \\
\hline 650 & 17,000 \\
\hline
\end{tabular}

For ease in picking a pipe wall thickness, table 3.41 gives standard pipe diameter characteristics.

Table 3.41- Data for different pipe sizes

\begin{tabular}{|c|c|c|c|c|c|c|}
\hline $\begin{array}{c}\text { Nominal } \\
\text { size, in. }\end{array}$ & $\begin{array}{l}\text { OD, } \\
\text { in. }\end{array}$ & $\begin{array}{l}\text { Nominal wall } \\
\text { thickness, in. }\end{array}$ & ID, in. & $\begin{array}{c}\text { Nominal } \\
\text { weight per } \\
\text { foot, lb/ft }\end{array}$ & $\begin{array}{l}\text { Weight } \\
\text { Class }\end{array}$ & $\begin{array}{c}\text { Schedule } \\
\text { No. }\end{array}$ \\
\hline \multirow[t]{6}{*}{1.5} & \multirow[t]{6}{*}{1.9} & 0.065 & 1.770 & 1.274 & - & - \\
\hline & & 0.109 & 1.682 & 2.085 & - & - \\
\hline & & 0.145 & 1.610 & 2.718 & STD & 40 \\
\hline & & 0.200 & 1.500 & 3.631 & XS & 80 \\
\hline & & 0.281 & 1.338 & 4.859 & - & 160 \\
\hline & & 0.400 & 1.100 & 6.408 & XXS & - \\
\hline \multirow[t]{3}{*}{2} & \multirow[t]{3}{*}{2.375} & 0.218 & 1.939 & 5.02 & $\mathrm{XS}$ & 80 \\
\hline & & 0.344 & 1.687 & 7.46 & - & 160 \\
\hline & & 0.436 & 1.503 & 9.03 & XXS & - \\
\hline \multirow[t]{6}{*}{$21 / 2$} & \multirow[t]{6}{*}{2.875} & 0.083 & 2.709 & 2.48 & - & - \\
\hline & & 0.120 & 2.635 & 3.53 & - & - \\
\hline & & 0.203 & 2.469 & 5.79 & STD & 40 \\
\hline & & 0.276 & 2.323 & 7.66 & XS & 80 \\
\hline & & 0.375 & 2.125 & 10.01 & - & 160 \\
\hline & & 0.552 & 1.771 & 13.69 & XXS & - \\
\hline \multirow[t]{6}{*}{3} & \multirow[t]{6}{*}{3.500} & 0.083 & 3.334 & 3.03 & - & - \\
\hline & & 0.120 & 3.260 & 4.33 & - & - \\
\hline & & 0.216 & 3.068 & 7.58 & STD & 40 \\
\hline & & 0.300 & 2.900 & 10.25 & XS & 80 \\
\hline & & 0.438 & 2.624 & 14.32 & - & 160 \\
\hline & & 0.600 & 2.300 & 18.58 & XXS & - \\
\hline
\end{tabular}


- $\quad$ ANSI B31.8 - Gas Transmission and Distribution Piping Systems

The wall thickness specified by ANSI B 31.8 and by 49 CFR 192 for a given pipe can be calculated by:

$\mathrm{t}=\frac{\mathrm{Pd}_{\mathrm{o}}}{2(\mathrm{FETS})}$

Where:

$\mathrm{t}=$ required wall thickness, in.

$\mathrm{P}=\quad$ internal pipe pressure

$\mathrm{d}_{\mathrm{o}}=$ pipe outside diameter, in.

$\mathrm{S}=\quad$ minimum yield strength of pipe material, psi

$\mathrm{F}=\quad$ design factor (Table 3.42)

$\mathrm{E}=\quad$ longitudinal weld joint factor (1.00 for seamless, 0.85 for ERW)

$\mathrm{T}=\quad$ temperature derating factor (Table 3.43)

Table 3.42- Design factor, $F$

\begin{tabular}{|l|l|c|l|}
\hline \multicolumn{1}{|c|}{ Location Class } & Class Location & $\begin{array}{c}\text { Design } \\
\text { Factor, F }\end{array}$ & \multicolumn{2}{|c|}{ General Description } \\
\hline Location Class 1, Division 1 & Not Applicable & 0.8 & $\begin{array}{l}\text { Sparsely populated areas, } \\
\text { farmland, deserts }\end{array}$ \\
\hline Location Class I, Division 2 & Class Location 1 & 0.72 & $\begin{array}{l}\text { Sparsely populated areas, } \\
\text { farmland, deserts }\end{array}$ \\
\hline Location Class 2 & Class Location 2 & 0.6 & $\begin{array}{l}\text { Fringe areas around cities and } \\
\text { towns }\end{array}$ \\
\hline Location Class 3 & Class Location 3 & 0.5 & $\begin{array}{l}\text { Residential and industrial } \\
\text { areas }\end{array}$ \\
\hline Location Class 4 & Class Location 4 & 0.4 & $\begin{array}{l}\text { Dense areas with multi-story } \\
\text { buildings }\end{array}$ \\
\hline
\end{tabular}

Table 3.43- Temperature derating factor, $T$

\begin{tabular}{|c|c|}
\hline Temperature, ${ }^{\mathbf{}} \mathbf{F}$ & Derating Factor, $\mathbf{T}$ \\
\hline-20 to 250 & 1.000 \\
\hline 300 & 0.967 \\
\hline 350 & 0.933 \\
\hline
\end{tabular}


Equations 3.53 and 3.54 are slightly different in form because of the different types of piping system each was intended to cover. In equations 3.54, no special provision is made for corrosion or thread allowance, furthermore it does not account for threaded or grooved joints as it assumes that all pipe is welded.

Most gas transmission lines handle a relatively "clean" product and so no specific wall thickness allowance is suggested for internal corrosion in ANSI B 31.8. ANSI B 31.3 specifically states that an allowance should be included for corrosion and erosion. API Recommended Practice 14E for Offshore Production Platform Piping Systems suggests that a corrosive/mechanical strength allowance of 0.05 in. be used for carbon steel piping.

The two criteria were used to determine the minimum thickness required for the two manifolds using a range in nominal pipes. The internal diameter for the air and water line should be within the ranges specified in section 3.4.3.1.1. The results are presented in Table 3.44.

Table 3.44- Results in wall thickness using ANSI B31.3 and ANSI B31.8

\begin{tabular}{|l|c|c|c|c|c|c|}
\hline \multirow{2}{*}{$\begin{array}{l}\text { Nominal } \\
\text { pipe size, } \\
\text { in. }\end{array}$} & \multicolumn{4}{|c|}{ Wall thickness, in. } & \multicolumn{2}{c|}{ Internal diameter, in. } \\
\cline { 2 - 7 } & $\begin{array}{c}\text { ANSI } \\
\text { B31.3 }\end{array}$ & $\begin{array}{c}\text { Pipe } \\
\text { STD }\end{array}$ & $\begin{array}{c}\text { ANSI } \\
\text { B31.8 }\end{array}$ & $\begin{array}{c}\text { Pipe } \\
\text { STD }\end{array}$ & $\begin{array}{c}\text { ANSI } \\
\text { B31.3 }\end{array}$ & ANSI B31.8 \\
\hline 1 & 0.173 & 0.179 & 0.032 & 0.065 & 0.957 & 1.185 \\
\hline $\mathbf{1 . 5}$ & $\mathbf{0 . 1 8 4}$ & $\mathbf{0 . 2 0 0}$ & $\mathbf{0 . 0 4 6}$ & $\mathbf{0 . 0 6 5}$ & $\mathbf{1 . 5 0 0}$ & $\mathbf{1 . 7 7 0}$ \\
\hline 2 & 0.227 & 0.344 & 0.057 & 0.065 & 1.687 & 2.245 \\
\hline 2.5 & 0.236 & 0.276 & 0.069 & 0.083 & 2.323 & 2.709 \\
\hline $\mathbf{3}$ & $\mathbf{0 . 2 4 7}$ & $\mathbf{0 . 3 0 0}$ & $\mathbf{0 . 0 8 4}$ & $\mathbf{0 . 1 2 0}$ & $\mathbf{2 . 9 0 0}$ & $\mathbf{3 . 2 6 0}$ \\
\hline
\end{tabular}

Evaluating the results and considering the constraint in internal diameter imposed by the superficial velocity of the fluids, the nominal diameters recommended for the two manifolds are 1.5 and 3 Schedule 80 for air and water respectively. As for the criteria to use, none of the methods fully fits the conditions at TAMU TowerLab; however, we suggest ANSI B31.3 which represents the most conservative approach. 


\subsubsection{Pressure drop in manifold}

For liquid lines, it is common to express the energy contained in a fluid in terms of the potential energy contained in an equivalent height or head of a column of the fluid. Using this convention, Bernoulli's theorem breaks down to the total energy at a point in terms of:

- The head due to its elevation above an arbitrary datum of zero potential energy.

- A pressure head due to the potential energy contained in the pressure in the fluid in that point.

- A velocity head due to the kinetic energy contained within the fluid.

Assuming that no energy is added to the fluid by a pump or compressor, and that the fluid is not performing work, the law of conservation of energy requires the energy at point " 2 " in the piping system downstream of point " 1 " must equal the energy at point "1" minus the energy loss to friction and change in elevation.

$\mathrm{Z}_{1}+\frac{144 \mathrm{P}_{1}}{\rho_{1}}+\frac{\mathrm{V}_{1}{ }^{2}}{2 \mathrm{~g}}=\mathrm{Z}_{2}+\frac{144 \mathrm{P}_{2}}{\rho_{2}}+\frac{\mathrm{V}_{2}{ }^{2}}{2 \mathrm{~g}}+\mathrm{H}_{\mathrm{L}}$

Where:

$\mathrm{Z}=$ elevation head, $\mathrm{ft}$

$\mathrm{P}=$ pressure, $\mathrm{psi}$

$\rho=$ density, $\mathrm{lb} / \mathrm{ft}^{3}$

$\mathrm{V}=$ velocity, $\mathrm{ft} / \mathrm{s}$

$\mathrm{g}=\quad$ gravitation constant

$\mathrm{H}_{\mathrm{L}}=$ friction head loss

The Darcy equation or usually called the Darcy-Weisbach equation, states that the friction head loss between two points in a completely filled, circular cross section pipe is proportional to the velocity head and the length of pipe and inversely proportional to the pipe diameter. This can be written as: 
$\mathrm{H}_{\mathrm{L}}=\frac{\mathrm{fLV}}{2 \mathrm{gd}}$

Where:

$\mathrm{L}=\quad$ length of pipe, $\mathrm{ft}$

$\mathrm{f}=\operatorname{moody}$ friction factor

In most production facilities piping systems, the head differences due to elevation and velocity changes between two points can be neglected. Substituting Eq. 3.62 in 3.61 to get the pressure drop, we obtain the following expression:

$\Delta \mathrm{P}=0.0013 \frac{\mathrm{f} \rho \mathrm{LV}}{\mathrm{d}}$

Equation 3.57 can be rearranged as a function of flow rate:

$\Delta \mathrm{P}=11.5 \times 10^{-6} \frac{\mathrm{fLQ}_{\mathrm{l}}^{2} \gamma}{\mathrm{d}^{5}}$

Where:

$\gamma=\quad$ specific gravity of liquid relative to water

$\mathrm{d}=\quad$ pipe internal diameter, in.

$\mathrm{g}=$ gravitation constant

$\mathrm{Q}_{\mathrm{l}}=$ liquid flow rate, bpd

Calculating the Moody friction factor required the relative roughness of the pipe and the Reynolds number. Table 3.45 provides the pipe internal roughness for different materials and the friction factor can be obtained using Fig. 3.74.

Table 3.45- Pipe internal roughness

\begin{tabular}{|l|c|}
\hline Pipe material & Roughness, in. \\
\hline Commercial steel/welded steel & 0.0018 \\
\hline Riveted steel & $0.035-0.35$ \\
\hline PVC, drawn tubing, glass & 0.000059 \\
\hline
\end{tabular}




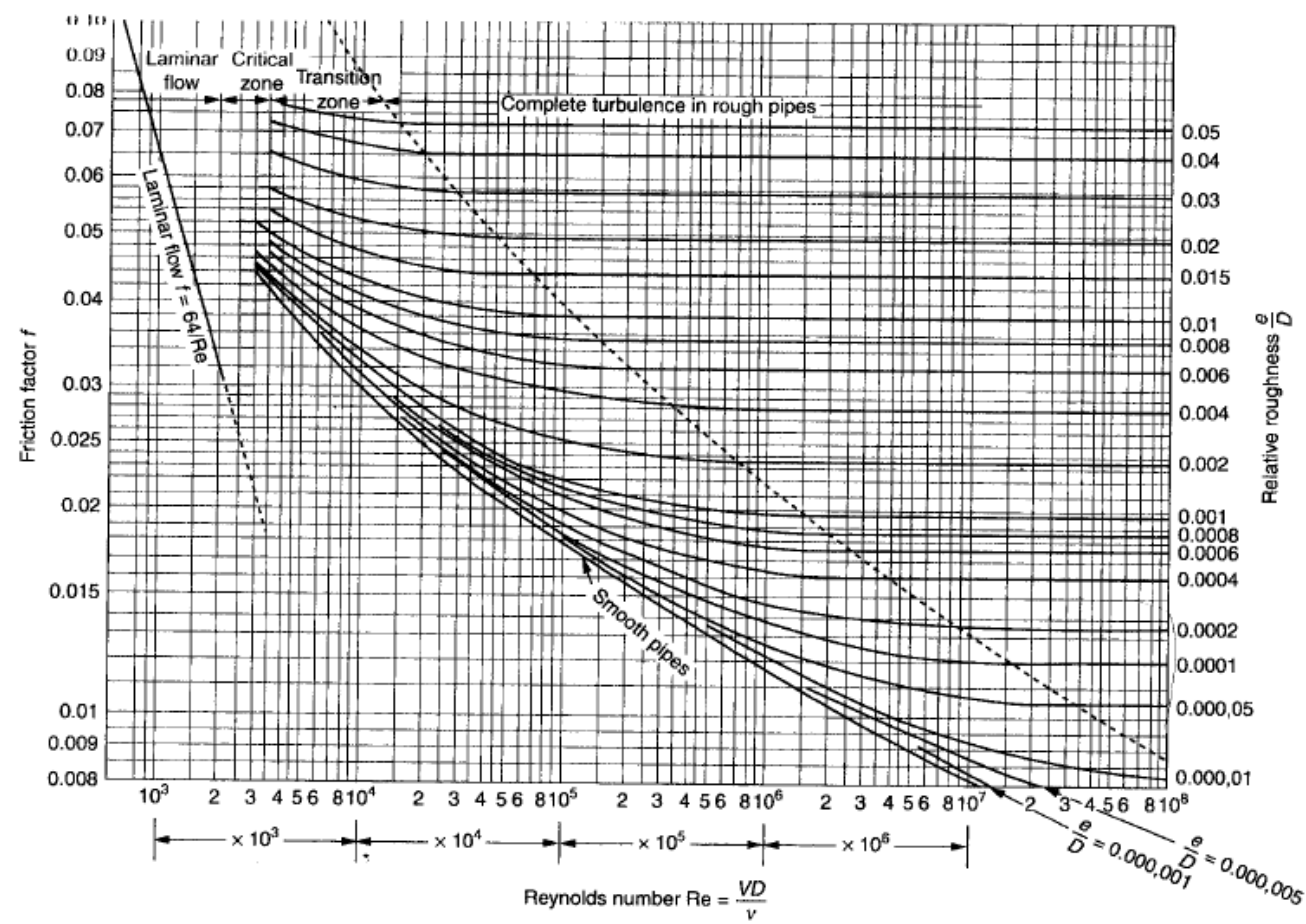

Fig. 3.74- Moody friction factor ${ }^{58}$

Results from this analysis where validated using a pressure drop software calculator. Based on the input data, the friction factor corresponds to 0.0207. The pressure drop as a function of pipe length is presented in Fig. 3.75.

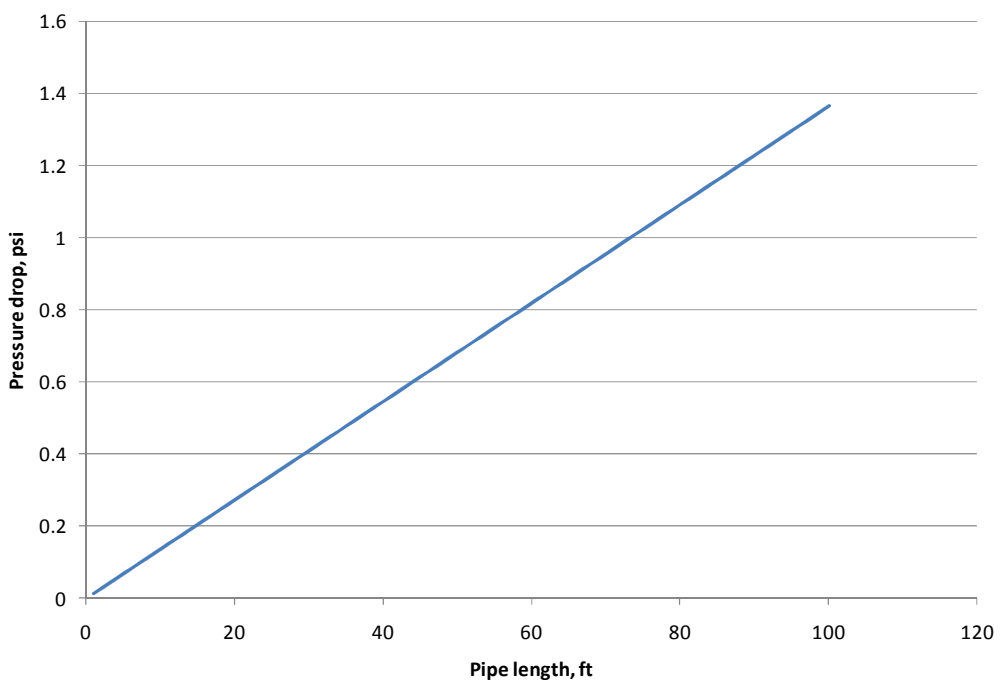

Fig. 3.75- Pressure drop as a function of pipe length (water) 
Table 3.46 presents the pressure drop in the manifold for the two designs under evaluation.

Table 3.46- Major pressure drops in manifold

\begin{tabular}{|c|c|c|c|c|c|}
\hline Manifold design & \multicolumn{5}{|c|}{ Pressure drop for water system, psi } \\
\hline & $0-1(4 \mathrm{ft})$ & $0-2(10 \mathrm{ft})$ & $0-3(10 \mathrm{ft})$ & $0-4(4 \mathrm{ft})$ & $0-0(28 \mathrm{ft})$ \\
\cline { 2 - 6 } & & & & & \\
& & & & & \\
& & & & & \\
\end{tabular}

In addition to pressure drop due to friction, minor losses can be produced as a result of piping components such as valves and fittings. Fittings include elbows, tees or Y-connections. The pipe nozzles exiting from the pressure vessel will have entrance and exit losses. Generally, minor losses are included in calculations by using the equivalent length of the valve or fitting. Table 3.47 shows the values obtained for the two manifolds following the equivalent length criteria.

Table 3.47- Pressure drop due to fittings

\begin{tabular}{|c|c|c|c|c|c|c|}
\hline \multicolumn{7}{|c|}{ Equivalent length } \\
\hline Manifold design & Fitting & Qty & L/D & $\mathbf{L}_{\mathbf{e}}, \mathbf{f t}$ & $\begin{array}{c}\text { Total } \\
\text { length, ft }\end{array}$ & $\Delta_{\mathbf{0}} \mathbf{0}, \mathbf{p s i}$ \\
\hline & Tee & 4 & 60 & 60 & 88 & 1.2 \\
\hline & $\begin{array}{c}\text { Mitre } \\
\text { bends }\end{array}$ & 6 & 15 & 15 & 101 & 1.378 \\
\hline & Tee & 4 & 60 & 60 & & \\
\hline
\end{tabular}


For gas pipelines, several empirical gas flow equations have been developed. These equations are patterned after the general flow equation, but make certain assumptions as to avoid solving for Moody friction factor. The correlations are the ones developed by Weymouth, Panhandle and Spitsglass.

Weymouth's equation is suitable for most piping within the production facility; however, the friction factor used by Weymouth is generally too low for large diameter or low velocity lines where the flow regime is more properly characterized by the slope portion of the Moody diagram.

The correlation developed by Panhandle is used for commonly large diameter, long pipelines where the Reynolds number is on the straight line portion of the Moody diagram where as Spitzglass's is often applied for near atmospheric pressure lines.

The empirical gas flow equations use various coefficients and exponents to account for efficiency and friction factors. These equations represent the flow condition upon which they were derived, but may not be accurate under different conditions. The recommended approach is to use the general flow gas equation "Darcy-Weiscbach" for most general cases.

For compressed air systems, the equation to get the pressure drop can be expressed as:

$\mathrm{P}_{1}^{2}-\mathrm{P}_{2}^{2}=25.1 \frac{\mathrm{SfLQ}_{\mathrm{g}}{ }^{2} \mathrm{ZT}}{\mathrm{d}^{5}}$

Where:

$\mathrm{P}_{1}=\quad$ specific gravity of liquid relative to water

$\mathrm{P}_{2}=$ pipe internal diameter, in.

$\mathrm{Q}_{\mathrm{g}}=$ liquid flow rate, $\mathrm{bpd}$

An approximation of Eq. 3.59 can be made when the change in pressure is less than $10 \%$ of the inlet pressure. If this is true, the following assumption is valid: $\mathrm{P}_{1}^{2}-\mathrm{P}_{2}^{2} \cong 2 \mathrm{P}_{1}\left(\mathrm{P}_{1}-\mathrm{P}_{2}\right)$ 
Substituting in Eq 3.59 we get:

$\Delta \mathrm{P}=12.6\left[\frac{\mathrm{SfLQ}_{\mathrm{g}}{ }^{2} \mathrm{ZT}}{\mathrm{P}_{1} \mathrm{~d}^{5}}\right]$

The friction factor based on the Reynolds number and the relative roughness is 0.0209. Figure 3.76 illustrates the pressure drop versus length for different correlations used in gas pipelines. However as previously mentioned, the Darcy-Weisbach method is the one considered to estimate pressure drops in the system. As it is observed, the values are less than $10 \%$ of the inlet pressure; therefore the initial assumption to obtain Eq. 3.60 is valid.

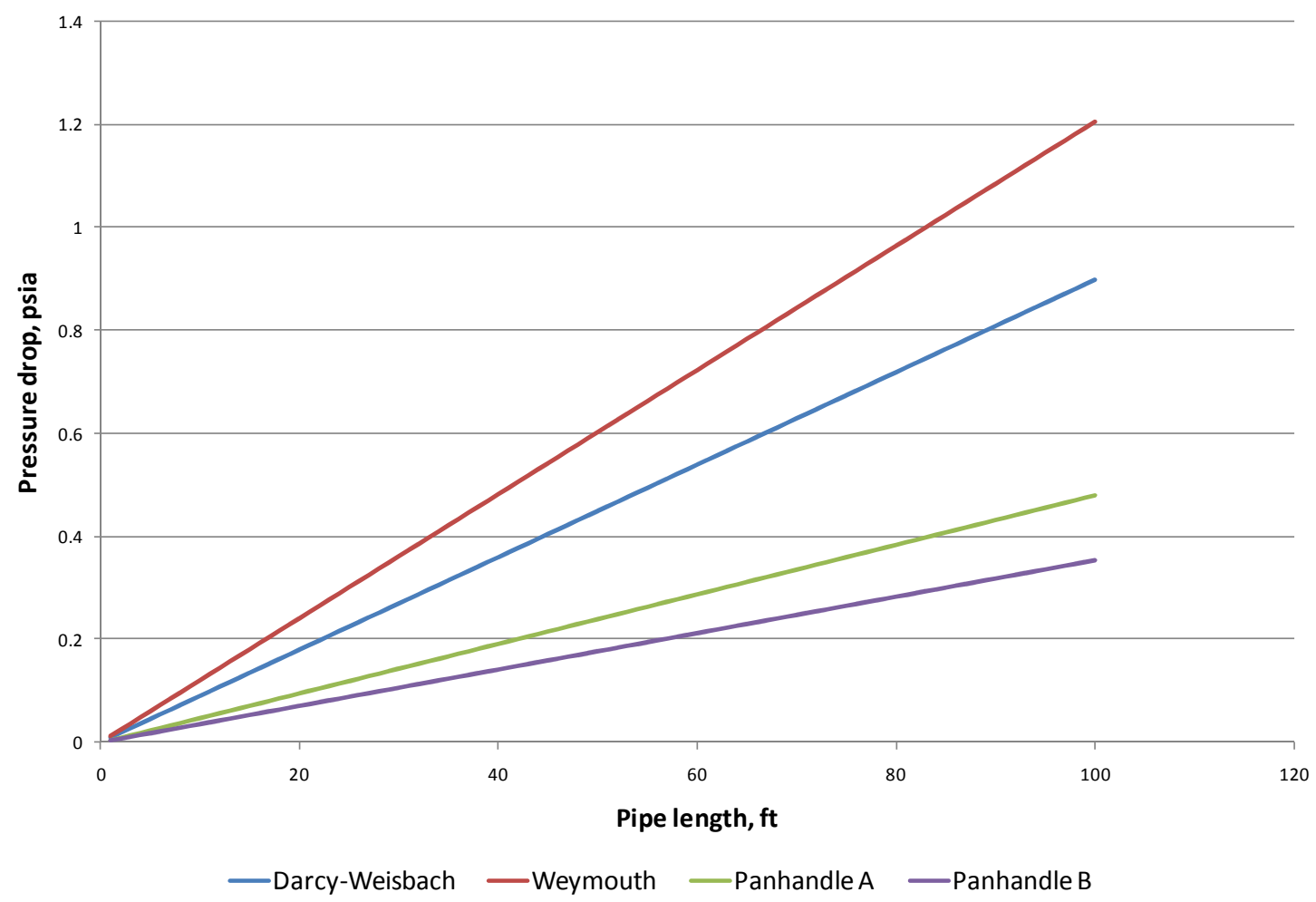

Fig. 3.76- Compressed air system pressure drop due to friction 
Tables 3.48 and 3.49 show the major and minor pressure drops in the air manifold.

Table 3.48- Pressure drop in the manifold for air flow

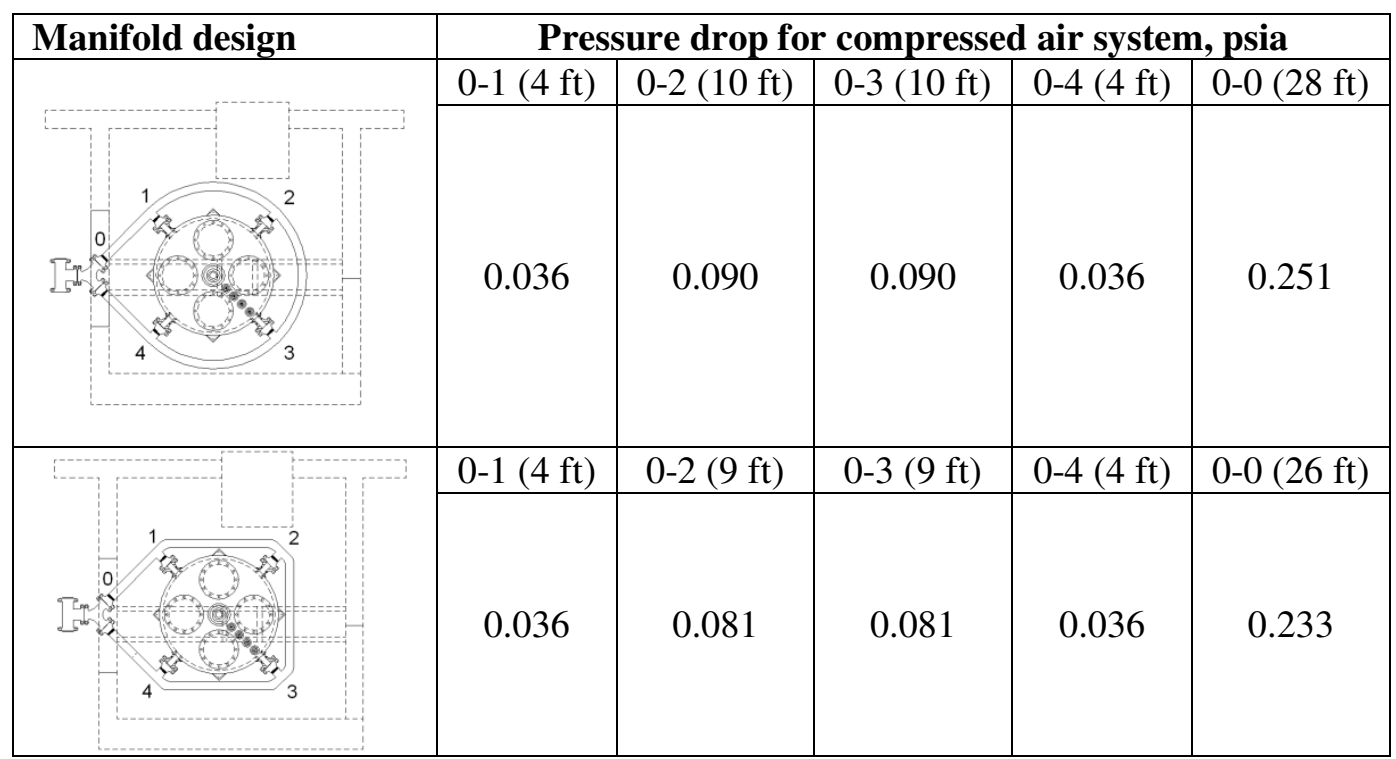

Table 3.49- Pressure drop due to fittings

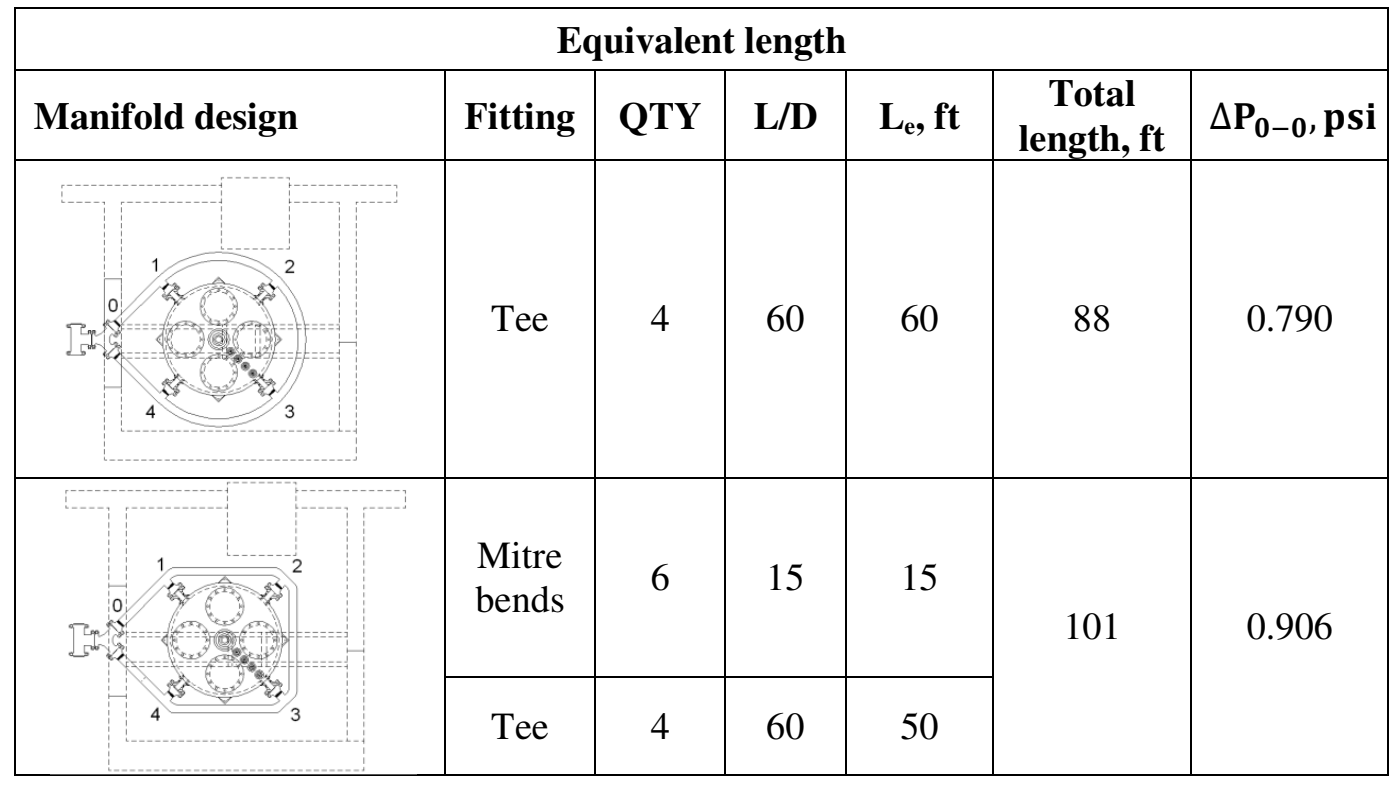




\subsubsection{Piping system}

Pressure drop is a particularly important criterion for long lines or those flowing between pieces of equipment operating in the same or nearly the same pressure. Neither of these situations is being faced at the TowerLab since the estimated distance for the piping network connecting both the compressed air and water system to the pressure vessel corresponds approximately to $215 \mathrm{ft}$. A schematic of the proposed piping system for water and air flow is depicted in Figure 3.77.

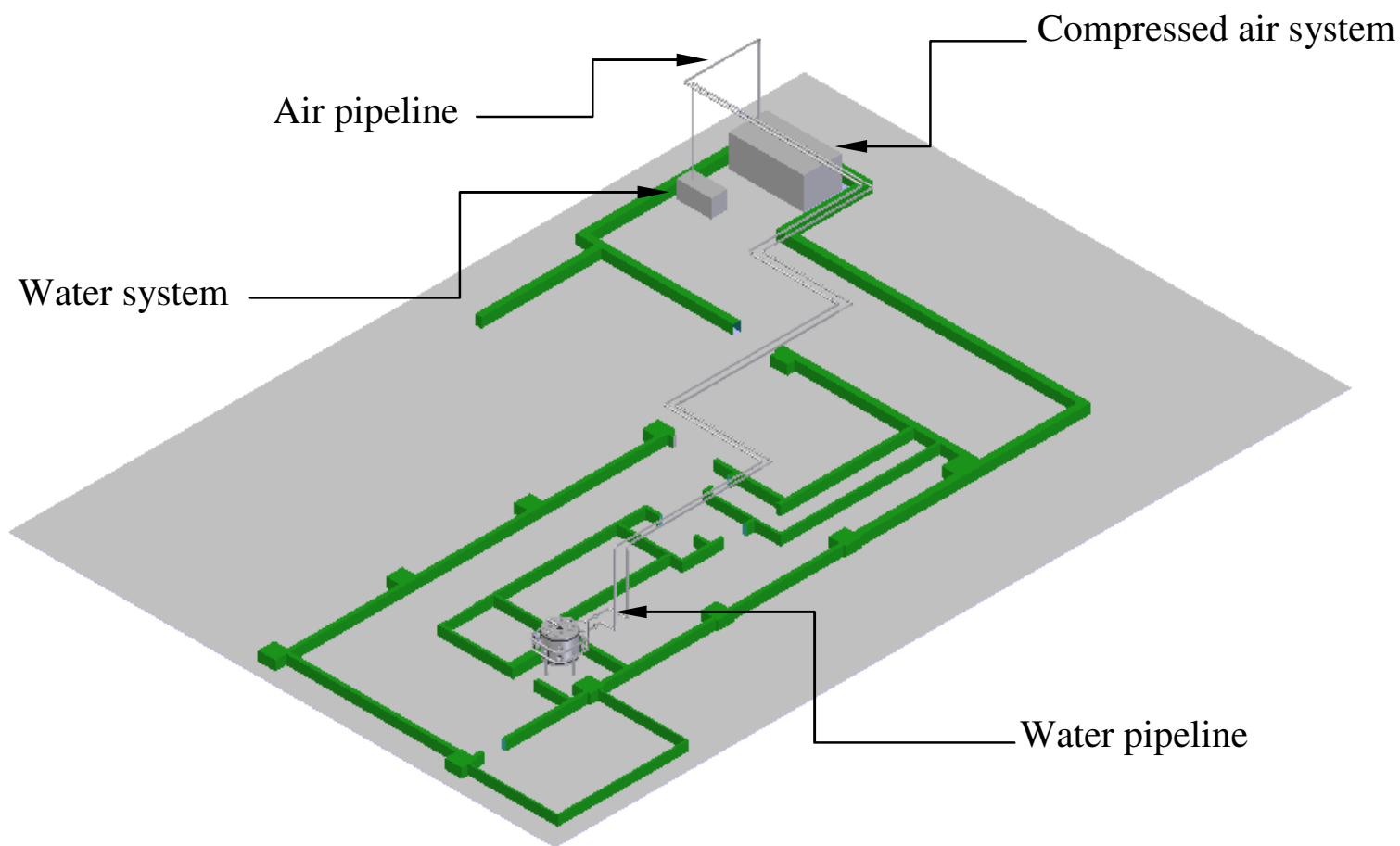

Fig. 3.77- Schematic of piping system

As indicated for the injection manifold, the line diameters had to be sized for achieving the required superficial velocities needed to conduct the experiments and to allow the installation of flow control and monitoring devices such as valves and meters. 


\subsection{Liquid line}

Water supply at the TowerLab is to be provided using a high pressure water pump. The pipe transporting the liquid flows independently and connects to its manifold as illustrated in Fig. 3.78.
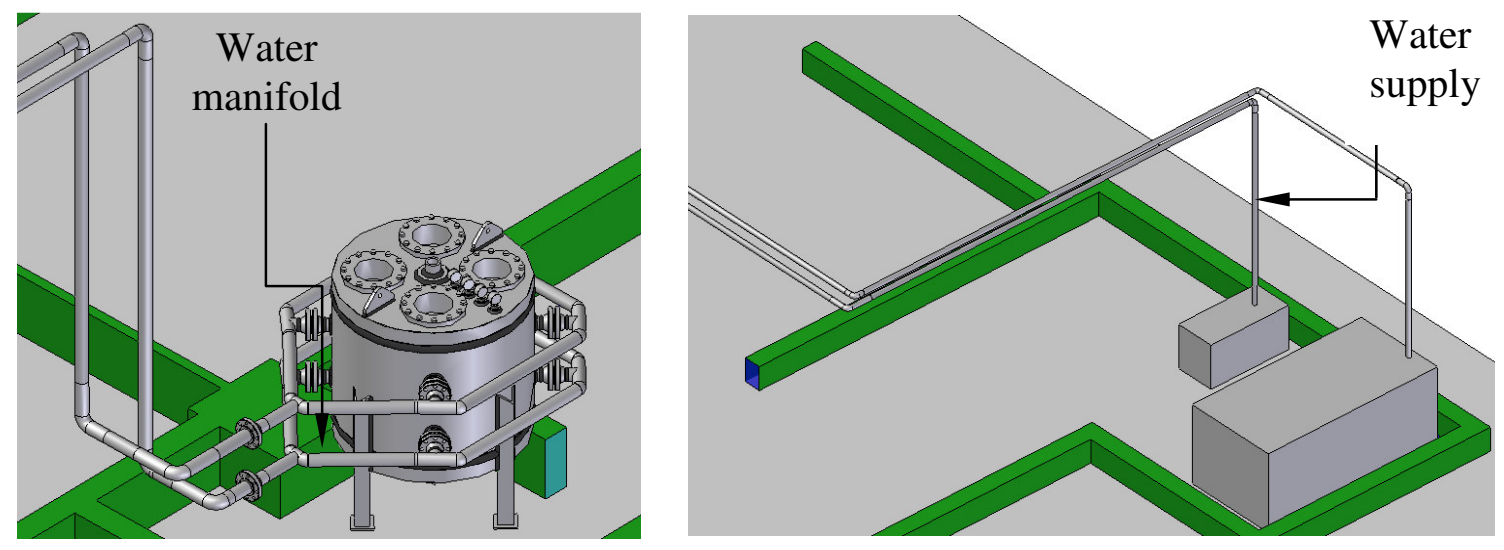

Fig. 3.78- Water supply system

The water pipe line will have the same size as indicated for the injection manifold. Based on the latter, the pipe will be a 3 in. pipe schedule 80 which will accommodate long radius elbows, valves and flowmeters. Depending on the selection of the water pump, enlargements or reductions in the line may also be necessary.

The total length of straight pipeline is approximately $157 \mathrm{ft}$; however, the minor losses due to the installation of 8 long radius elbows increase the total equivalent length to $190 \mathrm{ft}$. Based on this result the total pressure drop in the water line is 2.6 psi.

\subsection{Air line}

The air pipeline is connected to the discharge of the compressed air system and covers a similar path as compared to the water line. As a result, the same number of fittings will be required to drive the flow from upstream to downstream conditions, that is, from compressor discharge to manifold. Figures 3.79, shows the proposed layout for the compressed air piping system. 

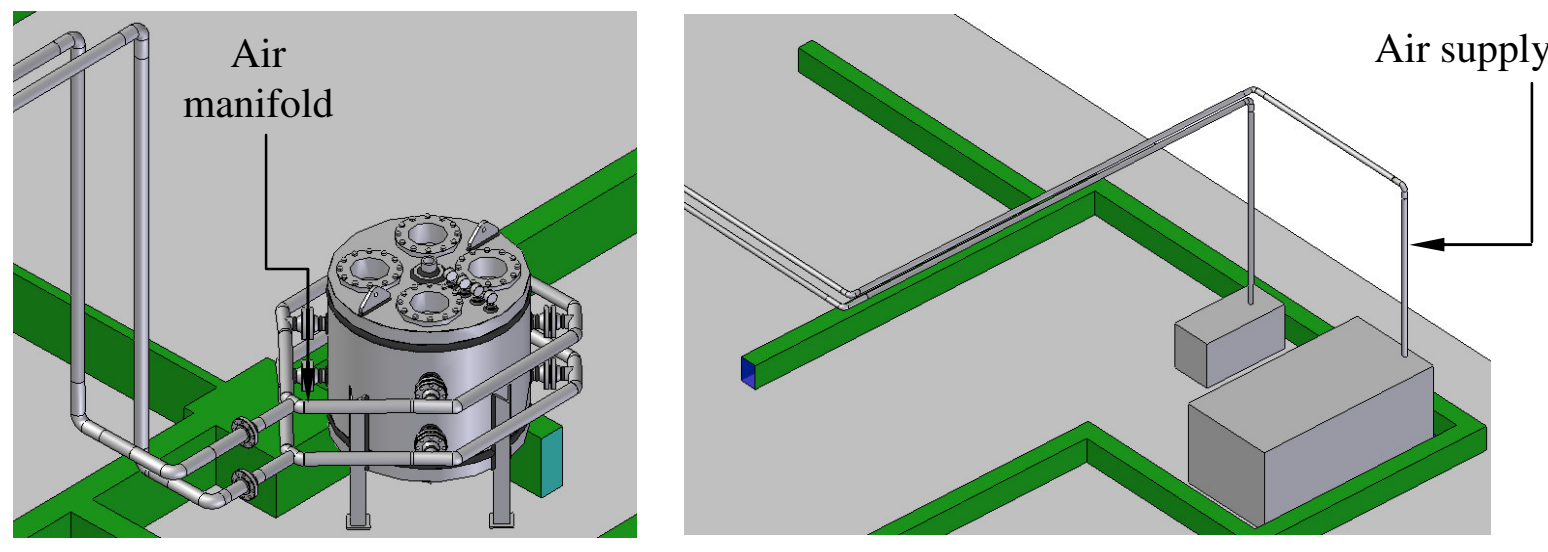

Fig. 3.79- Air supply system

The total length in straight pipes is expected to be $170 \mathrm{ft}$, increasing to $188 \mathrm{ft}$ with the installation of long elbows. Additionally, an enlargement will be located at the exit of the compressed air system which corresponds to 1 in. in size; however, the pressure drop at this point can be considered as negligible. The final estimated pressure drop is 1.685 psia.

\subsection{Pipe joints}

A joint is required each time it is necessary for piping to be connected either to itself, a fitting, or a piece of equipment. It must be able to withstand the greatest possible pressure exerted upon it by either the design or test pressure of the individual system. The selection of the jointing methods will be determined by the type of pipe used, the type of fittings available for the pipe, the highest pressure expected in the system, and the possible need for disassembly.

Steel pipe is joined by threading, welding, or grooving. Threaded fittings are made of cast iron, malleable iron, ductile iron, and forged steel for joining pipe up to NPS 8 (DN 200). It is an industry standard; however, to change to welding or grooving at sizes above NPS 2 (DN 50). Threaded joints are made using ASME B1.20.1 standard taper pipe threads. Pipe lighter than standard weight must not be threaded.

Welding is normally used to join steel pipe from NPS 2-1/2 (DN 65) and up. Welding fittings (ASME B16.9) are made to match steel-pipe diameters and wall 
thicknesses. They are furnished with a standard bevel end for butt welding to the pipe. Weld fittings have the same pressure-temperature ratings as the equivalent thickness seamless pipe.

For smaller sizes, forged steel socket-weld fittings (ASME B16.11) can be used for high-pressure service. Properly made welded joints are as strong as the pipe, do not deteriorate, and have a smooth inside contour to minimize friction losses.

The two lines at TAMU TowerLab are 1.5 and 3 in. nominal pipe schedule 80. The recommended type of joints for both cases will be socket welding and welding respectively. Fig. 3.80 (a) and (b) show the two configurations.

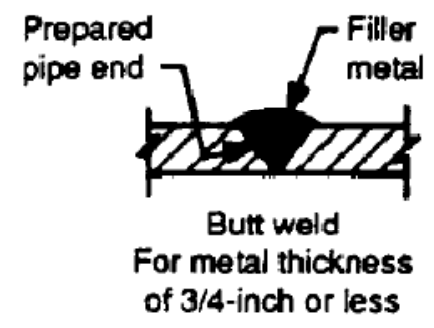

(a)

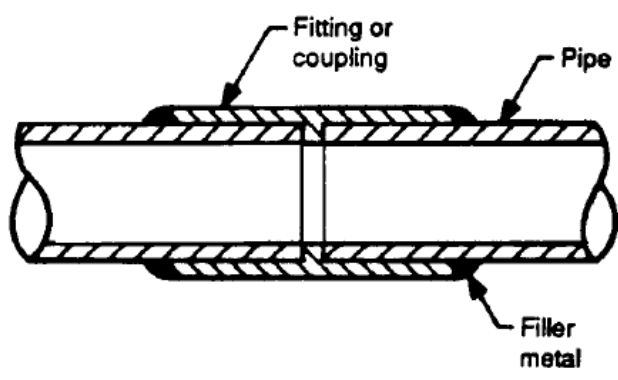

(b)

Fig. 3.80- Typical welded joints. (a) Butt weld; (b) socket weld ${ }^{59}$

Additionally, bolted or flanged joints will be recommended to install valves and flow metering devices, as well as, to facilitate the disassembly of the pipe network. A typical flanged joint is illustrated in Fig. 3.81.
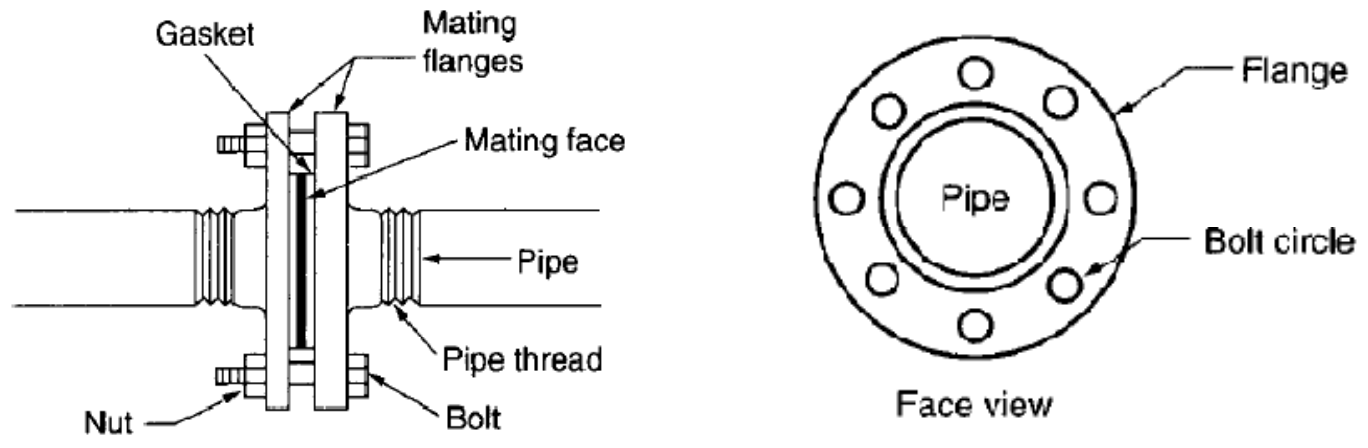

Fig. 3.81- Flanged joint ${ }^{59}$ 


\subsection{Pipe support and hangers}

The pipe support is an assembly of components including a device or method used as a direct attachment to the structure. Typical attachment include: an insert, a beam clamp or brackets. Fig. 3.82 illustrates the three methods.

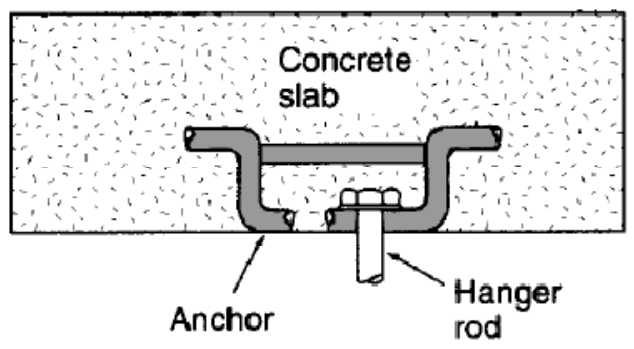

(a)

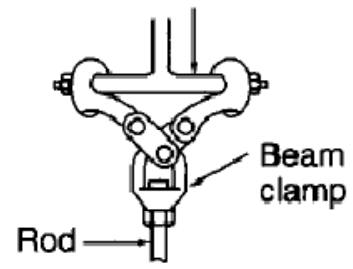

(b)

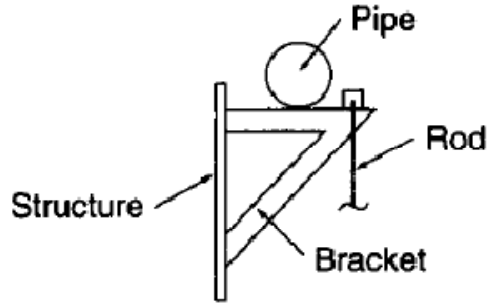

(c)

\section{Fig. 3.82- Typical structure attachments. (a) Insert; (b) beam clamp; (c) bracket b9 $^{59}$}

The hanger rod is usually threaded and connects the attachment to the hanger that is threaded to receive the rod. The diameter of the rod is selected by the amount of weight it will support. Table 3.50 presents the requirements for minimum acceptable rod diameters for various sizes supporting individual pipes.

Table 3.50- Recommended rod size for individual pipes

\begin{tabular}{|c|c|}
\hline Pipe size, in. & Rod size, in. \\
\hline 2 and smaller & $3 / 8$ \\
\hline $2-1 / 2$ to $3-1 / 2$ & $1 / 2$ \\
\hline 4 and 5 & $5 / 8$ \\
\hline
\end{tabular}


A hanger is the device used to secure the pipe to the hanger rod. It must not distort, cut, or abrade any pipe while allowing free movement. There is a wide variety to choose as depicted by Fig. 3.83.

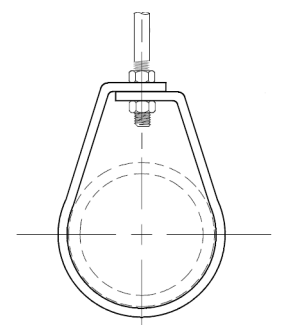

(a)

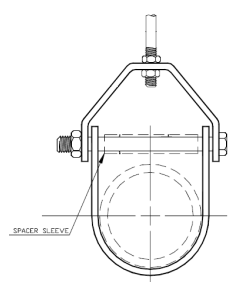

(b)

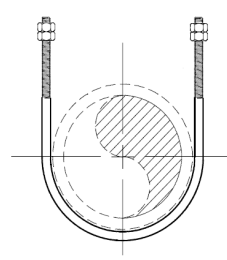

(c)

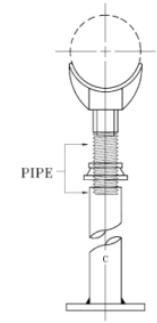

(d)

Fig. 3.83- Typical pipe hanger types. (a) Adjustable band hanger; (b) adjustable clevis hanger; (c) long tangent $U$-bolt and (d) saddles

The horizontal spacing of hangers depend on the size of the pipe and the requirement to prevent sagging based on the weight of the pipe filled with water, and other heavy valves and fittings on the pipe run. For pipe where no other requirement exist, the recommended maximum spacing is $12 \mathrm{ft}$ for the case of steel pipe of size 1/4 and larger.

Any of the models in Fig. 3.80 is suitable for the pipe lines at the TowerLab. The price per unit and the quantity required is shown in Table 3.51.

Table 3.51- Pipe hanger prices

\begin{tabular}{|l|c|c|c|c|}
\hline Item & Size, in. & Qty & Unit price, $\$$ & Total price, $\$$ \\
\hline \multirow{2}{*}{ (a) } & $1-1 / 2$ & 18 & 2.07 & 37.26 \\
\cline { 2 - 5 } & 3 & 16 & 3.70 & 59.20 \\
\hline \multirow{2}{*}{ (b) } & $1-1 / 2$ & 18 & 3.43 & 61.74 \\
\cline { 2 - 5 } & 3 & 16 & 3.70 & 59.20 \\
\hline \multirow{2}{*}{ (c) } & $1-1 / 2$ & 18 & 3.73 & 67.14 \\
\cline { 2 - 5 } & 3 & 16 & 7.13 & 114.08 \\
\hline (d) & 3 & 6 & 316.65 & $1,899.9$ \\
\hline
\end{tabular}

The injection manifold might also need a support device for which the adjustable pipe saddle design could be selected. Fig. 3.83 (d) shows the component. 


\section{CHAPTER IV}

\section{MONITORING AND INSTRUMENTATION}

\subsection{Pressure gauges}

The installation of pressure gauges in a research flow loop is of vital importance, not only to monitor the pressure at which experiments are conducted but also to evaluate and assess pressure drops in the system and equipment performance. Table 4.1 presents the location and number of gauges planned to be installed at the TowerLab:

Table 4.1- Location and quantity of pressure gauges for TAMU TowerLab

\begin{tabular}{|l|c|}
\hline Location & Quantity \\
\hline Air line prior arrival at injection manifold & 1 \\
\hline Entry points of injection manifold & 4 \\
\hline Water line prior arrival at injection manifold & 1 \\
\hline Top surface of pressure vessel & 4 \\
\hline
\end{tabular}

Since the accuracy of most pressure gauges is better in the middle portion of a gauge, the range should be about two times the maximum anticipated pressure. Furthermore, the maximum operating pressure should not exceed $80 \%$ of the full pressure range of the gauge. Based on the maximum operating pressure of 580 psi given by the CompAir system, the range for the pressure gauge should be $0-1000$ psi.

Standard gauge connections are male NPT and they are located at the bottom or back of the gauge case. The ports designed at the top of the pressure vessel, as well as, the glands that will accommodate the gauges for both the manifold, and water and air line correspond to $1 / 4$ NPT.

Pressure gauges are manufactured to different accuracies. The higher the quality of the gauge, the more accurate it is. Gauges that are more accurate tend to be more complex and cost more to manufacture. Higher quality gauges last longer and some can even be repaired or recalibrated. The pressure gauges to be selected for the TowerLab will be the digital type, and the accuracy will have to at least be $0.25 \%$; however, if it is economically feasible to install 0.1 or $0.05 \%$, then the more accurate the measurements 
will be. Table 4.2 presents information from several manufacturers of digital pressure gauges and Fig. 4.1 illustrates the range in costs as a function of accuracy for 1/4 socket size, battery charged and 0-1000 psi pressure gauges.

Table 4.2- Comparison of different pressure gauges

\begin{tabular}{|c|c|c|c|c|}
\hline Manufacturer & Model & Accuracy (+/-) & Operating temperature, ${ }^{\circ} \mathrm{F}$ & Price, $\$$ \\
\hline \multirow{10}{*}{ Omega } & DPG9100/9100/9200 & 0.25 & $14-140$ & 415 \\
\hline & DPG1100 & 0.1 & $(-) 67-203$ & 499 \\
\hline & DPG4000 & 0.05 & $14-131$ & 759 \\
\hline & DPG5500 & 0.25 & $(-) 4-185$ & 289 \\
\hline & DPG5600 & 0.25 & $(-) 4-185$ & 319 \\
\hline & DPG7000 & 0.05 & $0-150$ & 960 \\
\hline & DPG7010/7025 & 0.1 & $0-150$ & 840 \\
\hline & DPG8000 & 0.25 & $14-140$ & 329 \\
\hline & DPG6000L & 0.3 & $0-180$ & 500 \\
\hline & DPG6000L & 0.6 & $0-180$ & 500 \\
\hline \multirow{4}{*}{ Ashcroft } & EW-68338-72 & 0.25 & $14-140$ & 604 \\
\hline & EW-68338-12 & 0.05 & $0-150$ & 840 \\
\hline & EW-68339-85 & 0.25 & $14-140$ & 488 \\
\hline & EW-68332-14 & 0.5 & $14-140$ & 210 \\
\hline \multirow{2}{*}{ Honeywell } & $\mathrm{CK}$ & 0.25 & $(4)-185$ & 386 \\
\hline & $\mathrm{JK}$ & 0.2 & $30-160$ & 860 \\
\hline \multirow{3}{*}{$\begin{array}{l}\text { Aschcroft } \\
\text { (BriceBarclay) }\end{array}$} & 2089 & 0.05 & $0-150$ & 929.07 \\
\hline & 2086 & 0.1 & $0-150$ & 812.82 \\
\hline & 2084 & 0.25 & $0-150$ & 696.57 \\
\hline
\end{tabular}

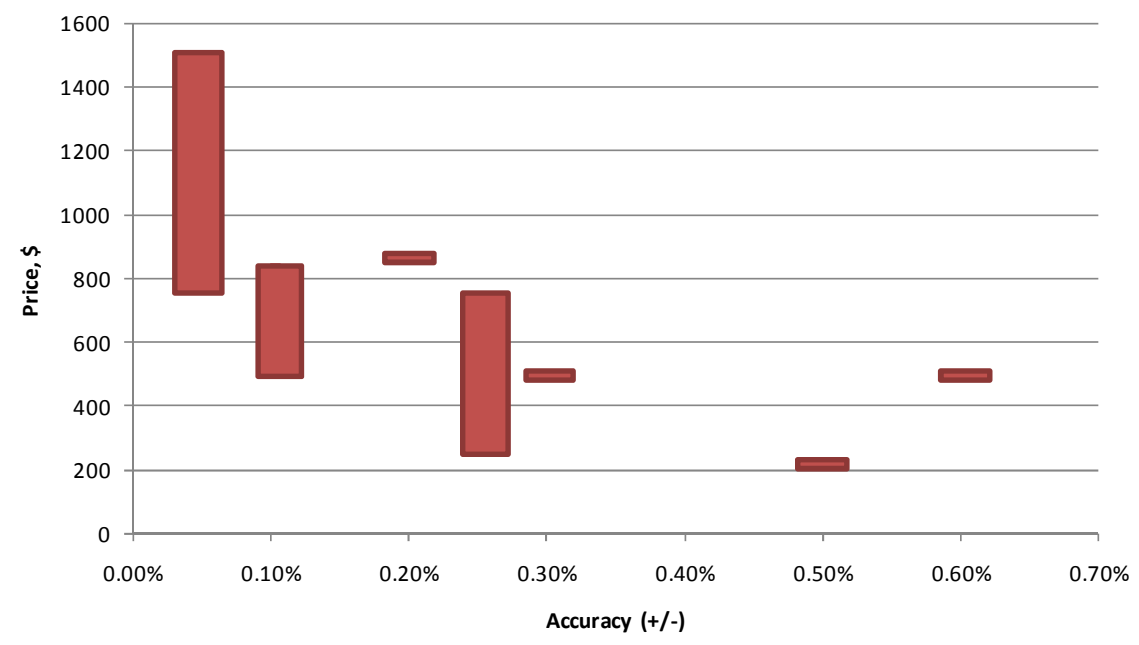

Fig. 4.1- Range in price as a function of accuracy 
The final decision on the model to purchase will have to agree the technical aspects considered in the facility design. Fig. 4.2 illustrates the installation of model DPG7000 (accuracy of $0.05 \%$ ) on the vessel. It can be seen that no major issues with space availability are anticipated.

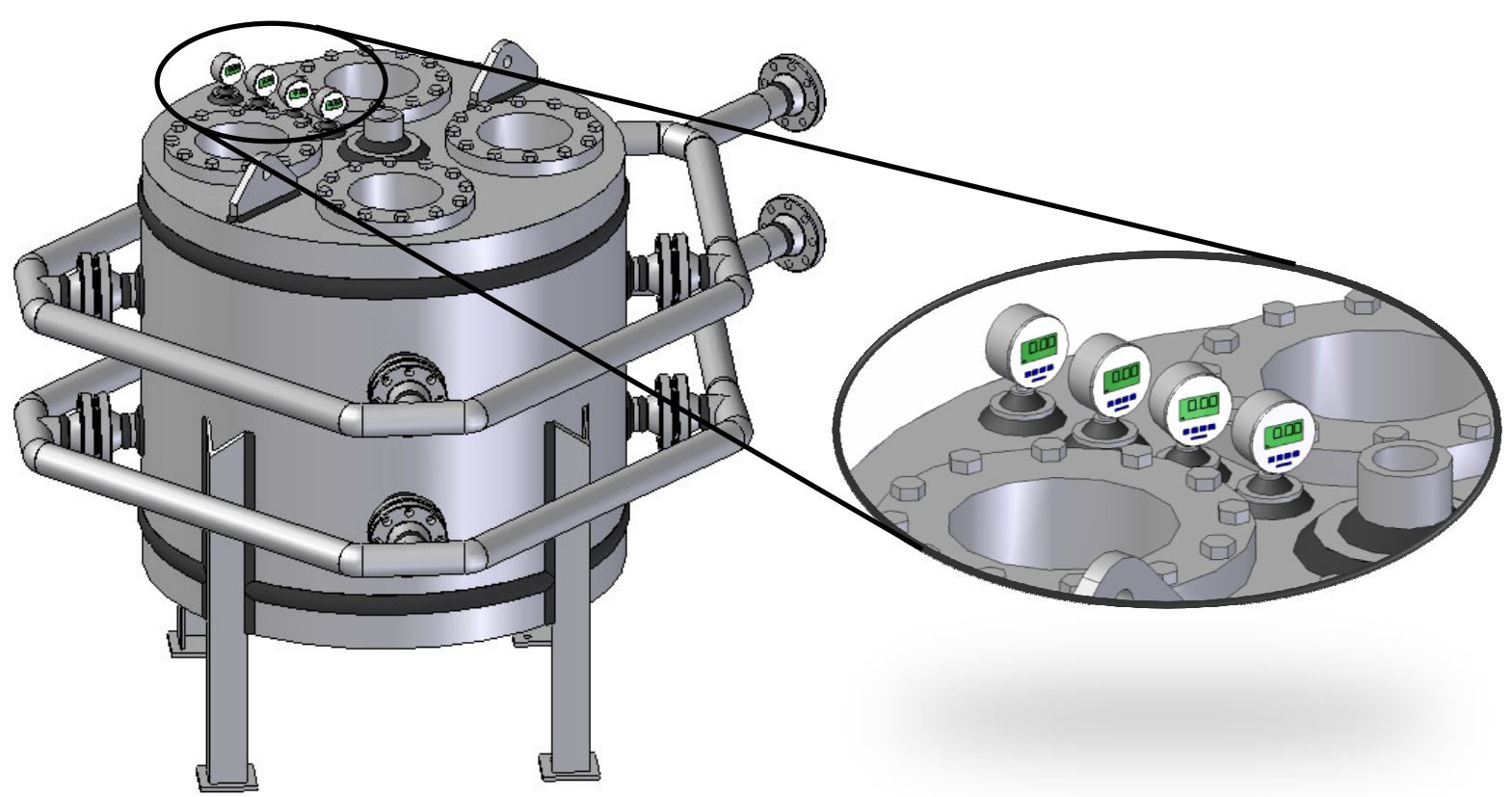

Fig. 4.2- Installation of model DPG7000 on pressure vessel

\subsection{Flowmeters}

Flow measurement is recognized as one of the "need to know" process parameters alongside temperature and pressure. Accurate measurement of both air and water is critical in the operation of a research flow loop.

Numerous types of flowmeters are available for closed-piping systems. In general, the equipment can be classified as differential pressure, positive displacement, velocity, and mass meters. Furthermore, they can be grouped into general categories, some of which may overlap one another but nonetheless are useful in describing some of the factors involved in flowmeter selection. These categories are: 
I. Flowmeters with wetted moving parts:

- Positive displacement

- Hydraulic wheatstone bridge

- Turbine

- Variable area

II. Flowmeters with no wetted moving parts

- Differential pressure

- Oscillatory

- Target

- Thermal

III. Obstructionless flowmeters

- Coriolis mass

- Magnetic

- Ultrasonic

IV. Flowmeters with sensors mounted external to the pipe.

- Clamp-on ultrasonic

Today there is a tendency to distinguish between "new technologies" and "traditional technologies", grouping coriolis, magnetic, ultrasonic and vortex flowmeters under the new category, with methods such as DP, turbine, positive displacement and variable area (VA) under the old one.

Flowmeters selection is generally a process of elimination based on technical criteria such as pressure, temperature, specific gravity or density, viscosity and flow range; however specific technologies have been recognized to be more adequate for research facilities. Based on the literature review in CHAPTER II, a tendency for using coriolis, vortex and turbine meters was evidenced. Moreover, the coriolis technique in particular, has been often described as the near-perfect measuring principle for gas measurement. However, coriolis flowmeters are the most expensive meters made in terms of average selling price. The average selling price of coriolis flowmeters is between $\$ 5,000$ and $\$ 6,000$. Some suppliers have introduced low-cost coriolis 
flowmeters in the $\$ 3,000$ range. Table 4.3 presents a general comparison between the different technologies used in metering.

Table 4.3- Comparison between different flowmeter elements

\begin{tabular}{|c|c|c|c|c|c|}
\hline Flowmeter Element & $\begin{array}{l}\text { Recommended } \\
\text { Service }\end{array}$ & Rangeability & $\begin{array}{l}\text { Pressure } \\
\text { Loss }\end{array}$ & Typical Accuracy(\%) & Relative Cost \\
\hline Venturi tube & $\begin{array}{l}\text { Clean, dirty and } \\
\text { viscous liquids; } \\
\text { some slurries }\end{array}$ & 4 to 1 & Low & \pm 1 of full scale & Medium \\
\hline Flow nozzle & $\begin{array}{l}\text { Clean and dirty } \\
\text { liquids }\end{array}$ & 4 to 1 & Medium & \pm 1 to \pm 2 of full scale & Medium \\
\hline Pitot tube & Clean liquids & 3 to 1 & Very low & \pm 3 to \pm 5 of full scale & Low \\
\hline Target meter & $\begin{array}{l}\text { Clean, dirty viscous } \\
\text { liquids; some } \\
\text { slurries }\end{array}$ & 10 to 1 & Medium & \pm 1 to \pm 5 of full scale & Medium \\
\hline Positive Displacement & $\begin{array}{l}\text { Clean, viscous } \\
\text { liquids }\end{array}$ & 10 to 1 & High & \pm 0.5 of rate & Medium \\
\hline Turbine & $\begin{array}{l}\text { Clean, viscous } \\
\text { liquids }\end{array}$ & 20 to 1 & High & \pm 0.25 of rate & High \\
\hline Vortex & Clean, dirty liquids & 10 to 1 & Medium & \pm 1 of rate & High \\
\hline Electromagnetic & $\begin{array}{l}\text { Clean, dirty, } \\
\text { viscous conductive } \\
\text { liquids and slurries }\end{array}$ & 40 to 1 & None & \pm 0.5 of rate & High \\
\hline Ultrasonic(Doppler) & $\begin{array}{l}\text { Dirty, viscous } \\
\text { liquids and slurries }\end{array}$ & 10 to 1 & None & \pm 5 of full scale & High \\
\hline Mass (Coriolis) & $\begin{array}{l}\text { Clean, dirty viscous } \\
\text { liquids; some } \\
\text { slurries }\end{array}$ & 10 to 1 & Low & \pm 0.4 of rate & High \\
\hline Mass (Thermal) & & 10 to 1 & Low & \pm 1 of full scale & High \\
\hline
\end{tabular}

Initially, two flowmeters will be required for the air and water lines prior the arrival of fluids at the independent manifolds. Regardless of economic issues, it is recommended to install a coriolis or vortex meter for the air line, and for the water, a turbine or vortex could be accommodated. Based on the installation of a coriolis meter for the air line, the estimated cost will be $\$ 6,768$ with pressure drops varying from 0.043 to $13.2 \mathrm{psi}$; however, if turbine meters are considered for the two lines, the cost is approximately $\$ 2,569$ for air, and $\$ 1,808$ for water with anticipated pressure drops of 0.108 and 2 psi respectively. Finally for the case of vortex meters, the cost will be $\$ 4,790$ and $\$ 2,490$ for air and water with less than 3.25 psi pressure drop for both cases. 


\section{CHAPTER V}

\section{CONCLUSIONS AND RECOMMENDATIONS}

\subsection{Conclusions}

A new dedicated facility to be located at the Richardson building has being designed for the experimental investigation of liquid loading in gas wells. From this investigation we conclude the following:

1. A technical and economic analysis of the design options initially considered for the compression and pumping system showed that using a compressor and a water pump results in the most feasible option for the TowerLab as compared to the multiphase pump or pressurized tank alternatives.

2. For the compressed air system, several arrangements were analyzed including the installation of single or multiple units; however, after conducting a weighted score selection process based on the proposals gathered from different manufacturers, CompAir resulted in the most attractive candidate with threereciprocating units working in parallel. Each machine provides 580 psig and 168 scfm and they are conveniently balanced with minimal vibration and low structure and air borne noise.

3. The pressure vessel located at the bottom of the vertical section of the tubing was designed not only to minimize costs and weight but also to facilitate its installation in the basement. This resulted in a top and bottom flat head cover with multiple openings welded to the cylindrical shell body. These openings will allow access to the internal space in the vessel to accommodate the glass beads. The vessel also incorporates eight openings to attach the injection manifolds, fittings to install pressure gauges and lugs for lifting and installation purposes.

4. The required superficial velocities for air and water showed the need of independent manifolds to be connected to the pressure vessel. Two options are recommended for the geometry of the device; however, costs and ease for 
fabrication should be addressed. The pipes chosen correspond to 3 in and 1.5 in schedule 80 for water and air respectively.

5. The air and water pipelines were selected based on the superficial velocities required at the entry points at the pressure vessel. The lines, as for the manifolds, correspond to 3 in and 1.5 in for water and air. They will accommodate different types of fittings, such as: long radius elbows and reduction/expansion joints, as well as, pressure gauges and flow metering devices. Welded joints are recommended; however, flanged joints will be required to install valves, flowmeters and to allow disassembly of the lines. The total pressure drop in the system was estimated to be below 3 psi for both lines including their respective manifolds. In addition, different pipe hangers and pipe supports are recommended for which prices were obtained from a manufacturer.

6. Digital pressure gauges with a minimum range in accuracy from 0.05 to $0.25 \%$ are to be installed on the fittings located at the top of the pressure vessel, as well as, the eight entry points in the injection manifold. In addition, two flowmeters will measure the flowrate of air and water. The selected technologies are coriolis or vortex for the air line and turbine meters for the water line.

\subsection{Recommendations}

1. Get quotations to start the fabrication of the pressure vessel and make sure the company follows ASME Section VIII, Division 1 standards.

2. Proceed with the purchase of the compressed air package supplied by CompAir including the control system to suit our application. This suggests the addition of an air receiver (accumulation/storage tank) for blow off control.

3. CompAir's proposal indicates that approximately $90 \%$ of the heat generated by compression will be dissipated into the cooling water, and approximately $10 \%$ will be radiated heat; however, proper ventilation of the equipment should guarantee minimum increase in room temperature. 
4. Select a water pump capable of delivering the same pressure as given by the compressed air system covering the flowrate range obtained in the sensitivity analysis located in Appendix C.

5. Select the best design for the injection manifolds depending on the costs and ease of fabrication.

6. If single injection is desired, more studies should be conducted to analyze the phase distribution through a single line coming from a commingling point through a tee joint prior the arrival of fluids at the entry points located in the vessel.

7. The flow loop requires the installation of different valves to protect mechanical equipment in the piping system from reversal fluid flow. This is particularly important in the case of pumps and compressors, where back flow could damage the internals of the equipment and cause unnecessary shutdown of the system. For the air pipeline a compressor-type check valve could be installed and for the water pipeline, the required closing speed should be calculated before selecting the type of valve.

8. In addition to check valves, the flowrates need to be controlled through valves. For the air and water pipelines, glove balls could be a good choice since they are not only able to control flow, but also can be used to stop and start flow and they are recommended for frequent valve operation. They work well with gases and liquids free of solids as is the case for the testing fluids to be used at the TowerLab.

9. Additional valves are also needed to facilitate intervention and maintenance operations of the piping system and the devices to be installed in the lines.

10. This investigation did not include the recirculation system at the top of the vertical tubing of the flow loop; however, the installation of a separator for air release and water recirculation is an attractive choice. 


\section{REFERENCES}

1. Yamamoto, H. and Christiansen R.: "Enhancing Liquid Lift from Low Pressure Gas Reservoirs," paper SPE 55625 presented at the Rocky Mountain Regional Meeting held in Gillette, Wyoming 15-18 May 1999.

2. P. Toma., E.Vargas., E. Kuru.: "Predicting Slug to Annular Flow Pattern Transitions for Reducing Risks of Gas Lift Instabilities and Effective Gas/Liquid Transport from Low Pressure Reservoirs," paper SPE 100615 presented at the Gas Technology Symposium held in Calgary, Alberta, Canada 15-17 May 2006.

3. Girija, E., Christiansen, R. and Miller, M.: "Experimental Study of Critical Flow Rates in the Tubing-Casing Annulus of Natural Gas Wells," paper SPE 109609 presented at the 2007 Annual Technical Conference and Exhibition held in Anaheim, California 11-14 November 2007.

4. Lea, J.F., Nickens, H.V., and Wells, M.: Gas Well De-Liquefaction, first edition, Elsevier Press, Cambridge, MA (2003).

5. Poe, B.: "Optimized Tubing String Design Modeling for Improved Recovery," paper SPE 102583 presented at the 2006 Annual Technical Conference and Exhibition held in San Antonio, Texas 24-27 September 2006.

6. Coleman, S. and McCurdy D.: "A New Look at Predicting Gas-Well Load-Up," paper SPE 20280, JPT March 1991.

7. Neves, T. and Brimhall R.: "Elimination of Liquid Loading in Low-Productivity Gas Wells," paper SPE 18833 presented at the SPE Production Operations Symposium held in Oklahoma City, Oklahoma, 13-14 March 1989.

8. Sutton, R., Cox, S., Williams, E., Stoltz, R. and Gilbert, J.: "Gas Well Performance at Subcritical Rates," paper SPE 80887 SPE Production Operations Symposium held in Oklahoma City, Oklahoma, 22-25 March 2003. 
9. Dousi, N., Veeken, C.A.M., Currie, and P.K.: "Modeling the Gas Well Liquid Loading Process," paper SPE 95282 presented at the Offshore Europe Conference 2005 held in Aberdeen, Scotland U.K, 6-9 September 2005.

10. Bondurant, A., Cox, S., Williams, E., Dotson, B. and Oyewole, P.: "Getting the Last Gap: Deliquification of Challenging Gas Wells," paper IPTC 11651 presented at the International Petroleum Technology Conference held in Dubai, UAE, 4-6 December 2007.

11. Stephenson, G., Rouen, R., and Rosenzweig, M.: “Gas-Well Dewatering: A Coordinated Approach," paper SPE 58984 presented at the International Petroleum Conference and Exhibition held in Villahermosa, Mexico, 1-3 February 2000.

12. Guo, B., Ghalambor, A. and Xu, C.: "A Systematic Approach to Predicting Liquid Loading in Gas Wells," paper SPE 94081 presented at the Production Operations Symposium. Oklahoma City, Oklahoma, 17-19, April 2005.

13. Turner, R., Hubbard, M. and Dukler A.: "Analysis and Prediction of Minimum Flow Rate for the Continuous Removal of Liquids from Gas Wells,” paper 2198, JPT, November 1969.

14. Coleman, S., Clay, H., McCurdy, D. and Norris III, L.: "Understanding Gas-Well Load-Up Behavior,” paper SPE 20281, JPT, March 1991.

15. Nosseir, M.A., Darwich, T.A., Sayyouh, M.H., and El Sallaly, M.: “A New Approach for Accurate Prediction of Loading in Gas Wells Under Different Flowing Conditions," paper SPE 37408 presented at the 1997 SPE Production Operations Symposium, Oklahoma City, Oklahoma, 9-11, March 1997.

16. Gool, F. And Currie, P.: "An Improved Model for the Liquid Loading Process in Gas Wells," paper SPE 106699 presented at the Production Operations Symposium. Oklahoma City, Oklahoma, 31-3, March 2007.

17. Solomon, F. and Fernandez, J.: "Design and Construction of a Dedicated Facility for the Experimental Study of Liquid Loading in Gas Wells," Interim report for the Crisman Institute, College Station, Texas, 5, November 2007. 
18. Falcone, G., Teodoriu, C., Reinicke, K. and Bello, O.: "Multiphase Flow Modeling Based on Experimental Testing: A Comprehensive Overview of Research Facilities Worldwide and the Need for Future Developments," paper SPE 110116 presented at the 2007 Annual Technical Conference and Exhibition held in Anaheim, California, 11-14, November 2007.

19. Norris, H., Fuchs, P., Malnes, D. and Klemp, S.: "Developments in the Simulation and Design of Multiphase Pipeline Systems," paper SPE 14283 presented at the Annual Technical Conference and Exhibition held in Las Vegas, Nevada, 22-25 September 1985.

20. http://www.sintef.no/Home/Petroleum-and-Energy/SINTEF-PetroleumResearch/Wellstream-Technology/Laboratories/. Sintef Multiphase Flow Laboratories, Tor Erling Unander, October 12 $2^{\text {th }}, 2007$.

21. http://www.ife.no/laboratories/well_flow_loop/index_html-en/view, IFE's Well Flow Loop, Morten Langsholt, October $19^{\text {th }}, 2007$.

22. Robole, B., Kvandal, H. And Chuller, R.: “The Norsk Hydro Multiphase Flow Loop. A high pressure flow loop for real three-phase hydrocarbon systems," Flow Measurement and Instrumentation 17 (2006) 163-170.

23. http://www.swri.org/4org/d18/mechflu/fluiddyn/pdfs/flowloop.pdf, $\quad$ SwRI Multiphase Flow Loop, Robert Hart, October 20 2007.

24. Steven, R.: "Wet gas metering with a horizontally mounted Venturi meter," Flow Measurement and Instrumentation 12 (2002) 361-372.

25. http://www.flowprogramme.co.uk/facilities/wetgas.asp, Wet Gas Test Facility, January $21^{\text {st }}, 2008$.

26. http://www.tuvnel.com/content/pdfs/wet_gas_facility.pdf, Wet Gas Test Facility, January $21^{\text {st }}, 2008$.

27. Kegel, T., and Kinney, J.: "Wet Gas Metering Facility at CEESI," Nunn, Colorado, Internal Report.

28. http://www.ceesi.com/research_mp.aspx, Multiphase Research Loop, January $21^{\text {st }}, 2008$. 
29. Bosio, J., Wilcox, P. And Sembsmoen, O.: "Gas-metering test and research facility to meet North Sea needs," OGJ Report, Measurement and Control Technology 86, December 1988, 33-39.

30. Corteville, J., Grouvel, M. and Lagiere, M.: "Experimentation des Ecoulements Diphasiques en Conduuites Petrolieres: Boucles d'essais de Boussens," Revue de l'Institute Francaise du Petrole, (March-April 1983) 38, No.2, 143-151.

31. Vilagines, R. and Hall, A. "A comparative Behavior of Multiphase Flowmeter Test Facilities," Oil and Gas Science and Technology - Rev. IFP, (2003) 58, No.6, 647-657.

32. Falcone, G., Hewitt, G. and Richardson, L.: “ANUMET: A Novel Wet Gas Flowmeter," paper SPE 84504 presented at the Annual Technical Conference and Exhibition held in Denver, Colorado, 5-8 October 2003.

33. Constantini, A., Falcone, G., Hewitt, G. and Alimonti, C.: "Using Transient Inflow Performance Relationships to Model the Dynamic Interaction Between Reservoir and Wellbore During Pressure Testing," paper OMAE 2007-29524 presented at the 26th International Conference on Offshore Mechanics and Arctic Engineering held in San Diego, California 10-15 June 2007.

34. Jonge, R. and Tousis U.: "Liquid Unloading of Depleted Gas Wells in North Sea and Continental Europe, Using Coil Tubing, Jointed Pipe Velocity/Insert String and Microstrings," paper SPE 107048 presented at the Coiled Tubing and Well Intervention Conference and Exhibition held in The Woodlands, Texas, 20-21, March 2007.

35. Green, R. and Korzekwa, C.: "Dewatering a Deep gas Well With a Gas Lift System - A Case History," paper SPE 12590 presented at the Permian Basin Oil \& Gas Recovery Conference held in Midland, Texas, 8-9 March 1984.

36. G.H. Schwall, G.: "Case Histories: Plunger Lift Boosts Production in Deep Appalachian Gas Wells, paper SPE 18870 presented at the Production Operations Symposium. Oklahoma City, Oklahoma, 13-14, March 1989. 
37. Foo, D.: "Production Optimization of Gas Wells by Automated Unloading: Case Histories," paper SPE 59748 presented at the SPE/CERI Symposium held in Calgary, Alberta, 3-5 April 2000.

38. Lisigurski, O., and Rowe, G.: "Practical Steps to Increase Production and Reserves in Mature Fields: Hugoton and Panoma, Texas County, Oklahoma, U.S.A.," paper SPE 102259 presented at the Annual Technical Conference and Exhibition held in San Antonio, Texas, 24-27 September 2006.

39. Moe, G., Johnson, C., Ingle, R., and Smith, R.: "Innovative Well Completion Harnesses Deep, Hot South Texas Gas," paper SPE 84514 presented at the Annual Technical Conference and Exhibition held in Denver, Colorado, 5-8 October 2003.

40. Flowers, J., Hupp, M., and Ryan, J.: "The Results of Increase Fracture Conductivity on Well Performance in a Mature East Texas Gas Field," paper SPE 84307 presented at the Annual Technical Conference and Exhibition held in Denver, Colorado, 5-8 October 2003.

41. Costantini, A., DYNAMIC INTERACTION BETWEEN THE RESERVOIR AND THE WELL DURING WELL TESTING, Dip. Ing. thesis, University "La Sapienza" of Rome \& Imperial College, October 2005.

42. H. Alshuraiqui, C. Grattoni, A. Muggeridge.: "Laboratory and Numerical Studies of First Contact Miscible WAG Displacement: The Effects of WAG Ratio and Flowrate," paper presented at the 12th European Symposium on Improved Oil Recovery, Kazan, Russia, 8-10 September 2003.

43. Bornemann Multiphase pumping systems manual for pump model MW 6.5zk-37, Obernkirchen, Germany.

44. Megyesy, E.: Pressure Vessel Handbook, Eighth Edition, Tulsa Oklahoma, (1989), 213.

45. American Society of Mechanical Engineers, Boiler and Pressure Vessel Code, ASME, Section VIII: Rules for Construction of Pressure Vessels, Division 1, New York (2004) 15-364. 
46. Bednar, H.: Pressure Vessel Design Handbook, Van Norstrand Reinhold Company, New York (1986), 290.

47. Moss, D.: Pressure Vessel Design Manual, Elsevier, Third Edition, Burlington, Massachusetts (2004) 7, 446.

48. ASME B-16.5: Pipe Flanges and Flanged Fittings, New York (2004)

49. Thompson, G.: An Engineer's Guide to Pipe Joints, Professional Engineering Publishing, London (1998), 47-56.

50. Flitney, R. Seals and Sealing Handbook, Elsevier, Fifth Edition, Burlington, Massachusetts (2007), 17.

51. Improving Compressed Air System Performance: A sourcebook for Industry, U.S. Department of Energy's Office of Energy Efficiency and Renewable Energy (EERE), Washington D.C. (2003), 4.

52. Ingersoll Rand Industrial Technologies: Air Quality Guide, UK (2007), 4-10.

53. Energy Savings in the Selection, Control and Maintenance of Air Compressors, Carbon Trust, UK Department of Environment Transport and Regions, Good practice Guide 241 (1998), 5.

54. Davidson, J., Bertele, O.: Process Fan and Compressor Selection, Mechanical Engineering Publications, London (1996), 13.

55. Snow, D.: Plant Engineer's Reference Book, Elsevier, Second Edition, Burlington, Massachusetts (2003), 26.

56. Shah, H.: "Energy is crucial in compressor selection," Plant engineering (2005), 68-70.

57. Energy Efficient Compressed Air Systems, Carbon Trust, UK Department of Environment Transport and Regions, Good Practice Guide 385 (1998), 3.

58. Menon, S.:Piping Calculations Manual, McGraw-Hill, New York, New York (2005), 17.

59. Frankel, M. Facility Piping Systems Handbook, McGraw-Hill, $2^{\text {nd }}$ Edition, New York, New York (2002), 2.41, 2.82. 


\section{APPENDIX A}

PRESSURE VESSEL EQUATIONS

\section{A.1) Shell thickness}

(1) Tensile stress

(a) Circumferential stress $U G-27(c)(1)$

$\mathrm{t}_{\mathrm{c}}=\frac{\mathrm{PR}_{\mathrm{i}}}{\mathrm{SE}_{\mathrm{c}}-0.6 \mathrm{P}}+\frac{\frac{\mathrm{Hg}}{144} \mathrm{R}_{\mathrm{i}}}{\mathrm{SE}_{\mathrm{c}}-0.6\left(\frac{\mathrm{Hg}}{144}\right)}$

(b) Longitudinal stress $U G-27(c)(2)$

$\mathrm{t}_{\mathrm{l}}=\frac{\mathrm{PR}_{\mathrm{i}}}{2 \mathrm{SE}_{\mathrm{l}}+0.4 \mathrm{P}}-\frac{\mathrm{W}_{\mathrm{v}}+\mathrm{W}_{\mathrm{c}}}{\pi \mathrm{D}_{\mathrm{i}} \mathrm{SE}_{\mathrm{l}}} \pm \frac{\mathrm{M}}{\pi \mathrm{R}_{\mathrm{i}}^{2} \mathrm{SE}_{\mathrm{l}}}$

(2) Compressive stress UG-23(b)

$\mathrm{R}_{\mathrm{o}}=\mathrm{R}_{\mathrm{i}}+\mathrm{t}_{\mathrm{c}}$

$\mathrm{A}=\frac{0.125}{\mathrm{R}_{\mathrm{o}} / \mathrm{t}_{\mathrm{c}}}$

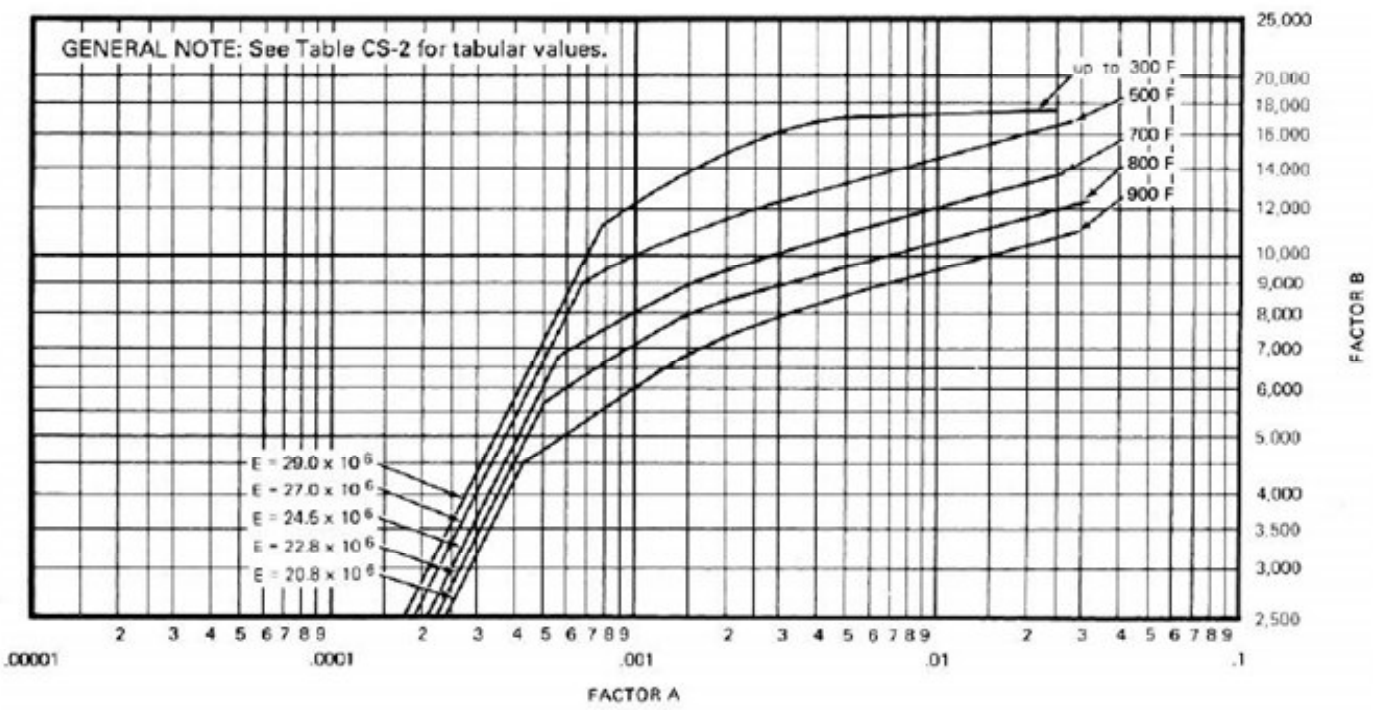

Fig. A.1- Chart for determining Shell thickness of components under external pressure when constructed of carbon or low alloy steel ${ }^{47}$ 


\section{A.2) Bottom flat head}

(1) For circular heads:

$t=d \sqrt{\frac{C P}{S E}}$

The configuration for the attachment to the shell is the following:

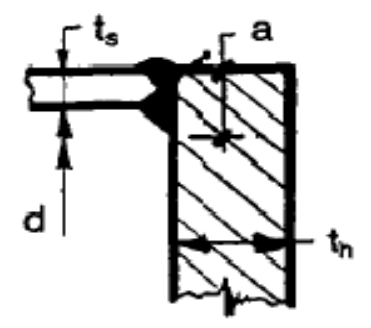

$$
\begin{gathered}
C=0.33 \mathrm{~m} \\
\mathrm{~m}=\frac{\mathrm{t}_{\mathrm{r}}}{\mathrm{t}_{\mathrm{s}}}
\end{gathered}
$$

Fig. A.2- Attachment configuration ${ }^{49}$

\section{A.3) Nozzles \\ (1) Area required \\ $A=d t_{r} F+2 t_{n} t_{r} F\left(1-f_{r 1}\right)$}

(2) Calculations without a reinforcement element

Fig. A.3 shows the configuration for a nozzle attached to the vessel wall without a pad.

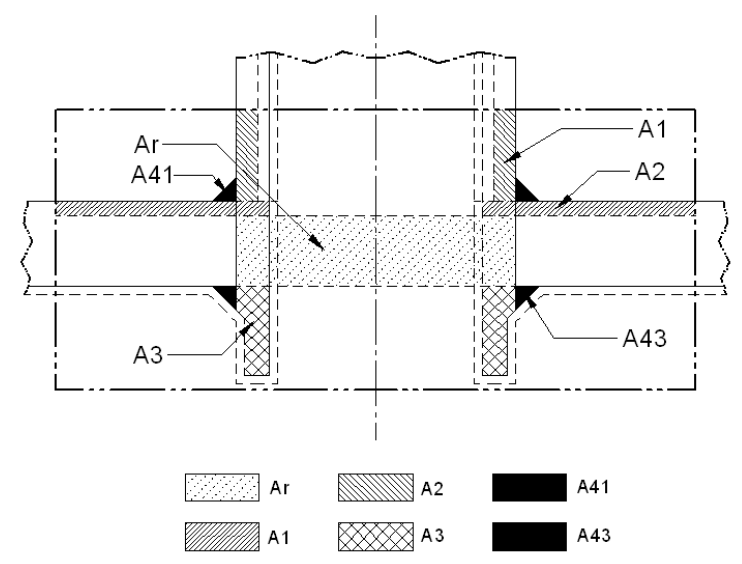

Fig. A.3- Area available for reinforcement without pad 
Area available in shell:

Larger value from the following equations:

$A_{1}=\left\{\begin{array}{c}=d\left(E_{1} t-F t_{r}\right)-2 t_{n}\left(E_{1} t-F t_{r}\right)\left(1-f_{r 1}\right) \\ =2\left(t+t_{n}\right)\left(E_{1} t-F t_{r}\right)-2 t_{n}\left(E_{1} t-F t_{r}\right)\left(1-f_{r 1}\right)\end{array}\right.$

Area available in nozzle projecting outward; use smaller value

$A_{2}=\left\{\begin{array}{l}=5\left(t_{n}-t_{r n}\right) f_{r 2} t \\ =5\left(t_{n}-t_{r n}\right) f_{r 2} t_{n}\end{array}\right.$

Area available in outward weld

$\mathrm{A}_{41}=2(0.5)(\text { weld size })^{2}$

Total area

$\mathrm{A}_{\text {available }}=\mathrm{A}_{1}+\mathrm{A}_{2}+\mathrm{A}_{41}$

(3) Calculations including a reinforcement element

Area available in shell:

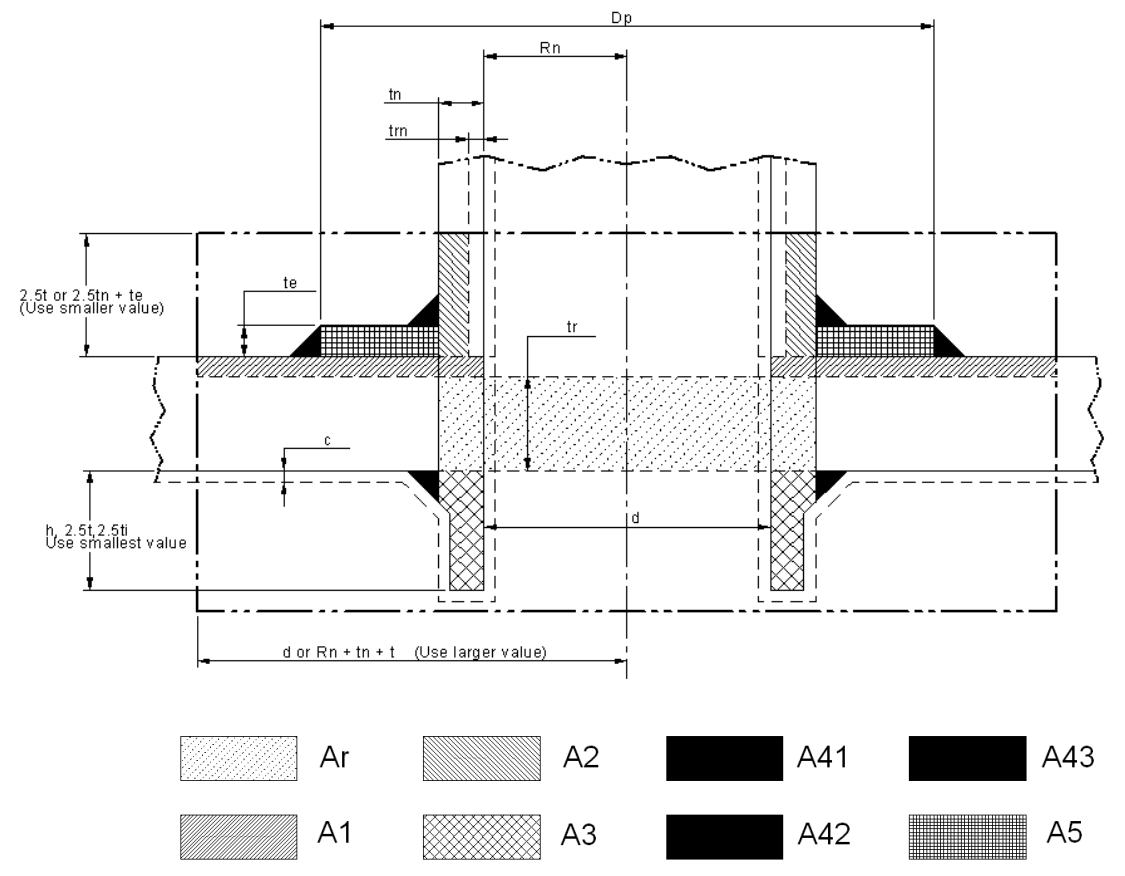

Fig. A.4- Area for available for reinforcement without pad 
Use the larger value from the following equations:

$\mathrm{A}_{1}=$ Same as above

Area available in nozzle projecting outward; use smaller value:

$A_{2}=\left\{\begin{array}{c}=5\left(t_{n}-t_{r n}\right) f_{r 2} t \\ =2\left(t_{n}-t_{r n}\right)\left(2.5 t_{n}+t_{e}\right) f_{r 2}\end{array}\right.$

Area available in outward weld:

$\mathrm{A}_{41}=2(0.5)(\text { weld size })^{2}$

Area available in outer element weld:

$\mathrm{A}_{42}=2(0.5)(\text { weld size })^{2}$

Area available in element

$A_{5}=\left(D_{p}-d-2 t_{n}\right) t_{e} f_{r 2}$

Total area:

$\mathrm{A}_{\text {available }}=\mathrm{A}_{1}+\mathrm{A}_{2}+\mathrm{A}_{41}+\mathrm{A}_{42}+\mathrm{A}_{5}$

(4) Checking minimum neck thickness required by UG-45

UG-45(a) requires the minimum nozzle wall thickness to be not less than that computed for the applicable loading plus corrosion allowance

UG-45(b) requires determining the one applicable wall thickness from (b)(1), (b)(2), or (b)(3), comparing that with the thickness from (b)(4) and then choosing the smaller of those two values

UG-45(b)(1) requires the minimum nozzle wall thickness to be not less than the required for internal pressure of the head or shell or shell where the nozzle is located but in no case less than that thickness required by UG-16(b).

UG-45(b)(2) and (b)(3) applies to vessels designed for both internal and external pressure therefore they do not apply in our case. 
UG-45(b)(4) requires minimum nozzle wall thickness of standard wall pipe accounting for undertolerance plus the thickness added for corrosion allowance. Undertolerance for pipe manufactured in accordance with ASME B36.10M is $12.5 \%$ and standard wall thickness is $0.375 \mathrm{in}$. Thus, the minimum wall thickness is

$0.375(1-0.125)+c . a \cdot(0.125)=0.453$ in

Therefore the minimum nozzle wall thickness required by UG-45(b) is the smaller of (b)(1) or (b)(4), or 0.453 in.

The minimum nozzle wall thickness required by UG-45 is the larger of UG-45(a) or UG-45(b).

(5) Size of weld required (UW-16(d); Fig. UW-16.1 Sketch $(q)^{47}$

Inner (reinforcing element) fillet weld

$\mathrm{t}_{\mathrm{c}}=$ not less than the smaller of $1 / 4$ or $0.7 \mathrm{t}_{\text {min }}$

$t_{\min }=$ the smaller of 0.75 or the thick. of the thinner of the parts joined by a fillet

Outer (reinforcing element) fillet weld

$\mathrm{t}_{\mathrm{w}}=1 / 2 \mathrm{t}_{\min }$

Upper groove weld

$\mathrm{t}_{\mathrm{w}}=0.7 \mathrm{t}_{\min }$

Lower groove weld

$\mathrm{t}_{\mathrm{w}}=0.7 \mathrm{t}_{\min }$

(6) Limits of reinforcement

Parallel to the vessel wall, the smaller of:

$d$ or $R_{n}+t_{n}+t$

Normal to the vessel wall, the smaller of:

2.5 t or $2.5 \mathrm{t}_{\mathrm{n}}+\mathrm{t}_{\mathrm{e}}$ 
(7) Loads to be carried by welds

Per UG-41 (b)(1)

$\mathrm{W}_{1-1}=\left(\mathrm{A}_{5}+\mathrm{A}_{2}+\mathrm{A}_{41}+\mathrm{A}_{42}\right) \mathrm{S}_{\mathrm{v}}$

$\mathrm{W}_{2-2}=\left(\mathrm{A}_{2}+\mathrm{A}_{41}+2 \mathrm{t}_{\mathrm{n}} \mathrm{tf}_{\mathrm{r} 1}\right) \mathrm{S}_{\mathrm{v}}$

$\mathrm{W}_{3-3}=\left(\mathrm{A}_{2}+\mathrm{A}_{5}+\mathrm{A}_{41}+\mathrm{A}_{42}+2 \mathrm{t}_{\mathrm{n}} \mathrm{tf}_{\mathrm{r} 1}\right) \mathrm{S}_{\mathrm{v}}$

Per UG-41(b)(2)

$\mathrm{W}=\left[\mathrm{A}-\mathrm{A}_{1}+2 \mathrm{t}_{\mathrm{n}} \mathrm{tf}_{\mathrm{r} 1}\left(\mathrm{E}_{1} \mathrm{t}-\mathrm{Ft}_{\mathrm{r}}\right)\right] \mathrm{S}_{\mathrm{v}}$

(8) Unit stresses (UW-15(c) and UG-45(c)

Outward nozzle weld shear

$=0.49 \mathrm{~S}_{\mathrm{v}}$

Outer element weld shear

$=0.49 \mathrm{~S}_{\mathrm{v}}$

Nozzle wall shear

$=0.70 \mathrm{~S}_{\mathrm{n}}$

Element groove weld tension

$=0.74 \mathrm{~S}_{\mathrm{v}}$

Nozzle groove weld tension

$=0.74 \mathrm{~S}_{\mathrm{v}}$

Groove weld shear

$=0.60 \mathrm{~S}_{\mathrm{v}}$

(9) Strength of connection elements

Outward nozzle weld shear

$=\pi / 2 \times$ nozzle 0. D. $\times$ weld leg $\times$ fillet weld shear

Outer element weld shear

$=\pi / 2 \times$ reinfocerment element 0. D. $\times$ weld leg $\times$ fillet weld shear 
Nozzle wall shear

$=\pi / 2 \times$ mean nozzle diam. $\times t_{n} \times$ nozzle wall shear

Element groove weld tension

$=\pi / 2 \times$ nozzle 0. D. $\times t_{e} \times$ element groove weld tension

Nozzle groove weld tension

$=\pi / 2 \times$ nozzle $0 . \mathrm{D} . \times \mathrm{t} \times$ nozzle groove weld tension

(10) Strength path verifications

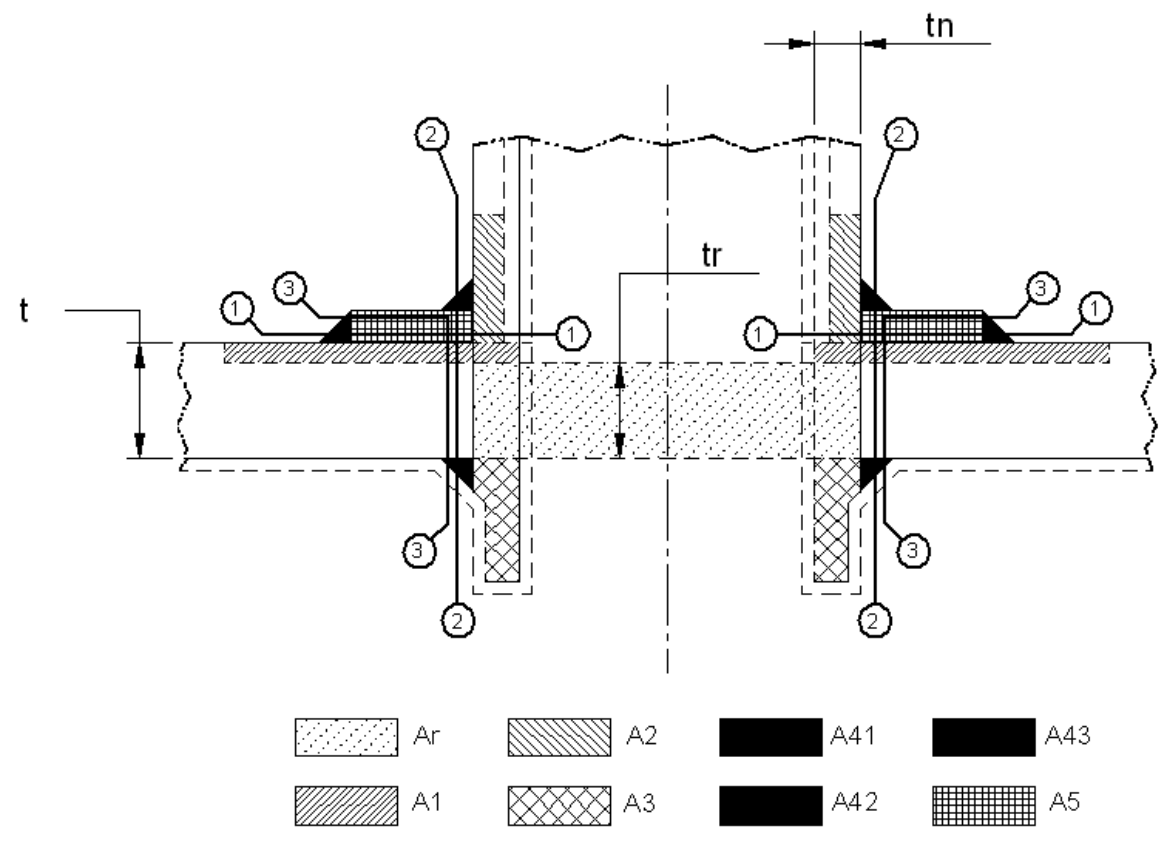

Fig. A.5- Nozzle attachment weld loads and weld strength paths to be considered

$$
\begin{aligned}
& \mathrm{W}=\left[\mathrm{A}-\mathrm{A}_{1}+2 \mathrm{t}_{\mathrm{n}} \mathrm{fr}_{1}\left(\mathrm{E}_{1} \mathrm{t}-\mathrm{Ft}_{\mathrm{r}}\right)\right] \mathrm{S}_{\mathrm{v}} \\
& \mathrm{W}_{1-1}=\left(\mathrm{A}_{2}+\mathrm{A}_{5}+\mathrm{A}_{41}+\mathrm{A}_{42}\right) \mathrm{S}_{\mathrm{v}} \\
& \mathrm{W}_{2-2}=\left(\mathrm{A}_{2}+\mathrm{A}_{3}+\mathrm{A}_{41}+\mathrm{A}_{43}+2 \mathrm{t}_{\mathrm{n}} \mathrm{tf}_{\mathrm{r} 1}\right) \mathrm{S}_{\mathrm{v}} \\
& \mathrm{W}_{3-3}=\left(\mathrm{A}_{2}+\mathrm{A}_{3}+\mathrm{A}_{5}+\mathrm{A}_{41}+\mathrm{A}_{42}+\mathrm{A}_{43}+2 \mathrm{t}_{\mathrm{n}} \mathrm{tf}_{\mathrm{r} 1}\right) \mathrm{S}_{\mathrm{v}}
\end{aligned}
$$




\section{A.4) Gasket information}

Table A.1- Flange surface finish ${ }^{51}$

\begin{tabular}{|c|c|}
\hline Gasket material & $\begin{array}{l}\text { Flange surface finish: } \\
\text { Ra and CLA }\end{array}$ \\
\hline $\begin{array}{l}\text { Compressed asbestos fibre } \\
\text { (all grades above } 1 \mathrm{~mm} \text { thickness) }\end{array}$ & $\begin{array}{l}3.2 \mu \mathrm{m} \text { to } 12.5 \mu \mathrm{m} \mathrm{Ra} \\
(125 \mu \mathrm{in} \text { to } 500 \mu \mathrm{in} \text { CLA })\end{array}$ \\
\hline $\begin{array}{l}\text { Asbestos free carbon fiber with nitrile rubber } \\
\text { bonder }\end{array}$ & $\begin{array}{l}3.2 \mu \mathrm{m} \text { to } 12.5 \mu \mathrm{m} \mathrm{Ra} \\
(25 \mu \mathrm{in} \text { to } 500 \mu \mathrm{in} \text { CLA })\end{array}$ \\
\hline Graphite laminate & $\begin{array}{l}3.2 \mu \mathrm{m} \text { to } 12.5 \mu \mathrm{m} \mathrm{Ra} \\
(125 \mu \mathrm{in} \text { to } 500 \mu \mathrm{in} \text { CLA })\end{array}$ \\
\hline $\begin{array}{l}\text { Spiral-wound gasket with graphite filler } \\
\text { General duty }\end{array}$ & $\begin{array}{l}3.2 \mu \mathrm{m} \text { to } 5.1 \mu \mathrm{m} \mathrm{Ra} \\
(125 \mu \mathrm{in} \text { to } 200 \mu \mathrm{in} \text { CLA })\end{array}$ \\
\hline $\begin{array}{l}\text { Spiral-wound gasket with graphite filler } \\
\text { Critical duty }\end{array}$ & $\begin{array}{l}3.2 \mu \mathrm{m} \mathrm{Ra} \\
(125 \mu \mathrm{in} \text { CLA })\end{array}$ \\
\hline $\begin{array}{l}\text { Spiral-wound gasket with graphite filler } \\
\text { Vacuum duty }\end{array}$ & $\begin{array}{l}2.0 \mu \mathrm{m} \mathrm{Ra} \\
(80 \mu \mathrm{in} \text { CLA })\end{array}$ \\
\hline $\begin{array}{l}\text { Solid flat metal gasket } \\
\text { General duty }\end{array}$ & $\begin{array}{l}1.6 \mu \mathrm{m} \mathrm{Ra} \\
(60 \mu \mathrm{in} \text { CLA })\end{array}$ \\
\hline $\begin{array}{l}\text { Solid flat metal gasket } \\
\text { Critical duty }\end{array}$ & $\begin{array}{l}0.8 \mu \mathrm{m} \text { Ra minimum preferably better } \\
(30 \mu \mathrm{in} \text { CLA })\end{array}$ \\
\hline Metal ring joint gaskets & $\begin{array}{l}1.6 \mu \mathrm{m} \mathrm{Ra} \\
(60 \mu \mathrm{in} \text { CLA })\end{array}$ \\
\hline
\end{tabular}

\section{A.5) Flange design}

(1) Required bolt load to initially seat the gasket:

$\mathrm{W}_{\mathrm{m} 2}=\mathrm{b} \pi \mathrm{Gy}$

(2) Required bolt operating load:

$\mathrm{W}_{\mathrm{m} 1}=\frac{\pi \mathrm{G}^{2}}{4} \mathrm{P}+2 \mathrm{~b} \pi \mathrm{GmP}$ 
Table A.2- Effective gasket width ${ }^{49}$

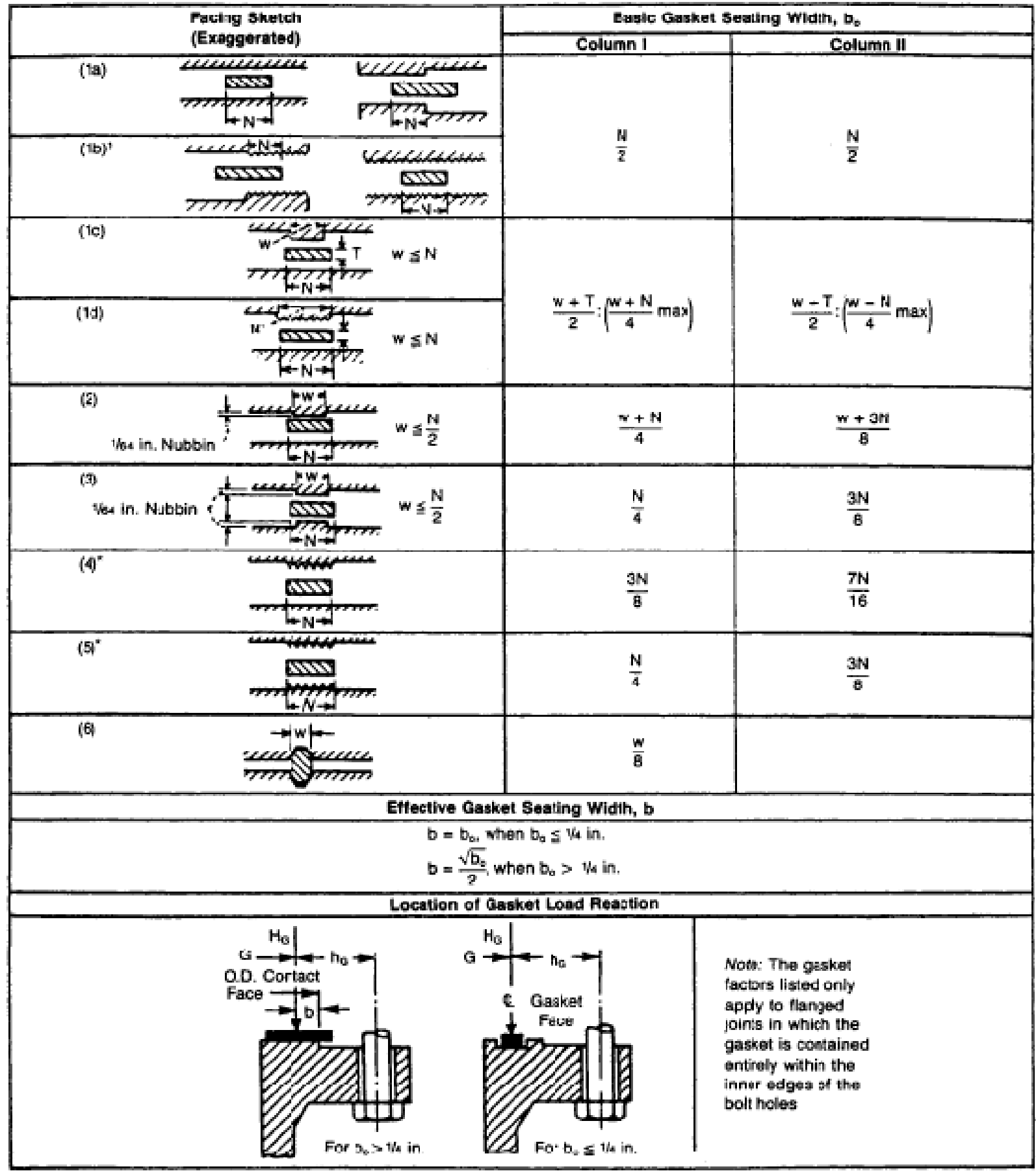


Table A.3- Gasket materials and contact facings ${ }^{49}$

\begin{tabular}{|c|c|c|c|c|c|c|}
\hline \multirow{2}{*}{\multicolumn{2}{|c|}{$\begin{array}{cc}\text { Gasket Material } \\
\end{array}$}} & \multirow{2}{*}{$\begin{array}{l}\text { Gasket } \\
\text { Factor } \\
m\end{array}$} & \multirow{3}{*}{$\begin{array}{c}\text { Min. } \\
\text { Design } \\
\text { Seating } \\
\text { Stress } \\
y \\
\\
\\
0 \\
\end{array}$} & \multirow{3}{*}{$\begin{array}{c}\text { Sketches } \\
\text { and } \\
\text { Notes }\end{array}$} & \multirow{2}{*}{\multicolumn{2}{|c|}{$\begin{array}{l}\text { Use } \\
\text { Fueing } \\
\text { Stretch Use- } \\
\text { Refier to Table 2-4 } \\
\end{array}$}} \\
\hline & & & & & & \\
\hline $\begin{array}{l}\text { Sell-energizing types: O rings, } \\
\text { metallic, elastomer or other } \\
\text { gasket types considered as } \\
\text { sell-sealing }\end{array}$ & & 0 & & & & \\
\hline $\begin{array}{l}\text { Elastomers without fabric or a } \\
\text { high percentage of asbestos } \\
\text { tlber: } \\
\text { Below 75.A Shore Ounometer } \\
\text { 75A or higher Shore } \\
\text { Durometer }\end{array}$ & & $\begin{array}{l}0.50 \\
1.00 \\
\end{array}$ & $\begin{array}{r}0 \\
200 \\
\end{array}$ & & \multirow{5}{*}{$\begin{array}{l}\text { (1a), (1b). } \\
\text { (1e), (1d). } \\
\text { (4). (5) }\end{array}$} & \\
\hline $\begin{array}{l}\text { Asbeastós with à suitable bindèr } \\
\text { for the oparating conditions }\end{array}$ & $\begin{array}{l}\text { Wh thick } \\
\text { Wo thick } \\
\text { W2 thick }\end{array}$ & $\begin{array}{l}2.00 \\
2.75 \\
3.50 \\
\end{array}$ & $\begin{array}{l}1.600 \\
3.700 \\
6.500 \\
\end{array}$ & & & \\
\hline $\begin{array}{l}\text { Elastomers with cotton tabric } \\
\text { insertion }\end{array}$ & & 1.25 & 400 & & & \\
\hline $\begin{array}{l}\text { Elastomers with asbestos fabric } \\
\text { insertion, with or without wire } \\
\text { reinforcement }\end{array}$ & $\begin{array}{l}\text { 3-ply } \\
\text { 2-ply } \\
\text { 1-ply }\end{array}$ & $\begin{array}{r}2.25 \\
2.50 \\
2.75 \\
\end{array}$ & $\begin{array}{l}2,200 \\
2.900 \\
3.700 \\
\end{array}$ & & & \\
\hline Vegetable fiber & & 1.75 & 1.100 & & & \\
\hline $\begin{array}{l}\text { Spiral-wound metal, asbestos } \\
\text { filled }\end{array}$ & $\begin{array}{l}\text { Carbon } \\
\text { Stainless or Monel }\end{array}$ & $\begin{array}{l}2.50 \\
3.00\end{array}$ & $\begin{array}{l}10,000 \\
10,000\end{array}$ & & & \\
\hline $\begin{array}{l}\text { Corrugated metai, asbestos } \\
\text { inserted or corrugated metal, } \\
\text { jacketed asbestos filled }\end{array}$ & $\begin{array}{l}\text { Sott aluminum } \\
\text { Solt copper or brass } \\
\text { Iron or soit steel } \\
\text { Monel or } 4 \%-696 \text { chrome } \\
\text { Stainiess steels }\end{array}$ & $\begin{array}{l}2.50 \\
2.75 \\
3.00 \\
3.25 \\
3.50 \\
\end{array}$ & $\begin{array}{l}2.900 \\
3.700 \\
4.500 \\
5.500 \\
6.500 \\
\end{array}$ & & & \\
\hline Corrugated metal & $\begin{array}{l}\text { Solt aluminum } \\
\text { Solk copper or brass } \\
\text { Iron or sot steel } \\
\text { Monel or } 4 \%-696 \text { chrome } \\
\text { Stainless stepels }\end{array}$ & $\begin{array}{l}2.75 \\
3.00 \\
3.25 \\
3.50 \\
3.75 \\
\end{array}$ & $\begin{array}{l}3,700 \\
4,500 \\
5,500 \\
6,500 \\
7,600 \\
\end{array}$ & & $\begin{array}{l}\text { (1a). (1b) } \\
\text { (1c). (1d) }\end{array}$ & \\
\hline $\begin{array}{l}\text { Flat metal jacketed asbestos } \\
\text { filled }\end{array}$ & $\begin{array}{l}\text { Solt aluminum } \\
\text { Soft copper or brass } \\
\text { Iron or soit steel } \\
\text { Monel or } \\
496-6 \% \text { chrome } \\
\text { Staintess steeis } \\
\end{array}$ & $\begin{array}{l}3.25 \\
3.50 \\
3.75 \\
3.50 \\
3.75 \\
3.75 \\
\end{array}$ & $\begin{array}{l}5,500 \\
6,500 \\
7,600 \\
8,000 \\
9,000 \\
9,000 \\
\end{array}$ & & $\begin{array}{l}(1 a),(1 b) \\
(1 c) .(1 d) \\
(2)^{2}\end{array}$ & \\
\hline Grooved metal & $\begin{array}{l}\text { Solt aluminum } \\
\text { Soft copper or brass } \\
\text { Iron or solt steei } \\
\text { Monel or } 4 \%-6 \% \text { chrome } \\
\text { Stainiess steels }\end{array}$ & $\begin{array}{l}3.25 \\
3.50 \\
3.75 \\
3.75 \\
4.25 \\
\end{array}$ & $\begin{array}{r}5,500 \\
6,500 \\
7,600 \\
9,000 \\
10,100 \\
\end{array}$ & & $\begin{array}{l}\text { (1a). (1b). } \\
\text { (1c). (1d). } \\
\text { (2). (3) }\end{array}$ & \\
\hline Solid tlat metal & $\begin{array}{l}\text { Soft aluminum } \\
\text { Solt copper or bitass } \\
\text { Iron or sott steel } \\
\text { Monel or } 4 \% 6-6 \% \text { chrome } \\
\text { Stainless steels }\end{array}$ & $\begin{array}{l}4.00 \\
4.75 \\
5.50 \\
6.00 \\
6.50 \\
\end{array}$ & $\begin{array}{r}8,000 \\
13,000 \\
18,000 \\
21,800 \\
26,000 \\
\end{array}$ & & $\begin{array}{l}(1 \mathrm{a}),(1 \mathrm{~b}) \\
\text { (1c), (1d). } \\
\text { (2). (3). } \\
\text { (4). (5) }\end{array}$ & \\
\hline Ring joint & $\begin{array}{l}\text { Iron or solt steel } \\
\text { Monel or } 496-69 \% \text { chrome } \\
\text { Stainiess steels }\end{array}$ & $\begin{array}{l}5.50 \\
6.00 \\
6.50 \\
\end{array}$ & $\begin{array}{l}18,000 \\
21,800 \\
26,000\end{array}$ & & (6) & \\
\hline
\end{tabular}


(3) Total cross-sectional area of bolts at root of thread (Operating Conditions)

$A_{\mathrm{m} 1}=\frac{W_{\mathrm{m} 1}}{\mathrm{~S}_{\mathrm{b}}}=106.37 \mathrm{in}^{2}$

(4) Total cross-sectional area of bolts at root of thread (Gasket Seating)

$\mathrm{A}_{\mathrm{m} 2}=\frac{\mathrm{W}_{\mathrm{m} 2}}{\mathrm{~S}_{\mathrm{a}}}=1.42249 \mathrm{in}^{2}$

Table A.4- Dimensional data for bolts and flanges ${ }^{49}$

\begin{tabular}{|c|c|c|c|c|c|c|c|c|c|c|}
\hline \multirow[b]{2}{*}{ Bolt Size } & \multicolumn{2}{|c|}{ Standard Thread } & \multicolumn{2}{|c|}{ 8-Thread Series } & \multicolumn{2}{|c|}{ Bolt Spacing } & \multirow{2}{*}{$\begin{array}{c}\text { Minimum } \\
\text { Radial } \\
\text { Distance } \boldsymbol{A}\end{array}$} & \multirow[b]{2}{*}{$\begin{array}{c}\text { Edge } \\
\text { Distance } E\end{array}$} & \multirow{2}{*}{$\begin{array}{c}\text { Nut } \\
\text { Dimension } \\
\text { (across flats) }\end{array}$} & \multirow{2}{*}{$\begin{array}{l}\text { Maximum } \\
\text { Fille1 Radius } \\
\text { at base of hub }\end{array}$} \\
\hline & $\begin{array}{l}\text { No. of } \\
\text { Threads }\end{array}$ & $\begin{array}{l}\text { Root } \\
\text { Area }\end{array}$ & $\begin{array}{l}\text { No. of } \\
\text { Threads }\end{array}$ & $\begin{array}{l}\text { Root } \\
\text { Area }\end{array}$ & $\begin{array}{c}\text { Minimum } \\
B_{s}\end{array}$ & Preferred & & & & \\
\hline 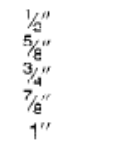 & $\begin{array}{c}13 \\
11 \\
10 \\
9 \\
8\end{array}$ & $\begin{array}{l}0.126 \\
0.202 \\
0.302 \\
0.419 \\
0.551\end{array}$ & $\begin{array}{l}\text { No. } 8 \text { thread } \\
\text { series below ' } 1\end{array}$ & 0.551 & $\begin{array}{l}11 / 4^{\prime \prime} \\
11 / 2 \\
13 / 4 \\
21 / 16 \\
21 / 4\end{array}$ & $\begin{array}{l}3^{\prime} \\
3 \\
3 \\
3 \\
3\end{array}$ & $\begin{array}{c}13 / 96 \\
15 / 16 \\
11 / e \\
11 / 4 \\
13 / 9\end{array}$ & $\begin{array}{c}5 / 9 " \\
3 / 4 \\
13 / 10 \\
15 / 16 \\
11 / 16\end{array}$ & 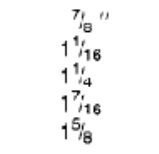 & $\begin{array}{l}1 / 41 \\
5 \% 16 \\
3 / 8 \\
3 / 8 \\
7 / 16\end{array}$ \\
\hline $\begin{array}{l}11^{1 / 2} \\
1 \frac{2^{\prime \prime}}{4^{\prime \prime}} \\
13 / e^{\prime \prime} \\
1 \frac{1}{a^{\prime \prime}}\end{array}$ & $\begin{array}{l}7 \\
7 \\
6 \\
6\end{array}$ & $\begin{array}{l}0.693 \\
0.890 \\
1.054 \\
1.294\end{array}$ & $\begin{array}{l}8 \\
8 \\
8 \\
8\end{array}$ & $\begin{array}{l}3.728 \\
0.929 \\
1.155 \\
1.405\end{array}$ & $\begin{array}{l}21 / 2 \\
2^{13 / 16} \\
31 / 16 \\
31 / 4\end{array}$ & $\begin{array}{l}3 \\
3\end{array}$ & $\begin{array}{l}11 / 2 \\
13 / 4 \\
17 / 8 \\
2\end{array}$ & $\begin{array}{l}1 \frac{1 / 6}{1 / 4} \\
1 \frac{4}{13 / 2} \\
1 \frac{1}{2}\end{array}$ & $\begin{array}{c}1^{13 s_{16}} \\
2 \\
2^{3 / 16} \\
2^{3 / 6}\end{array}$ & $\begin{array}{l}7 / 10 \\
0 / 16 \\
9 / 16 \\
5 / 8\end{array}$ \\
\hline $\begin{array}{l}15 / \varepsilon^{\prime \prime} \\
13 / 4^{\prime \prime} \\
17 / e^{\prime \prime} \\
2^{\prime \prime}\end{array}$ & $\begin{array}{r}5 \frac{1}{2} \\
5 \\
5 \\
1 \frac{1 / 2}{}\end{array}$ & $\begin{array}{l}1.515 \\
1.744 \\
2.049 \\
2.300\end{array}$ & $\begin{array}{l}8 \\
8 \\
8 \\
8\end{array}$ & $\begin{array}{l}1.680 \\
1.980 \\
2.304 \\
2.652\end{array}$ & $\begin{array}{c}31 / 2 \\
3^{3 / 4} \\
4 \\
11 / 4\end{array}$ & & $\begin{array}{l}21 / 8 \\
21 / 4 \\
2^{3 / / 8} \\
2^{1 / 2}\end{array}$ & $\begin{array}{l}15 / 3 \\
13 / 4 \\
17 / 8 \\
2\end{array}$ & $\begin{array}{c}2^{9 / 16} \\
2^{3 / 4} \\
2^{15} i_{16}^{3} \\
3^{1 \%}\end{array}$ & $\begin{array}{c}5 / 3 \\
5 / 3 \\
5 / 3 \\
11 / 10\end{array}$ \\
\hline $\begin{array}{l}21 / 4^{\prime \prime} \\
21 / 2^{\prime \prime} \\
23 / 4 \\
3^{\prime \prime \prime}\end{array}$ & $\begin{array}{r}4 \frac{1}{2} \\
4 \\
4 \\
4\end{array}$ & $\begin{array}{l}3.020 \\
3.715 \\
\angle 618 \\
5.621\end{array}$ & $\begin{array}{l}8 \\
8 \\
8 \\
8\end{array}$ & $\begin{array}{l}3.423 \\
4.292 \\
5.259 \\
5.234\end{array}$ & $\begin{array}{l}4^{3} / 4 \\
51 / 4 \\
5 \% / 4 \\
61 / 4\end{array}$ & & $\begin{array}{l}2^{3 / 4} \\
3^{1 / 16} \\
3^{3 / 8} \\
3^{5 / 8}\end{array}$ & $\begin{array}{l}2 \% / 9 \\
23 \% \\
2 \% \\
2 \% / 8\end{array}$ & $\begin{array}{l}31 / 2 \\
37 / 8 \\
41 / 4 \\
4 \%\end{array}$ & $\begin{array}{c}11 / 16 \\
13 / 16 \\
7 / 3 \\
15 / 16\end{array}$ \\
\hline
\end{tabular}

\section{(5) Flange moment calculations}

In the calculation of flange stress, the moment of a load acting on the flange is the product of the load and its moment arm (see table A.5). The moment arm is determined by the relative position of the bolt circle with respect to that of the load producing the moment.

For the operating conditions the total flange moment $\mathrm{M}_{\mathrm{o}}$ is the sum of the three individual moments and based on the flange design load $\mathrm{W}_{\mathrm{m} 1}$.

$M_{o}=M_{D}+M_{G}+M_{T}$ 
For the gasket seating, the total flange moment $\mathrm{M}_{\mathrm{o}}$ is based on the flange design bolt load $\mathrm{W}_{\mathrm{m} 2}$.

Table A.5- Level arms and moments equations

\begin{tabular}{|l|l|l|}
\hline \multicolumn{3}{|c|}{ Operating conditions } \\
\hline \multicolumn{1}{|c|}{ Loads } & \multicolumn{1}{|c|}{ Lever Arm } & Moments \\
\hline $\mathrm{H}_{\mathrm{D}}=\frac{\pi \mathrm{B}^{2}}{4} \mathrm{P}$ & $\mathrm{h}_{\mathrm{D}}=\mathrm{R}+0.5 \mathrm{~g}_{1}$ & $\mathrm{M}_{\mathrm{D}}=\mathrm{H}_{\mathrm{D}} \mathrm{h}_{\mathrm{D}}$ \\
\hline $\mathrm{H}_{\mathrm{G}}=\mathrm{W}_{\mathrm{m} 1}-\frac{\pi \mathrm{G}^{2}}{4} \mathrm{P}$ & $\mathrm{h}_{\mathrm{G}}=0.5(\mathrm{C}-\mathrm{G})$ & $\mathrm{M}_{\mathrm{G}}=\mathrm{H}_{\mathrm{G}} \mathrm{h}_{\mathrm{G}}$ \\
\hline $\mathrm{H}_{\mathrm{T}}=\frac{\pi \mathrm{G}^{2}}{4} \mathrm{P}-\mathrm{H}_{\mathrm{D}}$ & $\mathrm{h}_{\mathrm{T}}=0.5\left(\mathrm{R}+\mathrm{g}_{1}+\mathrm{h}_{\mathrm{G}}\right)$ & $\mathrm{M}_{\mathrm{T}}=\mathrm{H}_{\mathrm{T}} \mathrm{h}_{\mathrm{T}}$ \\
\hline Gasket seating & $\mathrm{h}_{\mathrm{G}}=0.5(\mathrm{C}-\mathrm{G})$ & $\mathrm{M}_{\mathrm{O}}{ }^{\prime}=\mathrm{H}_{\mathrm{G}} \mathrm{h}_{\mathrm{G}}$ \\
\hline $\mathrm{H}_{\mathrm{G}}=\mathrm{W}$
\end{tabular}

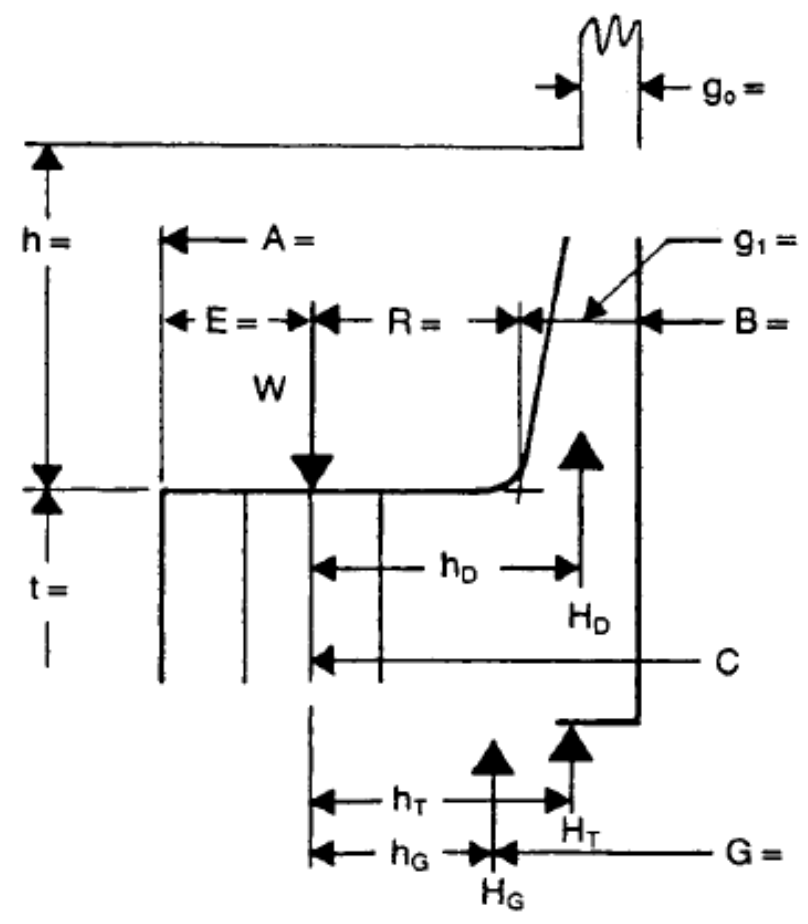

Fig. A.6- Dimensional Data and Forces for a Weld Neck Flange ${ }^{49}$ 
(6) $K$ and Hub factors

These factors are calculated to be used in determining the longitudinal, radial and tangential flange stresses.

$\mathrm{K}=\frac{\mathrm{A}}{\mathrm{B}}$

Table A.6- Table of coefficients ${ }^{49}$

\begin{tabular}{|c|c|c|c|c|c|c|c|c|c|c|c|c|c|c|c|c|c|c|c|}
\hline K & $\mathbf{T}$ & $z$ & $\mathbf{V}$ & $\mathbf{U}$ & K & $\mathbf{T}$ & $z$ & $\mathbf{Y}$ & $\mathbf{u}$ & $\mathbf{K}$ & $\mathbf{T}$ & z & $v$ & $\mathbf{u}$ & $K$ & $T$ & $\mathbf{z}$ & $v$ & $\mathbf{u}$ \\
\hline 1.182 & 1.85 & 6.04 & 11.70 & 12.86 & 1.278 & 1.81 & 4.16 & 8.05 & 8.85 & 1.434 & 1.74 & 2.89 & 5.56 & 6.10 & 1.75 & 1.60 & 1.97 & 3.64 & 4.00 \\
\hline 1.184 & 1.05 & 5.78 & 11.58 & 12.73 & 1281 & 1.01 & 4.12 & 7.03 & 8.77 & 1.438 & 1.74 & 2.87 & 5.52 & 6.05 & 1.76 & 1.60 & 1.95 & 3.61 & 3.96 \\
\hline 1.186 & 1.85 & 5.92 & 11.47 & 12.61 & 1284 & 1.80 & 4.08 & 7.91 & 8.69 & 1.442 & 1.74 & 2.85 & 5.48 & 6.01 & 1.77 & 1.60 & 1.94 & 3.57 & 3.93 \\
\hline 1.188 & 1.85 & 5.86 & 11.36 & 12.49 & 1287 & 1.80 & 4.05 & 7.84 & 8.61 & 1.446 & 1.74 & 2.83 & 5.44 & 5.97 & 1.78 & 1.59 & 1.92 & 3.54 & 3.89 \\
\hline 1.190 & 1.84 & 5.81 & 11.26 & 12.37 & 1290 & 1.80 & 4.01 & 7.77 & 8.53 & 1.450 & 1.73 & 2.81 & 5.40 & 5.93 & 1.79 & 1.59 & 1.91 & 3.51 & 3.85 \\
\hline 1.192 & 1.84 & 5.75 & 11.15 & 12.25 & 1.293 & 1,80 & 3.98 & 7.70 & 8.46 & 1.454 & 1.73 & 2.80 & 5.36 & 5.89 & 1.80 & 1.58 & 1.89 & 3.47 & 3.82 \\
\hline 1.194 & 1.84 & 5.70 & 11.05 & 12.14 & 1.296 & 1.80 & 3.94 & 7.63 & 8.39 & 1.458 & 1.73 & 2.78 & 5.32 & 5.85 & 1.81 & 1.58 & 1.88 & 3.44 & 3.78 \\
\hline 1.196 & 1.84 & 5.65 & 10.95 & 12.03 & 1299 & 1.80 & 3.91 & 7.57 & 8.31 & 1.462 & 1.73 & 2.76 & 5.28 & 5.80 & 1.82 & 1.58 & 1.86 & 3.41 & 3.75 \\
\hline 1.198 & 1.84 & 5.60 & 10.85 & 11.92 & $1 \mathrm{~m} 2$ & 1.80 & 388 & 7.50 & 824 & 1.466 & 1.73 & 2.74 & 5.24 & 5.76 & 1.83 & 1.57 & 1.85 & 3.38 & 372 \\
\hline 1.200 & 1.84 & 5.55 & 10.75 & 11.81 & 1305 & 1.80 & 3.84 & 7.44 & 8.18 & 1.470 & 1.72 & 2.72 & 5.20 & 5.71 & 1.84 & 1.57 & 1.84 & 3.35 & 3.69 \\
\hline 1.202 & 1.84 & 5.50 & 10.65 & 11.71 & 1.308 & 1.79 & 3.81 & 7.38 & 8.11 & 1.475 & 1.72 & 2.70 & 5.16 & 5.66 & 1.85 & 1.56 & 1.83 & 3.33 & 3.65 \\
\hline 1.204 & 1.84 & 5.45 & 10.56 & 11.61 & 1.311 & 1.79 & 3.78 & 7.32 & 8.05 & 1.480 & 1.72 & 2.68 & 5.12 & 5.61 & 1.85 & 1.56 & 1.81 & 3.30 & 3.62 \\
\hline 1.206 & 1.84 & 5.40 & 10.47 & 11.51 & 1.314 & 1.79 & 3.75 & 7.26 & 7.90 & 1.485 & 1.72 & 2.66 & 5.08 & 5.57 & 1.87 & 1.56 & 1.00 & 3.27 & 3.59 \\
\hline 1.208 & 1.84 & 5.35 & 10.38 & 11.41 & 1.317 & 1.70 & 3.72 & 7.20 & 7.92 & 1.490 & 1.72 & 2.64 & 5.04 & 5.53 & 1.88 & 1.65 & $1.7 \theta$ & 3.24 & 3.56 \\
\hline 1.210 & 1.84 & 5.31 & 10.30 & 11.32 & 1320 & 1.79 & 369 & 7.14 & 7.85 & 1495 & 171 & $26 ?$ & 5.00 & 549 & 189 & 155 & 178 & 322 & 354 \\
\hline 1.212 & 1.83 & 5.27 & 1021 & 1122 & 1.323 & 1.79 & 3.67 & 7.09 & 7.79 & 1.500 & 1.71 & 2.60 & 1.96 & 5.15 & 1.90 & 1.51 & 1.77 & 3.19 & 3.61 \\
\hline 1.214 & 1.83 & 5.22 & 1012 & $111 ?$ & 1.206 & 1.79 & 3.64 & 7.0 .3 & 7.73 & 1.50 .5 & 1.71 & 2.58 & 4.92 & 5.41 & 1.91 & 1.54 & 1.75 & 3.17 & 3.48 \\
\hline 1.216 & 1.83 & 5.18 & 10.04 & 11.03 & 1.329 & 1.78 & 3.61 & 6.98 & 7.67 & 1.510 & 1.71 & 2.56 & 4.88 & 5.37 & 1.92 & 1.54 & 1.74 & 3.14 & 3.45 \\
\hline 1.218 & 1.83 & 5.14 & 9.96 & 10.94 & 1.332 & 1.78 & 3.58 & 6.92 & 7.61 & 1.515 & 1.71 & 2.54 & 4.04 & 5.33 & 1.93 & 1.53 & 1.73 & 3.12 & 3.43 \\
\hline 1.220 & 1.83 & 5.10 & 9.89 & 10.87 & 1.335 & 1.78 & 3.56 & 6.87 & 7.55 & 1.520 & 1.70 & 2.53 & 4.80 & 5.29 & 1.94 & 1.53 & 1.72 & 3.09 & 3.40 \\
\hline 1.222 & 1.83 & 5.05 & 9.80 & 10.77 & 1.338 & 1.78 & 3.53 & 6.82 & 7.50 & 1.525 & 1.70 & 2.51 & 4.77 & 5.25 & 1.95 & 1.53 & 1.71 & 3.07 & 3.38 \\
\hline 1.224 & 1.83 & 5.01 & 9.72 & 10.63 & 1.341 & 1.78 & 3.51 & 6.77 & 7.44 & 1.530 & 1.70 & 2.49 & 4.74 & 5.21 & 1.95 & 1.52 & 1.70 & 3.05 & 3.35 \\
\hline 1.226 & 183 & 498 & 965 & 10.60 & 1.344 & 1.78 & 3.48 & 6.72 & 7.39 & 1.535 & 1.70 & 2.47 & 4.70 & 5.17 & 1.97 & 1.52 & 1.69 & 3.03 & 3.33 \\
\hline 1.228 & 1.83 & 4.94 & 9.57 & 10.52 & 1.347 & 1.78 & 3.46 & 6.68 & 7.33 & 1.540 & 1.69 & 2.46 & 4.66 & 5.13 & 1.93 & 1.51 & 1.68 & 3.01 & 3.30 \\
\hline 1.230 & 1.83 & 4.90 & 9.50 & 10.44 & 1.350 & 1.78 & 3.43 & 6.63 & 7.28 & 1.545 & 1.69 & 2.44 & 4.63 & 5.09 & 1.99 & 1.51 & 1.68 & 2.98 & 3.28 \\
\hline 1.232 & 1.83 & 4.86 & 9.43 & 10.35 & 1.354 & 1.77 & 3.40 & 6.57 & 7.21 & 1.55 & 1.69 & 2.43 & 4.60 & 5.05 & 2.00 & 1.51 & 1.67 & 2.96 & 3.26 \\
\hline 1.234 & 1.83 & 4.83 & 9.36 & 10.23 & 1.358 & 1.77 & 3.37 & 6.50 & 7.14 & 1.56 & 1.69 & 2.40 & 4.54 & 4.99 & 201 & 1.50 & 1.66 & 2.94 & 3.23 \\
\hline 1.236 & 1.82 & 4.79 & 9.29 & 10.20 & 1.362 & 1.77 & 3.34 & 6.44 & 7.08 & 1.57 & 1.68 & 2.37 & 4.48 & 4.92 & 2.02 & 1.50 & 1.65 & 2.92 & 3.21 \\
\hline 1.238 & 1.82 & 4.76 & 9.22 & 10.13 & 1.365 & $1.1 /$ & 3.31 & 6.38 & 1.01 & 1.58 & 1.68 & 2.34 & 4.42 & 4.86 & 204 & 1.49 & $1.6 \mathrm{~s}$ & 2.88 & $3.1 /$ \\
\hline 1.240 & 1.82 & 4.72 & 9.15 & 10.05 & 1.370 & 1.77 & 3.28 & 6.32 & 6.95 & 1.59 & 1.67 & 2.31 & 4.36 & 4.79 & 203 & 1.48 & 1.62 & 2.85 & 3.13 \\
\hline 1.242 & 1.82 & 4.69 & 9.08 & 9.93 & 1.374 & 1.77 & 3.25 & 6.27 & 6.89 & 1.50 & 1.67 & 2.28 & 4.31 & 4.73 & 203 & 1.48 & 1.60 & 2.81 & 3.09 \\
\hline 1.244 & 1.82 & 4.65 & 9.02 & 9.91 & 1.378 & 1.76 & 3.22 & 6.21 & 6.82 & 1.61 & 1.66 & 2.26 & 4.25 & 4.67 & 2.10 & 1.47 & 1.59 & 2.78 & 3.05 \\
\hline 1.246 & 1.82 & 4.62 & 8.95 & 0.84 & 1.382 & 1.76 & 3.20 & 6.16 & 6.77 & 1.62 & 1.65 & 2.23 & 4.20 & 4.61 & 2.12 & 1.46 & 1.57 & 2.74 & 3.01 \\
\hline 1.248 & 1.82 & 4.59 & B.89 & 9.77 & 1.386 & 1.76 & 3.17 & 6.11 & 6.72 & 1.63 & 1.65 & 2.21 & 4.15 & 4.56 & 214 & 1.46 & 1.56 & 2.71 & 2.97 \\
\hline 1.250 & 1.82 & 4.56 & B.83 & 9.70 & 1.390 & 1.76 & 3.15 & 6.06 & 6.66 & 1.64 & 1.63 & 2.18 & 4.10 & 4.50 & 216 & $1.4 b$ & 1.55 & 2.67 & 2.94 \\
\hline 1.252 & 1.82 & 4.52 & B.77 & 9.64 & 1.394 & 1.76 & 3.12 & 6.01 & 6.60 & 1.65 & 1.65 & 2.16 & 4.05 & 4.45 & 2.18 & 1.44 & 1.53 & 2.64 & 2.90 \\
\hline 1.254 & 1.82 & 4.49 & 8.71 & 9.57 & 1.398 & 1.75 & 3.10 & 5.96 & 6.55 & 1.66 & 1.64 & 2.14 & 4.01 & 4.40 & 220 & 1.44 & 1.52 & 2.61 & 2.87 \\
\hline 1.256 & 1.82 & 4.46 & B.65 & 9.51 & 1.402 & 1.75 & 3.07 & 5.92 & 6.49 & $1.0 ?$ & 1.64 & 2.12 & 3.96 & 4.35 & 222 & 1.43 & 1.51 & 2.58 & 2.84 \\
\hline 1.258 & 1.81 & 4.43 & & 0.44 & 1.406 & 1.75 & 3.05 & 5.87 & 6.44 & 1.68 & 1.63 & 2.10 & 3.92 & 4.30 & 224 & 1.42 & 1.60 & 2.56 & 2.81 \\
\hline 1.260 & 1.81 & 4.40 & 8.53 & 9.38 & 1410 & 175 & 3. .7 & $58 D$ & 6.39 & 1.69 & 163 & 208 & 3.87 & 4.26 & 2 กิ & 1.41 & 1.49 & 2.53 & 278 \\
\hline 1.263 & 1.81 & 4.36 & 8.4 & 9.28 & 1.414 & 1.75 & 3.00 & 5.77 & 6.31 & 1.70 & 1.63 & 2.05 & 3.83 & 1.21 & 223 & 1.41 & 1.48 & 2.50 & 2.75 \\
\hline 1. 26็ & 1.81 & $4.3 ?$ & & & 1.418 & 1.75 & 2.98 & 5.72 & $6.2 \mathrm{~s}$ & 1.71 & 1.62 & 2.04 & 3.79 & 4.17 & 230 & 1.40 & 1.47 & 2.48 & 2.72 \\
\hline 1.269 & 1.81 & 4.28 & 8.29 & 9.11 & 1.422 & 1.75 & 2.96 & 5.68 & 6.25 & 1.72 & 1.62 & 2.02 & 3.75 & 4.12 & 2.32 & 1.40 & 1.46 & 2.45 & 2.69 \\
\hline 1.272 & 1.81 & 4.24 & 8.21 & 9.02 & 1.426 & 1.74 & 2.94 & 564 & $6.2 \mathrm{C}$ & 1.73 & 1.61 & 2.00 & 3.72 & 4.08 & 234 & 1.39 & 1.45 & 2.43 & 2.67 \\
\hline 1.275 & 1.81 & 4.20 & 8.13 & 8.93 & 1.430 & 1.74 & 2.91 & 5.60 & 6.15 & 1.74 & 1.61 & 1.99 & 3.68 & 4.04 & 2.35 & 1.38 & 1.44 & 2.40 & 2.64 \\
\hline
\end{tabular}

Assuming a flange thickness, the following parameters are calculated:

$\underline{g_{1}}$

go

$\mathrm{h}_{\mathrm{o}}=\sqrt{\mathrm{Bg}_{\mathrm{o}}}$

$\mathrm{h} / \mathrm{h}_{\mathrm{o}}$ 
V, $F$ and $f$ are also calcultaed using Figs. A-7, A-8 and A-9

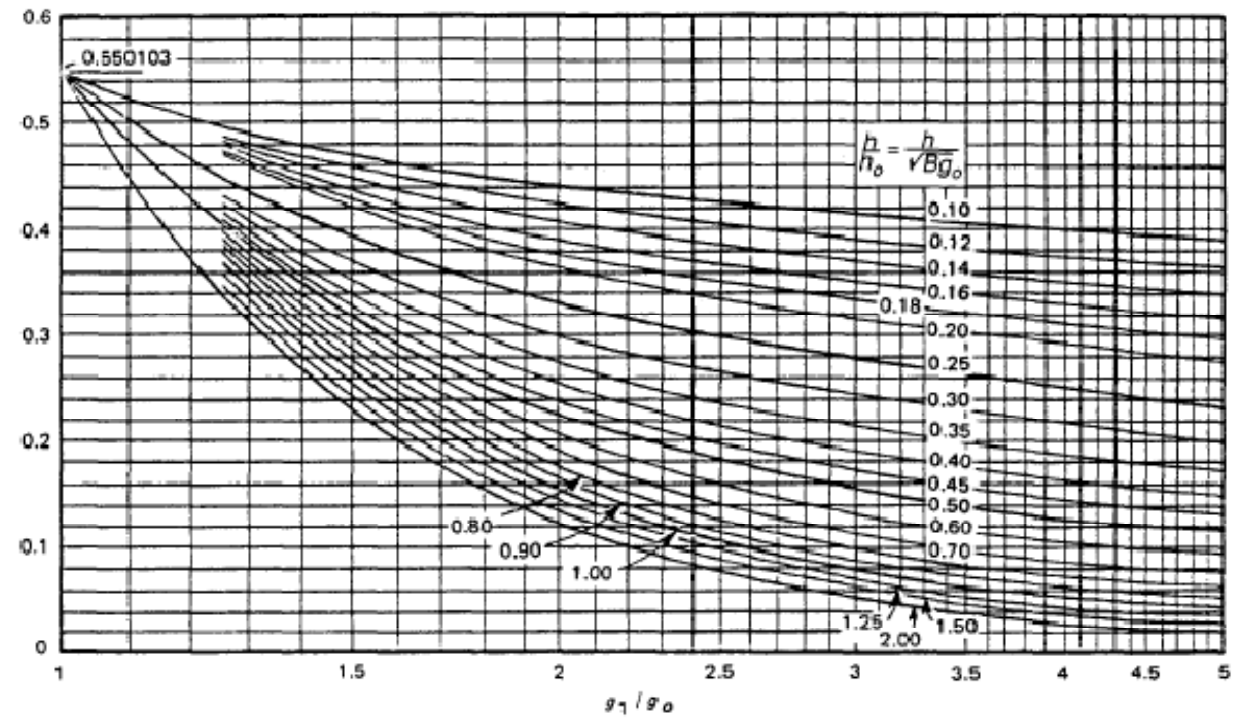

Fig. A.7-Values of $\mathrm{V}$ (Integral flange factors) ${ }^{49}$

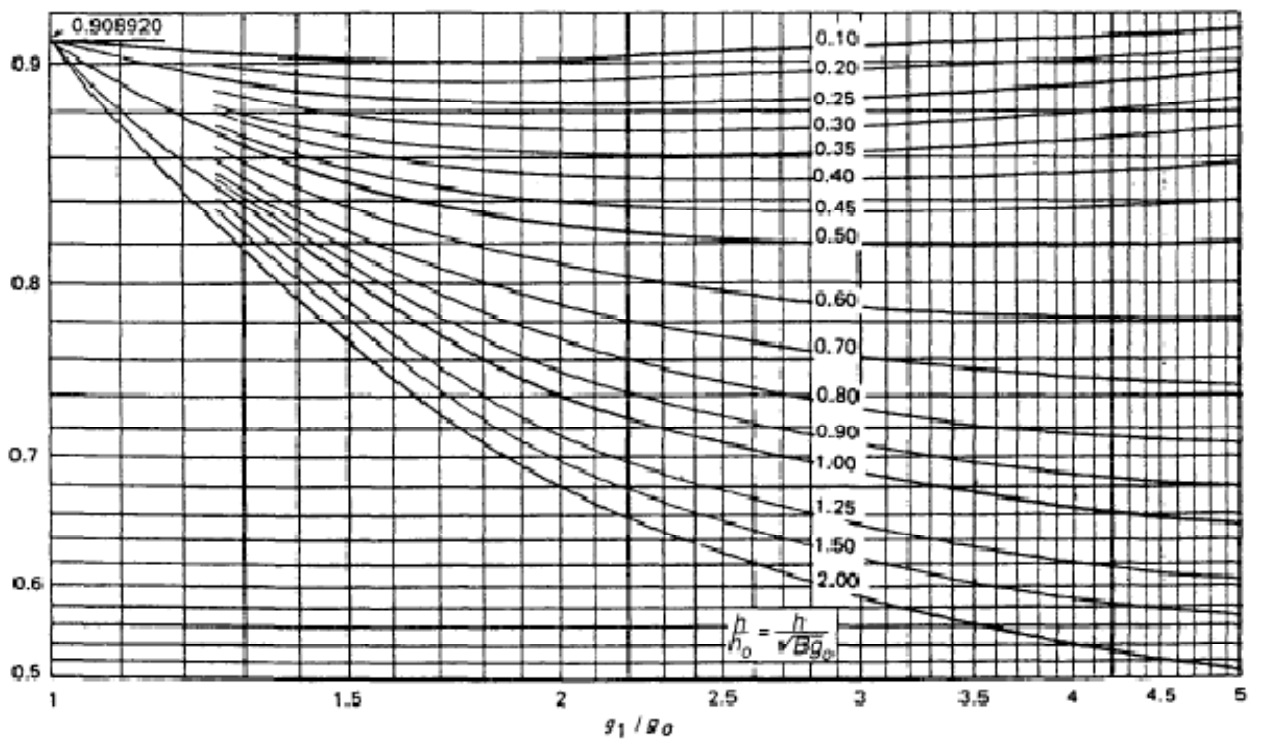

Fig. A.8-Values of F (Integral flange factors) ${ }^{49}$ 


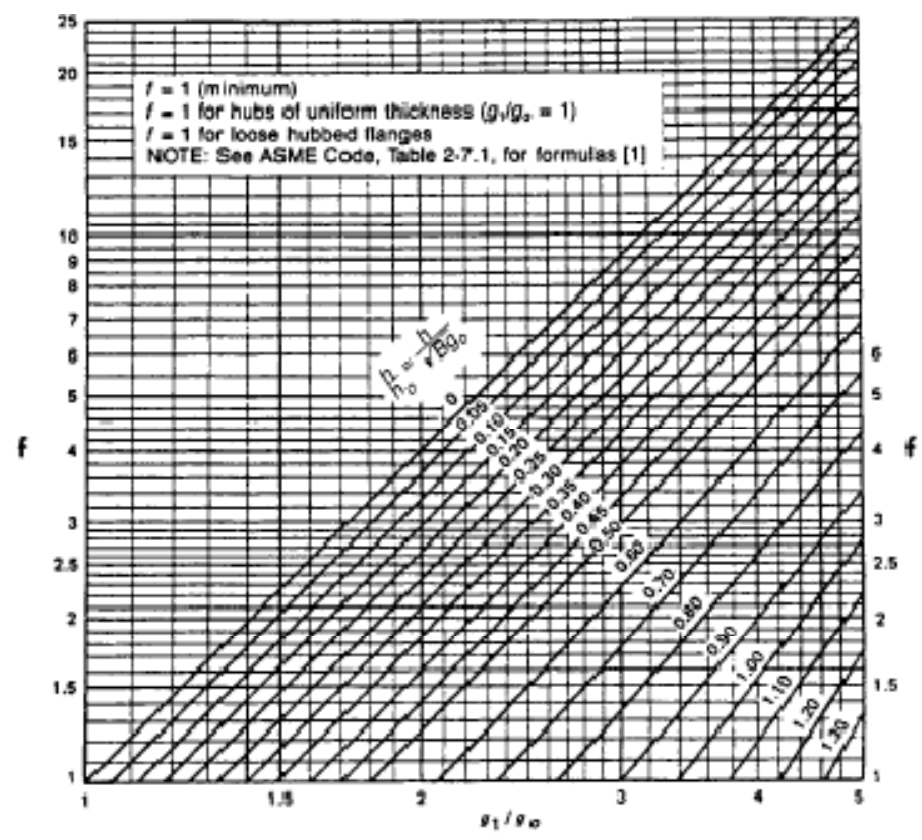

Fig. A.9- Values of $f\left(\right.$ hub stress correction factor) ${ }^{49}$

Finally:

$\mathrm{e}=\frac{\mathrm{F}}{\mathrm{h}_{\mathrm{o}}}$

$\mathrm{L}=\frac{\mathrm{te}+1}{\mathrm{~T}}+\frac{\mathrm{t}^{3}}{\mathrm{~d}}$

\section{(7) Calculation of flange stresses}

The stresses in the flange are determined for both the operating conditions and gasket seating condition, whichever controls, in accordance with the following equations:

Longitudinal hub stress

$\mathrm{S}_{\mathrm{H}}=\frac{\mathrm{fM}_{\mathrm{o}}}{\operatorname{Lg}_{1}^{2} \mathrm{~B}}$

Radial flange stress

$S_{R}=\frac{(1.33 t e+1) M_{o}}{L^{2} B}$ 
Tangential flange stress

$\mathrm{S}_{\mathrm{T}}=\frac{\mathrm{YM}_{\mathrm{o}}}{\mathrm{t}^{2} \mathrm{~B}}-\mathrm{ZS}_{\mathrm{R}}$

(8) Allowable flange design stresses

The flange stresses must not exceed the following values: ${ }^{47}$

- Longitudinal hub stress $\mathrm{S}_{\mathrm{H}}$ not greater than the smaller of $1.5 \mathrm{~S}_{\mathrm{f}}$ or $2.5 \mathrm{~S}_{\mathrm{n}}$ for integral type flanges with hub welded to the neck, pipe or vessel wall.

- Radial flange stress $S_{R}$ not greater than $S_{f}$

- Tangential stress $\mathrm{S}_{\mathrm{T}}$ not greater than $\mathrm{S}_{\mathrm{f}}$

- $\left(\mathrm{S}_{\mathrm{H}}+\mathrm{S}_{\mathrm{R}}\right) / 2$ and $\left(\mathrm{S}_{\mathrm{H}}+\mathrm{S}_{\mathrm{T}}\right) / 2$ not greater than $\mathrm{S}_{\mathrm{f}}$

(9) Flange maximum allowable pressure

The following moments are calculated:

$$
\begin{aligned}
& \mathrm{M}_{1}=1.5 \mathrm{~S}_{\mathrm{fo}} \frac{\mathrm{g}_{1}{ }^{2} \mathrm{BL}}{\mathrm{f}} \\
& \mathrm{M}_{2}=\frac{\mathrm{S}_{\mathrm{fo}} \mathrm{Lt}^{2} \mathrm{~B}}{(1.33 \mathrm{te}+1)} \\
& \mathrm{M}_{3}=\frac{\mathrm{S}_{\mathrm{fo}} \mathrm{Lt}^{2} \mathrm{~B}}{\mathrm{YL}-\mathrm{Z}(1.33 \mathrm{te}+1)} \\
& \mathrm{M}_{4}=\frac{2 \mathrm{~S}_{\mathrm{fo}} \mathrm{Lt}^{2} \mathrm{Bg}_{1}{ }^{2}}{\mathrm{ft}^{2}+\left(1.33 \mathrm{te}^{2}+1\right) \mathrm{g}_{1}{ }^{2}} \\
& \mathrm{M}_{5}=\frac{2 \mathrm{~S}_{\mathrm{fo}} \mathrm{Lt}^{2} \mathrm{Bg}_{1}{ }^{2}}{\mathrm{ft}^{2}+\mathrm{YLg}_{1}{ }^{2}-\mathrm{Z}(1.33 \mathrm{te}+1) \mathrm{g}_{1}{ }^{2}}
\end{aligned}
$$

Then, the maximum allowable moment $M_{\max }$ is the lesser of $M_{1}-M_{5}$ 
Calculating $\mathrm{A}_{\mathrm{m}(\max )}$

$\mathrm{A}_{\mathrm{m}(\max )}=\frac{2 \mathrm{M}_{\max }(@ \text { Ambient temperature })}{\mathrm{h}_{\mathrm{G}} \mathrm{S}_{\mathrm{a}}}-\mathrm{A}_{\mathrm{b}}>\mathrm{A}_{\mathrm{m} 2} \rightarrow \mathrm{OK}$

For the Operating Condition:

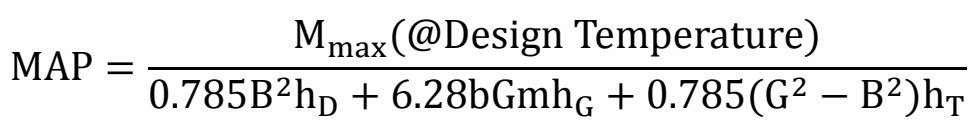

For Gasket Seating

$\mathrm{MAP}=\frac{\mathrm{S}_{\mathrm{b}} \mathrm{A}_{\mathrm{m}(\max )}}{6.28 \mathrm{bGm}+0.785 \mathrm{G}^{2}}$

\section{A.6) Top flat head}

Since the top cover of the pressure vessel will provide an opening for the production tubing, the design will be based on the analysis for a blind flange with an opening as depicted in Fig. A.10.

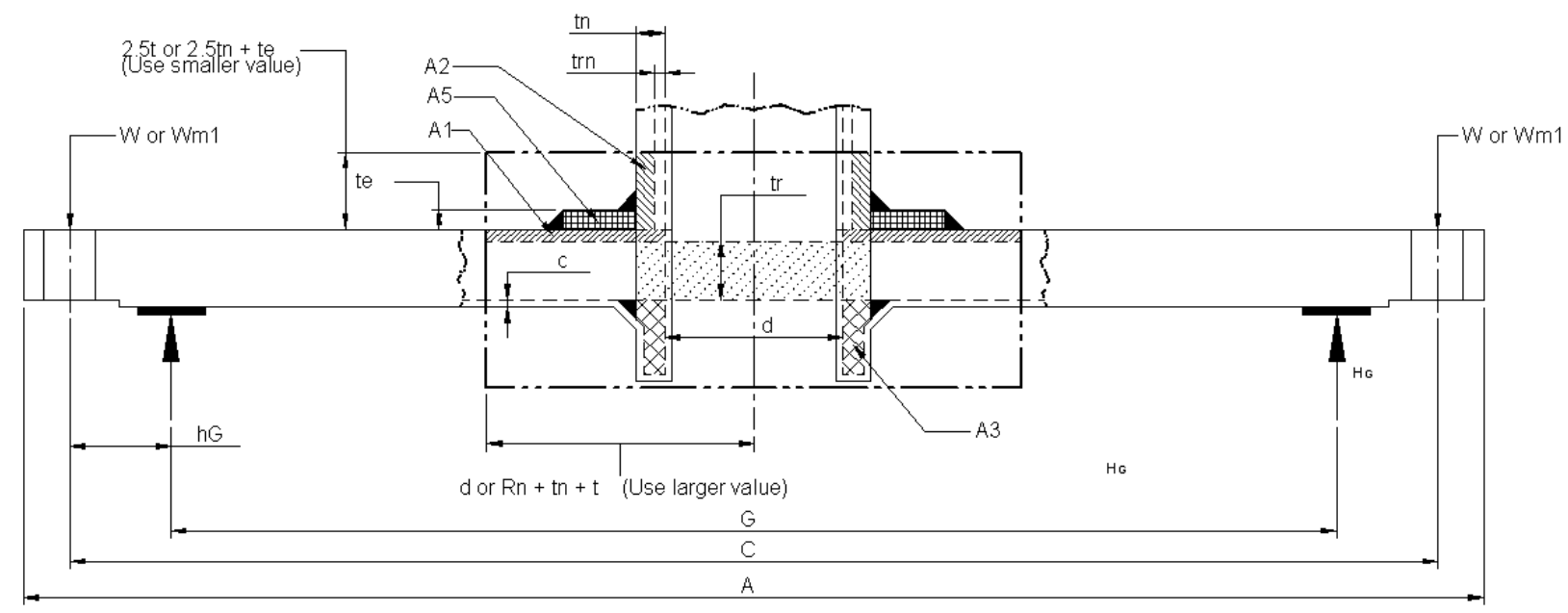

Fig. A.10- Opening for a blind flange 
(1) Thickness for the operating and gasket seating condition

$$
\begin{aligned}
& \mathrm{t}_{\mathrm{r}}=\mathrm{G} \sqrt{\frac{0.3 \mathrm{P}}{\mathrm{S}_{\mathrm{fo}}}+\frac{1.9 \mathrm{~W}_{\mathrm{m} 1} \mathrm{~h}_{\mathrm{G}}}{\mathrm{S}_{\mathrm{fo}} \mathrm{G}^{3}}} \\
& \mathrm{t}_{\mathrm{G}}=\mathrm{G} \sqrt{\frac{1.9 \mathrm{Wh}_{\mathrm{G}}}{\mathrm{S}_{\mathrm{fa}} \mathrm{G}^{3}}}
\end{aligned}
$$

(2) Reinforcement required for opening in flat heads (UG-39)

$$
\mathrm{t}_{\mathrm{rn}}=\frac{\mathrm{PR}_{\mathrm{n}}}{\mathrm{SE}-0.6 \mathrm{P}}
$$

Flat heads that have an opening with a diameter that does not exceed one-half of the diameter or shortest span, as defined in UG-34, shall have a total cross-sectional area of reinforcement not less than:

$\mathrm{A}_{\mathrm{r}}=0.5 \mathrm{dt}_{\mathrm{o}}+\mathrm{tt}_{\mathrm{n}}\left(1-\mathrm{f}_{\mathrm{r} 1}\right)$

Fig. A.4 illustrates the areas that can be used for reinforcement

(3) Calculating the areas available for reinforcement without a reinforcement element Follow the same procedure indicated in A.3

(4) Maximum allowable pressure for flat head and nozzle

$$
\text { MAP }=\frac{\mathrm{S}_{\mathrm{fo}}}{0.3}\left(\frac{\mathrm{t}_{\mathrm{o}}{ }^{2}}{\mathrm{G}^{2}}-\frac{1.9 \mathrm{~W}_{\mathrm{m} 1} \mathrm{~h}_{\mathrm{G}}}{\mathrm{S}_{\mathrm{fo}} \mathrm{G}^{3}}\right)
$$

$\mathrm{MAP}=\frac{\mathrm{t}_{\mathrm{n}} \mathrm{SE}}{\mathrm{R}_{\mathrm{n}}+0.6 \mathrm{t}_{\mathrm{n}}}$

\section{A.7) Weight reduction alternatives}

Using CAD software 6 options were considered for the vessel design as shown in Table A.7. 
Table A.7- Options for the configuration of the pressure vessel

\begin{tabular}{|c|c|c|c|c|c|c|c|c|c|}
\hline & & Weig1 & of vessel & & & & Weig & of vessel & \\
\hline & Option & Part & $\begin{array}{l}\text { Weight } \\
\text { (lbm) }\end{array}$ & Figure & & Option & Part & $\begin{array}{l}\text { Weight } \\
\text { (lbm) }\end{array}$ & Figure \\
\hline 1. & Flat head & Shell & 913.489 & & 4. & Flat head & She ll & 913.489 & \\
\hline & bolted cover & Nozzle-4 & 120.00 & & & $\begin{array}{l}\text { bolted cover } \\
\text { with openines }\end{array}$ & Nozzzle-4 & 120.00 & \\
\hline & on bottom & Top & $5,572,214$ & & & and & Top & $4,525,06$ & \\
\hline & & Nozzle-1 & 35.237 & & & $\begin{array}{l}\text { torispherical } \\
\text { head on }\end{array}$ & Nozzle-1 & 32.237 & \\
\hline & & Bottom & $2,459.22$ & & & botbm & Bottom & $1,002.243$ & \\
\hline & & Flange & $2,228,932$ & & & & Fange & $2,228.932$ & \\
\hline & & $\begin{array}{l}\text { Bolts\& } \\
\text { Nuts }\end{array}$ & 867.072 & & & & $\begin{array}{l}\text { Bolts\& } \\
\text { Nuts }\end{array}$ & 867.072 & \\
\hline & & Total & $12,206.164$ & & & & Total & $9,699.033$ & \\
\hline 2. & Hat head & Shell & 913.489 & & 5. & Welded flat & Shell & $1,360.728$ & \\
\hline & bolted cover & Nozzle-4 & 120.00 & & & $\begin{array}{l}\text { head cover } \\
\text { withopenings }\end{array}$ & Nozzle-4 & 120.00 & \\
\hline & torispherical & Top & $5,572.214$ & & & and flat head & Top & $1,961.445$ & \\
\hline & $\begin{array}{l}\text { head on } \\
\text { bottom }\end{array}$ & Nozzle-1 & 35,237 & & & on bottom & Nozzle-1 & 32.237 & \\
\hline & & Bottom & $1,002.243$ & & & & Bottom & $2,459.22$ & \\
\hline & & Flange & $2,228,932$ & & & & Total & $5,933.63$ & \\
\hline & & $\begin{array}{l}\text { Bdts\& } \\
\text { Nuts }\end{array}$ & 867.072 & & & & & & \\
\hline & & Total & $10,479.187$ & & & & & & \\
\hline 3. & Fat head & Shell & 913.489 & & 6. & Welded flat & Shell & $1,360.728$ & \\
\hline & $\begin{array}{l}\text { bolted sover } \\
\text { with copenings }\end{array}$ & Nozze-4 & 120.00 & & & $\begin{array}{l}\text { head cover } \\
\text { with openings }\end{array}$ & Nozzle-4 & 120.00 & \\
\hline & and flat head & Top & $4,525.06$ & & & and & Top & $1,961.445$ & \\
\hline & & Nozzle-1 & 35.237 & & & $\begin{array}{l}\text { torisphe rical } \\
\text { head on }\end{array}$ & Nozzle-1 & 32.237 & \\
\hline & & Battom & $2,450.22$ & & & bottom & Bottom & $1,002.243$ & \\
\hline & & Flange & $2,228.932$ & & & & Total & $4,476.653$ & \\
\hline & & $\begin{array}{l}\text { Bolts\& } \\
\text { Nu ts }\end{array}$ & 867.072 & & & & & & \\
\hline & & Total & $11,159.01$ & & & & & & \\
\hline
\end{tabular}


A.8) Weld sizes for base plate

Table A.8- Properties of welds ${ }^{46}$

\begin{tabular}{ll}
\hline $\begin{array}{l}\text { Outline of welded jönt } \\
\boldsymbol{b}=\text { width, } \boldsymbol{d}=\text { depth }\end{array}$ & $\begin{array}{l}\text { Bending } \\
\text { (about horizontal axis } x-x \text { ) }\end{array}$ \\
\hline
\end{tabular}

\section{A.9) Lifting lugs}
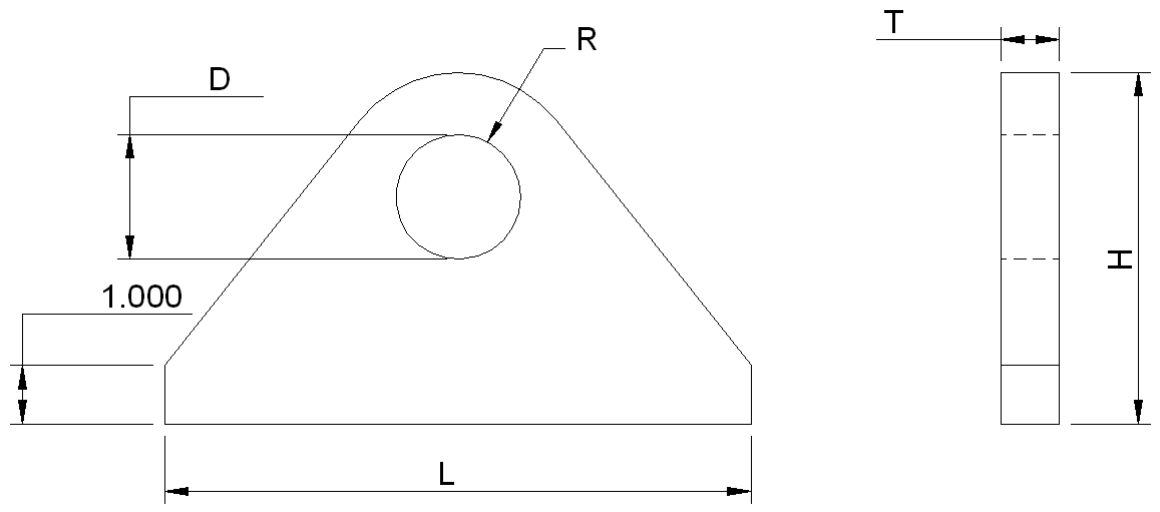

Table A.9- Lifting lug dimensions ${ }^{46}$

\begin{tabular}{|c|c|c|c|c|c|c|}
\hline Vessel weight & $D$, in & $T$, in & $R$, in & $\mathbf{H}$, in & $\mathbf{L}$, in & Weld (min) \\
\hline 12,000 & 1 & $1 / 2$ & $1-1 / 2$ & 5 & 10 & \multirow{4}{*}{ 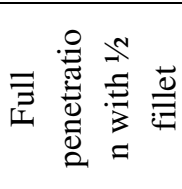 } \\
\hline 20,000 & $1-1 / 8$ & $3 / 4$ & 2 & 6 & 10 & \\
\hline 30,000 & $1-3 / 8$ & 1 & $2-1 / 8$ & 6 & 10 & \\
\hline 50,000 & $1-5 / 8$ & $1-1 / 4$ & $2-1 / 2$ & 7 & 12 & \\
\hline 70,000 & $2-1 / 8$ & $1-1 / 4$ & $3-1 / 2$ & 8 & 12 & \multirow{6}{*}{ 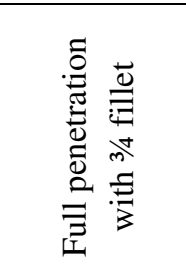 } \\
\hline 100,000 & $2-1 / 2$ & $1-1 / 2$ & $4-1 / 2$ & 9 & 16 & \\
\hline 150,000 & 3 & $1-3 / 4$ & 5 & 10 & 16 & \\
\hline 200,000 & 4 & 2 & 6 & 12 & 18 & \\
\hline 250,000 & $4-1 / 4$ & 2 & $6-1 / 2$ & 13 & 18 & \\
\hline 300,000 & $4-1 / 2$ & $2-1 / 2$ & 7 & 14 & 20 & \\
\hline
\end{tabular}




\section{APPENDIX B}

\section{COMPARISON OF COMPRESSOR PROPOSALS}

\section{B.1) Power required}

\begin{tabular}{|l|c|c|c|}
\hline Manufacturer & Equipment & HP & Efficiency \\
\hline \multirow{2}{*}{ Sullair } & Rotary screw & 300 & 0.962 \\
\cline { 2 - 4 } & Reciprocating & 75 & 0.941 \\
\hline Cameron & Centrifugal & 800 & 0.958 \\
\hline \multirow{2}{*}{ Atlas Copco } & Rotary screw & 150 & 0.958 \\
\cline { 2 - 4 } & Reciprocating & 125 & 0.958 \\
\hline \multirow{2}{*}{ Kaesser } & Rotary screw & 250 & 0.955 \\
\cline { 2 - 4 } & Reciprocating & 60 & 0.955 \\
\hline \multirow{2}{*}{ Ingersoll Rand } & Rotary screw & 125 & 0.945 \\
\cline { 2 - 4 } & Reciprocating & 50 & 0.945 \\
\hline CompAir & Reciprocating & 300 & 0.947 \\
\hline
\end{tabular}

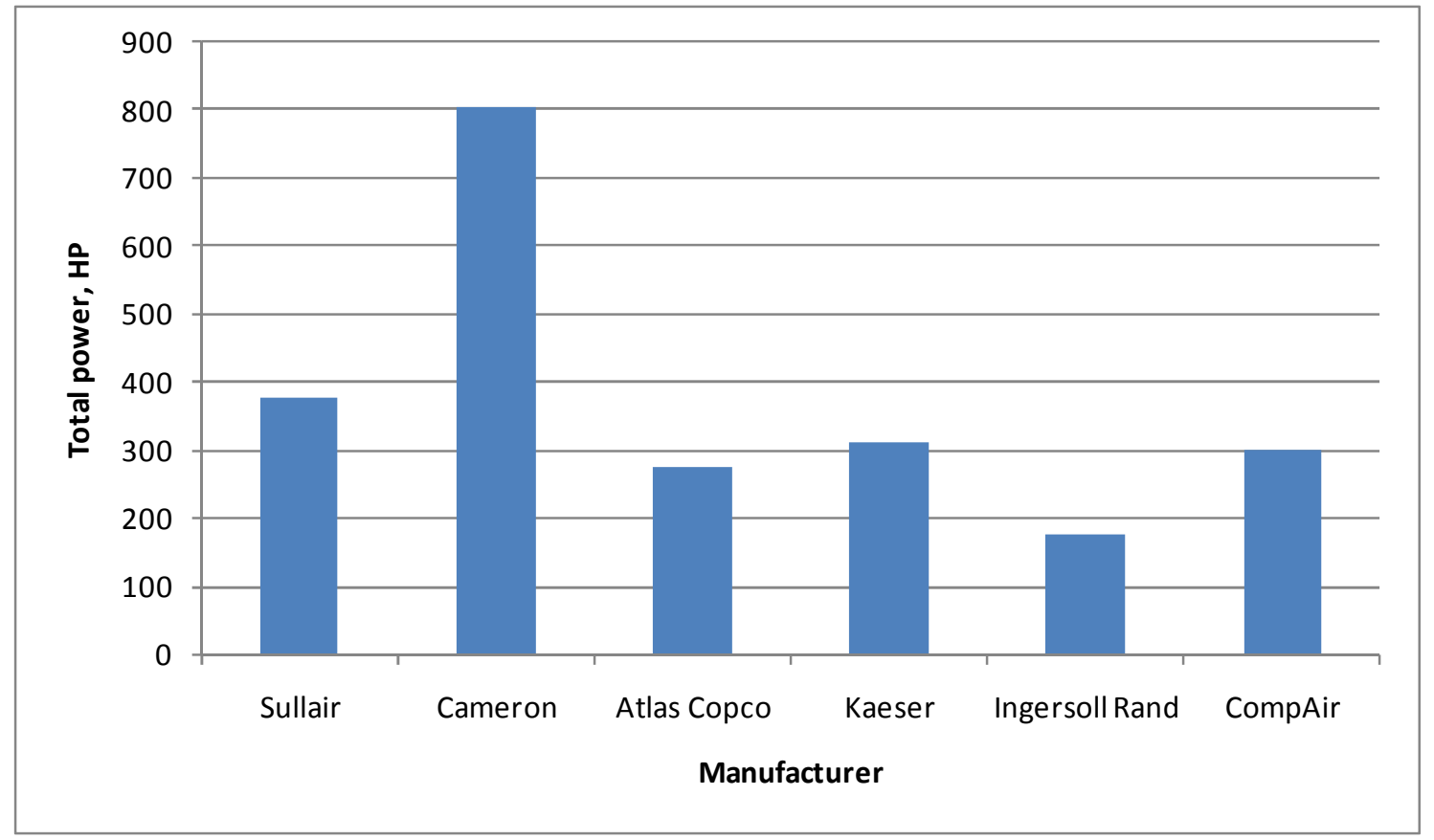

Fig. B.1- Power required for each manufacturer 


\section{B.2) Initial and operating costs}

\begin{tabular}{|l|c|c|c|c|}
\hline Manufacturer & Equipment & Initial cost & Total $^{* *}$ & Operating cost $^{* * *}$ \\
\hline \multirow{2}{*}{ Sullair } & Rotary screw & $99,166.00$ & $196,961.00$ & $68,421.00$ \\
\cline { 2 - 3 } & Reciprocating & $97,795.00$ & & \\
\hline Cameron & Centrifugal & $350,000.00$ & $350,000.00$ & $145,923.00$ \\
\hline \multirow{2}{*}{ Atlas Copco } & Rotary screw & $65,100.00$ & \multirow{2}{*}{$228,900.00$} & $50,161.00$ \\
\cline { 2 - 3 } & Reciprocating & $163,800.00$ & & \\
\hline \multirow{2}{*}{ Ingersoll Rand } & Rotary screw & $61,969.41$ & \multirow{2}{*}{$149,153.84$} & $56,723.00$ \\
\cline { 2 - 3 } & Reciprocating & $49,252.94$ & \\
\cline { 1 - 3 } compare & Rotary screw & $57,123.00$ & \multirow{2}{*}{$137,795.00$} & $32,360.00$ \\
\cline { 2 - 3 } & Reciprocating & $68,744.00$ & & $55,357.00$ \\
\hline
\end{tabular}

* Initial cost includes compressor only

** Total cost includes additional components

*** Based on one year of operation

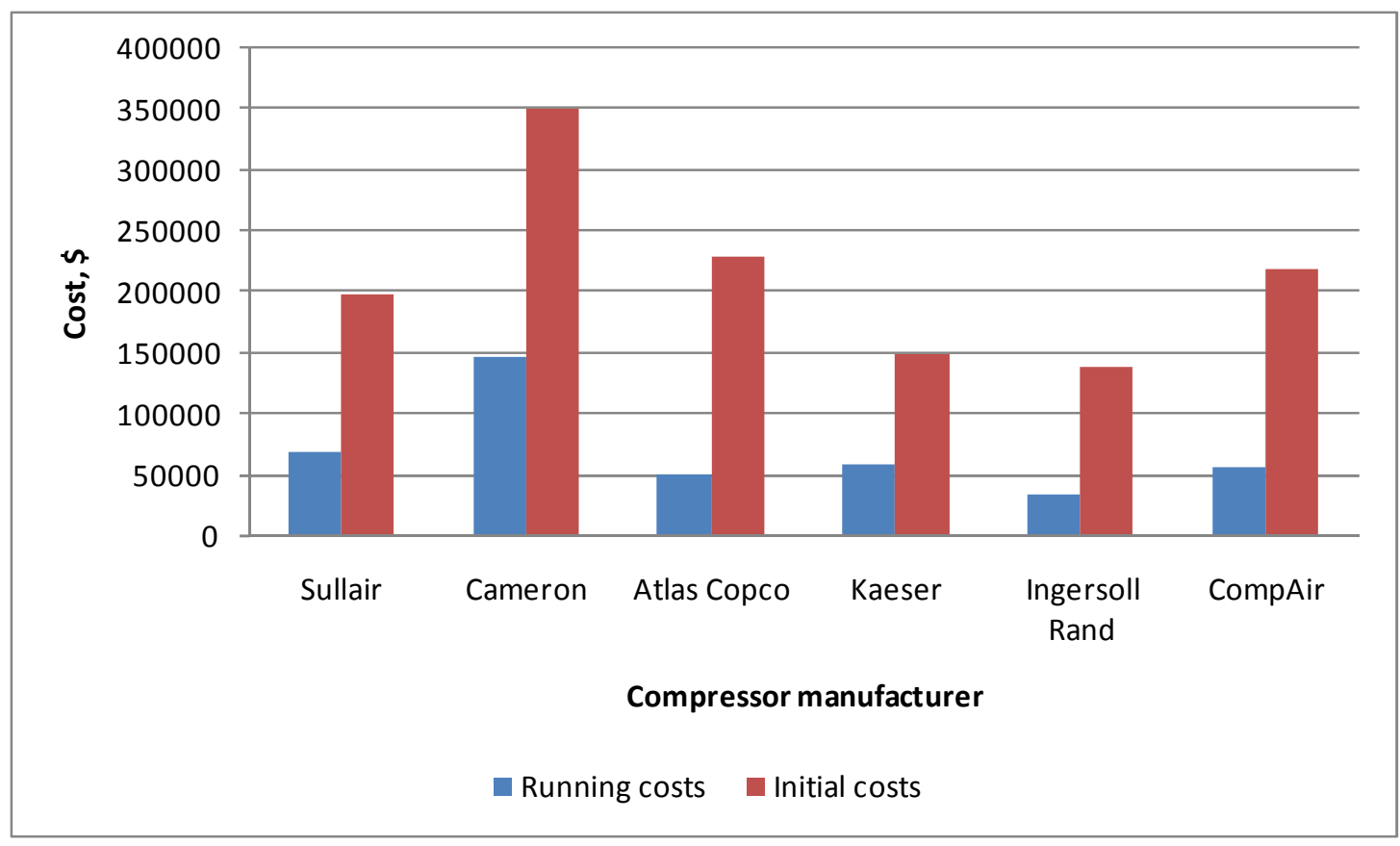

Fig. B.2- Initial and operating costs for each manufacturer 
B.3) Foundation requirements, footprint and weight

\begin{tabular}{|c|c|c|c|c|c|c|}
\hline Manufacturer & Equipment & Footprint ${ }^{*}$ & Total & Weight** & Total & $\begin{array}{l}\text { Foundation } \\
\text { required? }\end{array}$ \\
\hline \multirow[t]{2}{*}{ Sullair } & Rotary screw & 8,640 & \multirow{2}{*}{17,280} & 9,000 & \multirow{2}{*}{16,000} & No \\
\hline & Reciprocating & 8,640 & & 7,000 & & Yes \\
\hline Cameron & Centrifugal & $13,308.33$ & $13,308.33$ & 20,000 & 20,000 & No \\
\hline \multirow{2}{*}{ Atlas Copco } & Rotary screw & 6,867 & \multirow{2}{*}{16,947} & 6,946 & \multirow{2}{*}{14,746} & No \\
\hline & Reciprocating & 10,080 & & 7,800 & & Yes \\
\hline \multirow[t]{2}{*}{ Kaesser } & Rotary screw & $7,982.18$ & \multirow{2}{*}{$11,053.43$} & 8,267 & \multirow{2}{*}{10,692} & No \\
\hline & Reciprocating & $3,071.25$ & & 2,425 & & No \\
\hline \multirow{2}{*}{ Ingersoll Rand } & Rotary screw & 7,749 & \multirow{2}{*}{15,695} & 6,770 & \multirow{2}{*}{10,420} & No \\
\hline & Reciprocating & 7,946 & & 3,650 & & Yes \\
\hline CompAir & Reciprocating & 2,976 & 8,930 & 3,640 & 10,920 & No \\
\hline
\end{tabular}

* Only compressors

** Only compressors

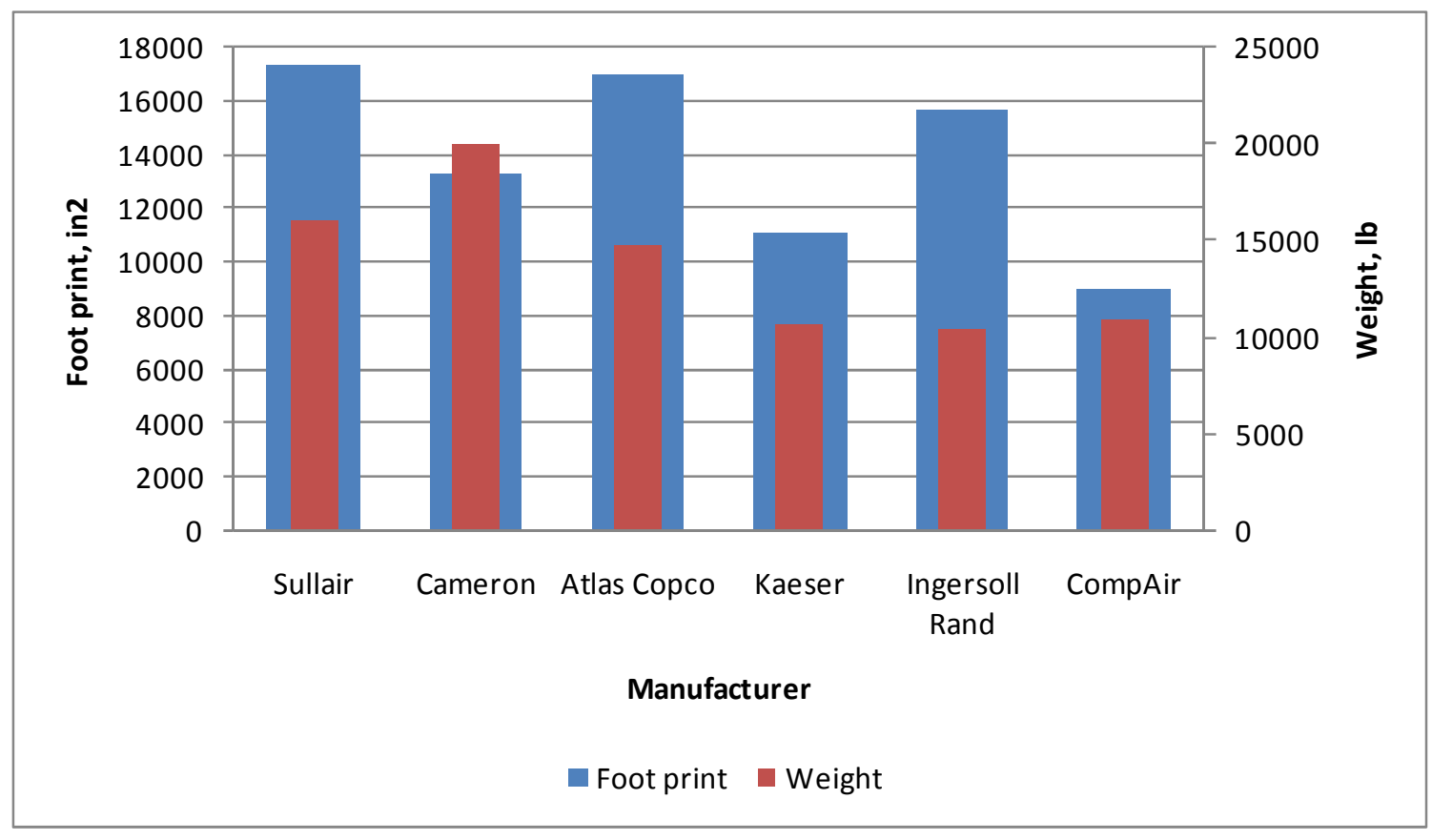

Fig. B.3- Total footprint and weight for each manufacturer 


\section{B.4) Selection process}

There are numerous aspects on which decision aids can differ. The following six major characteristics help evaluate the decision aids. ${ }^{\text {CPS }}$

- Resource requirements:

- Depth of Analysis/Complexity

- Logical rigor

- Group focus

- Quantitativeness

- $\quad$ Track Record

Resource Requirements: include the amount of budget, time, and effort required to perform an analysis with the decision aid.

Depth of Analysis/Complexity: refers to the detail and explicitness with which important aspects of the problem are addressed, and the simplicity with which the aid can perform a complete and thorough analysis of the decision.

Logical Rigor: is concerned with the logical and mathematical soundness of the analysis and the ability to avoid incorrect conclusions.

Group Focus: refers to the ability of the decision air to incorporate group judgments, handle group decision making, and address widely divergent concerns or objectives.

Quantitativeness: is the ability of the decision aid to provide a quantitative basis for the decision, to address sensitivity analysis, and to address decisions such as resource allocation problems, that are inherently quantitative.

Track Record: has to do with how long the decision aid has been available, how many times it has been applied to process safety decisions and similar types of decisions, how widely the aid is accepted, and how much documentation is available on the quality and practicality of the method. 
Table B.1 shows an overview of the characteristics of commonly used decision aids.

Table B.1- Overview of decision aids and their characteristics

\begin{tabular}{|c|c|c|c|c|c|c|}
\hline & $\begin{array}{c}\text { Resource } \\
\text { Requirements }\end{array}$ & $\begin{array}{c}\text { Depth of } \\
\text { Analysis/ } \\
\text { Complexity }\end{array}$ & $\begin{array}{l}\text { Logical } \\
\text { Rigor }\end{array}$ & $\begin{array}{l}\text { Group } \\
\text { focus }\end{array}$ & $\begin{array}{c}\text { Quantitativen } \\
\text { ess }\end{array}$ & $\begin{array}{l}\text { Track } \\
\text { Record }\end{array}$ \\
\hline $\begin{array}{l}\text { Analytic } \\
\text { Hierarchy } \\
\text { Process }\end{array}$ & Moderate & Moderate & Moderate & Moderate & Moderate & Limited \\
\hline $\begin{array}{l}\text { Compromise } \\
\text { Programming }\end{array}$ & Extensive & Extensive & Moderate & Limited & Extensive & Limited \\
\hline $\begin{array}{l}\text { Cost-Benefit } \\
\text { Analysis }\end{array}$ & Moderate & Moderate & Moderate & Limited & Extensive & Extensive \\
\hline $\begin{array}{l}\text { Decsion } \\
\text { Analysis }\end{array}$ & Extensive & Extensive & Extensive & Moderate & Extensive & Moderate \\
\hline Ganne Theory & Muderate & Linniled & Moderate & Limited & Moderate & Limited \\
\hline $\begin{array}{l}\text { Coal } \\
\text { Programming }\end{array}$ & Extensive & Extensive & Moderate & Limited & Extensive & Limited \\
\hline $\begin{array}{l}\text { Kepner-Tregoe } \\
\text { Decision } \\
\text { Analysis }\end{array}$ & Moderate & Moderate & Moderate & Moderate & Moderate & Moderate \\
\hline $\begin{array}{l}\text { Mathematical } \\
\text { Programming }\end{array}$ & Extensive & Extensive & Moderate & Limited & Extensive & Limited \\
\hline $\begin{array}{l}\text { Multiattribute } \\
\text { Utility Analysis }\end{array}$ & Extensive & Extensive & Extersive & Moderate & Extensive & Limited \\
\hline $\begin{array}{l}\text { Nominal } \\
\text { Group } \\
\text { Technique }\end{array}$ & Moderate & Limited & Limited & Extensive & Limited & Umited \\
\hline $\begin{array}{l}\text { Outranking } \\
\text { Methods }\end{array}$ & Moderate & Moderate & Limited & Limired & Moderate & Limited \\
\hline $\begin{array}{l}\text { Payoff Matrix } \\
\text { Analysis }\end{array}$ & Moderate & Moderate & Moderate & Moderate & Moderate & Limited \\
\hline $\begin{array}{l}\text { Portfolio } \\
\text { Analysis }\end{array}$ & Extensive & Moderate & Moderate & Limited & Extensive & Limited \\
\hline $\begin{array}{l}\text { Screening' } \\
\text { Ranking } \\
\text { Methods }\end{array}$ & Limited & Limited & Moderate & Moderate & Moderate & Limited \\
\hline SMART & Moderate & Moderate & Moderate & Limited & Moderate & Limited \\
\hline Voting & Limited & Limited & Limited & Extensive & Limited & Limited \\
\hline
\end{tabular}


Six problem classes are useful for identifying the appropriate decision aid for a problem. Table B.2 summarizes the class, distinguishing problem aspects and the recommended decision aid.

Table B.2- Problem classes and appropriate decision aids

\begin{tabular}{|c|c|c|c|c|c|c|}
\hline \multirow{2}{*}{ Problem Class } & \multicolumn{5}{|c|}{ Distinguishing Problem Aspects } & \multirow{2}{*}{$\begin{array}{l}\text { Well-Suited } \\
\text { Decision Aids }\end{array}$} \\
\hline & $\begin{array}{l}\text { Resource } \\
\text { Availability }\end{array}$ & $\begin{array}{c}\text { Problem } \\
\text { Complexity }\end{array}$ & $\begin{array}{l}\text { Impor- } \\
\text { tance/ } \\
\text { Scrutiny }\end{array}$ & $\begin{array}{l}\text { Croup In- } \\
\text { volvement }\end{array}$ & $\begin{array}{l}\text { Need for } \\
\text { Quantif- } \\
\text { ication }\end{array}$ & \\
\hline 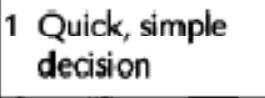 & Low & Low & Low & Low & Low & Screening/Ranking \\
\hline $\begin{array}{l}2 \text { Quick group } \\
\text { decision }\end{array}$ & Low & - & - & High & Low & Voting \\
\hline $\begin{array}{l}3 \text { More } \\
\text { thorough } \\
\text { group decision }\end{array}$ & High & - & - & High & Low & $\begin{array}{l}\text { Nominal Group } \\
\text { Technique }\end{array}$ \\
\hline $\begin{array}{l}4 \text { Quick, highly } \\
\text { quantitative } \\
\text { nongroup } \\
\text { decision }\end{array}$ & Low & - & - & Low & High & $\begin{array}{l}\text { Analytic Hierarchy } \\
\text { Process } \\
\text { Kepner-Tregoe } \\
\text { Decision Analysis } \\
\text { Payoff Matrix } \\
\text { Analysis } \\
\text { Outranking } \\
\text { SMART }\end{array}$ \\
\hline $\begin{array}{l}5 \text { Quick, highly } \\
\text { quantitative } \\
\text { group decision }\end{array}$ & Low & - & - & High & High & $\begin{array}{l}\text { Payoff Matrix } \\
\text { Analysis } \\
\text { Kepner-Tregoe } \\
\text { Decision Analysis } \\
\text { Analytic } \\
\text { Hierarchy Process }\end{array}$ \\
\hline $\begin{array}{l}6 \text { Complex, } \\
\text { quantitative } \\
\text { decision with } \\
\text { high importance } \\
\text { and resource } \\
\text { availability }\end{array}$ & High & High & High & - & High & $\begin{array}{l}\text { Cost-Benefit } \\
\text { Analysis } \\
\text { Compromise } \\
\text { Programming } \\
\text { Decision Analysis } \\
\text { Coal Programming } \\
\text { Multiattribute } \\
\text { Utility Analysis } \\
\text { Mathematical } \\
\text { Programming }\end{array}$ \\
\hline
\end{tabular}


For this investigation the Kepner-Tregoe decision analysis and the Analytic Hierarchy process have been selected to identify the potential supplier for the compressed air system.

\section{a) Kepner-Tregoe Decision Analysis}

The steps followed by this methodology can be summarized in 9 steps:

1. Construct a decision statement

2. List the objectives of the decision

3. Divide the objectives into MUSTS and WANTS

4. Assign weights to the WANTS

5. Evaluate the alternatives on the MUSTS

6. Find a total weighted score for each alternative

7. Make a "tentative" choice

8. Examine the possible negative consequences

9. Make a final decision

A key strength of Kepner-Tregoe decision analysis is its simple, structured methodology. It is easy to implement and avoids complicated mathematical computations. Decisions can be more easily communicated and justified by showing that a formal methodology is used. However, one weakness of the method is that the numbers can be easily manipulated to favor an alternative. Nevertheless, the approach taken in the following analysis and the inherent nature of the project justifies the weights considered for making the final decision. 

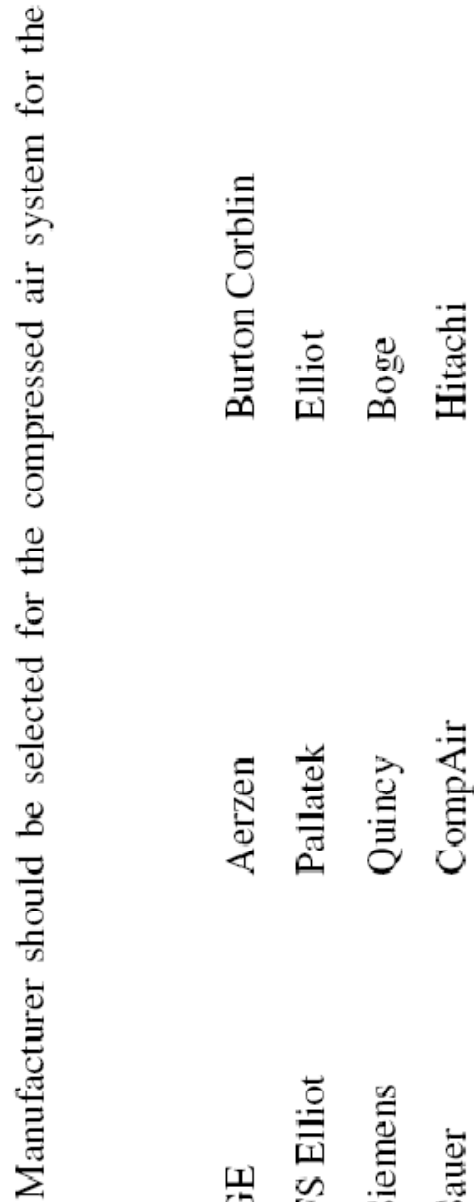

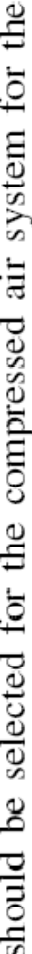

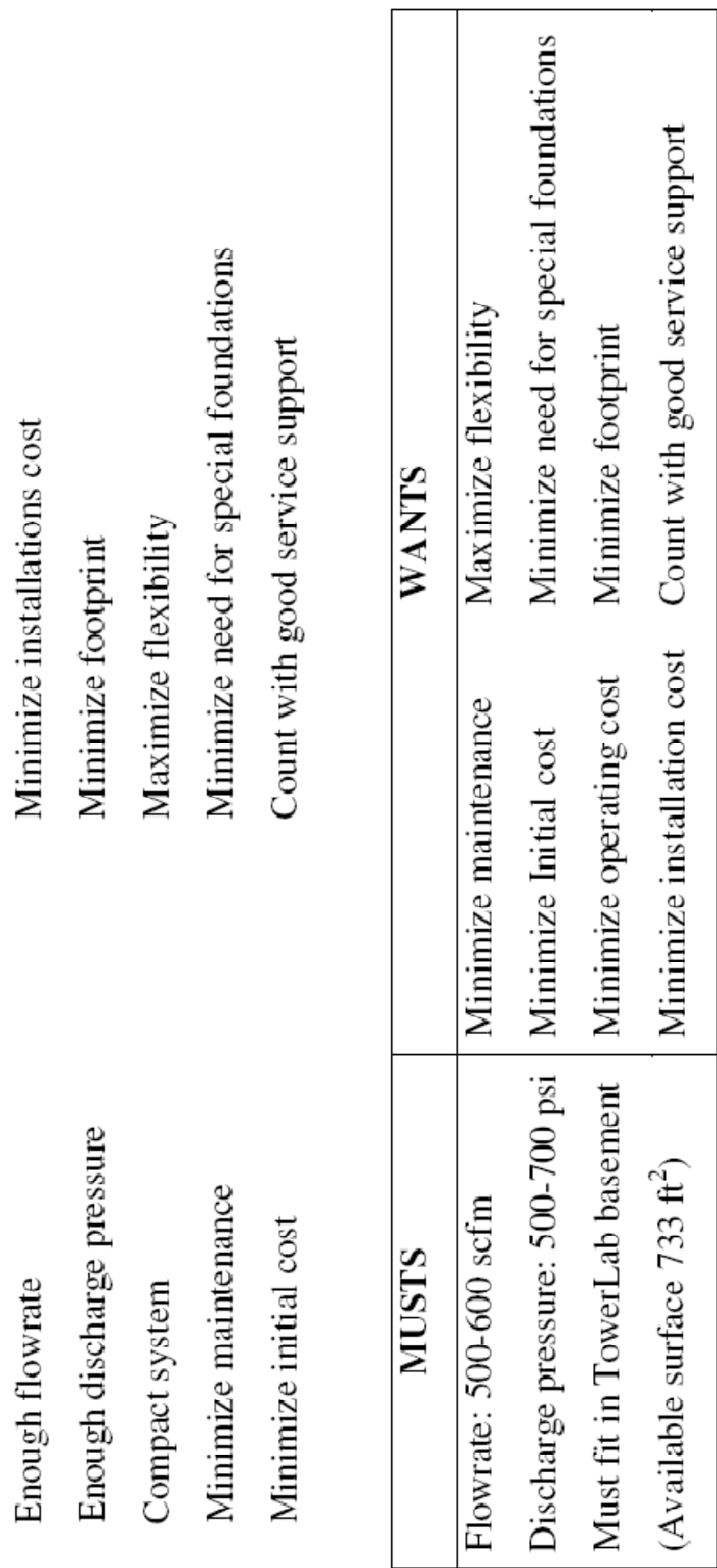

تُ

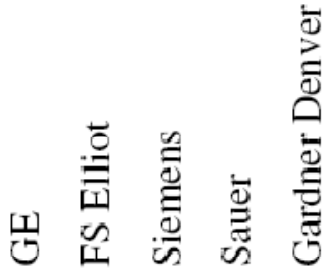

Q

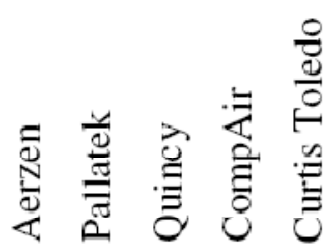

童

言苛

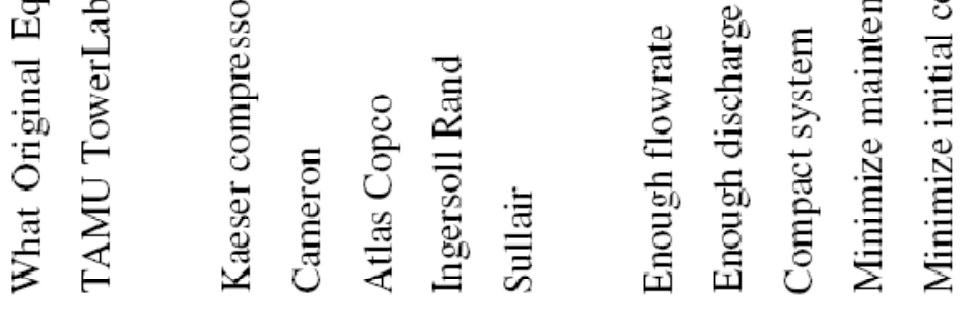

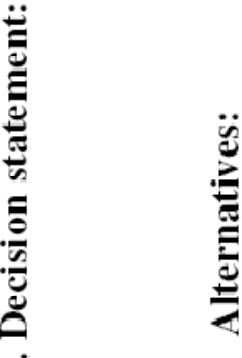

苞
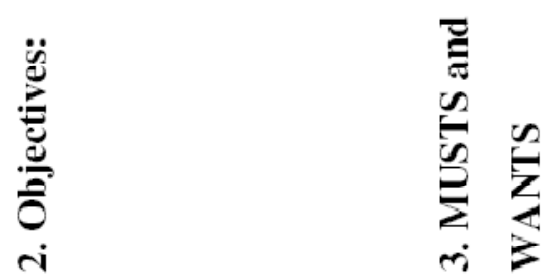


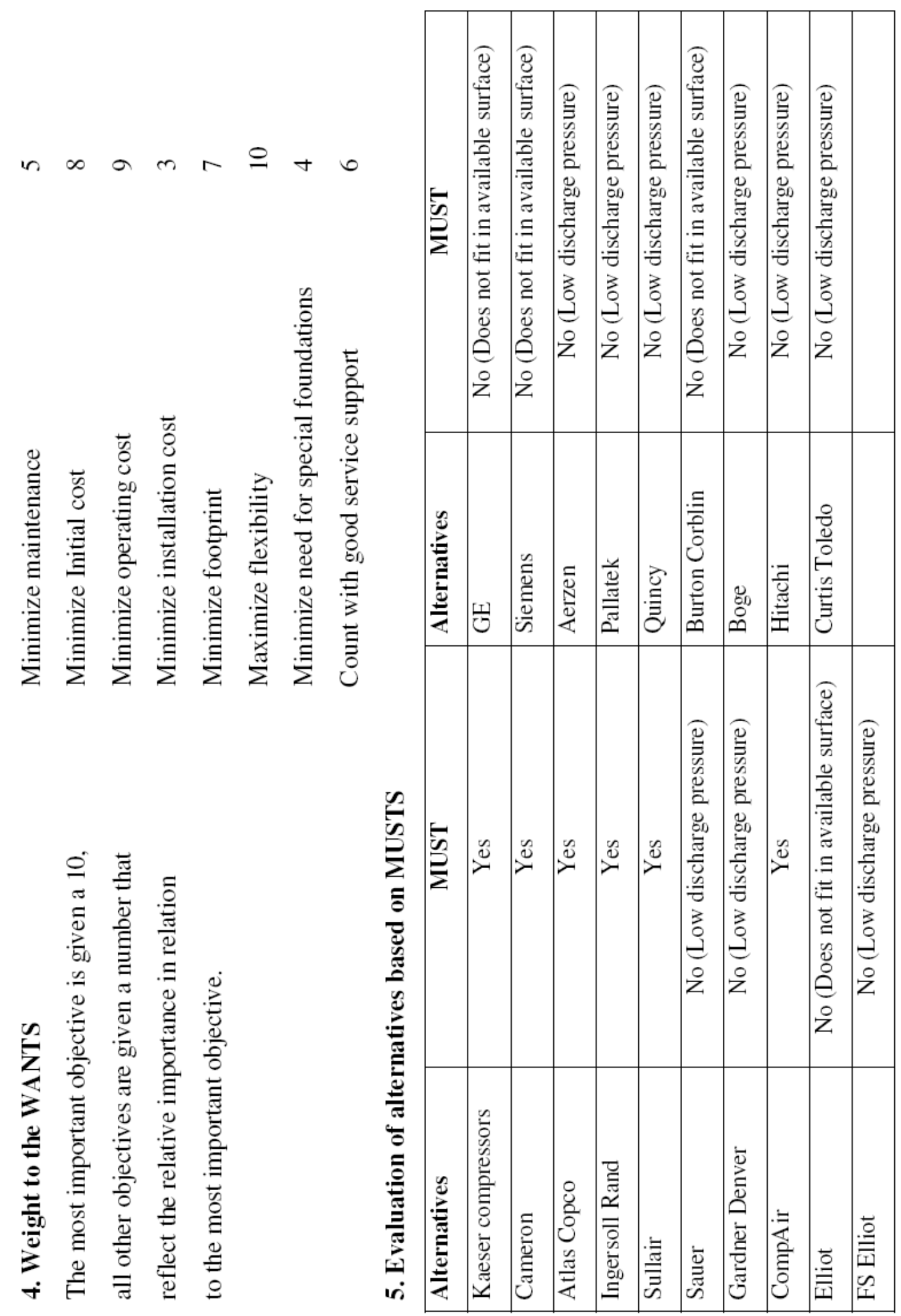



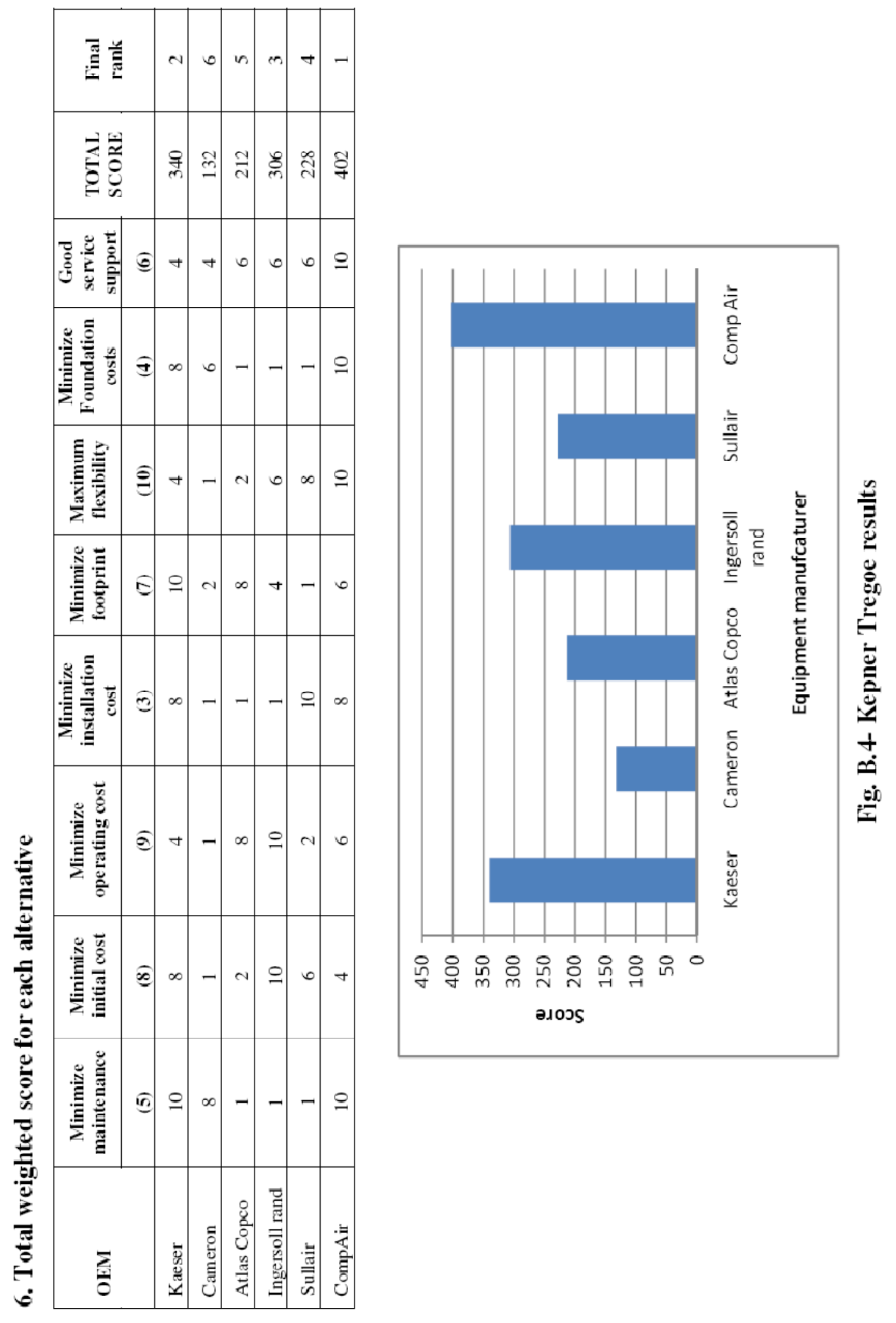


\section{Tentative choices}

- CompAir

- Kaeser

- Ingersoll Rand

\section{Negative consequences}

No negative consequences are expected since all the options comply with the technical requirements needed for the TowerLab.

\section{Final decision}

CompAir is the potential provider for the compressed air system for the TAMU TowerLab.

\section{b) Analytic Hierarchy Process}

The analytic hierarchy process is designed to structure decision problems hierarchically. The hierarchy consists of an overall objective at the top level, criteria that lead to the overall objective in the middle levels, and at the bottommost level, the set of possible alternatives.

For each level, weights are determined for each criterion that appears in that level. These weights are called prioirities and they express how important each criterion in that level is in fulfilling the criterion just above it. These priorities are derived from a series of pairwise comparisons made between the elements on one level with respect to their contribution to the elements on the next highest level. At the most bottommost level, priorities are determined for the alternatives themselves. These priorities express the degree to which each alternative satisfies each criterion in the next level up. The priorities of the alternatives on the bottom level are weighted by the priorities of the objectives in the other levels to find an overall priority for each alternative. This overall priority is the total weighted score.

As with Kepner-Tregeo, the analytic hierarchy process provides the steps needed to be followed to make the final decision: 
1. Define the decision problem

2. Structure a decision hierarchy

3. Construct pairwise comparison matrices

4. Calculate the priorities for the elements of the hierarchy

5. Find the overall priority for each alternative

6. Evaluate the results and make a decision

1. Decision problem: Which compressed air system supplier should be selected for the TAMU TowerLab?

\section{Decision hierarchy:}

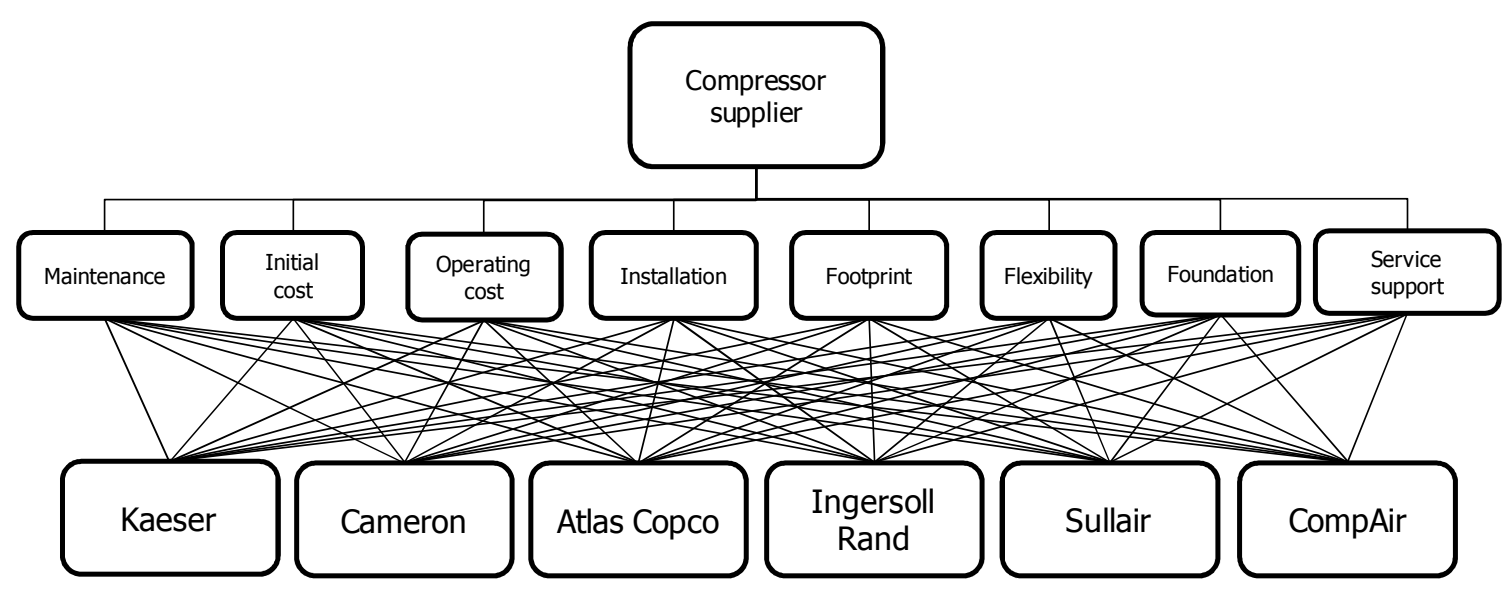

Fig. B.5- Decision hierarchy for compressed air system supplier 


\begin{tabular}{|c|c|c|c|c|c|c|c|c|c|}
\hline 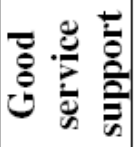 & $\cong$ & $\stackrel{ \pm}{ \pm}$ & - & $\stackrel{n}{=}$ & $\stackrel{n}{=}$ & $n$ & $\stackrel{n}{=}$ & - & $\frac{\infty}{\infty}$ \\
\hline 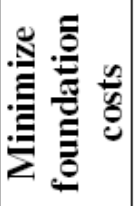 & $m$ & N & $n$ & $N$ & $\stackrel{ \pm}{ \pm}$ & $n$ & - & $n$ & $\begin{array}{l}n \\
2 \\
2 \\
\end{array}$ \\
\hline 帮竞 & $\stackrel{n}{=}$ & $\stackrel{n}{=}$ & $\stackrel{n}{=}$ & $\stackrel{n}{=}$ & $\stackrel{n}{=}$ & - & $\stackrel{n}{=}$ & $\stackrel{n}{=}$ & $\begin{array}{l}\stackrel{\circ}{+} \\
\text { i }\end{array}$ \\
\hline 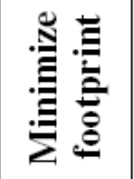 & in & $\nabla$ & $n$ & $m$ & - & $n$ & $\nabla$ & $n$ & $\begin{array}{l}8 \\
\text { in } \\
\text { r }\end{array}$ \\
\hline 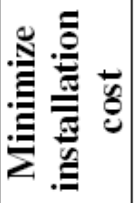 & $m$ & $m$ & $n$ & - & $\stackrel{\varrho}{=}$ & in & $\stackrel{N}{S}$ & $n$ & 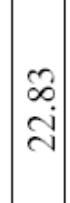 \\
\hline 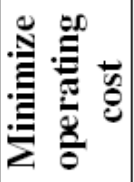 & $\stackrel{n}{=}$ & $\stackrel{\curvearrowleft}{=}$ & - & $\stackrel{n}{=}$ & $\stackrel{n}{=}$ & $n$ & $\stackrel{n}{=}$ & $\stackrel{N}{S}$ & in \\
\hline 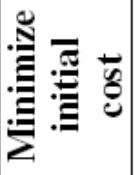 & $n$ & - & $n$ & $\stackrel{\varrho}{=}$ & \pm & $n$ & $\stackrel{\Omega}{I}$ & - & $\begin{array}{c}\infty \\
\stackrel{0}{0} \\
\infty\end{array}$ \\
\hline 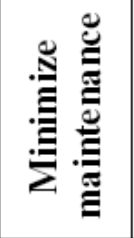 & - & $\stackrel{n}{=}$ & $n$ & $\stackrel{?}{S}$ & $\stackrel{n}{=}$ & $n$ & $\stackrel{9}{S}$ & $m$ & $\begin{array}{l}0 \\
1 \\
1\end{array}$ \\
\hline & 胥 & 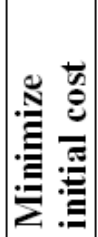 & 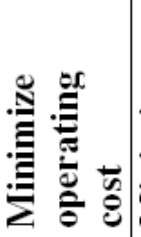 & 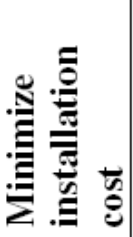 & 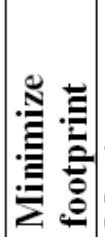 & 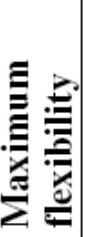 & 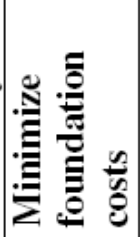 & 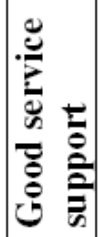 & $\frac{n}{\frac{\pi}{6}}$ \\
\hline
\end{tabular}




\begin{tabular}{|c|c|c|c|c|c|c|c|c|c|}
\hline 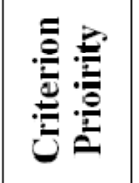 & $\begin{array}{l}\stackrel{ \pm}{=} \\
0\end{array}$ & $\begin{array}{l}\stackrel{8}{\circ} \\
\stackrel{0}{0}\end{array}$ & $\frac{\sigma}{\sigma}$ & $\begin{array}{l}0 \\
0 \\
0 \\
0\end{array}$ & 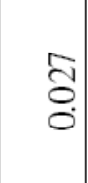 & $\begin{array}{l}\tilde{b} \\
\vdots \\
0\end{array}$ & $\begin{array}{l}\text { fo } \\
\text { : } \\
0\end{array}$ & $\frac{9}{0}$ & \\
\hline 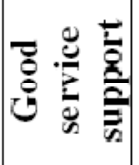 & 守 & $\overline{8}$ & $\frac{\tilde{I}}{0}$ & $\begin{array}{l}\text { İ } \\
\stackrel{0}{0}\end{array}$ & $\begin{array}{l}\stackrel{1}{2} \\
\text { o. }\end{array}$ & $\begin{array}{l}\vec{\sigma} \\
\stackrel{0}{0}\end{array}$ & 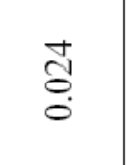 & $\frac{d}{0}$ & 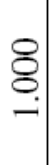 \\
\hline 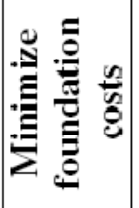 & $\begin{array}{l}\text { ปे } \\
\text { : }\end{array}$ & $\begin{array}{l}0 \\
\stackrel{0}{0} \\
0\end{array}$ & $\frac{n}{\stackrel{n}{c}}$ & $\begin{array}{l}0 \\
\stackrel{0}{0} \\
\stackrel{0}{0}\end{array}$ & $\vec{\sigma}$ & $\frac{n}{2}$ & $\frac{9}{0}$ & $\frac{n}{3}$ & $\underset{8}{\stackrel{8}{8}}$ \\
\hline 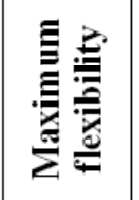 & $\begin{array}{l}0 \\
0 \\
O \\
0\end{array}$ & 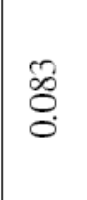 & Oी. & $\begin{array}{l}0 \\
0 \\
O \\
0\end{array}$ & 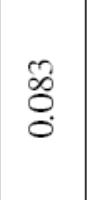 & $\stackrel{5}{ \pm}$ & $\begin{array}{l}\mathscr{O} \\
\mathscr{O} \\
O \\
0\end{array}$ & $\begin{array}{l}\mathscr{O} \\
\stackrel{0}{0}\end{array}$ & $\underset{8}{8}$ \\
\hline 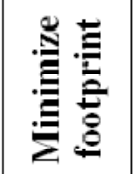 & $\frac{0}{\stackrel{2}{0}}$ & $\frac{a}{3}$ & $\frac{\mathscr{L}}{\circ}$ & $\begin{array}{l}\text { ta } \\
\text { : } \\
0\end{array}$ & $\bar{\delta}$ & $\frac{\circ}{0}$ & $\stackrel{2}{\stackrel{2}{0}}$ & $\frac{\mathscr{0}}{0}$ & 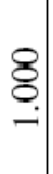 \\
\hline 苞高 & $\frac{\overrightarrow{2}}{0}$ & $\stackrel{\bar{a}}{0}$ & $\frac{\curvearrowright}{\hat{\sigma}}$ & $\begin{array}{l}\frac{J}{0} \\
\stackrel{0}{0}\end{array}$ & $\stackrel{n}{\frac{n}{0}}$ & $\frac{\ddot{c}}{\hat{\sigma}}$ & तิ & $\frac{\vartheta}{\tilde{\sigma}}$ & \&. \\
\hline 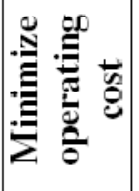 & ڤ్ర & 홍 & $\stackrel{m}{\stackrel{0}{0}}$ & ठิ & ठิ & ¿̊: & ठิ & 8 & $\stackrel{8}{8}$ \\
\hline 总毵 & 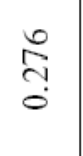 & $\begin{array}{l}n \\
8 \\
0 \\
0\end{array}$ & $\begin{array}{l}\stackrel{\circ}{\text { s }} \\
\text { s. }\end{array}$ & $\stackrel{\infty}{\frac{\infty}{0}}$ & $\frac{\Delta}{0}$ & $\begin{array}{l}\stackrel{0}{\Delta} \\
\text { ș } \\
0\end{array}$ & 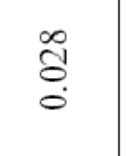 & $\begin{array}{l}n \\
\text { ñ } \\
0 \\
0\end{array}$ & $\stackrel{8}{8}$ \\
\hline 苋 & $\begin{array}{l}\stackrel{\circ}{\circ} \\
:\end{array}$ & $\stackrel{2}{\circ}$ & ๙ึ? & तี & $\stackrel{m}{\circ}$ & ֶै? & तี & $\frac{\sigma}{\sigma}$ & $\begin{array}{l}8 \\
\varnothing \\
\end{array}$ \\
\hline & 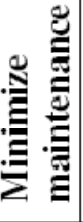 & 产 & 照 & . & |气 & 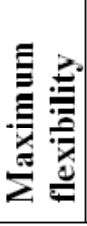 & 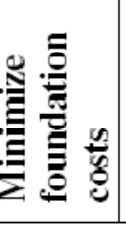 & 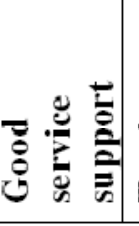 & \\
\hline
\end{tabular}



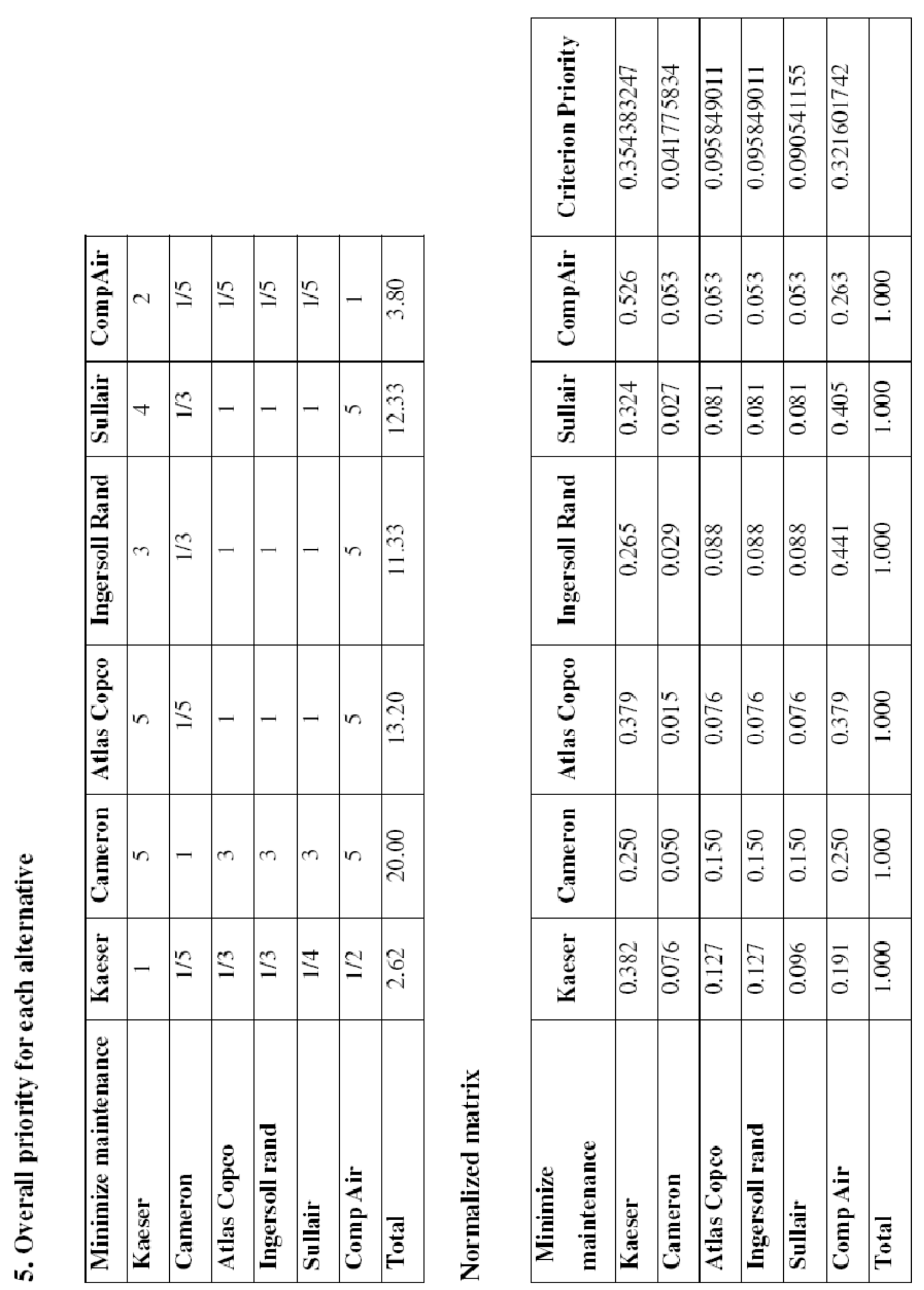

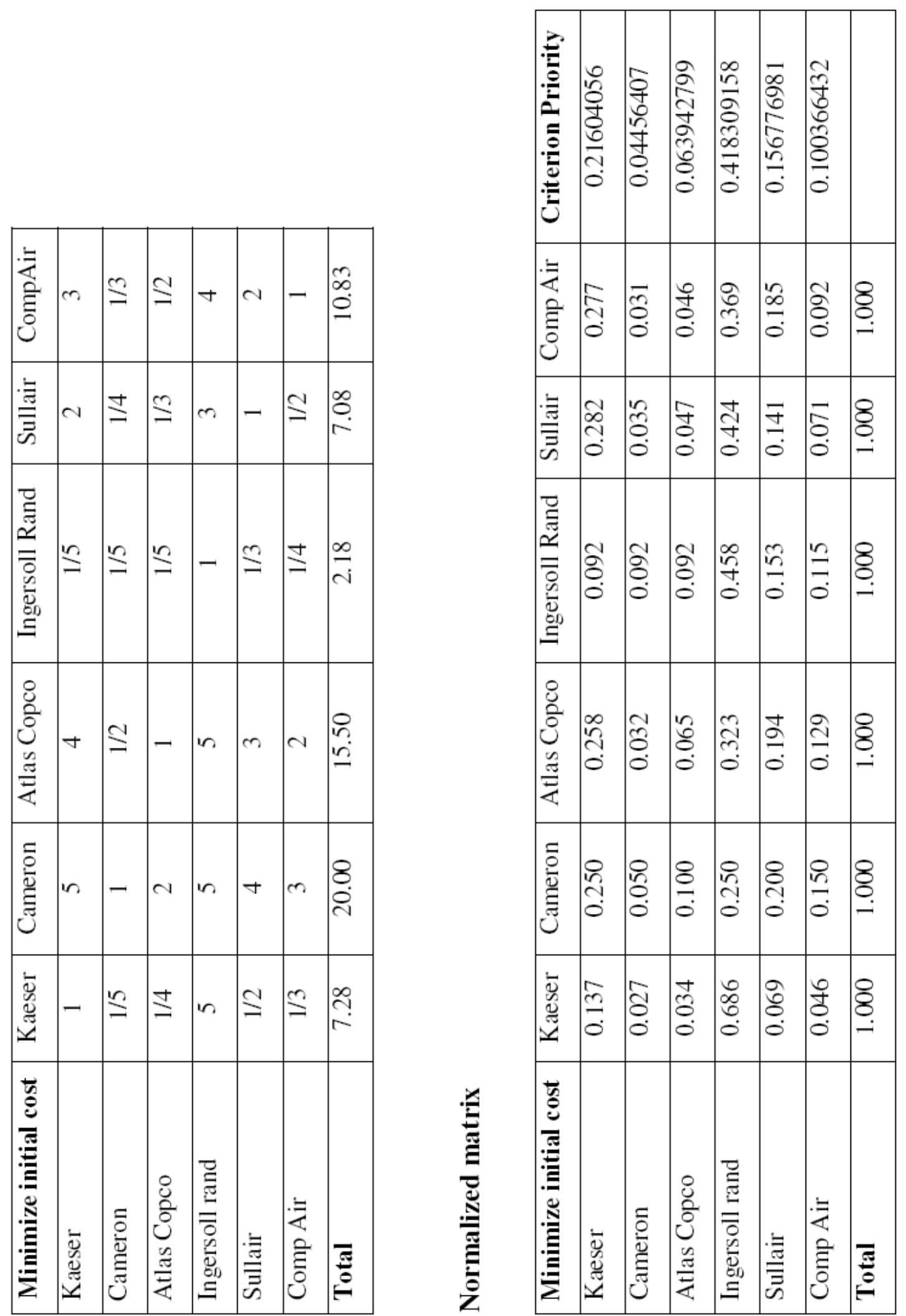


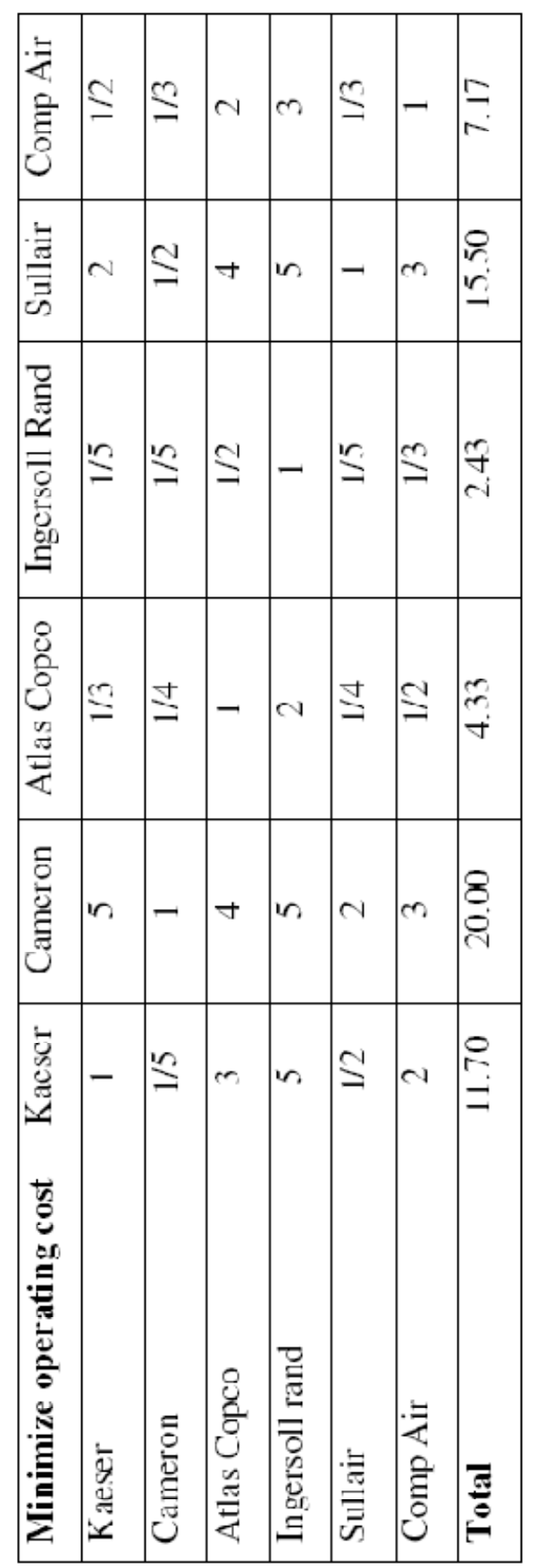

\begin{tabular}{|c|c|c|c|c|c|c|c|}
\hline 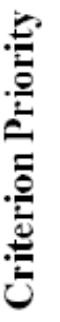 & 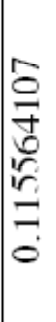 & 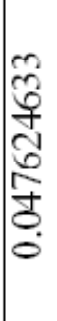 & 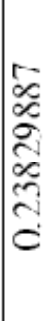 & 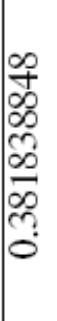 & 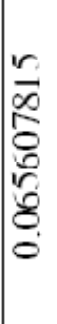 & 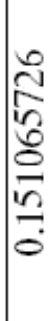 & \\
\hline 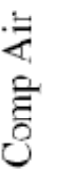 & $\stackrel{8}{0}$ & 寺 & $\stackrel{尺}{\curvearrowright}$ & $\frac{\theta}{\partial}$ & 吉 & $\frac{9}{\dot{9}}$ & 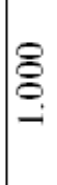 \\
\hline 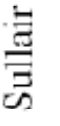 & $\frac{\partial}{\stackrel{-1}{0}}$ & 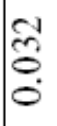 & $\begin{array}{l}\stackrel{c}{\sim} \\
\stackrel{2}{c} \\
c\end{array}$ & $\begin{array}{l}\hat{y} \\
\text { ma } \\
0\end{array}$ & $\begin{array}{l}8 \\
0 \\
0\end{array}$ & $\frac{\dot{\sigma}}{\overrightarrow{0}}$ & $\underset{\mathscr{E}}{\mathscr{E}}$ \\
\hline 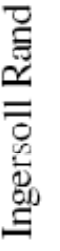 & 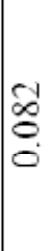 & $\mid \begin{array}{l}\infty \\
\infty \\
0 \\
0\end{array}$ & $\frac{n}{2}$ & 声 & $\begin{array}{l}\text { cy } \\
0 \\
0 \\
0 \\
0\end{array}$ & $\frac{\pi}{0}$ & $\underset{\varrho}{\varrho}$ \\
\hline 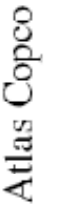 & 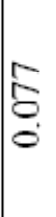 & $\begin{array}{l}\infty \\
2 \\
\vdots \\
0 \\
0\end{array}$ & $\sqrt{2}$ & \begin{tabular}{l} 
Co \\
\multirow{0}{0}{} \\
0
\end{tabular} & $\begin{array}{l}\infty \\
v \\
0 \\
0\end{array}$ & $\frac{n}{0}$ & $\underset{-}{\S}$ \\
\hline 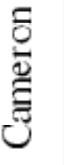 & 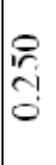 & $\frac{\hat{n}}{\hat{0}}$ & 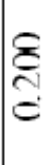 & مि & $\frac{\hat{\sigma}}{0}$ & $\frac{\vec{n}}{0}$ & 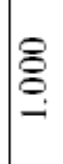 \\
\hline 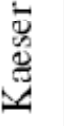 & 点 & $\frac{2}{0}$ & $\stackrel{c}{2}$ & $\frac{5}{y}$ & $\frac{\text { On }}{0}$ & $\frac{F}{0}$ & $\underset{\wp}{\varnothing}$ \\
\hline 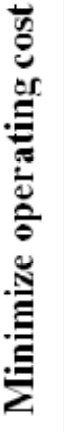 & 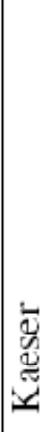 & 竞 & 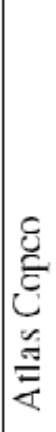 & 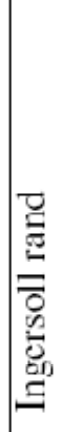 & 言 & $\begin{array}{l}\exists \\
\tilde{\Xi} \\
\tilde{\Xi} \\
0\end{array}$ & 홍 \\
\hline
\end{tabular}




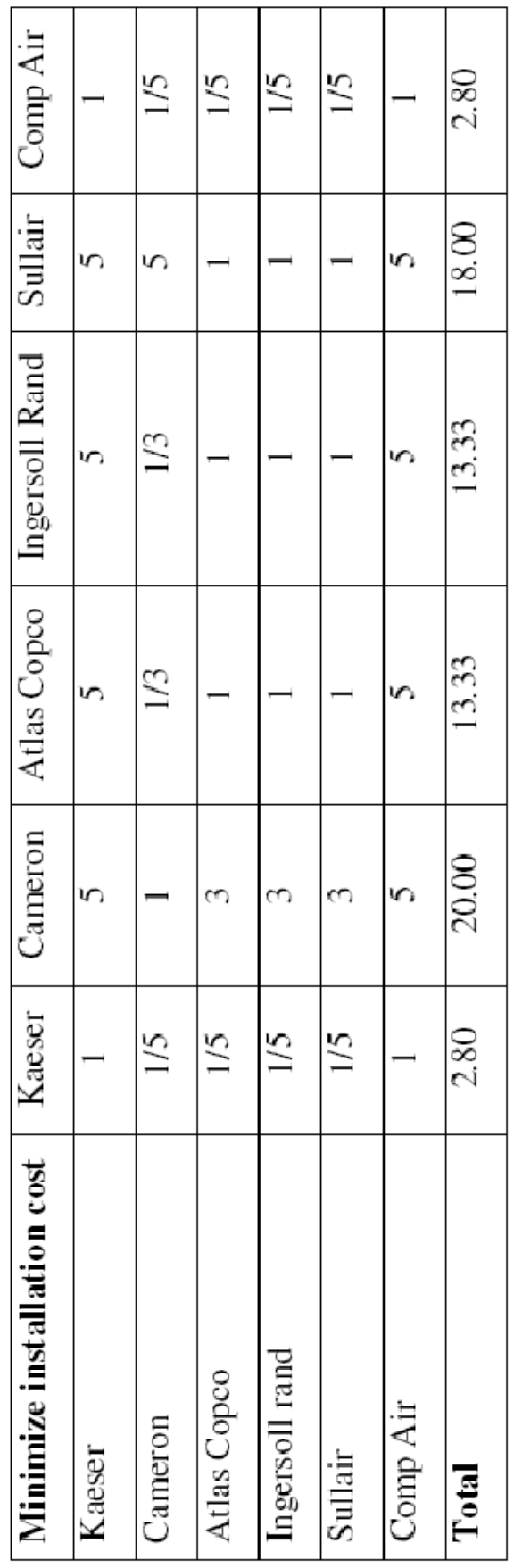

\begin{tabular}{|c|c|c|c|c|c|c|c|}
\hline 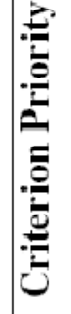 & 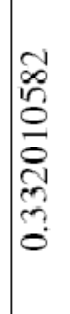 & 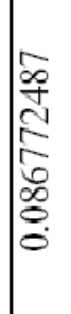 & $\begin{array}{l}\infty \\
\infty \\
\infty \\
\infty \\
0 \\
0 \\
\infty \\
\infty \\
0 \\
0 \\
0\end{array}$ & $\begin{array}{l}\hat{\infty} \\
\infty \\
0 \\
0 \\
0 \\
\infty \\
0 \\
0 \\
0\end{array}$ & $\begin{array}{l}0 \\
\infty \\
\infty \\
0 \\
0 \\
0 \\
0 \\
\infty \\
0 \\
0 \\
0\end{array}$ & 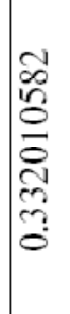 & \\
\hline 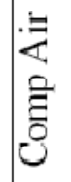 & $\begin{array}{l}\hat{n} \\
\tilde{n} \\
0\end{array}$ & $\overline{0}$ & $\overline{0}$ & $\underline{\bar{\theta}}$ & $\underline{\mathbf{S}}$ & శn & $\stackrel{8}{8}$ \\
\hline 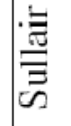 & 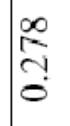 & 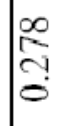 & مُ & 吕 & $\begin{array}{l}0 \\
0 \\
0 \\
0 \\
0\end{array}$ & 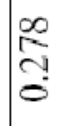 & $\underset{8}{8}$ \\
\hline 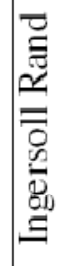 & ?n & ڤ̂̃ & $\frac{n}{0}$ & $\frac{1}{0}$ & مo & $\frac{2}{2}$ & $\underset{8}{\mathscr{8}}$ \\
\hline 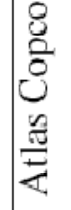 & $\stackrel{n}{2}$ & $\stackrel{1}{2}$ & $\frac{1}{6}$ & $\frac{n}{0}$ & $\stackrel{n}{0}$ & $\frac{12}{3}$ & 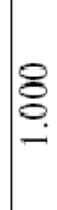 \\
\hline 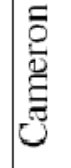 & م્ & $\begin{array}{l}0 \\
0 \\
0 \\
0\end{array}$ & $\frac{8}{0}$ & $\frac{0}{0}$ & $\frac{n}{0}$ & 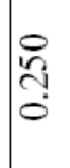 & ஜ \\
\hline 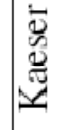 & ñ & 官 & 官 & $\overline{0}$ & $\overrightarrow{\hat{O}}$ & $\begin{array}{l}\tilde{n} \\
\tilde{2} \\
0\end{array}$ & $\underset{-}{8}$ \\
\hline 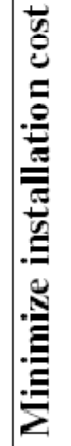 & 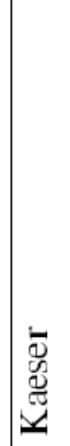 & 卷 & 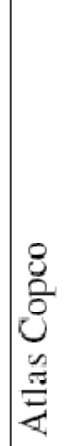 & 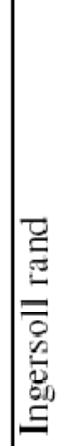 & $\frac{\sqrt{\Xi}}{\bar{E}}$ & 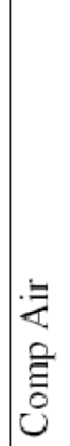 & 홍 \\
\hline
\end{tabular}




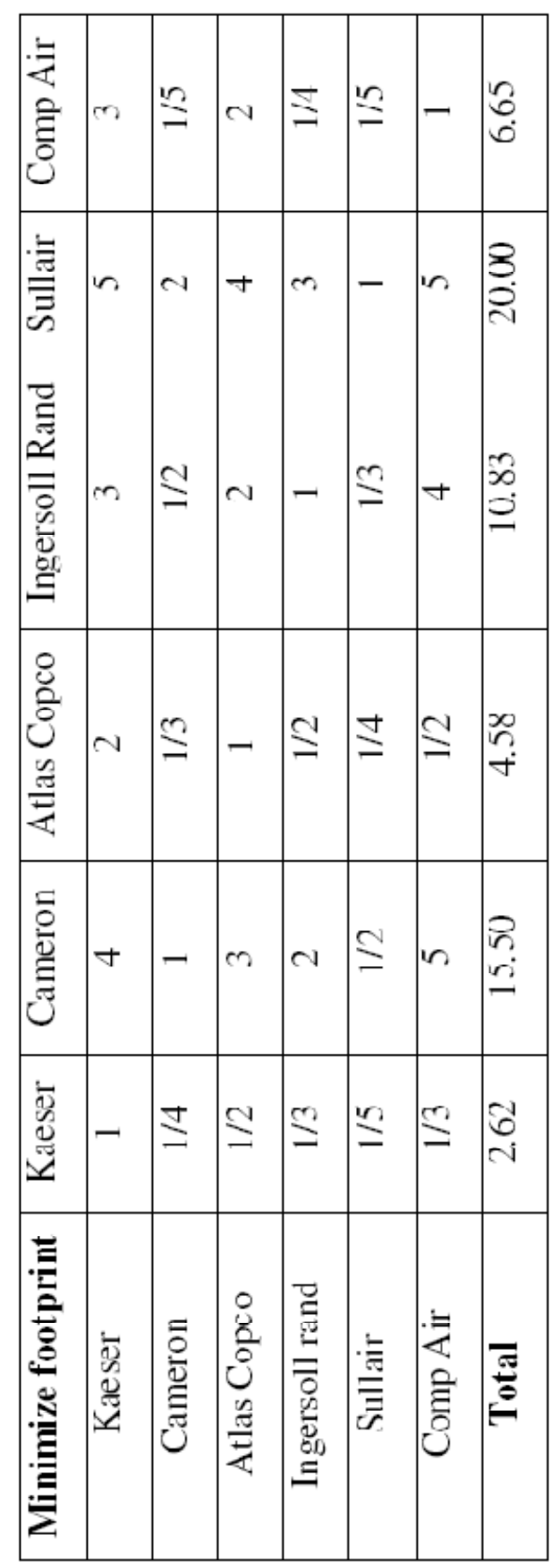

\begin{tabular}{|c|c|c|c|c|c|c|c|}
\hline 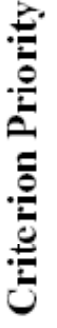 & 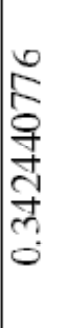 & 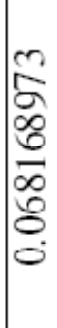 & 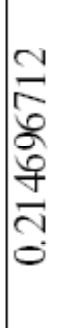 & 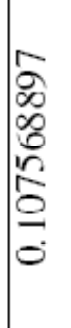 & $\begin{array}{l}0 \\
\frac{1}{0} \\
0 \\
0 \\
0 \\
10 \\
0 \\
0 \\
0\end{array}$ & 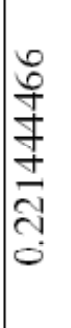 & \\
\hline 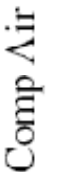 & 离 & ڤ్ & ळ) & $\begin{array}{l}\infty \\
0 \\
0 \\
0\end{array}$ & 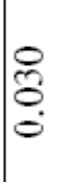 & $\stackrel{0}{0}$ & 8 \\
\hline$\underset{\tilde{\Xi}}{\stackrel{\vec{\Xi}}{\bar{\Xi}}}$ & 号 & $\frac{8}{8}$ & \&્y & $\frac{0}{0}$ & 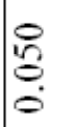 & $\begin{array}{l}0 \\
0 \\
0 \\
0 \\
0\end{array}$ & $\underset{8}{8}$ \\
\hline 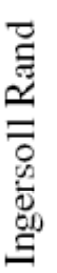 & 송 & 叧 & $\frac{\infty}{0}$ & $\hat{O}_{0}^{1}$ & $\vec{\beta}$ & $\begin{array}{l}\hat{b} \\
\text { ? } \\
0\end{array}$ & ○్ \\
\hline 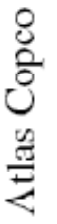 & $\frac{0}{\stackrel{7}{+}}$ & $\stackrel{m}{\stackrel{0}{0}}$ & $\frac{\infty}{\stackrel{1}{c}}$ & $\frac{8}{0}$ & $\begin{array}{l}n \\
0 \\
0 \\
0\end{array}$ & $\frac{8}{\frac{8}{0}}$ & § \\
\hline 妾 & $\begin{array}{l}\infty \\
\stackrel{n}{n} \\
\end{array}$ & $\stackrel{10}{\circ}$ & $\frac{1}{0}$ & $\frac{2}{0}$ & लै & 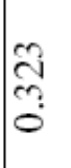 & 8 \\
\hline 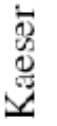 & $\begin{array}{l}\infty \\
\infty \\
\infty \\
0\end{array}$ & $\stackrel{\circ}{\circ}$ & $\frac{\bar{a}}{0}$ & $\frac{\widehat{0}}{0}$ & $\frac{0}{O}$ & $\frac{\vec{N}}{0}$ & 8 \\
\hline 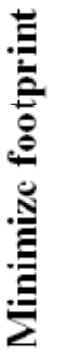 & 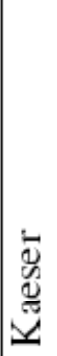 & 离 & 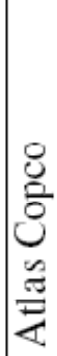 & 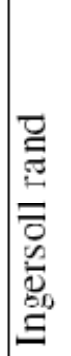 & 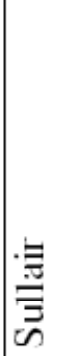 & 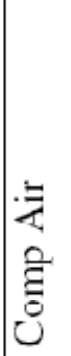 & 吾 \\
\hline
\end{tabular}



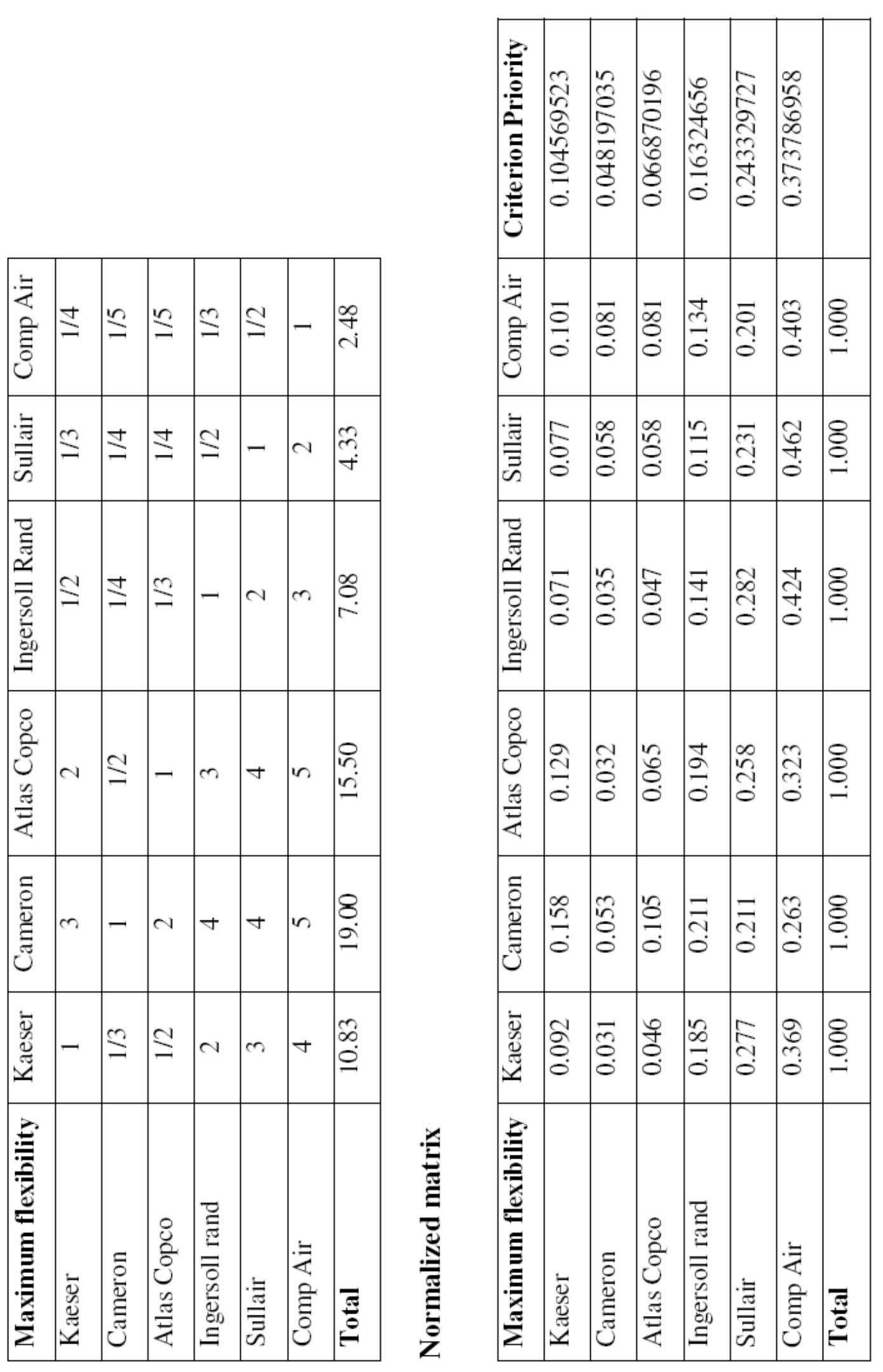

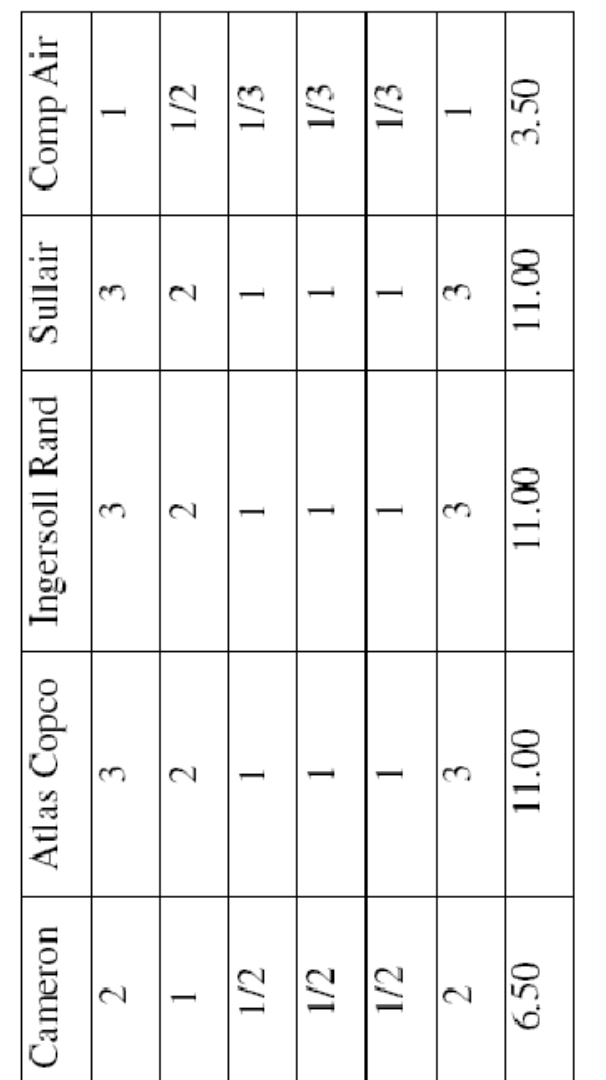

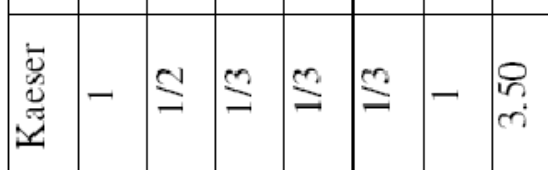

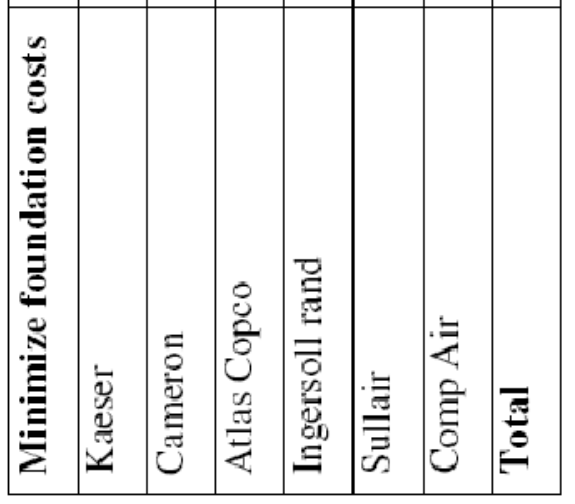

\begin{tabular}{|c|c|c|c|c|c|c|c|}
\hline 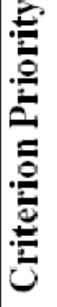 & $\begin{array}{l}\infty \\
\infty \\
2 \\
\infty \\
\infty \\
\infty \\
\infty \\
\sim \\
0 \\
0\end{array}$ & $\begin{array}{l}\frac{1}{0} \\
8 \\
0 \\
\frac{1}{0} \\
0 \\
0\end{array}$ & $\begin{array}{l}\frac{8}{0} \\
\frac{1}{8} \\
8 \\
\frac{8}{0} \\
0\end{array}$ & $\begin{array}{l}\frac{8}{0} \\
\frac{1}{8} \\
\frac{8}{8} \\
0 \\
0\end{array}$ & $\begin{array}{l}\frac{8}{0} \\
\\
\frac{1}{8} \\
0 \\
0 \\
0 \\
0\end{array}$ & $\begin{array}{l}m \\
\infty \\
\infty \\
\infty \\
\infty \\
\infty \\
\infty \\
\infty \\
0 \\
0\end{array}$ & \\
\hline 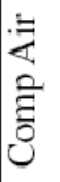 & \begin{tabular}{l}
0 \\
$\infty$ \\
\multirow{2}{0}{} \\
0
\end{tabular} & $\frac{9}{ \pm}$ & $\stackrel{2}{8}$ & 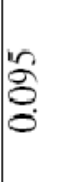 & $\frac{2}{8}$ & 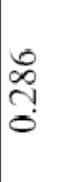 & 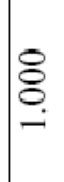 \\
\hline 言 & $\frac{\sigma}{\delta}$ & $\frac{\infty}{0}$ & 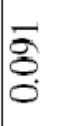 & $\overline{8}$ & $\bar{a}$ & $\frac{m}{2}$ & 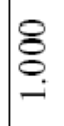 \\
\hline 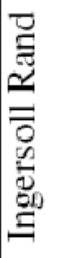 & ָㅗㅇ & $\frac{\infty}{0}$ & $\overline{8}$ & 要 & $\stackrel{\bar{g}}{\circ}$ & $\frac{\pi}{2}$ & $\underset{8}{8}$ \\
\hline 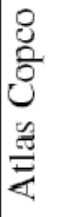 & $\stackrel{\mathrm{v}}{\mathrm{\sigma}}$ & $\frac{\infty}{\infty}$ & $\begin{array}{l}\overline{8} \\
0 \\
0\end{array}$ & $\begin{array}{l}\overline{8} \\
\dot{0}\end{array}$ & $\begin{array}{l}\overline{8} \\
0 \\
0\end{array}$ & लn & 8 \\
\hline 总 & $\stackrel{\substack{\infty \\
0}}{0}$ & $\frac{1}{\circ}$ & $\frac{\hat{\sigma}}{0}$ & $\frac{\mathbb{5}}{0}$ & $\stackrel{5}{0}$ & $\begin{array}{l}\infty \\
0 \\
? \\
0 \\
0\end{array}$ & $\underset{8}{8}$ \\
\hline 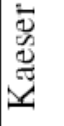 & $\begin{array}{l}\infty \\
\infty \\
\\
0 \\
0\end{array}$ & $\frac{\infty}{ \pm}$ & $\frac{1}{8}$ & $\frac{1}{8}$ & $\frac{n}{2}$ & 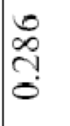 & $\stackrel{8}{8}$ \\
\hline 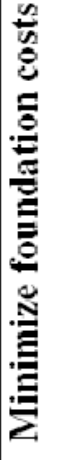 & 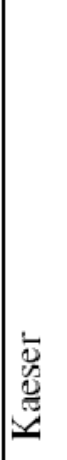 & 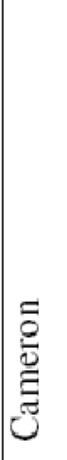 & 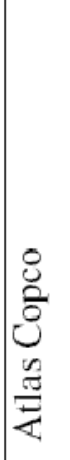 & 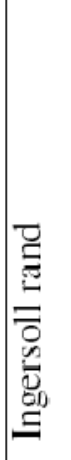 & $\begin{array}{l}\bar{\Xi} \\
\overline{\bar{E}} \\
\bar{E}\end{array}$ & $\begin{array}{l}\bar{z} \\
\bar{z} \\
\bar{\Xi} \\
ن\end{array}$ & 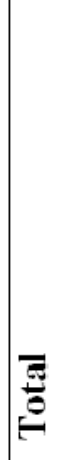 \\
\hline
\end{tabular}



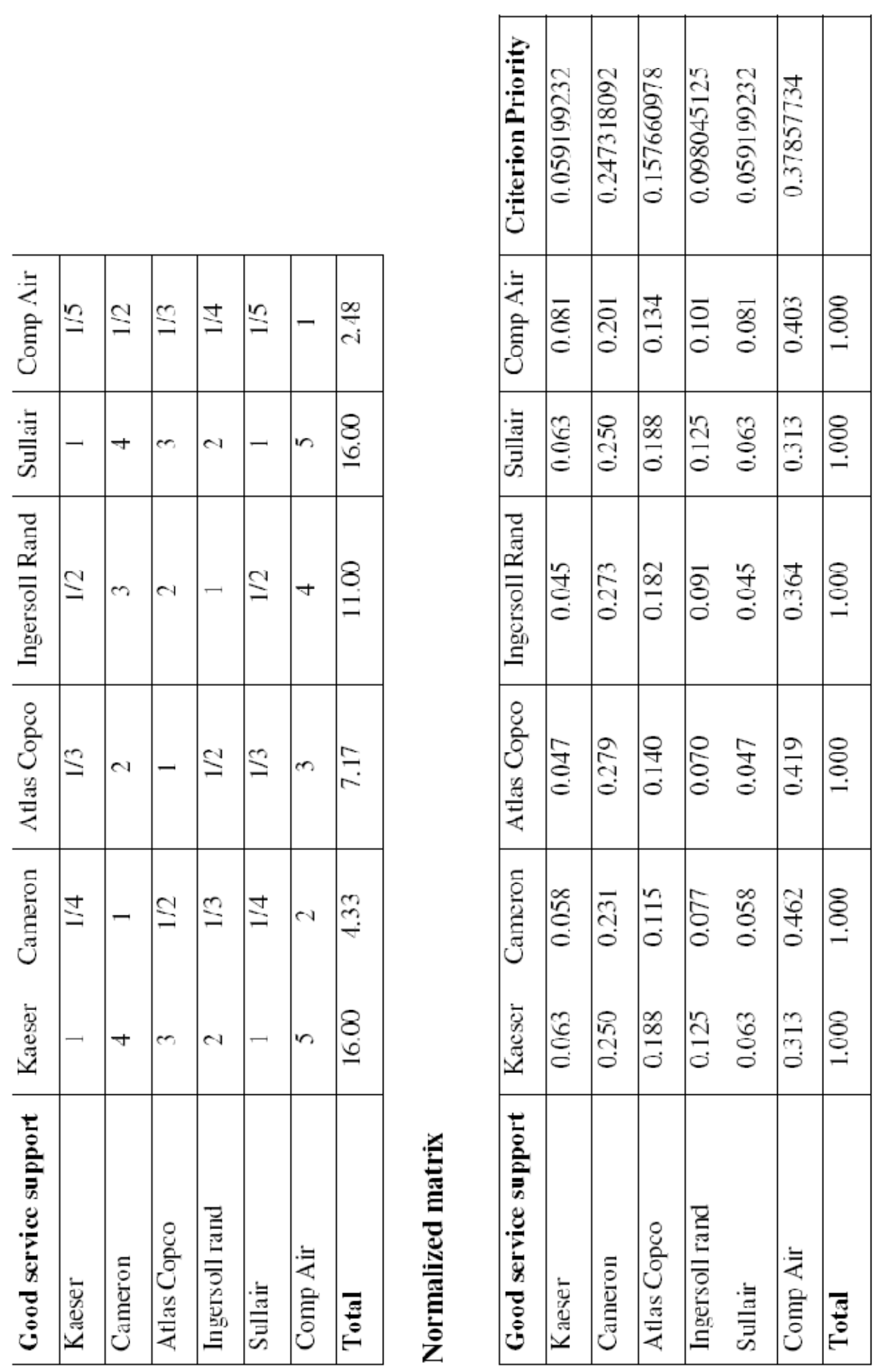


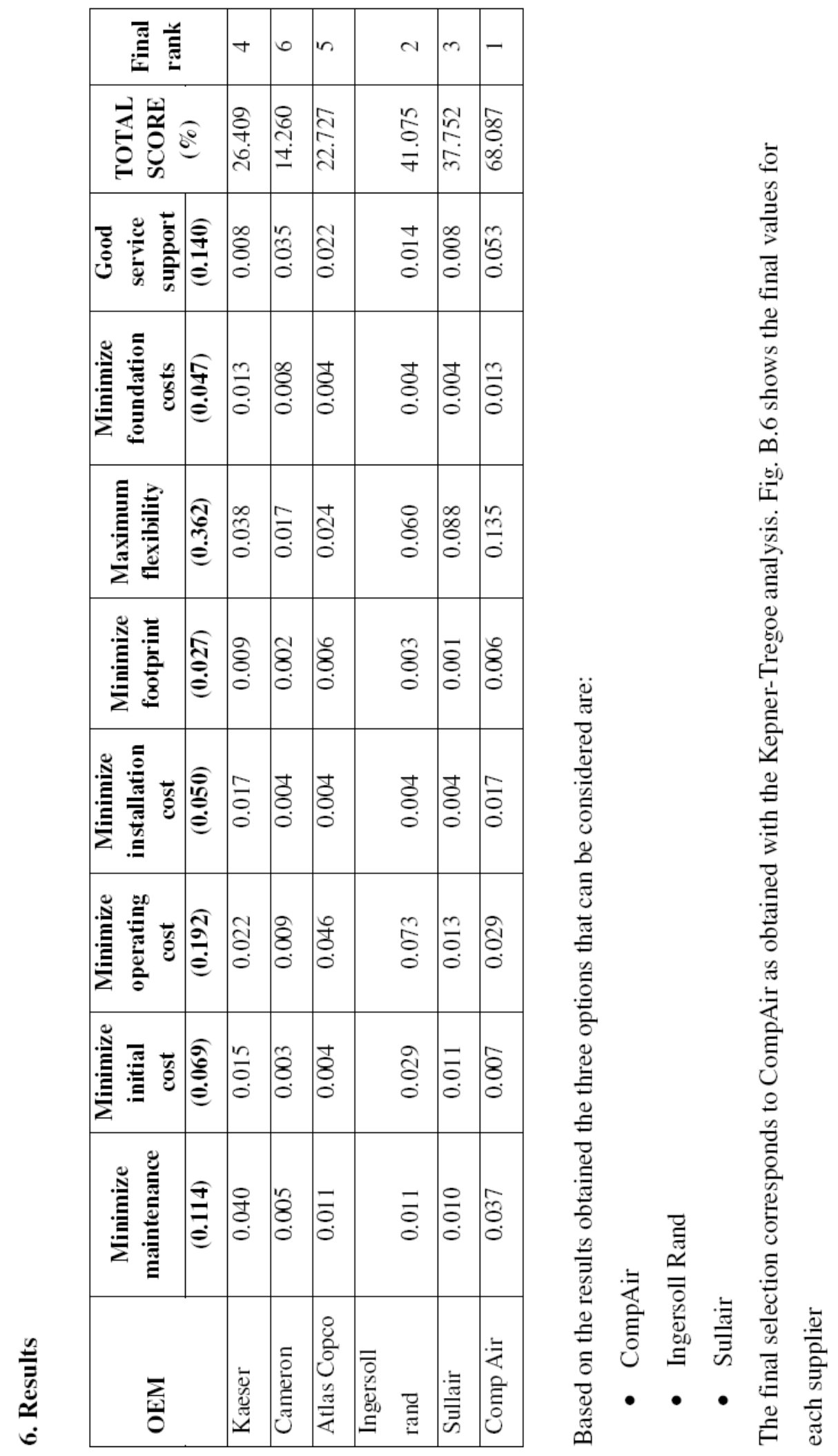




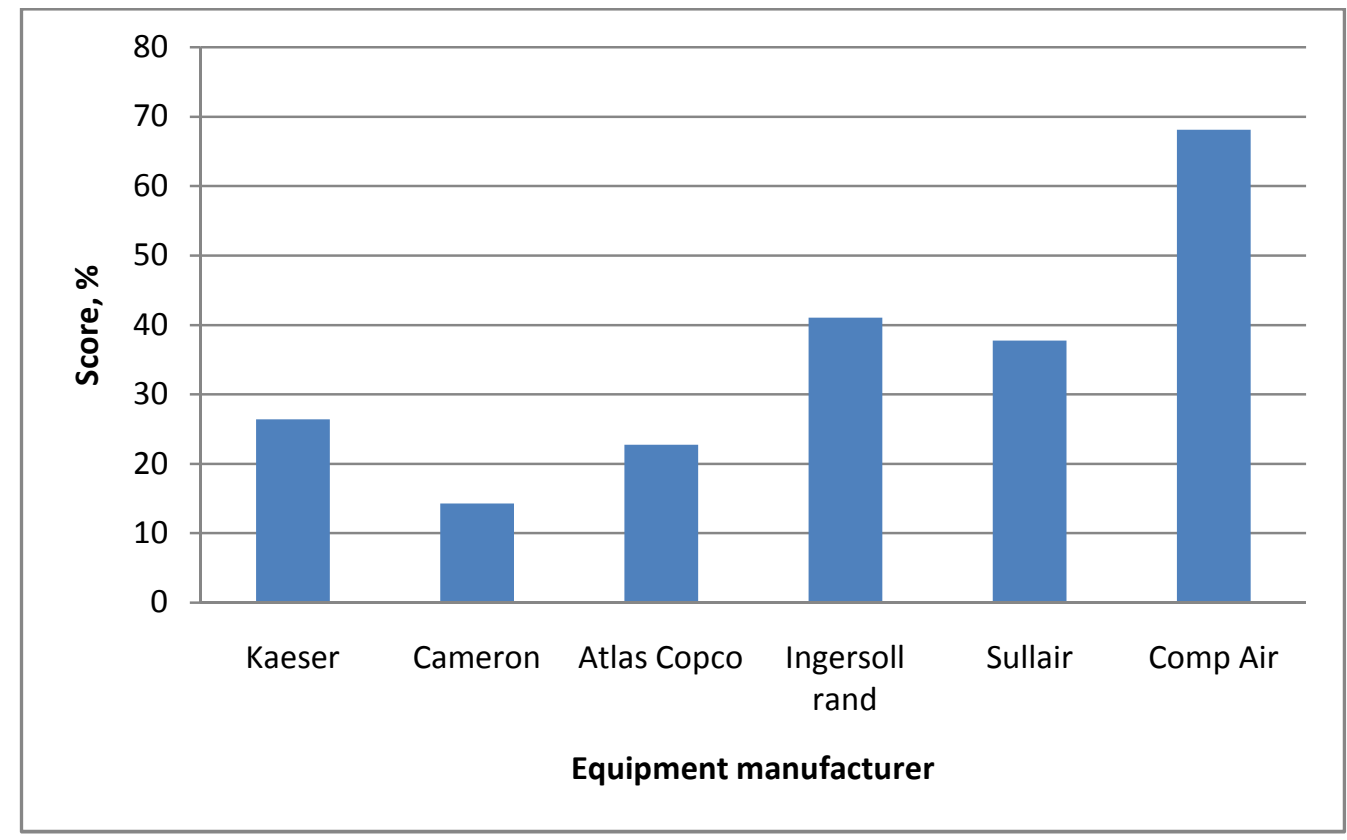

Fig. B.6- Results from the AHP 


\section{APPENDIX C}

PARAMETERS AND RESULTS FROM SENSITIVITY ANALYSIS ${ }^{17}$

\section{C.1) Design factors}

\begin{tabular}{|l|c|}
\hline Factor & Numerical Range \\
\hline Permeability, Darcy & 0.01 to 6000 \\
\hline Tubing Diameter, in & 2.72 to 4.5 \\
\hline Compressor discharge pressure, psia & 472 to 650 \\
\hline Pressure vessel radius, $\mathrm{ft}$ & 1 to 6 \\
\hline Pressure vessel height, ft & 0.7 to 3 \\
\hline $\begin{array}{l}\text { Water Gas Ratio (constant) } \\
\text { STB/SCF , 98\% GVF }\end{array}$ & 3300 \\
\hline Surface choke pressure, psia & 20 to 400 \\
\hline
\end{tabular}

\section{C.2) Fluid properties}

\begin{tabular}{|l|l|}
\hline Fluid type & Air \\
\hline Z-factor & 0.97 \\
\hline Viscosity, cp & 0.0187 \\
\hline Fluid Type & Water \\
\hline Viscosity, cp & 0.862 \\
\hline Density, Ib/ft ${ }^{3}$ & 62.2 \\
\hline Temperature, ${ }^{0} \mathrm{~F}$ & 80 \\
\hline Standard Conditions & \\
\hline Pressure, psi & 14.7 \\
\hline Temperature, ${ }^{0} \mathrm{~F}$ & 60 \\
\hline
\end{tabular}

C.3) Constraints for multiple optimizations on system variables to minimize costs

\begin{tabular}{|l|l|}
\hline Factor & Goal \\
\hline Permeability, md & 29,000 \\
\hline Tubing size, in & 3 \\
\hline Pressure, psia & 500 \\
\hline Radius, ft & Maximize \\
\hline Choke, maximize & Maximize \\
\hline Height, ft & 2 \\
\hline Production, MMscf/d & Maximize \\
\hline Cost, $\$$ & $920,808.00$ \\
\hline
\end{tabular}




\section{C.4) Responses and results options}

\begin{tabular}{|c|c|c|c|c|c|c|c|c|}
\hline Number & $\begin{array}{c}\text { Perm, } \\
\text { md }\end{array}$ & $\begin{array}{c}\text { Tubing, } \\
\text { in }\end{array}$ & $\begin{array}{c}\text { Pressure, } \\
\text { psia }\end{array}$ & $\begin{array}{c}\text { Radius, } \\
\mathbf{f t}\end{array}$ & $\begin{array}{c}\text { Choke, } \\
\text { psia }\end{array}$ & $\begin{array}{c}\text { Height } \\
\mathbf{f t}\end{array}$ & $\begin{array}{c}\text { Production } \\
\text { MMscf/day }\end{array}$ & $\begin{array}{c}\text { Cost } \\
\mathbf{\$}\end{array}$ \\
\hline 1 & $29,000.15$ & 3.00 & 500.00 & 4.82 & 100.00 & 2.00 & 0.67 & $\$ 961,465.38$ \\
\hline 2 & $29,000.14$ & 3.00 & 500.00 & 4.79 & 100.00 & 2.00 & 0.68 & $\$ 961,339.74$ \\
\hline 3 & $29,000.07$ & 3.00 & 500.00 & 4.85 & 100.00 & 2.00 & 0.67 & $\$ 961,640.31$ \\
\hline 4 & $29,000.60$ & 3.00 & 500.00 & 4.87 & 100.00 & 2.00 & 0.67 & $\$ 961,770.39$ \\
\hline 5 & $29,015.32$ & 3.00 & 500.00 & 4.78 & 100.00 & 2.00 & 0.68 & $\$ 961,279.60$ \\
\hline 6 & $29,000.36$ & 3.00 & 500.00 & 4.89 & 100.00 & 2.00 & 0.67 & $\$ 961,886.27$ \\
\hline 7 & $29,000.71$ & 3.00 & 500.00 & 4.73 & 100.00 & 2.00 & 0.68 & $\$ 960,990.77$ \\
\hline 8 & $29,000.13$ & 3.00 & 500.00 & 4.69 & 100.00 & 2.00 & 0.69 & $\$ 960,790.27$ \\
\hline 9 & $29,000.54$ & 3.00 & 500.00 & 4.65 & 100.00 & 2.00 & 0.69 & $\$ 960,538.48$ \\
\hline 10 & $29,000.10$ & 3.00 & 500.00 & 4.61 & 100.00 & 2.00 & 0.70 & $\$ 960,326.96$ \\
\hline 11 & $28,999.91$ & 3.00 & 500.00 & 4.70 & 100.00 & 2.00 & 0.69 & $\$ 960,814.20$ \\
\hline 12 & $29,283.70$ & 3.00 & 500.00 & 4.42 & 100.00 & 2.00 & 0.73 & $\$ 959,296.31$ \\
\hline 13 & $30,490.66$ & 3.00 & 500.00 & 4.60 & 100.00 & 2.00 & 0.74 & $\$ 960,326.12$ \\
\hline
\end{tabular}

C.5) PROSPER generated system plot: The plot shows that the Turner criteria has not been satisfied at the operating points indicated (0.64495 MMscf/day and $219 \mathrm{psi})$, hence revealing possible flow rates for simulating liquid loading in the proposed multiphase flow facility.

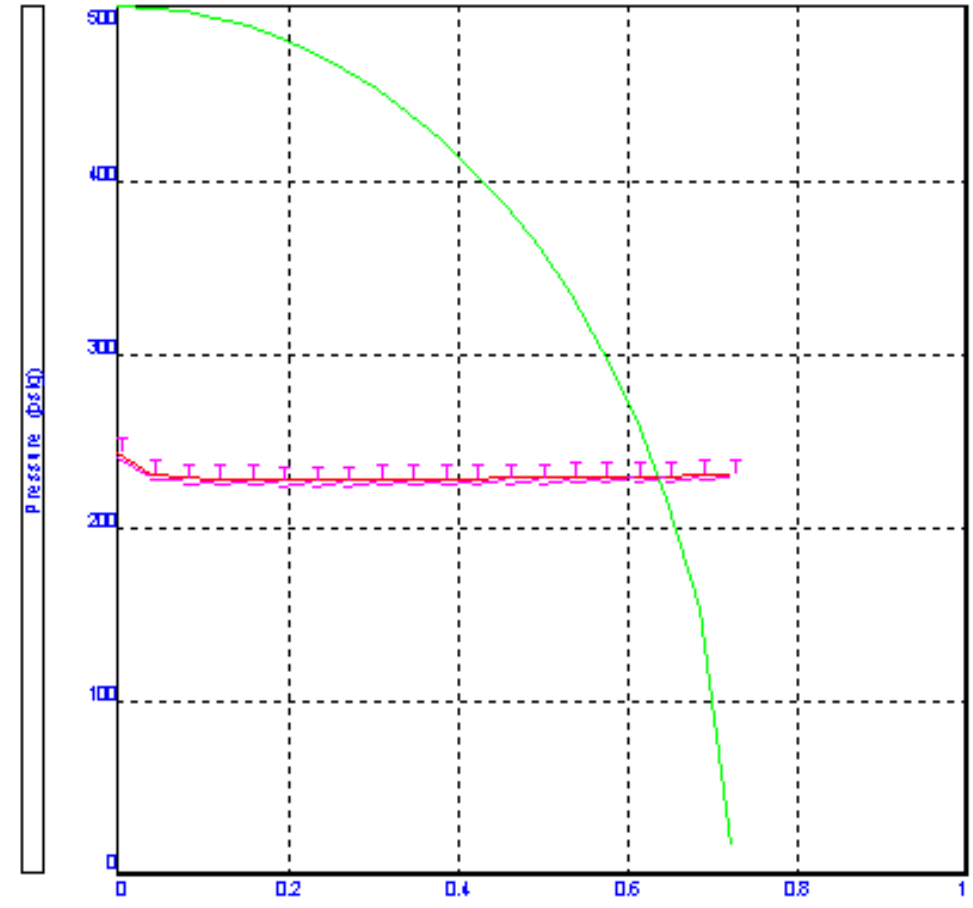




\section{C.4) Superficial velocities from PROSPER}

\begin{tabular}{|c|c|c|c|c|c|c|c|c|c|c|c|c|c|}
\hline run & $\begin{array}{l}\text { Perm, } \\
\text { md }\end{array}$ & \begin{tabular}{|c|} 
Pressure, \\
psi
\end{tabular} & $\begin{array}{c}r_{w}, \\
f t\end{array}$ & $\begin{array}{c}\text { Choke, } \\
\text { psi }\end{array}$ & Height, ft & $\begin{array}{c}\text { Tubing } \\
\text { ft }\end{array}$ & $\mathrm{C}$ & $\begin{array}{l}\text { Vsl, } \\
\mathrm{ft} / \mathrm{s}\end{array}$ & $\begin{array}{l}\mathrm{d}, \\
\text { in }\end{array}$ & $\begin{array}{l}\text { Vsg, } \\
\mathrm{ft} / \mathrm{s}\end{array}$ & $\begin{array}{l}\text { d, } \\
\text { in }\end{array}$ & $\begin{array}{c}Q_{\mathrm{G}}, \\
\mathrm{sefm}\end{array}$ & $\begin{array}{c}\mathrm{Q}_{\mathrm{L}}, \\
\mathrm{gpm}\end{array}$ \\
\hline 1 & 10000 & 400 & 2 & 200 & 5 & 0.25 & 1.726 & 2.82 & 2.887 & 10.3 & 1.565 & 415.144 & 57.539 \\
\hline 2 & 30000 & 575 & 2 & 110 & 0.5 & 0.25 & 0.518 & 1.4 & 2.865 & 8.2 & 1.028 & 202.909 & 28.123 \\
\hline 3 & 30000 & 225 & 2 & 110 & 3.5 & 0.25 & 3.624 & 3.33 & 2.898 & 19.6 & 1.628 & 493.968 & 68.464 \\
\hline 4 & 50000 & 750 & 2 & 200 & 5 & 0.25 & 8.629 & 27.64 & 2.979 & 98.5 & 1.204 & 4331.339 & 600.323 \\
\hline 5 & 30000 & 575 & 2 & 110 & 3.5 & 0.25 & 3.624 & 9.7 & 2.879 & 57 & 1.032 & 1420.365 & 196.862 \\
\hline 6 & 10000 & 400 & 2 & 20 & 5 & 0.25 & 1.726 & 3.35 & 2.845 & 55.8 & 0.722 & 478.768 & 66.357 \\
\hline 7 & 50000 & 400 & 2 & 200 & 2 & 0.25 & 3.451 & 5.54 & 2.913 & 19.76 & 1.598 & 830.289 & 115.078 \\
\hline 8 & 10000 & 750 & 2 & 20 & 2 & 0.25 & 0.69 & 2.51 & 2.847 & 41.788 & 0.532 & 359.398 & 49.812 \\
\hline 9 & 30000 & 575 & 2 & 110 & 3.5 & 0.25 & 3.624 & 9.6 & 2.894 & 56.9 & 1.033 & 1420.365 & 196.862 \\
\hline 10 & 50000 & 400 & 2 & 200 & 5 & 0.25 & 8.629 & 12.8 & 3.03 & 45.8 & 1.66 & 2075.724 & 287.695 \\
\hline 11 & 10000 & 400 & 2 & 20 & 2 & 0.25 & 0.69 & 1.3 & 2.888 & 22.5 & 0.719 & 191.507 & 26.542 \\
\hline 12 & 50000 & 750 & 2 & 20 & 2 & 0.25 & 3.451 & 12.2 & 2.888 & 204.4 & 0.538 & 1796.99 & 249.062 \\
\hline 13 & 10000 & 575 & 2 & 110 & 3.5 & 0.25 & 1.208 & 3.27 & 2.863 & 19.21 & 1.026 & 473.455 & 65.62 \\
\hline 14 & 10000 & 750 & 2 & 20 & 5 & 0.25 & 1.726 & 6.22 & 2.859 & 103.55 & 0.535 & 898.495 & 124.531 \\
\hline 15 & 30000 & 575 & 2 & 110 & 3.5 & 0.25 & 3.624 & 9.7 & 2.879 & 56.9 & 1.033 & 1420.365 & 196.862 \\
\hline 16 & 30000 & 925 & 2 & 110 & 3.5 & 0.25 & 3.624 & 15.67 & 2.889 & 92.12 & 0.82 & 2311.411 & 320.361 \\
\hline 17 & 30000 & 575 & 2 & 110 & 3.5 & 0.25 & 3.624 & 9.7 & 2.879 & 56.9 & 1.033 & 1420.365 & 196.862 \\
\hline 18 & 30000 & 575 & 2 & 110 & 3.5 & 0.25 & 3.624 & 9.7 & 2.879 & 56.9 & 1.033 & 1420.365 & 196.862 \\
\hline 19 & 10000 & 750 & 2 & 200 & 2 & 0.25 & 0.69 & 2.39 & 2.865 & 8.51 & 1.158 & 346.507 & 48.025 \\
\hline 20 & 50000 & 750 & 2 & 20 & 5 & 0.25 & 8.629 & 29.1 & 2.956 & 484.8 & 0.552 & 4492.476 & 622.657 \\
\hline 21 & 10000 & 400 & 2 & 200 & 2 & 0.25 & 0.69 & 1.13 & 2.884 & 4.03 & 1.583 & 166.057 & 23.015 \\
\hline 22 & 50000 & 400 & 2 & 20 & 5 & 0.25 & 8.629 & 15.7 & 2.938 & 261.9 & 0.745 & 2393.841 & 331.786 \\
\hline 23 & 70000 & 575 & 2 & 110 & 3.5 & 0.25 & 8.456 & 21.4 & 2.961 & 126.1 & 1.06 & 3314.185 & 459.346 \\
\hline 24 & 30000 & 575 & 2 & 290 & 3.5 & 0.25 & 3.624 & 8.3 & 2.919 & 21.19 & 1.588 & 1249.564 & 173.189 \\
\hline 25 & 30000 & 575 & 2 & 110 & 6.5 & 0.25 & 6.73 & 17.5 & 2.921 & 102.6 & 1.048 & 2637.821 & 365.602 \\
\hline 26 & 10000 & 750 & 2 & 200 & 5 & 0.25 & 1.726 & 5.9 & 2.883 & 21.2 & 1.16 & 866.267 & 120.064 \\
\hline 27 & 50000 & 400 & 2 & 20 & 2 & 0.25 & 3.451 & 6.6 & 2.866 & 110.3 & 0.726 & 957.536 & 132.714 \\
\hline 28 & 30000 & 575 & 2 & 70 & 3.5 & 0.25 & 3.624 & 9.8 & 2.88 & 80.9 & 0.871 & 1436.328 & 199.075 \\
\hline 29 & 30000 & 575 & 2 & 110 & 3.5 & 0.25 & 3.624 & 9.7 & 2.879 & 56.9 & 1.033 & 1420.365 & 196.862 \\
\hline 30 & 50000 & 750 & 2 & 200 & 2 & 0.25 & 3.451 & 11.8 & 2.883 & 49.9 & 1.07 & 1732.535 & 240.129 \\
\hline
\end{tabular}




\section{APPENDIX D}

\section{INSTALLATION AND MANUFACTURING DRAWINGS}

This appendix contains the installation and manufacturing drawings of the components of the pressure vessel hosting the artificial porous medium in the TowerLab. Below is a list that includes the description and part number of every element:

\begin{tabular}{|c|c|}
\hline$\underline{\text { Part number }}$ & $\underline{\text { Description }}$ \\
\hline DPC-INST-001 & Installation drawing \\
\hline DPC-FAB-001 & $\mathrm{Pad}$ \\
\hline DPC-FAB-002 & Cover plate \\
\hline DPC-FAB-003 & Nozzle pipe \\
\hline DPC-FAB-004 & Pipe \\
\hline DPC-FAB-005 & Nozzle pipe \\
\hline DPC-FAB-006 & Pipe \\
\hline DPC-FAB-007 & $\mathrm{Pad}$ \\
\hline DPC-FAB-008 & Pipe \\
\hline DPC-FAB-009 & $\mathrm{Pad}$ \\
\hline DPC-FAB-010 & Tubing centralizer \\
\hline DPC-FAB-011 & Plate \\
\hline DPC-FAB-012 & Bottom head \\
\hline DPC-FAB-013 & Shell \\
\hline DPC-FAB-014 & Top head \\
\hline DPC-FAB-015 & $\mathrm{Pad}$ \\
\hline DPC-FAB-016 & Pipe gauge \\
\hline DPC-FAB-017 & Nozzle \\
\hline
\end{tabular}




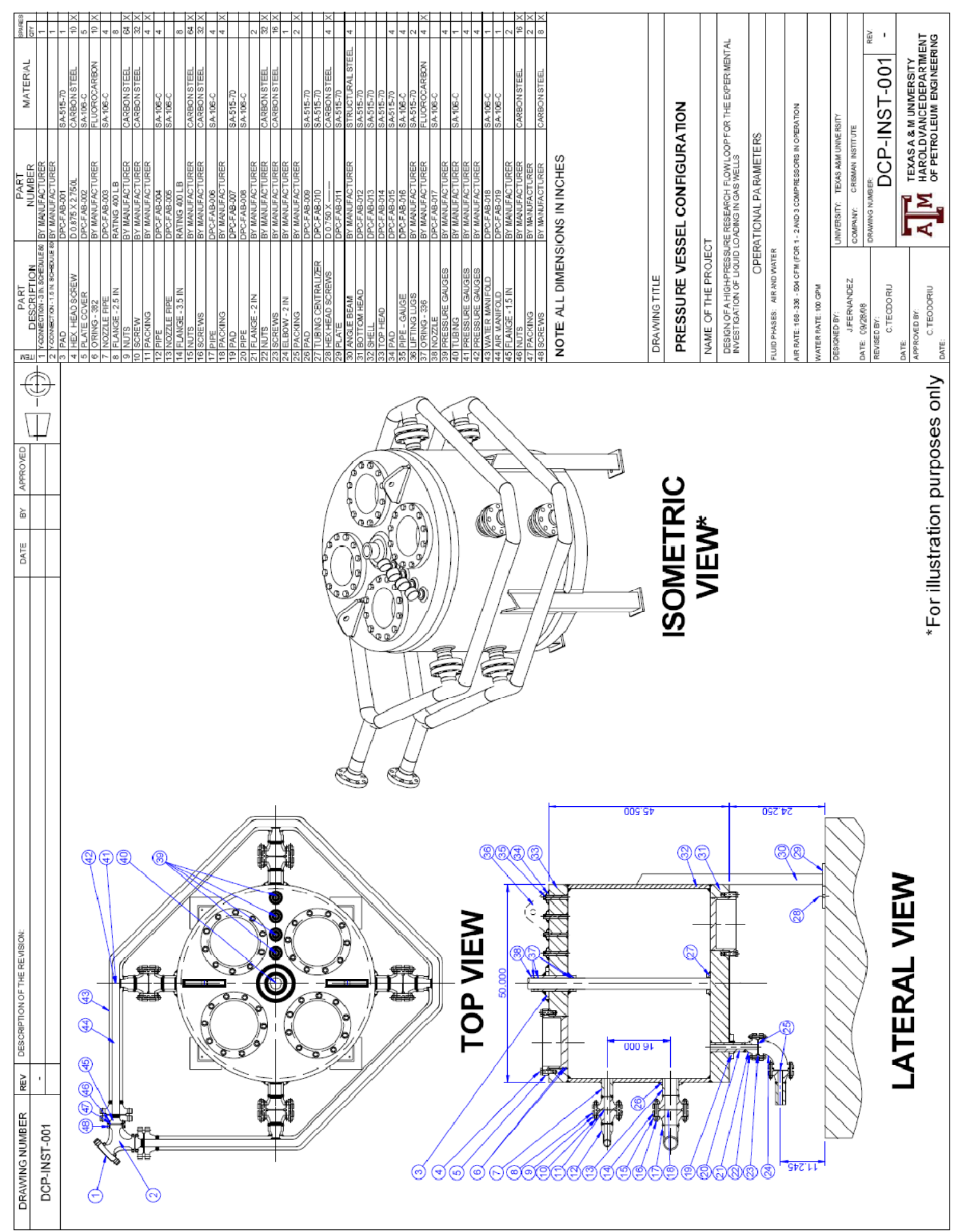




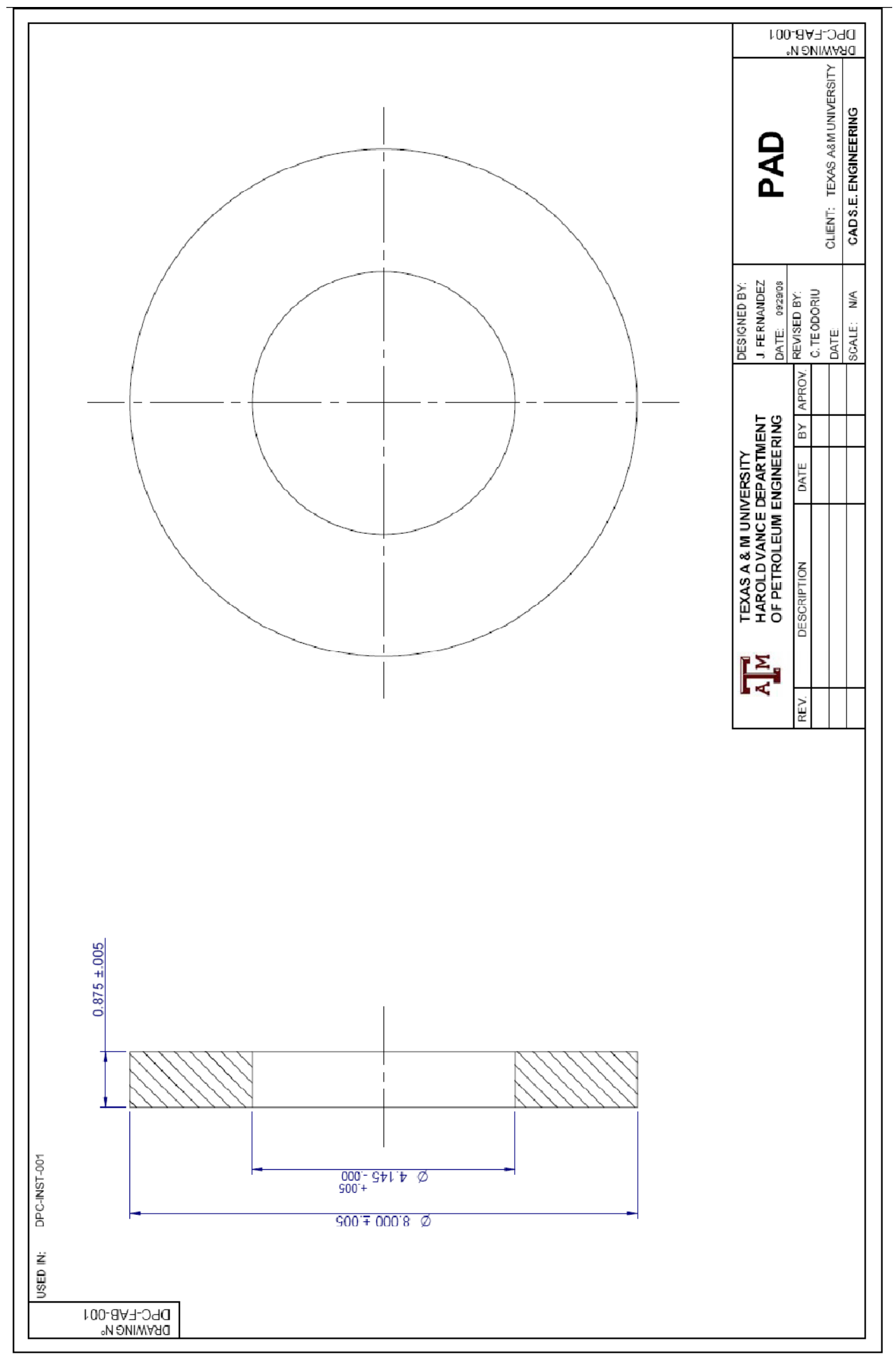




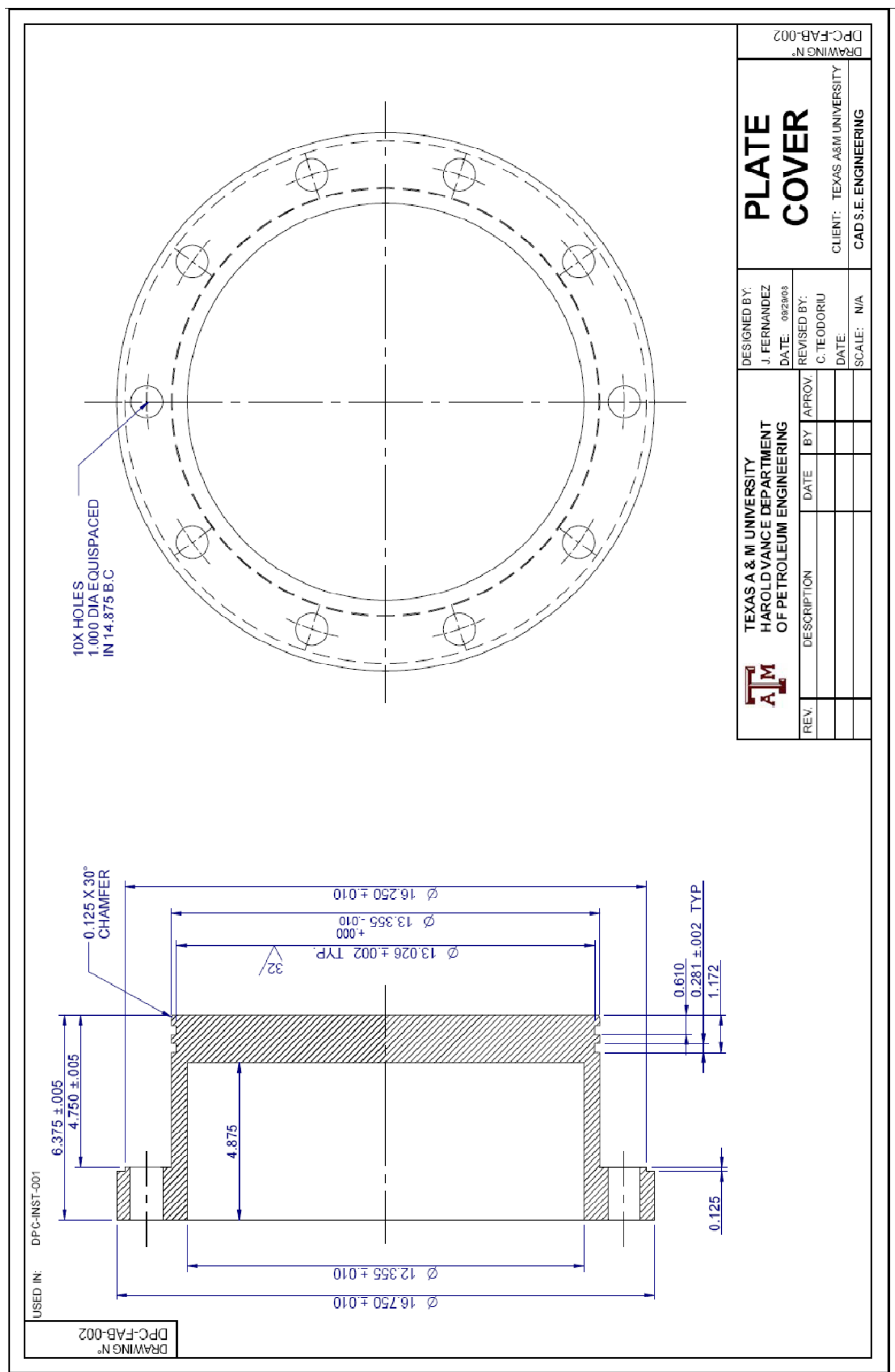




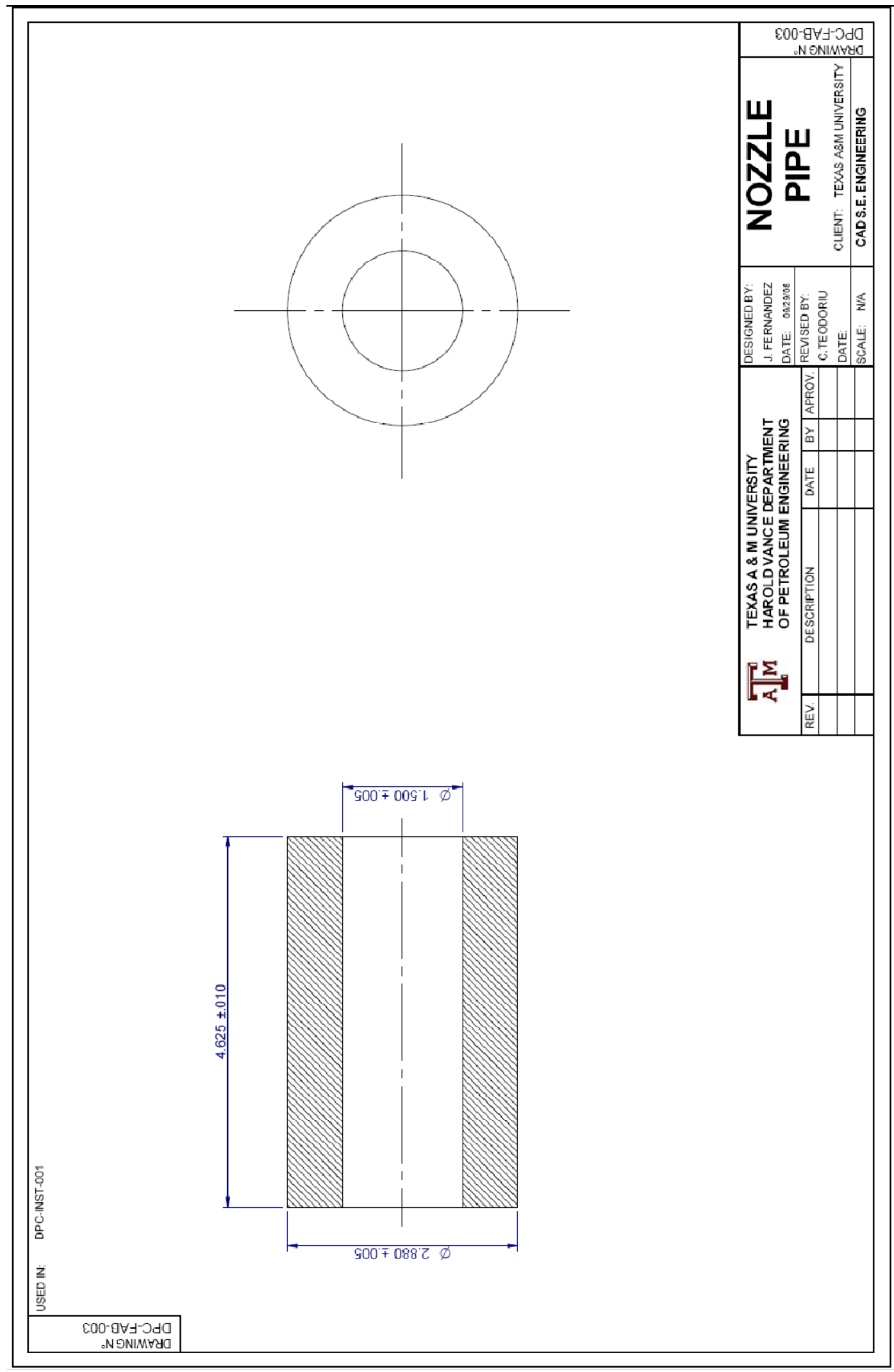




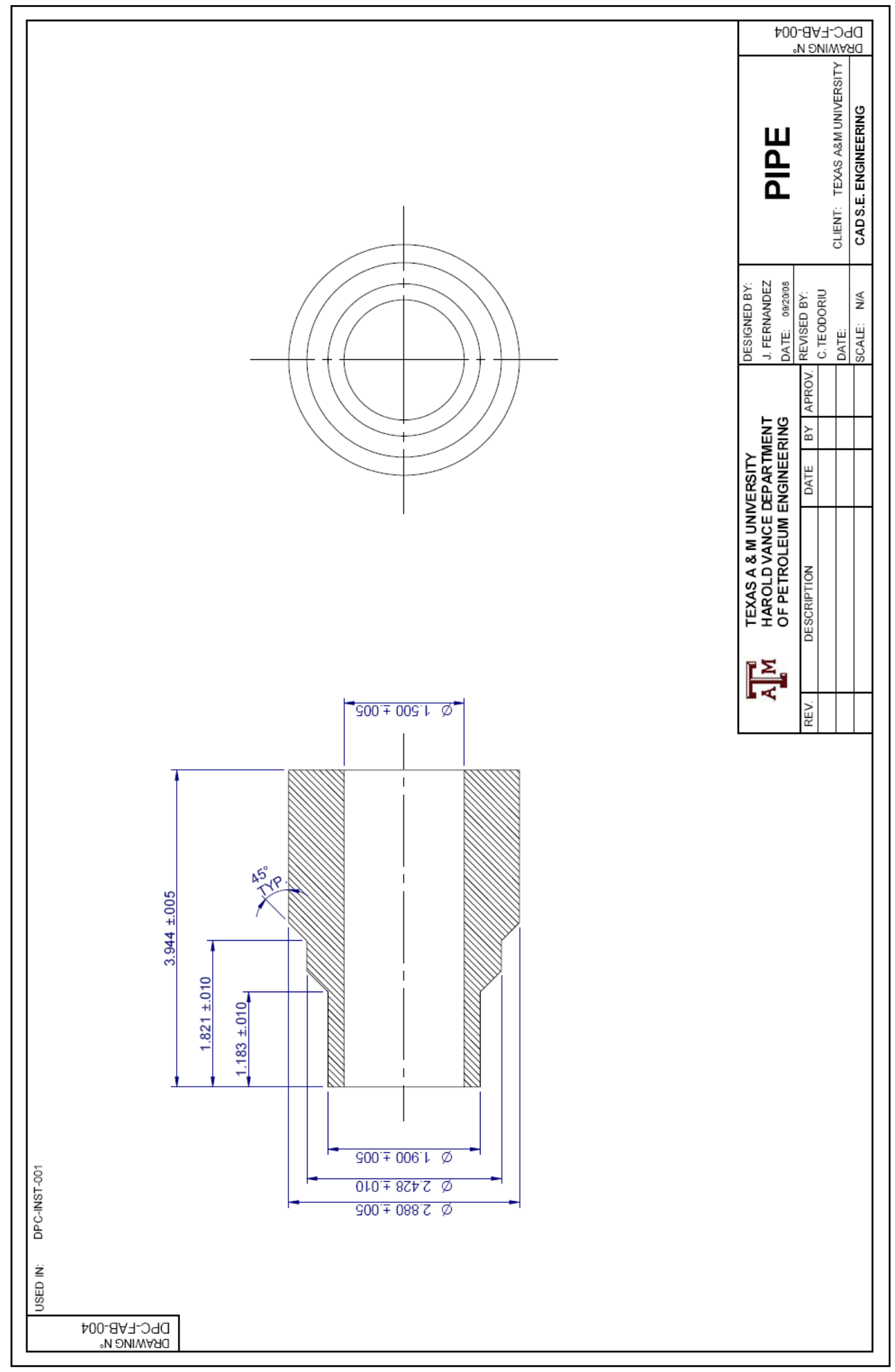




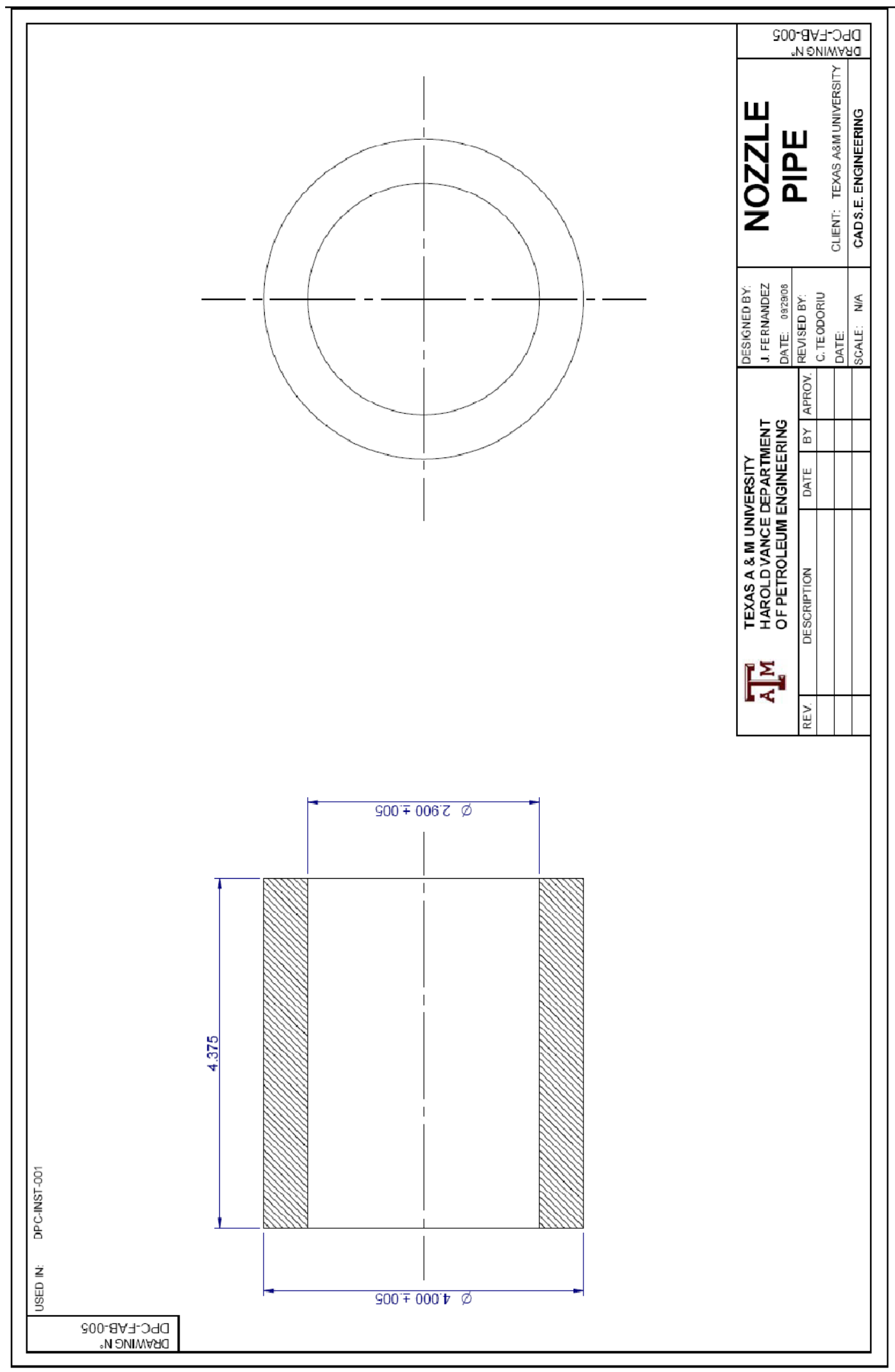




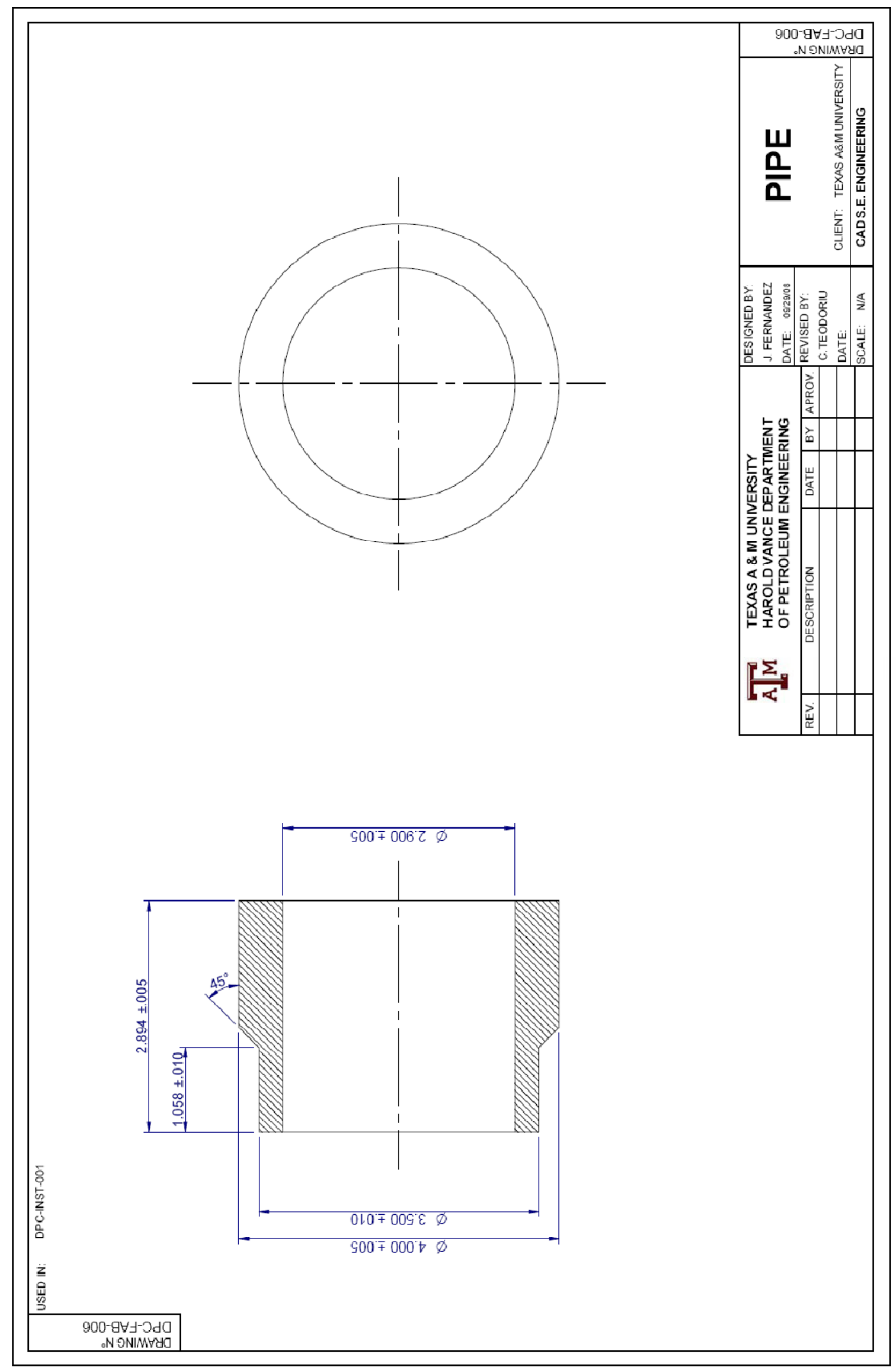




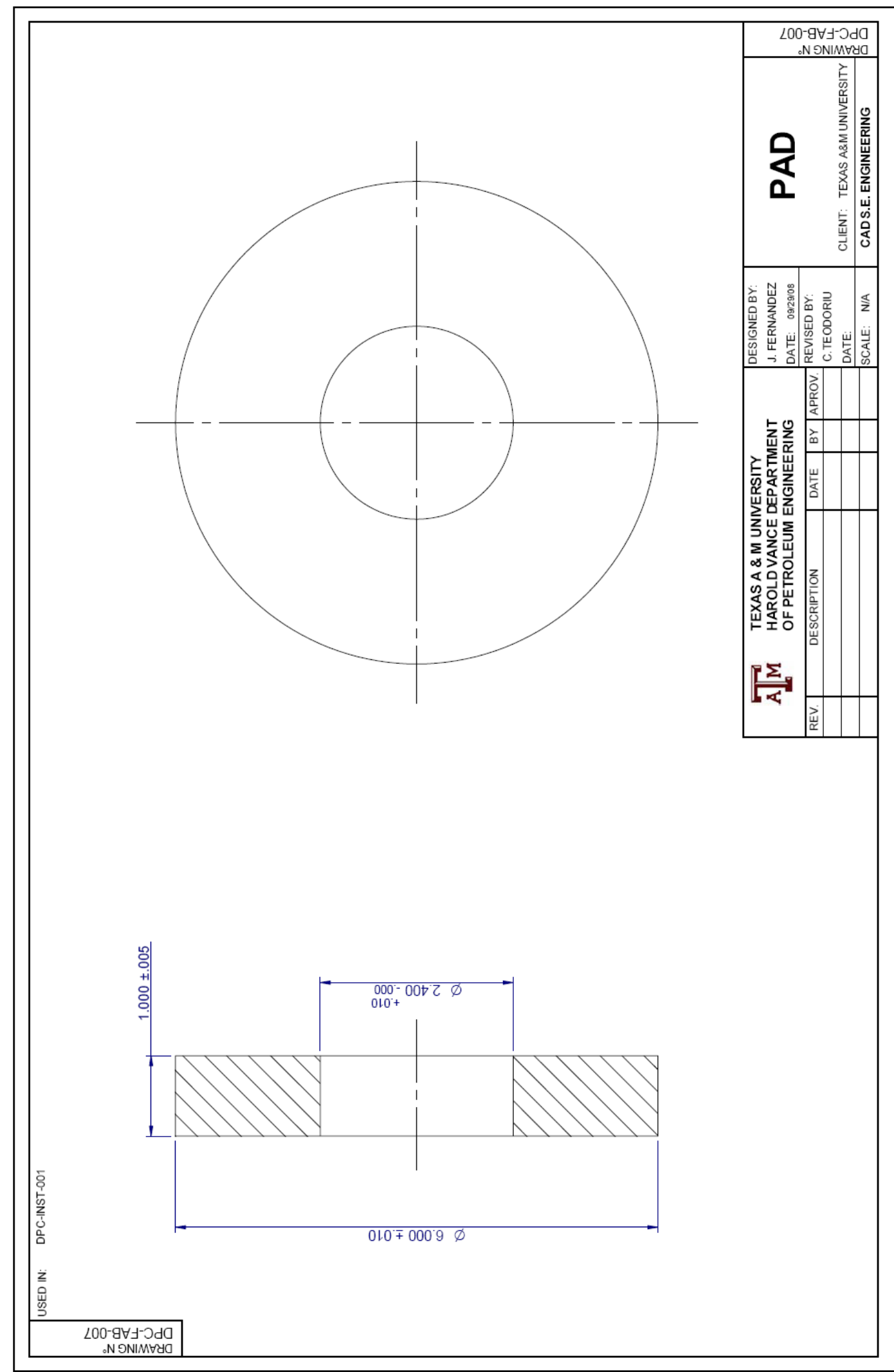




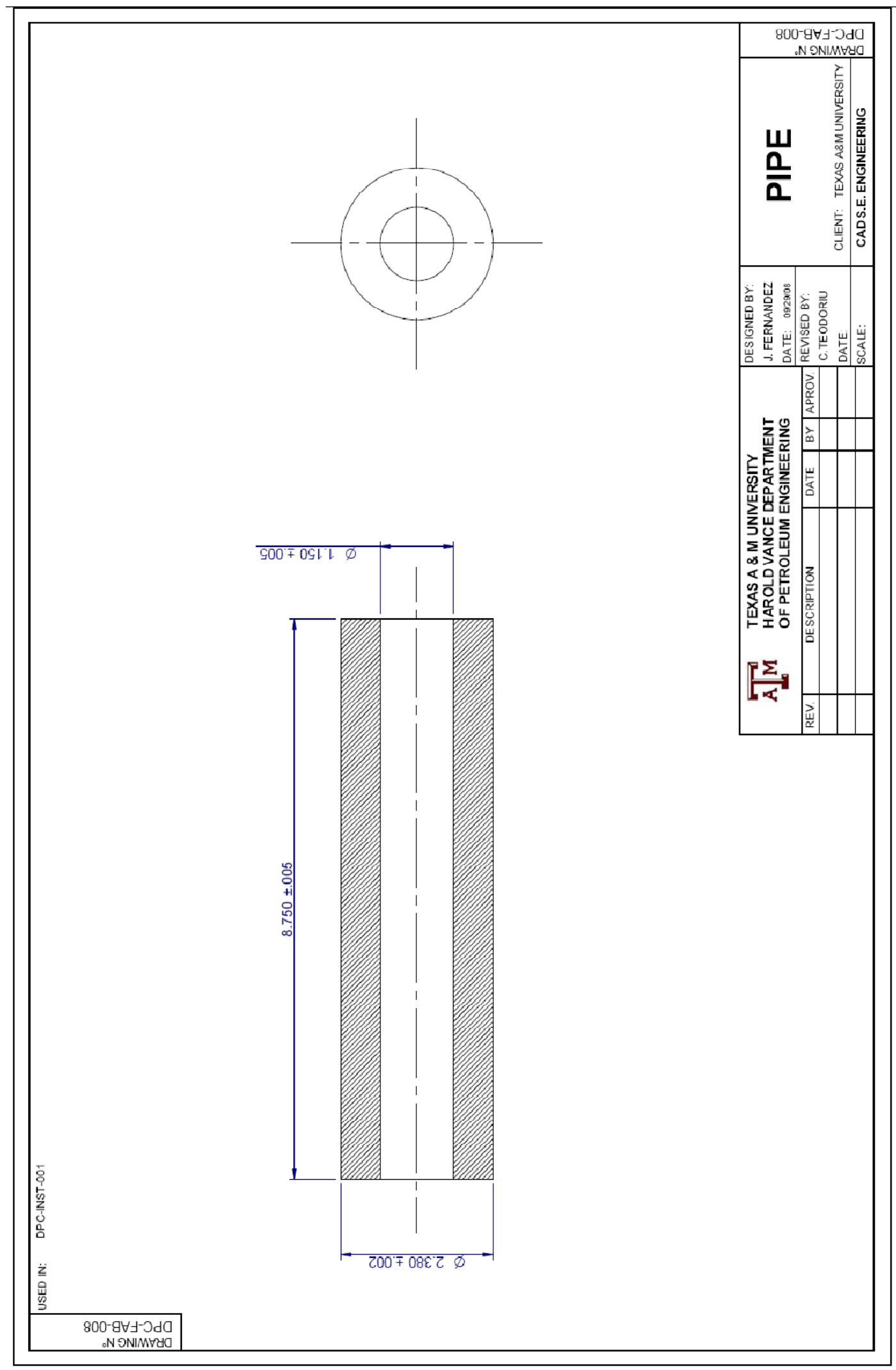




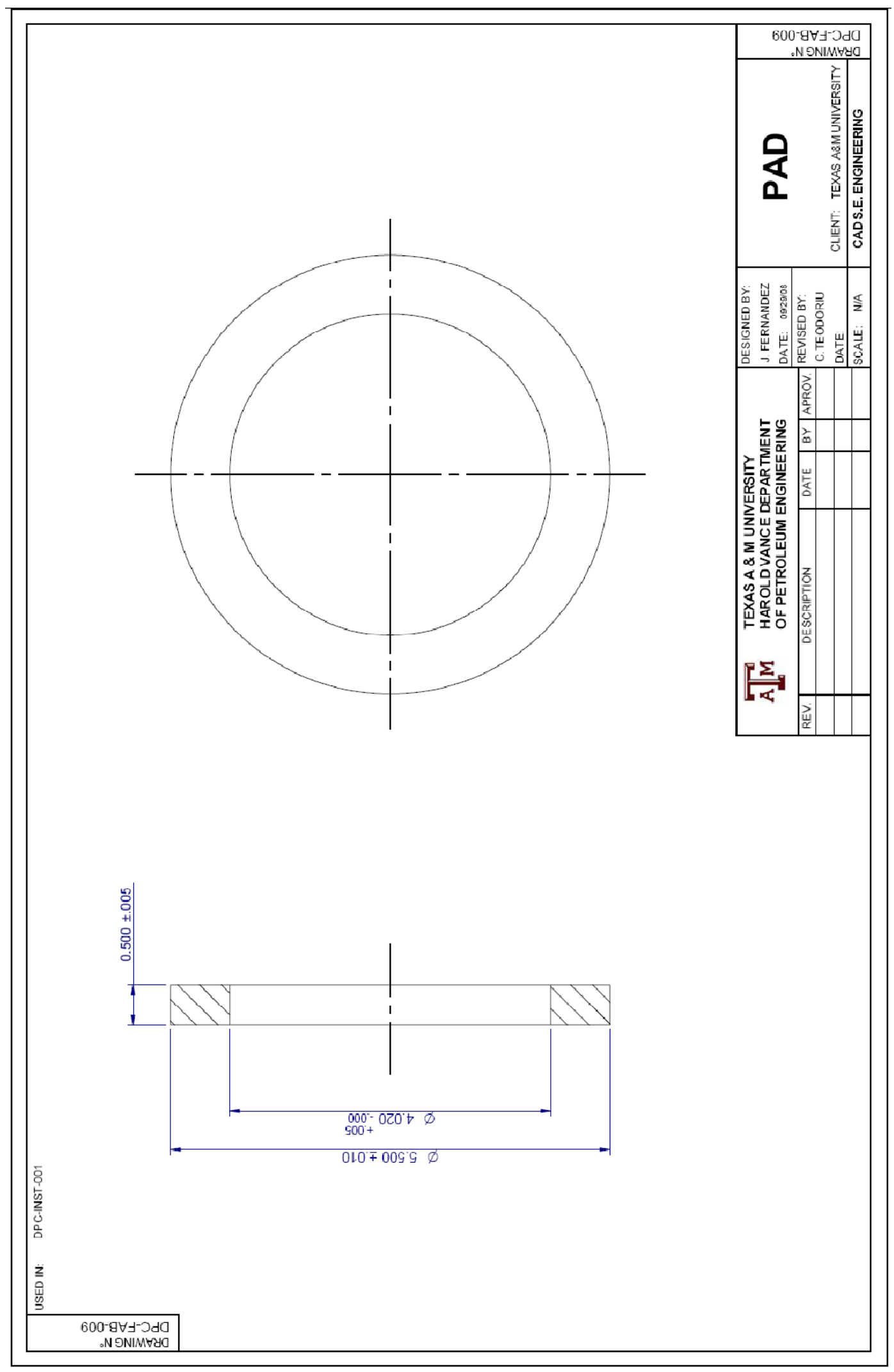




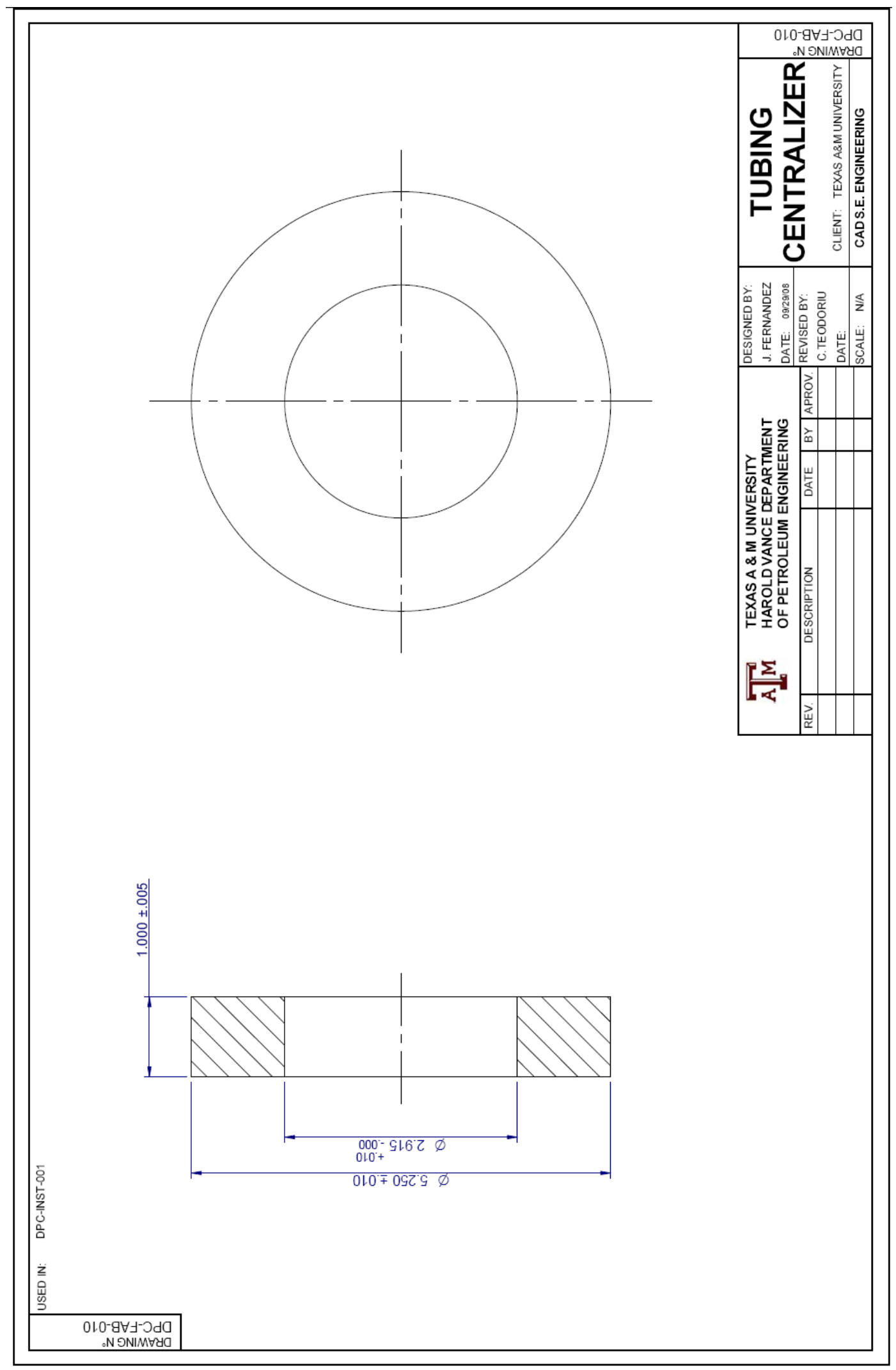




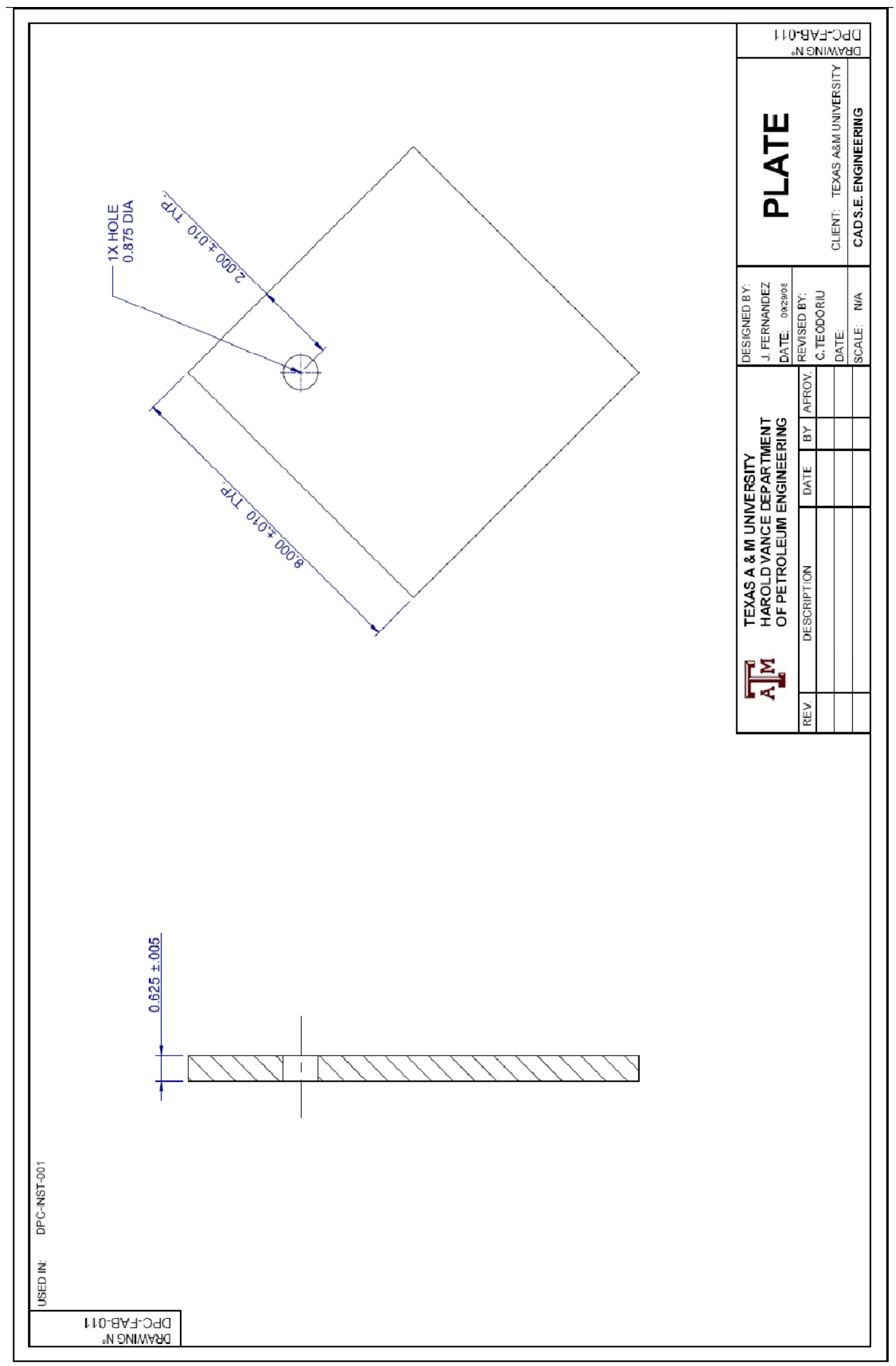




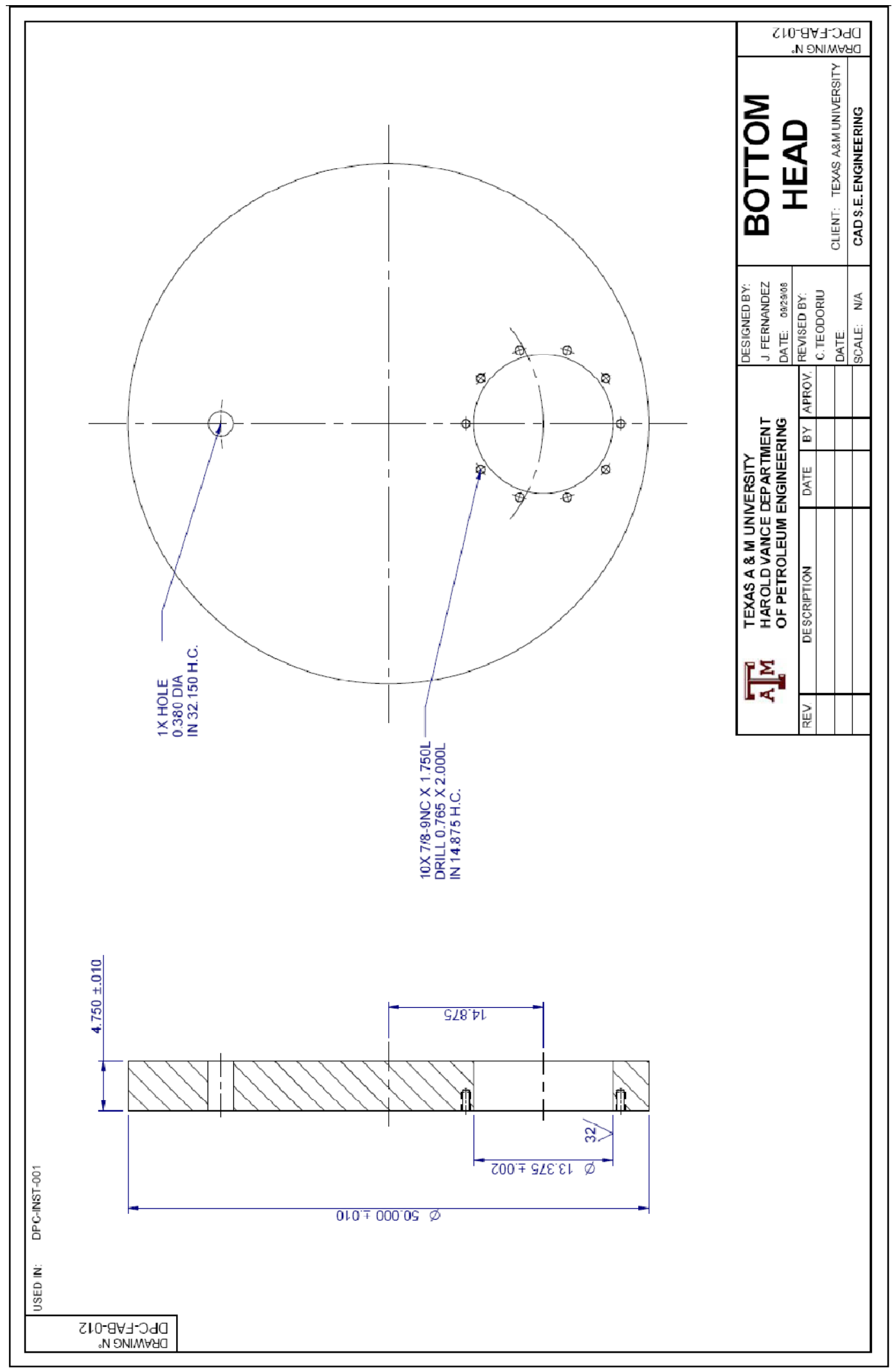




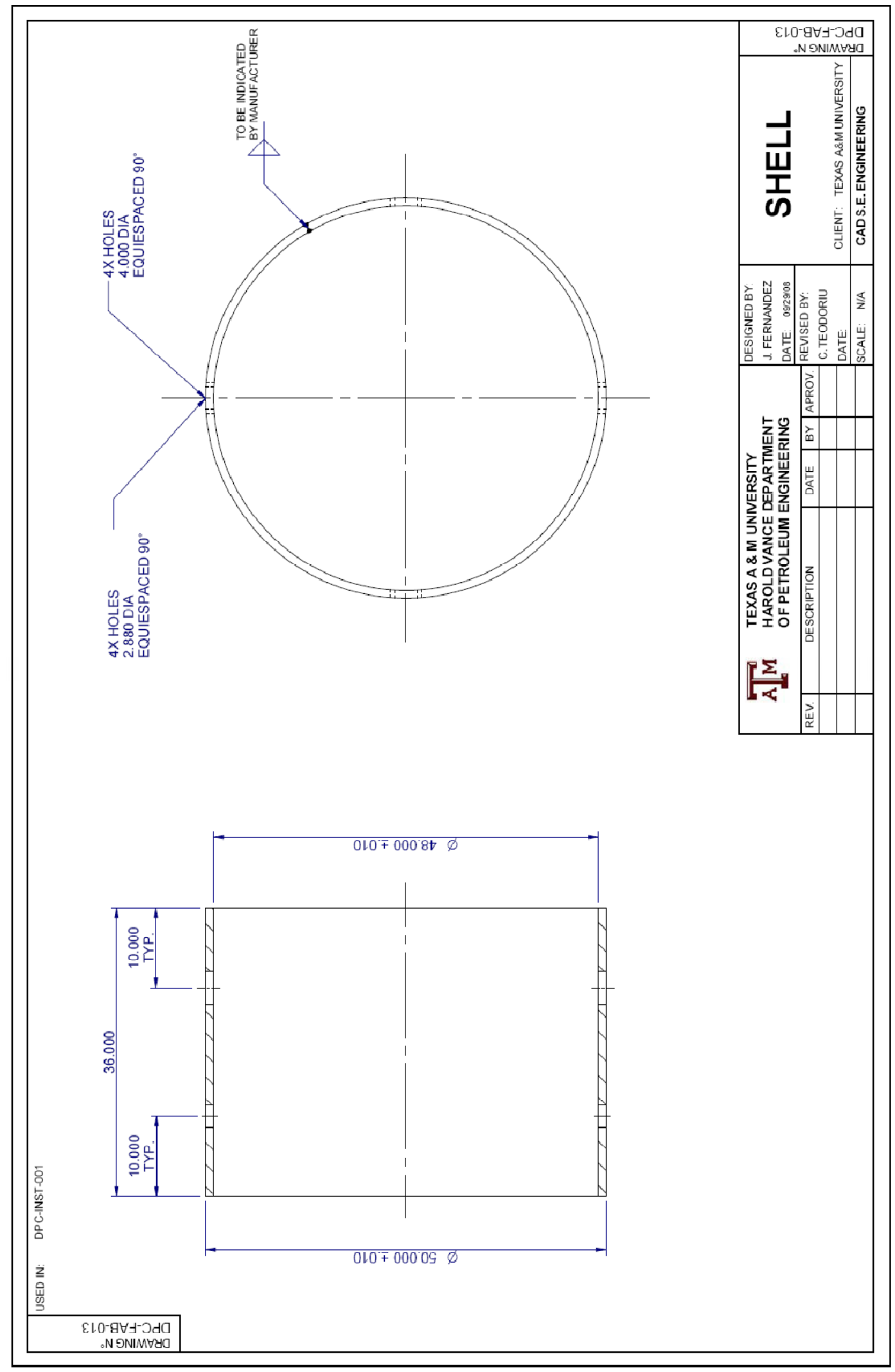




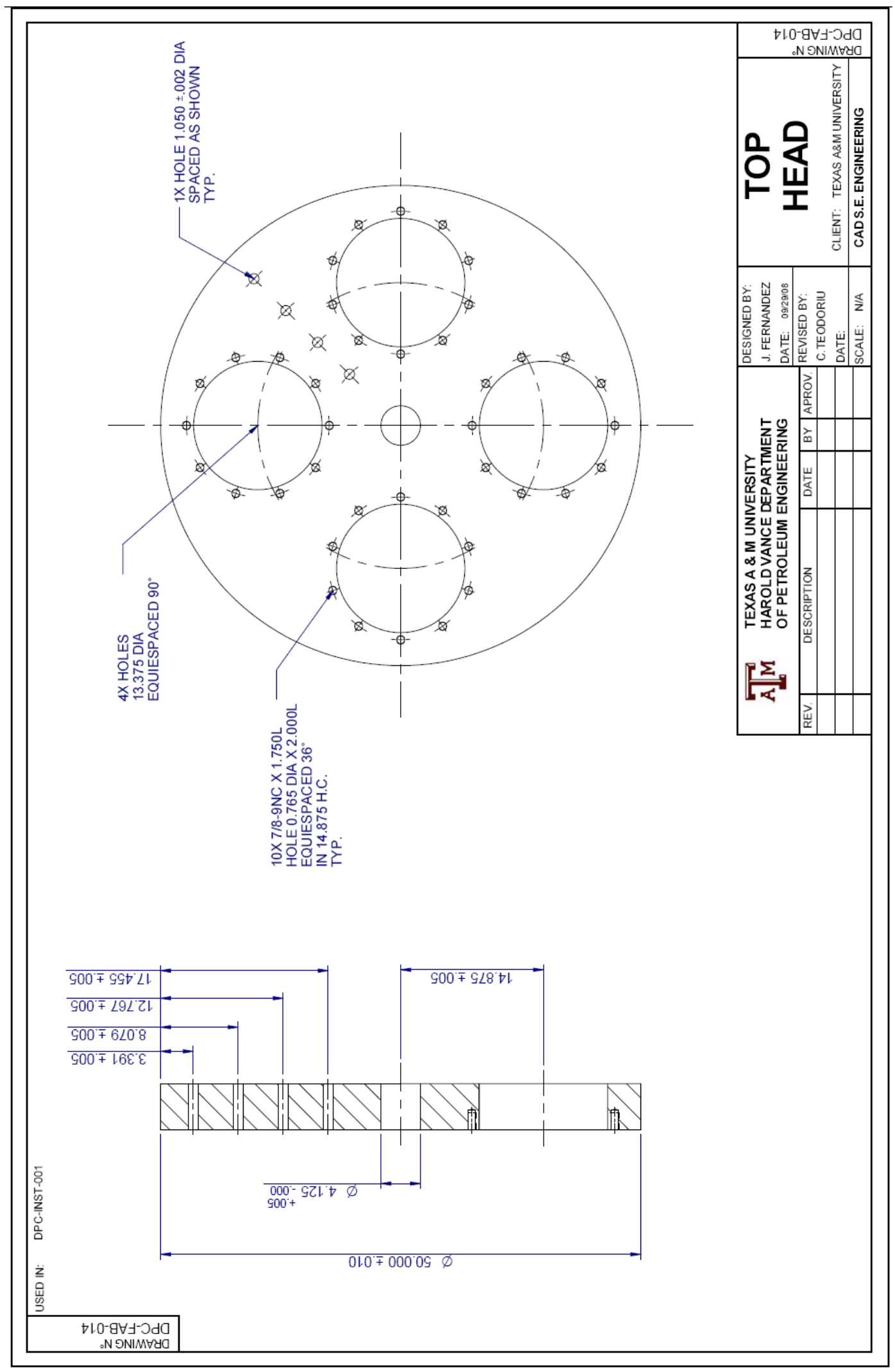




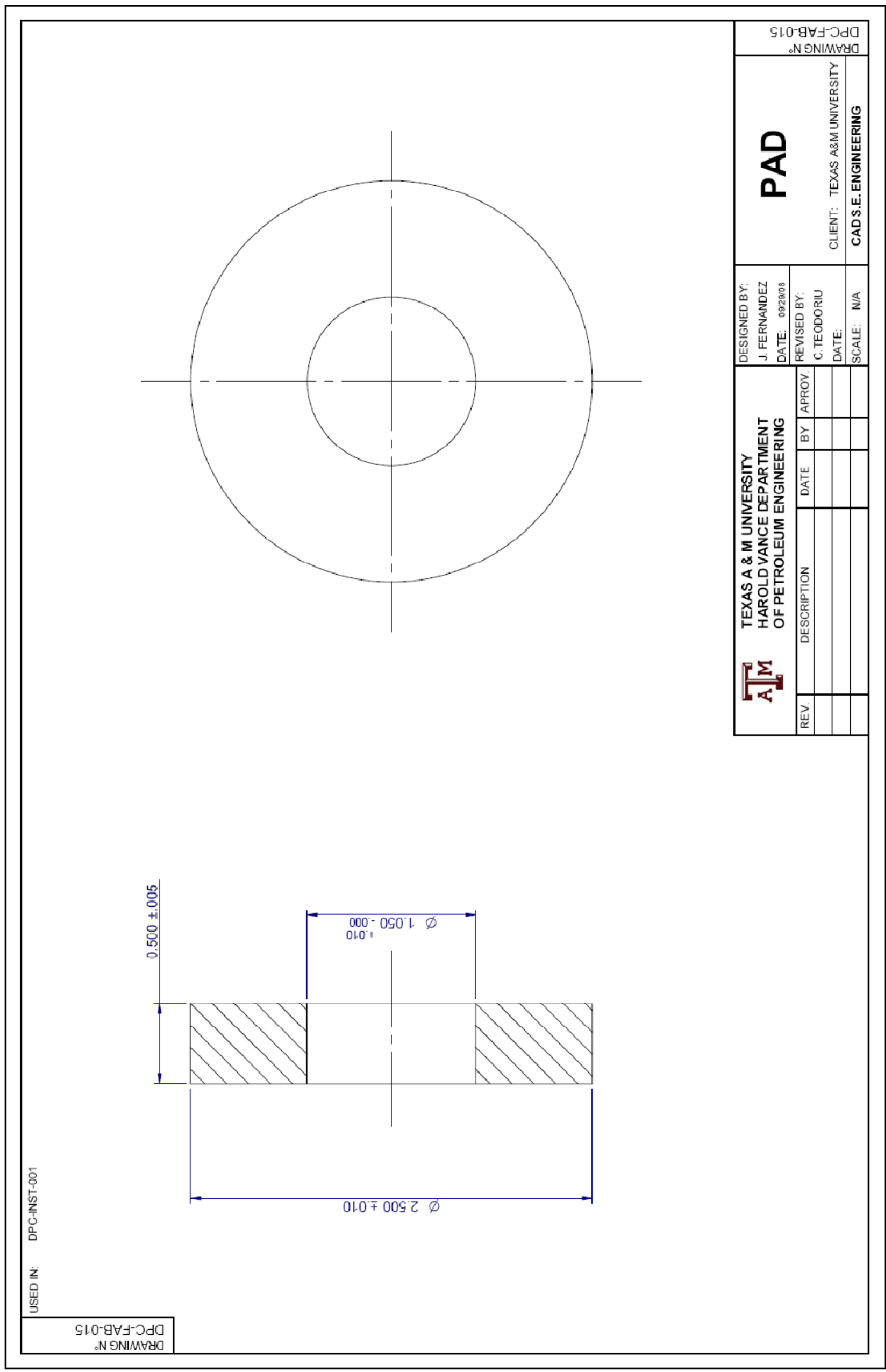




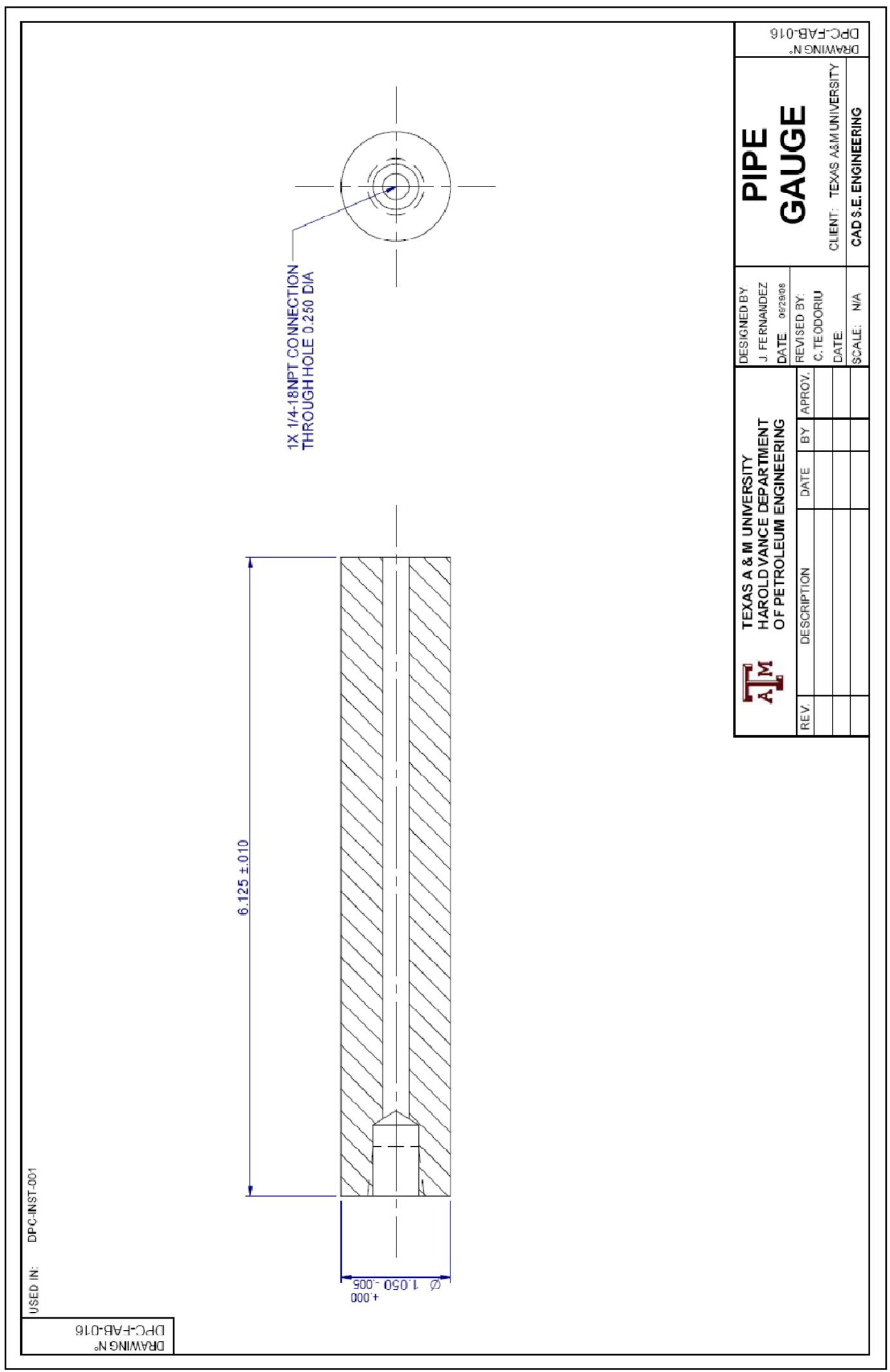




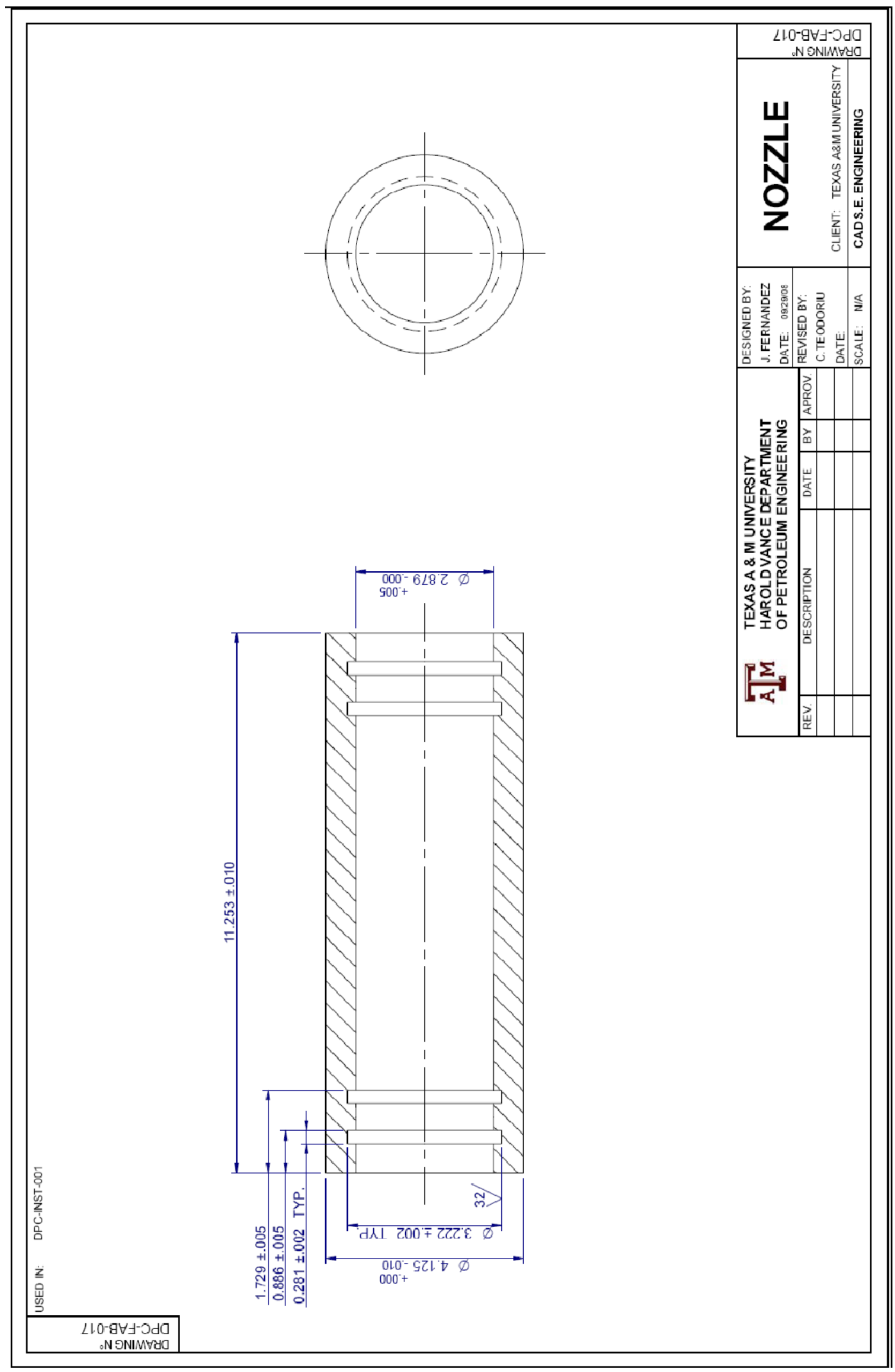




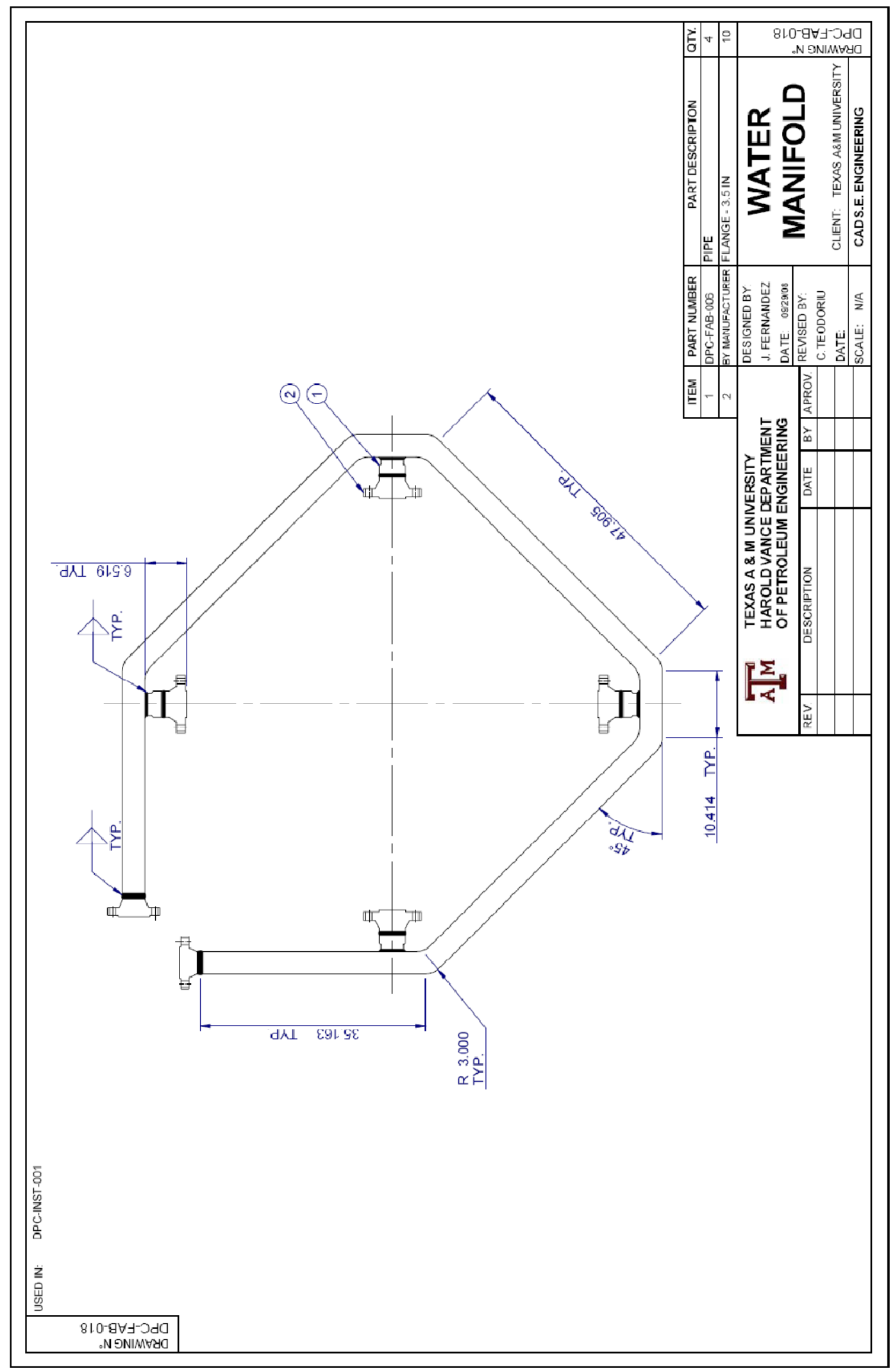




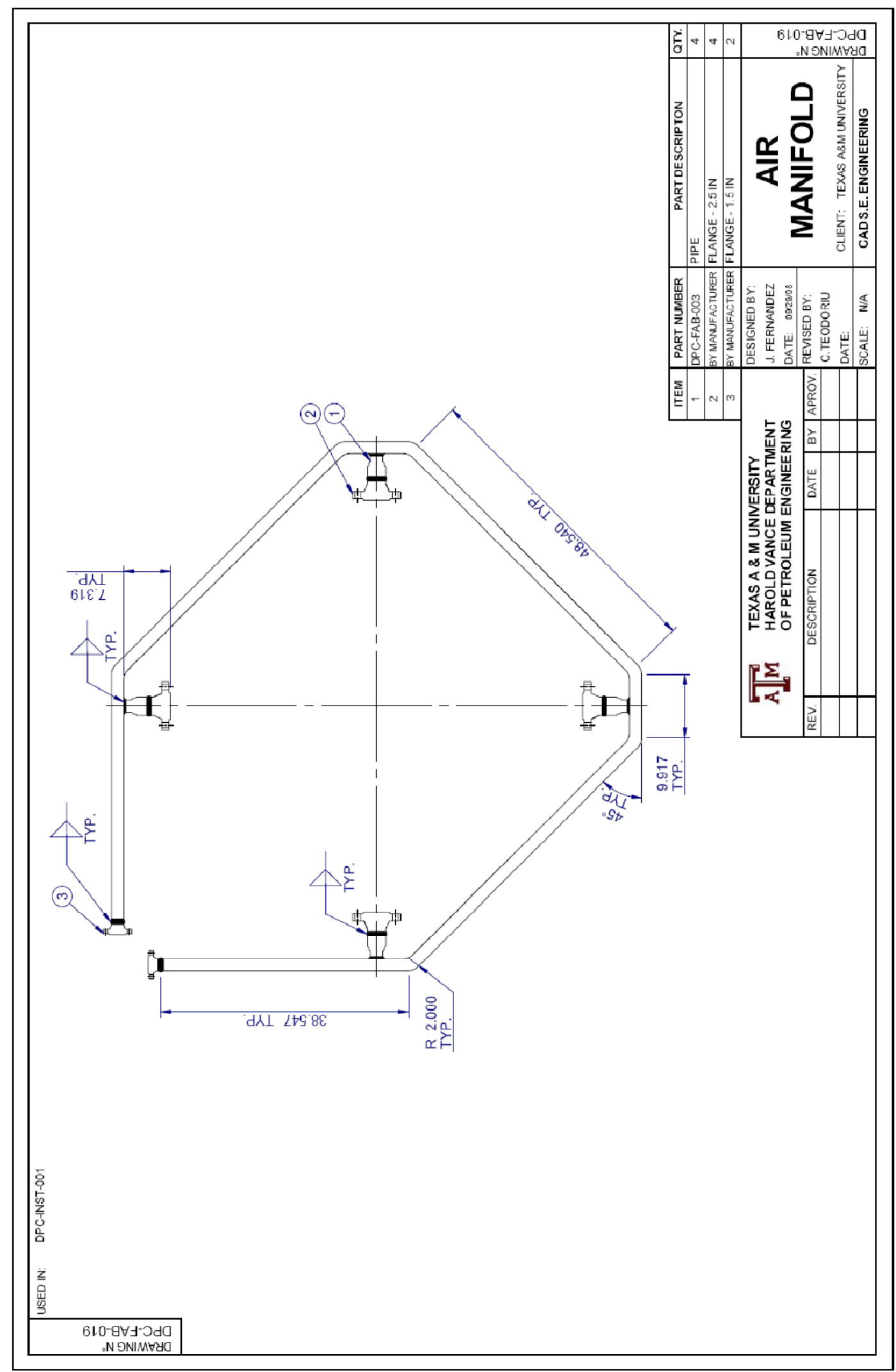




\section{VITA}

Name:

Address:

Email Address:

Education:
Juan Jose Fernandez Alvarez

3116 TAMU 602

Department of Petroleum Engineering

College Station, TX, 77843

Phone: 979-845-7081

juan.fernandez@pe.tamu.edu

M.S., Petroleum Engineering

Texas A\&M University, 2008

College Station, Texas

B.S., Mechanical Engineering

La Universidad del Zulia, 2004

Maracaibo, Venezuela 